


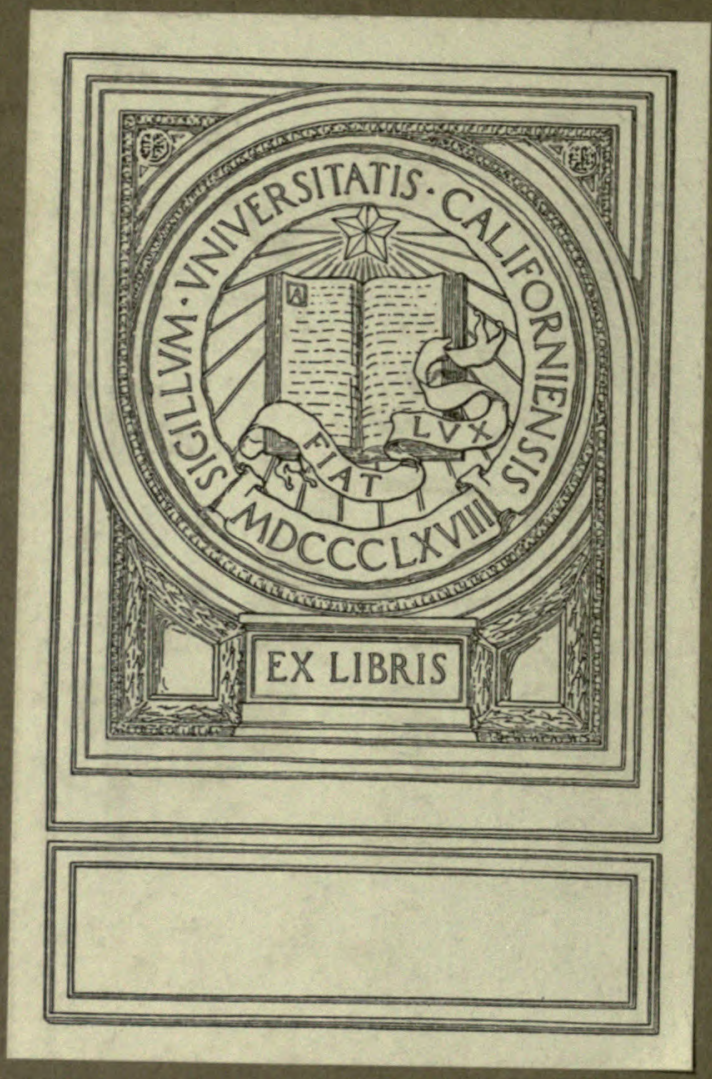




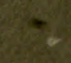

-9. (3) 

SMITHSONIAN CONTRIBUTIONS TO KNOWLEDGE PART OF VOLUME XXXIV

\title{
Thoogkíns jfund
}

\section{A CONTINUOUS RECORD OF ATMOSPHERIC NUCLEATION}

\author{
BY \\ C A R L B A R US \\ HAZARD PROFESSOR OF PHYSICS IN BROWN UNIVERSITY, PROVIDENCE, R. I.
}

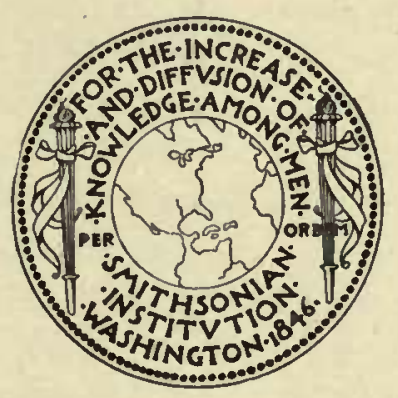

(No. 1651)

CITY OF WASHINGTON

PUBLISHED BY THE SMITHSONIAN INSTITUTION

1905 
Commission to whom this memoir has been referred:

WILDER D. BANCROFT, EDGAR F. SMITH. 


\section{ADVERTISEMENT.}

In the present memoir, entitled "A Continuous Record of Atmospheric Nucleation," the author further discusses his researches on the nucleus, as published in Experiments with Ionized Air, Smithsonian Contributions to Knowledge, vol. xxix, I9or, and in Structure of the Nucleus, issued as part of the same volume in 1903. The investigation has been carried on with the aid of a grant from the Hodgkins Fund of the Smithsonian Institution.

Doctor Barus describes the nucleus as a dust particle small enough to float in the air, but larger than the order of molecular size. Such a particle precipitates condensation in an atmosphere saturated with water vapor in its immediate vicinity. When these nuclei occur approximately of uniform size in thousands and millions, they give rise to condensational phenomena of transcendent beauty and importance. By far the greater number of nuclei are initially ionized, or at least carry electric charge.

In addition to mechanical, thermal, and chemical processes, high potential is shown to be a fruitful source of nuclei. Certain kinds of radiation, like ultraviolet light, or the $\mathrm{X}$-rays, or radioactive bodies, may also generate nuclei in the dust-free air through which the radiation passes.

The term "nucleation" is here used to denote the number of nuclei per cubic centimeter regardless of their source or special properties.

The scope of the present memoir is summarized by the author in his preface.

In accordance with the rule adopted by the Smithsonian Institution, the manuscript has been submitted for examination to a Committee consisting of Professor Wilder D. Bancroft, of Cornell University, and Professor Edgar F. Smith, of the University of Pennsylvania, and, having been recommended for publication, it is herewith presented in the series of Contributions to Knowledge.

S. P. LANGLEY, SECRETARY.

Smithsonian Institution,

Washington, May, 1905. 



\section{PREFACE.}

"What is a nucleus?" asks my friend, smiling incredulously. The conviction has become prevalent that only the ions induce condensation, and my own consistent adhesion to the nucleus since my report to the U. S. Weather Bureau (Bull. I2) in 1893 is often looked upon as heretical obstinacy. But this is quite unjust, unjust even to those who have with such brilliancy maintained the occurrence of condensation in ions. The nucleus has not left the field in discomfiture. It has merely been forced reluctantly and under conditions of extreme supersaturation to share its functions in this respect with the ubiquitous and irrepressible ion.

But to reply: The nucleus is at the outset simply a dust particle, small enough to float in the air, but much larger than the order of molecular size. Such a particle precipitates condensation in an atmosphere supersaturated with water vapor in its immediate vicinity, for the reasons long ago (1880) pointed out by Lord Kelvin in his brief but epoch-making paper. The support of this explanation was established experimentally by Coulier (I 875 ), Kiessling ( 1884 et seq.), von Helmholtz (1886, 1887), and others, and, with particular ingenuity and breadth of view, by John Aitken (1 880, particularly i 888 et seq.)

A single nucleus, however, would be of but little interest. It is when the nuclei occur approximately of uniform size in thousands and millions that they give rise to condensational phenomena of transcendent beauty and importance. To produce these legions of nuclei is not impossible by rechanical means, just as we can, for instance, triturate a solid to a remarkable degree of fineness; but the impalpable powders are perhaps best produced by chemical or at least by very refined physical processes. Similarly, though a class of interesting nuclei may be produced by vigorously shaking liquids, or, better, by mutually impinging jets or by jets impinging on a solid obstacle, nuclei are more abundantly produced by ignition or combustion. Such ignition, moreover, should be unaccompanied by any kind of smoke, as the gross particles in this case are an efficient means of absorbing nuclei. A clear non-luminous bunsen flame, a red-hot metal or any other solid, like glass, for instance, is a powerful nucleator. It is not even necessary that the solid be red-hot. Phosphorus is subject to a peculiar kind of chemical reaction, whereby nuclei are produced at $13^{\circ}$ or a little below, and are then produced from the smokeless body in maximum 
abundance. Fuming phosphorus is a relatively weaker nucleator. Many gaseous sulphides, on mixture with air, become good nucleators. Even dust-free coal gas and dust-free air, on commingling, set free nuclei.

Certain hygroscopic liquids like concentrated sulphuric acid are remarkable nucleators. They probably make up a class by themselves. At least it is not improbable that 1,000 or $1,000,000$ molecules per cubic centimeter may escape from such a body by ordinary evaporation, in spite of the low vapor pressure. Since each such molecule is hygroscopic, stable nuclei may be formed in a saturated atmosphere by condensation of the water vapor. Sulphide and sulphur nuclei are in turn probably oxydized to sulphuric acid.

In addition to mechanical, thermal, and chemical processes, high potential is a fruitful source of nuclei. A metal highly charged with electricity, or even a glass insulator, or the nodal points in the metallic pathway of a stationary electric wave are a source of nuclei. There is probably always an electric glow present in such cases, though there need be no spark.

Finally (and here we reach debatable ground), certain kinds of radiation, like ultra-violet light, or the $\mathrm{X}$-rays, or radioactive bodies, generate nuclei in the dust-free air through which the radiation passes.

Air originally made quite dust-free by filtration or otherwise, if exposed to any of these sources becomes more or less filled with a freight of nuclei, fleeting or persistent, and we may for brevity introduce the term nucleation to denote the number of nuclei per cubic centimeter, regardless of kind or origin or other properties possessed, and considered solely with respect to their tendency to promote the condensation of water vapor in supersaturated moist air. If the supersaturation is sufficiently pronounced the air molecules in successively greater numbers as the supersaturation increases must themselves become nuclei, probably beginning with the more complex systems. This, for instance, occurs in the blues, opaques, and the succeeding browns and yellows of the first order of the axial colors of the steam jet. The importance of experiments in the spontaneous condensation of dust-free moist air was pointed out in my report to the Weather Bureau in 1893, p. 48 et seq.; they were first carried out in an independent manner and with exquisite finish by C. T. R. Wilson (I897).

The nucleus as an inert excessively small body, just transcending the order of molecular dimensions, and occurring in immense numbers, has an interest of its own; but this interest becomes much enhanced when it is found that by far the greater number of nuclei are initially ionized, or at least carry electric charge. The cases in which this does not occur are sufficiently exceptional to prove the rule, though such nuclei need not for this reason be less efficient. They probably admit of a categorical classification, such as has been suggested above for concentrated sulphuric acid and sulphides. Apart from these, all nuclei produced by ignition, by high potential, by the X-rays, or by radiation are powerfully ionized. So marked is the quality that certain investigators (in particular the younger von Helmholtz, r887) have endeavored to find in the ionization a sufficient cause for the condensation of supersaturated moist air, or at least an 
additional tendency to promote it. J. J. Thomson (1888) was the first to adduce theoretical reasons for the suspected condensational activity of the ion. In the time since, so large in number and so important have been the researches in which the precipitation of supersaturated water vapor on ions enters as an argumentative premise, that insistence on the functions of the nucleus has dwindled by comparison. I must nevertheless claim the right of an independent investigator to interpret my work in a way which seems to me inevitable; and I have therefore ventured to believe that, so far as experimental evidence goes, the occurrence of electrical excitation is quite without influence in promoting condensation of moisture in supersaturated air. However ionization may be produced in the laboratory, whether by X-rays, or by ignition, or by high potential, by chemical means, or even by excessively vigorous trituration as in jets, it is always accompanied by nucleation. The average size of the nucleus resulting depends for a given medium on the time of exposure to the exciting cause and its intensity; or, in general, upon the number of nuclei produced per cubic centimeter per second. Roughly speaking, if the conditions producing ionization are sufficient and if they are maintained, there will be continuous growth in the number and size of the nuclei. On this question I have already expressed myself at some length in Nature (vol. Lxix, 1903, p. 103), believing that "out of all systems eventually issues a stable nucleation." "Why," I ask, "may one condense on a nucleus from which the soul has fled, and still be permitted to call it an ion? Why, indeed, does the nucleus persist after the ionization has vanished; why does one not get back to dust-free air?" I conclude that "electrification, if present simultaneously with nucleation, is an incidental accompaniment with no immediate bearing on the condensation produced, and for this reason I have endeavored to account for the nucleus at the outset, chemically." It is therefore merely necessary to summarize the point of view in the following statement. Whenever ionization and nucleation are associated in the outcome of any process, physical or chemical, the former is generated proportionally to the latter, in such a way that each is produced at its own rate, depending on incidental conditions. The subsequent life histories of the nucleation and the ionization are distinct, nuclei being often surprisingly persistent, ions by contrast characteristically fleeting. Hence it seems to me best in keeping with all the data in hand, to regard the nucleation as the product which owes its growth or origin to the expulsion (possibly also to the absorption) of the corpuscles representing the concomitant ionization. Moreover ionization should be present only during the period in which the nucleation varies, and a high order of nucleation may be associated with a very low or even vanishing order of ionization. Many phenomena met with in the case of dustfree air seem to be favorable to this view. Ignition and high potential nuclei, $\mathrm{X}$-ray and radiation nuclei in general, phosphorus and water nuclei, produced throughout in dust-free air, all admit of this account of their occurrence and properties; and there is no observable case of a process producing ionization free from nucleation, although there are many cases of nucleation free from ionization. 
What becomes of the ionization is a pertinent question: the ions probably vanish by recombination, as they possess strong affinities for each other. Ejected not by atomic but by molecular disintegration, we can scarcely attribute to them phenomenal velocity. They may under favorable circumstances produce fresh nuclei by absorption, by collision, but experiment does not show any appreciable increase of nucleation during the period in which the ionization vanishes. If, however, the velocity of the ion is incremented by the presence of an electric field, the production of fresh nuclei by collision may become perceptible, and the result would then appear as if the nuclei themselves moved in the electric field, whereas they are actually the inert residues left in the wake of a fleeting electron.

Finally, it should be noticed that to produce condensation on X-ray nuclei after long exposure, less than a double supersaturation is needed, whereas in Wilson and Thomson's case of condensation on ions, the supersaturation required is three- to four-fold. Thus the two views of condensation on nuclei and condensation on ions would not in any case be mutually exclusive. Furthermore, if initially (i.e., for short exposures, and nuclei in the extreme state of fineness antedating growth) the nucleation is supposed to have ejected but one electron per nucleus (an assumption which in one form or another must be made in any other explanation), the present view is in no way incompatible with J. J. Thomson's method of measuring the charge of one electron.

If a nucleus like that of phosphorus, for instance, shows a continued tendency to grow, until it finally appears as part of a visible smoke, there may be continuous ejection of electrons within certain limits, as the growth matures. In such a case, electric conduction through a gas freighted with these nuclei would obey Ohm's law, as is actually the case for phosphorus. ${ }^{1}$

To return from this digression to the present volume: the contents of the first two chapters bear on my "Experiments with ionized air." The second chapter originally carried the working hypothesis into further development, but as I have not been able to supply the requisite numerical detail, I have retained the experimental parts only. These chapters, like the earlier work, show, I think, that whereas ionization vanishes with characteristic rapidity, the nucleation has a long lease of life by contrast. At the same time the ionization and nucleation produced in any given process are proportional quantities. The

I In the time elapsed since these experiments were made, I have carried them much further than stated in the text (cf. Science, xxı., r905, pp. 275 and $56_{3}$; American Journ. of Sci. (4), 1905, xIx, pp. 75 and 349; Physical Review, July, 1905), showing among other things that persistent $\mathrm{X}$-ray nuclei pass into fleeting nuclei on removal of the X-ray tube to greater distances from the outside of the fog chamber or on loss of intensity. Such fleeting nuclei become persistent water nuclei on solution. For the case of radium in a sealed aluminum tube surrounded by a wall of lead $\mathbf{r} \mathrm{cm}$. in thickness, the nucleation is reduced by but $30 \%$ of the value obtained when the lead envelope is absent. Hence the gamma rays which produce but a few per cent. of the ionization are accountable for the greater part of the nucleation. 
corresponding cases for nuclei produced by the $\mathrm{X}$-rays are given in the earlier volume and elsewhere.

The following chapters, III, IV, V, VI, have been written to draw a variety of conclusions from the data in my work on the Structure of the Nucleus, which escaped me in the earlier volume, as well as to correct certain errors relating to the quantity of water precipitated under given conditions, and to the diffusion of nuclei, to which I have already called attention elsewhere. Chapter VI, relating to periodic distribution in the colors of successive coronas, shows under what conditions the angular diameter of a ring of a given color may be used for the estimation of the number of particles producing the observed diffraction pattern. In Chapter VIII a definite practical application is made, for use in the last chapter of the book.

Chapter VII shows a method by which fog particles, even of minutest size, may be measured under the microscope, or microscopically photographed. The peculiar difficulties encountered in the interpretation of these results, in spite of the fact that fog particles are obtained in definite sizes and numbers, are considered critically.

The chief results of the book, however, are given in the last chapter, which is a record of over two years of observation of the number of nuclei present per cubic centimeter of the atmosphere of the city of Providence. The nuclei are abundantly represented, particularly in the winter months. Curiously enough, the maxima and minima appear at about the time of the winter and summer solstices respectively. The reason for this cannot be sought in the astronomical circumstances involved, but must be atmospheric in character. I have supposed that in addition to rain, light pressure, which must be more effective as the days are longer, may have something to do with this. Under any circumstances a highly nucleated medium is an interesting medium. Since much of the nucleation must be of local origin and referable to combustion, the question arises, what has become of the ionization which was simultaneously generated? It has probably vanished as does the ionization in the experimental condensation chamber in the laboratory.

To reply to these questions systematically, observations have now for nearly a year been taken at Providence and at Block Island simultaneously. The latter station has many of the meteorological elements of Providence, but Block Island lies sufficiently in the sea, and is in winter at least sufficiently free from human habitation to present entirely different conditions as to nucleation. I have also in progress a continuous series of observations on the changes in the lapse of time of the nucleation of dust-free (filtered) air, i. e., of air free from foreign nuclear ingredients. The results will be reported in due course elsewhere.

BROWN UNIVERSITY, January 9, I905.

Carl Barus. 



\section{TABLE OF CONTENTS.}

\section{Cilapters I-II.-Relating to "Experiments with Ionized Air," Smitilsonian Contri- BUTIONS TO KNOWLEDGE, VOL. XXIX, No. I 309 .}

\section{CHAPTER I.}

The Relation of Ionization to Nucleation in Air after Contact with Phosphorus.........

1. Introductory. 2. Method proposed. 3. Water nuclei. 4. Comparison of the steam jet and the condensation chamber. 5. Decay and absorption. 6. Apparatus. 7. Manipulations. 8. Data for phosphorus ionization. 9. Further data. 10. Effect of different charges in the condenser. 11. Dried emanation. 12. Wet emanation. 13. Residual ionization after one hour. 14. Nucleation partially precipitated. '15. Ionization of dry phosphorus nuclei. 16. Inferences.

\section{CHAPTER II.}

The Relation of the Ionization and the Nucleation Associated with Water Nuclei,

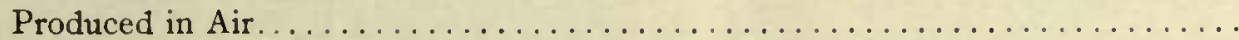

Ioniz ation Produced by Shaking Solutions.

I. Introduction. 2. Apparatus. 3. Results.

Efficiency of Nuclei-Producing Jets.

4. Powerful methods of comminution. 5. Results

The Ionization of Water Nuclei.

6. Introductory. 7. Apparatus. 8. Results. Initial charges. 9. Comparison with coronas. 1o. Evanescence of the charges of water nuclei. II. Results with an Elliott electrometer. 12. Further data. I3. Jets self-shattering or impinging on water. I4. Summary of the relative degree of ionization and nucleation. 15. Spontaneous time loss of nuclei. 16. Effect of condensation on ionization. 17. Effective condenser length.

Summary and Inferences.

18. Working hypothesis. I9. Charge and conduction. 20. Comparison of phosphorus and water nuclei.

Chapters III-ViI.-Relating to "Structure of the Nucleus," Smithonian ContriBUtIONS TO KNOWLEDGE, No. 1373.

\section{CHAPTER III.}

Prcliminary Survey of the Apertures of Coronas in Relation to the Number of Nuclei

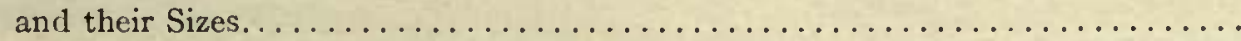

1. Introductory. 2. Apparatus and preliminary results. 3. Diameter of cloud particle. 4. Nucleation. 5. Cause of periodicity. 6. Effect of temperature. 7. Pressure decrement. 8. Summary. 9. Plate-glass apparatus. 


\section{CHAPTER IV.}

The Number of Nuclei Produced by Shaking Different Liquids, and Allied Experiments.

PAG 1. Explanation. 2. Data. 3. Coronas in general. 4. Axial colors. 5. Carbon disulphide.

\section{CHAPTER V.}

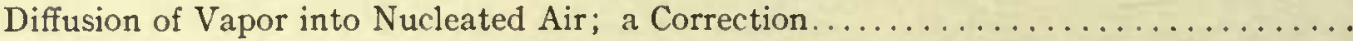
r. Apparatus and manipulation. 2. Equation. 3. Application and data. 4. Conclusions. 5. Diffusion from greater to less saturation. 6. Crucial experiment and conclusion. 7. Nuclei produced by mixture of coal gas and air.

\section{CHAPTER VI.}

Periodic Color Distributions in Relation to the Coronas of Cloudy Condensation, with a

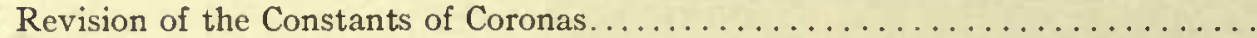
Introduction.

1. Purpose and plan. 2. Apparatus. 3. Color distributions. 4. Apertures.

Data for the Welsbach Gas Burner.

5. Explanation of the tables and notation. 6. Charts. 7. Tables.

Method of Reduction.

8. Constants of the geometric sequence. 9. Time losses. 10. Exhaustion losses. I I Losses attributed to subsidence. 12. The optic constant. Diffraction methods. 13. The optic constant. Subsidence methods. 14. Summary of optic constants. I5. Resulting equations applied. 16. Remarks on the tables and graphs. 17. Diameter of fog particles

Data with Electric and Mono-Chromatic Light. 18. Tables. 19. Charts. 20. Diameters of cloud particles.

Miscellaneous Experiments with Deep Vessel.

21. Aperture of white dise. 22. Tables for the coronas. 23. Remarks on the table and graphs. 24. Condensation chamber remote from the eye.

Long and Shallow Condensation Chambers.

25 Apparatus. 26. Reduction of data and tables. $\delta p=17 \mathrm{~cm}$. 27. Smaller and larger pressure differences. 28. Remarks on the tables. 29. Nucleation of the green coronas.

Other Causes of Change in the Types of Coronas.

30. Thickness of cloud layer. 3r. Obliquity of diffraction. 32. Effect of wave length.

Different Speeds of Exhaustion for a Given Pressure Difference.

33. Increased suddenness of eondensation. 34. Results. 35. Growth of nuclei. 36. Subsidence. 37. Exhaustion ratio. 38. Inferences. 39. Different exhaustion rates for moderate nucleations. 40. Conclusion.

\section{CHAPTER VII.}

Direct Micro-metric and Micro-photographic Measurement of Fog Particles, with a Summary of all Relevant Data.................................

I. Micrometry of Fog Particles.

Earlier Methods.

1. Introductory. 2. Apparatus. 3. Behavior of the precipitated droplets. 4. Preliminary data. 
Improved Method.

5. Number of droplets. 6. Diameters of droplets. 7. Graded particles.

II. Micro-photography of Fog Particles.

General Results.

8. Preliminary. 9 Apparatus and method. Io. Incidental phenomena. Pitting

11. Dew. 12. Evaporation. 13. Moving and floating globules. 14. Graded particles.

Specific Results.

15. Photographic plates. T6. Tabulated results. 17. Remarks on the tables.

Inferences.

18. Precipitation per cubic centimeter. 19. Diancters and numbers. 20. Explanation of discrepancies. 21. Summary.

III. Results from Subsidenee.

22. Object and method. 23. Results. 24. Remarks on the tables. 25. Further results.

IV. Summary of all the Results Obtained for the Relation between Nucleation and Coronal Diameter.

26. Preparation of a table of reduction.

\section{Chapters Vili-IX.-Applications.}

\section{CHAPTER VIII.}

The Coronal Method of Estimating Atmospheric Nucleation................

3. Introductory. 2. Apparatus. 3. Diffusion from two opposed surfaces. 4. Miscellaneous tests. 5. Diffusion from a single surface. 6. Absorption and decay of nuclei. 7. Effect of pressure differencc. 8. Precipitation per cubic centimeter. 9. Relation of nucleation to aperture of corona. to. Absence of electrification in cases of sudden condensation and of sudden evaporation. 11. Conclusion.

\section{CHAPTER IX.}

The Variation in the Nucleation of the Atmosphere of the City of Providence.......

Introduction.

1. Preliminary. 2. Apparatus, 3. Classification.

First Group of Observations.

4. Early observations: 5. Plate-glass apparatus. New apertures.

Second Group of Observations.

6. Plan of tables and graphs. 7-16. Successive monthly data for 1903 . 17-25. Successive monthly data for 1904 .

General Inferences.

26. Efficiency of Apparatus. 27. Variability. 28. Wind effect. 29. Rain effect.

30. Snow effect. 3r. Cloud effect. 32. Solar effect absent. 33. Temperature effect. Cold-wave effect. 34. Local effect.

Summary and Conelusions.

35. Mean daily nucleations. 36. Mean monthly nucleations. 37. Occurrence of maxima and minima of nucleation during the winter and the summer solstices, respectively. 38 . Conclusion. 



\section{LIST OF FIGURES.}

\section{CHAPTER I.}

FIGURE I. Apparatus for comparing nucleation and ionization......

FigurE 2. Modification of preceding apparatus, with side influx tube and removable desiccator.... . 5

\section{CHAPTER II.}

FiGURE I. Apparatus for comparing nucleation and ionization................. I

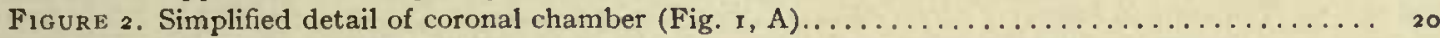

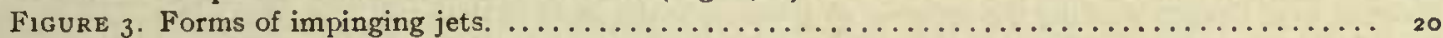

Figures 4 to 6 . Curves showing electrometer deflection (leakage), after consecutive half-minutes, for

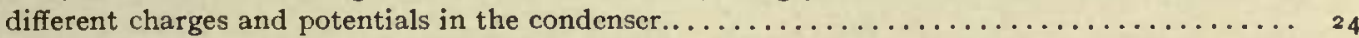

Figures 7 and 8. Curves showing radial currents (amperes) for different charges and potentials (volts)

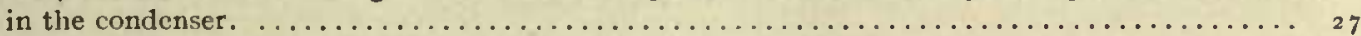

Figure 9. Tube condenser witlı sliding and removable outer coating..................... 34

\section{CHAPTER III.}

FIGURE $x$. Chart giving curves showing relations of apertures computed from successive exhaustions $(1,2)$, and the relation of diameter of fog particles computed from successive exhaustions $(4,5)$

FIGURE 2. Chart giving curves showing ratios of nucleation computed from successive exhaustions and from measured apcrtures $(8,9,10,11,12)$, and corresponding diameters of fog particles in

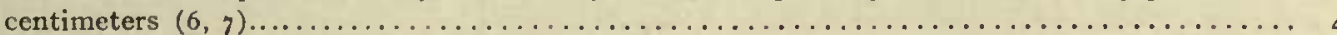

\section{CHAPTER V.}

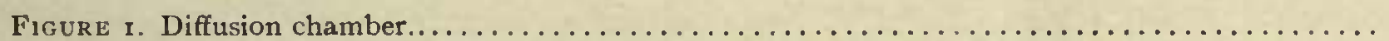

FigURE 2. Chart showing the vapor pressures at different heights in the lapse of time when water vapor

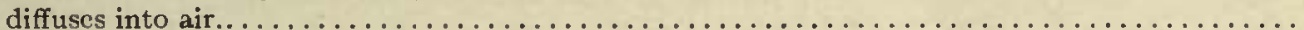

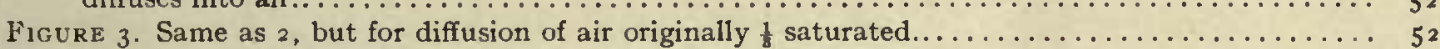

\section{CHAPTER VI.}

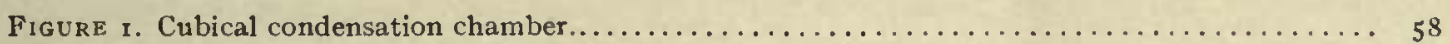

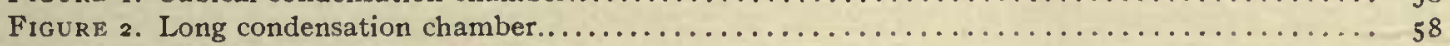

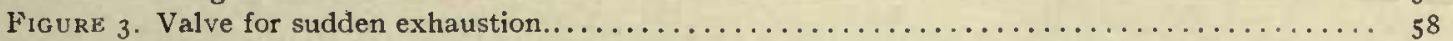

FIGURE 4. Chart for Table $I$, showing the relation of nucleation and of diameter of fog particle in

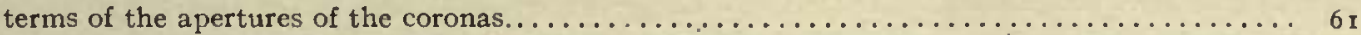

Frgure 5. Chart for Table 3 , showing the relation of relative nucleation to the number or order of

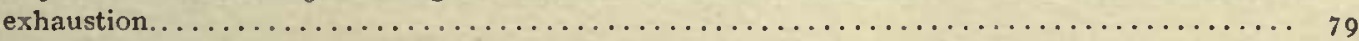

FIGURE 6 . Chart for Table 8 , showing the relation of nucleation and of diameter of fog particle in

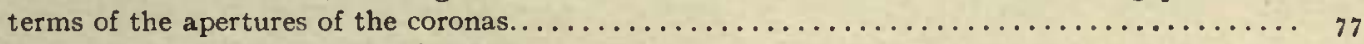

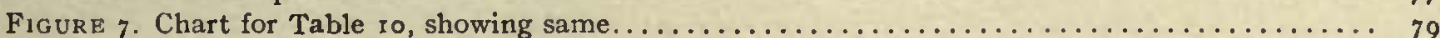

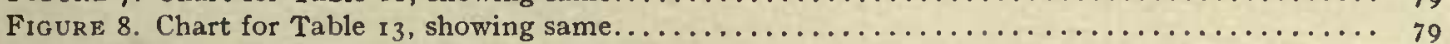

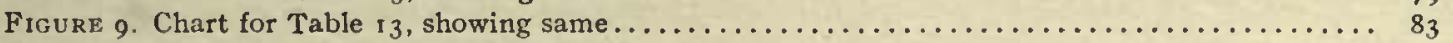

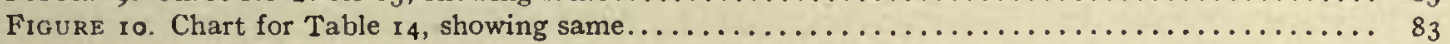




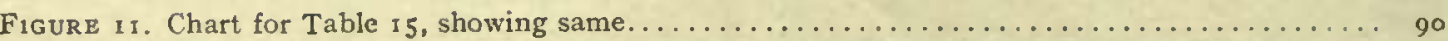
FiguRES I 2 and 13. Charts for Table 18 , showing the relation of the apertures of the coronas to the

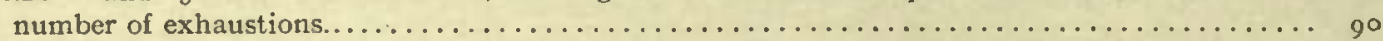

FiguRE 14. Chart showing the effects of different valves with nuclcations increasing in the lapse of time.

\section{CHAPTER VII.}

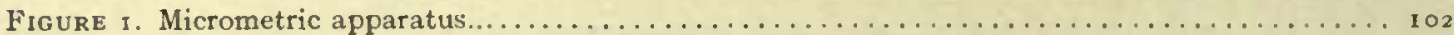

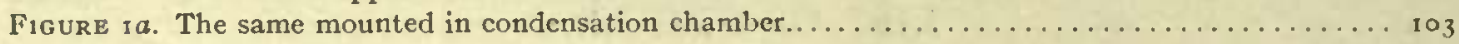

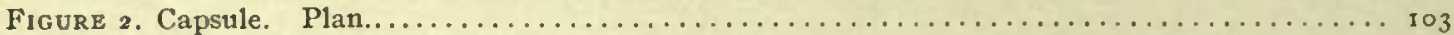

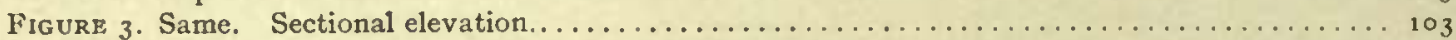

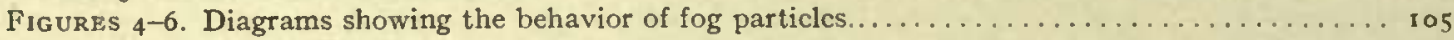

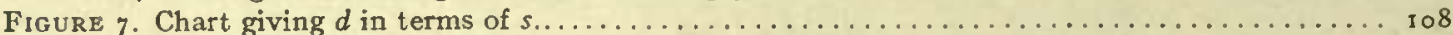

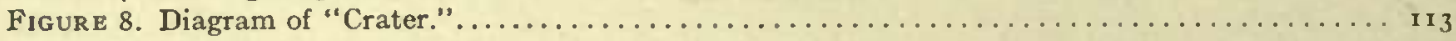

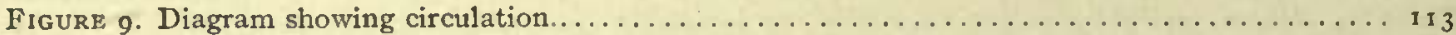

Figure 10. Micro-photographs of fog particles $\ldots \ldots \ldots \ldots \ldots \ldots \ldots \ldots \ldots \ldots \ldots \ldots \ldots \ldots \ldots \ldots \ldots$ I 6

\section{CHAP'TER VIII.}

FIGURE I. Condensation chamber. Sectional elevation........................ I 29

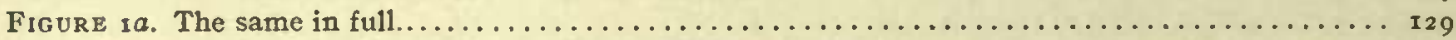

FIGURES 2-4. Graphs showing the progress of diffusion in the lapse of time..............

Figure 5. Apparatus to detect ionization produced by sudden condensation............... 137

\section{CHAPTER . IX.}

PIGURE I. Diagram showing general disposition of apparatus......................... 140

Charts I-48. Daily observations of the atmospheric nucleation of the city of Providence........ I I I

Charts 49 and 50. Mean daily nucleations in thousands per cubic centimeter..................... 23

Chart 5r. Mean monthly nucleations from Octoher, igo2, to October, 1904 , in thousands per cubic

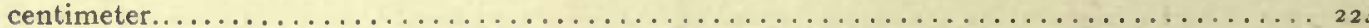




\title{
A CONTINUOUS RECORD OF ATMOSPHERIC NUCLEATION.
}

\author{
By Carl Barus, \\ HAZARD PROFESSOR OF PHYSICS AT BROWN UNIVERSITY.
}

\section{CHAPTER I.}

THE RELATION OF IONIZATION TO NUCLEATION IN AIR AFTER CONTACT WITH PHOSPHORUS.

I. Introductory. - The opinion was expressed in ny earlier papers, that wherever there is sufficiently intense iomization, there one may also expect to find active nucleation; for it is hardly probable that a group of dissociated molecules, neutral as a whole, can ultimately escape combination with each other and the medium in which they are suspended. If these combinations occur in the presence of water vapor, and particularly in a saturated atmosphere, the nuclei due to solution may result. When the nuclei are produced from dilute solutions by shaking, evaporation of the fog particle to the nuclear diameter might be inferred; similarly, when the solute is produced by any kind of radiation or emanation, each trace of solute may grow in bulk by absorbing water to the nuclear stage. In case of an intense emanation like that from phosphorus, this process may actually continue until a visible cloud is produced and the nuclei attain the size of fog particles. But it is to be borne in mind that the nucleus, dissolved or not, is present initially, and is in case of phosphorus producible in dry air; whereas, in case of water nuclei produced by shaking or by jets, there is no evidence of evaporation from the comminuted water particles, nor is it certain that the nuclei here are mere water dust. One must keep in mind that a nucleus may be the residue after the corpuscles representing the ionization have been expelled.

2. Method proposed.- If the original emanation, highly ionized though neutral as a whole, is put through the process of condensation, then, if the negative ions are more efficient as condensation nuclei than the positive ions, the nuclei after condensation, or even after remaining in a saturated atmosphere, 
should become continually more positive,-assuming that a greater number of negative ions are removed by condensation.

The investigation would therefore consist in testing the ionization immediately coming from phosphqrus as to its power in dissipating positive and negative charges, and in comparing these results with the degree of ionization after the emanation has produced condensation. In other words, it is to be ascertained whether the nuclei after a succession of condensations become continually more positive.

The results to be discussed in the following paragraphs have made this apparently straightforward investigation of little avail: for after the emanation has reached the nuclear stage, scarcely 3 per cent. of the original ionization is left. The residue is then so small that a decision of a possible excess of positive or negative ionization is difficult, because the whole is now of the same order as the normal leakage of the electrometer.

In fact, the decision as to whether the positive or negative ionization is in excess is now of very secondary interest, for the nuclei introduced into the condensation chamber have already lost all but a trifle of their original charges. The successive and even the initial condensations thus virtually proceed without electrification.

The initial intense ionization nearly vanishes even in a moderately dry atmosphere. Indeed, it is hard to understand how a neutral, intensely ionized emanation can be produced from a body like phosphorus. It appears to me that the emanation is a molecular body which is stable in the presence of an

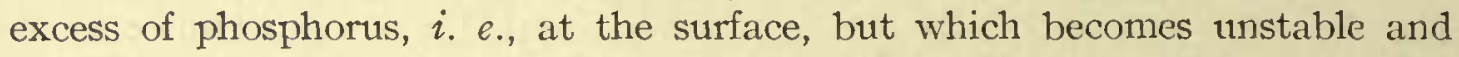
breaks to pieces in presence of an excess of air, on leaving the phosphorus. The observed ionization is the accompaniment of this dissociation, and occurs on the passage from the first environment to the second. If the ions were produced by phosphorus directly, one would expect them to be either positive or negative, but not neutral.

3. Water nuclei.-After finishing the work with phosphorus, correlative experiments with water nuclei were undertaken. It was found necessary, however, to produce them in greater number than is possible by mere shaking, to obtain marked effects. Hence jets were resorted to and studied in some detail, as will be shown in succeeding chapters. The results obtained, though closely resembling the phosphorus data in the main, differed from them inasmuch as the currents above a certain potential difference were constant, and independent of the electromotive force of the condenser, while the ionization or charge is not, neutral as a whole. Nucleation again remained equally effective after the ionization had all but vanished in the speedy way observed for phosphorus. Here, too, however, it may be plausibly argued that the nucleus is the stable product after the corpuscles representing the ionization have been extruded.

4. Comparison of the steam jet and the condensation chamber.-A digression may here be made relative to the indications of the colors of the steam jet and 
of the coronal phenomena, in relation to the number of nuclei concerned. The usual and strong response of the steam jet is for axial blues and greens as far as the purples of the second order. These are already too weak to be of effective service for measurement. But at this stage of smallness of fog particle, the coronal display has but begun. The strong blues of the axial colors correspond to the diffuse gray fogs of the condensation chamber out of which the coronas are gradually evoked when the number of particles ${ }^{1}$ has sufficiently diminished. The two instruments are thus in a measure supplementary; the condensation chamber gives intense evidence of the presence of nuclei long after the steam jet would imply their absence. It is for this reason that ordinary smokes like sal ammoniac do not affect the steam jet ${ }^{2}$ where a number of nuclei exceeding a certain lower limit is necessary. The latter again is particularly active for those intense and fresh nucleations which produce the browns and yellows of the first order, implying conditions which it is impossible to produce in the condensation chamber at all, until the lower limit of spontaneous condensation of dust-free moist air has been exceeded.

5. Decay and absorption.-To account for the rapid diminution of the number of nuclei in the phosphorus emanation in the lapse of time, two hypotheses are prominent. With finely divided and in so far highly potentialized matter (possibly ionized positively and negatively), combinations of nuclei may occur to the detriment of the number of independent nuclei. Such a decrease would take place as the square of the number. On the other hand, it is equally probable that the initial and very small nuclei are in rapid motion much like molecules, and that the loss takes place by absorption or arrest at the walls of the vessel. In my memoir on the subject, I included both hypotheses in the computation; but finding that the phenomena could be adequately explained by the latter, I ignored all spontaneous decay. Though this policy would not be generally admissible, it is unlikely that nuclei can vanish initially at the same rapid rate as the ionization. Indeed, evidence will show that it does not. In case of water nuclei, which are much the more sparsely distributed, the original number of nuclei can be proved by coronas to have varied but little in the short time in which the ionization falls off to a few per cent. Hence the nuclei must be regarded as parting with their charges more rapidly than they are themsclves absorbed in the lapse of time, and one will have to distinguish between the velocity of the uncharged and of the charged nucleus in the electric field, the latter being incremented by the electric forces. The cases will be worked out in the chapters below.

6. Apparatus. - The apparatus used in the present experiments was capable of a great number of variations. The essential purpose is to enable the observer either to introduce phosphorus emanation at once into the electrical condenser or else to introduce it after it has been saturated with water, suddenly

${ }^{1}$ Phil. Mag. (6), iv, p. 24, I902; cf. Structure of the Nucleus, Chapter III.

2 Thus smoke due to sal ammoniac if introduced in to the steam tube will actually clear the blue field produced by phosphorus nuclei, $i . e$. , will wipe out the condensation. 
cooled, or otherwise treated. The following diagram, figure $\mathbf{1}$, will make the adjustments clear.

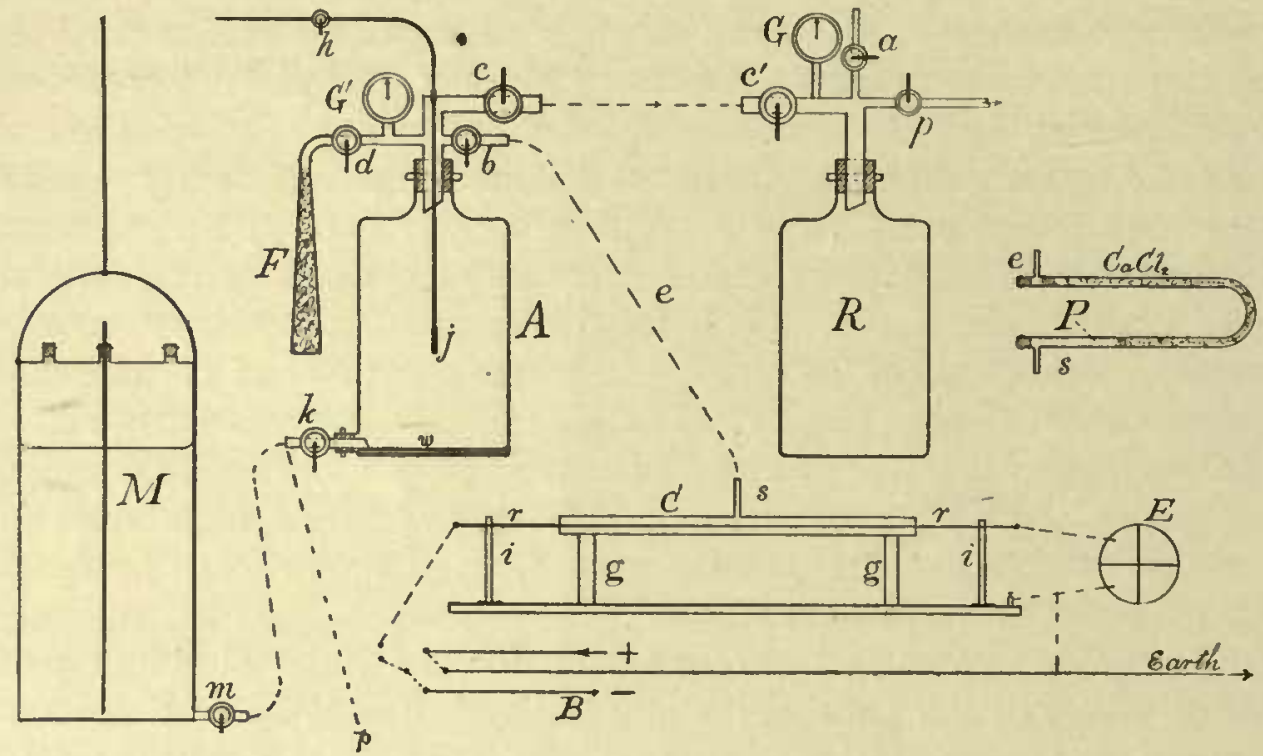

Figure r.-Apparatus for Comparing Nucleation and Ionization. $A$, Coronal Cihamber; $k$, Exhaustion Reservoir; $M$, Mariotte Flask; $C$, Tubular Condenser; $P$ (to be Inserted in tuie Conveyance Tube, $e$ ), Phosphorus Ionizer; $G$ and $G$, Vacuum Gauges; $F$, Cotton Filter; $B$, Storage Battery Terminals. Tube $p$ to Suction Pump, $a$ to Atmosphere, $c$ and $c$ for Exiaustion, $e$ for Conveying Nuclei into Condenser, $d$ for Filtration, $h$ from the Hydrant. Supports $g, g, a r e$ Metallic, $i, i$, Insulating.

The parts of the train of apparatus are the large copper Mariotte flask $M$, with a supply of water sufficient for aspiration, the condensation chamber $A$, used both for producing coronas and for the aspiration and storage of air laden with phosphorus nuclei, the exhaustion chamber, $R$, the phospliorus ionizer, $P$, the tubular electrical condenser, $C$, and the electrometer, $E$. An accessory desiccator, $D$, of the tower form may be inserted on the way, when dry air is needed, as shown in figure 2.

$R$ is connected through a stopcock with the suction pump at $p$, with the atmosphere at $a$, and by wide tubing with the condensation chamber, $A$. It carries a vacuum gauge, $G$.

$A$ is connected with a stopcock with the cotton filter, $F$, with the ionizer by $b$ (where the tall desiccator may be inserted), with $R$ by $c$, and also carries a vacuum gauge, $G^{\prime}$. A is further joined by stopcocks with the Mariotte flask, $M$, for aspiration, and is graduated in liters on its side. It holds about ro liters.

The ionizer, $P$, is a large $U$ tube containing calcic chloride for desiccation, kept in place by loose cotton plugs. One shank is nearly empty, and at $g$ carries thin pellets of phosphorus between strips of wire gauze.

The condenser is tubular, 2.10 and $.64 \mathrm{~cm}$. in diameter and $50 \mathrm{~cm}$. long, with the outer mantel permanently put to earth. The core is a brass rod, sup- 
ported on hard rubber insulators, $i$, about $15 \mathrm{~cm}$. long, and at a distance of ro $\mathrm{cm}$. from the end of the tube. This rod is highly charged from the storage battery, $B$, and the leakage found from the electrometer, $E$, one pair of quadrants of which are in connection with $r, r$, and the other pair put to earth. A commutator enables the observer to use either the positive or the negative pole of the storage battery for charging the system.

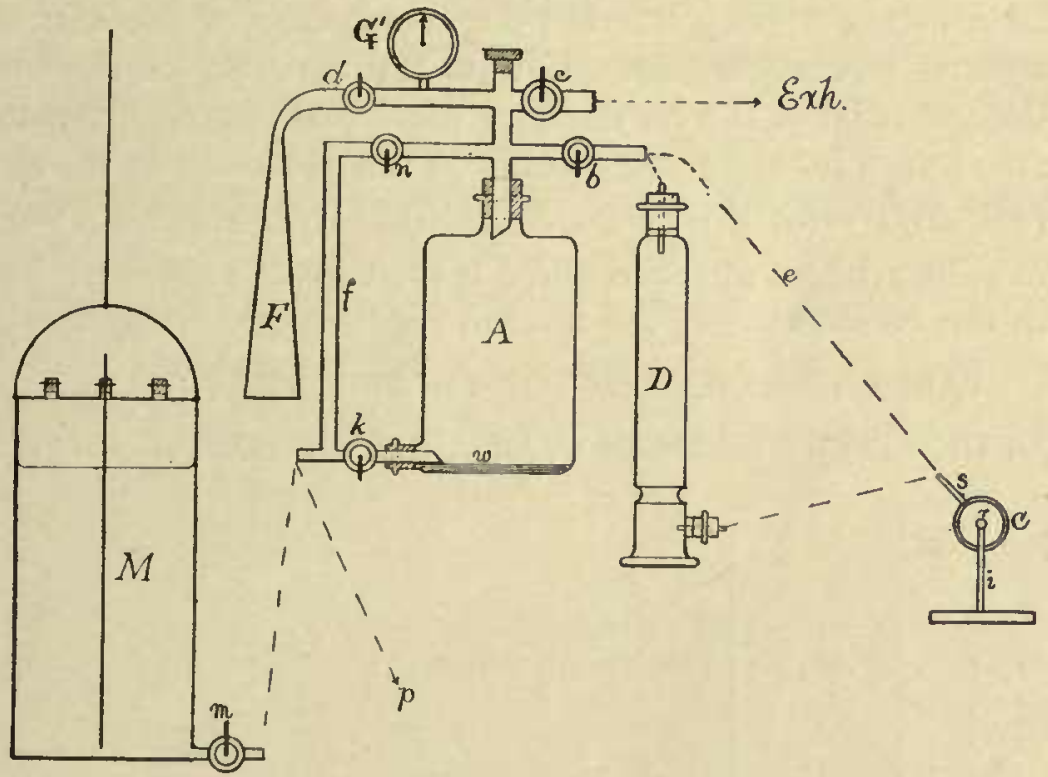

Figure 2.-Miodification of I'receding Apparatus with Sine Influx Tube, $k, f, n$, for Maintaining Constant Pressure in $A$, and Removabie Desiccator, $D$.

The electrometer was specially built for the present purposes, and the needle was kept charged by a water battery of 48 volts, one pole of which was earthed. The suspension is a silk bifilar moistened by a dilute solution of any hygroscopic salt, and the battery charge is conveyed through the fibers. The quadrants are supported on hard rubber insulators ro $\mathrm{cm}$. long. Difficulties were encountered in using this apparatus as will appear below. In figure 2 a side tube, $f$, between stopcocks $k$ and $n$ has been added, with the object of securing greater constancy of pressure in $A$, the flow of water from $M$ being via $m f n$.

7. Manipulations.-On raising the Mariotte flask, $M$, and opening appropriate stopcocks, the emanation passes directly from the ionizer into the condenser, and its ionization may be measured. A tower desiccator is here to be inserted before $P$, to dry the air.

On lowering $M$, removing $D$, and reversing $P$, the emanation passes into A. Here its nucleation may be tested by condensation, and it may thereafter be introduced into $C$ at once or after a number of condensations. The nucleation may also be stored in a dry vessel between $D$ and $P$ reversed, and subsequently transferred from the dry vessel into the condenser.

Finally, the phosphorus ionizer $P$ and $D$ may be quite removed and replaced with a pipe connection, while the tube $n j$ is adjusted for spraying. The 
hydrant water passing $h$ under high pressure reaches the jet $j$, placed suitably within $A$. The water nuclei thus producible may be tested either by coronas or electrically by passing them into the condenser, $C$. Compare Chapter II.

\section{IONIZATION OF PHOSPHORUS NUCLEI COLLECTED OVER WATER.}

8. Data.-The current through the condenser follows a law similar to Ohm's. The constant appropriate for the comparison of the data may therefore be computed logarithmically. If $C$ is the capacity of the apparatus in parallel, $E$ the potential of the condenser, $i$ the radial electric current through it, $R$ its ohmic resistance for the given medium, $s$ the deflection at the electrometer,

$$
(d E / d t) / E=(d s / d t) / s=\mathrm{r} / C R \text {, or } d(\log s) / d t=.434 / C R=a
$$

where common logarithms are used and $a$ is the constant sought. Thus $a$ varies directly with the conduction of the ionized medium traversing the condenser.

TABLE I.-IONIZATION OF PHOSPHORUS NUCLEI.

$d V / d t$ (IN LITERS PER MIN.) VARIABLE. NEGATIVE CHARGE.

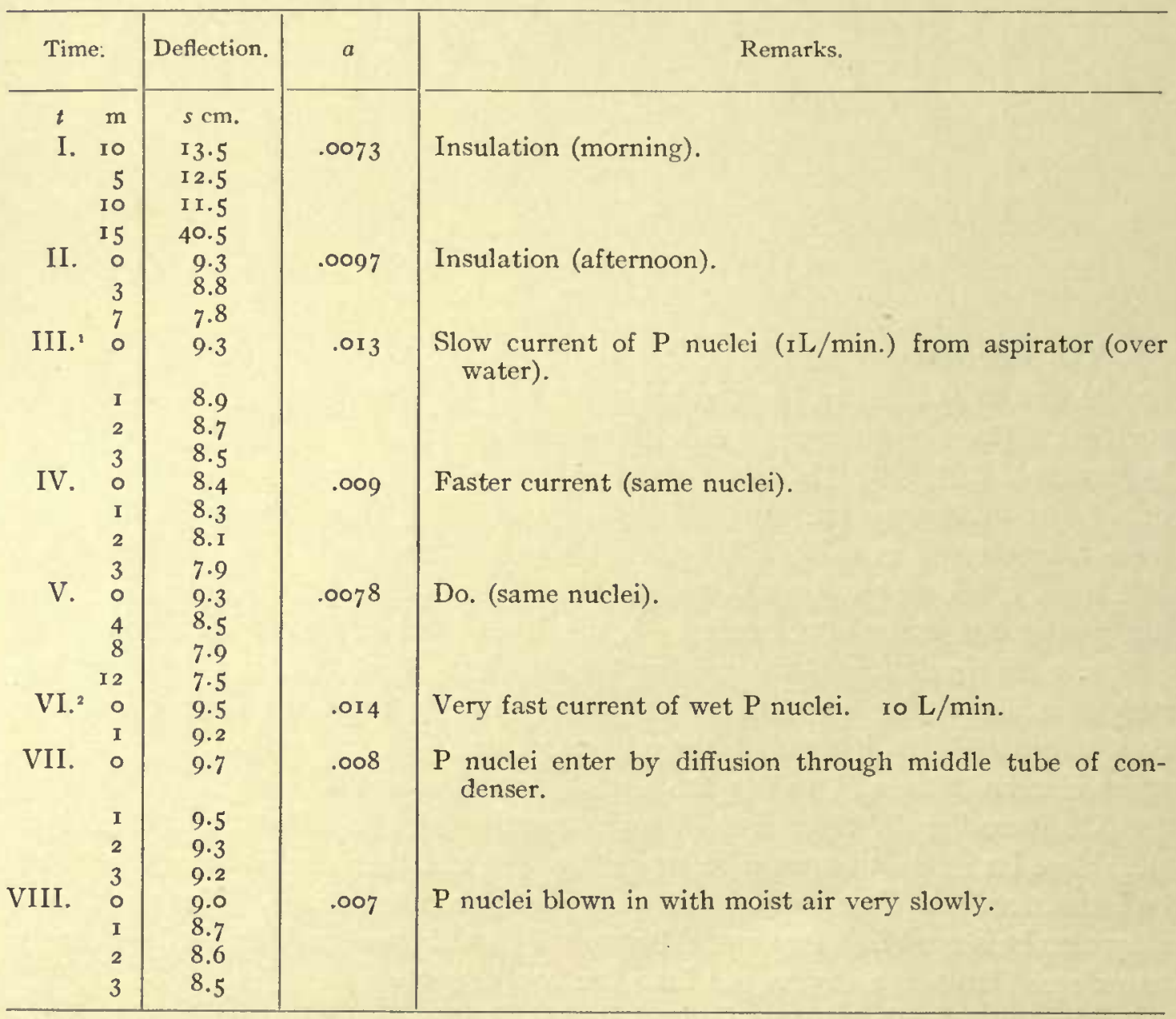

1. Nuclei put into receiver (A) by preliminary exhaustion.

2 In this and the other cases fresh nuclei were aspirated into A by passing room air over phosphorus. 
TABLE I.-Continued.

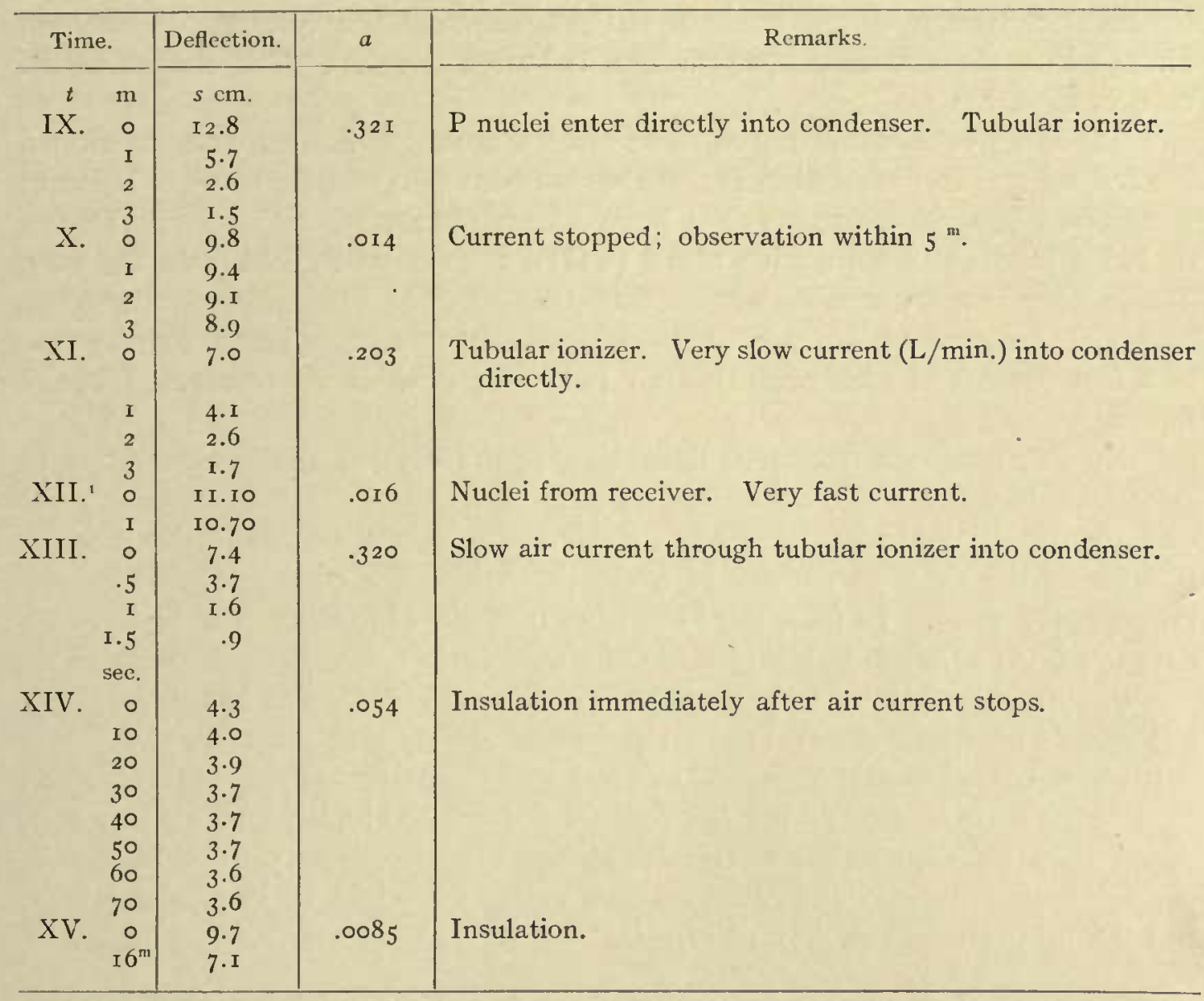

In this and the other cases fresh nuclei were aspirated into $\mathrm{A}$ by passing room air over phosphorus.

TABLE 2.-SUMMARY OF THE PRECEDING. NUCLEI INTRODUCED INTO RECEIVER BY ASPIRATING ROOM AIR OVER PHOSPHORUS.

\begin{tabular}{|c|c|c|}
\hline & Remarks. & $a \times 10^{3}$ \\
\hline I. & Insulation (morning). & 7 \\
\hline II. & (afternoon)...... & IO \\
\hline V. & Slow current of phosphorus nuclei (liters per min.) in saturated moist air.. & 8 \\
\hline VI. & 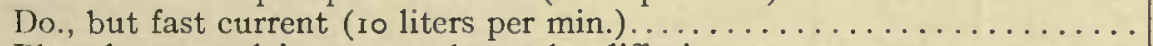 & 14 to 16 \\
\hline VII. & 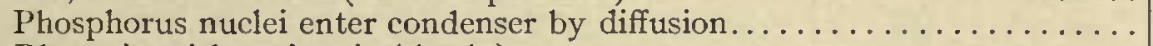 & 8 \\
\hline VIII. & 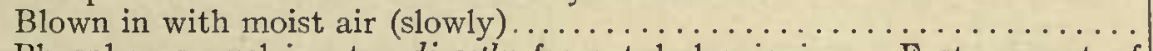 & 7 \\
\hline IX. & $\begin{array}{l}\text { Phosphorus nuclei enter directly from tubular ionizer. Fast current of } \\
\text { moist air. } \ldots \ldots \ldots \ldots \ldots \ldots \ldots \ldots \ldots \ldots \ldots \ldots \ldots \ldots \ldots \ldots \ldots \ldots\end{array}$ & 320 \\
\hline $\mathrm{X}$. & 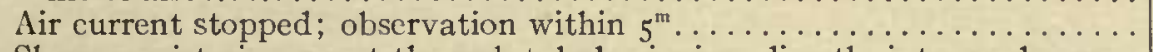 & 14 \\
\hline XI. & Slower moist air current through tubular ionizer directly into condenser. & 200 \\
\hline XIII. & Do. Slow moist air current, but fresher phosphorus. . . . . . . . . . . & 320 \\
\hline XIV. & Ionized air current stopped: immediately after. . . . . . . . . . . . . & 54 \\
\hline XV. & 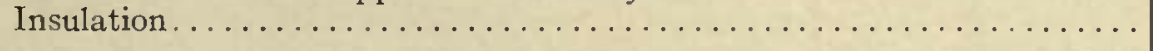 & 8 \\
\hline \multicolumn{3}{|c|}{$\begin{array}{l}\text { Cases V, VI, tested for coronas, gave the usual intense and full series, beginning } \\
\text { with diffuse dense fogs. }\end{array}$} \\
\hline
\end{tabular}


Table I contains the direct observations, time in minutes, and deflections, $s$, of the electrometer in centimeters. $d V / d t$, denoting the volume of nucleated air put into the condenser per minute, is here estimated. The charge of the core is negative.

Table 2 gives a summary of these results, among which may be mentioned the following: The insulation is not above $a=$. ox and usually lower. It is not exceeded when phosphorus nuclei have access merely by diffusion (VII), nor on being blown in from a wide vessel (VIII), the charge vanishing on the way. The leakage is not exceeded when a slow current of highly nucleated air stored over water is passed through the condenser (V), and but slightly for the case of a fast current of such nucleated air (VI) taken out of the receiver $A$ in the figure.

By contrast, the excessive ionization $(a=.2-.3)$, if the nucleation is at once introduced into the condenser, is striking enough. Hence scarcely 3 per cent. of the original ionization has survived after short storage in the receiver in spite of the extreme density of nucleation which the coronas would cotemporaneously show. In fact, the ionization dies out almost at once in the condenser (X-XIV), even in the absence of water vapor.

9. Further data.- The experiments were now repeated as in table 3 , with the résumé of results shown in table 4 . These are substantially like the above. With fresh phosphorus the residual ionization of the nuclei-bearing air after short storage over water is but a few per cent. of the original ionization. It was supposed that on drying the nucleated air over phosphorus pentoxide, before passing it into the condenser, the original ionization might be in part regained, but the table shows not a trace of this.

TABLE 3.-IONIZATION OF PHOSPHORUS NUCLEI. $d V / d t=$ ABOUT 2 LIT./MIN. NEGATIVE CHARGE.

\begin{tabular}{c|c|c|l}
\hline Time. & Potential. & $a$ & \\
\cline { 2 - 3 }$t \mathrm{~m}$. & $s$ & & \\
0 & 15.8 & .009 & Room air (insulation). \\
2 & 15.2 & & \\
4 & 14.6 & & \\
6 & 13.9 & & \\
0 & 20.5 & .024 & Slow eurrent of P nuclei in damp air. \\
1 & 19.4 & & \\
2 & 18.5 & & \\
3 & 17.6 & & \\
0 & 18.9 & .011 & Filtered moist air. \\
1 & 18.4 & & \\
2 & 17.9 & & \\
3 & 17.5 & & \\
0 & 21.1 & .010 & Room air. \\
$\mathrm{I}$ & 20.7 & & \\
2 & 20.2 & & \\
3 & 19.7 & & \\
\hline
\end{tabular}


TABLE 3.-Continued.

\begin{tabular}{|c|c|c|c|}
\hline Time. & Potential. & $a$ & Remarks. \\
\hline$t \mathrm{~m}$ & $s$ & & \\
\hline$\circ$ & I9.6 & .021 & Current of $\mathrm{P}$ nuclei in damp air passes over $\mathrm{P}_{2} \mathrm{O}_{5}$. \\
\hline I & & & \\
\hline 2 & 17.7 & & \\
\hline 3 & 17.0 & & \\
\hline$\circ$ & 10.8 & .017 & $\mathrm{P}$ nuclei in damp air without $\mathrm{P}_{2} \mathrm{O}_{5}$ (small deflection). \\
\hline $\begin{array}{l}1 \\
2\end{array}$ & $\begin{array}{l}10.4 \\
10.0\end{array}$ & & \\
\hline 3 & 9.6 & & \\
\hline ○ & 23.1 & .008 & Room air. \\
\hline I & 22.6 & & \\
\hline 2 & 22.2 & & \\
\hline 3 & 21.9 & & \\
\hline$\circ$ & 20.9 & .018 & P nuclei in damp air (large deflcction). \\
\hline I & 20.2 & & \\
\hline 2 & I 9.4 & & \\
\hline 3 & 18.4 & & P nurai dirontly from ionizer \\
\hline o & 24.7 & .264 & P nuclei directly from ionizer. \\
\hline I & I 3.4 & & \\
\hline 2 & 7.I & & \\
\hline 3 & 4.I & & \\
\hline o & 26.0 & .286 & Do. Aspirator more constant. \\
\hline I & 14.4 & & \\
\hline 2 & 6.9 & & \\
\hline 3 & 3.9 & & \\
\hline
\end{tabular}

TABLE 4.-RÉSUMÉ.

\begin{tabular}{|c|c|c|}
\hline & $a \times 10^{3}$ & $a \times 10^{3}$ corrected. \\
\hline 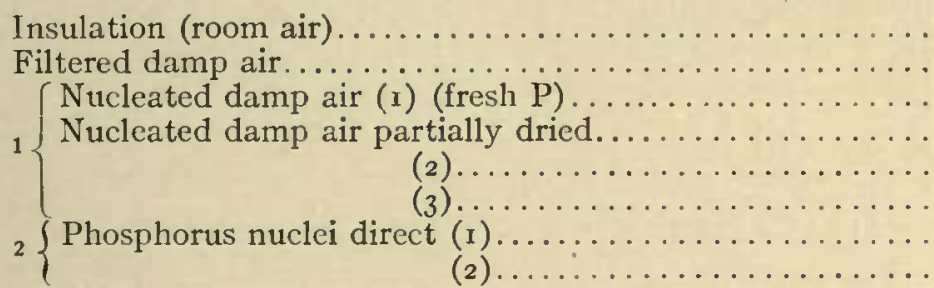 & $\begin{array}{r}8-10 \\
11 \\
24 \\
21 \\
17 \\
18 \\
260 \\
290\end{array}$ & $\begin{array}{r}0 \\
14 \\
11 \\
7 \\
8 \\
250 \\
280\end{array}$ \\
\hline
\end{tabular}

1 From condensation chamber.

2 From ionizer.

The charge in the condenser is negative as before. It should be more rapidly dissipated if negative ions are precipitated more rapidly in the receiver, than a positive charge. To obtain dense nucleation, room air was again aspirated over phosphorus into the receiver, from which it was then discharged as expeditiously as possible, the time taken being from $5^{- \text {ro minutes. }}$

Io. . Effect of different charges in the condenser. - In the next experiments the sign of the eharge was varied. To find comparable results it was thus necessary to maintain a definite current through the condenser, and about 2.5 liters per minute was adopted compatibly with the dimensions of the apparatus. 
Nuelei were again aspirated into the receiver over phosphorus. The leakage was apparently different for charges of opposed sign; but this was due to the insulation of the condenser, which is greater for negative than for positive charges. Deducting this, the values of the ionization, $a$, differ by quantities which lie within the errors of observation.

As the receiver in the course of the efflux of nucleated air shows fogs of continually inereasing density, the spontaneous precipitation must have been equally effective for positive and for negative nuelei.

TABLE 5--IONIZATION OF PHOSPHORUS NUCLEI. CHARGE IN CONDENSER AT 10 VOLTS. $d V / d t=2.5$ LITERS/MIN.

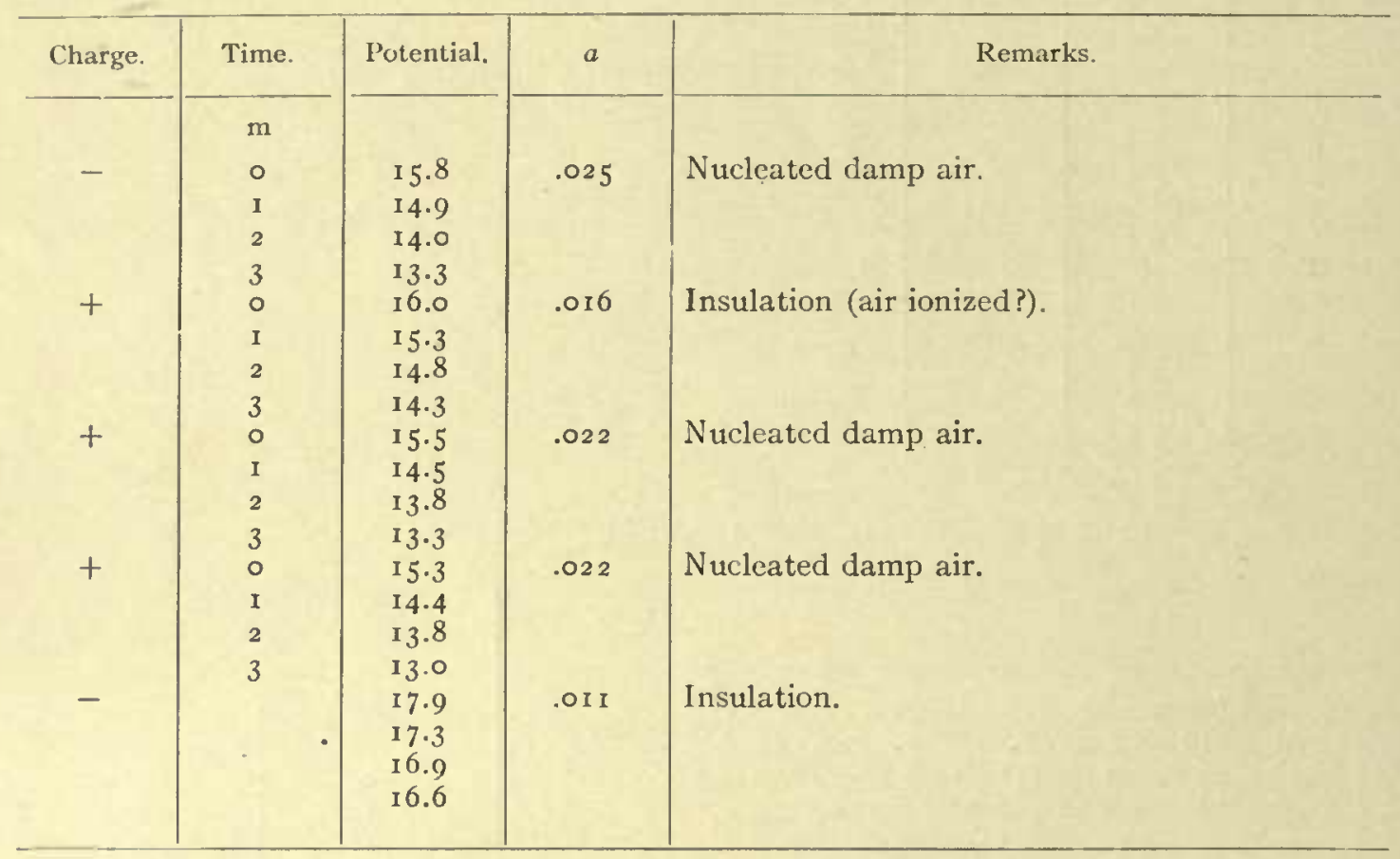

TABLE 6.-RESULTS.

\begin{tabular}{c|c|c|c}
\hline & $a \times 10^{3}$ & $\begin{array}{c}a \times 10^{3}(\text { cor- } \\
\text { rected.) }\end{array}$ & Insulation $a \times 10^{3}=10-16$. \\
\hline+ & & -9 \\
+ & 25 & +6 \\
+ & 22 & +6 \\
+ & 22 & -8 \\
- & 18 & +8 & \\
\hline+ & 24 & -7 & \\
\hline
\end{tabular}

In tables 7,8 , similar results are given, but with the insulation tested after each passage of nucleated air through the condenser. The results taken consecutively are shown in table 8. 
A CONTINUOUS RECORD OF ATMOSPHERIC NUCLEATION.

TABLE 7.-IONIZATION OF PHOSPHORUS NUCLEI. $d V / d t=2.5$ LITERS/MIN.

CHARGE $\Lambda$ T 20 VOLTS, $19.6 \mathrm{~cm}$. DEFLECTION.

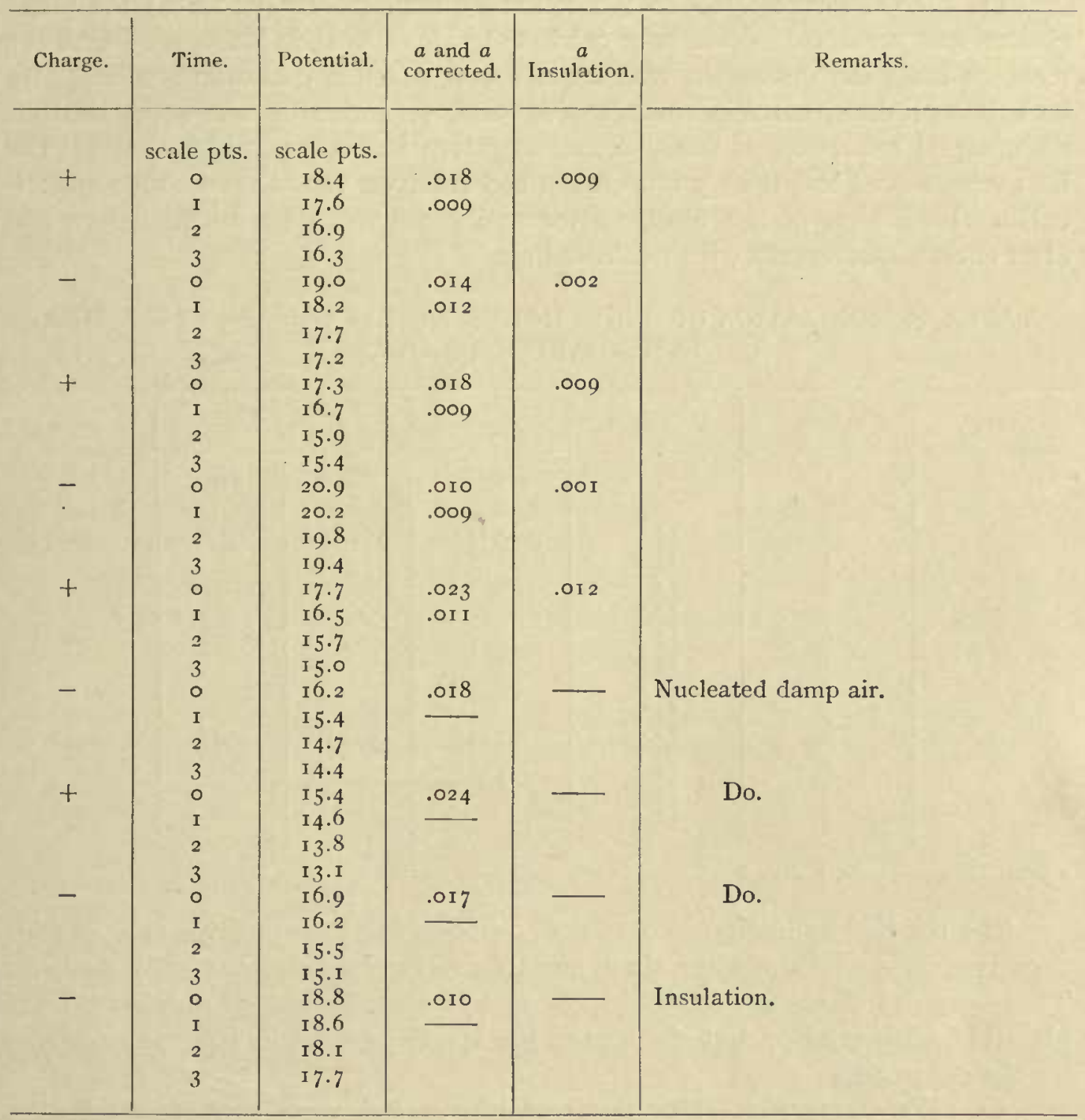

TABLE 8.-RÉSUMÉ.

$$
\left.\begin{array}{rr}
\text { Charge }+ & a=.009 \\
- & \text { 1 } 2 \\
+ & 09 \\
- & 09 \\
+ & \text { I I }
\end{array}\right\} \text { Mean .010 }=a
$$

There is slight excess of leakage for negative charges; but as the insulation was $\mathrm{ro}^{3} \times a=9^{-\mathrm{I} 2}$ for positive, and $\mathrm{I}-2$ for negative charges, these differences are within the uncertainties of observation. One may again note that if the 
dense spontaneous fogs in the receiver were associated with selective condensation, then negative charges should be more rapidly dispelled.

II. Dricd emanation. - In the following experiments (table 9) the phosphorus was previously dried over calcic chloride, and then introduced into the receiver from the desiccator and the shortest possible connecting tube. Dry ions thus suddenly came in contact with water vapor, and it was supposed that an unequal reduction of positive and negative ionization might ensue. The ions were stored less than 5 minutes in the receiver, the shortest time practicable. Insulation of the electrometer and parts was determined before and after each mcasurement with nucleated air.

TABLE 9.-IONIZATION OF PHOSPHORUS NUCLEI.* $d V / d t=2.5$ LIT./MIN. TESTED AFTER 4-5 MIN.

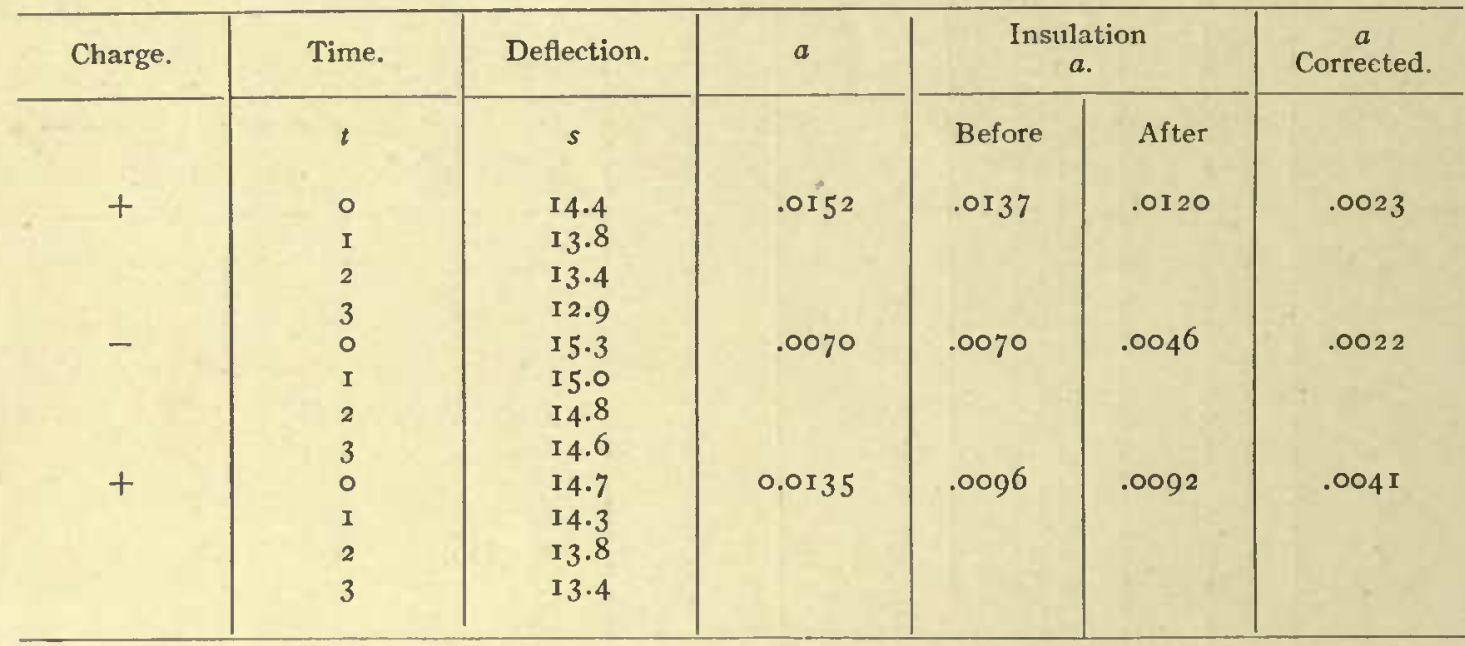

* Phosphorus nuclei dried over ealcic chloride and conveyed by a dry current of air, before storing over water

The residual ionization so obtained, $a=.002-.004$ for positive and .002 for negative charges, is smaller than heretofore, but again practically neutral. Thus very dry phosphorts nuclei seem to lose their ionization quicker than if placed in ordinary air; but changes of the activity of the ionizer may account for the difference.

12. Wet cmanation.-For contrast, the nuclei were conveyed into the receiver (table Io) in a wet current of air passing over phosphorts. A U-tube was used, one leg of which contained wet sponges and the other the phosphorus grid, the damp air from the former sweeping over the latter into the receiver. The ionization found is distinctly greater ${ }^{1}$ than the dry air data of the last table, though it does not exceed the usual values for room air. The difficulty of keeping the ionizing activity constant is again involved. According to the table, positive charges are more rapidly discharged than negative charges, which would indicate an excess of negative ions comparable to the case of water nuclei in the next chapter.

\footnotetext{
I American Fournal of Science (4), XI1, p. 327, Igor.
} 
A CONTINUOUS RECORD OF ATMOSPHERIC NUCLEATION.

TABLE $10 .-$ IONIZATION OF PHOSPHORUS NUCLEI.' $d V / d t=2.5$ LIT./MIN. TESTED AFTER 5-10 MIN.

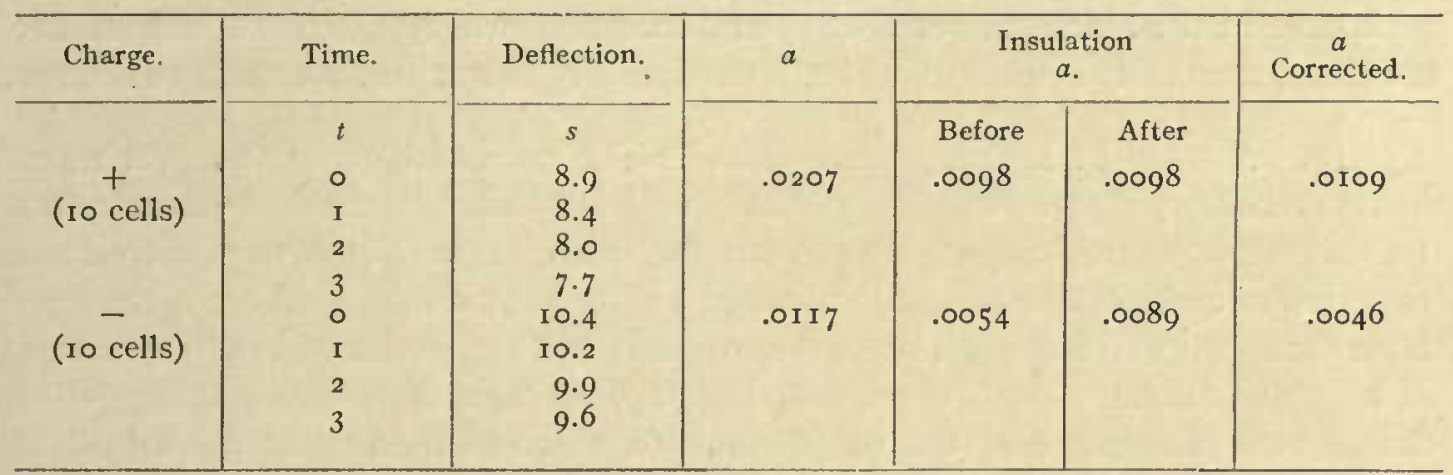

1 Phosphorus nuclei conveyed in a damp current of air. Tested for coronas, the nucleated air of the receiver shows the usual strength.

13. Residual ionization after one hour.-The storage of phosphorus nuclei over water in the above experiments did not exceed ro-I5 minutes. It was thought that by giving the fog particles more time to subside, the sign of the residual ionization might become apparent, supposing that more negative nuclei are precipitated. The results in table II are peculiar: whereas the positive charges in the presence of the nucleation vanish more slowly than for room air, the negative charge vanishes much faster. This would make an

TABLE II.-RESIDUAL IONIZATION OF PHOSPHORUS NUCLEI AFTER ONE HOUR. $d V / d t=2.5$ LIT./MIN. CHARGE AT ABOUT 20 VOLTS, OR LESS.

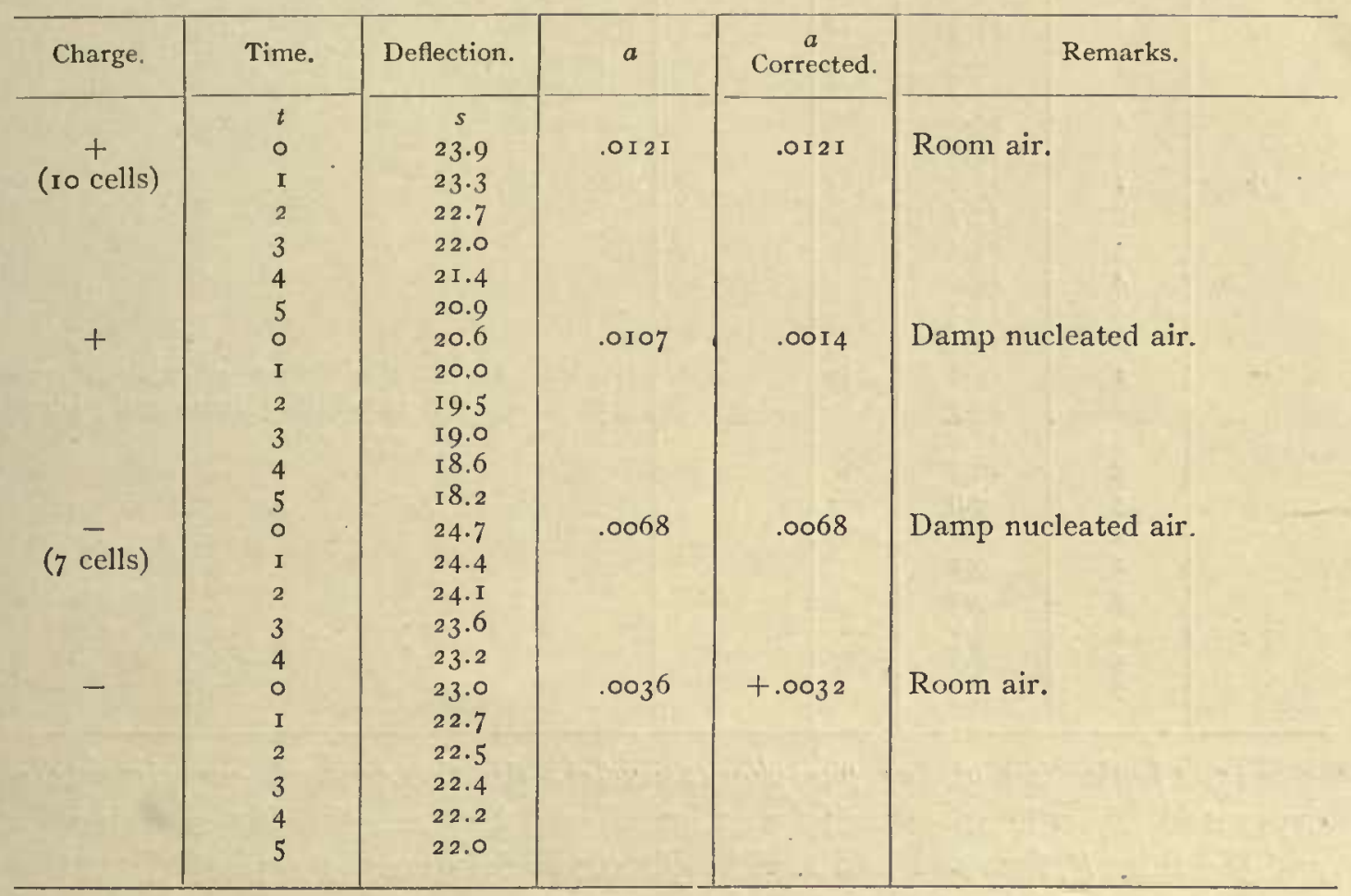


excess of $a=.005$ of residual positive nucleation over the negative nucleation, and it would follow that the negative nuclei are precipitated faster. But with a leakage in the electrometer of .OI I and .004 respectively, without any ionized medium, the result is not guaranteed, particularly as the positive leakage is large.

I4. Nucleation partially precipitated.-The nucleated air stored over water in the receiver, $A$, was suddenly cooled and allowed to subside $5^{- \text {Io minutes. }}$ In this way greater chance was given for the differentiation of positive and negative nuclei. The corrected values of $a$ show that positive charge is removed faster than negative charge, by $a .=.0010$ and .004 respectively, implying excess of negative nuclei. With the insulation varying from .003-.o 10 and .003-.013, before and after some of the measurements, this result is again doubtful. It should be noticed that it is the reverse of the preceding.

TABLE I2.-RESIDUAL IONIZATION AFTER PARTIAL PRECIPITATION. $d V / d t=2.5$ LIT./MIN. CHARGE AT IO VOLTS.

\begin{tabular}{|c|c|c|c|c|c|}
\hline Charge. & Time. & Deflection & $a$ & $\begin{array}{c}a \\
\text { Corrected. }\end{array}$ & Remarks. \\
\hline & $\begin{array}{l}t \\
0\end{array}$ & $\begin{array}{c}s \\
13.0\end{array}$ & \multirow[t]{4}{*}{.013} & & \multirow{3}{*}{$\begin{array}{l}\text { Nucleated air. Subsiding } 4^{\mathrm{m}} \text { without ex- } \\
\text { haustion and } 6^{\mathrm{m}} \text { with exhaustion. Insu- } \\
\text { lation before } .003=a \text {. }\end{array}$} \\
\hline \multirow{2}{*}{ (5 cells) } & I & I 2.9 & & \multirow{2}{*}{.004} & \\
\hline & 2 & $\times 2.4$ & & & \\
\hline \multirow{3}{*}{ - } & 3 & I 2.0 & & & \\
\hline & $\begin{array}{l}\mathrm{I} \\
2\end{array}$ & $\begin{array}{l}\text { I } 1.0 \\
\text { I0. } 6\end{array}$ & \multirow[t]{2}{*}{.015} & \multirow[t]{2}{*}{0} & \multirow{2}{*}{ Room air. } \\
\hline & 3 & 10.3 & & & \\
\hline \multirow[t]{2}{*}{+} & $\begin{array}{l}4 \\
1 \\
2 \\
3\end{array}$ & $\begin{array}{r}9.9 \\
17.4 \\
16.6 \\
\text { I } 5.6\end{array}$ & \multirow[t]{2}{*}{.022} & \multirow[t]{2}{*}{.014} & \multirow[t]{2}{*}{$\begin{array}{l}\text { Nucleated air. Subsiding } 4^{\mathrm{m}} \text { without ex- } \\
\text { haustion and } 8^{\mathrm{m}} \text { with exhaustion. Insu- } \\
\text { lation before } .002=a \text {. }\end{array}$} \\
\hline & 4 & I 5.0 & & & \\
\hline \multirow[t]{4}{*}{$t$} & $\begin{array}{l}5 \\
1\end{array}$ & $\begin{array}{l}\text { I } 4.1 \\
\text { I } 3.8\end{array}$ & \multirow[t]{4}{*}{.013} & & \multirow[t]{4}{*}{ Room air. } \\
\hline & $\begin{array}{l}2 \\
2\end{array}$ & I $3 \cdot 7$ & & & \\
\hline & $\begin{array}{l}3 \\
4\end{array}$ & $\begin{array}{l}13.4 \\
13.0\end{array}$ & & & \\
\hline & $\begin{array}{l}5 \\
6\end{array}$ & $\begin{array}{l}\text { I } 2.4 \\
\text { I } 2.1\end{array}$ & & & \\
\hline \multirow[t]{4}{*}{ - } & I & I I. 8 & \multirow[t]{2}{*}{.019} & \multirow[t]{2}{*}{.010} & \multirow{3}{*}{$\begin{array}{l}\text { Nucleated air. Subsiding } 4^{\mathrm{m}} \text { without } \mathrm{cx} \\
\text { haustion and } \mathrm{I} 2^{\mathrm{m}} \text { with exhaustion. Insu- } \\
\text { lation before } .0079=a \text {. }\end{array}$} \\
\hline & 2 & II. 5 & & & \\
\hline & $\begin{array}{l}3 \\
4\end{array}$ & $\begin{array}{l}\text { II.0 } \\
\text { I0.5 }\end{array}$ & & & \\
\hline & 5 & 9.9 & \multirow{5}{*}{.009} & & \multirow{5}{*}{ Room air. } \\
\hline \multirow{4}{*}{ - } & I & 9.6 & & & \\
\hline & 3 & 9.2 & & & \\
\hline & 4 & $\begin{array}{l}9.0 \\
88\end{array}$ & & & \\
\hline & 6 & 8.5 & & & \\
\hline
\end{tabular}

I5. Ionization of $d r y$ phosphorus nuclei.-In the present experiments a dry vessel of ro liters capacity was introduced between the tower desiccator. and the tube, $P$, figures I and 2. Phosphorus nuclei were now aspirated into this 
vessel. They were discharged after 5 minutes into the condenser after removing the phosphorus tube. Table $\mathrm{r} 3$ shows that almost all the ionization is lost by this dry storage as the excess of leakage due to the discharge of the dry air through the condenser is $a=.000$ and .007 , respectively. Thus dry air shows no preservative effect.

TABLE I3.-RESIDUAL IONIZATION AFTER DRY STORAGE OF PHOSPHORUS NUCLEI. ${ }^{1} d V / d t=2.5$ LIT./MIN. CHARGE AT ro VOLTS.

\begin{tabular}{|c|c|c|c|c|c|}
\hline Charge. & Time. & $\begin{array}{c}\text { Deflection. } \\
s .\end{array}$ & $a$ & $\stackrel{a}{\text { Corrected. }}$ & Remarks. \\
\hline & $t$ & & & & \\
\hline- & $\circ$ & 14.4 & .006 & .000 & Phosphorus stored dry $2-5^{\mathrm{mI}}$ \\
\hline ( 5 cells) & I & 14.1 & & & \\
\hline & 2 & I 3.9 & & & \\
\hline & 3 & I $3 \cdot 7$ & & & \\
\hline 一 & $\circ$ & II.7 & .006 & & Room air. \\
\hline & I & I I. 6 & & & \\
\hline & 2 & I I.4 & & & \\
\hline & 3 & I I.3 & & & \\
\hline+ & $\circ$ & I $3 \cdot 7$ & $.01 \mathrm{I}$ & .007 & Phosphorus stored dry $2-5^{\mathrm{m}}$ \\
\hline & I & I $3 \cdot 4$ & & & \\
\hline & 2 & I 3.0 & & & \\
\hline+ & $\begin{array}{l}3 \\
0\end{array}$ & $\begin{array}{l}\text { I } 2.6 \\
\text { I I. } 7\end{array}$ & .004 & & Room air. \\
\hline & I & I 1.6 & & & \\
\hline & 2 & I r. 5 & & & \\
\hline & 3 & II.4 & & & \\
\hline
\end{tabular}

Intense antecoronal fogs obtained in the exhausted receiver, $A$, due to back motion of the nuclei into the receiver through the aspirators.

In table 14 , by a modification of the apparatus, a current of dry air passes from the desiccator over phosphorus, and then into one end of a dry vessel of Io liters capacity. At the other end of the vessel the air is continually discharged into the condenser. But the usual negative result appears.

TABLE I4.-DRIED PHOSPHORUS IONS PASSED INTO A DRY VESSEL AND THEN CONTINUOUSLY INTO THE CONDENSER. $d V / d t=2.5$ LIT./MIN. CHARGE AT Io AND 20 VOLTS.

\begin{tabular}{|c|c|c|}
\hline Charge. & $a$ & Remarks. \\
\hline $\begin{array}{c}- \\
\text { (Io cells) } \\
\text { ( } 5 \text { cells) } \\
-\end{array}$ & $\begin{array}{l}.017 \\
.000 \\
.012 \\
.000\end{array}$ & $\begin{array}{l}\text { P ions. } \\
\text { Room air before and after. } \\
\mathrm{P} \text { ions. } \\
\text { Room air before and after. }\end{array}$ \\
\hline
\end{tabular}

I6. Inferences. - The chief result of the investigation is the enormously rapid reduction of the ionization of the phosphorts emanation in contrast with the persistence of the nucleation. In other words, only a few per cent. of the 
original ions are associated with the nuclei by which the dense fogs and coronal sequences are produced, even at the outset. It was with a view to possibly restoring some of this lost ionization that the great variety of experiments detailed in the chapter were undertaken; but not a trace of restoration was observable in any case.

Moreover, the whole of the original ionization vanishes symmetrically, for the nuclei as a whole are neutral throughout. At least with so insignificant a residue of the original ionization, the decision as to whether more positive or more negative ions have vanished is a delicate one and of trifling interest in this connection. For the phenomena are now all of the order of the leakage of the electrometer and appurtenances. If when roo ionized nuclei of the phosphorus emanation are suddenly introduced into an atmosphere saturated with water vapor, the ionization of 96 has vanished without a record, while the remaining 4 are in equal number positive and negative, it is unlikely that negative ions can have greater affinity for water vapor or be more remarkable in their efficiency as condensation nuclei than positive ions.

Finally, it does not appear that the ionization lost so soon after the removal of the emanation from the phosphorus surface can ever be restored, notwithstanding the fact that in the condensation chamber nuclei may be made to pass from the fog particle to the nuclear stage of size and density an indefinite number of times. Hence it follows that it is the ante-nuclear stage by which the ionization is introduced.

In one respect the experiments with phosphorus are unsatisfactory: it takes some time before the condensation chamber can be adjusted for condensation on the phosphorus nucleus as it is necessary to introduce the nucleation from without. Placed within the chamber over water, phosphorus emits a dense filament of smoke, and is relatively inefficient. With water nuclei there is no such difficulty; for here the nuclei are most efficiently produced in the condensation chamber itself, while the ionization may be studied without loss of time. The results are given in the succeeding chapter.

As a whole, the experiments agree well with the original hypothesis, that nuclei and ions are distinct entities; that the former constitute the residual product left after the corpuscles representing the ionization have been expelled. Radio-activity in case of the relatively gentle breakdown of molecular structure here in question can hardly be anticipated. If a nucleus, like that of phosphorus, for instance, shows a tendency to grow continuously, until it finally appears as part of a visible smoke, there may be continuous ejection of electrons. In such a case electric conduction through the gas freighted with these nuclei would follow Ohm's law, as is actually the case for phosphorus. 


\title{
CHAPTER II.
}

\author{
RELATION OF THE IONIZATION AND THE NUCLEATION ASSOCIATED WITH WATER \\ NUCLEI, PRODUCED IN AIR.
}

\section{IONIZATION PRODUCED BY SHAKING SOLUTIONS.}

I. Introduction.-In the present chapter there will be three subjects for consideration. The endeavor must first be made to detect ionization in water nuclei, produced as in the former memoir, ${ }^{1}$ by shaking solutions. As the ionization so recognized proves to be inadequate, the problem next in order will

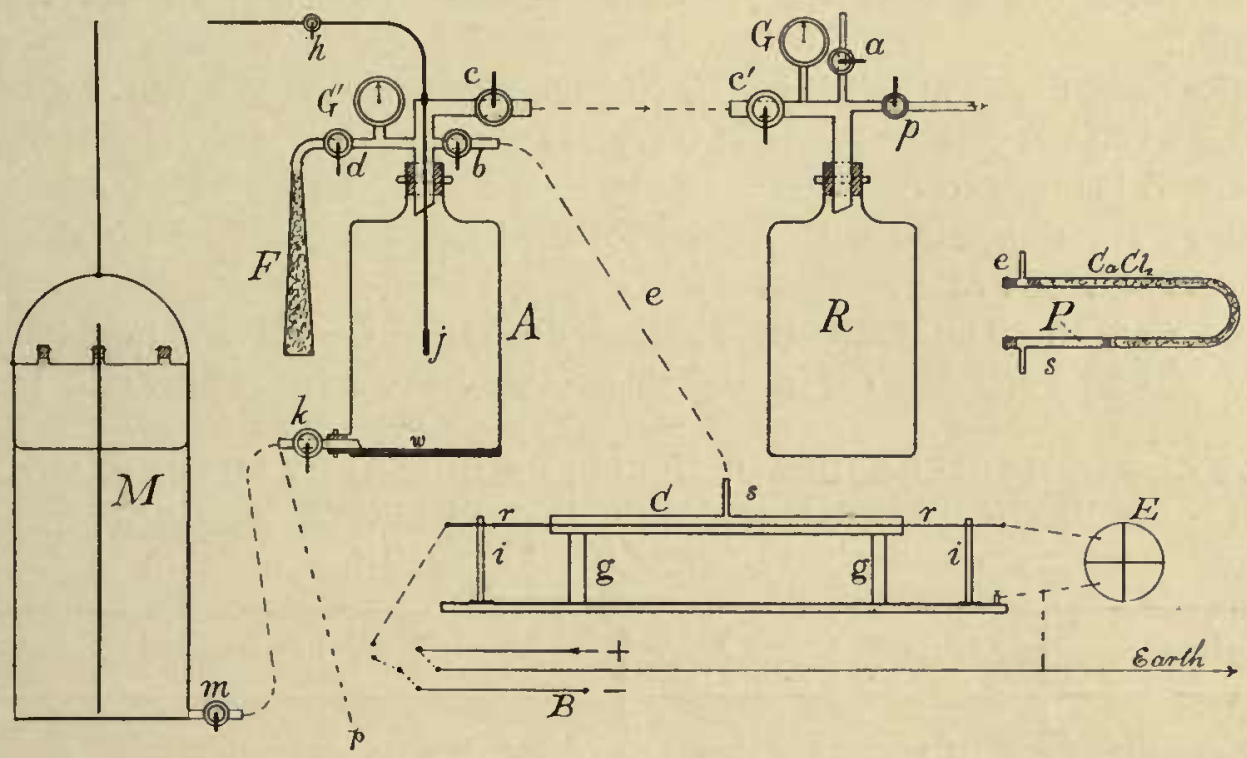

Figure I.-Apparatus for Comparing Nucleation and Ionization. $A$, Coronal Chamber; $R$ Exhaustion Reservoir; $M$, Mariotte Flask; $C$, Tubular Condenser; $P$, (to be Insertedin the Conveyance Tube, $e$ ), Phosphorus Ionizer; $G, G$, Vacuum Gauges; $F$, Cotton Filter; $B$, Storage Battery Terminals. Tubes, $p$ to Suction Pump, $a$ to Atmosphere, $c, c$, for Exhaustion, $e$ for Conveying the Nuclei into the Condenser, $d$ for Filtration, $h$ from the Hydrant. Extremely fine Jets of Water Shoot out from time Needle-pricked Lead Pipe, $j$. Supports $g, j$, are Metallic, $i, i$, InsuLATING.

be the production of the maximum number of nuclei per cubic centimeter possible, and will be considered in the second section of the chapter. The third will then take up and examine the ionization produced along lines very similar

' Structure of the Nucleus, Chap. V. 
to those of the preceding chapter, and will lead to an examination of the inferences already drawn for phosphorus nuclei.

2. Apparatus.-To detect the electrification of nuclei produced by shaking, a ro per cent. solution of sodic sulphate was employed, as this had been shown to produce the nuclei in largest numbers under otherwise like conditions. ${ }^{1}$ The solution was used both to generate the nuclei by vigorously shaking the large aspirator flask, $A$, figure $\mathrm{I}$, containing a small amount of the solution at the bottom, $w$, as well as to discharge the nuclei into the condenser, $C$, by filling $A$ with the liquid coming from the large Mariotte flask, $M$. Raising or lowering the latter enabled the observer either to add liquid to $A$, or to withdraw it. When a constant pressure was needed the device shown in the preceding chapter (figure 2) was used, and the liquid passed along a side tube. The nucleated air is discharged by the pipe, $e s$, into the tubular condenser, $C$, entering at $s$. The core of the condenser is highly charged and connected with one pair of quadrants of the electrometer, $E$, the other pair being earthed and the needle kept charged by a water battery, whose other pole is also earthed. The supports, $i i$, are of hard rubber, those $(g g)$ of the outer tube of the condenser are metallic, and tugether with the base of the apparatus and the pipe, $e s$, are kept at zero potential.

The operations are evident. Having charged the core, the rate of discharge is found when the current of nuclei traverses the condenser, the volume supplied per second being read off on a scale attached to $A$. Results for nucleated air (shaking) are alternated with results for dust-free air obtained by aid of the filter and stopcock at $F$.

3. Results.-The results (two independent series) are given in the following tables. The season was damp and unsuitable for electrostatic work, but the

TABLE 1.-ELECTRIC CHARGES OF NUCLEI PRODUCED BY SHAKING A DILUTE SOLUTION OF $\mathrm{Na}_{2} \mathrm{SO}_{4}$. CHARGE IN CONDENSER, 40 VOLTS. $a=d(\log s) / d t ; d V / d t$ IN LIT./MIN.

\begin{tabular}{|c|c|c|c|}
\hline Condenser at & $d V / d t$ & $a$ & Remarks. \\
\hline $\begin{array}{l}+40 \text { volts } \\
-40 \\
+40 \\
-40\end{array}$ & $\begin{array}{l}\text { I. } 7 \\
\text { I. } 7 \\
\text { I. } 7 \\
\text { I. }\end{array}$ & $\begin{array}{l}.020 \\
.022 \\
.020 \\
.022\end{array}$ & $\begin{array}{l}\text { After vigorous shaking. } \\
\text { " } \\
\text { Without shaking. }\end{array}$ \\
\hline
\end{tabular}

TABLE 2.-Same as preceding.

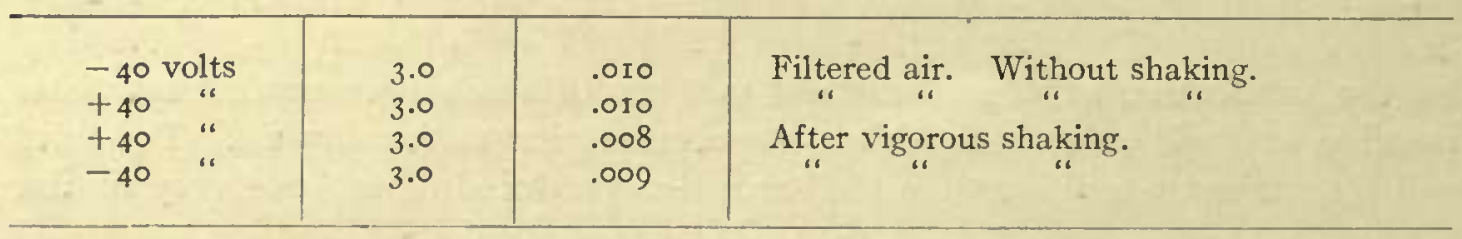

'Structure of the Nucleus, p. I 2 I. 
data are sufficient to indicate the extreme smallness of the effect to be observed. Ohm's law being assunced as usual, as a rough approximation to the truth, the readings in scale parts, $s$, are read off, at the successive times, $t$, of observation. The constant then follows as $a=d(\log s) / d t$ and $d V / d t$ denotes the number of liters of air, nucleated or not as stated, put through the condenser. In table I the leakage, $a$, is larger for negative than for positive charges, but the effect is equally large no matter whether the influx is dust-free and filtered or whether the nuclei produced by shaking traverse the condenser. Shaking is thus without an electrical effect, so far as can here be discerned. What has been observed in both cases is a continuous drift of the needle.

In table 2, for another adjustment, the positive and negative charges leak out equally fast when dust-free air constitutes the medium of the condenser. When shaken nuclei circulate through it, the negative charge leaks out more rapidly than the positive charge, implying positively charged nuclei. But as the result is no larger than for dust-free air, it is again probable that the mere drift of the needle is being observed.

The results from both tables are therefore negative, showing that the excess of leakage for a charge of one sign over that of another must be of the order of $.00 \mathrm{I}$ when referred to minutes, if the nuclei in question are produced by shaking. This result, however, is not unexpected; for it is not more than about a thousand nuclei that are here available, and with the necessarily small charge residing on each, a detection of the effect will not easily be accomplished in connection with moist air. The following pages, moreover, will show how quickly the charge vanishes, and I am not sure that the experiments were made expeditiously enough. Hence I waived the experiments temporarily, to be resumed with a more efficient method of producing water nuclei and during the dryer atmospheric conditions of winter.

THE EFFICIENCY OF NUCLEI-PRODUCING JETS.

4. Powerful methods of comminution.-It appears from the preceding section and elsewhere that the number of nuclei produced by shaking is relatively very small, and the coronas correspondingly simple. To obtain more nuclei a much more violent method of comminution must be resorted to, such as is given if fine jets of water, generated under high pressure, are shattered either against a solid obstacle or against each other. Fortunately, ordinary hydrant water contains enough solute to answer the requirements, and the construction of the jet is thus a straightforward problem. It will be found that for each jet there is a maximum of productivity, and one is thus able to make a series of jets, each corresponding to a definite number of nuclei under like conditions. Each jet has a definite saturation number, and while the maximum saturation producible in this way is naturally far below the efficiency of phosphorus and other chemical ionizers, so far as nuclei are concerned, the aggregate ionization is not very different. 
In table 3 the results obtained with jets of a variety of patterns and numbers are given in detail. They were all fed with hydrant water at $60-70$ pounds pressure. In these experiments the jet, $j$, replaced the phosphorus, the nuclei being actually produced from the spray in the vessel, $A$, figure $I$. Details of a simplified form are given in figure 2, where $j$ is the jet to be tested, screwed to a brass pipe, $f$, joined by gas couplings (unions at $U$, etc.) to the pipe $h$ from the hydrant. In many of the jets the spray is broken against the sides of the vessel, this being the most efficient mode of comminution. The excess of water is carried off by the cock, $k$, for which there is a side branch, $p$, with a special stopcock. When $k$ is closed, the jet may often be used to discharge its own

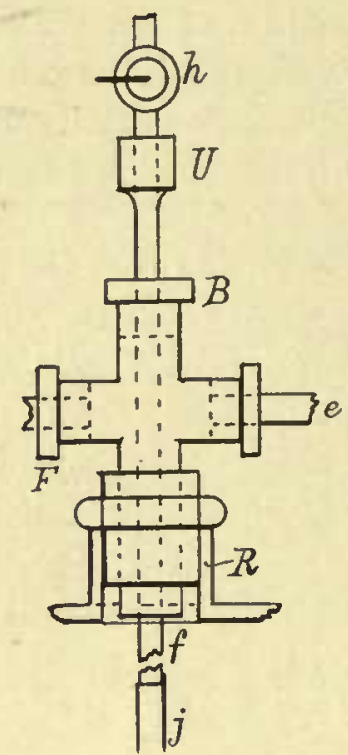

Figure 2.-Detail (Simplified) of $A$, Fig. I

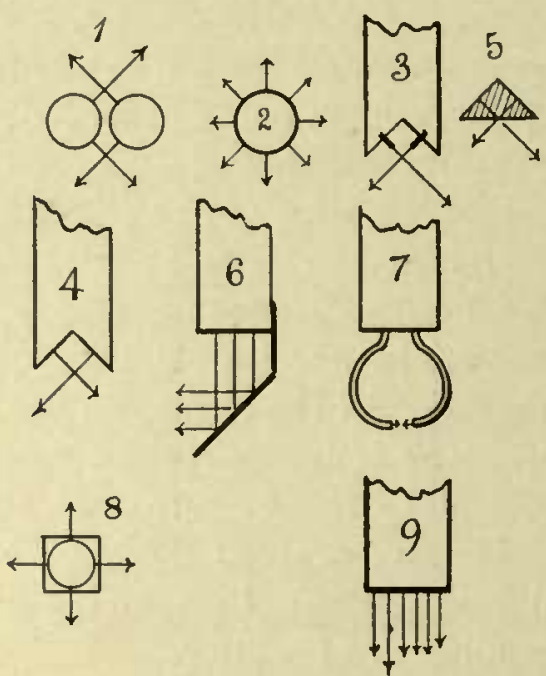

Figure 3.-ForMS OF IMPINGING JETS.

nuclei into the condenser without the intervention of the Mariotte flask, $M$, figure $\mathrm{r}$.

The types of jets used are shown in figure 3 in cross section, and the numbers in the table correspond to those of the figure. In No. I, two parallel eighthinch lead pipes emit jets from their sides in such a way as to impinge on each other nearly at right angles. It was not possible to completely or continually shatter both jets mutually in this way. In No. 2, radial jets from a quarterinch lead pipe impinge on the walls of the receiver; in No. 8 the pipe has been thinned. In No. 3 two capillary adjutages produce jets which impinge on each other, and the same is the case, with evident modifications, in Nos. 4 and 7 . In No. 6 an oblique lead buffer has been added, while in No. 9 the jets issue from a finely perforated copper plate, and impinge turbulently on the pool of water below. In addition to these, ordinary lava tips (No. 5) and other steatite jets were used.

The holes were usually pricked with fine cambric needles. Of all the jets, 
Nos. 2 and 8 were not only the simplest but the most efficient relative to the quantity of water consumed. Adjutages introducing resistances gave inferior results, but are needed for special purposes. To change the efficiency at will, the number of holes may be varied from two to fifty.

5. Results.-In table 3 the description of the jet, the corona produced and its serial number, together with the number of nuclei per cubic centimeter corresponding, are given in each case. Certain data of the aperture, $s$, are sometimes added for identification. For convenience in comparisons, a brief table of coronas is subjoined to the next table.

TABLE 3.-NUMBER OF NUCLEI PRODUCED BY DIFFERENT JETS. WATER PRESSURE, 60-70 lbs.

\begin{tabular}{|c|c|c|c|c|c|}
\hline & Kind of Jet. & Corona. & $s$ & $n$ & Remarks. \\
\hline $\begin{array}{l}\text { No. } \\
\text { ", } \\
\text { ", } \\
\text { ", } \\
\text { ", } \\
\text { ", } \\
\text { ", } \\
\text { " } \\
\text { ", } \\
\text { ", }\end{array}$ & $\begin{array}{l}\text { I. One jet. } \\
\text { I. Two impinging jets. } \\
\text { I. " " } \\
\text { 1. " } \\
\text { I. Twenty . " } \\
\text { 2. Fifty radial jets. } \\
\text { 2. Ioo " } \\
\text { 3. Short adjutages. } \\
\text { 3. Do. Mouths flat. } \\
\text { 3. Finer holes. } \\
\text { 3. " } \\
\text { 4. Six jets. } \\
\text { 6. Threc " } \\
\text { 7. Two " } \\
\text { 8. Thirty " }\end{array}$ & $\begin{array}{l}\text { Simple } \\
\text { ". } \\
\text { ". } \\
g^{\prime} \text { b r } \\
g^{\prime} \text { b r } \\
\text { g b r } \\
\text { gy o b } \\
\text { w c g } \\
\text { w r } \mid g \\
\text { w r g } \\
\text { g'|b|r } \\
\text { w r } \mid g \\
\text { w r g } \\
\text { w p b g r }\end{array}$ & $\begin{array}{l}2.5 \\
4.3 \\
4.2 \\
4.5 \\
6.0\end{array}$ & $\begin{array}{r}6000 \\
30000 \\
29000 \\
40000 \\
80000 \\
100000 \\
80000 \\
80000 \\
50000 \\
30000 \\
30000 \\
40000 \\
30000 \\
25000 \\
100000\end{array}$ & $\begin{array}{l}\text { After } 5_{10^{\mathrm{m}}} \text { spraying. } \\
\text { Jets .04 } \mathrm{cm} \text {. diam. } \\
\text { (Lead walls too thick.) } \\
\text { Jets . I I cm. diam. } \\
\text { Jets .05 } \mathrm{cm} \text {. diam. } \\
\text { Punctured sheet lead. } \\
\text { Pe3 } \\
\text { Jets .05 } \mathrm{cm} \text {. diam. } \\
\text { ".05 " }\end{array}$ \\
\hline
\end{tabular}

It is frequently difficult to place the coronas, and for this reason, several cases, one of which is given in table 4 , were investigated by successive exhaustions, as explained in the preceding memoir. The identification of the coronas is then more easily possible, beginning with the green corona. The table also contains direct measurements of the successive apertures, with the number of particles computed therefrom. The results show a peculiar periodic discrepancy, the nature of which will be treated at length elsewhere. ${ }^{1}$ For the present, the apertures serve merely for the identification of coronas.

The results obtained for jets will conveniently be discussed in the next section in connection with the electrical data there set forth. Here I need only point out that the maximum nucleation obtainable with jets is obviously dependent on their pressure, number, fineness, etc., and probably on the degree with which the air current simultaneously generated has been removed. The presence of this current eventually sweeps out the nuclei as fast as they are generated, and for this reason it makes no difference whether one begins with 
filtered or with room air. A jet free from air currents should be most efficient, cat. par. Curiously, the maximum nucleation obtainable with jets lies just TABLE 4.-EFFECTS OF SUCCESSIVE EXHAUSTION. WATER NUCLEI. ESTIMATED NUCLEATION.

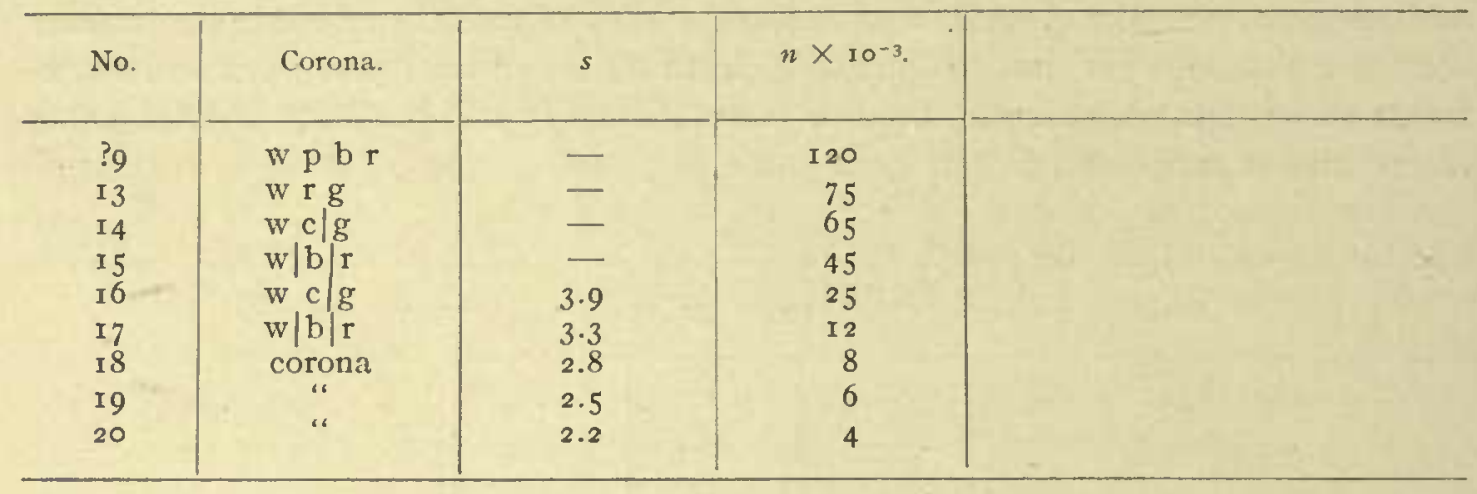

Nucleations corresponding to successive coronas:

\begin{tabular}{r|l|l|l|l|l}
\hline No. 5 & olive & - & No. I3 & w, rg & 75000 \\
6 & w y & 200000 & I & w clg & 65000 \\
7 & w o & I80000 & I & w p cor & 55000 \\
8 & w r & I60000 & I6 & g' b p & 45000 \\
9 & w c & I40000 & I & w r g & 35000 \\
I0 & w p & I20000 & I8 & w c g & 25000 \\
I I & g b p & I00000 & I9 & coronas & 20000 \\
I2 & g'o & 90000 & & & \\
\hline
\end{tabular}

below the maximum nucleation of atmospheric air as found in the winter observations. Probably this is a mere coincidence.

THE IONIZATION OF WATER NUCLEI.

6. Introductory.-In my report to the Smithsonian Institution (August, 1902) and elsewhere, ${ }^{1}$ I pointed out the desirability of further investigations on the Lenard ${ }^{2}$ effect. While my work in this direction was in progress, a paper due to J. J. Thomson ${ }^{3}$ appeared covering similar ground. Nevertheless, I shall venture to publish the following results, since the subject is looked at from a somewhat different point of view, obtained from coronal and other measurements. My chief purpose, however, was to find in what degree the theory given in my experiment with Ionized Air ${ }^{4}$ was to be modified to meet the conditions

'Science, $\mathrm{xvI}$, p. 633,1902 .

2 Lenard, Wied. Ann., XLvi, p. 584, 1892.

${ }^{3} \mathrm{~J}$. J. Thomson, "Experiments with Induced Radioactivity of Air, and on the Electrical Conduction Produced in Gases when they Pass through Water," Phil. Mag. (6), Iv, p. 352, September, 1902.

- Smithsonian Contributions to Knowledge, I309, Igor. 
for water nuclei. I have not, however, with the data now in hand been able to complete this to my satisfaction, and have for this reason confined the present chapter to experimental work, to the exclusion of theoretical considerations of the kind given tentatively elsewhere. ${ }^{1}$

7. Apparatus.-If in the receiver, or condensation chamber, $A$, the metallic pipe, $e$, joining at $b$, leads directly to the tubular condenser, $C$ (radii I.05 and .34 cm., length, $50 \mathrm{~cm}$.), the apparatus, figures I, 2, takes the form adapted for measuring the initial ionization of the nuclei. If the cocks $k$ and $d$ (filter) are closed and the fine radial jets are put in action by opening the water faucet $h$, the charged air is gradually expelled through $b$ as the water level in $A$ rises. When an efficient jet is used the rate is usually about 2 liters/minute. This velocity may be increased or diminished by aid of the flask, $M$, figure $I$, attached at $k$.

Since the jets, $j$, impinge on the walls of the vessel, this is kept uniformly moist or better coated with water, and therefore continually put to earth by the hydrant connection. Similarly the pipe, $e$, leading to the outer coating of the condenser is with this continually put to earth. The core of the condenser, insulated by long hard rubber supports, retains charge well even at high potentials, and in spite of the damp gases, because of the remoteness of the supports.

8. Results. Initial charges.-The following table gives the data obtained, when the nuclei generated by the spray are at once passed into the tubular condenser, whose inner surface is charged as stated, the outer being put to earth.

TABLE 5.-IONIZATION OF WATER NUCLEI. $d V / d t=2$ LIT./MIN. TO'TAL CAPACITY OF ELECTROMETER AND CONDENSER, $72 \mathrm{~cm}$.

\begin{tabular}{|c|c|c|c|c|}
\hline Condenser charge. & Insulation before. & $a$ & Insulation after. & $d s / d t . \mathrm{cm} . / \mathrm{min}$ \\
\hline+20 volts & $a=.013$ & $\begin{array}{l}.33^{8} \\
.490\end{array}$ & $a=.010$ & $\begin{array}{l}.79 \\
.71\end{array}$ \\
\hline-20 volts & $a=.020$ & $\begin{array}{r}.072 \\
.091 \\
.108\end{array}$ & $a=.002$ & $\begin{array}{r}.32 \\
.38 \\
.40\end{array}$ \\
\hline & & . 124 & & .40 \\
\hline+20 volts & $a=.022$ & $\begin{array}{r}.223 \\
.335 \\
.498 \\
.872\end{array}$ & $a=.015$ & $\begin{array}{l}.79 \\
.78 \\
.71\end{array}$ \\
\hline
\end{tabular}

The method consisted in testing the insulation of the condenser, immediately before and after the introduction of water nuclei. The table gives the deflection in centimeters, after intervals of I min., $\frac{1}{2}$ min., and I min., in each of the cases, respectively. The conduction, $a=\delta(\log s) / \delta t$, is computed by assuming Ohm's law; but in case of the medium of water nuclei, it is seen at once that Ohm's law does not apply, and that the conduction, $a$, increases enormously as 
the charge on the condenser vanishes. The average value of the conduction, moreover, is quite of the order of values found for phosphorus nuclei in the above tables, under similar circumstances. As the data in the table show $a$ for successive minutes, its variation in the last series is from .223 to .872 in 3 minutes.

Since 2.3 $a C=\mathrm{I} / R$, where $C=8 / \mathrm{ro}^{\mathrm{II}}$ farads is the capacity of the condenser and appurtenances, the initial and final resistance would be

$$
R=25 \times 10^{\circ} \text { and } R=6 \times 10^{\circ} \mathrm{ohms} \text {. }
$$

It follows then that if the equation of the current be taken, or $i \propto n(U+V) e(E / l)$ in the usual notation, the number of charged nuclei, $n$,
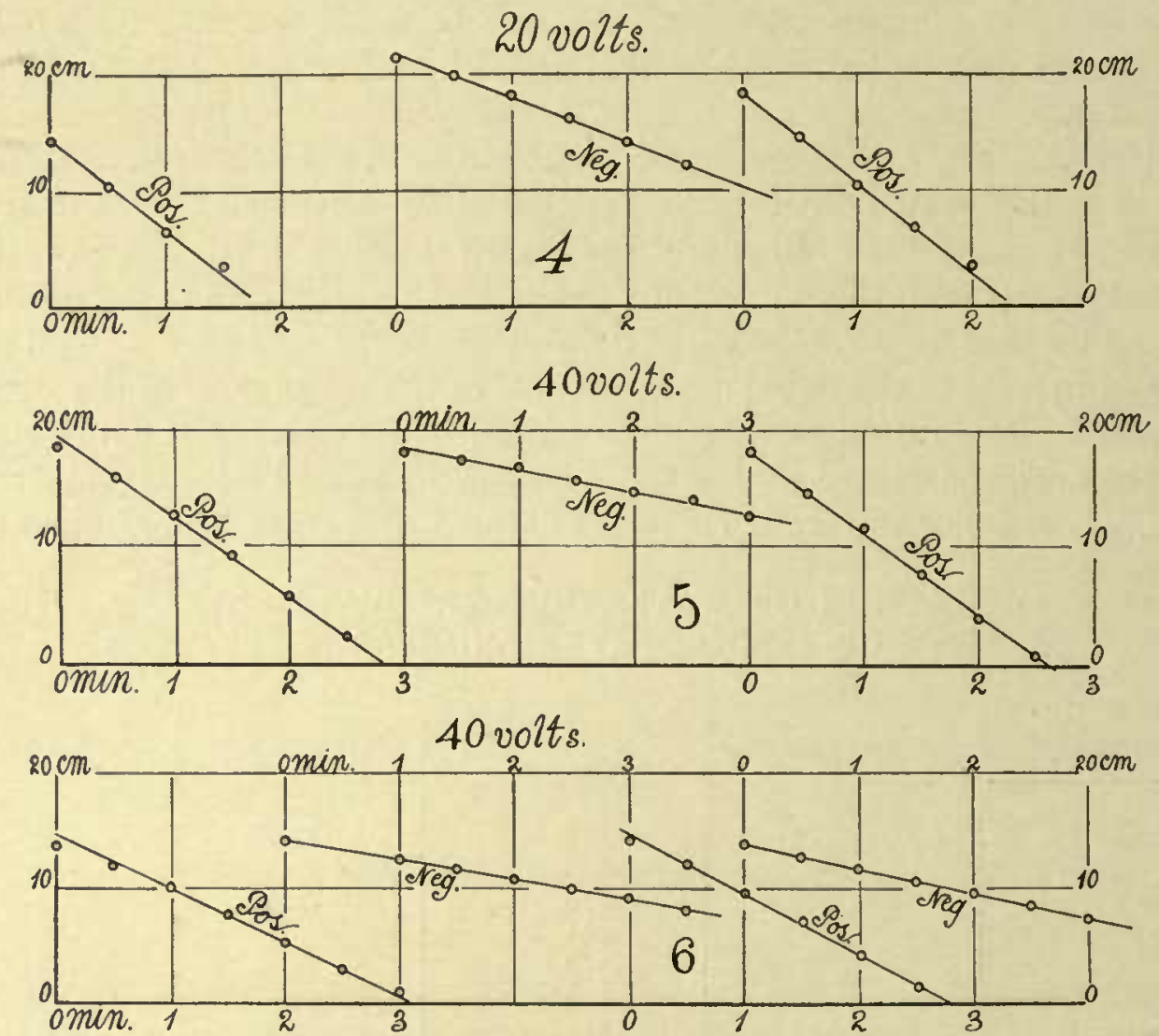

Figures 4, 5, 6.-Curves Showing Electrometer Deflection (Leakage) after consecutive Half Minutes, for Different Charges and Potentials in the Condenser.

increases as the potential difference, $E$, diminishes. The same is true for the negative current with a smaller coefficient.

The results for the conduction, $a$, become more interesting if the electrometer deflections are charted graphically in relation to time as in the annexed figures $4,5,6$. It is thus seen that the current is surprisingly constant, while the initial potential difference of about 20 volts gradually quite vanishes.

9. Comparison with coronas. - The number of ions which may be computed from the given currents is excessive when compared with the number of 
nuclei, particularly when considered in parallel with the corresponding case of phosphorus. The contrast would be even more striking if the water nuclei could be tested immediately after production. Several inferences are thus suggested: either the charge of each nucleus is many electrons, or nuclei are lost at the outset at a rapid rate (this is disproved by experiment), or each nucleus emits many electrons.

ro. Evanescence of the charges of water nuclei.--The same remarkable contrast between the initial charges and the subsequent charges on the nuclei that has been already pointed out for phosphorus will now be observed, if only a little time is allowed to intervene. Table 6 refers to nuclei produced in the receiver, $A$, figure $\mathrm{I}$, by allowing the accumulating water to run off by the cock, $k$. They were then conveyed to the condenser, $C$, about 5 or Io minutes later, by aid of the Mariotte flask, $M$. The conduction is enormously reduced, though for positive charges in the condenser it is greater than for negative charges, showing that an excess of negative nuclei has persisted.

TABLE 6.--IONIZATION OF WATER NUCLEI AFTER 5 MIN. $a=\delta(\log s) / \delta t$.

\begin{tabular}{|c|c|c|c|c|c|}
\hline Condenser at & Time $t$. & Deflection $s$. & Observed $a$ & Insulation $a$. & Corrected $a$. \\
\hline $\begin{array}{r}\text { volts. } \\
+30\end{array}$ & $\begin{array}{c}\min . \\
0 \\
1 \\
2 \\
3\end{array}$ & $\begin{array}{r}\mathrm{cm} . \\
\text { I } 8.9 \\
.1 \\
\text { I } 7.3 \\
\text { I } 6.6\end{array}$ & .019 & -.009 & .010 \\
\hline-30 & $\begin{array}{l}0 \\
\text { I } \\
2 \\
3\end{array}$ & $\begin{array}{r}\text { I } 8.9 \\
.7 \\
.5 \\
.2\end{array}$ & .005 & +.002 & .007 \\
\hline
\end{tabular}

In table 7 the data refer to nuclei which were left in the vessel $A$ for about one hour after they were produced. The original ionization has all but

TABLE 7--IONIZATION OF WATER NUCLEI AFTER I HOUR. $a=\delta(\log s) / \delta t$.

\begin{tabular}{|c|c|c|c|c|c|}
\hline Condenser at & Time $t$. & Deflection $s$. & Observed $a$ & Insulation $a$. & Corrected $a$. \\
\hline $\begin{array}{l}\text { volts. } \\
+20\end{array}$ & $\begin{array}{c}\min . \\
0 \\
I \\
2 \\
3\end{array}$ & $\begin{array}{l}\mathrm{cm} . \\
20.0 \\
\text { I } 9.3 \\
\text { I } 8.8 \\
\text { I } 8.3 .\end{array}$ & .013 & .009 & .004 \\
\hline-20 & $\begin{array}{l}0 \\
\text { I } \\
2 \\
3\end{array}$ & $\begin{array}{r}26.1 \\
26.1 \\
25.8 \\
-\quad 25.7\end{array}$ & .003 & -.001 & .002 \\
\hline
\end{tabular}


vanished; nevertheless there is still an excess of negative nuclei, as shown by the greater leakage of positive charges in the condenser. If negative nuclei had been more rapidly precipitated in the intervening hour in $A$, the reverse should have been the case; there should have been an excess of positive nuclei, and negative charges in the condenser should vanish more rapidly.

Tested for coronas, even after about one hour, 50,000 were left, or ove1 $\frac{1}{3}$ of the original $10^{5}$ to $2 \times 10^{5}$, a result quite out of proportion with the loss of ionization. The electrical and the condensational phenomena are thus distinctly separated.

I1. Results with an Elliott electrometer.-For reasons which need not be stated, the electroreter of modern type in which the charge is imparted to the needle through the suspension, notwithstanding its sensitiveness and low capacity, was not adapted for further experiments. Accordingly, the data of the following table were obtained with an ordinary electrometer, with the quadrants permanently charged with a water battery. The core of the tubular condenser communicated with the needle. This adjustment was chosen because the leakage here was relatively smaller, though the high capacity of needle, jar, and condenser is unfavorable to sensitiveness. The table contains results in which the potential of the core of the condenser was altered in steps of one half. Care was taken to determine the insulation immediately before and after each measurement with the nucleated medium. The leakage is seen to be always greater at the beginning than at the end, which is the usual phenomenon of absorption and release of charge in the insulators. If any trace of radioactivity occurred it would be obscured by this phenomenon.

The results of this table may be summarized.

TABLE 8. CHARGES OF WATER NUCLEI. $d V / d t=2$ LIT. $/$ MIN. CAPACITY $409 \mathrm{~cm}$. DEFLECTION OF THE ELECTROMETER, $s$.

\begin{tabular}{|c|c|c|c|c|}
\hline \multirow[b]{2}{*}{$\begin{array}{l}\text { Electrometer } \\
\text { charge. }\end{array}$} & \multicolumn{3}{|c|}{ Leakage $d s / d t$ per $2^{\mathrm{m}}$} & \multirow[b]{2}{*}{$\begin{array}{c}\text { Current } \\
C(d E / d t) \times \mathrm{ro}^{\mathrm{II}} . \\
\text { amperes. }\end{array}$} \\
\hline & $\begin{array}{l}\text { Before. } \\
\mathrm{cm} .\end{array}$ & $\begin{array}{c}\text { During } \\
\text { nucleation. } \\
\mathrm{cm} .\end{array}$ & $\begin{array}{l}\text { After. } \\
\mathrm{cm} .\end{array}$ & \\
\hline $\begin{array}{l}\text { +at } 8 \text { I volts } \\
\text { - at } 8 \text { I volts } \\
\text { +at } 40 \text { volts } \\
\text { - at } 40 \text { volts } \\
\text { + at } 20 \text { volts } \\
\text { - at } 20 \text { volts }\end{array}$ & $\begin{array}{l}.13 \\
.17 \\
.10 \\
.11 \\
.08 \\
.06\end{array}$ & $\begin{array}{l}.64 \\
.39 \\
.67 \\
.34 \\
.63 \\
.27\end{array}$ & $\begin{array}{l}.09 \\
.06 \\
.06 \\
.05 \\
.03 \\
.02\end{array}$ & $\begin{array}{r}\mathrm{I} .33 \\
.67 \\
\mathrm{I} .47 \\
.64 \\
\mathrm{I} .45 \\
.57\end{array}$ \\
\hline
\end{tabular}

The ionization here is somewhat greater than the preceding, but the difference is at once referable to the gradually increasing size of the holes in the lead jet as the result of long spraying. Slight changes in $\mathrm{V}$ lit./min. are now of importance because of the rapid loss of the charges in the influx tube of the condenser. 
Whereas the current for positive charges decreases with the potential, the current for negative charges increases; but this, too, is due to incidental reasons of the kind mentioned. Seen in the light of the results preceding and following it, the general evidence of the table is rather to the effect that the current in the condenser is constant, independent of the electromotive force when the gradient exceeds about 20 volts per radial centimeter.

These results are given in the chart, figure 7 .
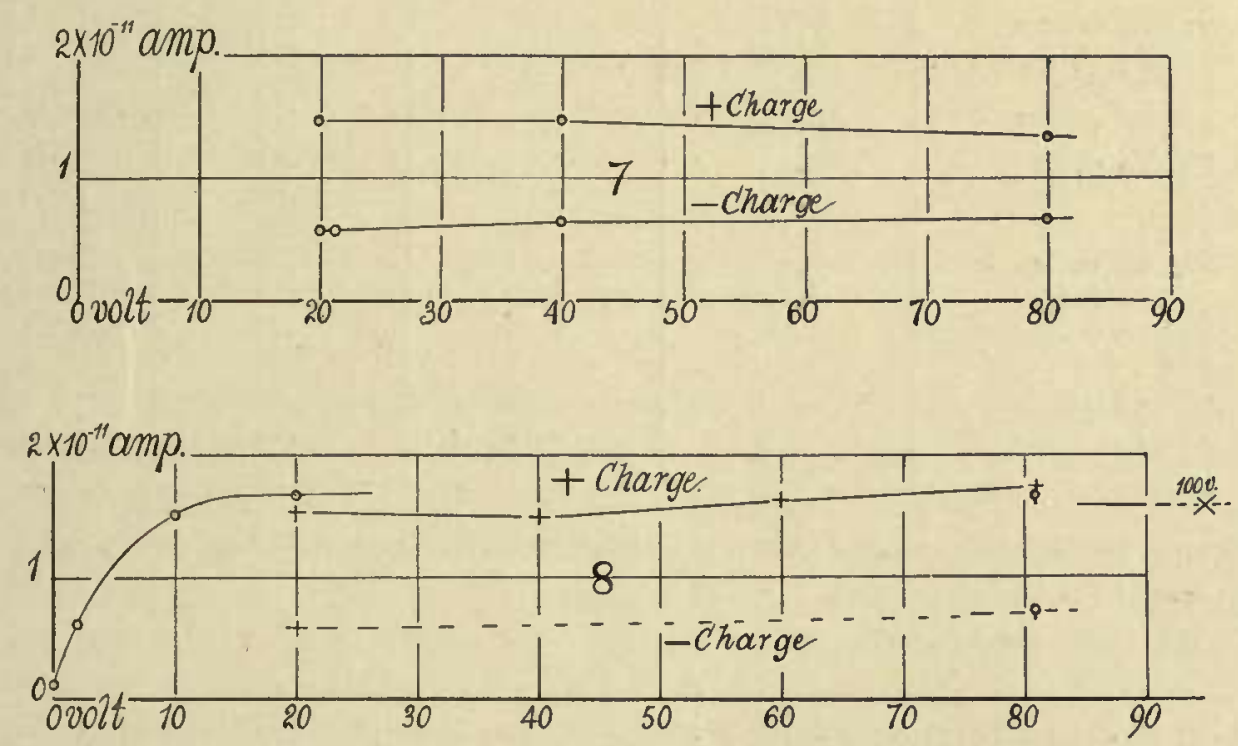

Figures 7 8. - Curves Showing Radial Currents (Amperes) for Difperent Charges and Potentials (Volts) in the Condenser.

I2. Further data.-A series of results quite similar to the last, but with a more sensitive electrometer, is given in the next table. As a rule, positive charges were taken in succession, though a number of incidental data accompany the table. The insulation of the electrometer was found before and after each measurement with nuclei. Having been taken on different days and not in a single sweep, the results cannot be quite coincident, because of jet differences, water pressures, etc., as already stated.

These results are summarized.

The currents are given in the chart marked figure 8. They show that above so volts the currents are practically constant, remembering that any change in $\mathrm{V}$ due to water pressure, etc., will convey the nuclei more rapidly into the condenser, and from their exceedingly rapid decay at the outset the currents will necessarily be variable. Below ro volts the current decreases with the potential, but remains quite appreciable even when the potential is zero with the absence of charge in the condenser. The two observations made for the negative charge indicate similar relations, when taken in connection with the preceding results. 
TABLE 9.-CHARGES OF WATER NUCLEI. $d V / d t=2$ LIT. $/$ MIN. CAPACITY $409 \mathrm{~cm}$. ELECTROMETER DEFLECTIONS, s.

\begin{tabular}{|c|c|c|c|c|}
\hline \multirow[b]{2}{*}{$\begin{array}{l}\text { Electrometer } \\
\text { charge. }\end{array}$} & \multicolumn{3}{|c|}{ Leakage $d s / d t$ per $2^{\mathrm{m}}$. } & \multirow[b]{2}{*}{$\begin{array}{c}C(d E / d t) \\
\times \text { Ion. } \\
\text { amperes. }\end{array}$} \\
\hline & $\begin{array}{l}\text { Before. } \\
\mathrm{cm} \text {. }\end{array}$ & $\begin{array}{l}\text { During } \\
\text { nucleation. } \\
\mathrm{cm} .\end{array}$ & $\begin{array}{l}\text { After. } \\
\mathrm{cm} .\end{array}$ & \\
\hline $\begin{array}{l}\text { +at } 81 \text { volts } \\
\text { - at } 80 \text { volts } \\
+ \text { at } 81 \text { volts } \\
\text { +at } 60 \text { volts } \\
\text { +at } 40 \text { volts } \\
\text { +at } 20 \text { volts } \\
\text { - at } 20 \text { volts } \\
\text { +at } 20 \text { volts } \\
\text { +at } 20 \text { volts } \\
\text { + at } 10 \text { volts } \\
\text { +at } 2 \text { volts } \\
\text { tno charge } \\
\text { +at } 100 \text { volts }\end{array}$ & $\begin{array}{l}.21 \\
.23 \\
.22 \\
.11 \\
.05 \\
.13 \\
.08 \\
.08 \\
.02 \\
.01 \\
.00 \\
.16 *\end{array}$ & $\begin{array}{r}1.88 \\
.98 \\
1.98 \\
1.79 \\
1.55 \\
1.57 \\
.68 \\
1.56 \\
1.46 \\
1.29 \\
.52 \\
.11 \\
.88\end{array}$ & $\begin{array}{l}.17 \\
.19 \\
.22 \\
.13 \\
.04 \\
.00 \\
.05 \\
.02 \\
.05 \\
.00 \\
.00 \\
.00 \\
.12\end{array}$ & $\begin{array}{r}2.17 \\
.95 \\
1.74 \\
1.62 \\
1.48 \\
1.54 \\
.59 \\
1.51 \\
1.66 \\
1.51 \\
.61 \\
.13 \\
1.59\end{array}$ \\
\hline
\end{tabular}

Corona on immediate condensation: white, crimson, green, being No. 9 with about I 50,000 nuclei per $\mathrm{cm}^{3}$.

* Smaller electrometer factor.

The average number of ions in those cases where positive and negative charges were observed are again found to be slightly larger than the preceding, due to further enlargement of the holes of the jet, whereby fresher nuclei are put into the condenser.

The table states that the most advanced corona obtainable did not exceed the middle green-blue-purple type of my series, ${ }^{1}$ throughout the whole of the work. It makes little difference whether the corona is taken instantly or a few minutes after the jet is shut off. The number of nuclei therefore is constant throughout the experiments, being about $10^{5}$.

I3. Jets self-shattering or impinging on water.-To make sure preliminarily that no induced radioactivity is demonstrable within the limiting potentials to be employed, the experiments of the following table ro were devised. Here the large vertical jet (No. 9, with I 8 needle holes, discharging about 8 liters per minute into the water below and violently churning it) was put in action, and the air above the water in the aspirator discharged into the condenser by the rise of level due to the jet. The table shows the insulation before and after the passage of nucleated air, for different potentials in the condenser. The data are given in centimeters of deflection per minute $(d s / d t)$. Hence the currents are $i=d s / d t \times 2.4 \times 10^{-11}$ amperes.

1 Phil. Mag. (6), III, pp. 80-91, I902, corrected in Am. J., xvi, I903, p. 325, and in Boltzmann's Jubelband, p. 204, I904. Cf. Chap. VI. 
TABLE Io. -ABSENCE OF APPRECIABLE RADIOACTIVITY. VERTICAL JET NO. 9 IMPINGING ON WATER WITH VIOLENT CHURNING.

\begin{tabular}{|c|c|c|c|}
\hline $\begin{array}{l}\text { Leakage ' before } \\
\text { expt. }\end{array}$ & $\begin{array}{l}\text { Condenser charge while nuclei } \\
\text { are passing. }\end{array}$ & $\begin{array}{l}\text { Leakage \& after } \\
\text { expt. } \\
\text { positive charge. }\end{array}$ & $\begin{array}{c}\text { Leakage : after } \\
\text { expt } \\
\text { negative eharge. }\end{array}$ \\
\hline $\begin{array}{l}.07 \\
.00 \\
.06 \\
.03 \\
.06 \\
.10 \\
.20 \\
.15 \\
.18\end{array}$ & $\begin{array}{rl} & 0 \\
+ & \text { volts } \\
+10 & " 1 \\
-10 & " 1 \\
+20 & " 1 \\
-20 & " 1 \\
+60 & " 1 \\
-60 & " 1 \\
+80 & " 1 \\
-80 & \end{array}$ & $\begin{array}{l}.14 \\
.05 \\
.00 \\
.13 \\
.08 \\
.07 \\
.18 \\
.10 \\
.24\end{array}$ & $\begin{array}{l}.23 \\
.06 \\
.01 \\
.12 \\
.02 \\
.21 \\
.09 \\
.3 x \\
.07\end{array}$ \\
\hline
\end{tabular}

${ }_{1}$ Condenser at 80 volts in first row. Positive and negative charges follow charges of same sign during passage of nuclei.

As it is the purpose of the present section to find the nucleation and the ionization of jets when shattering against a solid obstacle is avoided, the experiments were all made with jets either discharging vertically down into water or with jets impinging upon each other either vertically or horizontally.

The table shows that the insulation is, as a rule, better (smaller leakage) after than before the passage of nuclei, for potentials of the same sign, so that no induced radioactivity can be detected in comparison with the absorption and release of charges by the apparatus. The absorption phenomenon is strongly narked when the sign of the charge is changed, as in the two experiments after nucleation.

TABLE II.-IONIZATION AND NUCLEATION OF WATER NUCLEI.

\begin{tabular}{|c|c|c|c|c|c|}
\hline Jet. & $d V / d t$ & $\begin{array}{c}\text { Condenser } \\
\text { at }\end{array}$ & $i \times 10^{I I}$ & $\begin{array}{l}i \times \mathrm{ro}^{\mathrm{Ix}} \text { per } \\
2 \text { lit./min. }\end{array}$ & $\begin{array}{c}\text { Original } \\
\text { nucleation. }\end{array}$ \\
\hline 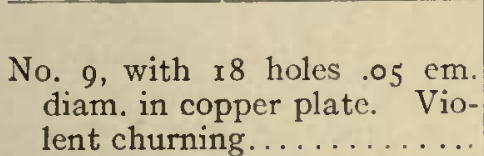 & $\begin{array}{c}\text { lit./min. } \\
8 \\
8\end{array}$ & $\begin{array}{l}\text { volts. } \\
+8 I \\
-8 I\end{array}$ & $\begin{array}{c}\text { amperes. } \\
2.2 \\
1.0\end{array}$ & $\begin{array}{c}\text { amperes. } \\
.55 \\
.25\end{array}$ & 30000 \\
\hline $\begin{array}{l}\text { No. 9, with } 8 \text { finer holes in lead } \\
\text { plate. Slight churning... }\end{array}$ & 2 & +100 & .12 & .12 & $\begin{array}{l}\text { corona } \\
\text { small }\end{array}$ \\
\hline No. $7 \ldots \ldots \ldots \ldots \ldots \ldots$ & 3 & $\begin{array}{l}+80 \\
+80\end{array}$ & 1.0 & $\begin{array}{l}.66 \\
60\end{array}$ & 30000 \\
\hline No. $3 \ldots$. & $\begin{array}{l}3 \\
3 \\
6\end{array}$ & $\begin{array}{l}+80 \\
+80\end{array}$ & .8 & $\begin{array}{l}.50 \\
.80\end{array}$ & 80000 \\
\hline No. 2 & 2 & $\begin{array}{l}+8 I \\
+8 I \\
+8 I\end{array}$ & - & $\begin{array}{l}1.33 \\
2.17 \\
1.74\end{array}$ & I 20000 \\
\hline
\end{tabular}

The ionization of the air from this turbulently discharging jet was now tested as in table II. The coronas obtained did not go further than to correspond 
to about 30,000 nuclei, whereas those of the radial jet, No. 2, correspond to $10^{5}$ or more. Hence weak ionization is to be expected, if the two occurrences go in parallel. The table (part 1 ) shows the positive and negative currents when 8 liters of nucleated air pass through the condenser per minute. Hence for $2 \mathrm{lit} . / \mathrm{min}$.

$$
\begin{array}{ll}
\text { at }+80 \text { volts, } & i=.55 \times 10^{-11} \text { amperes, } \\
\text { at }-8 \text { I volts, } & i=.25 \times 10^{-11}
\end{array}
$$

giving a mean current $i=.40 \times 10^{-11}$, as compared with $1.6 \times 10^{-11}$ amperes for the radial jet. Thus the mean current is about 4 times smaller, and the mean nucleation also about 4 times smaller, as nearly as can be ascertained. Therefore, the reduction of nucleation and of ionization run in parallel.

For the flat-bottomed lead jet with 8 very fine needle holes, the ionization was almost inappreciable. The current was about $10^{-12}$ amperes, not much exceeding the ordinary leakage of the electrometer. The corona was correspondingly small.

The next experiments, made with two capillary threads of water impinging on each other (jet No. 7), are given in the third part of the table. The different data for the mean current in case of a positive charge in the condenser and a supply of $2 \mathrm{lit} . / \mathrm{min}$. of nucleated air passing through it show that $i=.6 \times 10^{-\mathrm{r}}$ amperes. The coronas correspond to about 20,000 or 40,000 nuclei, evidencing a relatively large ionization.

The last experiments of the table were made with the large oblique jet No. 3 , and the coronas here obtained are just inferior to those of the radial jet, corresponding to about 80,000 nuclei per cubic centimeter. The positive currents are about $i=.7 \times 10^{-1 r}$ amperes. When $d V / d t=3$, almost half the ions are lost in transfer.

Experiments were made at somewhat.greater length with two oblique capillary threads of water shattering each other, as in jet No. 3, above. Special care was taken to prevent the jet from striking the walls of the vessel. As the self-shattering was very complete, the spray reached the water with but little churning. In spite of the small amount of water used, however, relatively many nuclei were produced, the number estimated from coronas being 40,000 per cubic centimeter.

Moreover, to keep the conditions more uniform, the water level in $A$, figure I, was kept constant (efflux from $k$ (Fig. I) being just as large as the water coming from the spray), and the nuclei were removed by a current of air flowing through $A$ into the condenser, $C$, at the rate of 2 liters per minute. The currents so obtained are relatively large as compared with the number of nuclei, which is due to the condition that the space in which the nuclei are produced is less than $\frac{1}{3}$ as large as usual above, and that therefore nuclei are fresher on entering the condenser. The mean positive and negative currents were

$$
\begin{array}{ll}
\text { at }+80 \text { volts, } & i=1.1 \times 10^{-11} \text { amperes, } \\
\text { at }-80 \text { volts }, & i=.3 \times 10^{-11} \text { amperes, }
\end{array}
$$

the mean, $.7 \times 10^{-11}$ amperes, being about half as large as the cuirents for radial 
jets, whereas the nucleation is deficient. The reason will presently appear (\$ I4). The currents for negative charges in the condenser, moreover, are exceptionally small relatively to the positive currents.

No charge was imparted to the condenser by the spray, the current vanishing with the potential; but this also occurs at times with the radial jet.

TABLE 12.- SPRAY FOUND IN SMALL SPACE AND REMOVED BY AUXILIARY AIR CURRENT. JET NO. 3, CAPILLARY THREADS, SELF-SHATTERING.

\begin{tabular}{|c|c|c|c|c|c|c|}
\hline $\begin{array}{l}\text { Condenser } \\
\text { charged to }\end{array}$ & $d V / d t$ & $\begin{array}{l}i \times \text { ron } \\
\text { observed. }\end{array}$ & $\begin{array}{l}\text { Mean } i \times 10^{1 r} \\
\text { corrected. }\end{array}$ & $d s / d t$ & $\begin{array}{l}i \times 10^{\mathrm{n}} \text { per } \\
2 \mathrm{lit} . \mathrm{min} .\end{array}$ & Nucleation. \\
\hline $\begin{array}{l}\text { volts. } \\
+80 \\
+80 \\
+80 \\
+80 \\
+80\end{array}$ & $\begin{array}{c}\text { lit./min. } \\
2 \\
2 \\
2 \\
2 \\
2\end{array}$ & $\begin{array}{l}\text { ampercs. } \\
\text { I.03 } \\
\text { I.32 } \\
\text { I.I } 9 \\
\text { I.00 } \\
\text { I.I } 6\end{array}$ & $\begin{array}{l}\text { amperes. } \\
\text { I.I4 }\end{array}$ & $\overline{-}$ & $\begin{array}{c}\text { amperes. } \\
\text { - } \\
\text { - } \\
\text { - }\end{array}$ & 40000 \\
\hline $\begin{array}{l}-80 \\
-80\end{array}$ & $\begin{array}{l}2 \\
2\end{array}$ & $\begin{array}{l}\cdot 35 \\
.32\end{array}$ & 30 & & 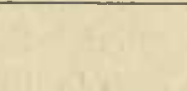 & \\
\hline
\end{tabular}

TABLE I3.-SPRAY FORMED IN GRADUALLY DIMINISHING SPACE AND REMOVED BY RISING SURFACE OF WATER.

\begin{tabular}{c|c|c|c|c|c|c}
\hline+80 & 3.5 & I.19 & I.04 & $\left\{\begin{array}{l}.38 \\
.44 \\
.53\end{array}\right.$ & .62 & 40000 \\
+80 & 3.5 & .99 & & $\left\{\begin{array}{l}.37 \\
.42 \\
.45\end{array}\right.$ & & \\
\hline-80 & 3.5 & - & .35 & - & .20 & \\
\hline+80 & 2.5 & - & .72 & - & $.5^{8}$ & \\
\hline+80 & I.0 & - & .29 & - & $.5^{8}$ & \\
\hline
\end{tabular}

I4. Summary of the relative degree of ionization and nucleation.-The number of nuclei, and the electric conduction of the nucleated air, are quantities which increase and decrease together. Nuclei and ionization are produced whether the jet is shattered by a solid obstacle, by two impinging jets, or by jets impinging on a surface of water, but the efficiency of the spraying arrangement depends on the degree of comminution produced, and in this respect the jets shattered at the highest velocity by a solid obstacle are preferable. When the jet is shattered on itself or on a surface of water, the electrical current vanishes with the potential difference in the condenser, so far as can be seen; or at least is less than 5 per cent. of the constant positive current. When the 
jet is shattered against a solid, the charging current, for the condenser at zero potential, is about 8 per cent. of the constant current; but it may also be absent.

The ratio of the ionization to the nucleation does not always appear as a fixed quantity; from which it follows that the mean charge per nucleus depends on incidental conditions of freshness, the nature of the jet, its impact, etc. Similarly, the ratio of positive to negative ionization does not seem to be a fixed quantity, but to vary under the same conditions. Nuclei generated in a small space are more highly charged because they can be more swiftly transferred to the condenser.

Finally, the maximum nucleation for any jet is reached when as many nuclei are produced per second as are lost in the same time. Unquestionably the air current accompanying the action of a violent jet contributes to this loss, by washing the air against the sides of the vessel and the surface of water. Hence jets with a strong single direction, even if made up of filimentary jets, produce few nuclei.

Finally, the reason for the unique efficiency in the capillary oblique jet was specially verified. Supposing that the high ionization relatively to the nucleation in this case is due to keeping the water level near the jet and expelling the nuclei by an auxiliary air current from a small volume, I made the following experiments in which the nuclei were discharged by a rising surface of water by aid of the Mariotte flask. Table 13 shows that on successive half minutes the currents $d s / d t$ increase rapidly, as was supposed. Moreover, when referred to an efflux of 2 liters per minute, the amperes are now actually of the low order corresponding to the nucleation of the jet.

The effect of different volumes is also seen from the table, which proves that proportionality is roughly admissible. This also follows necessarily from the equation of the phenomenon given elsewhere ( $c f . \S \mathrm{I} 8)$, and has. been carefully verified for phosphorus.

I5. Spontaneous time loss of nuclei.-The following table (14) shows the spontaneous loss of nuclei in the lapse of time. The nuclei were produced in the receiver in the usual way, and their number was then determined by the condensation produced after a stated interval. The approximate number or order of the corona in my series is nevertheless somewhat difficult to determine, and the number of nuclei estimated therefrom not quite definite. As this number is an exponent, arithmetical progression indicates geometric progression in the number of nuclei.

The radial jet, No. 2, shattering itself against the sides of the vessel is strongest as a nuclei producer, and the large oblique jet, No. 3, considerably below it in efficiency. The capillary oblique jet, No. 3 , is remarkably efficient relatively to the quantity of water used. The vertical large copper jet, No. 9, used in tables ro and II, is a very poor producer of nuclei, though using about 8 liters of water per minute and in spite of the turbulent churning of the pool below. 
TABLE 14.-LOSS OF WATER NUCLEI IN LAPSE OF TIME. ESTIMATED.

\begin{tabular}{|c|c|c|c|c|}
\hline & Jet. & Lapse of time. & Corona. & Nucleation. \\
\hline No. 2. & Radial. & $\begin{array}{r}\min . \\
.3 \\
.3 \\
6.0 \\
10.0 \\
.3\end{array}$ & $\begin{array}{l}\text { w bribg } \\
\text { w r g } \\
\text { g br b r } \\
\text { w r g g } \\
\text { wibrbg }\end{array}$ & $\begin{array}{r}\text { I } 200000 \\
\text { I } 400000 \\
100000 \\
75000 \\
\text { r } 200000\end{array}$ \\
\hline No. 3 . & Large. 1 & $\begin{array}{r}.3 \\
.3 \\
6.0 \\
.3 \\
10.0 \\
.3 \\
.3\end{array}$ & $\begin{array}{l}g \mathrm{~g} b \mathrm{br} \\
\text { gy brb r } \\
y^{\prime} \mathrm{cg} \\
\text { gy br b } \\
\text { weg } \\
\text { wr b g } \\
y^{\prime} \text { rg }\end{array}$ & $\begin{array}{r}100000 \\
90000 \\
70000 \\
90000 \\
65000 \\
75000 \\
80000\end{array}$ \\
\hline No. 3 . & Capillary. & $\begin{array}{r}.3 \\
.3 \\
10.0\end{array}$ & $\begin{array}{l}\text { gy r b r } \\
\text { gy br b r } \\
\text { gy br b r }\end{array}$ & $\begin{array}{l}50000 \\
45000 \\
45000\end{array}$ \\
\hline
\end{tabular}

${ }_{1}^{1}$ Note that the second jet gradually loses efficiency.

16. Effect of condensation on ionization.-The following table shows the cffect of precipitation on the ionization of the water nuclei. The original current (without condensation) is given both for positive and for negative charges. The condensation was produced by exhaustion immediately after the jet was shut off and but a few minutes allowed for subsidence of the fog. Only a small number of nuclei relatively to the total number therefore can have been removed. On the other hand, however, the original ionization has vanished as the result of condensation, for the residual currents are below those corresponding to the normal leakage of the condenser and electrometer, and are thus mere errors. One may note that both the positive and the negative ionization is completely removed by condensation, even though subsidence of fog particles has been all but excluded.

TABLE I5.-IONIZATION OF WATER NUCLEI AFTER PARTIAL PRECIPITATION.

\begin{tabular}{c|c|c|c|c}
\hline Condenser charge at & $d V / d t$ & $\begin{array}{c}\text { No. of precipita- } \\
\text { tions. }\end{array}$ & $i \times$ ro corrected. & $\begin{array}{c}\text { Electrometer } \\
\text { leakage } i \times \text { I }^{11} .\end{array}$ \\
\hline volts. & lit./min. & & ampcres. & ampcres. \\
+80 & 2 & 0 & I.34 & .34 \\
+80 & I.5 & I I & .07 \\
-80 & 2 & 0 & .77 & .03 \\
-80 & I & I & .06 & .06 \\
-80 & 2 & I & .03 & .12 \\
\hline
\end{tabular}


Another peculiarity may here be referred to: when the radial jet is very active it is capable of charging the neutral condenser. This, however, is not usually the case with a jet striking water. The following table, in which $s$ denotes the galvanometer deflection, shows that the radial jet, when shattered on a rigid obstacle, does not always convey charge.

TABLE 16.-CONDENSER 1 CHARGED BY RADIAL JET. $d V / d t=2 \mathrm{lit} . / \mathrm{min}$.

\begin{tabular}{|c|c|c|c|c|c|c|c|}
\hline Time, $=$ & o & $\mathrm{I}^{\mathrm{m}}$ & $2^{\mathrm{m}}$ & $3^{m}$ & $4^{m}$ & $5^{\mathrm{m}}$ & $6^{\mathrm{m}}$ \\
\hline $\begin{array}{l}\text { Charge, } s= \\
\text { Leakage, } s=\end{array}$ & $\overline{.01}$ & .00 & .01 & +.00 & +.02 & $\begin{array}{l}.04 \\
.00\end{array}$ & .00 \\
\hline
\end{tabular}

$d V / d t=4.5 \mathrm{lit} . / \mathrm{min}$.

\begin{tabular}{l|c|c|c|c|c|c|c}
\hline $\begin{array}{l}\text { Charge, } s= \\
\text { Leakage, } s=\end{array}$ & - & .00 & -.01 & -.01 & - & \\
\hline
\end{tabular}

1 Combined capacity $4 \circ 9 \mathrm{~cm}$.; 5 volts per scale part.

In these experiments non-symmetrical charge was not detected when the nuclei from the radial jet passed through the uncharged and insulated condenser. This was even true when the air current carrying the nuclei was increased to nearly 5 liters per minute. Reasons for this diversity of behavior have yet to be sought.

I 7. Effective condenser length.-It is finally desirable to ascertain whether the charges of water nuclei are actually lost to a few per cent. in the first few centimeters of the condenser, very near the influx tube. A condenser was therefore constructed the length of which could be varied by placing earthed tubes, $2 r_{2}=2.1 \mathrm{~cm}$. in diameter and of different lengths, $l=60,30$, and $15 \mathrm{cms}$., around a fixed charged insulated core, $2 r_{1}=.64 \mathrm{~cm}$., concentrically, with the usual precautions.

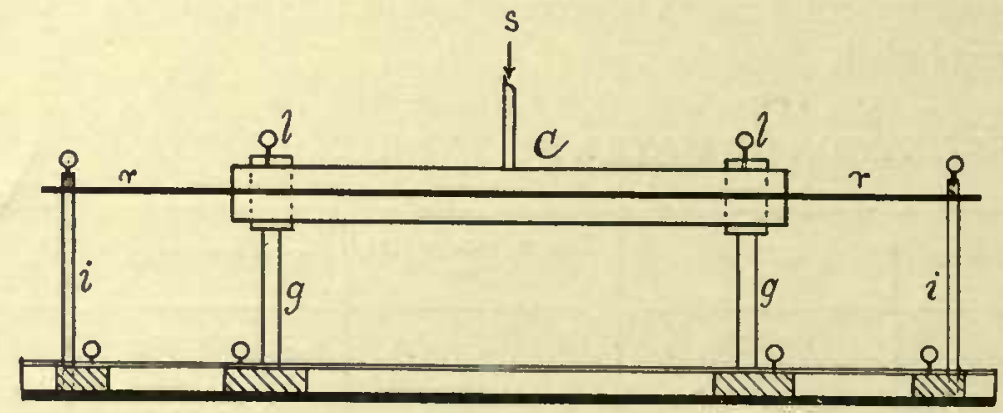

Figure 9.-Tube Condenser with Sliding and Removable Outer Coating.

Figure 9 shows the apparatus where $r$ is the inner and $C$ the outer coating of the condenser, the latter held in the sleeves, $l l$. The insulators and the 
metallic supports are capable of sliding to and fro in the base plate and of being clamped in any position. Influx of nuclei occurs at $s$. To change $C$, the sleeves $l l$ are loosened and the rod $r r$ removed, after which the tube $C$ may be slid off and another inserted. Set screws and clamp screws complete the adjustment as shown in the figure.

Table I7 shows the results in which the insulation of the condenser was determined before and after each measurement with the nucleated medium. The condenser lengths, 60 and $15 \mathrm{cms}$., are inserted as a sufficient contrast.

TABLE 17.-EFFECT OF LENGTH OF TUBULAR CONDENSER. $d V / d t=1.9$ LIT./ MIN. $A=3.5$ VOLTS $/ \mathrm{cm} . \quad C=409 / 9 \times 10^{\mathrm{II}}$ FARADS.

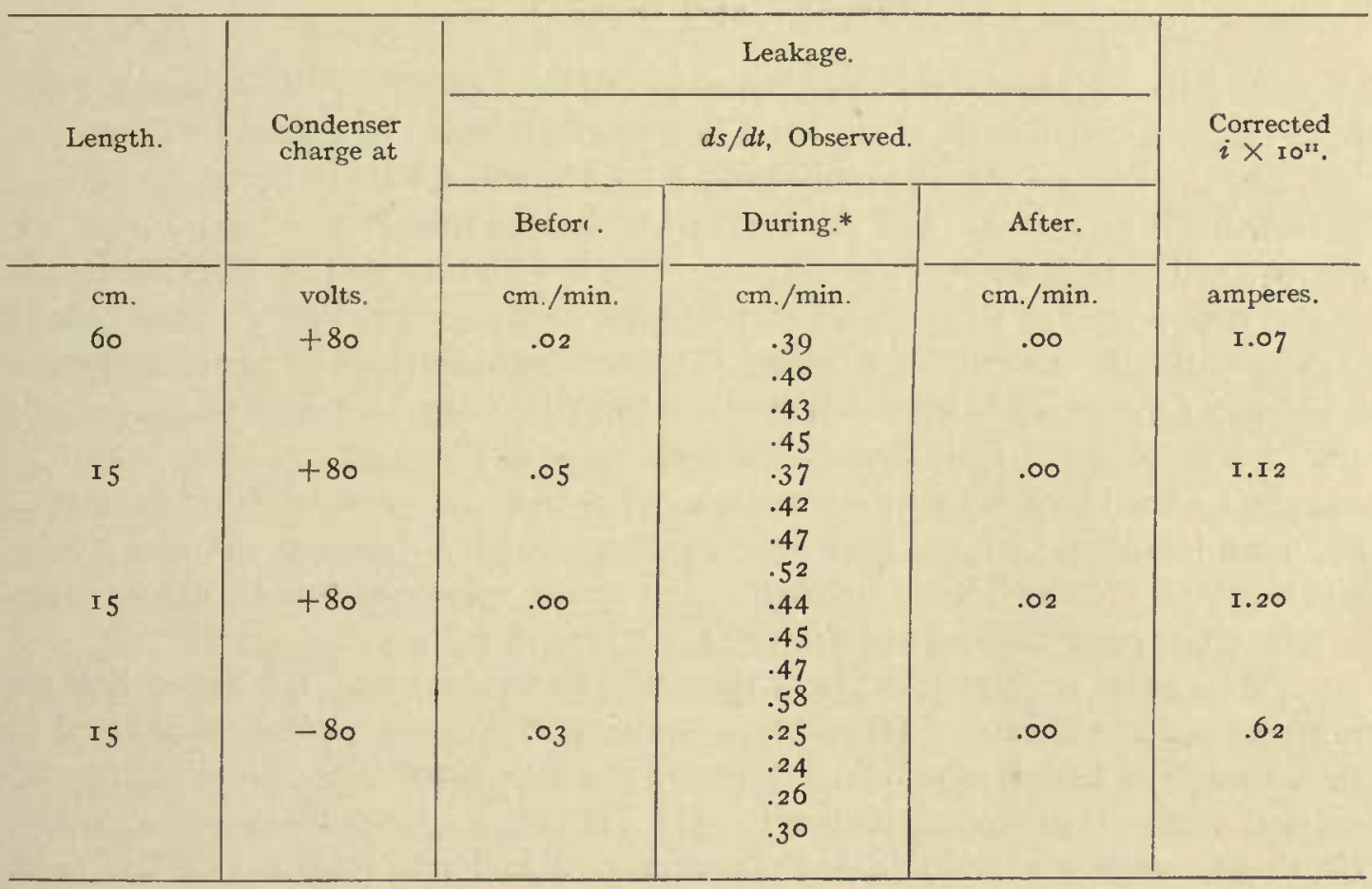

* During the passage of nucleated air through condenser.

It is seen that the currents are certainly quite as large, cat. par., when the length of the tube condenser is I 5 as when it is $60 \mathrm{~cm}$. It is actually larger at I 5 cms., due to the gradual enlargement of the needle holes ${ }^{1}$ in the lead jet, whereby fresher nuclei are conveyed into the condenser. The currents for positive and for negative charges have the usual relation to each other.

The table shows another interesting fact, already pointed out above, that the current, $d s / d t$ (per min.), increases as the water level in the receiver rises, or as the discharge into the condenser is fresher. One naturally inquires what the maximum charge of each nucleus would be if there were no conveyance tube.

1 The fine holes clog with lead hydrate when the jet is left standing in a damp atmosphere, and the obstruction is gradually removed by the friction of the water. Old jets long unused therefore show small electrical currents as compared with new jets. 
The successive values of the table (one minute apart) correspond on the average to about 16 per cent. per minute. In the present installation this jet was unable to charge the condenser, the charging current being less than $10^{-\mathrm{r} 2}$ amperes, about of the same order as the leakage.

One may conclude, therefore, that the loss of charge per minute, $i$. $e$., the electrical current radially traversing the condenser, is practically independent of its length if the latter exceeds a few centimeters, for the air current and width given. All but a few per cent. of the charge are lost in the first few centimeters ahead of the influx tube of the condenser. The experiments are thus in keeping with the surmise of the preceding paragraphs.

\section{SUMMARY AND INFERENCES.}

I 8. Working hypothesis. - In conclusion a brief summary of the working hypothesis from which most of my work has proceeded may be added for reference.

Let the ions be regarded as charged nuclei, and let there be an average of $q$ electrons per nucleus. Let the loss of ions be due merely to absorption of the charges at the boundary of the region. This is virtually stating that the loss is as the first power of the number $n$, per cubic centimeter. Whether the charge travels with the nucleus, or whether it travels from nucleus to nucleus along a highway of nuclei, as it were, is left open, but the charges are lost at the boundary at a more rapid rate than the nuclei.

To fix the ideas, let a tube condenser of radii $r_{2}>r_{1}$, and length, $l$, be given, and let $v(\mathrm{~cm} . / \mathrm{sec}$.) be the velocity of the air current bearing charged nuclei longitudinally through the condenser. If $V$ is the volume of this air in liter $/ \mathrm{min}$. entering the condenser at one end, $\pi\left(r_{2}^{2}-r_{1}^{2}\right) v=16.7 \mathrm{~V}$.

The loss of nuclear charges is then due to two causes: (I) These charges have a specific velocity, $k$ (absorption velocity in a given cardinal direction) in the absence of the electric field. Charges are lost in pairs by this non-directed motion without producing current. (2) The nuclei have a second velocity, $U^{\prime}$, in the same direction per electron carried and per volt $/ \mathrm{cm}$. of the field. Hence the number of nuclei, $n$, at the section $l \mathrm{~cm}$. from the influx end, where $n=n_{\circ}$, is given per unit of length by

$$
n=n_{0} \varepsilon^{-2 K l / v\left(r_{2}+r_{1}\right)}=n_{0} \varepsilon^{-a} \ldots .
$$

where $K=k+q E U^{\prime} /\left(r_{2}-r_{1}\right)$ and $\alpha=.377 l K\left(r_{2}+r_{\mathrm{r}}\right) / V$.

The radial current at the same section, if the potential difference between the surfaces $r_{2}$ and $r_{x}$ of the tube condenser is $E$, will not depend on $k$, but on $U^{\prime}$, so that

or eventually

$$
-d i=2 \pi\left(r_{2}+r_{1}\right) n e U^{\prime} q^{2}\left(E /\left(r_{2}-r_{1}\right)\right) d l
$$

$$
-C d E / d t=\frac{16.7 V n_{0} e q^{2}\left(l-\varepsilon^{-a}\right)}{q+k /\left(U^{\prime} E /\left(r_{3}-r_{x}\right)\right)} \ldots
$$

where $C$ is the capacity of the condenser and $e$ the charge of one electron, while $q$ such charges travel per nucleus. 
Experiment shows that the currents are of about the same order when charged water nuclei from an intense high-pressure jet and when charged phosphorus nuclei are passing longitudinally through the condenser. But as the number of water nuclei as tested by coronas are, even in the condensation chamber, not above $10^{5}$ per cubic centimeter, while the number of phosphorus nuclei may reach $10^{7}$, the charge $q$ in electrons per nucleus is large for water nuclei and small for phosphorus. Similarly one may expect the water nucleus derived by a mechanical process to be larger than the initial phosphorus nucleus derived chemically, so that $k$ is larger in the latter case.

Hence it is assumed that in case of water nuclei, $k$ is negligible in comparison with $q E U^{\prime} /\left(r_{2}-r_{\mathrm{s}}\right)$ and equation (2) becomes, if $q U^{\prime}=U$

$$
-C d E / d t=16.7 V n_{\mathrm{o}}(e q)\left(\mathrm{I}-\varepsilon-.377 l\left(r_{2}+r_{\mathrm{x}}\right) U E / V\left(r_{2}-r_{x}\right)\right) .
$$

This equation, which fits the phenomena very well, predicts saturation as the exponent is essentially dependent on $E$.

On the other hand, in case of phosphorus nuclei, $k$ is large in comparison with $q E U^{\prime} /\left(r_{2}-r_{1}\right)$, for here a single electron travels with many nuclei. The exponential term in (2) vanishes or

$$
-C d E / d t=\mathrm{I} 6.7 V n_{\mathrm{o}} c q^{2} E U^{\prime} / k\left(r_{2}-r_{\mathrm{I}}\right)
$$

which is virtually Ohm's law.

An endeavor has thus been made to explain the two types of conduction in question, the charged water nucleus type and the phosphorus nucleus type, by a simple self-contained hypothesis. I have not, however, been able to complete the numerical details to my satisfaction, and will therefore leave the subject here without further comment.

I 9. Charge and conduction.- The data have shown that positive as well as negative charges are dissipated by water nuclei, immediately after they have been produced, and that the ionization, if it may be so called, is quite of the order of that of phosphorus, while the nucleation is much smaller. After being stored but a few minutes, the nucleation loses all but a few per cent. of this property of conduction, behaving in this respect again like phosphorus nuclei. The number of nuclei does not appreciably vary in the same time. The character of the ionization (whether positive or negative nuclei are in excess) remains intact so long as it can be observed. Hence the large initial and the eventual very small conduction (a few per cent. of the original value) may be regarded as two successive phases of a single continuous phenomenon, either of charge or ionization or conduction. It seems to me therefore that it is not necessary to distinguish the initial charges from the initial ionization. The experiment as a whole shows an attenuation of the Lenard effect, continuously through infinite time. One is at liberty to refer the conduction either to charged nuclei or to ionized nuclei unless some distinctive definition is adopted. Both occurrences are similarly reduced. The present case of river water is one in which there is an excess of negative over positive nuclei. In other cases (pure water) the reverse may be the case, or, again, there may be an absence of an excess of 
either sign. If the nuclei were without charges, however, the medium would not conduct. In a condenser, positive or negative charges are sooner dissipated according as there is excess of negative or positive nuclei in the medium, respectively.

20. Comparison of phosphorus and water nuclei.-Between phosphorus and water nuclei there is in the first place the essential difference that whereas the current in the first case obeys Ohm's law, roughly, it does not do so in the second, being more and more independent of the electromotive force as $E$ increases above about 15 volts per $\mathrm{cm}$. Similarly, the coronas for water nuclei usually terminate with the middle $\mathrm{g}-\mathrm{b}-\mathrm{p}$ type, whereas in case of phosphorus they go to indefinitely higher orders, beyond the first in the series. Parallel to this there may run a difference in the size of nuclei. The inference is warranted that phosphorus nuclei are small as compared with water nuclei, inasmuch as the latter owe their origin to mechanical conditions, while the phosphorus nuclei arise under molecular conditions and molecular dimensions. As the observed electric currents are about of the same order in both cases, it follows that the charges per nucleus are very much larger for water nuclei than for phosphorus. If water nuclei could be examined immediately after production, i. e.; in the same degree of freshness as is customary for the phosphorus nuclei, the contrast would be enormous.

In both cases, however, whenever ionization and nucleation are associated phenomena, the number of ions generated varies directly with the concomitant number of nuclei.

In other respects there is great similarity in the behavior of the two types of nuclei. The enormous charges of ionizations at the beginning vanish to a residuum of a few per cent. in a few minutes if confined by a receptacle, while the nuclei are not affected either as to number or condensational properties by the presence or absence of the primitive charge. It is not unreasonable to suspect, therefore, that the water nucleus, like the phosphorus nucleus, may be the permanent residue produced by the expulsion of the electrons representing the ionization: for whenever nucleation and ionization arise in a common source, any increment of the former is accompanied by a corresponding increment of the latter. 


\section{CHAPTER III.}

\section{PRELIMINARY SURVEY OF THE APERTURES OF CORONAS, IN RELATION TO THE NUMBER OF NUCLEI AND THEIR SIZES.}

I. Introductory.-Throughout my earlier work with coronas, I have relied chiefly upon the color sequences, and have taken the data for numbers and sizes of cloud particles (a fixed degree of supersaturation presupposed) from the tables given elsewhere. ${ }^{1}$ When apertures were measured this was done chiefly for the identification of the series to which the corona belongs. There is no doubt, however, that an expression for the diameters of particles in terms of the aperture of the coronas would be a great and immediate convenience, particularly as facility in using the color sequences is apt to be lost, unless one is at work with them continually. Apart from this, the colors represent steps of progress, while the apertures should be continuously, even if irregularly, variable. The purpose is then to find under what conditions the discrepancies of aperture may be reduced to a minimum.

If the supersaturation is constant throughout, the diameters of cloud particles and their distance apart will in general be proportional quantities. Let $m$ be the grammes of water precipitated, $n$ the number of particles per cubic centimeter, $D=n^{-x / 3}$ their distance apart, $d$ the diameter of each, $s$ the aperture of the corona. If, therefore, for normal coronas $d=a / s$, where $a$ is a constant found by purely optical experiments,

$$
n=\left(6 m / \pi a^{3}\right) s^{3}=(6 m / \pi) / d^{3}=\mathrm{x} / D^{3} \text {, and } d=D(\pi / 6 m)^{1 / 3} .
$$

But it is doubtful if these equations are true even for normal coronas; they must certainly be a very crude approximation for coronas of the higher orders, where $d$ and $D$ are possibly both implicated in producing coronal effects. If one builds up a system of glass plates each sprinkled with lycopodium particles, the diffraction pattern, which is finely multi-annular for a single plate, is a mere blur for ro plates placed within a linear foot, for instance, without changing the aperture appreciably. If the source of light and the eye are both distant, the coronas gradually lose sharpness and soon cease to be measurable as the number of plates increases. This indicates that greater uniformity of distribution and equality of diameter must be met with in case of cloud particles, but it leaves the question open whether the distance apart of particles is not from the outset a consideration.

${ }_{1}^{1}$ Am. Fourn. of Science (4), xirr, p. 8r, r902; Phil. Mag. (6), Iv, p. 26, r902; cf. Structure of the Nucleus, Smithsonian Contributions, 1903, Chapter III. 
What is further menacing is the distortion produced by spherical and cylindrical vessels, the surfaces of which are rarely quite concentric. In my work with globes, I assumed that if the annuli showed no distortion and were small in aperture as compared with the aperture of the globe, distortion could be neglected. It is questionable, however, if this observation is vouched for, since the apertures of coronas are peculiarly sensitive to refraction, particularly when the distances of eye and source from the receiver are purposely chosen large.

Again, the quantity $m$ is dependent on temperature. It is necessary therefore to refer coronas to a standard temperature as well as to a given degree of supersaturation, and the correction is important if the coronas are to be used in estimating the number of particles.

Finally, the ratio of densities before and after exhaustion is a serionsly difficult datum to determine, for it depends on the degree to which adiabatic conditions have been attained. It is here that the work is liable to be discrepant. Hence a determination of apertures has an ulterior value, for it is not improbable that the two series of results will mutually interpret each other. The present chapter bears out this surmise, though it is merely to be regarded as a rough test of my earlier results $(l . c$.$) . An independent survey is made in$ Chapter VI with plate-glass vessels.

2. Apparatus and preliminary results.-The following charts contain a preliminary survey of the sequence of coronas, their apertures, and the number of particles of specified diameter encountered. The data for diameter and number, $d$ and $n$, are taken from my work on successive exhaustion $(l . c$.), where the experiments are largely non-optical, and they are compared with the corresponding data $d^{\prime}$ and $n^{\prime}$ which follow from measurements of aperture. The eye and source of light are distant $\mathrm{x}$ and 3 meters, respectively, from the condensation chamber between them. This was here a long cylindrical vessel of as clear glass as possible, $50 \mathrm{~cm}$. long and I $3 \mathrm{~cm}$. in diameter. The observations werc made parallel to the axis, absence of distortion being assumed for the axial plane, an assumption which was justified by trial comparisons with plate-glass apparatus, though the latter was not quite large enough for the complete survey. The method of work was otherwise the same as that described in the earlier papers.

The results of the work may be given without tables in the accompanying charts, figures $\mathrm{I}, 2,4,5,8,9$, in which the old results for $d$ and $n$ (computed from successive exhaustions) are laid off horizontally, the new results $d^{\prime}$ and $n^{\prime}$, computed (as stated) from the observed aperture, vertically. The discrepancy of the two sets of data is enormous, and the curves all show sustained periodicity: All measurements of aperture, $s$, are made to the inner edge of the red ring, and show the diameter of the central disc.

3. Diameter of cloud particle.-The variations of $d$ and $d^{\prime}$ are on the average $\delta d=\mathrm{x} .4 \delta d^{\prime}$, from curve 4 , and $\delta d=\mathrm{r} .6 \delta d^{\prime}$ from curve 5 . In other words, the diameters obtained for coronas by computation from the conditions of suc- 
cessive exhaustion are about $\mathbf{I} .5$ times larger than the same data estimated directly from the apertures of the coronas.

Moreover, the new values of diameter $d^{\prime}$ show a curious periodicity which must be peculiar to them, since the old values from the manner in which they

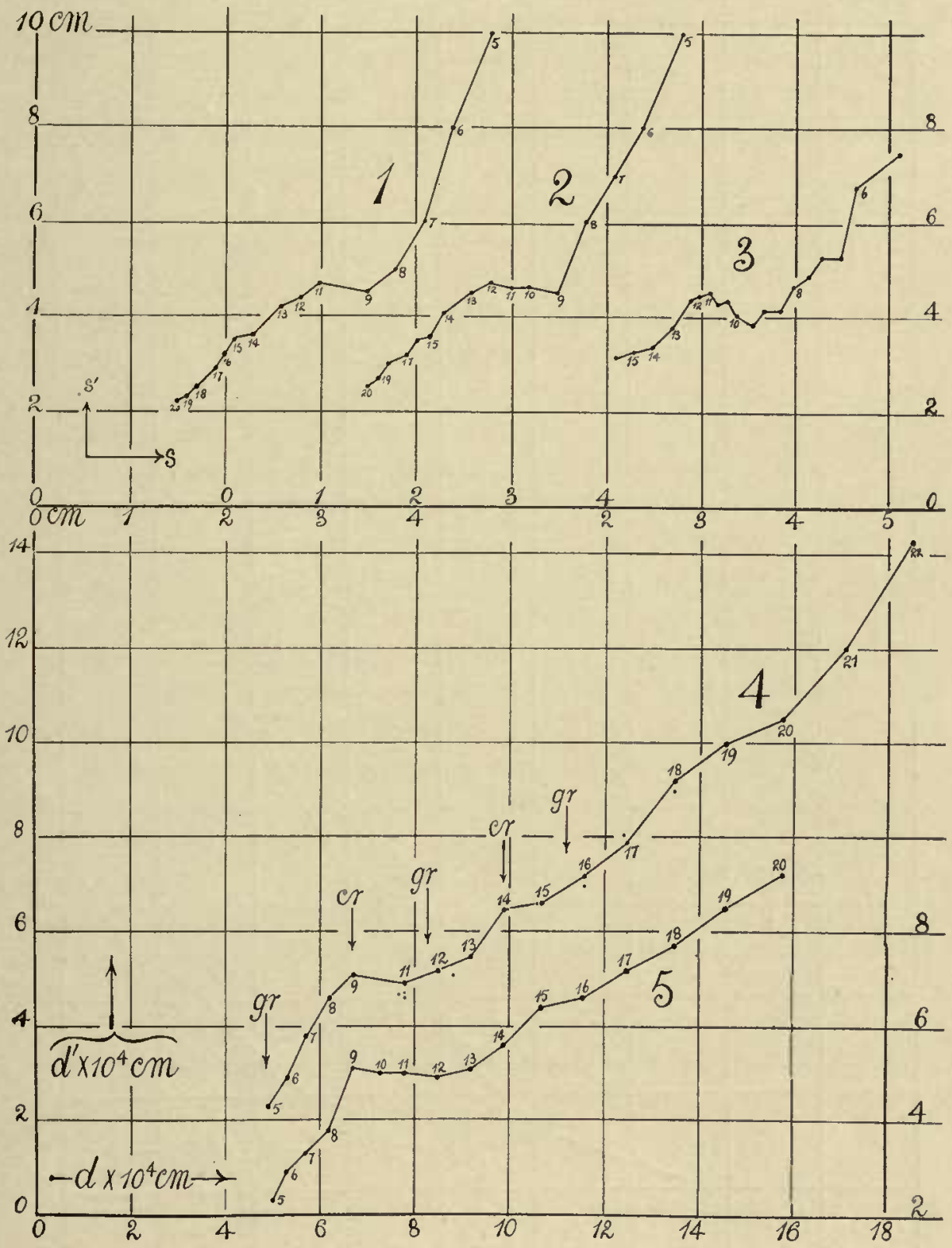

Chart I.-Curves i and 2, Relations of Apertures Computed from Successive Exhaustions $(s)$, and Directly Measured $\left(s^{\prime}\right)$. Long Cylindrical Receiver, 3 cm. in Diameter. Curve 3, the Same for Plate-Glass Apparatus $20 \mathrm{Cm}$. Deep. Coronas here Difficult to Place.

Curves 4 and 5, Relation of Diameter of Fog Particle Computed from Successive Exhaustions (d), and from Measurements of Aperture ( $\left.d^{\prime}\right)$, both Given in Centimeters. Long Cylindrical, Receiver. The Types of Coronas are Marked gr (Green Centered), cr (White-Crimson Centered). Curve 5 Dropped .0002 cu. Small Dots Refer to a Special Series. 
were obtained (geometric progression) cannot be periodic. There is accelerated increase of diameter toward the crimson types, Nos. 9 and $\mathrm{I}_{4}$, and a falling off which may even be a retrogression toward the green types, Nos. 4-5, I I-I 2, I5-I6, these being respectively the crests and troughs of the wave. The

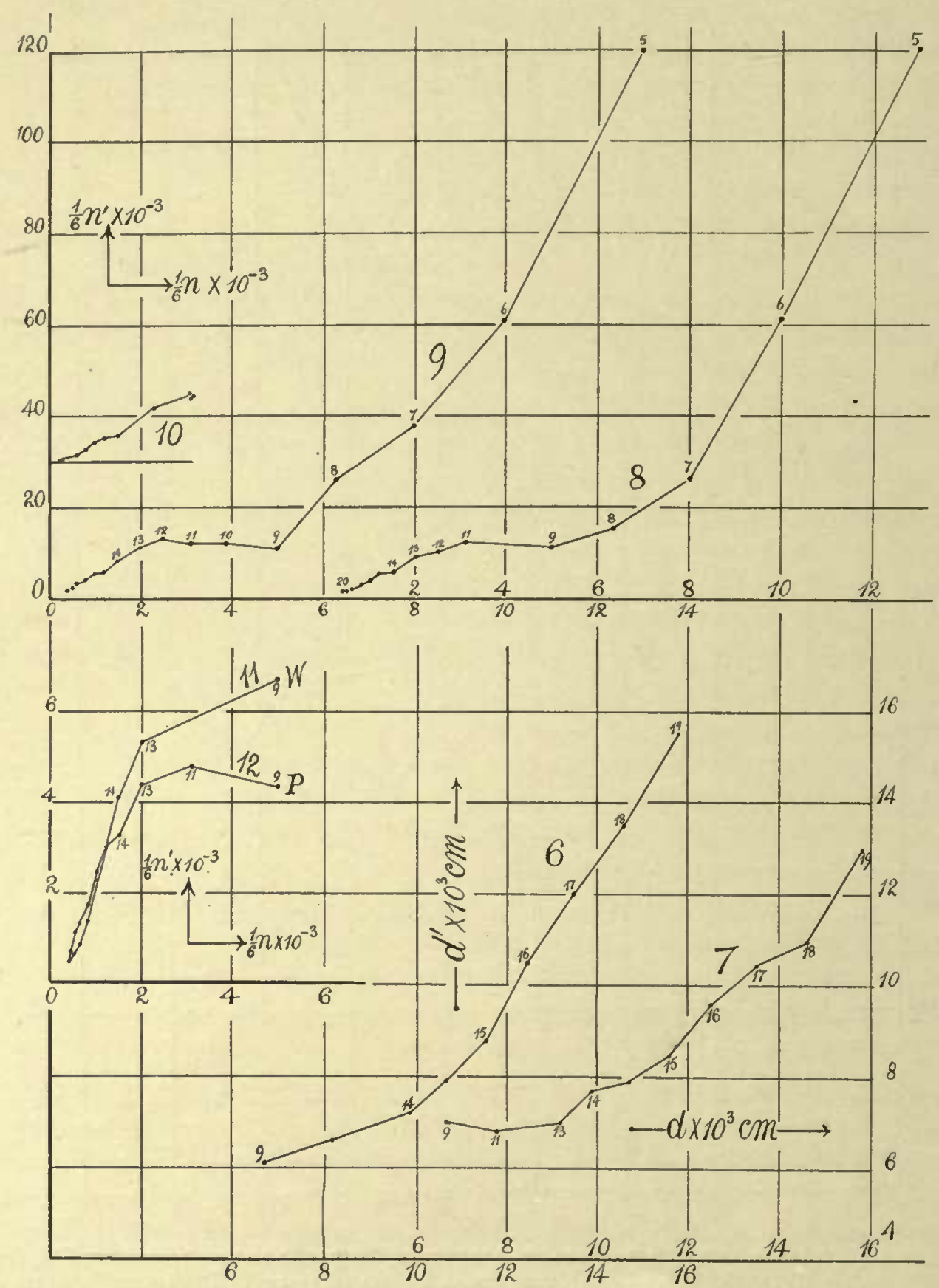

Chart 2.-Curves 8, 9, ro, Ratios of Nucleation Computed from Successive Exhaustions ( $n$ Particles per cub. cm.), and from Measured Apertures $\left(n^{\prime}\right)$. Long Cylindrical Receiver.

Curves i and I2, the Same por another Cylindrical Receiver, 20 cm. Deep. Curve it Refers to Water Nuclei, Curve 2 to Phosphorus Nuclei.

Curves 6 and 7 , Corresponding Diameters of fog Particles in Centimeters. 
undulation continues even beyond this, but it is then difficult to identify it, as the annuli become crowded into the normal coronas. The results are similar in all the curves. In other data obtained during experiments with jets in Chapter II, s? $4,5, \mathrm{I} 7$, and given in the present figures $6,7, \mathrm{I}$, I 2 , the same undulatory line is encountered both for phosphorus and for water nuclei, with maxima at the 9th and I th coronas. Here a different vessel (aspirator $32 \mathrm{~cm}$. high, $22 \mathrm{~cm}$. in diameter) was used, and the ratio, $\delta d=\mathrm{x} \cdot 3 \delta d^{\prime}$, is distinctly smaller for this case, showing the marked influence of distortion due to the vessel. The same ratio (x.3) will be adduced below in connection with the preliminary experiments with plate-glass apparatus.

4. Nucleation.- Since $n d^{3}$ is constant, remarks of the same general character may be made for the nucleation, $n$, except that the discrepancy will be reciprocal in character and enormously exaggerated. If on the average $d=I \cdot 5 d^{\prime}$, $n^{\prime}=3.4 n$; if $d=\mathrm{r} .3 d^{\prime}, n^{\prime}=2.2 n$, but the undulations have now become so sweeping that a ratio can only be inferred for the small coronas.

5. Cause of periodicity. - If one inquires into the cause of the periodic discrepancies, it appears that the crimson coronas are too small or else the green coronas too large, for the data computed from exhaustions cannot be periodic. The former being white-centered with a diffuse red margin, it is impossible to mistake the outside edge of the first ring for the inside edge. The blue-green coronas, however, show a uniformly colored disc, and here the first ring may be of the same color as the disc, and the corona would then be measured to the outside margin of the first ring. From this point of view only the crimson coronas are adapted for measurement, and both curves would then give $d=\mathrm{r} \cdot 3 d$, and $n^{\prime}=2.2 n$. Since the curves actually give evidence of diminishing aperture while the droplets certainly decrease in size, this explanation is plausible, though it does not agree well with the evidence from normal coronas. The red and crimson coronas are the only ones which retain the white center, and the phenomenon may in so far be regarded as similar to the case of normal coronas.

6. Effect of temperature.-The explanation of the discrepancy between $d$ and $d^{\prime}$ (computed from exhaustions and measured from apertures, respectively) reduces in the most favorable case to $d=\mathbf{r} \cdot 3 d^{\prime}$, and for this two explanations must be examined. Supposing that one does not inadvertently measure into a ring, the value of $m$ which enters into the computation of $d$ is very variable with temperature. For $\delta p=I 7 \mathrm{~cm}$., for instance,

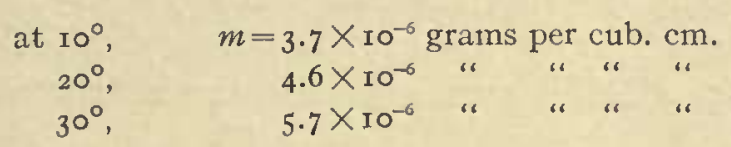

Since $d$ varies as $m^{1 / 3}$, for the same nucleation the values of $d$ at $10^{\circ}, 20^{\circ}$, $30^{\circ}$, will be in the ratio of $56,60,64$, respectively, and the coronas will be in the same degree smaller. Per degree between $20^{\circ}$ and $30^{\circ}$ this amounts to about .8 per cent. of the value at $20^{\circ}$. Hence to bring the values of $d$ computed from successive exhaustions into coincidence with the data computed from 
apertures would require a temperature excess of nearly $30^{\circ}$, which is out of the question.

7. Pressure decrement.-As none of the explanations are satisfactory, light of a different character may be thrown upon the discrepancy by computing by the approximate method of the earlier memoirs the density ratio, $y^{\prime}$, of the gas after and before exhaustion, corresponding to the observed values, $s^{\prime}$. Since if $n$ is the nucleation, $z$ the order of the corona in a geometric series, $b$ the coefficient of time loss, $t$ the time interval between exhaustions,

$$
\log n=z(\mathrm{I}+b t) \log y,
$$

the equation corresponding to a different exhaustion ratio would be

$$
\log n^{\prime}=z(\mathrm{I}+b t) \log y^{\prime}
$$

if the same corona, $z$, and time interval, $t$, is implied.

Hence $\log n / \log n^{\prime}=\log y / \log y^{\prime}$, while $n=\left(6 m / \pi a^{3}\right) s^{3}=A s^{3}$. Therefore, $\log y^{\prime} / \log y=\left(\log A+3 \log s^{\prime}\right) /(\log A+3 \log s)$.

The computed values $s=a \sqrt{n \pi / 6 m}$ are given and in the chart, figures I and 2. From the latter for $s=5.0, s^{\prime}=8.0$ to $9.0 \mathrm{~cm}$. From the earlier memoir, ${ }^{1}$ the value computed for $y$ was .819 . Hence

$$
\begin{array}{ll}
s^{\prime}=8, & y^{\prime}=.807, \\
s^{\prime}=9, & y^{\prime}=.804,
\end{array}
$$

whereas $y=.819$ was the value computed in my work on coronas for the exhaustion $76-58 \mathrm{~cm}$.

Since, roughly, $y=\left(p / p_{0}\right)^{1 / \gamma}$, where $p=76$ and $\gamma=1.4$, the following values of $\delta p$ obtain:

$$
\begin{array}{lc}
s=5.0 \mathrm{~cm} ., \delta p= & \text { I } 8.0 \mathrm{~cm} . \\
s^{\prime}=8.0 & \text { I } 9.1 \\
s^{\prime}=9.0 & \text { I } 9.4
\end{array}
$$

Thus if the pressure decrement on exhaustion had been taken I $\mathrm{cm}$. higher than the observed value, the apertures computed from successive exhaustions in the former memoir would agree with the average apertures directly measured in the present paper. Observationally this is out of the question, but it is nevertheless difficult to know just what pressure is effective in the adiabatically cooled receiver (cf. Structure of the Nucleus pp. 35, 38), since neither the isothermal nor the adiabatic conditions will rigorously suffice. The memoir shows that isothermally $y=.764$; adiabatically $y=.825$; adiabatically with allowance for condensed water $y=.8 \mathrm{I} 9$, as already specified. The aperture data demand $y=.805$, which is even nearer to the isothermal $y$ than the value taken.

Incidentally one may note the precision with which $y$ must be entered or the pressure difference determined, if the observations are to be sufficiently close to admit of a computation of $d$ and $n$. In other words, it is probable that the ratio $y$ may be determined with greater accuracy from the successive aper-

'Structure of the Nucleus, Chapters III and IV. 
tures as a whole, notwithstanding their periodic character, than by direct measurement. This is what I meant by stating that the two sets of observations would probably sustain each other, for nobody would be justified in using the apertures of abnormal coronas, unless such use was suggested and guided by independent evidence. The subject will be resumed in Chapter VI, and treated from a point of view different from the present, which is merely tentative.

8. Summary.-The result of this paper is then favorable to the use of the: apertures of coronas in place of the colors of the annuli, for estimating the number of particles corresponding to a given degree of supersaturation at a given temperature. Full allowance must, however, be made for the occurrence of periodic variations of aperture in relation to the diameter of the fog particles; in other words, a given aperture is only of value when qualified by the type of corona (whether of the crimson or green order) to which the aperture belongs. Thus it will not in any case be possible to dispense completely with the color pattern. It was with the object of finding these corrections systematically that I began a series of experiments (Chapter VI) with new forms of plate-glass apparatus, and I shall there refer to other developments. Homogeneous light, though in nany respects desirable, gives effects so faint as to be useless in practice.

With the above data I am able to make an independent estimate of the number of particles in the saturated phosphorus emanation. The number found for the first fog of the series was (Phil. Mag. (6), IV, pp. 25-26, I902) $n=6 \times 83,000 ;$ since $n^{\prime}=2.2 n, n^{\prime}=6 \times 1_{8} 3,000$ particles per cub. cm. Now the density ratio before and after exhaustion is $y$, so that $I-y$ is the volume of saturated emanation added. As this has passed directly and slowly over excess of phosphorus, it must be very nearly saturated, becoming diluted on mixture with the dust-free air of the receiver. Hence, if $n_{\circ}$ particles per cub. $\mathrm{cm}$. correspond to saturation, $n(\mathrm{I}-\mathrm{y})=n_{0}=6 \times 183,000 ;$ or $n_{\circ}=10^{6} \times 6$. There must therefore be at least 6 million nuclei ${ }^{1}$ per cub. cn. of the air in contact with a surface of phosphorus. The value following from my electrometer work was $n_{0}=2 \times 10^{6}$. The two methods are absolutely distinct, but lead to data of the same order. It is because of the general reasonableness of the data which have followed from my simple hypothesis throughout a very wide territory of observation that I have felt bound to adhere to it.

\section{PLATE-GLASS APPARATUS.}

9. Description.- To test the results just adduced, the apparatus shown in Chapter VI, figure $I$, and in Chapter VII, figure $I a$, was constructed. The frame, $20 \mathrm{~cm}$. deep, $35 \mathrm{~cm}$. long, $27 \mathrm{~cm}$. high, was of wood, nicely joined, and covered within and without with a mixture of burgundy pitch and beeswax while hot. The front and rear faces are of $\mathrm{I} / 4$-inch plate-glass, cemented on by the same resinous mixture, and further held in place by the wooden clamps,

1 The factor 6 is introduced in conformity with the work of Chapter VI. 
secured by the brass bolts at their ends (see Chapter VII). $F$ is the filtering attachment with a cock, $E$ the exhausting attachment, $P$ the nucleator. Thermometers show the temperature both of the air within the chamber and of the water at its bottom. The goniometer is in front and the source of light in the rear of the apparatus, and the exhaustions are made in. the way frequently described in these memoirs.

In the preliminary results, $\delta p=4.5 \mathrm{~cm}$. was the pressure decrement on exhaustion, $t=23^{\circ}$, the temperature of the saturated air and water. The eye and the light are at distances 85 and $235 \mathrm{~cm}$. from the central plane of the apparatus. Since $s_{\circ}$ for lycopodium is by experiment $.75 \mathrm{~cm}$. and $d_{0}=.0032 \mathrm{~cm}$., $a=d_{\circ} s_{\circ}=$ .0024 . Hence at $20^{\circ} d=.0024 / s$ and $n=6 m s^{3} / \pi a^{3}$.

The results, $s$ and $s^{\prime}$, are constructed in the chart, figure 3 , and show the same general character as the results already discussed. Moreover, since $s^{\prime}=1.3 s$, the two sets of data are more nearly in correspondence here than was the case with the cylinder above. Definite results of this character for higher values of $\delta p$ will presently be given (Chapter VI), after a few incidental questions have been disposed of. 


\section{CHAPTER IV.}

ON THE NUMBERS OF NUCLEI PRODUCED BY SHAKING DIFFERENT LIQUIDS AND ON ALLIED DATA.

I. Explanation. - In my report on the nucleus, ${ }^{1}$ I showed that the number produced in a given mode of comminution was least in pure water, greater in dilute organic solutions, and still greater in dilute inorganic solutions, all of the same strength. Results were also given for other solvents than water, in particular for benzol; but I was unable to reduce the data to the same scale as for aqueous solvents, as the data needed for the reductions were not at hand. I have since found that the method of Wilson and Thomson ${ }^{2}$ lends itself to benzol, and have therefore computed the data over again, as shown in table I.

TABLE I.-NUMBERS OF NUCLEI PRODUCED BY VIGOROUSLY SHAKING DIFFERENT SOLUTIONS IN THE SAME MANNER. CONCENTRATION I \%.

\begin{tabular}{|c|c|c|}
\hline Solvent. & Solute. & Number of nuclei per cub. $\mathrm{cm}$. \\
\hline 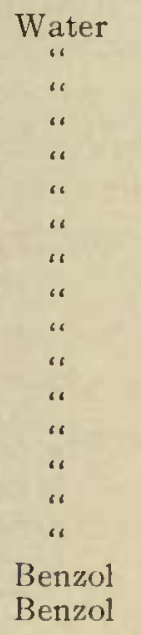 & $\begin{array}{l}\text { (Pure water) } \\
\text { Sucrose } \\
\text { Glucose } \\
\text { Glycerin } \\
\text { Urea } \\
\text { Tartaric Acid } \\
\mathrm{Na}_{2} \mathrm{SO}_{4} \\
\mathrm{~K}_{2} \mathrm{SO}_{4} \\
\mathrm{Alum} \\
\mathrm{CaCl}_{2}, \mathrm{FeCl}_{3} \\
\mathrm{NaCl}_{3} \mathrm{HCl} \\
\mathrm{Ca}_{2} \mathrm{NO}_{3} \\
\mathrm{H}_{4} \mathrm{~N} \mathrm{NO}_{3} \\
\mathrm{Al}_{3} \mathrm{NO}_{3} \\
\mathrm{Fe}_{3} \mathrm{NO}_{3} \\
\mathrm{Na}_{3} \mathrm{PO}_{4} \\
\mathrm{Naphthalene} \\
\text { Paraffine }\end{array}$ & $\int_{3500}{ }^{1300}$ \\
\hline
\end{tabular}

2. Data.-The pressure reduction used to effect the condensations was throughout $\delta p=\mathrm{I} 6 \mathrm{~cm}$. Hence at about $20^{\circ}$ the adiabatic fall of temperature in case of a benzol-air medium should be as far as $-10.2^{\circ}$, the rise of temperature

'Smithsonian Contributions to Knowledge, No. I373, Chap. V, 1903.

${ }^{2}$ Phil. Mag. (5), xLvi, p. 538, I898. 
thereafter (due to condensed liquid) to $\mathrm{rr} \cdot 3^{\circ}$, and consequently the liquid benzol precipitated per cubic centimeter $m=30.4 \times 10^{-6}$ grams. The goniometer factor was $a=.003 \mathrm{I}=d s$, being the product of the diameter $d$ of the fog particle and the aperture $s$ of the corona. Hence the number of nuclei per cubic centimeter is finally $n=\mathrm{I} .95(\mathrm{IOS})^{3}$, all the coronas in question being normal, excessively intense and brilliant.

This may be compared with water. The corresponding temperature reduction of the water-air medium is to $-7.6^{\circ}$, the rise of temperature due to the ensuing condensation as far as $9.5^{\circ}$, so that $m=4.5 \times 10^{-6}$ grams per cubic centimeter almost 7 times smaller than the corresponding datum for benzol. When the same goniometer as above is used, therefore, $n=.29$ (ros) ${ }^{3}$.

The curious result thus appears that the number of nuclei produced by a definite amount of shaking is least for water, about 5 times greater for dilute organic solutions in water, about Io times greater for dilute inorganic solutions in water, and about 30 to 40 times greater for dilute solutions of non-conductors like naphthalene and paraffine in benzol. It is difficult to even conjecture a reason for this behavior.

3. Coronas in general.-The coronas in benzol for the above pressure differences, $\delta p$, are all normal, even if nucleation from sulphur, phosphorus, etc., is introduced. From the slow diffusion of the vapor they soon become distorted during successive exhaustions unless the vessel is shaken between them. It is interesting to show, however, that in spite of the normal coronas the high initial nucleation is fully accounted for. To do this I shall select a series of observations for coronas in benzol vapor at random (l. c., p. 56). Sulphur nuclei were used and the vessel shaken between observations. The table gives the results.

TABLE 2.-CORONAS IN BENZOL VAPOR. SULPHUR NUCLEI. $\delta p=18 \mathrm{~cm}$. $n=6 m / \pi d^{3} . \quad m=33 \times 10^{-6} \mathrm{~g}$. Per cub. cm. $d=.00144 / \mathrm{s}$.

\begin{tabular}{|c|c|c|c|}
\hline Exhaustion No. & Observed $d \times 10^{8}$ & Computed $d \times 10^{8}$ & Computed $n$. \\
\hline 0 & Fog & .2 & 6800000 \\
\hline I & “ & .3 & 3200000 \\
\hline 2 & “ & .4 & 1400000 \\
\hline 3 & " & .5 & 610000 \\
\hline 4 & " & .6 & 270000 \\
\hline 5 & .8 & .8 & 120000 \\
\hline 6 & 1.0 & I.I & 52000 \\
\hline $\begin{array}{l}7 \\
8\end{array}$ & $\begin{array}{l}1.2 \\
1.8\end{array}$ & $\begin{array}{l}1.4 \\
1.8\end{array}$ & $\begin{array}{l}23000 \\
10000\end{array}$ \\
\hline 9 & 2.6 & 2.4 & 4400 \\
\hline 10 & 3.7 & 3.2 & 1900 \\
\hline I I & 4.2 & 4.2 & 850 \\
\hline
\end{tabular}

Computed exponentially the initial nucleation would run up into the millions. The observations are not, however, in keeping with such a locus, and 
conform more closely to $\mathrm{I}=d\left(\mathrm{I} / d_{\circ}-z \sigma / a\right)$ or $s=s_{\circ}-\sigma z$ and $d s=a$. For present purposes this is near enough. I shall therefore lay off the aperture, $s$, as a linear function of the number of the exhaustion, $z$, for which the observations show per unit of $z$, in case of sulphur nuclei, $\delta s=.28$, and in case of punk nuclei, $\delta s=$.I9. The initial aperture computed herefrom as the mean of six series, in each of which the nucleation was introduced independently, is for sulphur, $s_{\circ}=3.4$ and for punk, $s_{\circ}=2.2$. Hence $n_{\circ}=840,000$ in the former case and $n_{0}=230,000$ in the latter.

Since the pressure ratio was in each case 1.36 , the nuclei in the influx air passing over burning sulphur or glowing punk must have been 3.8 times more numerous. Thus there were nearly 3,000,000 sulphur nuclei and nearly 900,000 punk nuclei per cubic centimeter in the laden air currents entering the condensation chamber.

I shall show in Chapter VI that the equation applicable to the present experiments is

$$
n_{z}=n_{Z} \text { IO }{ }^{(z-Z) \log y_{Z}^{z-1}}\left(\mathrm{I}-S / s^{2}\right)
$$

where $n_{z}$ is the initial nucleation, $y$ the volume ratio on exhaustion, $z$ the number of the exhaustion, and $S$ an appropriate subsidence constant. The function $\Pi$ is a product of the terms $\left(I-S / s_{Z}^{2}\right)\left(I-S / s_{Z+1}^{2}\right) \ldots\left(I-S / s_{z-1}^{2}\right)$, so that $Z$ is the number of the exhaustion in which the first corona is seen and $\Pi=\mathrm{x}$. When the particles are as large as is the case for benzol the subsidence function is of prevailing importance and masks the exponential function as all the observations for benzol show. I have carried this method out for water vapor, obtaining consistent results throughout. The present observations for benzol are scarcely systematic enough to make it worth while to compute $S$, and the experiments should be such in which the diffusion and homogeneity of vapor is insured by continued rotation of the vessel rather than by shaking. But there can be no doubt that, with proper precautions in this respect, the number of nuclei furnished per cubic centimeter by any given nucleator can be determined with benzol vapor as the coronas are all normal, even for large values of $\delta p$, with certainty.

4. Axial colors.--It is because of the relatively great number of relatively large particles in case of benzol and similar hydrocarbon vapors, that the axial colors are seen, and may be traced into much higher orders than is the case with water vapor. The yellows, browns, etc., of the first order may be easily obtained with the steam jet, though they cannot be produced in the condensation chamber by any means except by pressure differences causing intense spontaneous condensation in moist air. The subsequent violets, blues, etc., however, are here distinctly seen as far as the orange red of the second order, after which the admixture of white light makes recognition of color more and more difficult. With hydrocarbon liquids like gasolene, benzine, etc., the axial colors are seen much farther along the series even through a short column, and they are intense in the drum. The difficulty encountered in observation is due to 
the slow diffusion and consequent absence of homogeneous vapor. I hope, however, by keeping the drum in rotation around the axis of vision, as already suggested, to counteract this discrepancy, and correspondingly to prolong the series.

5. Carbon disulphide. - The vapor of this reagent is another in which coarse normal coronas usually appear. The endeavor to produce the higher coronas with sulphur, punk, or air nuclei fails if the pressure differences are of the same order as those used for water. Particles of the fog are usually about $d=.00 \mathrm{I} \mathrm{cm}$. in diameter for strong nucleation, and the strong coronas produced on shaking showed diameters of the order of $d=.00$ I 5 under the given conditions of exhaustion. Relatively large coronas were obtained with nuclei which apparently rise from this reagent spontaneously. Thus after about 2 hours $d=.002$, after 6 to $I_{5}$ hours $d=.0012 \mathrm{~cm}$. was observed. The fact that the coronas increase in size in the lapse of time suggests other explanations than the slow diffusion of vapor or the difficulty in keeping it uniformly saturated when successive exhaustions are made. For in this case coronas would decrease and the size of particles increase, whereas the reverse is observed.

The computation of the number of nuclei per cubic centimeter for carbon disulphide is more precarious in view of the high vapor pressures and the deficiency of data applying throughout the range of temperatures involved. For the case of a pressure decrement of $\delta p=18 \mathrm{~cm}$., from $76 \mathrm{~cm}$., and at $20^{\circ}$, the adiabatic fall of temperature would be as far as $-34^{\circ}$, the rise thereafter due to condensed liquid as far as $5^{\circ}$. This implies $53 \times 10^{-6}$ grams of moisture per cubic centimeter, whence with the above goniometer the number of nuclei per cubic centimeter would be $n=34(\text { Ios })^{3}=.10 /(\text { I od })^{3}$.

The coronas obtained by spontaneous nucleation thus correspond to $n=\mathrm{I} 3,000$ after 3 hours and $n=50,000$ after 6 hours or more. Finally, punk nuclei after two or three exhaustions with shaking were still present to the number of $n=75,000$ per cubic centimeter. 


\section{CHAPTER V.}

\section{THE DIFFUSION OF VAPOR INTO NUCLEATED AIR; A CORRECTION.}

I. Apparatus and manipulation.-The apparatus with which experiments of the present kind are made is conveniently described by aid of the accompanying diagram. The appurtenances necessary in practice are given in my report on the "Structure of the Nucleus" (Smithsonian Contributions, No. 1373, 1903), to which reference has frequently been made. A, Figure $\mathrm{I}$, is a tall glass vessel about one meter high, either cylindrical or rectangular in section, in the latter case with opposed plate-glass sides. The liquid, $L$, whose vapors are to be tested, is placed in the bottom. The wide tube, $c$, is used for sudden exhaustion, while a vacuum gauge, $g$, registers the pressure differences. The tubes $a$ and $b$ to the top and the bottom of $A$ serve for the admission either of filtered air or of nucleated air. They are used together, one for influx and the other for efflux, in connection with the suction of an aspirator.

When the diffusion of the necessarily heavy vapors from $L$ is to be measured, the air in $A$ is first cleansed of vapor by a current of nucleated air from $a$ to $b$. Thereafter the stopcocks are closed at a stated time. If now at a subsequent time a sudden exhaustion is made in $A$ through $c$, for a stated pressure difference, $\delta p^{\prime}$, shown at $g$, the progress of the diffusion may be computed from the height of the fog-bank after an allowance is made for the rise due to the exhaustion.

On the other hand, if the aspirating current is of filtered air and moves in the direction from $b$ to $a$, over the surface of the volatile liquid, the receiver, $A$, should become uniformly saturated to a high degree throughout. If nuclei are added at a stated time below near the surface of the liquid, the corresponding height of the fog-bank seen on exhaustion at a later time should indicate the rate at which the nuclei diffuse, if they diffuse more slowly than the residual concentration of vapor. This method for nuclei, which I pursued with entire confidence, leads, however, to erroneous results, as the present paper will show; for the diffusion of the nuclei is a much more rapid process than the accompanying complications of vapor diffusion.

2. Equation.-To state the case specifically, let $p$ be the vapor pressure relative to the saturation pressure at the temperature $s_{0}$, at a time $t$ after diffusion of vapor commences and at a height $x$ above the surface of the liquid in the receiver, $A$. Then from well-known principles it may be shown that

$$
\text { (1) } \quad p=\mathrm{I}-\frac{2}{\sqrt{\pi}} \int_{2}^{x / 2 \sqrt{2} q^{2}} d q
$$

where $k$ is the coefficient of pressure diffusion. 
If the exhaustion at the time, $t$, is made from air pressure, $p^{\prime}$ to $p^{\prime}$, corresponding to the temperatures $\vartheta_{0}$ and $q^{\prime}$, the relation is approximately $q^{\prime} / \vartheta_{0}$ $=\left(p^{\prime} / p_{0}^{\prime}\right)^{(k-1) / k}$ where a correction for precipitated liquid, etc., is needed.

The vapor pressure corresponding to the reduced temperature, $9^{\prime}$, so obtained after division by the saturation pressure at $q_{0}$, is, then, the value of $p$ in equation ( $\mathrm{I}$ ), which therefore, like $x$ and $t$, is known, so that $k$ may be computed.

3. Application and data.- In order to have an example for use in the discussion below, I computed the case for water vapor, which though unsuitable from its lightness for experiment, is convenient for comparison with other vapors, almost all of which are heavier than air. The well-known expansion of ( 1 ),

$$
p=I-\frac{2}{1 / \pi}\left\{x / 2(k t)^{1 / 2}-x^{3} / 3 \times 8(k t)^{3 / 2}+x^{5} / 3 \times 5 \times 32(k t)^{5 / 2}\right.
$$

judiciously manipulated is sufficient for the purpose, though I afterwards availed myself of the tables in Dienger's Method of Least Squares in the absence of larger tables.

The results for water vapor were given in a table, with the time, $t$, in minutes and the height of the fog-bank, $x$, in centimeters.

The table also contained a second series of data, for the case in which the diffusion takes place into a vapor $\frac{1}{3}$ saturated, to which reference will be made below. The results of the table may be constructed graphically, showing respectively the advance of diffusion at a given height and at a given time. The

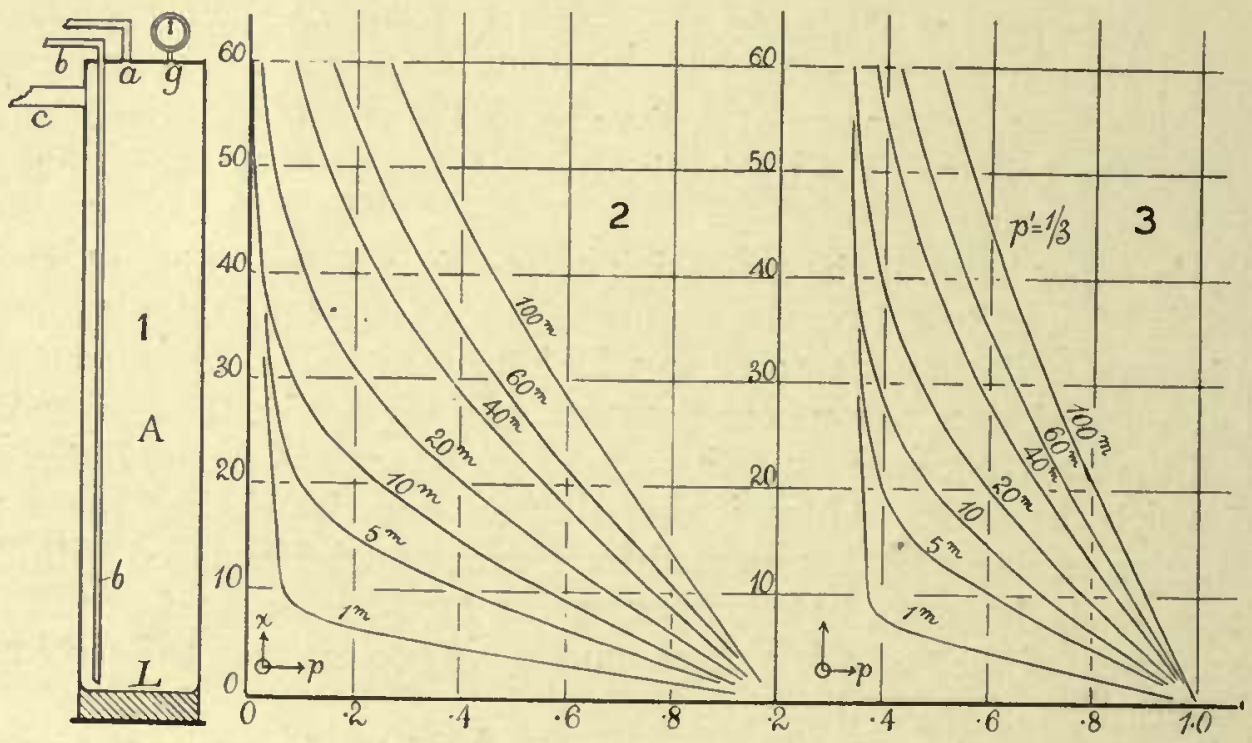

Figure i.-Diffusion Chamber.

Figure 2.-Chart Showing the Vapor Pressures, $p$, at Different Heights, $x$, in tile Lapse of Time, when Water Vapor Diffuses into Air.

Figure 3.-The Same, for Diffusion into Air Originally $\frac{1}{3}$ Saturated.

latter are exhibited in figure 2 , in connection with figure I. From either set of curves the parabolas which show the rise of a given vapor pressure in the lapse of time may be obtained by graphic interpolation. 
If the exhaustion chosen is such as to reduce the vapor pressure to $\frac{1}{3}$ (this corresponds roughly to a pressure difference of $\delta p^{\prime}=\mathrm{I} 7$ ), the intersection of the vertical line of the figure with the successive curves will show the heights of the fog-banks on condensation. Thus after 20,40 , or 60 minutes the fogbanks, having attained heights of, roughly, 22, 33, and 40 centimeters, will be in good position for observation.

For any other vapor than water, the times will increase inversely as the coefficients of diffusion. Thus for benzol the time intervals should be increased about $2 \frac{1}{2}$ times.

4. Conclusions. - The striking feature of these curves is the extreme slowness of diffusion even for water vapor. At but 20 centimeters above the liquid surface it takes half an hour to reach semi-saturation. The case is accentuated for other liquids where the coefficients are smaller, as, for instance, for the following liquids at about $20^{\circ}$ :

$\begin{array}{lcccccc}\text { Vapor, } & \mathrm{H}_{2} \mathrm{O} & \mathrm{CH}_{4} \mathrm{O} & \mathrm{C}_{2} \mathrm{H}_{6} \mathrm{O} & \mathrm{C}_{3} \mathrm{H}_{8} \mathrm{O} & \mathrm{C}_{6} \mathrm{H}_{6} & \mathrm{CS}_{2} \\ k= & .23 & .16 & . \mathrm{I} 2 & .07 & .09 & . \mathrm{I}\end{array}$

If, therefore, the fog particles are relatively numerous, large, and subside rapidly, the air will soon become highly de-saturated. In other words, if the air in the receiver $A$ is cleaned of nuclei by condensation, there is no vapor available to replace the moisture lost.

In case of water vapor the fog particles are sinall and subside slowly while the vapor is lighter than air. Hence the latter is liable to be reheated from the rapid radiation of gases assisted by convection, as stated, before much de-saturation takes place, unless the vessel is very long and the sides dry. Precisely the opposite is the case for the hydrocarbon vapors in spite of their volatility, since the fog particles for the same nucleation are larger and fall rapidly, and where the vapors are heavier than air. After successive precipitations at a given pressure difference the vapor may be so far de-saturated that it nearly ceases to condense even if nuclei are present. That it can quite cease to respond is impossible, for some vapor must return to the air after condensation almost instantaneously; but it is not improbable that a vapor exhausted to a slightly higher pressure difference will fail to respond thereafter at the original pressure difference. Thus there is considerable chance for error, and what is taken for the diffusion of nuclei added near the surface of the liquid may actually be the diffusion of the liquid itself. This will even be the case if an aspiration current, as in $\$ \mathrm{I}$, falls sufficiently short of saturation, supposing always that the velocity of nuclei is relatively large. True, in the experiments which I made, the two sets of results for nuclei and for vapors differ radically in order of values, in distribution among different vapors, while for carbon disulphide the gradually increasing apertures of the coronas is certain evidence of greater concentration of nuclei. But these and other occurrences may each in their turn be explained away.

5. Diffusion from greater to less saturation.-To facilitate the discrimination 
in question, the diffusion of vapor into partially saturated vapor may be computed, as has already been done since

$$
p=I+\frac{2}{V \pi}\left(p_{0}-x\right) \int_{0}^{x / 2 V k \bar{\varepsilon}} \varepsilon^{-q^{2}} d q
$$

and the initial saturation is $p_{0}=\frac{1}{3}$ at $t=0$. The results are constructed in figure 3 , and from them the parabolas showing the rise of the levels of successively increasing saturation may be derived.

An inspection of figure 3 shows that if the exhaustion were carried somewhat further than corresponds to the lower limit $p=\frac{1}{3}$, the fog-banks would be capped at a definite height, and that the latter would be enormously influenced by slight changes of pressure decrement on exhaustion. Experiment bears this out. Even for fixed pressure differences $(\delta p)$ the condensation must progress with a sweep from the bottom upward, and if the very small particles last formed evaporate fast enough, an upper demarcation of the fogbank will again show itself which would easily be mistaken as a true case of the diffusion of nuclei. In this way the diffusion of about semi-saturation $(p=.5)$ into benzol vapor initially about $\frac{1}{3}$ saturated would fully account for the apparent diffusion of nuclei into benzol vapor shown in the niemoir cited.

6. Crucial experiment and conclusion.-Special experiments must therefore be made to decide whether, when nuclei are added at the bottom of a homogeneous column of nearly saturated vapor, the observed diffusion is that of nuclei through the vapor, or of a greater concentration of vapor through homogeneous nucleation. For this purpose it is sufficient to add the nuclei in successive experiments at the top and at the bottom of the receiver, $A$, figure $\mathrm{I}$. The nuclei in such a case must diffuse alternately downward and upward, while the vapor diffuses upward only. Such experiments since made with care showed that the addition of nuclei above or below the column of vapor is without effect on the observed diffusion. Hence it follows not only that the diffusion of the vapor and not of the nuclei has been observed, but that the nuclei must diffuse much more rapidly than the vapor. Indeed, in the time in which the nuclei travel from top to bottom of the tall vessel nearly $\mathrm{I}$ meter high, the vapor has scarcely risen, and the fog-bank seen on exhaustion lies close to the surface of the liquid.

An attempt to measure this rapid diffusion of the nucleus in benzol vapor by the present direct method failed, chiefly because all attempts to rigorously saturate the air in the receiver with the heavy vapor in a reasonable time were seriously hampered by convection. The results merely showed that the velocity of the nucleus in benzol vapor must be quite of the same order as in water vapor, but sharp data could not be obtained.

A curious observation, obtained particularly in the case of coronas from alcoholic fog particles, deserves mention. Here the tendency to irregular coronas decreases as the number of nuclei becomes smaller. The final coronas are generally regular, though small. It follows from this that the diminished 
saturation in the upper parts of the vessel, due to the precipitation of fog particles with relatively slow diffusion of vapor, eventually becomes more and more negligible. Hence the small region from which each nucleus draws its liquid charge is apparently limited by the low rate of diffusion relatively to the rate of subsidence of the nucleus. In large coronas, subsidence is slow, and the region from which vapor reaches the nucleus is correspondingly large. In small coronas, subsidence is rapid, and the region from which vapor is received dwindles in local extent.

7. Nuclei produced by the mixture of coal gas and air.-Some time ago. I noticed that if coal gas is examined by the steam jet or color tube, as described elsewhere, ${ }^{1}$ a faint pink flush is seen in the field of the tube. This indicates the presence of nuclei to the extent of many thousands per cubic centimeter in the gas. Inasmuch as such nuclei could not be retained in the gas pipes (they would soon be lost either by subsidence or diffusion), an explanation of the phenomenon was difficult to suggest. Recently I examined the question by the aid of the present method of coronas. Coal gas stored over water and suddenly cooled shows no condensation. It is therefore free from nuclei, as would be anticipated. Filtered air under the same conditions behaves in the same way. If, however, coal gas and filtered air are mixed and then examined, nuclei are abundantly present, to the extent of several thousand per cubic centimeter, showing that chemical reaction (attributable to the presence of sulphide gas as an impurity) has taken place.

If the air is introduced above the lighter coal gas, the nuclei are obtained at once as a result of the mixture, by convection. If the coal gas is introduced above the air, nuclei are not at first in evidence, but they appear later as the result of diffusion at the surface of contact. The case of the steam tube is now obvious, seeing that the gas is here necessarily introduced in contact with air. These nuclei are not ionized, as special experiments with a condenser showed. Very probably the product of the oxidation is sulphuric acid.

see Experiments with Ionized Air, Smithsonian Contributions to Knowledge, No. I309, I901. 


\section{CHAPTER VI.}

PERIODIC COLOR DISTRIBUTIONS IN RELATION TO THE CORONAS OF CLOUDY CONDENSATION, WITH A REVISION OF THE CONSTANTS OF CORONAS.

\section{INTRODUCTION.}

I. Purpose and plan.-The growing importance of cosn ic dust ${ }^{1}$ in relation to geophysic phenomena suggested the need of developing a method by which the atmospheric dust contents could be speedily and systematically determined. An appropriate method for this purpose was tested in a number of my earlier papers ${ }^{2}$ which gave promise of being in a measure independent of merely local or accidental dust distributions. It is based on the measurement of the angular apertures of the coronas produced on suddenly cooling moist atmospheric air under definite conditions. Observations of atmospheric nucleation made in this way for about two years show results of considerable interest.

There is some difficulty, however, in reducing these data to absolute values (number of nuclei per cubic centimeter), inasmuch as the coronas obtained with lamp light very frequently pass beyond the ordinary white centered normal type into the more complex forms corresponding to very small particles. I have therefore been obliged to make an extended study of coronas. ${ }^{3}$ The method pursued consisted in highly nucleating the air stored within a given receiver over water (with adequate provision for continued saturation), and then withdrawing definite amounts of it by successive partial exhaustions. If the nucleated air is replaced by filtered air free from nuclei, the residual number of nuclei in the receiver must decrease in geometric progression with the number of partial exhaustions. The latter, moreover, produce the sudden cooling by which the coronas are obtained. Let $m$ be the moisture precipitated per cubic centimeter, in any exhaustion, $n$ the number of cloud particles contained, $d$ the diameter of each: then $n=6 \mathrm{~m} / \pi d^{3}$. Since for the successive partial exhaustions $m$ is constant, $n$ follows from $d$, and vice versa.

Two methods are available for the absolute measurement of $d$. One may

- The pioneering work of Aitken is well known and cited in my earlier papers.

${ }^{2}$ Science, xv1, p. 948, 1902; Physical Review, xv1, p. 193, 1902; ibid., xvir, p. 233, 1903.

s Phil. Mag. (6), Iv, p. 24, 1902; American Journ. of Science (4), xIII, p. 81, 1902; ibid., xv, p. 335, 1903; Physical Review, 1. c.; Smithsonian Contributions to Knowledge, No. I $373, \mathrm{XXIX}, \mathrm{pp} . \mathrm{I}-\mathrm{x} 76, \mathrm{x} 903$. 
determine the apertures of the coronas (so long as these are normal) by a suitable goniometer, or one may find the rate of subsidence of the cloud particles. Both are approximate and limited in scope, as they fail in the cases of the higher transient coronas. Two methods, furthermore, are available for measuring the nucleation, $n$, or at least relations of $n$. Aitken's direct dust counter or a similar apparatus may be applied (work ${ }^{1}$ with this end in view is given in Chaps. VII, VIII), or the values of $n$ may be made to decrease geometrically in the way just specified until normal coronas are obtained, for which $d$ follows from aperture. For the last of these methods I have already published data; but in the course of over a year's additional experimentation a number of new developments have shown themselves which it is my purpose here to elucidate. In the first place the method formerly used for determining $m$ gave results much too small. These are corrected in the present paper. In the second place, the coronas were supposed to be observed under adiabatic conditions of temperature; direct experiments in this paper show that the air temperatures during which the coronas are observed are nearly isothermal. Moreover, the new results prove that in addition to the systematic loss of nuclei by exhaustion, as thus fully computed, there is an additional loss which has hitherto escaped me. Each exhaustion, in fact, is accompanied by a definite loss of nuclei for which reasons must be investigated (\$ IO).

Finally, I have in this chapter used both electric- and mono-chromatic light as a source, as well as the Welsbach mantel employed for practical purposes. Naturally from the introduction of intense violets the coronas become more complicated, but it is only in this way that their true nature may be detected.

2. Apparatus.-The apparatus in which the present experiments were made differs from the earlier forms merely in the employment of plate-glass condensation chambers. A variety of forms were used, some bulky and nearly cubical, like figure I $(20 \mathrm{~cm}$. deep, $25 \mathrm{~cm}$. high, $35 \mathrm{~cm}$. long), others (figure 2) long and narrow ( $5 \mathrm{~cm}$. deep, I I cm. high, $55 \mathrm{~cm}$. long). Practically an apparatus $25 \mathrm{~cm}$. deep, ro $\mathrm{cm}$. high, or less, and $60 \mathrm{~cm}$. long would be most generally suitable. They were all lined, except on the opposed plate-glass faces, with a double layer of cotton on a copper frame. The chamber is to be mounted on trunnions, $E, t$, so as to admit of easy rotation around a horizontal axis at right angles to the line of vision. Holes, $A, A^{\prime}$, must be provided so that the plate-glass may be cleaned within, with a probang. The chamber carries a stopcock, $F$, leading to a cotton filter, and another, $P$, leading to the nucleator (preferably phosphorus). The trunnions are wide and hollow, and exhaustion is made through one of them, $E$, while the other, $t$, is either closed or may serve for the admission of a thermometer. To produce a definite amount of

${ }^{1}$ Aitken's dust counter may be dispensed with, and the intensity of the nucleator determined by condensation in benzol vapor, in which the coronas are all normal. See Smithsonian Contributions, 1. c., p. 55 et seq. 


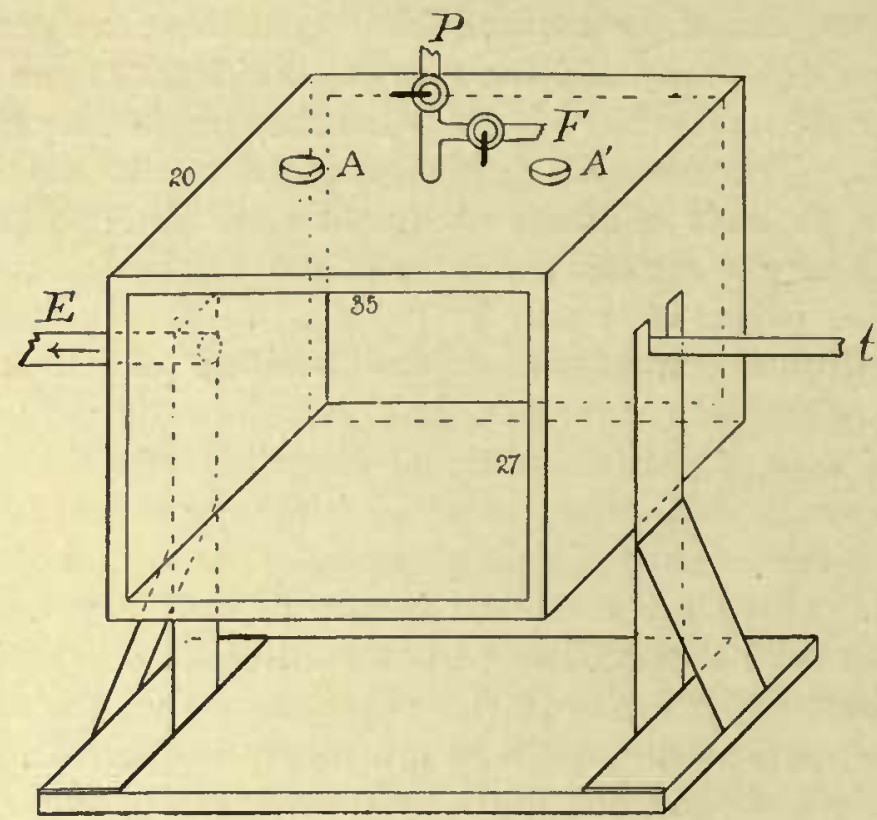

Figure i.-Cubical Condensation Chamber.

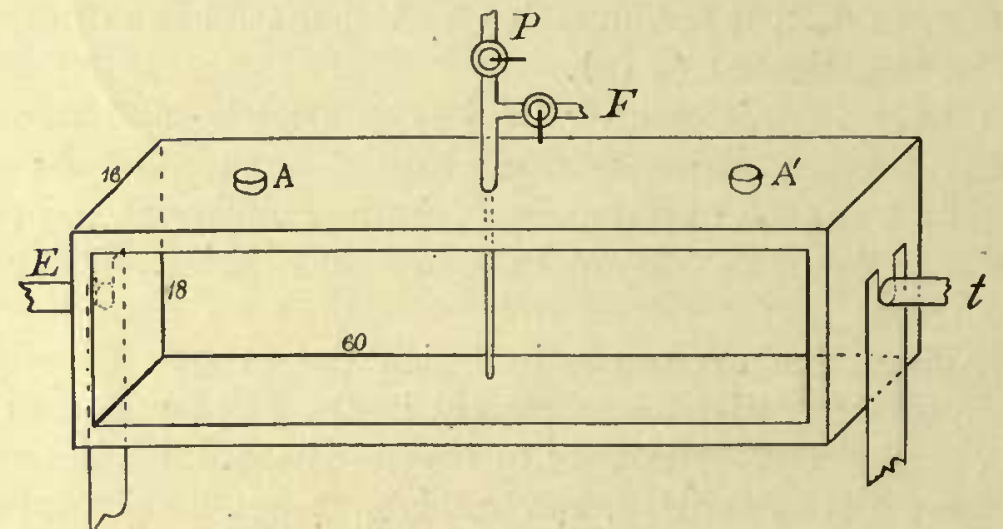

figure 2.-Long Condensation Chamber.

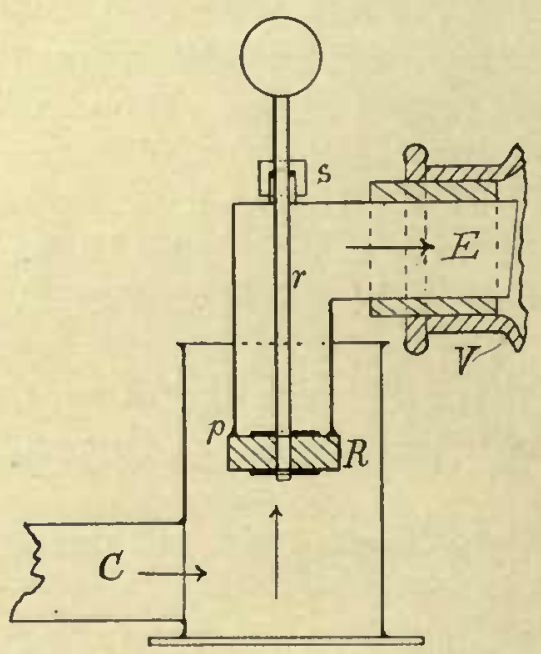

Figure 3.-VALVe for Sudden Exhaustion. 
exhaustion an external vacuum chamber provided with a mercury gauge and a wide stopcock suffices. A jet pump is suitable for evacuation.

The condensation chamber is placed between the goniometer and the source of light, nearer the former if large coronas are to be obtained. In my experiments the distances were usually 84 and $250 \mathrm{~cm}$., or about as $\mathrm{r} / 3$. The eye at the goniometer is focussed unconsciously on the distant source (334 cm.).

The exhaustions must be made systematically in connection with a seconds clock, to admit of allowance for the time losses. The time during which the fog remains suspended is particularly important, and must be uniform and as short as possible. ${ }^{1}$

3. Color distributions. - In classifying the coronas, a statement of the colors of the first two or three annuli, counted from the center, will usually suffice. For the case of the electric light the central patch remains white, or at least opalescent or bluish. With the Welsbach lamp a central disc of vivid green or green-yellow, or even yellow, is frequently observed; but the use of the electric light in parallel series shows this to be due to the absence of strong complimentary blues and violet.

For convenience in specifying color, the following abbreviations will be used throughout: w, white; p, purple; c, crimson; r, orange-red; br, brown; $\mathrm{o}$, orange; $\mathrm{y}$, yellow; g, green; $\mathrm{b}$, blue; $\mathrm{v}$, violet. Mixed colors are written together; thus bg is blue-green, rv red-violet. An accent denotes an approximation to the color; thus $b^{\prime}$ is bluish, which has been otherwise indeterminable. A dot or capital denotes a deep or dark color; thus $\dot{b}$ or $B$ is dark blue. A mere line denotes a color ring too narrow or dark to be recognized. This is the frequent transition from red to green, marked $\mathrm{w} r \mid \mathrm{g}$.

Beginning with the most intense nucleation obtainable, $i . e_{\text {., with particles }}$ of the least size producible, the following coronas appear in succession, at first filmy and fleeting, but eventually brilliant and dense. The numerals attached to the series are arbitrary.

I. $\quad \mathrm{w}^{\prime} \mathrm{O}^{\prime}$

II. $w \vee g^{\prime} ; b^{\prime} \dot{b} r^{\prime} ; w^{\prime} g \dot{v} ; w y v b g^{\prime} ; w$ yo $v g^{\prime} ; w$ c yg $v^{\prime}$

There is thus an obvious tendency for the colors succeeding white to follow each other in the order of wave length, as the particles continually increase in diameter. All intermediate gradations are represented. The second cycle is nearly complete, the first (?) cannot be obtained except in the opalescent orange tint, unless the steam jet is employed. The second annulus of any corona is apt to vary in width so as to be unequally important.

The next series (III) for successively larger particles is a contraction of the preceding. There is obviously much overlapping. The following types of coronas may be cited. The colors are very brilliant. The second "green" 
corona is particularly characteristic, consisting of three broad color bands. The disc is green with the Welsbach lamp.

$$
\text { III. } w \text { vp b } g^{\prime} \mathrm{r}^{\prime} \text {; w } \mathrm{g} \dot{\mathrm{b}} \mathrm{p} \text {; w yo (b) gb } \mathrm{r} \text {; } w \dot{\mathrm{r}}(\mathrm{b}) \mathrm{g} \mathrm{r}^{\prime} \text {. }
$$

The next series (IV) is a variation of $\mathrm{w} \mathrm{r}^{\prime} \mathrm{b} g \mathrm{r}$, approaching the steady normal coronas of the next cycle. The colors are very closely packed together, so that it is difficult to produce definite types of them at will. Very small differences of diameter of cloud particle materially change the details of the color scheme. Incidentally, however, the "green" corona, $\mathrm{wg}^{\prime} \dot{\mathrm{b}} \mathrm{p}$ is obtained particularly with the Welsbach lamp; the red of the first ring changes from $\mathrm{y}^{\prime}$ to $\mathrm{br}^{\prime}$. $\mathrm{w} \mathrm{r} / \mathrm{g}$ is frequent.

In succeeding coronas the normal type is practically permanent and the observable variation is merely in diameter.

4. Apertures.-For the measurement of the relative apertures $(s)$ of the coronas the inner edge of the first ring or the diameter of the white patch is unsuitable, because this demarcation is usually vague. On the other hand, the demarcation between the first and second color rings is usually very sharp and the colors in contrast. Most of the measurements have therefore been made to the outer edge. of the first ring. Naturally there will be periodicity from the fluctuation of wave length specified, but this periodicity persists when homogeneous light is employed. Unfortunately, the coronas are usually so faint that the simple means for homogeneous light are not available and electric or sunlight must be employed. For practical purposes, colored annuli are thus inevitable.

The opalescent colors of the series marked II above soon fadc. Evaporation takes place while the partially exhausted air is regaining its original temperature. Particles become irregular with no markedly preponderating size. Initial coronas are fleeting, final coronas washed. The evaporation effect is much less evident in Series III and the succeeding series.

\section{DATA OBTAINED WITH THE WELSBaCH BURNER.}

5. Explanation of tables. - To correlate the present with my earlier investigations I will give a series of results found by using a small circular part of the Welsbach mantel as a source of light. Coronas in this case are more easily identified because of the simplified color scheme, to the practical advantages of which I have already referred.

In table $1, z$ denotes the number of the partial exhaustions each of volume ratio, $y$, and made in succession, $t$, the current time in minutes (the interval being about 3 minutes to allow for adjustments and for diffusion), $s$ the chord of the angular radius, $\phi$, at radius $R$, so that $s / R=2 \sin q$. The eye and source of light were at distances 85 and $250 \mathrm{~cm}$. from the intervening condensation chamber, and the former was focussed for long distances. The pressure and temperature of the atmosphere were $P$ and $\theta$, and the fixed pressure decrement 
on exhaustion uniformly $\delta p=\mathrm{I} 7 \mathrm{~cm}$, , nearly, so that the precipitate per cub. $\mathrm{cm}$. is $m=4.7 \times 10^{-6}$. Measurements were made to the outer edge of the first ring. In the column marked "coronas," the color of the annuli is specified from within outward, using the abbreviations stated above. The nucleation is marked $n^{\prime}$ if computed from the aperture $s$ standardized with lycopodium, $n^{\prime \prime}$ if computed from $s$ standardized by: subsidence measurements, $N$ if computed relatively as a geometric progression, $n$ when the latter is reduced as shown below, and the absolute values corrected for time and exhaustion losses, etc. The initial nucleation is shown under $n_{0}$, and corresponds to $z=4$. The other coefficients, $\beta$, referring to time losses, $\alpha$ referring to exhaustion losses, $S$ referring to subsidence losses, will be presently explained. Though $n$ is measured for the partially exhausted receiver, a final correction $(I / y)$ need not be added, for the influx of filtered air leaves the nucleation undisturbed. The ratio $n^{\prime} / N$ $=275 \mathrm{~s}^{3} / \mathrm{IO}^{z \log y}$ constructed in the charts shows the wide departure from the constancy which would be anticipated. Diameters of the fog particles are given under $d$, the accents referring to the method of computation. All data will be fully discussed below.

6. Charts.-The charts show the relations of important quantities in the tables. Thus $r=n^{\prime} / N$ may be laid off in relation to the number of the exhaustion, $z$, as in figure 5 ; but, generally, the nucleations, $n$, and finally the diameters, $d$, of the fog particles, are given in terms of the apertures, $s$, where the angular radius $\phi=s / 60$.

7. Tables. - The data investigated for the Welsbach burner follow.

TABLE I.-CONSTANTS OF CORONAS. WELSBACH LAMP. CONDENSATION CHAMBER, $20 \mathrm{~cm}$. BROAD, $25 \mathrm{~cm}$. HIGH, $35 \mathrm{~cm}$. LONG; DISTANCES OF EYE AND SOURCE OF LIGHT FROM CHAMBER, $85 \mathrm{~cm}$. and $250 \mathrm{~cm}$., RESPECTIVELY; $\theta=22^{\circ}$; BAROM., $75.34 \mathrm{~cm} . ; \delta p=16.9 ; y=.77 ; \alpha=.064 ; \beta=0 ; S=2.65$; $a^{\prime \prime}=.0029$ (SUBSIDENCE); $a^{\prime}=.0032$ (LYCOPODIUM) $; n_{0}=209000 ;$ PHOSPHORUS NUCI.EI. $\quad d^{\prime \prime}=.0029 / \mathrm{s} ; m=4.7 \times 10^{-6}$. MEASUREMENT OF $s$.TO OUTER EDGE OF FIRST RING.

\begin{tabular}{|c|c|c|c|c|c|c|c|c|c|}
\hline$z$ & $t$ & $s$ & Corona. & $n^{\prime}=275 s^{3}$ & $n^{\prime \prime}=370 \mathrm{~s}^{3}$ & $N \Pi\left(1-\frac{s}{s^{2}}\right)$ & $n_{0}$ & $n$ & $\begin{array}{c}d=.021 \\
n^{-1 / 3}\end{array}$ \\
\hline ० & min. & $\mathrm{cm}$. & & $\times 10^{-3}$ & $\times 10^{-3}$ & $(2.84)$ & $\times 10^{-3}$ & 600000 & $\mathrm{~cm}$. \\
\hline I & I7 & - & & - & - & (2.19) & & 460000 & .000270 \\
\hline 2 & 20 & - & $b^{\prime} r^{\prime}$ & - & - & $(1.69)$ & & 355000 & 290 \\
\hline 3 & 23 & - & $\mathrm{g}^{\prime}$ & - & - & (1.30) & & 273000 & 320 \\
\hline 4 & 27 & I0.3 & y o b & 300 & 403 & 1.000 & & 210000 & $35^{\circ}$ \\
\hline 5 & 30 & 8.3 & w C g & I 57 & 2 I 2 & $.75 \mathrm{I}$ & & I 58000 & 385 \\
\hline 6 & 34 & 6.8 & $w p b^{\cdot} g^{\prime}$ & 86.3 & I I 6 & .557 & & I I 7000 & 425 \\
\hline 7 & 37 & 6.0 & $\mathrm{~g} \mathrm{~b} \mathrm{~b}^{\circ} \mathrm{p}$ & $59 \cdot 4$ & 79.9 & .403 & & 84600 & 475 \\
\hline$\dot{S}$ & $4 \mathrm{I}$ & 5.8 & $\mathrm{w} \mathrm{rg}$ & 53.6 & 72.2 & .289 & & 60700 & 530 \\
\hline 9 & 44 & 4.6 & w br b $\cdot p$ & 26.7 & $3^{6.0}$ & .204 & & 42800 & 595 \\
\hline I0 & 48 & 4.2 & w r g & 20.4 & 27.4 & .137 & 200 & 28800 & 680 \\
\hline I I & $5 \mathrm{I}$ & 3.7 & corona & I 3.9 & I 8.8 & .090 & 208 & 19000 & 780 \\
\hline I 2 & 54 & 3.2 & " & 9.0 & T2. I & .055 & 221 & I I 500 & 920 \\
\hline 13 & 57 & 2.6 & “" & 4.8 & 6.5 & .032 & 205 & 6700 & .001 I IO \\
\hline 14 & 60 & 2.0 & “" & 2.2 & 3.0 & .015 & 199 & 3 I00 & 1430 \\
\hline I 5 & 63 & I. 3 & " & .6 & .8 & .004 & 220 & 800 & 2250 \\
\hline
\end{tabular}


TABLE I. -SECOND SERIES. $n_{0}=190000 ; a=.06 \mathrm{r}$.

\begin{tabular}{|c|c|c|c|c|c|c|c|c|c|}
\hline$z$ & $t$ & $s$ & Corona. & $n^{\prime}=275 s^{3}$ & $n^{n}=370 \mathrm{~s}^{3}$. & $N \Pi\left(\mathrm{I}-\mathrm{S}_{\mathrm{S}^{2}}\right)$ & $n_{\circ}$ & $n$ & $d=.021$ \\
\hline 0 & - & - & - & - & & $(2.84)$ & & 540000 & .000256 \\
\hline I & 4 & - & - & - & & $(2.19)$ & & 416000 & 278 \\
\hline 2 & 7 & - & $\mathrm{b}^{\prime} \mathrm{b}^{-}$ & - & & $(1.69)$ & & 321000 & 304 \\
\hline 3 & 10 & - & $\mathrm{g}^{\prime}$ & - & & (I.30) & & 247000 & $33 \mathrm{I}$ \\
\hline 4 & I 3 & 10.6 & yob & 327 & 440 & I. 000 & & I90000 & $3^{62}$ \\
\hline 5 & 16 & 8.3 & w c g $g^{1}$ & 157 & 212 & $.75^{\mathrm{I}}$ & & 143000 & 398 \\
\hline 6 & 19 & 6.0 & $\mathrm{~g} \mathrm{~b} \cdot \mathrm{r}$ & $59 \cdot 4$ & 79.9 & .557 & & 106000 & $44^{\circ}$ \\
\hline 7 & 22 & 5.6 & yg o bg & 48.4 & $65 . x$ & .398 & & 75600 & 492 \\
\hline 8 & 25 & $5 \cdot 3$ & w C g & 41.0 & $55 . \mathrm{I}$ & .280 & & 53200 & 555 \\
\hline 9 & 28 & $4 \cdot 4$ & $\mathrm{~g}^{\prime} ! \mathrm{b}^{\circ} \mid \mathrm{p}$ & 24.2 & 32.6 & . 196 & & 37200 & 623 \\
\hline 10 & $3 \mathrm{I}$ & 4.0 & w c g & $\times 7.6$ & 23.7 & .130 & I 82 & 24700 & $7 \times 5$ \\
\hline I I & 34 & 3.6 & w br b'g'r & 12.8 & $77 \cdot 3$ & .083 & 207 & I 5800 & 830 \\
\hline$x_{2}$ & 37 & 2.9 & corona & 6.7 & 9.0 & $.05^{\circ}$ & I 81 & 9500 & 980 \\
\hline I 3 & 40 & 2.4 & " & 3.8 & $5 . \mathrm{I}$ & .027 & 189 & $5^{100}$ & .001200 \\
\hline I4 & 43 & 1.9 & “ & I.9 & 2.5 & $.0 I I$ & 230 & 2100 & r6 630 \\
\hline 15 & $4^{6}$ & 1.5 & " & .9 & r. 3 & .002 & - & 400 & 2850 \\
\hline 16 & 49 & 1.0 & “ & .3 & .4 & & - & $\circ$ & - \\
\hline
\end{tabular}

- Gap in coronal sequences probably due to accidental delay. Similar difficulties at end of series. Timed fog intervals desirable.

\section{METHOD OF REDUCTION.}

8. Constants of the geometric progression.-To determine whether the factor of the geometric progression of successive nucleations, $z$, was to be computed isothermally or adiabatically, a series of direct temperature measurements was deemed necessary. These were made by aid of a thermocouple of extremely thin wires $(.007 \mathrm{~cm}$. in diameter), of copper and german silver. The junction within the receiver was not soldered, but the flexible copper wire looped once around the other. In this way the variation of the instantaneous air temperature in the receiver could be closely followed. It was necessary to use a sensitive astatic galvanometer, and the measurements are thus subject to the fluctuations of the earth field. As it is the immediate purpose of these data to determine about how soon the isothermal conditions are re-established by radiation from without, the irregularities are of little consequence.

Table 2 contains three series of results, the upper end of each row corresponding to the period of exhaustion, the lower to that of (slow) refilling. Readings were taken in intervals of half a minute. The table shows that after the lapse of one minute following the sudden exhaustion the temperature has been regained to within a degree. As the coronas can hardly be observed and measured within this time, the exhaustion ratio may be computed isothermally. I have therefore computed the density ratio of nucleation $\rho / \rho^{\prime}=n / n^{\prime}$, before and after exhaustion, as follows.

Since $p=R \rho 9$ in the usual notation of Boyle's law, and $p=P-p^{\prime}$ where $P$ 
is the reduced reading of the mercury gauge and $p^{\prime}$ the vapor pressure of water vapor,

$$
\rho / \rho^{\prime}=(P-p)(1-\delta 9 / 9) /\left(P^{\prime}-p^{\prime}\right) \text {. }
$$

The correction $\delta 9 / 9$, being by the table $.7 / 293=.0024$ or about $\frac{1}{4}$ per cent., may be neglected. Hence $y=\rho^{\prime} / \rho=1-\delta p /\left(P-p^{\prime}\right)$, where $\delta p$ is the pressure difference selected. Thus in table $I, N$, not corrected for time losses, etc., would be

$$
N=y^{z}=10^{z \log y} \text { where } y=.77 \text { and } N=10^{-.1135^{z}} \text {. }
$$

In this way the auxiliary ratios $n^{\prime} / N$ in the tables were constructed. This is the first departure from the method of my earlier work (Smithsonian Contributions, No. 1373).

TABLE, 2.-AIR TEMPERATURE + IN CONDENSATION CHAMBER DURING AND

\begin{tabular}{|c|c|c|c|}
\hline Time after exhaustion. & Ist Series ${ }^{\circ} \mathrm{C}$. & $2 \mathrm{~d}$ Series ${ }^{\circ} \mathrm{C}$. & $3 \mathrm{~d}$ Series ${ }^{\circ} \mathrm{C}$ \\
\hline $\begin{array}{l}0^{\mathrm{m} 2} \\
.5 \\
1.0 \\
1.5 \\
2.0\end{array}$ & $\begin{array}{r}-10.7^{\circ} \\
=\quad .7 \\
=\quad .7 \\
=\quad .7 \\
-\quad .7\end{array}$ & $\begin{array}{l}=10.7^{\circ} \\
=\quad .8 \\
=\quad .8 \\
=\quad .7 \\
=\quad .4\end{array}$ & $\begin{array}{l}-10.8^{\circ} \\
=\quad 1.1 \\
=\quad .7 \\
=\quad .7 \\
=\quad .5\end{array}$ \\
\hline 2.5 & $+1.4^{3}$ & $-\quad \cdot 3$ & -.5 \\
\hline 3.0 & I. 4 & $+1.2^{3}$ & -.4 \\
\hline $\begin{array}{l}3.5 \\
4.0 \\
4.5\end{array}$ & $\begin{array}{l}.7 \\
.4 \\
-\end{array}$ & $\begin{array}{r}2.0 \\
1.1 \\
.5\end{array}$ & $\begin{aligned}+ & \text { I. } .^{3} \\
& \text { I.I } \\
& \text { I.I }\end{aligned}$ \\
\hline 5.0 & & .3 & .4 \\
\hline $\begin{array}{l}5.5 \\
6.0\end{array}$ & & & $\begin{array}{l}.3 \\
.1\end{array}$ \\
\hline
\end{tabular}
AFTER SUDDEN EXHAUSTION AND DURING INFLUX.

1 Irregular march after exhaustion due to unsteady earth field.

2 Throw on sudden exhaustion.

${ }^{3}$ March on filling slowly with filtered air.

9. Time losses.-Nuclei apparently decay spontaneously in the lapse of time, $t$, and a correction is to be added to $N$. Since this loss is relatively small in view of the short time intervals occurring above, $n=n_{1} \mathrm{IO}^{\beta\left(t-t_{1}\right)}$ may be assumed for convenience. Hence if $n$ be the nucleation due to a given corona seen at low pressure or the identical nucleation at atmospheric pressure after filtered air has been added, the next corona after $z$ exhaustions and $t$ minutes will correspond to $n_{\mathrm{I}}=n \mathrm{ro}^{z \log \mathrm{y}+\beta\left(t-t_{1}\right)}$ whence

$$
\beta=\left(\mathrm{r} /\left(t-t_{\mathrm{r}}\right)\right) \log \left(n_{\mathrm{r}} / n y^{2}\right) \text {. }
$$


Thus it is merely necessary to know the relative values of $n$ or the nucleation ratios, and these may be obtained by any method of smoothing, by plotting $n^{\prime}$ or $N$ in terms of $s$.

In table $\mathrm{x}, N$ has been found for each aperture, $s$, and $\beta$ computed as stated. The values of $\beta$ so found are of the mean order of .002. They apparently decrease toward the end of each series as the number of nuclei grows smaller; but this conclusion is not vouched for, as it will presently be shown that a specific loss of nuclei accompanies each exhaustion. In other words, $n=N r_{\circ}(\mathrm{I}-a z)$, where $r_{\circ}$ is a constant, or the numbers diminish faster than is attributable to time loss alone. The effect will be particularly marked for the smaller initial time intervals of the original nucleations.

Accordingly I have made another computation, writing approximately

$$
n / n^{\prime}=\left(N / N^{\prime}\right)\left(1+\alpha\left(z^{\prime}-z\right)\right)
$$

and finding $\beta$, from this by the equation already given. These values of $\beta$ are inserted in the last column of the table. They are irregular in distribution and of the mean value $\beta=.0016$.

The chief result of this paragraph is the relatively small value of the coefficient of time loss of nuclei. Its effect on the results may therefore be neglected (tested), particularly as the effect $10^{.0016 z}=\mathrm{I} .004$ for $z=\mathrm{I}$ is in part compensated by the temperature factor $\delta 9 / 2=.0024$ of the preceding paragraph. Direct experiments below $(\$ 27,28)$, where identical results are obtained for different time intervals ( $2 \mathrm{~min}$. and $4 \mathrm{~min}$.), vouch for this conclusion. Measurements of coronal aperture of the present kind, hampered as they are by the difficulties of observation, of reduction, of subsidence, etc., cannot offer more than an estimate of the conditions sought.

ro. Exhaustion losses.-I shall next consider the independent destruction of nuclei which accompanies each exhaustion, and for which it is difficult at the outset to assign a reason. It will be shown that it is due to the subsidence of fog particles. This loss did not appear in my original investigations, probably because the spherical receiver used was not lined with wet cloth.

From what has preceded, the relative number of nuclei after $z$ exhaustions is $10^{z \log y}$, whereas in the region of normal coronas the absolute number is certainly very nearly $n^{\prime}=C s^{3}$, where $C$ is a constant. This will be independently verified presently by special experiments. Hence the ratio $r=C s^{3} / 10^{z \log y}$ should be constant, whereas experiment and the charts, figure 5 , show (roughly) that $r=r_{\circ}(\mathrm{I}-\alpha z), \alpha$ being the coefficient of exhaustion loss and $r_{0}=C s_{0}{ }^{3}$ the arbitrary initial ratio for $z=0$, preceding the first corona observed. Thus

$$
\boldsymbol{\alpha} z=\left(\mathrm{I}-\left(s / s_{0}\right)^{3} / \mathrm{ro}^{z \log y}\right) \text {. }
$$

If $r=0$ for $z=z^{\prime}, \mathrm{I} / z^{\prime}=\alpha$, so that $\alpha$ appears as the reciprocal of the abscissa underlying the positive ordinates in the first quadrant, and may be taken at once from the graphs. These values are given at the head of each table. 
A CONTINUOUS RECORD OF ATMOSPHERIC NUCLEATION.

TABLE 3.-CONSTANTS OF CORONAS, COMPUTED BY SUPPOSING $\alpha=$ CONST. DISTANCES OF EYE AND SOURCE 85 AND $250 \mathrm{~cm}$. FROM RECEIVER, RESPECTIVELY. $\theta=22^{\circ} ; \quad \mathrm{P}=75.34 \mathrm{~cm} . ; \delta p=16.9 ; \quad y=.759 ; n=n_{0} \mathrm{IO}^{-. .12 z}(\mathrm{I}-\alpha z)$; $\alpha=.062 ; \beta=.002$. MEASURED TO OUTER RED, INNER BLUE OR GREEN. $n_{\mathrm{o}}=750000 ; m=4.7 \times 10^{-6} ; a=.0032 ; 6 \mathrm{~m} / \pi d^{3}=275^{3} . \quad P$ IONIZER.

First Series.

\begin{tabular}{|c|c|c|c|c|c|c|c|c|}
\hline$z$ & $t$ & $s$ & Corona. & $n^{\prime}=275^{3}$ & $N(\mathrm{1}-\alpha z)$ & $n_{0}$ & $\begin{array}{c}n=n_{0} 10^{-.12 x} \\
\times(1-\alpha z)\end{array}$ & $\begin{array}{c}r=n^{\prime} / N \\
\times 10^{3}\end{array}$ \\
\hline I & $\min$. & $\mathrm{cm}$ & nucleation. & & & & & 一 \\
\hline 2 & 20 & & $\mathrm{~b}^{\prime} \mathrm{r}^{\prime}$ & & .504 & & 460000 & - \\
\hline 3 & 23 & & $-g^{\prime}$ & & .355 & & 323000 & 一 \\
\hline 4 & 27 & 10.3 & y०b & 300000 & .249 & & 227000 & 300 \\
\hline 5 & 30 & 8.3 & W rc g & I 57000 & .173 & & 157000 & 204 \\
\hline 6 & 34 & 6.8 & $w p^{\prime} B g^{\prime}$ & 86300 & .120 & & 109000 & 146 \\
\hline 7 & 37 & 6.0 & $\mathrm{~g}^{\prime} \mathrm{BP}$ & 59400 & .082 & & 74700 & I 30 \\
\hline 8 & $4 I$ & 5.8 & $w r g$ & 53600 & .055 & & 50400 & I 52 \\
\hline 9 & 44 & 4.6 & w br $B \mid p$ & 26700 & .037 & & 33500 & 99 \\
\hline IO & $4^{8}$ & 4.2 & $\mathrm{w}$ or $\mathrm{g}$ & 20400 & .024 & $8_{5} 00000$ & 21800 & 98 \\
\hline I I & $5^{I}$ & 3.7 & corona & 13900 & .015 & 9100000 & I 3900 & 87 \\
\hline 12 & 54 & 3.2 & $"$ " & 9000 & .009 & 9700000 & 8500 & $74 \cdot 5$ \\
\hline I 3 & 57 & 2.6 & "“ & 4800 & .005 & 9000000 & 4900 & 51.0 \\
\hline I 4 & 60 & 2.0 & “ & 2200 & .003 & 8000000 & 2500 & 30.0 \\
\hline I 5 & $6_{3}$ & I. 3 & “" & 600 & .001 & 5400000 & 1000 & 10.7 \\
\hline & & & & & & mean, 9100000 & & \\
\hline
\end{tabular}

SeCOND Series.

\begin{tabular}{|c|c|c|c|c|c|c|c|c|}
\hline$z$ & $t$ & $s$ & Corona. & $n^{\prime}=275^{s^{3}}$ & $N(\mathrm{I}-\alpha z)$ & $n_{0}$ & $\begin{array}{c}n=n_{0} 10^{-.528} \\
\times(1-\alpha z)\end{array}$ & $\begin{array}{c}r=n^{\prime} / N \\
\times 10^{3}\end{array}$ \\
\hline I & $\min _{4}$ & $\mathrm{~cm}$. & nucleation. & & & & & \\
\hline 2 & 7 & & $\mathrm{~b}^{\prime} \mathrm{B}$ & & .504 & & 378000 & - \\
\hline 3 & I0 & & $\mathrm{g}^{\prime}$ & & .355 & & 266000 & 一 \\
\hline 4 & I 3 & 10.6 & y o b & 327000 & .249 & & I 86000 & 327 \\
\hline 5 & I6 & 8.3 & w c g & I 57000 & .173 & & I 30000 & 204 \\
\hline 6 & Ig & 6.0 & $\mathrm{~g} \mathrm{~B} \mathrm{r}$ & 59400 & . I 20 & & 90000 & 100 \\
\hline 7 & 22 & 5.6 & yg o bg & 48400 & .082 & & 61600 & 106 \\
\hline 8 & 25 & $5 \cdot 3$ & w c g & 41000 & .055 & & $4 I 500$ & I I 7 \\
\hline 9 & 28 & $4 \cdot 4$ & $\mathrm{~g}^{\prime} \mathrm{B} \mid \mathrm{p}$ & 24200 & .037 & & 27600 & 89.5 \\
\hline 10 & $3 I$ & 4.0 & w c g & 17600 & .024 & 740000 & I 8000 & 85.0 \\
\hline I I & 34 & 3.6 & $w$ br B $g^{\prime} r$ & I 2800 & .015 & 840000 & I I 500 & $79 \cdot 5$ \\
\hline 12 & 37 & 2.9 & corona. & 6700 & .009 & 720000 & 8400 & 55.5 \\
\hline 13 & 40 & 2.4 & $"$ & 3800 & .005 & 710000 & 4000 & 40.0 \\
\hline I 4 & 43 & I. 9 & $"$ & I 900 & .003 & 680000 & 2100 & 26.0 \\
\hline I 5 & 46 & I. 5 & “ & 930 & .001 & 830000 & 825 & I 6.6 \\
\hline 16 & 49 & I.O & " & 270 & .000 & - & - & 6.3 \\
\hline
\end{tabular}

Note:-In $N=10^{-12 x}$ the constant is corrected for time loss. This practice was later abandoned. $(\xi 9)$. The constant in $n^{\prime}$ is obtained from earlier measurements with lycopodium. The auxiliary column $n^{\prime} / N$ has been used in the charts. 
The occurrence of different slopes in the curves is an obvious result of the unavoidable differences of initial nucleation. The effect is a shifting of the $z$ values. Thus in two series for the same corona similarly observed, but with independent initial nucleations, $r=r_{0}(I-\alpha z)=r_{0}^{\prime}\left(I-\alpha^{\prime} z^{\prime}\right)$, where $z^{\prime}=z-z_{0}^{\prime}$; whence $\alpha^{\prime}=\alpha /\left(I-\alpha z^{\prime}{ }_{0}\right)$, the constant $z^{\prime}$ 。 being the index of the difference of initial nucleations.

A computation on the assumption of constancy of $\alpha$ was made for comparison, and is given in the preceding table, 3 , corresponding to table $I$ above, and with the same notation.

II. Exhaustion loss attributable to subsidence.-The misleading feature of $\alpha$ is its apparent constancy for a given receiver and a given scheme of observations. It will now be shown that this result is a mere approximation and that the phenomenon may be fully explained in terms of subsidence. In this case, ${ }_{10} 0^{4}=9 \sqrt{v}$, where $R$ is the radius of the water particle and $v$ its rate of subsidence. Since $2 R=d=.0032 / s$, approximately, $v=(1.78)^{2} / s^{2}$, or if $v^{\prime}$ refers to minutes, $v^{\prime}=190 / s^{2}$.

The, relative loss, $l$, per minute, is for a vessel of height $h$ and nucleation $n, l=v / h=190 / h s^{2}$. If, as in the above condensation chamber, the height is $h=26.5 \mathrm{~cm}, l=7.2 / \mathrm{s}^{2}$, or, in tabular form,

$$
\begin{array}{lllllll}
s= & I & 2 & 3 & 4 & 5 & \mathrm{~cm} . \\
l= & .7 .2 & \text { I. } 8 & .80 & .45 & .36 &
\end{array}
$$

numbers which are astonishingly large, but must be near the truth.

Let the time consumed in observation be $\mathrm{I} / 2 \mathrm{~min}$. and $\mathrm{I} / 4 \mathrm{~min}$., respectively; then the ratio $r$ in table I may be corrected in the region of normal coronas as follows:

TABLE 4. - CORRECTION OF $r=275^{3} / 10^{z \log y}$ IN TABLE 3 , SHOWING THE EFFECT OF SUBSIDENCE.

\begin{tabular}{|c|c|c|c|c|c|c|c|}
\hline$z$ & $r \times 10^{-3}$ & $s$ & $\frac{1}{2}$ & $\begin{array}{c}r \times 1^{-3} \\
\text { corrected. }\end{array}$ & $t$ & $\begin{array}{c}r \times \text { Io-3 }^{-3} \\
\text { corrected. }\end{array}$ & $\begin{array}{c}\text { Mean } r \times \text { ro-3 }^{-3} \\
\text { corrected }\end{array}$ \\
\hline IO & 98.0 & 4.2 & $.2 \mathrm{I}$ & 98.0 & .10 & 98.0 & 98.0 \\
\hline I I & 87.0 & 3.7 & .26 & 108.0 & . I 3 & 97.0 & 103.0 \\
\hline I 2 & 74.5 & 3.2 & .35 & I 18.5 & .18 & $95 \cdot 5$ & 107.0 \\
\hline I 3 & 51.0 & 2.6 & .51 & I 21.0 & .28 & 85.4 & 103.0 \\
\hline 14 & 30.0 & 2.0 & .90 & I 26.0 & .45 & 79.0 & 103.0 \\
\hline I 5 & 10.7 & I. 3 & - & 130.0 & .75 & 73.0 & 102.0 \\
\hline
\end{tabular}

The first of these values of $r$ is under-corrected, while the second $r$ is overcorrected. The mean of these corresponding to a time of observation of $\frac{3}{8}$ minute is constant. Now as the time during which the fog is left undispelled after exhaustion for measurement is actually of this order, there can be no question but that the error is due to subsidence. The equation should therefore read:

$$
n=C s^{3}=n_{0} 10^{z \log y}\left(\mathrm{I}-S / s_{0}^{2}\right)\left(\mathrm{I}-S / s_{1}^{2}\right) . .
$$


as will be more fully explained below. Here $S$ is an appropriate subsidence constant depending on the mean time of observation or on the time within which nuclei are suspended in the fog particles. Conformably with this view $\alpha$ or $S$ depends essentially on the height of the vessel in which the condensation is produced, being larger, ccet. par., in shallow vessels, seeing that $v / h$ is larger. ( $\$ 25)$.

12. The optic constant.- The proportionality of diameter with the inverse aperture may be assumed for normal coronas. The occurrence of periodicity in the higher coronas, even if merely a question of color were involved, would modify these simple conditions for these cases. It is well known that for a single particle, the masterly work of Lommel ${ }^{1}$ has given a complete treatment of the diffractions in terms of Bessel functions.

In meteorological work for a particle of diameter $d$ and for uniformly normal coronas, the equation $\sin \varphi=1.22 \lambda / d$ is usually assumed, if the angular radius of the corona is $\varphi$ and the wave length in question $\lambda$. Since in my goniometer $2 \sin \varphi=s / R$, where $R=30 \mathrm{~cm}$., $d s=a=73.2 \lambda$. 'Hence, for the successive spectrum colors, the following values obtain for the constant $a$ :

$$
\begin{array}{lccccccc}
\text { color, } & \mathrm{c} & \mathrm{r} & \mathrm{o} & \mathrm{y} & \mathrm{g} & \mathrm{b} & \mathrm{V} \\
a= & .005 \mathrm{I} & .0046 & .0044 & .0042 & .0039 & .0034 & .0029
\end{array}
$$

In view of the theoretical uncertainty of these values in the case of the distribution of particles met with in the above experiments, I have usually relied on the results of direct comparisons with the corona of lycopodium spores where $d_{0}=.0032 \mathrm{~cm}$. Placed in the position of the near plate of the coronal chamber, the corresponding aperture for lycopodium spores was $s_{0}=1.05 \mathrm{~cm}$., at the far plate, $s_{\circ}=1.03$. Hence $a=d_{\circ} s_{\circ}=.0034$ for measurements to the outer edge of the first ring. This corresponds to the preceding value for blue, though these apertures were measured through ruby glass. In the above tables, where merely relative results were in question, $a=.0032$, a datum of earlier measurements, was inserted. Reduction was deemed needless. For the case of measurements to the inner edge of the first ring or to the edge of the white disc, $s_{0}=.7$ to .8 , the demarcation being more vague. Assuming the latter, $a=.0026$ for such measurements. Still more troublesome is the measurement of $a$ when the condensation chamber is remote $(250 \mathrm{~cm}$.) from the goniometer and near $\left(85 \mathrm{~cm}\right.$.) the source. The datum was $a=.00$ I 25 from $s_{0}=.39$.

I3. The optic constant. Diameters from subsidence.-This is an independent method of standardizing $s$. In my earlier work the condensation chamber was not cloth-lined, and the subsidence data quite untrustworthy, showing rapid retardation of abnormally high initial values due to evaporation. In the present cloth-lined receiver kept wet on all sides, subsidence data are reasonably satisfactory. The coronas, however, change character during subsidence, and in case of the initial opalescent coronas (Series II above) all coronas vanish into a mere fog before subsidence is even appreciable. Finally, the upper plane

iLommel, Abhandl. der kön. Bayerischen Akad.der Wissensch., xv, 1886. 
boundary of the fog, which at the outset appears as a sharp horizontal line about $50 \mathrm{~cm}$. long, even after I or 2 min. becomes more and more vague. Subsidence is here accelerated. Hence it is chiefly for the normal coronas that subsidence data are available, and, fortunately, it is precisely here that they are wanted. In other cases the occurrence of periodicity and the rapid change of coronas makes the interpretation tedious and difficult.

The following table contains the results for subsidence. Stokes's well-known formula reduces to $R=9 \times 10^{-4}, \bar{v}$ where $R$ is the radius of the fog particle sought. Temperature is denoted by $\theta$ and current time by $t$, depth of the fog line by $x$, and rate of subsidence by $v$. The diameter computed from aperture is inserted for comparison. $2 R s=a$ is the new optical constant sought. The braces denote the observations of $x$ taken to compute $v$ whenever the corona changes character.

It appears from the earlier parts of this table that although the coronas may shrink and change appearance, this is not in the same measure the case with the rate of subsidence. In the latter parts of a given series of observations, therefore, the diameters from aperture are always in excess of those from subsidence. With the growth of droplets, the two phenomena refer to different particles, and hence only the original observations, $i$. $e_{\text {., }}$ those within the first minute, are of any value.

TABLE 5.-SUBSIDENCE OF FOG PARTICLES. PHOSPHORUS NUCLEI. VISCOSITY OF AIR, $\eta=.00019$.

First Series.

\begin{tabular}{|c|c|c|c|c|c|c|c|c|c|}
\hline 0 & & $t$ & Corona. & $s$ & $x$ & $v$ & $2 R \times 104$ & $104 \times \frac{.0032}{5}$ & ${ }_{2} R . s=a$ \\
\hline \multirow[t]{4}{*}{$\begin{array}{l}{ }^{\circ} \mathrm{C} \text {. } \\
{ }^{1} 5^{\circ}\end{array}$} & $\begin{array}{l}\text { h. } \\
\text { II }\end{array}$ & $\begin{array}{c}\mathrm{m} . \\
\mathrm{I} \\
2 \\
3 \\
4\end{array}$ & $\begin{array}{l}\text { w r g } \\
\text { shrunken } \\
\text { and foggy }\end{array}$ & $\begin{array}{l}\mathrm{cm} . \\
9 . \mathrm{I}\end{array}$ & $\left.\begin{array}{l}\mathrm{cm} . \\
0 \\
2 \\
4.5 \\
8.5\end{array}\right\}$ & $\begin{array}{c}\mathrm{cm} . / \mathrm{sec} \\
.030\end{array}$ & $\begin{array}{l}\mathrm{cm} . \\
3.1\end{array}$ & $\begin{array}{l}\mathrm{cm} . \\
3.5\end{array}$ & .0028 \\
\hline & & $\begin{array}{l}6 \\
7 \\
8 \\
9\end{array}$ & $\begin{array}{l}g b p \\
w b r b / p \\
w \circ b\end{array}$ & 5.8 & $\left.\begin{array}{l}3 \\
5.5 \\
8.5\end{array}\right\}$ & $\begin{array}{l}.047 \\
.042 \\
.047\end{array}$ & 4.0 & 5.0 & $\begin{array}{l}.0026 \\
(.0023)\end{array}$ \\
\hline & & $\begin{array}{l}\text { I3 } \\
\text { I4 } \\
\text { I5 } \\
\text { I6 }\end{array}$ & corona & $5 \cdot I$ & $\begin{array}{l}0 \\
5.5 \\
11.0 \\
15.0\end{array}$ & .092 & $5 \cdot 4$ & 6.3 & $.002 \pi$ \\
\hline & & $\begin{array}{l}2 \mathrm{I} \\
2 \mathrm{I} \cdot 5 \\
22\end{array}$ & corona & 2.8 & $\left.\begin{array}{r}0 \\
7 \\
17\end{array}\right\}$ & 2.4 & 8.8 & 10.0 & .0025 \\
\hline
\end{tabular}


Second Series.

\begin{tabular}{|c|c|c|c|c|c|c|c|c|c|}
\hline 0 & & 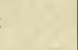 & Corona. & $s$ & $x$ & $v$ & $2 R \times 10^{4}$ & $10^{4} d$ & $2 R \cdot s=a$ \\
\hline \multirow[t]{5}{*}{$15^{\circ}$} & $\begin{array}{l}\text { h. } \\
\text { I I }\end{array}$ & $\begin{array}{l}\mathrm{m} . \\
43 \\
44\end{array}$ & $\begin{array}{l}g^{\prime} b^{\prime} p \\
w^{\prime} r^{\prime}\end{array}$ & 6.8 & $\begin{array}{l}\circ \\
\text { I. } 5\end{array}$ & .025 & 2.9 & $3-4.7$ & .0020 \\
\hline & & $\begin{array}{l}48 \\
49 \\
50\end{array}$ & $\begin{array}{l}\text { wog } \\
\text { foggy } \\
w^{\prime} r^{\prime}\end{array}$ & $\begin{array}{l}9.7 \\
.5 \cdot 5\end{array}$ & $\left\{\begin{array}{l}0 \\
\text { I.5 } \\
4.0\end{array}\right.$ & $\begin{array}{l}.025 \\
.042\end{array}$ & $\begin{array}{r}2.9 \\
3.8\end{array}$ & $\begin{array}{l}3 \cdot 3 \\
5.8\end{array}$ & $\begin{array}{l}.0032 \\
(.0021)\end{array}$ \\
\hline & & $\begin{array}{l}54 \\
55 \\
56 \\
57\end{array}$ & $\begin{array}{l}\mathrm{w} p \mathrm{~b} \\
\mathrm{w}^{\prime} \mathrm{o}^{\prime} \\
(\text { foggy) } \\
\mathrm{w}^{\prime} \mathrm{o}^{\prime}\end{array}$ & $\begin{array}{c}6.8 \\
(6.7) \\
6.5 \\
\\
5.2\end{array}$ & $\begin{array}{l}0 \\
2.5 \\
5.5 \\
9.0\end{array}$ & $\begin{array}{c}.042 \\
(.046) \\
.050 \\
.058\end{array}$ & 3.8 & 4.8 & .0025 \\
\hline & 12 & $\begin{array}{l}\text { I } \\
2 \\
3 \\
4\end{array}$ & wog & $\begin{array}{l}6.0 \\
5.5\end{array}$ & $\begin{array}{r}\circ \\
4.0 \\
8.5 \\
13.0\end{array}$ & $\begin{array}{l}.07 \mathrm{I} \\
.075\end{array}$ & 4.9 & $\begin{array}{l}5 \cdot 5 \\
6.0\end{array}$ & $\begin{array}{l}.0029 \\
(.0027)\end{array}$ \\
\hline & & $\begin{array}{l}\text { I } 3 \\
\text { I } 3.5 \\
\text { I } 4 \\
\text { I } 4.5\end{array}$ & $\begin{array}{l}\text { w cb g' } \mathrm{g}^{\prime} \\
\mathrm{w}^{\prime} o^{\prime} \\
\mathrm{w}^{\prime} \mathrm{o}^{\prime}\end{array}$ & $\begin{array}{l}4.2 \\
4.1 \\
4.0 \\
3.9\end{array}$ & $\begin{array}{l}\circ \\
4 \\
9 \cdot 5 \\
+4 .\end{array}$ & .158 & 7.2 & 7.8 & $\begin{array}{l}.0030 \\
(.0030)\end{array}$ \\
\hline
\end{tabular}

Third Series-Air Nuclet.

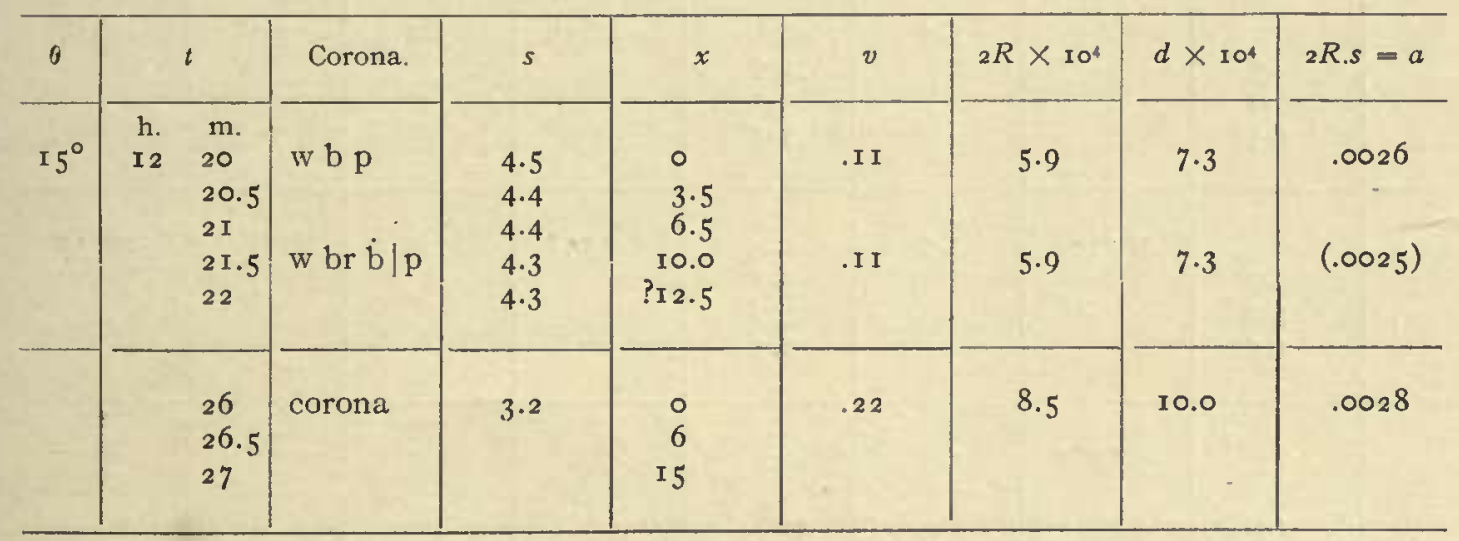


A CONTINUOUS RECORD OF ATMOSPHERIC NUCLEATION.

Fourth Series.

\begin{tabular}{|c|c|c|c|c|c|c|c|c|}
\hline$\theta$ & $t$ & Corona. & $s$ & $x$ & $v$ & ${ }_{2} R \times 104$ & $d \times 104$ & $2 R . s=a$ \\
\hline $15^{\circ}$ & $\begin{array}{l}0 \\
.5 \\
\text { I. } \\
\text { x. } \\
2.0 \\
\end{array}$ & $w$ br bg' $p$ & $4 \cdot 7$ & $\begin{array}{r}0 \\
3 \\
6 \\
9 \\
\mathbf{1 2} \\
\end{array}$ & .105 & 5.8 & 6.8 & .0027 \\
\hline & $\begin{array}{l}0 \\
.5 \\
1.0 \\
1.0\end{array}$ & corona & $3 \cdot 4$ & $\begin{array}{r}0 \\
5 \\
\mathrm{r} 3 \\
\mathrm{x} 9\end{array}$ & .225 & 8.5 & $9 \cdot 3$ & .0029 \\
\hline & $\begin{array}{l}0 \\
.5 \\
1.0 \\
2.5 \\
2.0\end{array}$ & $w b r \dot{b} g^{\prime} r$ & $5 \cdot I$ & $\begin{array}{c}0 \\
2.5 \\
5.2 \\
\\
\text { I0.0 }\end{array}$ & .083 & 5.2 & 6.3 & .0026 \\
\hline & $\begin{array}{l}0 \\
.5 \\
1.0 \\
1.5 \\
2.0\end{array}$ & wog & $5 \cdot 9$ & $\begin{array}{l}0 \\
2 \\
4 \\
7 \\
9\end{array}$ & .078 & 5.0 & $5 \cdot 4$ & .0029 \\
\hline & $\begin{array}{l}0 \\
.5 \\
1.0 \\
1.5\end{array}$ & wo b $g$ & 4.6 & $\begin{array}{l}0 \\
3.5 \\
7.5 \\
\text { I } 2.0\end{array}$ &. $\mathrm{x} 33$ & 6.6 & 7.0 & .0030 \\
\hline & $\begin{array}{l}? \\
.5 \\
1.0 \\
1.5\end{array}$ & corona & $3 \cdot 1$ & $\begin{array}{r}0 \\
6 \\
17 \\
25\end{array}$ & .300 & 9.9 & 10.3 & .0031 \\
\hline & $\begin{array}{l}0 \\
.5 \\
1.0 \\
1.5\end{array}$ & w c g & $5 \cdot 5$ & $\begin{array}{l}0 \\
2.5 \\
5.0 \\
7 \cdot 5\end{array}$ & .083 & $5 \cdot 2$ & $5 \cdot 7$ & .0029 \\
\hline & $\begin{array}{l}0 \\
.5 \\
1.0 \\
1.5\end{array}$ & $w r \mid g$ & $4 \cdot 5$ & $\begin{array}{l}0 \\
3 \cdot 7 \\
9.0 \\
1.3\end{array}$ & .153 & 7.0 & 7.1 & .0031 \\
\hline & $\begin{array}{l}0 \\
.5 \\
1.0 \\
1.5\end{array}$ & corona & 3.0 & $\begin{array}{c}0 \\
6 \\
17 \\
26+\end{array}$ & $\cdot 300$ & 9.9 & 10.5 & .0030 \\
\hline
\end{tabular}


It is nevertheless interesting to note that the values of $a$ obtained are of a reasonable order of values even for the higher coronas, in which periodicity supervenes. The corresponding graph (not shown) brings this out clearly. If the values of $a$ which correspond to normal coronas be selected, the following summary is obtained:

TABLE 6.-VALUES OF $a$ FROM APERTURE AND SUBSIDENCE.

\begin{tabular}{|c|c|c|c|c|}
\hline Series. & Corona. & $s$ & $a$ & Mean. \\
\hline $\begin{array}{c}\text { I } \\
\text { I } \\
2 \\
3 \\
3 \\
4 \\
4 \\
4 \\
4 \\
4 \\
4 \\
4 \\
\text { mean }\end{array}$ & $\begin{array}{c}\text { normal } \\
\text { “" } \\
\text { w b p } \\
\text { normal } \\
\text { “. } \\
\text { " } \\
\text { w o b g } \\
\text { normal } \\
\text { w r g } \\
\text { normal }\end{array}$ & $\begin{array}{l}5.1 \\
2.8 \\
4.2 \\
4.5 \\
3.2 \\
4.7 \\
3.4 \\
5.1 \\
4.6 \\
3.1 \\
4 \cdot 5 \\
3.0\end{array}$ & $\begin{array}{r}.0027 \\
25 \\
30 \\
26 \\
28 \\
27 \\
29 \\
26 \\
30 \\
3 \mathrm{I} \\
3 \mathrm{I} \\
30 \\
.00283\end{array}$ & $.0029^{x}$ \\
\hline
\end{tabular}

The mean of all the series is $a=.00283$; the mean of the fourth series, which is more uniform, $a=.0029 \mathrm{I}$. The latter datum will be taken in the following computations.

I4. Summary of optic constants. - The following series of values of $a=d s$ has been obtained when the measurements of aperture are made to the outer edge of the first ring.

$$
\begin{aligned}
& \text { Optically (blue), } \\
& \text { From lycopodium }\left(d_{\mathrm{o}}=.0032\right) \text {, } \\
& \text { From subsidence, }
\end{aligned}
$$$$
\begin{array}{r}
a=.00344 \\
.00336 \\
.00291
\end{array}
$$

The latter datum is decidedly the smaller, corresponding closely to optical puce-violet (.00293). If, in place of the above expression, the elementary optical equation $2 \sin \varphi=s / R=\lambda / d$ or $a=30 \lambda$ had been taken instead of $a=73.2 \lambda$, even the extreme red would show but $a^{\prime \prime} \doteq .0023$.

The datum for subsidence being simplest in character is apparently the most trustworthy. Since $n=\left(6 \mathrm{~m} / \pi a^{3}\right) s^{3}$, if the method of Wilson and Thomson ${ }^{1}$ be used for the computation of $m$ the following values in grams per cubic centimeter are applicable at the temperatures stated, for the pressure difference $\delta p=\mathrm{I} 7 \mathrm{~cm}$ :

$$
\begin{array}{llll}
\theta= & 10^{\circ} & 20^{\circ} & 30^{\circ} \\
m= & 3.7 \times 10^{-6} & 4.6 \times 10^{-6} & 5.7 \times 10^{-6}
\end{array}
$$


For the pressure difference $\delta p=22 \mathrm{~cm}$. which will occur below,

$$
m=\quad 4.2 \times 10^{-6} \quad 5.5 \times 10^{-6} \quad 6.7 \times 10^{-6}
$$

For $\delta p=8.5 \mathrm{~cm}$.
2. $1 \times 10^{-6}$
$2.6 \times 10^{-6}$
$2.8 \times 10^{-6}$

The effect of temperature on latent heat is not considered, since the data are not fully known. Its effect may be $I$ to 2 per cent. More important is the value to be taken for the heat ratio $\gamma=\mathrm{I} .4 \mathrm{I}$.

15. Resulting equations applied.-From what has been stated, it follows that the first quantity to be found is the initial nucleation, $n_{\circ}, i . c_{\text {. }}$, the nucleation which obtains when $z=Z$. This depends on incidental conditions, such as the intensity of the ionizer, the first corona seen $(Z)$, etc., and is therefore quite arbitrary. In table $\mathrm{I}$, for instance, $n_{4}=n_{\circ}$. Hence

$$
n_{z}=n_{\mathrm{o}} \mathrm{IO}^{(z-4) \log y}\left(\mathrm{I}-S / s_{4}^{2}\right)\left(\mathrm{I}-S / s_{5}^{2}\right)\left(\mathrm{I}-S / s_{6}^{2}\right) \ldots\left(\mathrm{I}-S / s_{(\mathrm{z}-\mathrm{I})}^{2}\right) \text {, }
$$

which will be abbreviated

$$
n_{2}=n_{\mathrm{o}} \mathrm{IO}^{(z-4) \log y_{4}^{z-3}} \prod_{4}\left(\mathrm{I}-S / \mathrm{s}^{2}\right) .
$$

This equation affords in the first place a means of computing $S$. For in the region of normal coronas $n$ is given by the apertures of the coronas. If, for brevity, $r_{z}=n_{z} / 10^{z \log y}$,

\begin{tabular}{|c|c|c|c|c|c|}
\hline & $r \times 10^{-3}$ & $\mathrm{r} / \mathrm{s}^{2}$ & $\mathrm{I} / s_{z-1}^{2} S_{z}^{2}$ & $S$ & Mean $S$. \\
\hline $\begin{array}{l}\text { SERIES } \mathrm{I} . \\
\text { TABLE I. }\end{array}$ & $\begin{array}{c}\text { I00 } \\
\text { I } 32 \\
68.4 \\
\text { I } 17 \\
40.5 \\
\text { I } 00 \\
\text { I } 44 \\
68.4\end{array}$ & $\begin{array}{l}.130 \\
.171 \\
.245 \\
.397\end{array}$ & $\begin{array}{r}.004 \mathrm{I} \\
7 \dot{I} \\
144 \\
367\end{array}$ & $\begin{array}{l}1.97 \\
2.70 \\
2.83 \\
2.44\end{array}$ & 2.66 \\
\hline $\begin{array}{l}\text { Series } 2 . \\
\text { TAble } 1 .\end{array}$ & $\begin{array}{l}39.4 \\
\text { I } 4 \\
53.7 \\
107 \\
397 \\
74.6 \\
31.3 \\
53.7 \\
26.3 \\
34.9\end{array}$ & $\begin{array}{l}.139 \\
.196 \\
.294 \\
.453 \\
.722\end{array}$ & $\begin{array}{r}.004^{8} \\
92 \\
.0208 \\
486 \\
.1230\end{array}$ & $\begin{array}{l}2.73 \\
2.88 \\
2.27 \\
1.84 \\
2.02\end{array}$ & 2.63 \\
\hline
\end{tabular}

$$
r_{\mathrm{z}+\mathrm{I}} / r_{\mathrm{z}-\mathrm{I}}=\left(\mathrm{I}-S / s_{\mathrm{z}-\mathrm{I}}^{2}\right)\left(\mathrm{I}-S / s_{z}^{2}\right) \text {, }
$$

from which $S$ is determinable in terms of pairs of values of $r$ and $s$; or $S$ may be even more simply found from two successive normal coronas. The following table shows the values found for the two series in table $I$.

TABLE 7.-VALUES OF $S$ FROM $r_{z+1} / r_{z-1}=I-S\left(I / s_{z-1}{ }^{2}+I / s_{z}^{2}\right)+S^{2} / s_{z-1}{ }^{2} s_{z}^{2}$. 
As both series were made in the same way, the mean value $S=2.65$ will be inserted. With this value of $S$ the data of the column $N \Pi\left(x-S / s^{2}\right)$ may be computed throughout. Then in the region of normal coronas the fundamental constant of the reduction follows as

$$
n_{\mathrm{o}}=370 \mathrm{~s}^{3} / N I I\left(\mathrm{I}-S / \mathrm{s}^{2}\right) \text {. }
$$

With this constant, the true value of the nucleation (number of particles per cub. cm.) is computed for all coronas as

$$
n=n_{0} N I I\left(\mathrm{I}-S / s^{2}\right) \text {. }
$$

All these data are found in table $\mathrm{I}$. It should be noticed that the coefficient 370 is obtained from subsidence.

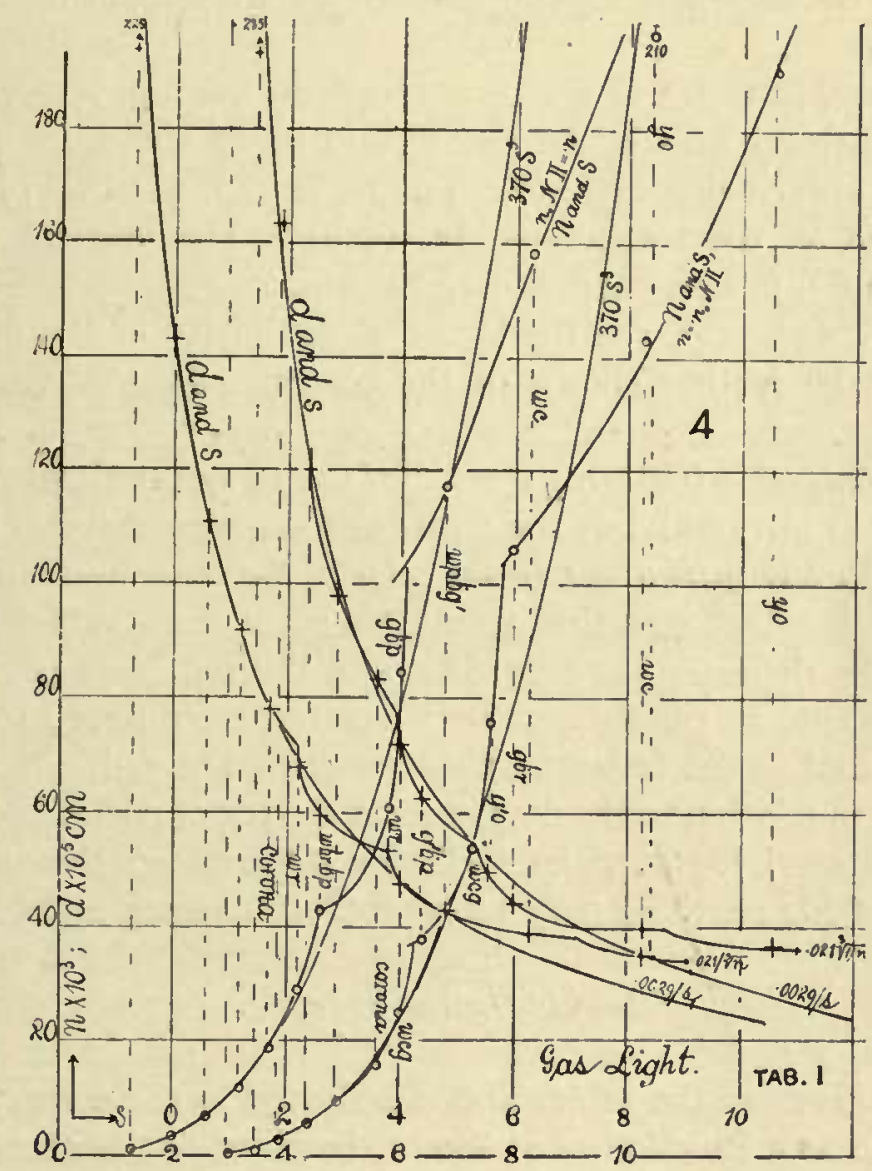

Figure 4.-Chart for Table i, Showing the Relation of Nucleation $(n)$ and of Diameter $(d)$ of Fog Particle in Terms of the Apertures ( $s$ ) of the Coronas.

16. Remarks on the tables and graphs.-The graphs, figure 4, for tables I and 3 , show four independent series of observations of diameter, $d$, and nucleation, $n$ (particles per cub. cm.), in terms of the relative aperture, $s=60 \sin \varphi$, where 
$\varphi$ is the angular radius. The partial exhaustion is to $17 \mathrm{~cm}$. and the standardization is by subsidence ( $\left(S_{14}\right)$. If standardized by diffraction, the $n$-data would be about .6 smaller, or the upper "green" corona, for instance, showing $n=98,000$, would then show $n=60,000$ nuclei. The $d$ effect is much smaller, being .2 larger.

Towards the end of any series the numbers diminish more slowly than the formula requires, but this is naturally the result of the evaporation seen, for instance, in the shrinkage of the coronas. On the other hand, when subsidence is exceptionally rapid, the time during which subsidence takes place, and which can in no case be sharply given (it was not directly timed), is seriously large. On the whole, the agreement is better than was anticipated, and certainly trustworthy. The results coincide in a general way, moreover, with the data found by assuming a constant as was done in $\$ \S 5,9$, and the latter equations may be regarded as approximations by expansion of those in this section.

The graphs, $n$ in terms of $s$, give evidence in the first series of three cycles, the lower two being merged. In the second series there are apparently four cycles, the two lower being distinct. The horizontal position of the cusps is as closely in accord as the measurements justify. The vertical position suffers from the shift and difficulty surrounding the absolute evaluation of $n$. Throughout their extent, however, the fundamental similarity of the graphs is unmistakable, as will be further shown in the corresponding curves for ruby light below.

Since $n^{\prime}=6 \mathrm{~m} / \pi d^{3}=\left(6 \mathrm{~m} / \pi a^{3}\right) s^{3}=23\left(s / \mathrm{IO}^{4} \lambda\right)^{3}$, approximately, the fluctuation of $n$ with $\lambda$ is obvious; but the feature of the phenomenon is none the less the occurrence of cyclic variations in the color of the innermost ring. The correction implied in the last equation would be more than sufficient. The violet coronas are to be depressed as regards $n$ and the red coronas raised in their $n$ values, showing that in the former the measurement referred to red surpassing the last violet, and in the latter to violet beyond the red. It is expedient to state these data in relation to the diameters of the fog particles under observation.

I7. Diameter of fog particles. - Having determined the true values of $n$, the diameters of fog particles may be computed for each aperture, since

$$
d=\sqrt[3]{6 m / \pi n}=.02 \mathrm{I} n^{-1 / 3} .
$$

The results are given in the tables and are plotted in the corresponding graphs. Each of these ( $d$ as a function of $s$ ) shows the three cycles already determined, and the cusps lie at $d=.0007$ to $.0008 \mathrm{~cm}$. and $d=.0005$ to $.00055 \mathrm{~cm}$., or that the intermediate and particularly luminous cycle covers a range corresponding to about ten times the wave lengths $(.00004$ to $.00008 \mathrm{~cm}$.) of the visible spectrum. But two of the cusps are unmistakably marked, while in other respects the graphs retain the hyperbolic contour, $d s=$ const.

Since $n^{-x / 3}$ is the cubical volume which contains one fog particle, $d / n^{-1 / 3}$ 
is the ratio of the diameter of particles to the distance between particles, constant throughout. The distance between centers is thus about 48 times the diameter of particles for the temperature and pressure conditions prevailing during the exhaustions.

One may note that the diameters found are independent of $m$; for in the above notation let $z$ and $D$ refer to the normal corona virtually used for standardization. Then, as the series stands,

$$
n_{\mathrm{z}}=n_{\circ} 1 \mathrm{O}^{(z-4) \log y^{z-x}} \Pi\left(\mathrm{x}-S / s^{2}\right)
$$

or after reduction, since the same equation also holds for $Z$,

$$
\mathrm{I} / d^{3}=\left(s_{0} / a\right)^{3} \mathrm{IO}^{(z-Z) \log y} \underset{z-\mathrm{I}}{\operatorname{six}}\left(\mathrm{I}-S / s^{2}\right)
$$

where $s_{\circ}$ is the aperture of the normal corona numbered $Z$. Thus $d$ depends on $a, y$, and $S$, and does not therefore differ much from my earlier values except in so far as $a$ and $y$ were differently determined and $S$ not observed.

Finally, since $n d^{3}=6 \mathrm{~m} / \pi=$ const., the relation of $n$ and $d$ are reciprocal, and maxima in $n$ thus correspond to minima in $d$. If $d$ is determined too large, $n$ will be too small. The curves bear this out. The periods indicated by the cusps in the $d$ curves are more appropriately referred to below. They may be placed as follows: in the first curve they lie at $d=.00069, .00053, .00039$; in the second at about $d=.00079, .00055, .00040 \mathrm{~cm}$. In conformity with the work below their mean position may be rated at $d=.00072, .00054, .00036$, or in the ratio of 4,3 , and 2 . In other words, they are, roughly, multiples of the cycle datum $.00018 \mathrm{~cm}$., and throughout large as compared with wave length.

\section{MONO-CHROMATIC LIGHT.}

18. Tables.-The coronas are too faint for effective observation with mono-chromatic light obtained from simple sources like the salt flame. I therefore used the electric arc as a point source and obtained sufficient limitation for the present with a double thickness of ruby glass. This arrangement has an ulterior advantage as it is thus possible to observe the colors of the annuli as well as their red diameter on interposing the colored glass. This greatly facilitates the reductions. The observations made with the large cubical condensation chamber are given in the following table arranged on the same plan as the preceding. The constant $a^{\prime \prime}$ is as before definitely determined from subsidence, while $a^{\prime}$ refers to preliminary standardization with lycopodium. The time loss $\beta$ is neglected. The subsidence loss has been separately computed and differs slightly from the above, showing more expeditious work. 
TABLE 8.-CONSTANTS OF CORONAS. ELECTRIC AND RUBY LIGHT. CONDENSATION CHAMBER AS IN TABLE I. DISTANCES AS IN TABLE $1 . \theta=15.5^{\circ}$; BAROM., $=75.8 \mathrm{~cm} . ; \delta p=16.9 \mathrm{~cm} . ; y=.77 ; \alpha=.0632 ; \beta=0 ; S=2.5 ; m=4.2 \times$ $10^{-6}$ GRAMS; $a^{\prime \prime}=.0029 ; a^{\prime}=.0032 ; n_{0}=188000 ;$ PHOSPHORUS NUCLEI; $d^{\prime \prime}=$ $.0029 / s$. MEASUREMENT TO OUTER EDGE OF FIRST RING. $\quad 2$ SIN $\phi=s / R$.

First Series.

\begin{tabular}{|c|c|c|c|c|c|c|c|c|c|}
\hline$z$ & $t$ & $s$ & Corona. & $\begin{array}{c}n^{\prime}=2505^{3} \\
\times 10^{-3}\end{array}$ & $\begin{array}{c}u^{\prime \prime}=335^{s^{3}} \\
\times 10^{-3}\end{array}$ & $N \Pi\left(\mathrm{I}-\frac{\mathrm{s}}{\mathrm{s}^{2}}\right)$ & $\begin{array}{l}n_{0} \\
\times 10^{-3}\end{array}$ & $n$ & $\begin{array}{c}d= \\
.020 n^{-2 / 3}\end{array}$ \\
\hline o & $\min$. & $\mathrm{cm}$ & & & & & & & \\
\hline I & $4 I$ & 一 & - & 一 & & $(2.2)$ & & 412000 & .000269 \\
\hline 2 & 44 & - & $v^{\prime} g^{\prime} r^{\prime}$ & - & & $(\mathrm{I}, 7)$ & & 318000 & 293 \\
\hline 3 & 48 & - & $\mathrm{w}^{\prime} \mathrm{y} \mathrm{g}^{\prime} \mathrm{v}^{\prime}$ & -- & $F$ & $(\mathrm{I} \cdot 3)$ & & 244000 & 320 \\
\hline 4 & $5^{I}$ & 9.0 & wopg & 182 & $244=$ & 1.000 & & I 88000 & 349 \\
\hline 5 & 55 & 8.3 & $w^{\prime} c y g$ & I 45 & 195 & .746 & & I 40000 & $3^{8} 5$ \\
\hline 6 & $5^{8}$ & 6.5 & $w g \dot{b} p$ & 70.2 & 94.1 & .554 & & 104000 & 426 \\
\hline 7 & 62 & $5 \cdot 7$ & w y $g$ b & $47 \cdot 5$ & 63.6 & .402 & & 75600 & 473 \\
\hline 8 & 65 & $5 \cdot 4$ & $\mathrm{wrg}$ & 39.2 & 52.6 & .286 & & 53800 & .531 \\
\hline 9 & 68 & $4 \cdot 7$ & $\mathrm{w} g \dot{\mathrm{b}} \mathrm{p}$ & 26.0 & 34.8 & $.20 \mathrm{I}$ & & 37800 & 595 \\
\hline 10 & 71 & 4.2 & $\mathrm{w} r \mathrm{~b}$ & 19.2 & $25 \cdot 7$ & .137 & 188 & $25^{800}$ & 676 \\
\hline I I & 74 & $3 \cdot 7$ & w y b g & 12.7 & 17.0 & .091 & I 86 & I 7200 & 775 \\
\hline 12 & 78 & 3.1 & wobg & 7.8 & 10.5 & .056 & I 86 & 10500 & 9 I 3 \\
\hline 13 & $8_{1}$ & 2.7 & corona & 4.9 & 6.6 & .033 & 200 & 6200 & .001090 \\
\hline 14 & 84 & 2.1 & " & 1.9 & 3.1 & .0I 7 & I 88 & 3100 & 1370 \\
\hline 15 & 87 & ?I.4 & " & .7 & 1.0 & .006 & I 80 & 1000 & 1960 \\
\hline
\end{tabular}

SECOND SERIES. - $n^{\circ}=200000 ; \alpha=.0610$.

\begin{tabular}{|c|c|c|c|c|c|c|c|c|c|}
\hline I & - & - & - & - & - & 2.2 & & 440000 & $.00026_{4}$ \\
\hline 2 & 54 & - & $w^{\prime} g^{\prime}$ & - & - & I. 7 & & 340000 & 287 \\
\hline 3 & 57 & 一 & w g v & - & - & I. 3 & & 260000 & 3 I 4 \\
\hline 4 & $6 I$ & $9 \cdot 5$ & w y g & 214 & 287 & 1.000 & & 200000 & 342 \\
\hline 5 & 64 & 8.8 & w c g & I 70 & 228 & .770 & & 149000 & 378 \\
\hline 6 & 68 & 7.1 & $\mathrm{wg} \mathrm{gr}$ & 89.5 & I 20 & .593 & & I I 2000 & 415 \\
\hline 7 & 71 & 6.0 & w g v r & 54.0 & 72.3 & .457 & & 81200 & 462 \\
\hline 8 & 74 & $5 \cdot 7$ & w og & 46.2 & 62.0 & $.35^{2}$ & & $5^{8600}$ & $5^{14}$ \\
\hline 9 & 77 & $5 \cdot 2$ & $w g$ b & 35.2 & 47.2 & .271 & & 4 I 600 & 576 \\
\hline Io & 80 & $4 \cdot 3$ & wob g & 19.9 & 26.6 & .208 & I 83 & 29000 & $65^{2}$ \\
\hline I I & 83 & $4 \cdot \mathrm{I}$ & $w \circ b g^{\prime} r^{\prime}$ & 17.2 & 23.1 & I61 & $23^{8}$ & $? 19400$ & 744 \\
\hline I 2 & 87 & $3 \cdot 3$ & corona & 9.4 & 12.6 & $.12 \mathrm{I}$ & 203 & I 2400 & 866 \\
\hline I 3 & 90 & 2.8 & “ & $5 \cdot 5$ & $7 \cdot 4$ & .095 & I 97 & 7500 & .001020 \\
\hline I4 & 93 & 2.2 & " & 2.6 & $3 \cdot 5$ & .073 & I 8 I & 3900 & 1270 \\
\hline I 5 & 96 & I. 6 & " & .I. I & I. 5 & .056 & 210 & 1400 & I 770 \\
\hline 16 & 99 & I. 2 & " & .4 & .6 & .043 & I 35 & 800 & 2130 \\
\hline
\end{tabular}

19. Graphs for nucleation.-The results for mono-chromatic light arc constructed in the chart, figure 6 , and show the three prominent cycles with suggestions of others crowded into the region of normal coronas; but the latter are uncertain. The two curves are more closely in agreement both in their horizontal and vertical dimensions than was the case above. All four curves agree, however, in their essential points, as closely as may be expected. The 
region of lower cusps is between $n=35,000$ and 45,000 , and of the upper cusps between $n=95,000$ and 105,000 , or at $s=4.5$ and $s=6.0$ in the two cases. These cusps mark the region of "green" coronas. The angular radius is $\varphi=4^{\circ}$ I $8^{\prime}$ and $\varphi=5^{\circ} 44^{\prime}$, respectively. Other remarks already made apply here.

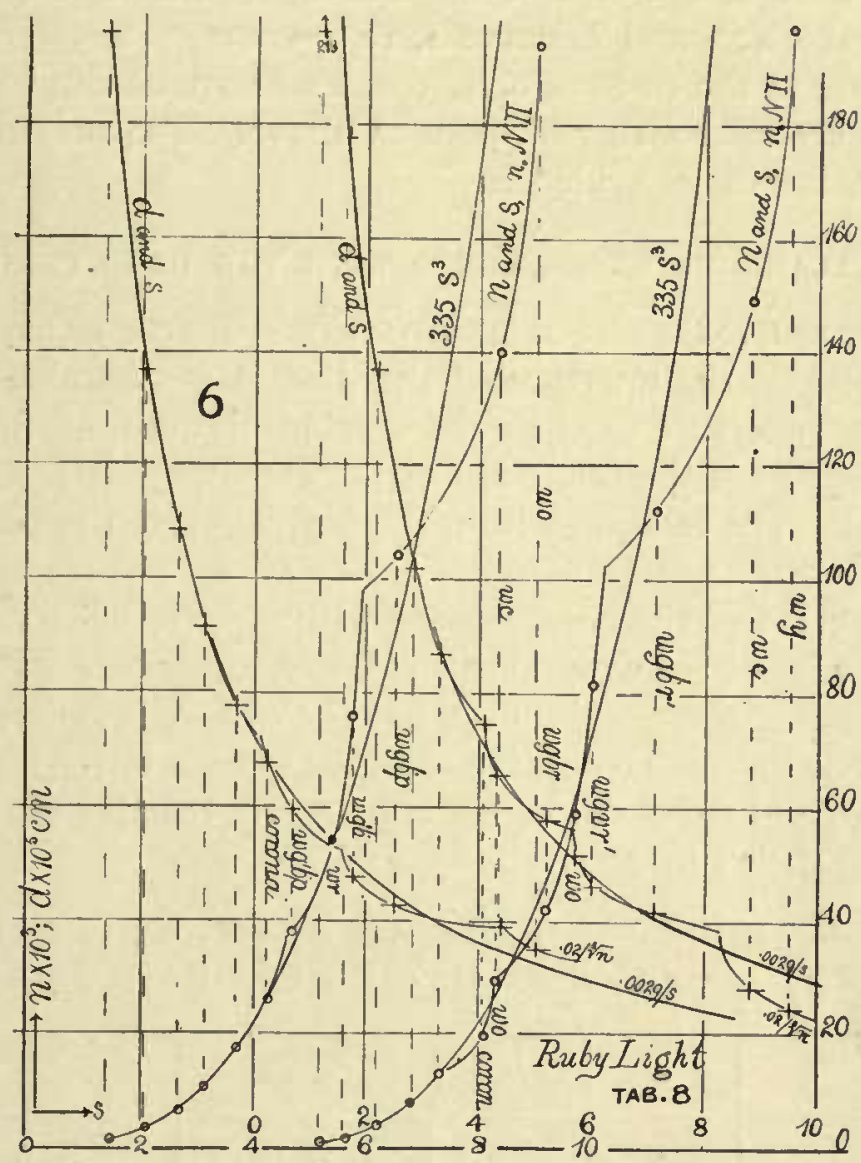

Figure 6.-Chart for Table 8, Showing the Relation of Nucleation $(n)$ and of Diameter of Fog Particle $(d)$ in Terms of the Apertures $(s)$ of the Coronas.

20. Graphs for diameter.-There is a slightly different constant involved here, as the temperature is lower. Since $\theta=\mathrm{r} 5.5^{\circ}$,

$$
d=\sqrt[3]{6 m / \pi n}=.020 n^{-x / 3} .
$$

Hence the edge of the cube containing one fog particle is just 50 times the diameter of the particle for the uniform conditions of condensation selected.

The curves for $d^{\prime \prime}$ (smooth hyperbola since $d^{\prime \prime} s=$ const.) and $d$ are drawn in relation to $s$ as usual, and the latter give evidence of three or more cusps with intervening minima. These in their maximum and minimum relations are reciprocal with the corresponding curves for $n$. Both curves agree with each other and with the preceding set for $d$.

Horizontal positions are naturally better than vertical positions because of 
the shifting attending the standardization of $n$ and $d$. If the horizontal position of the cusps be taken from the figure as $s=2.7,4.2,5.6,8.3$ in curve $\mathrm{I}$ on the left, and $s=2.8,4 . \mathrm{I}, 5.6,8.3$ in curve 2 on the right, the mean ratios are 2, $3,4,6$, so that $s=1.4$ or $p=x^{\circ}{ }_{1} 8^{\prime}$ repeats itself. Since $d=.0029 / s$ nearly, the $d$ ratios should be as $I / 2, I / 3, I / 4, I / 6$. The figure shows roughly in curve $I$ $10^{6} d=1100,710,540,400$, and in curve $2,10^{6} d=1050,740,55^{\circ}, 350$, the means of which values are in the ratio of $6,4,3,2$, as nearly as can be expected, the data being multiples of $.00018 \mathrm{~cm}$., here as above. The presence of a period between 4 and 6 is not in evidence.

\section{MISCELLANEOUS EXPERIMENTS WITH THE DEEP.VESSEL.}

21. Measurement to the edge of the white disc.-Before citing data obtained from measurements of the aperture of the disc, it is desirable to reduce the preceding data, $s_{\circ}$ (aperture to the outside edge of the first ring) to $s_{\mathrm{i}}$ (aperture to the inside edge of the first ring) by direct experiments. This is done in the next table (9). The relation within the normal region is practically linear and $s_{\mathrm{i}} / s_{\mathrm{o}}=.76$.

Careful inspection of the coronas seen with electric light shows that even when normal the edge of the white disc shades off through $y, o$, br, etc. There is no real demarcation of the white disc, but the $r^{\prime}$ edge is rather the beginning of a diffraction cycle in which (counting from the outside inward) $r, o, y$, merging into white, appear, and when the higher coronas are reached the missing $\mathrm{g}, \mathrm{b}, \mathrm{v}$ become evident in their turn.

TABLE 9.-OUTSIDE AND INSIDE DIAMETERS OF THE RED RING. DISC, w w' $y^{\prime}$ $\mathrm{br}^{\prime} \mathrm{vp}^{\prime}$ bं g y' r; EDGE, a w y HAZE. MEAN RATIO, $s_{\mathrm{i}} / s_{\mathrm{o}}=.77$.

\begin{tabular}{|c|c|c|c|c|c|c|c|}
\hline Corona. & $s_{i}$ & so & $s_{\mathrm{i}} / s_{\mathrm{o}}$ & Corona. & $s_{i}$ & $s_{0}$ & $s_{\mathrm{i}} / s_{\mathrm{o}}$ \\
\hline w y br b g y $y^{\prime}$ & $2 . I$ & 3.0 & .72 & $\mathrm{w} \mid \mathrm{rbg}$ & 3.1 & $4 \cdot 2$ & .74 \\
\hline Do. & 2.4 & $\begin{array}{l}2.9 \\
3.1 \\
3.0\end{array}$ & .78 & wobg & 2.8 & $\begin{array}{l}4 \cdot 2 \\
3 \cdot 5\end{array}$ & $.8 \mathrm{I}$ \\
\hline & & & & Do. & 2.2 & $\begin{array}{l}2.9 \\
3.0\end{array}$ & .75 \\
\hline w yob $\mid \mathrm{r}$ & 4.0 & $\begin{array}{l}5 \cdot I \\
4.8\end{array}$ & .80 & Do. & 2.7 & $\begin{array}{l}3 \cdot 4 \\
3 \cdot 4\end{array}$ & .79 \\
\hline $\begin{array}{l}\text { wg } \dot{b} \mid p \\
\text { change to wyg }\end{array}$ & 3.6 & $\begin{array}{l}4.6 \\
4.5\end{array}$ & & N. corona & 2.2 & 2.9 & .76 \\
\hline w o b g & 4.0 & $\begin{array}{l}4.9 \\
4.6\end{array}$ & .83 & “ & I. 8 & 2.5 & .74 \\
\hline$w y^{\prime} r \mid g$ & 3.2 & $\begin{array}{l}4 \cdot 3 \\
4.4\end{array}$ & .73 & " & I. 3 & I. 9 & .69 \\
\hline
\end{tabular}

Measurements of $s_{0}$ before and after $s_{\mathrm{i}}$.

Table 9 shows that if $d s_{\circ}=.0029$ I (subsidence), then

$$
a_{\mathrm{i}}=d s_{\mathrm{i}}=.0029 \mathrm{I} \times .77=.00223 \text { and } n=\left(6 \mathrm{~m} / \pi a_{\mathrm{i}}^{3}\right)(\mathrm{ros})^{3}=810 \mathrm{~s}^{3} .
$$


Direct measurements of the $a_{\mathrm{i}}$ by comparison with lycopodium gave $s_{\mathrm{i}}=.78$ and .84 for the near and far faces of the chamber whence $a_{i}=.0026$ (diffraction) and $n=5155^{3}$. This is in the usual way smaller, and $n / s^{3}$ larger than the subsidence datum $\left(a_{\mathrm{i}}=.00223\right)$, but the latter will be taken here as above. Both values are compared in the tables, the ratio being $.64=n^{\prime} / n^{\prime \prime}$.

22. A pertures of the discs of the coronas.-Table ro contains two independent series of results arranged as in the preceding tables. The source of light is the Welsbach mantel. The data are further shown in figure 7 .

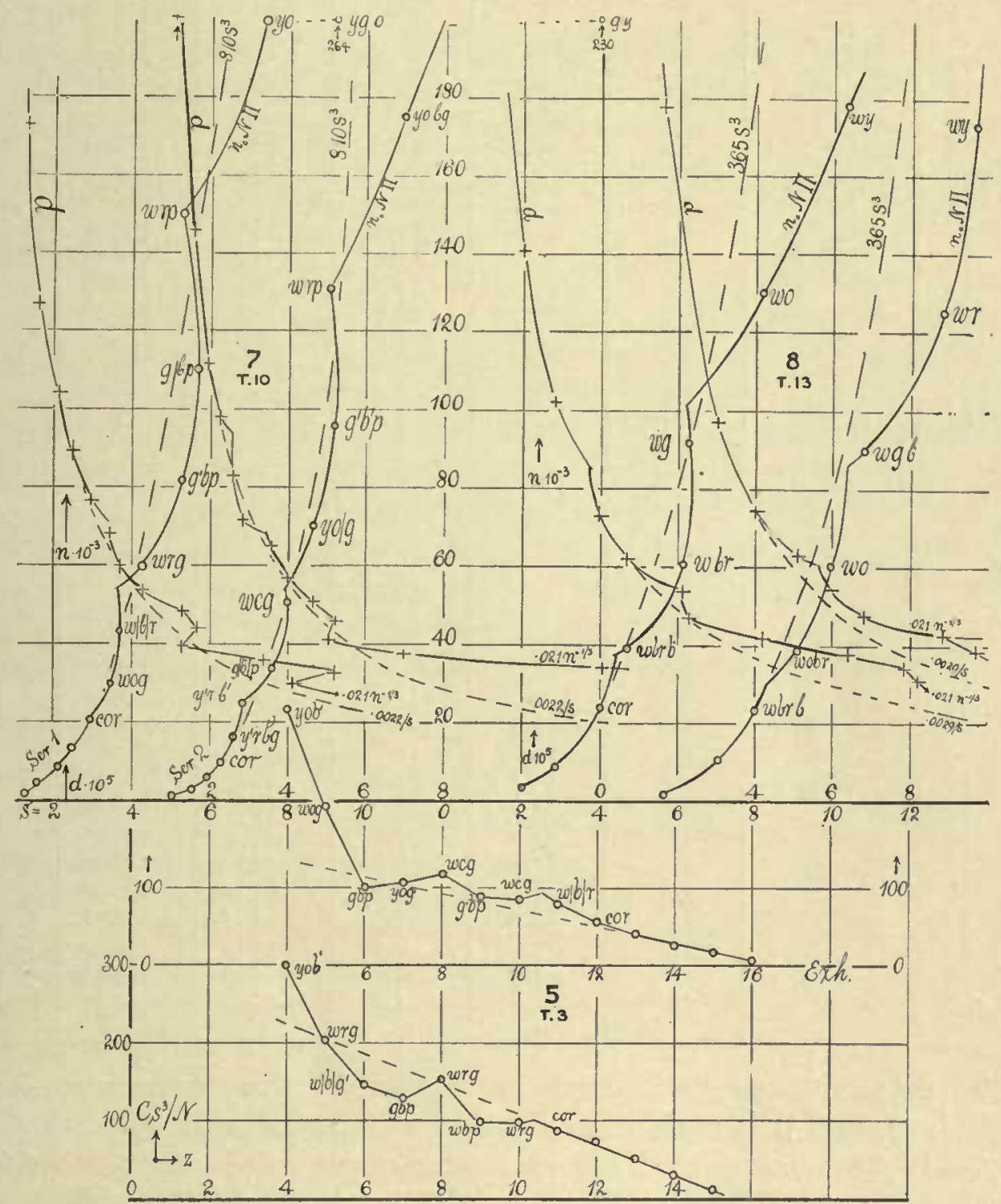

Figure 5.-Chart showing the Relation of Relative Nucleation $(r)$ to the Number $(z)$ or - Order of the Exhaustion for Table 3.

Figure 7.-Chart showing the Relation of the Actual Nucleation $(n)$ to the Number $(z)$, or Order of the Exhaustion, for Table io. Also the Diameter $d$ (in ro $0^{-5}$ cm.) of the Fog Particles in Terms of $\boldsymbol{z}$.

FIGURE 8.-Do. FOR TABLE 13 . 
TABLE 10.-CONSTANTS OF CORONAS. CHAMBER AND DISTANCES AS ABOVE. $\theta=2 \mathrm{I}^{\circ} ;$ BAROM., $75.3 \mathrm{~cm} . ; \delta p=16.9 \mathrm{~cm} . ; y=.77 ; \alpha=.062 ; \beta=0 ; S=1.3 ; a^{\prime}=$ $.0026 ; a^{\prime \prime}=.00223 ; m=4.7 \times 10^{-6}$ grams; $n_{0}=200000 ; s$ MEASURED TO INNER EDGE OF FIRST RING (APERTURE OF DISC); PHOSPHORUS NUCLEI; WELSBACH LAMP.

\begin{tabular}{|c|c|c|c|c|c|c|c|c|}
\hline$z$ & $t$ & Corona. & $s$ & $n^{\prime}=5^{1} 5^{53}$ & $n^{\prime \prime}=8 \operatorname{IOS} 3$ & $N \Pi\left(I-\frac{S}{s^{2}}\right)$ & $n=n_{0} N \Pi$ & $\underset{n^{-1 / 3}}{d=.02 \mathrm{I}}$ \\
\hline & $\min$. & & $\mathrm{cm}$. & $\times 10^{3}$ & $\times 10^{3}$ & & & $\mathrm{~cm}$ \\
\hline I & 9 & - & - & & & 2.8 & 560000 & .000255 \\
\hline 2 & I 2 & $\mathrm{~g}^{\prime} \mathrm{r}^{\prime}$ & - & & & 2.2 & 440000 & 277 \\
\hline 3 & 15 & $\mathrm{~g}^{\prime} \mathrm{b}^{\prime}$ & - & & & 1.7 & 340000 & 300 \\
\hline 4 & I 8 & yg o & 9.2 & 401 & $63 I$ & I. 32 & 264000 & 328 \\
\hline 5 & 22 & yo bg & $7 \cdot 4$ & 208 & 328 & 1.000 & 200000 & 360 \\
\hline 6 & 25 & w c/g & $5 \cdot 3$ & $7^{6.7}$ & I 2 I & $.75^{1}$ & I 50000 & 395 \\
\hline 7 & 29 & $g^{\prime}$ b p & $5 \cdot 7$ & $95 \cdot 3$ & I 49 & .552 & I I 0000 & 440 \\
\hline 8 & 32 & $g^{\prime} b p$ & $5 \cdot 3$ & 76.7 & I 2 I & .408 & 8 I 600 & $4^{8} 5$ \\
\hline 9 & 36 & w r g & $4 \cdot 3$ & 41.0 & $64 \cdot 4$ & .299 & 59800 & 537 \\
\hline IO & 39 & $\mathrm{w}$ br $\mathrm{b} \mid \mathrm{r}$ & $3 \cdot 7$ & 26.1 & 41.0 & .215 & 43000 & 600 \\
\hline I I & 43 & w olg & $3 \cdot 4$ & $2 \mathrm{I} .2$ & $33 \cdot 3$ & .149 & 29800 & 680 \\
\hline I 2 & $4^{6}$ & N. corona & 2.9 & 13.2 & 20.8 & .103 & 20600 & $7^{6} 5$ \\
\hline I 3 & 50 & “ & 2.5 & 8.0 & 12.6 & .066 & 13100 & 890 \\
\hline I 4 & 53 & $“$ & 2.1 & 5.1 & 8.0 & $.04 \mathrm{I}$ & 8200 & .001040 \\
\hline I 5 & 57 & “" & I. 6 & 2.3 & 3.6 & .022 & 4500 & 1270 \\
\hline I 6 & 60 & “" & $1 \cdot 3$ & I. I & I. 8 & .009 & I 800 & 1730 \\
\hline
\end{tabular}

SECOND SERIES. $-\alpha=.06 \mathrm{I} ; \mathrm{n}_{\mathrm{o}}=\mathrm{I} 75000$.

\begin{tabular}{|c|c|c|c|c|c|c|c|c|}
\hline I & & - & - & $\times 10^{3}$ & $\underbrace{\times 10^{3}}$ & 2.2 & 385000 & .000288 \\
\hline 2 & $5^{6}$ & - & - & - & - & I. 7 & 300000 & $3 \mathrm{I}_{4}$ \\
\hline 3 & 59 & gy & I 1.0 & & & I. 3 & 230000 & 343 \\
\hline 4 & 63 & yobg & 6.9 & I 73 & 272 & 1.000 & I75000 & 375 \\
\hline 5 & 66 & $\mathrm{w} c \mathrm{~b} \mathrm{~g}^{\prime}$ & 5.0 & 66.4 & 104 & .749 & I3 1000 & 4 I 4 \\
\hline 6 & 69 & $\mathrm{~g}^{\prime} \mathrm{b}$ p & 5.2 & 72.6 & I I 4 & .548 & 96000 & 460 \\
\hline 7 & 73 & y olg & 4.6 & 51.5 & 81.4 & .401 & 70200 & 510 \\
\hline 8 & 76 & w clg & 4.0 & 33.0 & 51.8 & .290 & 50800 & $57^{\circ}$ \\
\hline 9 & 79 & $g \mid b p$ & 3.6 & 24.0 & $37 \cdot 7$ & .206 & 34000 & $6_{50}^{\circ}$ \\
\hline I0 & 82 & $y^{\prime} c b g$ & 2.8 & I 1.9 & 18.7 & .142 & 24800 & 720 \\
\hline I I & 86 & $y^{\prime}+b g$ & 2.6 & 9. I & I 4.2 & .0924 & 16200 & 830 \\
\hline I2 & 89 & corona & 2.3 & 6.3 & 9.9 & $56 \mathrm{I}$ & 9800 & $9^{80}$ \\
\hline I3 & 93 & “" & I. 9 & 3.8 & 6.0 & 333 & 5800 & .001170 \\
\hline I 4 & 96 & “ & I. 5 & 1.9 & 3.0 & 169 & 2960 & 1460 \\
\hline I 5 & IOI & “ & I.O & .6 & 1.0 & .0060 & 1050 & 2060 \\
\hline
\end{tabular}

The subsidence constant must be smaller in this case, since $S_{z+1}=s_{s}^{2}$ $\left(I-s_{z+1}^{3} / y s_{z}^{3}\right)$, and it is clear that $S$ and $s^{2}$ must change in the same ratio nearly. It was shown that $s_{\mathrm{i}}=.77 s_{\circ}$, or $s_{\mathrm{i}}^{2}=.59 s_{\circ}^{2}$, and hence $S_{\mathrm{i}}=2.6 \times .59$ $=1.56$. An actual computation gave $S_{i}=1.3$, the difference being easily referable to incidental discrepancies.

23. Remarks on the table and graphs. - The green corona now shows the nucleations (II0,000 $+82,000$ ) $/ 2$ and independently 96,000 , in the two series, agreeing reasonably well with the above 85,000 , 106,000, 104,000, 96,000, the 
standardization being throughout by subsidence. This agreement is further substantiated below. The difference of values here is largely an actual difference in the positions of the coronas.

The curve $r=55^{5} s^{3} / \operatorname{IO}^{z \log y}$ if constructed in full shows the eventual linear march already instanced. As above $\alpha=.062$ nearly.

The curves of nucleation, $n$, in terms of the relative aperture, $s$, indicate the occurrence of four cycles, though the lower is uncertain. Both curves run in parallel as nearly as the observations allow, and they show actual cases of overlapping or reëntrance.

This reëntrance is particularly evident in the $d$ curves in which four distinct branches overlapping at their edges may be made out. The positions of the cusps, as indicated by the green coronas, may be placed at $10^{6} d=45^{\circ}, 65^{\circ}, 95^{\circ}, \mathrm{cm}$. or about in the ratio of 3,4 , and 6 times .000I $5 \mathrm{~cm}$. The corresponding number found above was larger than this in the ratio of $18 /{ }_{5} 5$, while the ratio of the red and blue wave lengths involved would be I.4.

24. Condensation chamber remote from the eye.-The distances are inverted in the following experiments, being $250 \mathrm{~cm}$. from the eye to the condensation chamber and $85 \mathrm{~cm}$. from this to the source. All coronas therefore are relatively small and the measurement of $s$ by the same method much more difficult, particularly in relation to standardization. Measurement of 's was naturally made to the outside of the first ring. Results with lycopodium seen through ruby glass and a source of electric light gave $\left(d_{\circ}=.0032\right), s_{\circ}=.344 \mathrm{~cm}$. Hence $a=d_{0} s_{0}=.00 \mathrm{II}$ and $n=675^{\circ s^{3}}$. If the value of $a$ be reduced to the conditions holding for subsidence, $a^{\prime \prime}=.001 \mathrm{I}\left(29 \mathrm{I} / 33^{6}\right)=.953 \times 10^{-3}$, whence $n=$ $10,400 s^{3}$. Table I computed with this value follows. Unfortunately, three important data were lost, and the series are deficient in this respect.

TABLE II.-CONSTANTS OF CORONAS. CHAMBER AS ABOVE. DISTANCES FROM EYE AND SOURCE, $25^{\circ} \mathrm{cm}$. AND $85 \mathrm{~cm}$. RESPECTIVELY. $\theta=2 \mathrm{I}^{\circ}$; BAROM., $75.3 \mathrm{~cm}$. $\delta p=16.9 \mathrm{~cm} . ; y=.77 ; \beta=0 ; S=.36 ; v_{0}=260000 ; a^{\prime}=.001 \mathrm{I} ; a^{\prime \prime}=.00095 ;$ $m=4.7 \times$ IO $^{-6}$ grams; $s$ MEASURED TO OUTER EDGE OF FIRST RING; PHOSPHORUS NUCLEI; WELSBACH MANTEL.

\begin{tabular}{|c|c|c|c|c|c|c|c|c|}
\hline$z$ & $t$ & Corona. & $s$ & $n^{\prime}=6750 s^{3}$ & $n^{\prime \prime}=10400 s^{3}$ & $N I I\left(\mathrm{I}-\frac{S}{s^{2}}\right)$ & $n=n_{0} N \Pi$ & $\begin{array}{c}d=.02 I \\
n-x / 3\end{array}$ \\
\hline I & $\min _{2 \mathrm{I}}$ & - & $\mathrm{cm}$. & $\times \mathrm{I0}^{3}$ & $\times 10^{3}$ & 2.2 & 570000 & $\begin{array}{c}\mathrm{cm} \\
.000254\end{array}$ \\
\hline 2 & 24 & $w^{\prime} r^{\prime}$ & 3.6 & 3 I 5 & 486 & I. 7 & 440000 & 276 \\
\hline 3 & 28 & $\mathrm{~g}^{\prime} \mathrm{b}^{\prime} \mathrm{p}^{\prime}$ & $3 \cdot 7$ & $35^{6}$ & 549 & I. 3 & 338000 & 302 \\
\hline 4 & 32 & y o bg & $3 \cdot 4$ & 265 & 409 & 1.000 & 260000 & 330 \\
\hline 5 & $3^{6}$ & w c g v & 3.0 & 182 & $28 \mathrm{I}$ & .747 & 194000 & 363 \\
\hline 6 & 40 & $w p b g^{\prime} r$ & 2.2 & 76.9 & I I 9 & $.55^{6}$ & I 44000 & 400 \\
\hline 7 & 44 & $\mathrm{gbp}$ & 2.3 & 82.3 & 127 & .404 & 105000 & 446 \\
\hline 8 & $4^{8}$ & w r $g$. & 2.0 & 58.0 & $89 \cdot 4$ & .290 & 75400 & 496 \\
\hline 9 & 52 & $w$ br b g g r & I. 7 & 33.1 & $5^{1.0}$ & .205 & 53300 & 560 \\
\hline ro & 56 & w y o b g & - & - & - & $.14 \mathrm{I}$ & 36700 & 633 \\
\hline I I & 60 & coronas & I. 2 & 13.2 & 20.4 & .094 & 24400 & 724 \\
\hline I 2 & 64 & "6 & I.I & 10.3 & I 5.9 & .059 & I 5300 & 850 \\
\hline I3 & 69 & " & I.O & 6.7 & 10.4 & .035 & 9100 & .001000 \\
\hline
\end{tabular}


SEcond SERIES. $-n=280000$.

\begin{tabular}{|c|c|c|c|c|c|c|c|c|}
\hline & & & & $\times \mathrm{IO}^{3}$ & $\times 103$ & & & $\because$ \\
\hline I & 24 & - & - & - & - & 2.2 & 620000 & .000246 \\
\hline 2 & 27 & $w^{\prime} r^{\prime}$ & 4.0 & 432 & 666 & 1.7 & 480000 & 268 \\
\hline 3 & 30 & $g^{\prime} b^{\prime} p^{\prime}$ & 3.6 & 328 & 505 & I. 3 & 360000 & 295 \\
\hline 4 & 34 & y o bg & - & - & - & 1.000 & 280000 & $32 \mathrm{I}$ \\
\hline 5 & 39 & w r g, & 3.I & $20 \mathrm{I}$ & 310 & .747 & 209000 & 354 \\
\hline 6 & 43 & w c $g^{\prime}$ & 2.4 & 99.2 & I 53 & $.55^{6}$ & I 56000 & 390 \\
\hline 7 & 47 & $\mathrm{~g} \mathrm{~b} \mathrm{p}$ & 2.2 & 71.5 & I I I & .404 & I I 3000 & 435 \\
\hline 8 & $5^{\mathrm{I}}$ & w ro $g$ & 2.2 & 71.5 & I I I & .290 & 81200 & 485 \\
\hline 9 & 55 & w c|g|r & 1.8 & 42.5 & 65.5 & .205 & 57400 & 544 \\
\hline I0 & 59 & $\mathrm{~g}^{\prime}|\mathrm{b}| \mathrm{p}$ & - & - & - & $.14 \mathrm{I}$ & 39500 & 616 \\
\hline I I & 62 & $w^{\prime} \operatorname{clg} r^{\prime}$ & I. 3 & 16.7 & 25.7 & .094 & 26300 & 705 \\
\hline I 2 & 65 & wobg & I. I & 10.3 & 15.9 & .059 & 16500 & 824 \\
\hline I3 & 70 & corona & 1.0 & 6.7 & 10.4 & .035 & 9800 & 980 \\
\hline
\end{tabular}

The $n$ values in their relation to $s$ work out similarly to the above data. The two cusps are in their usual location. The grecn coronas correspond to $n=105,000$ and II 3,000 nuclei, respectively, somewhat larger than the above order of values, but not more so than the difficulties of standardization would lead one to anticipate.

The $d$ values are difficult to interpret, as the curve is fairly uniform but for the break introduced in the $\mathrm{w} g \mathrm{~b}$ p region. Both curves are in close agreement, and under the circumstances it is impossible to locate the cusps and their relations.

LONG AND SHALLOW CONDENSATION CHAMBERS.

25. Apparatus. - The purpose of the present section is primarily to give greater scope for the observation of the higher coronas, $i$. $e$., those of large aperture. Incidentally it affords a means of testing the validity of the correction for subsidence, $S$.

To obviate fragility of apparatus and inconvenience in the exhaustions, the volume of the long vessel is suitably decreased by lessening the height. This does not interfere with the work, since only a diametral section of the color rings is needed while the apparatus becomes more manageable.

The vessel is shown in the diagram, figure 2 (above, p. $5^{8}$ ), and is (in the clear) $55 \mathrm{~cm}$. long, I $\mathrm{cm}$. high, and $\mathrm{r} 6 \mathrm{~cm}$. deep in the line of vision. Less height and even greater depth and length would have been preferable, but in such a case the correction for subsidence becomes unwieldy. As usual, there is a central brace and a lining of wet cloth, and the front and rearfaces are of plateglass $\frac{1}{4}$ inch thick. Exhaustion is effected through the wide hollow trunnion, $E$, the other being closed and holding a thermometer, or other accessories. $F$ is the filter and $P$ the nucleator, both attached with a cock. Rubber corks, $A, A^{\prime}$, close the wide holes necessary for cleaning. The external vacuum chamber was such that the pressure differences were reduced about one half on suddenly opening the wide cock into the condensation chamber. There was no leakage, and the 
easy means of wetting the whole inside by revolution of the apparatus around the trunnions was a great convenience. But in spite of this, color distortion of the coronas was an almost invariable occurrence, particularly when the interval between observations was short. Since in comparison with the case of the taller

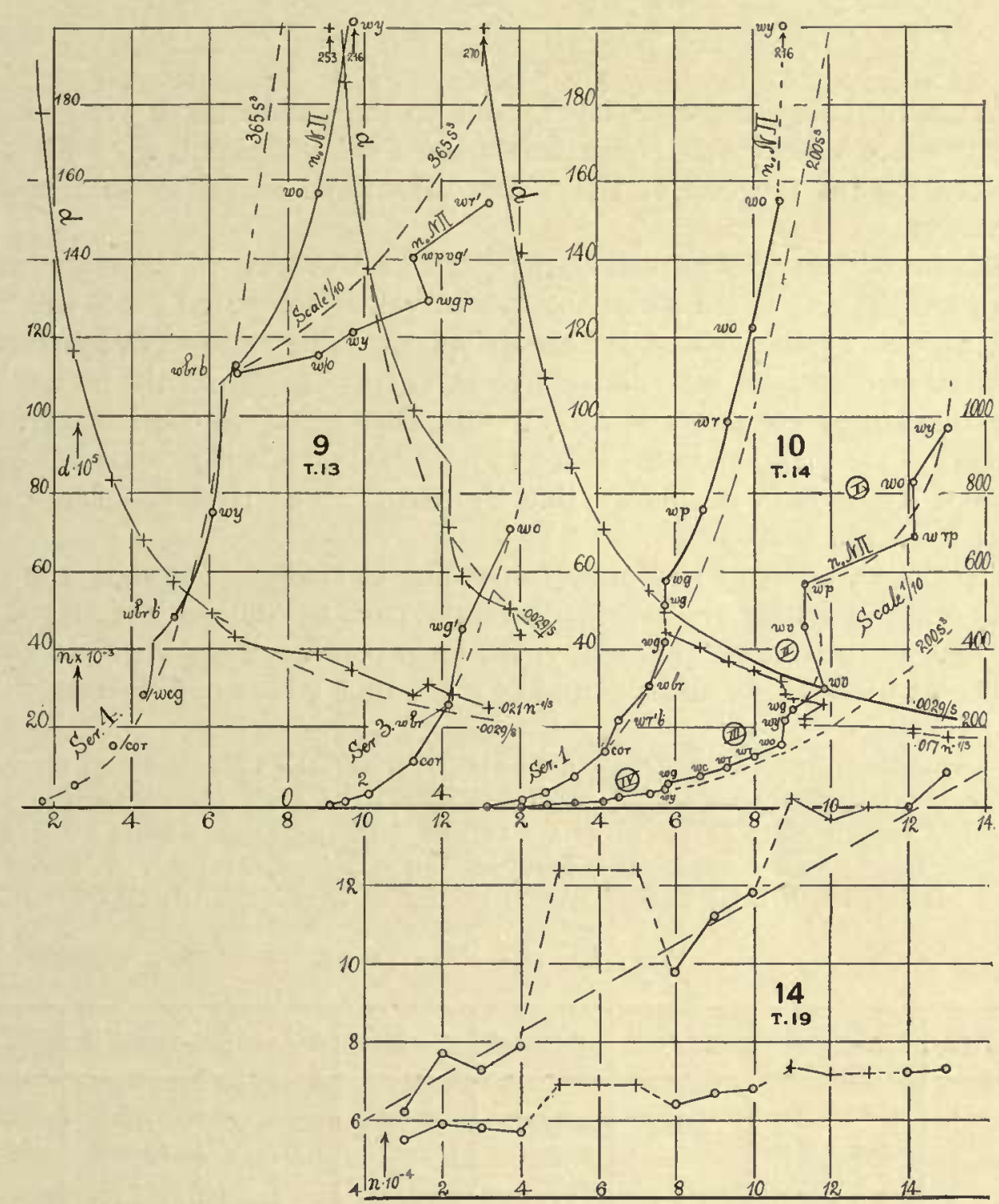

Figures 9, io.-Charts for Tables I3, I4, Showing the Nucleation $(n)$ and the Diameters $(d)$ of Fog Particles for Different Apertures $(s)$ of the Coronas.

Figure 14.-Chart showing the Effect of Different Valves with Nucleations Increasing in THE LAPSE OF TIME, for TABLE Ig.

vessel above, which showed no appreciable distortion, vapor diffusion is here much enhanced, the discrepancy must be referred to the diffusion of nuclei. This would have to take place unassisted by the convection of vapor.

26. Reduction of data.--For the pressure difference $\delta p=17 \mathrm{~cm}$. and at $20.7^{\circ}$, 
$m=4.7 \times 10^{-6}$ grams, and therefore, as above, $n=365^{3}$. The constancy of $S$ is less evident than above, being in the respective series

$\begin{array}{rrrrr}\text { I } & \text { II } & \text { III } & \text { IV } & \text { Mean } \\ 7.8 & 4.8 & 6.5 & 5.5 & 5.8 \\ 7.3 & 8.5 & 6.5 & 6.4 & \\ & 4.2 & 2.1 & 3.9 & \end{array}$

the variability being, however, largely due to the accidental delays inevitably encountered in observing. Nevertheless some other cause is at work simultaneously toward the end of the series. Here the nuclei are not removed apparently.

The mean value $S=5.8$ will be accepted for the body of the observations, but toward the end of the series the special values computed for $S$ will be inserted as well as the alternative mean values. The ratio of heights of the condensation chambers here and above is II $/ 26=.42$, while the inverse ratio of the subsidence constants is $2.6 / 5.8=.45$, showing close consistency for experiments of the present kind. Table I 3 for $\delta p=\mathrm{I} 7 \mathrm{~cm}$. will now be intelligible.

Since $n=6 \mathrm{~m} / \pi d^{3}$, it follows that $d=.02 \mathrm{I} n^{-1 / 3}$, so that the last column follows from the preceding.

The charts, figures 8 and 9, corresponding to these tables, show the cycles already mapped out above, but less distinctly, from the difficulty of maintaining homogeneous nucleation. Naturally, the large coronas, being vague in outline, are difficult to measure, and the middle cycle thus comes out clearest.

TABLE I3.-CONSTANTS OF CORONAS. CONDENSATION CHAMBER $55 \mathrm{~cm}$. LONG, II $\mathrm{cm}$. HIGH, I6 cm. DEEP; DISTANCES FROM EYE AND SOURCE, $85 \mathrm{~cm}$. AND $250 \mathrm{~cm}$. RESPECTIVELY; $\theta=2 \mathrm{I}^{\circ} ;$ BAROM., $75.6 \mathrm{~cm}$.; $\delta p=16.9 \mathrm{~cm} . ; y=.77$; $\beta=0 ; \quad S=5.8 ; \quad a^{\prime}=.0032 ; a^{\prime \prime}=.0029 ; \quad m=4.7 \times 10^{-6} ; n_{0}=173000 ; s$ MEASURED TO OUTER EDGE OF FIRST RING; PHOSPHORUS NUCLEI; ELECTRIC ARC.

First Series.-Ruby Light.

\begin{tabular}{|c|c|c|c|c|c|c|c|c|}
\hline$z$ & $t$ & Corona. & $s$ & $n^{\prime}=275^{s^{3}}$ & $n^{\prime \prime}=3^{6} 5^{s 3}$ & $N \Pi\left(1-\frac{s}{s^{2}}\right)$ & $n=n_{0} N \Pi$ & $d=\underset{n^{-1 / 3}}{.02 I}$ \\
\hline & $\min$. & & $\mathrm{cm}$. & $\times 10^{3}$ & $\times$ ro3 & & & \\
\hline I & 2 & w r g & - & - & - & I. 7 & 294000 & .000315 \\
\hline 2 & 4 & $\mathrm{vg} \mathrm{r}$ & - & - & 一 & 1.3 & 225000 & 345 \\
\hline 3 & 6 & w y p g & 9.7 & 251 & 333 & 1.000 & 173000 & 378 \\
\hline 4 & 8 & w rog & 8.8 & 191 & 253 & .722 & I 25000 & 420 \\
\hline $5^{1}$ & 10 & $w g b^{\prime}$ & 6.8 & 86.3 & I 15 & .515 & 89100 & $47^{\circ}$ \\
\hline 6 & I 2 & $w o g b p$ & $5 \cdot 9$ & $5^{8.0}$ & 77.0 & .347 & 60000 & 540 \\
\hline 7 & I 4 & wo b r g & $5 . I$ & 36.6 & 48.5 & .222 & 38400 & 625 \\
\hline 8 & I 6 & corona & 4.0 & I 7.6 & 23.4 & . I33 & 23000 & $74^{\circ}$ \\
\hline 9 & I 8 & “ & 3.0 & 7.8 & 10.4 & .059 & $10200^{2}$ & 970 \\
\hline 10 & 20 & “" & 1.6 & 1.2 & 1.6 & .010 & I $700^{2}$ & .001760 \\
\hline
\end{tabular}

1. Streaks of color and horizontal bands visible. $S$ for normal coronas specially computed.

2 Alternative, II 200,3300 . 
Second Series. - $n_{0}=327000$. White (Electric) Light.

\begin{tabular}{|c|c|c|c|c|c|c|c|c|}
\hline I & 0 & - & - & $\times 10^{3}$ & $\times 10^{3}$ & - & - & - \\
\hline 2 & 2 & w p g & I 2.2 & 499 & 664 & 1.000 & 327000 & .000305 \\
\hline 3 & 4 & w g p & I I. 8 & $45^{8}$ & 606 & .739 & 242000 & 337 \\
\hline 4 & 6 & w y v g & 10.4 & 309 & 409 & .545 & I 78000 & 373 \\
\hline 5 & 8 & v rog $g^{\prime}$ & 8.2 & I 5 I & 201 & .397 & I 30000 & 415 \\
\hline 6 & IO & $w g$ b $r^{\prime}$ & 6.3 & 68.8 & 91.3 & .279 & 91200 & 467 \\
\hline 7 & I 2 & $\mathrm{w}$ br $\mathrm{g} / \mathrm{p}$ & 6. I & 64.1 & 85.0 & .184 & 60200 & 536 \\
\hline 8 & I 4 & $w$ br b $g^{\prime} p$ & $.4 \cdot 7$ & 29.4 & 39.1 & . I I & 38900 & 620 \\
\hline 9 & 16 & N. coronas & 4.0 & I 7.6 & 23.4 & .072 & $23600^{1}$ & 730 \\
\hline 10 & I 8 & $"$ & 2.8 & 6.4 & 8.5 & .026 & $8700^{1}$ & .001020 \\
\hline I I & 20 & “ & 2.0 & 2.4 & 3.I & .010 & $3300^{1}$ & I 4 IO \\
\hline
\end{tabular}

1. Alternative, $22200,2800,0$.

Fourtii Series. ${ }^{2}-\mathrm{n}_{\mathrm{o}}=546000$. White Light.

\begin{tabular}{|c|c|c|c|c|c|c|c|c|}
\hline 0 & 2 & $\mathrm{w}^{\prime} \mathrm{r}^{\prime}$ & 13.2 & $\begin{array}{l}\times 10^{3} \\
6_{30}\end{array}$ & $\begin{array}{l}\times \mathrm{IO}^{3} \\
840\end{array}$ & 1.000 & 546000 & .000257 \\
\hline I & 4 & $\mathrm{w} p v \mathrm{~g}^{\prime}$ & I I.2 & 390 & 518 & .744 & 406000 & 285 \\
\hline 2 & 6 & w g p & I 1.6 & 434 & $5^{6} \mathrm{I}$ & .544 & 297000 & 315 \\
\hline 3 & 8 & w y pvg & $9 \cdot 7$ & $25 \mathrm{I}$ & 333 & .395 & 2 I 6000 & $35^{\circ}$ \\
\hline 4 & 10 & $\mathrm{w} / \mathrm{g}$ & 8.8 & 187 & 248 & .288 & I 57000 & $39 \circ$ \\
\hline 5 & I 2 & $w$ br $b$ & 6.7 & 82.7 & I IO & .206 & I I 3000 & 435 \\
\hline 6 & 14 & w y $g$ & 6.I & 62.4 & 82.9 & . $13^{8}$ & 75400 & 496 \\
\hline 7 & I6 & $\mathrm{w}$ br b g & 5.I & 36.6 & 48.5 & .089 & 48600 & 575 \\
\hline 8 & I 8 & $w p g$ & $4 \cdot 3$ & 21.9 & 29.0 & $.05^{2}$ & $28700^{3}$ & 685 \\
\hline 9 & 20 & corona & $3 \cdot 5$ & I I. 8 & I $5 \cdot 7$ & .029 & I $5800^{3}$ & 837 \\
\hline IO & 22 & $" “$ & 2.5 & $4 \cdot 3$ & $5 \cdot 7$ & .010 & $5730^{3}$ & .001170 \\
\hline I I & 24 & " & 1.6 & I. 2 & I. 6 & .003 & I $640^{3}$ & I 780 \\
\hline I 2 & 26 & $" 6$ & lost & & & & & \\
\hline
\end{tabular}

2 Cycles nearly absent. Third series omitted.

3 Alternative, $25300,10400,4900,0$.

The green coronas reproduce the orders of nucleation already attributed to them, showing $n=90,000$, I I0,000, $90,000,90,000$, a favorable result in view of the large differences of subsidence encountered.

27. Smaller and larger pressure differences.-To further elucidate the effect of the subsidence error and to exhibit the sequence of coronas more closely, experiments were now made with lower and higher exhaustions than the above. In view of the smaller steps of pressure, it was also thought that a more definite location of the cusps of the cycles would be possible. This, however, was not the case, for if the interval between observations is too small, the coronas are never free from distortion. Table $\mathrm{r}_{4}$ in particular, in which the interval is but one minute, gives evidence of this, but it is even present in the succeeding tables where the intervals are two minutes or more.

To reduce the data, the values of $m$ must first be computed. If the latent 
heat of steam is considered constant (Regnault's value, 606 at $0^{\circ} \mathrm{C}$.), and the ratio of specific heats is $\mathrm{I} .4 \mathrm{I}$, the following values may be derived:

$$
\begin{array}{rcrc}
\text { For } \delta p=22 ; \theta=10 & m=4.2 \times 10^{-6} & \text { For } \delta p=8.5 ; \theta=10 & m=21 \times 10^{-6} \\
20 & 5.5 & 20 & 26 \\
30 & 6.7 & 30 & 28
\end{array}
$$

The effect of a rise of temperature on latent heat would be an increment of the order of .05 per cent. per degree. The specific heat ratio is also variable, and for lack of data applying to the region of low temperature in question these subsidiary variations must be disregarded.

If the data for $\delta p=\mathrm{I} 7$ above be included, a table of double entry may be drawn up adapted for all pressure differences between 8 and $22 \mathrm{~cm}$. and for temperatures between $10^{\circ}$ and $30^{\circ}$.

The optic constant in the two cases becomes ( since $n=6 m s^{3} / \pi a^{3}$ )

For $\delta p=8.5 \mathrm{~cm} ., n=\mathrm{r} 22 s^{3}$ (diffraction) and $n=202 s^{3}$ (subsidence).

For $\delta p=22 \mathrm{~cm}$., $n=284 s^{3}$ (diffraction) and $n=470 s^{3}$ (subsidence).

Finally the volume ratio, $y$, is at $\delta p=8.5$,

$$
y=(57.7-8.5) /(75.6-17.9)=.853 \text {, }
$$

and at $\delta p=22 \mathrm{~cm}$.,

$$
y=(73.7-22.2) /(76.0-2.3)=.700,
$$

with which values a practical table is appropriately drawn up, time losses being ignored as above.

The subsidence constant computed for the different series shows the same falling off of value just before the coronas vanish, to which attention has already

\begin{tabular}{|c|c|c|c|c|c|c|c|c|c|c|}
\hline \multicolumn{7}{|c|}{ Low pressure, $\delta p=8.5 \mathrm{~cm}$} & \multicolumn{4}{|c|}{ Higher pressure, $\delta p=22 \mathrm{~cm}$} \\
\hline & $s$ & $S$ & $s$ & $S$ & $s$ & $S$ & $s$ & $S$ & $s$ & $S$ \\
\hline & $\begin{array}{l}4 . I-3.3 \\
3.3-2.6 \\
2.6-2.0 \\
2.0-1 . I\end{array}$ & $\begin{array}{l}6.4 \\
4.6 \\
3.2 \\
3.4\end{array}$ & $\begin{array}{l}5.4-4.5 \\
4.5-3.6 \\
3.6-2.8 \\
2.8-2.1\end{array}$ & $\begin{array}{l}8.5 \\
8.1 \\
6.2 \\
3.9\end{array}$ & $\begin{array}{l}5 . I-4.4 \\
4.4-3.6 \\
3.6-2.8\end{array}$ & $\begin{array}{l}6.3 \\
6.3 \\
6.2\end{array}$ & $\begin{array}{l}4.5-3.6 \\
3.6-2.7 \\
2.7-1.8 \\
r .8-.9\end{array}$ & $\begin{array}{l}6.0 \\
5 . I \\
3.9 \\
2.8\end{array}$ & $\begin{array}{l}4.7-3.6 \\
3.6-2.6 \\
2.6-1.8 \\
1.8-1.0\end{array}$ & $\begin{array}{l}8.5 \\
5 \cdot 5 \\
3 \cdot 9 \\
2.4\end{array}$ \\
\hline $\begin{array}{l}\text { Probable } S= \\
\qquad \delta t=\end{array}$ & & $\begin{array}{l}4.8 \\
\mathrm{I}^{\mathrm{m}}\end{array}$ & & $\begin{array}{l}6.7 \\
2^{\mathrm{mi}}\end{array}$ & & $\begin{array}{l}6.3 \\
2^{\mathrm{m}}\end{array}$ & & $\begin{array}{l}4 \cdot 5 \\
2^{\text {in }}\end{array}$ & & $\begin{array}{l}5.6 \\
4^{m}\end{array}$ \\
\hline
\end{tabular}
been called. The following cases may be instanced.

TABLE 12.-VALUES OF $S$ FOR SMALL CORONAS. 
The means of the best values are in the five cases $S=4.8,6.7,6.3$, and $S=4.5,5.6$, respectively, the mean of all of which is $S=5.6$. This value is practically the same as in the preceding table for $\delta p=17 \mathrm{~cm}$. The effect of waiting 2 or 4 minutes between observations does not appear in the individual results. The decay-effect of waiting I or 2 minutes is apparent, but this is due to the necessarily much more expeditious observation of coronas (time of fog suspension) in the former case. Curiously enough, at $\delta p=8.5 \mathrm{~cm} . S=6.5$ is larger than at $\delta p=22 \mathrm{~cm}$. where $S=5.0$.

The constants for computing diameters of fog particles $(d=\sqrt[3]{6 m / \pi n})$ are at $8.5 \mathrm{~cm} ., \mathrm{IO}^{2} \times d=\mathrm{I} .7 \mathrm{I}^{-\mathrm{x} / 3}$ and at $22 \mathrm{~cm}$., $\operatorname{10}^{2} \times d=2.27 n^{-\mathrm{x} / 3}$. Thus at $\delta p=8.5$ $\mathrm{cm}$. the ratio of distance between centers of cloud particles, $n^{-\mathrm{r} / 3}$, and their diameters is 58.4 , and at $\delta p=22 \mathrm{~cm}$. the corresponding ratio is 44.0 . It follows then that for the same corona, the distances apart are materially different in the two cases. Hence if the interstices enter into the character of the diffraction pattern and distribution of axial colors, these should be different for the two pressure differences in question.

TABLE I 4.-CONSTANTS OF CORONAS. ARC LIGHT, CONDENSATION CHAMBER, AND DISTANCES AS IN TABLE I $3 . \theta=2 \mathrm{I}^{\circ}$; BAROMETER, $75.6 \mathrm{~cm}$. $; \delta p=8.5 \mathrm{~cm}$; $y=.85 ; \beta=0 ; \quad S=4.8 ; a^{\prime}=.0034 ; a^{\prime \prime}=.0029 ; m=2.6 \times 10^{-6} ; n_{0}=825000 ; s$ MEASURED TO OUTER EDGE OF FIRST RING; PHOSPHORUS NUCLEI; $\delta t=\mathrm{I}^{\mathrm{m}}$.

\begin{tabular}{|c|c|c|c|c|c|c|c|}
\hline$z$ & $t$ & Corona. & $s$ & $n^{\prime \prime}=200 s^{3}$ & $N \Pi\left(\mathrm{I}-\frac{S}{s^{2}}\right)$ & $n=n_{0} N \Pi$ & $d=.0171$ \\
\hline & $\min$. & & $\mathrm{cm}$. & $=\times 103$ & & & \\
\hline$-\mathbf{I}$ & 3 & w y & I 3.0 & $44^{\circ}$ & I.I 7 & $9^{6} 5000$ & .000173 \\
\hline$\circ$ & 4 & wo & I 2.I & 354 & 1.000 & 825000 & I 82 \\
\hline I & 5 & W c & I 2. I & 354 & .830 & 685000 & I 94 \\
\hline 2 & 6 & w p & $9 \cdot 3$ & I6I & .690 & 569000 & 206 \\
\hline 3 & 7 & w v g & 9.3 & I6I & .554 & 457000 & 222 \\
\hline 4 & 8 & w bv g & - & - & $.44^{8}$ & 369000 & 239 \\
\hline 5 & 9 & $\mathrm{w} v \mathrm{~b}$ & 9.8 & I 88 & .360 & 297000 & 256 \\
\hline 6 & I0 & $w g p$ & 9.0 & I 46 & .292 & 241000 & 275 \\
\hline 7 & I I & w y g & 8.8 & 136 & .234 & 2 I 6000 & 285 \\
\hline 8 & I 2 & wo b g & 8.7 & 132 & .188 & I 55000 & 3 I 8 \\
\hline 9 & I 3 & $w \circ g$ & 8.0 & 102 & .150 & I 24000 & 343 \\
\hline 10 & I 4 & w r g & $7 \cdot 3$ & 79.4 & وII & 98200 & 370 \\
\hline I I & I 5 & w C g & 6.6 & 57.4 & .092 & 75900 & 404 \\
\hline I 2 & I 6 & $\mathrm{w} g \mathrm{~b} \mathrm{r}^{\prime}$ & $5 \cdot 7$ & 38.0 & .070 & 57500 & 443 \\
\hline I 3 & I 7 & $w \mathrm{~g} b \mathrm{r}^{\prime}$ & $5 \cdot 7$ & 38.0 & $.05 \mathrm{I}$ & 41900 & 493 \\
\hline I 4 & I 8 & $\mathrm{w}$ br $\mathrm{g}$ & $5 \cdot 3$ & 29.8 & .037 & 30500 & $55^{\circ}$ \\
\hline I 5 & I9 & $\mathrm{w} \mathbf{r} \mathrm{b} p$ & $4 \cdot 5$ & I 8.2 & .026 & 21600 & 6 I 7 \\
\hline I 6 & 20 & corona & 4. I & I 4.2 & .017 & I 4000 & 710 \\
\hline I 7 & 2 I & " & $3 \cdot 3$ & $7 \cdot 5$ & .009 & $7600^{1}$ & 868 \\
\hline I 8 & 22 & “" & 2.6 & 3.7 & .005 & $3700^{\prime}$ & .001100 \\
\hline I 9 & 23 & $"$ & 2.0 & 1.7 & .002 & $1730^{\circ}$ & I 420 \\
\hline 20 & 24 & " & I. I & .3 & .000 & $200^{\prime}$ & 2720 \\
\hline
\end{tabular}


TABLE 15-CONSTANTS OF CORONAS. CONDENSATION CHAMBER AND DISTANCES UNCHANGED. ARC LIGHT; PHOSPHORUS NUCLEI; $s$ TO OUTER EDGE OF FIRST RING; $\theta=2 \mathrm{I}^{\circ}$; BAROM., $75.6 \mathrm{~cm} . ; \delta p=8.5 \mathrm{~cm} . ; y=.85 ; \beta=0$; $S=6.7 ; a^{\prime}=.0034 ; a^{\prime \prime}=.0029 ; n_{0}=110000 ; m=2.6 \times 10^{-6}$.

FIRST SERIES. $-\delta t=2^{\mathrm{m}}$.

\begin{tabular}{|c|c|c|c|c|c|c|c|}
\hline$z$ & $t$ & Corona. & $s$ & $n^{\prime \prime}=2005^{3}$ & $N \Pi\left(1-\frac{s}{s^{2}}\right)$ & $n=n_{0} N \Pi$ & $d=\underset{n^{-1 / 3}}{.017 I}$ \\
\hline & $\min$. & & $\mathrm{cm}$ & $\times 10^{3}$ & & & $\mathrm{~cm}$. \\
\hline I & 0 . & - & - & - & 一 & & \\
\hline 2 & $2^{\circ}$ & w oy bg & 10.5 & 235 & 1.000 & 110000 & $.00035^{8}$ \\
\hline 3 & 4 & wog & 9.0 & 148 & .799 & 87900 & $3^{8} 5$ \\
\hline 4 & 6 & w c g & 8.1 & 106 & .627 & 69000 & $4 \mathrm{I} 8$ \\
\hline 5 & 8 & $w g b r^{\prime}$ & 6.3 & 50.0 & .478 & 52600 & 455 \\
\hline 6 & Io & $w o b^{1}$ & 5.6 & 36.0 & .340 & 37400 & 510 \\
\hline 7 & I 2 & w rp g & 5.4 & 3 I. 4 & .229 & 25200 & $5^{8} 5$ \\
\hline 8 & I4 & $w$ br b g & $4 \cdot 5$ & 18.8 & $.15 I$ & I6600 & 670 \\
\hline 9 & I 6 & corona & 3.6 & 9.7 & .087 & $9530^{2}$ & 807 \\
\hline 10 & I 8 & “ & 2.8 & 4.4 & .039 & $4340^{2}$ & $.00105^{\circ}$ \\
\hline I I & 20 & " & 2.1 & I. 9 & .017 & $1870^{2}$ & I 390 \\
\hline
\end{tabular}

1 Seen very obliquely this changes to $\mathrm{w} \mathrm{g}$.

$=$ Alternative $9530,4040,500$.

SeCOND SERIES. $-n_{\mathrm{o}}=680000 ; S=6.3 ; \delta t=2^{\mathrm{m}}$.

\begin{tabular}{|c|c|c|c|c|c|c|c|}
\hline & & & $\mathrm{cm}$. & $\times 10^{3}$ & & & \\
\hline $\begin{array}{l}\text { I } \\
2\end{array}$ & $\begin{array}{l}2 \\
4\end{array}$ & $\left.\begin{array}{l}w r^{\prime} \\
w \\
r^{\prime}\end{array}\right\}$ fog & - & & & & \\
\hline 3 & $\begin{array}{l}4 \\
6\end{array}$ & $\mathrm{w} \mathrm{r}^{\prime}$ & 一 & & & & \\
\hline 4 & 8 & $\mathrm{w} \mathrm{o}^{\prime}$ & 一 & & & & \\
\hline 5 & IO & w c & I 3.2 & 460 & 1.000 & 680000 & .000195 \\
\hline 6 & I 2 & w bv $g$ & I 2.4 & $3^{8 I}$ & .824 & 560000 & 208 \\
\hline 7 & I 4 & w vb g & I I .8 & 329 & .675 & 459000 & 222 \\
\hline 8 & I6 & $\mathrm{w} \mathrm{b} \mathrm{g}$ & I 1.0 & 266 & .549 & 373000 & $23^{8}$ \\
\hline 9 & I 8 & w g p & 10.7 & 245 & .446 & 303000 & 255 \\
\hline 10 & 20 & w yg vp & 10.2 & 212 & .360 & 245000 & 273 \\
\hline I I & 22 & w oylg & IO. I & 206 & .290 & I 97000 & 294 \\
\hline 12 & 24 & $\mathrm{w} / \mathrm{og}$ & 9.6 & I 80 & .232 & I 58000 & 316 \\
\hline I 3 & 26 & w r gy & 8.6 & I 27 & .185 & I 26000 & $34 \mathrm{I}$ \\
\hline I 4 & 28 & w c g g' & 7.8 & 95.0 & .145 & 98600 & 370 \\
\hline I 5 & 30 & $w g$ b $p$ & 6.4 & 52.4 & . I I I & 75500 & 404 \\
\hline 16 & 32 & w yg b p & 5.9 & 42.2 & .080 & 54400 & $45^{\mathrm{I}}$ \\
\hline 17 & 34 & w ro g & 5.8 & 39.0 & .056 & 37900 & 509 \\
\hline I 8 & 36 & w br b/g & 5.1 & 26.6 & .038 & 26200 & 575 \\
\hline I9 & 38 & w y bg|r & 4.4 & 17.0 & .025 & 17100 & 663 \\
\hline 20 & 40 & corona & 3.6 & 9.7 & .014 & 9790 & 800 \\
\hline $2 \mathrm{I}$ & 42 & (4) & 2.8 & $4 \cdot 4$ & .006 & $435^{\circ}$ & \\
\hline
\end{tabular}


A CONTINUOUS RECORD OF ATMOSPHERIC NUCLEATION.

TABLE I6.-CONSTANTS OF CORONAS. CONDENSATION CHAMBER, DISTANCES, MEASUREMENT OF $s$ UNCHANGED; ARC LAMP; $\theta=24.5^{\circ}$; BAROM., 76.0 $\mathrm{cm} . ; \quad \delta p=22 \mathrm{~cm} . ; \quad y=.70 ; \quad \beta=0 ; \quad S=4.5 ; \quad n_{0}=360000 ; m=6.1 \times 10^{-6} ; \quad a^{\prime}=.0032$; $a^{\prime \prime}=.00290 ;$ PHOSPHORUS NUCLEI; $\delta t=2^{\mathrm{m}}$.

\begin{tabular}{|c|c|c|c|c|c|c|c|c|}
\hline$z$ & $t$ & Corona. & $s$ & $n^{\prime}=360 s 3$ & $n^{\prime \prime}=470 s^{3}$ & $N \Pi\left(\mathrm{I}-\frac{S}{s^{2}}\right)$ & $n=n_{0} N \Pi$ & $d=.0227$ \\
\hline & $\min$. & & $\mathrm{cm}$. & $\times 10^{3}$ & $\times 103$ & & & $\mathrm{~cm}$. \\
\hline I & 2 & w o & - & - & - & - & - & - \\
\hline 2 & 4 & $\mathrm{w} \mathrm{c}^{\prime}$ & - & - & - & - & - & - \\
\hline 3 & 6 & w $g^{\prime} \mid p^{\prime}$ & I 3.2 & 828 & I 080 & 1.000 & 360000 & .000320 \\
\hline 4 & 8 & w y|vb & 10.5 & $42 I$ & $55^{\circ}$ & .683 & 246000 & 362 \\
\hline 5 & 10 & w c $g^{\prime}$ & 8.5 & 22 I & 289 & $.45^{6}$ & 164000 & 415 \\
\hline 6 & I 3 & wg $b \mid p$ & 6.0 & 79.6 & 104 & .297 & 107000 & 478 \\
\hline 7 & 15 & w ro $g^{\prime}$ & $5 \cdot 9$ & 73.8 & 96.3 & .183 & 65900 & 562 \\
\hline 8 & 17 & $\mathrm{w}$ y o b & $4 \cdot 5$ & 33.9 & $44 \cdot 3$ & $.1 I I$ & 40000 & 664 \\
\hline 9 & I 9 & $w$ br b & 3.6 & I 6.8 & 21.9 & $.06 \mathrm{I}$ & $22000^{\prime}$ & 8 Io \\
\hline IO & $2 I$ & corona & 2.7 & $7 \cdot 1$ & $9 \cdot 3$ & .026 & $9290^{1}$ & .001080 \\
\hline I I & 23 & $"$ " & I. 8 & 2.3 & 3.0 & .008 & $2920^{1}$ & I 590 \\
\hline 12 & 26 & “" & .9 & .3 & .3 & .001 & $360^{\prime}$ & 3200 \\
\hline
\end{tabular}

1 Alternative $22000,10500,2660,0$.

SECOND SERIES. $-n_{0}=4 \mathrm{r} 0000 ; S=5.6 ; \delta t=4^{\mathrm{m}}$.

\begin{tabular}{|c|c|c|c|c|c|c|c|c|}
\hline & $\min$. & & $\mathrm{cm}$ & $\times 10^{3}$ & $\times 10^{3}$ & & & $\mathrm{~cm}$ \\
\hline I & 4 & W V & J 2.7 & $73^{8}$ & 963 & 1.000 & 410000 & .000305 \\
\hline 2 & 8 & w g|v & 10. I & 371 & 484 & .675 & 277000 & $35^{\circ}$ \\
\hline 3 & I 2 & w r g & 8.8 & 245 & 320 & .444 & I 82000 & 400 \\
\hline 4 & I 6 & $\mathrm{w} g \mathrm{~b} \mid \mathrm{p}$ & 6.3 & 90.0 & 117 & .287 & I I 8000 & 462 \\
\hline 5 & 20 & w r g & 5.8 & 70.2 & 91.7 & .173 & 70900 & 548 \\
\hline 6 & 24 & w y b g & $4 \cdot 7$ & 37.4 & 48.9 & $.10 I$ & 41400 & 656 \\
\hline 7 & 28 & corona & 3.6 & 16.8 & $2 \mathrm{I} .9$ & .053 & 21600 & 814 \\
\hline 8 & 32 & “ & 2.6 & 6.7 & 8.7 & .021 & $8690^{2}$ & .001100 \\
\hline 9 & 36 & ". & I. 8 & $2 . I$ & 2.7 & .007 & $2790^{2}$ & 1610 \\
\hline Io & 40 & " & I.O & .4 & .5 & .001 & $490^{2}$ & 2900 \\
\hline
\end{tabular}

${ }^{2}$ Alternative $8690,1230,0$

28. Remarks on the tables.-Considering the graph, figure 10 , corresponding to table 14 , it is seen that the curve as a whole lies above the locus $n^{\prime}=2005^{3}$. This might be rectified by using a larger value of $S$ than the small one $(S=4.8$, while the mean value is 5.6) specially computed. The divergence of the $S$ effect is enhanced in view of the large number of exhaustions.

The graph $n=n_{0} N \Pi$ brings out for the first time the cycle of the first color series, $\mathrm{w} y$ to $\mathrm{w} \mathrm{p}$, which in the shorter apparatus above was not fully attainable. The successive colors of the first annuli vary in the order of wave length, though nothing was observed above the first $w y$. The next color cycle (enlarged Io-fold in the adjoining curve) is very complete, though irregular from the inevitable color distortion, the interval between observations being but one minute. The following color cycles III and IV, which include the vivid annuli, 
manifest the same relations already instanced above. The small value $n=$ 70,000 which belongs to the green corona, and the correspondingly small values for the other coronas, are in aecord with the small pressure difference, $\delta p=8.5$, chosen; but it will presently appear that this value is even smaller than would be predicted. The crests of the $n$-eycles may be loeated at $n=25,000,65,000$, and 550,000 , from which the corresponding $d$-values would be $d \times 10^{6}=585$, 430,2 I0 $\mathrm{cm}$, indieating diameters of fog particles in the ratio of $3: 2: \mathrm{I}$.

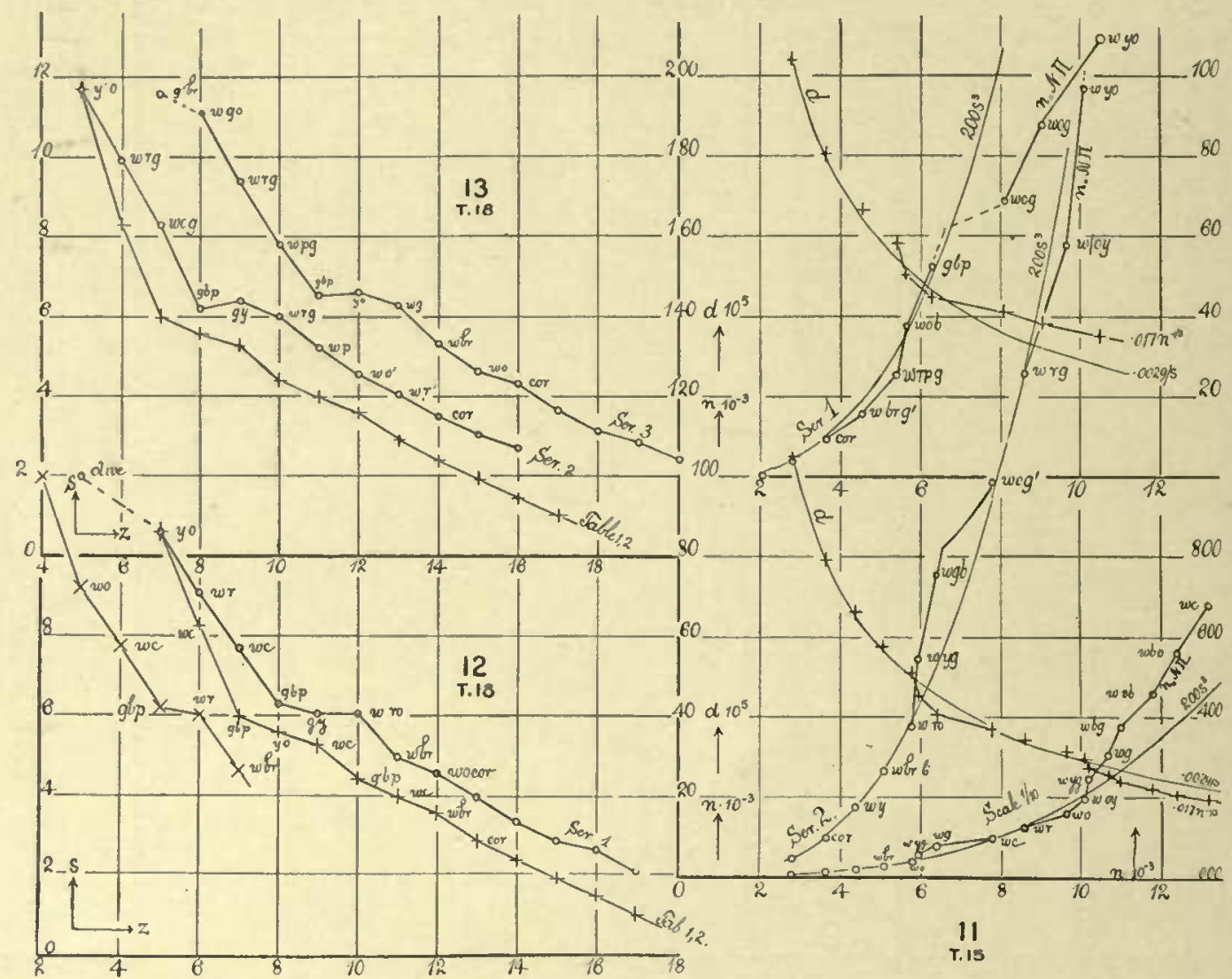

Figure il,-Charts for Table I5, showing the Nucleations $(n)$ and Diameters $(d)$ of the Fog Particles, in Terms of tie Apertures $(s)$ of the Coronas.

Figures I2, i3.-Charts for Table i8, Showing the Relation of tile Apertures, $s$, of tul Coronas to the Number, $z$, of Exhaustions.

The $d$-values are much more diffieult to correlate, although the main cycles are apparent. The appearance here is that of four independent loci which appear united as the result of color distortion. The view which ascribes to these curves different parameters is in many respects plausible. There are three green coronas discernible corresponding to $10^{6} \times d=275,443,617$, about in the ratio of $3: 5: 7$. But this result is again merely tentative.

Table I 5 contains two series of results in which larger times intervene between the exhaustions to insure more thorough diffusion and less distortion of color. The data are mapped out in the same chart, figure II, onc above the other. In the $n$-curves four eycles may be made out, the lower less distinctly, 
as this is concealed in a measure by the specially computed $S$-values. There is reasonable agreement with the graph $200 s^{3}$, though the upper cycle lies above it. In the $d$-curves the agreement with .0029/s is throughout as close as the difficulty of observation permits. Three sweeping marches from violet to deep red may be distinguished.

In the last table (I6) high pressure differences $\delta p=22 \mathrm{~cm}$. are brought to bear, and the time intervals $\delta t=2$ and 4 minutes intervene between the exhaustions. A definite effect of the latter does not appear, since the nucleation of the green coronas, for instance, is $n=107,000$ and I 8,000 , with more nuclei indicated for the larger time interval. The reverse would be the case if there were marked time loss. The corresponding charts may be drawn to show the relations in detail. The curve $470 \mathrm{~s}^{3}$ lies above the higher coronas, whereas in the preceding cases it lay below them, indicating the difficulty encountered in computing sufficiently correct values for the subsidence constant, $S$. While the $n$-values show the usual relations, the $d$-values are more difficult to interpret; but three cycles may be made out, with the middle one unusually bulging and contracted.

29. Nucleation of the green coronas.-These values, though difficult to obtain and suffering from cumulative errors, are nevertheless consistent; and the nucleation to produce the green corona seems to depend on the distance between particles. Put $d / n^{-1 / 3}=D$. Then the above results show that $n=10^{7}(D-.0114)$. Hence if the distance of particles is 87 times their diameter the green coronas should appear for vanishing nucleations. This implies a limit of values of $n$ by which green coronas may be evoked. Furthermore, since the apertures of this type of coronas remain within a range of values nearly the same for all conditions of nucleation, the value of $n$ may be found below which green coronas do not occur. For the diameter of fog particle is here about $d=.00046 \mathrm{~cm}$, and therefore $n=15,300$. One may argue, therefore, that at least I 5,000 nuclei per cubic centimeter must be present if coronas of the middle green type are to be possible.

TABLE I 7 - -NUCLEATION OF THE GREEN CORONAS. ${ }^{1} n$ (computed) $=10^{7}\left(d \mathbb{3}^{n}\right.$ $-.0 \mathrm{II} 4)$.

\begin{tabular}{|c|c|c|c|c|c|c|c|c|}
\hline Table. & $n \times 10^{-3}$ & $\delta t$ & Table. & $n \times 10^{-3}$ & $\delta t$ & Table. & $n \times 10^{-3}$ & $\delta t$ \\
\hline $\begin{array}{r}\text { I } 4 \\
\text { I } 5 \\
\text { I } 5 \\
\text { Mean }\end{array}$ & $\begin{array}{r}49.7 \\
52.6 \\
75.5 \\
57.0\end{array}$ & $\begin{array}{c}\min . \\
\text { I } \\
2 \\
2\end{array}$ & $\begin{array}{l}\text { I3 } \\
\text { I3 } \\
\text { I3 } \\
\\
\end{array}$ & $\begin{array}{r}89.1 \\
\text { I 10.0 } \\
91.2 \\
94.2 \\
96.1\end{array}$ & $\begin{array}{c}\min \\
2 \\
2 \\
2 \\
2\end{array}$ & $\begin{array}{l}16 \\
16\end{array}$ & $\begin{array}{l}107 \\
\text { I } 8\end{array}$ & $\begin{array}{c}\min \\
2 \\
4\end{array}$ \\
\hline
\end{tabular}

\begin{tabular}{|c|c|c|c|}
\hline $\begin{array}{l}10^{5} d= \\
\delta p= \\
d p^{3 / n}= \\
10^{-3 n}=\end{array}$ & $\begin{array}{l}45 \mathrm{~cm} \text {. (obs.) } \\
8.5 \mathrm{~cm} \text {. } \\
.017 \\
57 \text { (comp.) }\end{array}$ & $\begin{array}{l}46 \mathrm{~cm} . \\
\text { I } 7 \mathrm{~cm} \text {. } \\
.02 \mathrm{I} \\
96^{.02}\end{array}$ & $\begin{array}{r}47 \mathrm{~cm} . \\
22 \mathrm{~cm} . \\
.023 \\
\mathrm{I}_{3}\end{array}$ \\
\hline
\end{tabular}

1 Earlicr values, $\delta p=17 \mathrm{~cm}$. and cubical vessel, $10^{-3} n=85,106,104,96$, and 96,96 , the mean being $n=$ 97000 . 
While these inferences are essentially uncertain and might even seem attributable to the time coefficient, $\beta$, neglected, the observations do not warrant such exception. Thus at $\delta p=22 \mathrm{~cm}$. and $\delta t=4$ minutes, the total time consumed was 36 minutes, whereas in the case of $\delta p=8.5 \mathrm{~cm}$. it was only $3^{2}$ minutes for $\delta t=2$ minutes, and only 2 I minutes for $\delta t=I$ minute. The total amount of time loss-must therefore actually have been smaller in the latter cases.

\section{OTHER CAUSES OF CHANGE IN THE TYPES OF CORONAS.}

30. Thickness of cloud layer.-In passing from the cubical to the long apparatus, the thickness of the cloud layer decreased from 20 to $16 \mathrm{~cm}$. On tipping the former vessel on its trunnions, and looking through the chamber diagonally, the thickness of cloud layer could be increased to $30 \mathrm{~cm}$. In all these cases, the type of corona and its diameter showed no appreciable change. If symmetry about the center is maintained, the effect of the thickness of the cloud layer seems thus to be absent.

31. Obliquity of diffraction.-When the direction of the diffracted ray differs by a small angle from the direction of the normal ray of light, the type of corona does not change as to color of annuli. If, however, the angle of deviation due to diffraction is very large, the corona may change in character. Thus for great obliquity the orange-red corona was found to change into the preceding green corona. In other words, a larger particle on very oblique diffraction may produce the same corona as a smaller particle on less oblique diffraction.

32. Effect of wave length.-Since at $\delta p=I 7 \mathrm{~cm} ., a=73 \lambda$, and therefore $n=6 \mathrm{~ms}^{3} / \pi \alpha^{3}=6 \mathrm{~ms}^{3} / \pi \lambda^{3}(73)^{3}=23^{3} / 10^{1_{2}} \lambda^{3}$, the equation for different colors would be (denoting the colors by subscripts) $n=67 s_{\mathrm{c}}^{3}=97 s_{\mathrm{r}}^{3}=107 s_{\mathrm{v}}^{3}=\operatorname{II} 8 s_{\mathrm{y}}^{3}$ $=I 54 S_{\mathrm{g}}^{3}=22 \mathrm{I} S_{\mathrm{b}}^{3}=360 \mathrm{~S}_{\mathrm{v}}^{3}$, etc., while for other pressure differences the same relations would be preserved.

Testing this by the very full series in the second part of table 15 , and remembering that the measurement is made to beyond the first ring, the following data nay be deduced:

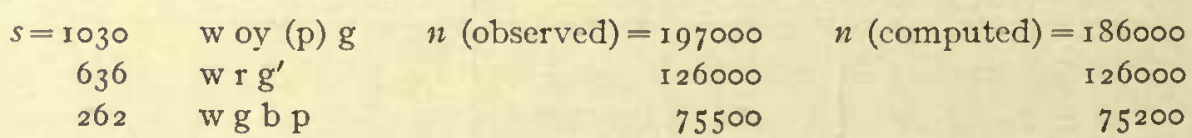

If the $w \mathrm{rg}^{\prime}$ corona is taken as correct, the $\mathrm{w} \mathrm{g}$ b $\mathrm{p}$ corona will also be, as well as the w oy ( $p$ ) g corona. But in the latter case the narrow purplish ring which intervenes before the green and the general difficulty of defining the mixed colors of the second ring makes inferences of the present kind precarious. Nevertheless it is probable that the rather sudden transition of green to blue in the colors of the second ring is associated with the underlying cause of periodicity. Examples of this kind might be multiplied. 
DIFFERENT SPEEDS OF EXHAUSTION FOR THE GIVEN PRESSURE DIFFERENCE.

33. Increased suddenness of condensation.-As a final test of the trustworthiness of the above sequences, it was necessary to repeat the results with some form of valve more nearly instantaneous in its action. In the case of air nuclei, special comparisons instanced below (Chap. IX, $\$ 3$ ) showed that for a reasonable relation between the sizes of the condensation chamber and the vacuum chamber, an ordinary plug stopcock was quite as serviceable as an instantaneous valve, the coronas observed with the air nuclei being in both cases the same. With phosphorus nuclei and at the outset of the experiment, however, this is not quite the case, at least when the nuclei are very numerous and very small.

The design of the new valve was very simple. In figure $3, p \cdot 58, V$ leads to the vacuum chamber (large aspirator flask), and $C$ to the condensation chamber.

TABLE I8.-THREE GEOMETRICAL SEQUENCES OF CORONAS, FOR INSTANTANEOUS VALVE. CHAMBER, $20 \times 26 \times 35 \mathrm{~cm}^{3}$; DISTANCES, 85 AND $250 \mathrm{~cm}$.; $\theta=22^{\circ}$; BAROM., $74.9 ; \delta p=\mathrm{x} 7 \mathrm{~cm} . ; y=.767 ; \beta=0 ; S=2.1 ; S^{\prime}=1.95 ; a$ (SUBSIDENCE) $=.0029 ; m=4.8 \times 10^{-6} g ; n_{0}^{\prime}=308000 ; n_{0}=340000 ;$ PHOSPHORUS NUCLEI; WELSBACH LAMP, AND $s$ MEASURED TO OUTER EDGE OF RED RING.

First Series.

\begin{tabular}{|c|c|c|c|c|c|c|c|c|}
\hline$z$ & $t$ & $s$ & Corona. & $n^{\prime}=376 s^{3}$ & $N \Pi\left(\mathrm{I}-\frac{S}{s^{2}}\right)$ & $\underset{n^{-I / 3}}{d=.02 I}$ & $n^{\prime}$ & $N \Pi^{\prime}$ \\
\hline 0 & $\min$. & $\mathrm{cm}$. & Nucleation. & $\times 10^{3}$ & & & & \\
\hline$I$ & I & & fog & & & & & \\
\hline 2 & 4 & $(13.0)$ & $\mathrm{w} \mathrm{r}^{\prime}$ & & & & & \\
\hline 3 & 7 & (II.O) & olive & & & 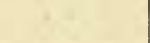 & & \\
\hline 4 & ro & & $\mathrm{g}^{\prime} \mathrm{r}^{\prime}$ & & & & & \\
\hline 5 & I 3 & 10.6 & y o bg & 534 & 1.000 & .00030 & 308000 & 1.000 \\
\hline 6 & 17 & $9 . x$ & $\mathrm{w} r \mathrm{~g}^{\prime}$ & 288 & .752 & 33 & 232000 & .754 \\
\hline 7 & 20 & $7 \cdot 7$ & w c g & I 7 I & .562 & 36 & I 74000 & .564 \\
\hline 8 & 23 & 6.3 & $\mathrm{~g} \mathrm{~b} \mathrm{p}$ & 94.0 & .416 & 40 & I 29000 & .419 \\
\hline 9 & 26 & $6 . x$ & gy br b' & 85.3 & .302 & 45 & 93900 & .305 \\
\hline I0 & 29 & 6.1 & w ro g & $85 \cdot 3$ & .218 & 50 & 68100 & $.22 \mathrm{I}$ \\
\hline I I & 32 & 5.0 & w br cor & 47.0 & $.15^{8}$ & $5^{6}$ & 49600 & $.16 \mathrm{I}$ \\
\hline I 2 & 35 & 4.6 & w o cor & 36.6 & . I I I & 62 & 35100 & I I 4 \\
\hline I 3 & $3^{8}$ & 4.0 & corona & 24.1 & .077 & 71 & 24600 & .080 \\
\hline I 4 & $4^{I}$ & $3 \cdot 4$ & $"$ & I 4.8 & $.05 \mathrm{I}$ & $8 \mathrm{I}$ & I 6300 & .053 \\
\hline I 5 & 44 & 2.9 & “ & 9.7 & .032 & 94 & 10500 & .034 \\
\hline I 6 & 47 & 2.6 & “" & 7.0 & .019 & .00112 & 6200 & .020 \\
\hline \multirow[t]{7}{*}{17} & 50 & $2 . I$ & “" & $3 \cdot 5$ & .010 & .00140 & 3400 & $.01 \mathrm{I}$ \\
\hline & 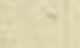 & & & & & & $321 \times 10^{3}$ & \\
\hline & & & & & & & 303 & \\
\hline & & & & & & & $\begin{array}{l}278 \\
285\end{array}$ & \\
\hline & & & & & & & 345 & \\
\hline & & & & & & & $3 \mathrm{I} 3$ & \\
\hline & & & & & & Mean: & 308000 & \\
\hline
\end{tabular}


$R$ is a soft rubber cork which can be raised or suddenly lowered (in the latter case by the blow of a swivelled hammer not shown in the figure) by actuating the knob or handle at the upper end of the rigid rod $r$. There is a stuffing box at $S$. The end of the tube $p$ has been turned off smoothly and serves as a seat for the plug, $R$. Stiff glycerin is used as a lubricant. All passageways and pipes are wide, the latter at least one inch in diameter. The valve has retained its efficiency after countless experiments, but it must be left open when not in usc.

34. Results. - The results are given in table 18 , on a plan similar to the above tables, and the calculations are made in the same way. All operations were strictly timed, and it was thought best to conpute $S$ from subsidence as

$$
S^{\prime}=s_{z}^{3}\left(\mathrm{I}-\frac{\mathrm{x}}{\mathrm{y}}\left(s_{\mathrm{z}+\mathrm{r}}^{3} / s_{\mathrm{z}}^{3}\right)\right) \text {. }
$$

The corresponding $n_{\circ}$ is written $n_{\circ}^{\prime}$. The result is $S^{\prime}=$ I.95. Computed from the observations themselves, $S=2.1,1.7$, for instance, in the first two parts of the table, a difference which can only be explained on the ground of observational error. Usually 3 minutes were consumed by the operations between the exhaustions, while the fog was dissipated (by the influx) within I 5 sec. after the exhaustion. In the last series but 2 minutes are allowed between the observations, but there seems to be no appreciable difference in the data so far as this cause is concerned.

Second Series. $-\theta=24^{\circ} ; \quad$ barom. $=75.08 \mathrm{~cm} . ; \quad \delta t=3^{\mathrm{m}} ; \quad y=.767 ; \quad n_{0}=2 \mathrm{I} 2000 ; \quad n_{0}^{\prime}=$ $305000 ; m=5.0 \times 10^{-6} \mathrm{~g} / \mathrm{cm}^{3} ; S=1.7 ; S^{\prime}=1.95$.

\begin{tabular}{|c|c|c|c|c|c|c|c|c|}
\hline$z$ & $t$ & $s$ & Corona. & $n^{\prime}=376 s^{3}$ & $N \Pi\left(\mathrm{I}-\frac{S}{s^{2}}\right)$ & $d=\underset{n}{n-1 / 3}$ & $n^{\prime}$ & $N \Pi^{\prime}$ \\
\hline I & 43 & & & $\begin{array}{c}390 \times s^{3}= \\
10^{-3} n^{\prime}\end{array}$ & & & & \\
\hline 2 & 45 & (13) & $w r^{\prime}$ & - & & & & \\
\hline 3 & 48 & (12) & $b^{\prime} B$ & - & & & & \\
\hline 4 & $5^{\mathrm{I}}$ & & g B & $\overline{624}$ & & & & \\
\hline 5 & 54 & II .7 & $y^{\prime} 0$ & $\begin{array}{l}624 \\
378\end{array}$ & $\begin{array}{r}1.000 \\
.758\end{array}$ & .00031 & $\begin{array}{l}305000 \\
231000\end{array}$ & 1.000 \\
\hline 6 & 57 & $9 \cdot 9$ & w r g & $\begin{array}{l}378 \\
223\end{array}$ & .758 & $\begin{array}{l}34 \\
38\end{array}$ & 173000 & $\begin{array}{r}.750 \\
-68\end{array}$ \\
\hline 7 & 00 & $\begin{array}{l}8.3 \\
6.2\end{array}$ & $\begin{array}{l}\text { w C g } \\
\text { g b }\end{array}$ & $\begin{array}{r}223 \\
02.8\end{array}$ & .571 & 30 & 120000 & .508 \\
\hline & $\begin{array}{l}3 \\
6\end{array}$ & $\begin{array}{l}0.2 \\
6.4\end{array}$ & $\begin{array}{l}g \text { b p } \\
g \text { y } h^{\prime}\end{array}$ & 102.2 & .313 & 46 & 03000 & $\begin{array}{r}.423 \\
208\end{array}$ \\
\hline 9 & 0 & $\begin{array}{l}0.4 \\
6.0\end{array}$ & $\begin{array}{l}g y \mid 0 \\
\text { w r g }\end{array}$ & 82.4 & .230 & $5 \mathrm{I}$ & 68600 & .308 \\
\hline & & & & & & & & .225 \\
\hline I I & 12 & 5.2 & w p cor & 55.0 & .108 & 57 & 49700 & .163 \\
\hline I 2 & 15 & 4.5 & $\mathrm{w}$ yo cor & $3^{6.8}$ & .121 & 64 & 35400 & .116 \\
\hline I3 & 18 & 4.0 & $\mathrm{w}$ br cor & 25.9 & .085 & 72 & 24700 & .08I \\
\hline I 4 & $2 \mathrm{I}$ & $3 \cdot 5$ & corona & 16.7 & .059 & 83 & 16500 & .054 \\
\hline I 5 & 24 & 3.0 & " & II 1.0 & .038 & 96 & 10500 & .035 \\
\hline I 6 & 27 & 2.7 & " & $5 \cdot 5$ & .024 & .00115 & 6400 & .021 \\
\hline & & & & & & & Note: $n_{0}^{\prime}=$ & \\
\hline & & & & & & & 320 & \\
\hline & & & & & & & 310 & \\
\hline & & & & & & & 3 I 5 & \\
\hline & & & & & & & & \\
\hline & & & & & & Mean & 305000 & \\
\hline
\end{tabular}


THIRD SERIES. $-\theta=24^{\circ} ;$ batom. $=75.08 \mathrm{~cm} . ; \delta t=2^{\mathrm{m}}$.

\begin{tabular}{|c|c|c|c|c|c|c|c|c|}
\hline$z$ & $t$ & $s$ & Corona. & $n^{\prime}=376 s^{3}$ & $N \Pi\left(\mathrm{r}-\frac{S}{S^{2}}\right)$ & $d=\underset{n^{-1 / 3}}{.021} \times$ & $n^{\prime}$ & $N \Pi^{\prime}$ \\
\hline I & 40 & & fog & & & & & \\
\hline 2 & 42 & & $\mathrm{w} \mathrm{r}^{\prime}$ & & & & & \\
\hline 3 & 44 & (13) & w p & & & & & \\
\hline 4 & 46 & (I 12$)$ & olive & & & & & \\
\hline 5 & 48 & (II) & $g^{\prime} r^{\prime}$ & & & & & \\
\hline 6 & $5^{\circ}$ & I I.I & w yo & 540 & 1.000 & .00030 & 346000 & 1.000 \\
\hline 7 & $5^{2}$ & $9 \cdot 4$ & w r g & 324 & $.75^{\circ}$ & 33 & 255000 & .739 \\
\hline 8 & 54 & 7.8 & w p g & 185 & $.55^{8}$ & 36 & 196000 & .566 \\
\hline 9 & $5^{6}$ & 6.5 & $g \mathrm{~b} \mathrm{p}$ & 107 & .408 & 40 & I 45000 & .420 \\
\hline Io & $5^{8}$ & 6.6 & yo & I I 2 & .293 & 44 & 106000 & .307 \\
\hline I I & 60 & 6.2 & w c g & 95.2 & .210 & 49 & 77800 & .225 \\
\hline 12 & 62 & $5 \cdot 3$ & $\mathrm{w}$ br & $5^{8.1}$ & .150 & 55 & 56700 & . 164 \\
\hline I 3 & 64 & 4.6 & wo cor & $3^{8.1}$ & .III & $6 I$ & 40500 & .117 \\
\hline I4 & 66 & $4 \cdot 3$ & corona & 31.2 & .070 & 69 & 28400 & .082 \\
\hline I 5 & 68 & 3.6 & “ & 18.2 & .046 & 78 & 19400 & .056 \\
\hline 16 & 70 & 3.I & " & II .5 & .028 & 90 & I 2600 & .0364 \\
\hline 17 & 72 & 2.8 & " & 8.54 & .0153 & .00107 & 7700 & .0223 \\
\hline \multirow[t]{8}{*}{ I8 } & 74 & 2.4 & & $5 \cdot 3^{8}$ & .0035 & 127 & 4500 & $.03 \mathrm{I}$ \\
\hline & & & & & & & Note: $n_{0}^{\prime}=$ & \\
\hline & & & & & & & $\begin{array}{l}323 \times 10 \\
380\end{array}$ & \\
\hline & & & & & & & 325 & \\
\hline & & & & & & & 320 & \\
\hline & & & & & & & $\begin{array}{l}383) \\
(420)\end{array}$ & \\
\hline & & & & & & & & \\
\hline & & & & & & Mean & 346000 & \\
\hline
\end{tabular}

$n^{\prime}$ and $S^{\prime}$ computed from subsidence and time.

To compare the new observations with the older ones above, it is convenient to lay off the aperture, $s$, in terms of the order of the exhaustion, $z$, as has been done in the charts, figures 12 and $I_{3}$. The following peculiarities are observed: Below the g-b-p coronas $(s=6$ to $6.5 \mathrm{~cm}$.) the general slope of all the curves is nearly the same. Above these coronas, the older results obtained with the plug valve correspond to a decidedly steeper slope than the new results. Curiously enough, therefore, a greater number of exhaustions are required in the case of the instantaneous valve to pass from a given corona to a given succeeding corona than in the case of the stopcock. The instantaneous valve thus removes a smaller relative number of nuclei per exhaustion than does the stopcock, so long as fresh nuclei or great numbers are in question. Subsidence of fog for the very fine particles here in question need not be considered in explanation. In fact, the recent work is even more rapid than the old.

35. Growth of muclei.-The simplest way of accounting for this result is to assume that there is a continual growth of nuclei in the interval between the observations, whereby those of extreme smallness come first within the range of action of the instantaneous valve. The excess of available nuclei obtained 
in this way would gradually decrease toward the end of the experiment so that the slopes of all curves compatibly with the observations would gradually be the same. Below the middle g-b-p corona, the curves if placed together would nearly coincide. The difficulty with this hypothesis is the absence of any obvious effect when the time between observations is varied. The curves are about the same for $\delta t=2 \mathrm{~min} ., 3 \mathrm{~min}$, or even $12 \mathrm{~min}$, so far as observed.

36. Subsidence. - The effect of errors in the subsidence constant may be estimated. Writing the equation $n_{z}=n_{0} 10^{(z-Z) \text { log } y} I I$, in the approximate form $n_{z}=n_{0} 10^{(z-Z) \log y}\left(I-S\left(\frac{1}{s_{z}^{z}}+\frac{x}{s_{z}^{z}}+x+\frac{x}{s_{z}^{z}}+z+\right)\right)$, whence if $N=10^{(z-Z) \log y}$, $\delta n_{z}=n_{0} N \delta S \Sigma\left(\frac{1}{s^{2}}\right)$.

It is preferable to use the inverse method, putting $n_{0}=n_{z} / N\left(\mathrm{I}-S \Sigma\left(\frac{1}{s^{2}}\right)\right)$, whence $\delta n_{0}=\frac{n_{n}}{N} \sum\left(\frac{x}{s^{2}}\right) \delta S$.

Putting $n_{\mathrm{z}}=10500, n_{0}=212000, N=.0703, \Sigma\left(\frac{1}{s^{2}}\right)=.265$,

$$
\delta n_{\circ}=40000 \delta S, \text { nearly. }
$$

Thus if $\delta S=.2, \delta n_{\circ}=8000$, or relatively $8000 / 2$ I $2000=.038$. It follows that by the error of .2 made in the estimate of $S, n_{\mathrm{o}}$ will not be affected more than about 4 per cent., which in no way accounts for the observed discrepancy of the new and the old results.

37. Exhaustion ratio.-Again if the above value of $n_{0}$ be taken, the effect of an error in the exhaustion ratio $y$ will be

$$
-\delta n_{\mathrm{o}} / n_{\mathrm{o}}=\frac{n_{0}}{y}(z-Z) \delta y \text {. }
$$

For $n_{0}=212000, z-Z=15-5, y=.767$, an average case in the preceding table, $\delta n_{\circ} / n_{\circ}=13 \delta y$, or for

$$
\begin{array}{rr}
\delta y=.1 & -\delta n_{0} / n_{0}=130 \% \\
.05 & 65 \% \\
.01 & \text { I } 3 \%
\end{array}
$$

showing that great care is needed in relation to $y$.

38. Inferences.-To reach an opinion as to the cause of the observed initial diversity of rates one may note that for equal pressure differences, smaller nuclei are necessarily caught by the instantaneous valve than by the stopcock. More nuclei are thus within reach in the former case: but apart from this, since all other conditions are the same for both cases, the rate of denucleation should be the same, even if the absolute number of efficient nuclei removed by exhaustion is greater for the instantaneous valve. If therefore one begins with the same corona which implies identity of diameter of particle and may be assumed to imply an identical number of available or effective nuclei in both cases, the two curves should be identical. In figures $\mathrm{I}_{2}$ and $\mathrm{I} 3$, considered first between the upper $w-y-o$ and the middle g-b-p corona, the new and the old curves cross at the large $\mathrm{w}-\mathrm{y}-\mathrm{o}$ corona. If under identical exhaustions the number of available nuclei are therefore successively greater for the instantaneous valve 
than for the stopcock, there must be an accession of nuclei between the exhaustions in the former case or a loss of nuclei for the case of the plug.

Between the middle $g-b-p$ corona and dust-free air, nuclei are removed at the same rate in the two cases, as the curves here have practically the same slope and would be brought to coincidence throughout if the upper coronas were to coincide. Inasmuch as in the case of atmospheric air the $g-b-p$ corona is not exceeded, it is thus immaterial which form of valve is used, and the direct experiments of Chapter IX bear this out.

The alternative that nuclei are generated by very sudden expansion is without correlative evidence. At least all my experiments to detect ionization produced by sudden expansion have failed. It is equally difficult to account for an abnormal loss of nuclei in the earlier stages of the experiment of table i 8. True, it is here that the ionization of phosphorus vanishes, though this evanescence is enormously rapid by comparison.

The nucleations of the g-b-p corona are by table I $8, n=$ I 29, 000 , I 29, 000 , and 145,000 , respectively, values decidedly larger than were found above, and due to the greater geometrical remove of the normal coronas from the $g-b-p$ corona. As about one more exhaustion is required on the average in the new data, the corresponding results for the same $z$ would be, $n=94,000,94,000$, 106,000, which agrees with the order of values above.

39. Different rates of exhaustion for moderate nucleations. - In this place it is interesting to insert a series of direct comparisons on the number of nuclei within the reach of a half-inch exhaust pipe with an ordinary plug stopcock, and the number caught when the valve is as in figure 3 with all pipes over one inch in diameter and less than one foot long. These are given in table I9, the

TABLE 19.-DIRECT COMPARISON OF RESULTS FOR STOPCOCK .5" DIAMETER, AND INSTANTANEOUS VALVE I" DIAMETER. LARGE CONDENSATION CHAMBER, AIR NUCLEI. $\delta p=\mathbf{r} 7 \mathrm{~cm}$.

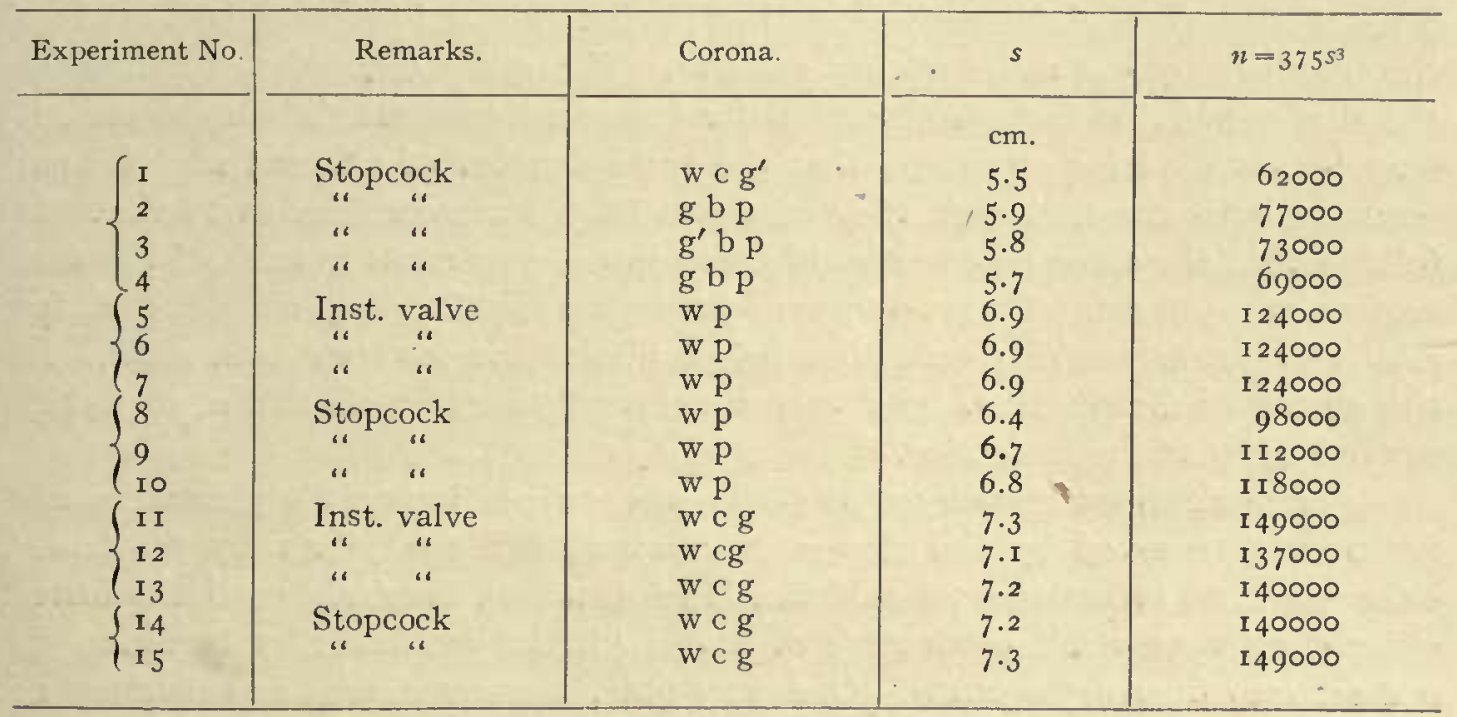


data for the stopcock alternating with data for the instantaneous valve. Unfortunately, the time intervals between observations were not quite equal, as the necessary adjustments consumed varying amounts of time.

These data are shown graphically on the chart, figure 14, p. 83 , the abscissa being merely distributive. If the mean data of each set be taken the results are

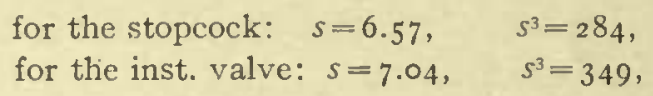

or the ratios of $s^{3}$ are about $8 \mathrm{I}$. Since, however, in view of periodicity, the g-b-p coronas show abnormally small values of $s$, it is better to conpare the red coronas only. In this case the mean values are

$$
\begin{aligned}
& \text { for the stopcock: } s=7.0, \quad s^{3}=343, \\
& \text { for the inst. valve: } s=7.2, \quad s^{3}=373,
\end{aligned}
$$

or the ratios of $s^{3}$ are .92 . Hence the two valves differ as to their data by less than Io per cent. If this value be ascribed by the value of $m$ assumed it would not suffice for the values obtained by photography.

In table 18 the ratios of $s^{3}$ for the large crimson coronas under like occurrence were on the average .42 , widely different from the present, showing that the discrepancy in table 18 enters with the very small nuclei of the very large coronas.

One may note that the nucleation of the large room is gradually increasing, due to a single small gas burner (source of light), although the air was all pumped into the condensation chamber from the floor of the room.

40. Conclusion.-As none of the explanations given are satisfactory, it seems well to restate the case in conclusion with additional remarks on a possible explanation. No matter which group of sizes of nuclei are efficient in producing coronas, the effect of successive identical partial exhaustions must be to reduce the numbers by the same relative amount. If a given corona is obtained for the slower and the faster exhaustion for the same pressure difference, etc., it may be assumed for argument that the same number of efficient nuclei must occur in both cases, though they may not be the same nuclei as to size. It follows that the same corona should occur in the two cases in each of the successive exhaustions. This is only true below the middle $\mathrm{g}$-b-p corona, $i . e$., for relatively fewer nuclei (nucleation below about 100,000 per cub. $\mathrm{cm}$.). In this region of distribution, both valves eventually remove all nuclei, and they remove them at the same rate.

For nucleations above 100,000, however, proportionately more nuclei are apparently removed by the slower exhaustion, cet. par., than by the faster exhaustion, so far as coronal evidence is in question, precisely as if the faster exhaustion were itself productive of nuclei (as, for instance, by breaking up coarser into finer aggregates). One may note, moreover, that in this stage of 
the work, the phosphorus nuclei are at first ionized. Subsidence is without effect. ${ }^{1}$

It is just here that another important question is suggested which may perhaps offer evidence in explanation. It was shown above $(\$ 8$, table 2$)$ that the moist air after exhaustion very nearly regains its original temperature after the lapse of even half a minute, while the coronas persist throughout this interval and much longer without appreciable change of character. It is difficult to understand why the fog particles do not evaporate proportionately to the rapid rise of temperature, unless there is rapid evaporation and diffusion from the relatively warm inner surface of the walls of the condensation chamber, immediately after exhaustion. In any case, the method above used for computing $m$, the moisture precipitated per cub. cm., will give a result too large, for it takes no account of the rapid increase of temperature in question after the fog particles are produced. The swifter the exhaustion the larger this discrepancy (which is probably indeterminable) is liable to be. Thus in the above case for the pressure difference $\delta p=I 7 \mathrm{~cm}$, at $20^{\circ}$, the cooling ideally as far as $-9.6^{\circ}$, rises to $8.8^{\circ}$, in consequence of condensation of fog particles, but within $\frac{1}{2}$ minute the temperature is nearly $20^{\circ}$ again. Hence the precipitated $4.6 \times 10^{-6} \mathrm{~g} / \mathrm{cm}^{3}$ at $8.8^{\circ}$ is to remain undisturbed while the moisture content of saturated air rises from about $8.7 \times 10^{-6}$ at $8.8^{\circ}$ to about $17.2 \times 10^{-6}\left(\mathrm{~g} / \mathrm{cm}^{3}\right)$ at about $20^{\circ}$, leaving an actual deficit of about $4 \times 10^{-6} \mathrm{~g} / \mathrm{cm}^{3}$. This would be out of the question unless moisture evaporated immediately from the damp and warmer walls and the pool of water in the bottom of the apparatus to supply the deficiency. But these conditions are vague, for this moisture may actually be precipitated on the colder fog particles. To some extent, therefore, a degree of uncertainty is left in the determination of $m$, the moisture precipitated per cub. $\mathrm{cm}$, inasmuch as the actual temperature at which the fog particles persist, and to which they have accommodated themselves, is left in doubt.

It is well to observe, however, that as there will presumably be more evaporation from the fog particles while the normal air temperature is being regained in case of very rapid than for the slow exhaustion because lower temperatures are in general associated with the former case, the discrepancies of plates $\mathrm{I}_{2}$ and $\mathrm{I} 3$ above the g-b-p corona may possibly be explained in this way. Compatibly with observation the effect would be less marked as the fog particles are larger. Finally, very large coronas are always more fleeting in character.

Experimentally and with a bearing on Chapter IX the question is easy of decision. So long as the pressure difference corresponding to the lower limit of spontaneous condensation of moisture from dust-free air is not approached (it will usually be reached at about $\delta p>20 \mathrm{~cm}$. for the above types of appa-

1 Recent experiments have shown that the very small nuclei associated with larger nuclei evaporate their loads of water after condensation in such a way as to form water nuclei. As the latter must be larger than the original nuclei now held in solution, a reason for the excess of nucleation detected by the instantaneous valve is suggested. 
ratus), and so long as the g-b-p corona is not exceeded (which again is the actual case), the degree of rapidity of condensation on the usually occurring atmospheric nuclei is a matter of little consequence. Thus the same coronas appear with non-filtered atmospheric air, cet. par., both for the $\frac{1}{2}$-inch plug valve and for the $\mathrm{I}$-inch instantaneous valve, even for large variation in the size of the condensation chamber. 


\section{CHAPTER VII.}

\section{MICROMETRIC MEASUREMENT OF FOG PARTICLES.}

\section{Earlier Methods.}

I. Before using the data computed for the dimensions of fog particles in the reductions of my observations of atmospheric nucleation, it seemed expedient to endeavor to obtain corroborative values by some straightforward method. Aitken's dust counter had naturally suggested itself early in the course of my work; but the results so obtained are essentially indirect, as the fog particles are not themselves observed. It was necessary, therefore, to devise apparatus by which the identical fog particles of a given corona could be directly entrapped and held for examination. This was eventually accomplished in a way admitting, apparently, both of the measurement of the diameters of the particles and of counting the number precipitated under known conditions. Moreover, the particles caught, however fine (even less than $.0003 \mathrm{~cm}$. in diameter), can often be kept in place for observation for some time, so that microscopic photography would appear to be at once applicable not only for the purpose of obtaining size but number.

Many investigations are thus suggested, as, for instance, a repetition. of Thomson's method for determining the charge of an ion. Again, while the corona gives merely the average size of the cloud particles, the microscope is particularly available for indicating variations of diameter for the particles of the same corona. In fact, the water particles of the coronas as caught on the plate are not of a size; they are graded, and hence the nuclei are probably also graded in size.

2. Apparatus.-Aitken's beautiful and highly ingenious instrument is well adapted for the purposes for which it was designed. Apart from this, it will furnish only an estimate of the dust contents sought. The droplets evaporate too rapidiy, and are often too numerous for exact counting. The need of mixing atmospheric air with dust-free air with shaking and stirring is an interference with the nucleation. In fact, water nuclei may even be generated in this way, possibly by the friction of air passing across damp surfaces. There is the tendency of the plate after long use to fog permanently or to collect droplets on its own account. Finally, it would be very difficult to remove the contents of the coronal chamber to the dust-counter without reducing the nucleation during the transfer. 
I therefore endeavored to ascertain whether the particles might not be made visible directly. The chances of success seemed small indeed, particularly as Assmann had failed to see the particles with magnifications of even 400 diameters. But after long trial, the result was eventually accomplished in a way that now seems surprisingly simple.

The compound microscope, $M$, magnifying about roo diameters, is provided with a filar ocular micrometer, $n$. The objective and the whole lower part of the microscope is submerged in the condensation chamber, being suspended for this purpose from the wide rubber cork, $C$. All lenses below $C$ are hermetically sealed with wax. The microscope originally carried a rigid stem, $r$, to which were attached the plate, $s$, to be examined, the mirror, $m$, and the metallic disc or shield, $p$. Afterwards the more flexible adjustment shown in the figure and described below was adopted. The lower side of $p$, which is flush with the objective, and the upper side of $s$ are covered with wet blotting-paper, the latter being perforated to admit light into the microscope through the thin cover-glass placed at $s$ and held sharply in focus by a suitable clip. The field within which drops are to be counted is bounded at pleasure by the wires of the micrometer.

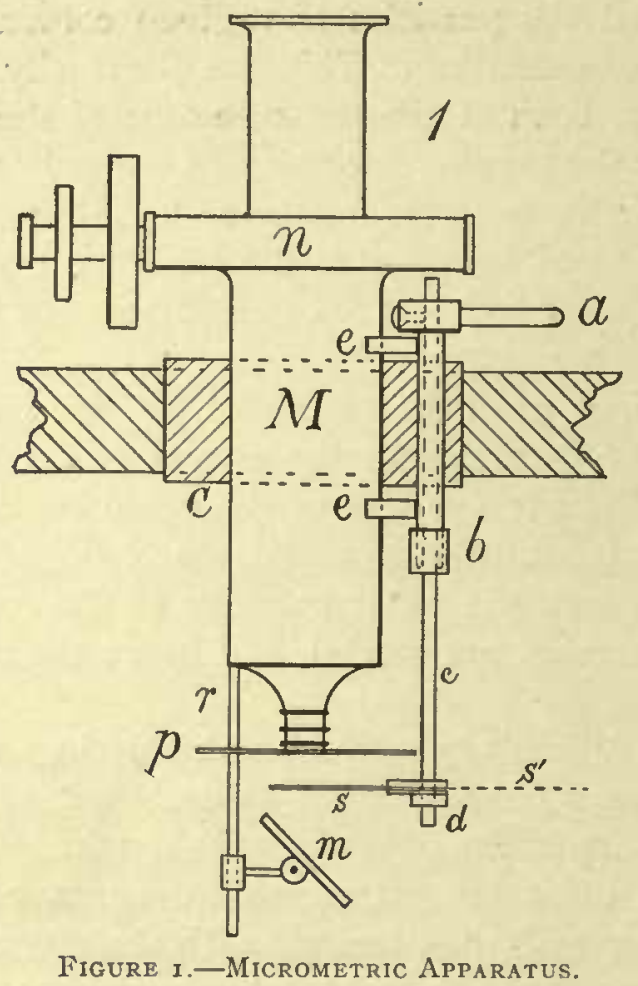

This apparatus was totally unsuccessful. Drops were but rarely seen to fall on exhaustion, while the dew soon gathered on the plate, $s$, in such a way as to be easily mistaken for droplets; for the dew evaporates like the latter when the microscope is removed, and the regularity of the pattern on the plate is the only distinguishing feature. 
Various modifications of this apparatus were then used, among them capsule forms, figures 2 and 3 , similar to Aitken's, but containing a very thin plate of glass or mica or celluloid slightly raised above the base on pellets of wax. It was supposed that this would counteract the tendency of the drops to vanish by evaporation from the warmer glass surface. Capillary metallic tubes led to the curl aneroid, $a$, the filter, $f$, and to the cock for influx of air, the only large

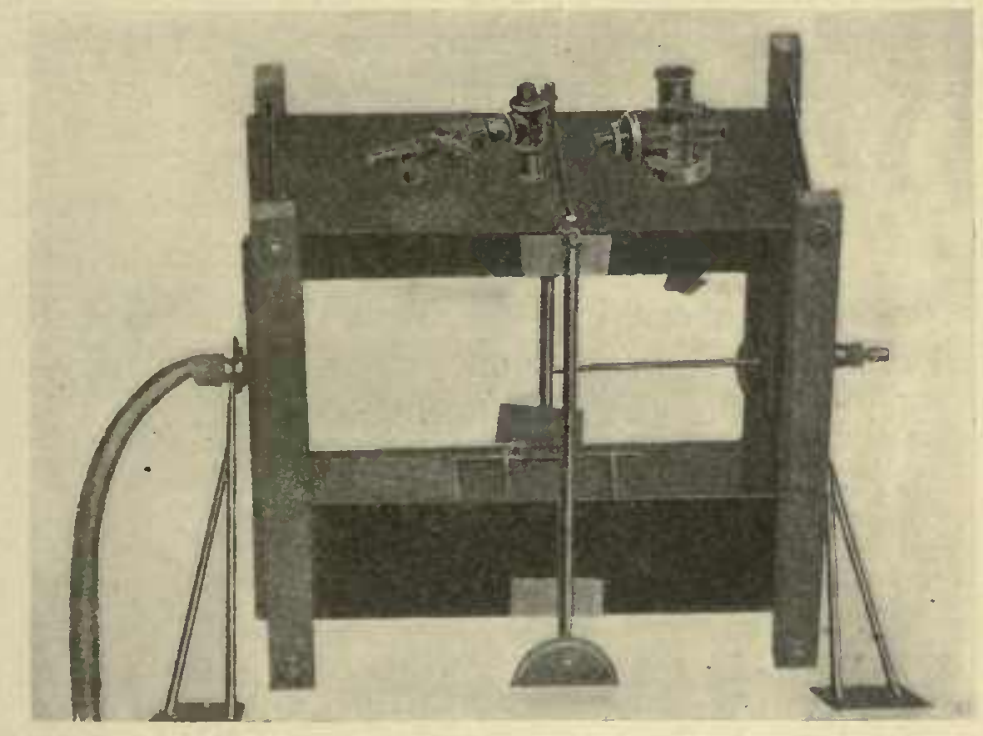

Figure ia. -The Same Mounted in Condensation Chamber.

tube being the exhaust pipe, $e$. Condensation again occurred as a microscopically granular deposit spontaneously on the raised surface, under all circumstances, and the experiments were failures. After oiling the filmy mica surface, $p$, however, droplets were often seen to fall and either to stick fast or to float. These could at times be counted (2000-5000 per cub. $\mathrm{cm}$.); but the rapid evan-

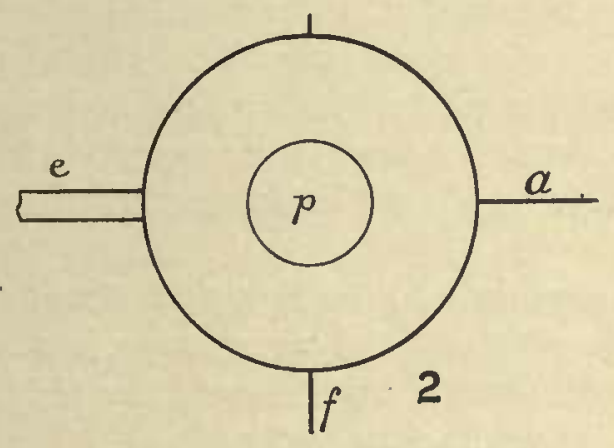

Figure 2.-Capsule. Plan.

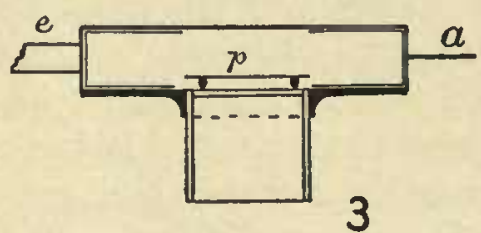

Figure 3.-Sane. Sectional Elevation.

escence of precipitated droplets and the failure of all attempts to reach systematic results induced me to abandon the capsule.

I therefore returned to the apparatus in figure $I$, using at $s$ a plate of thin microscopic glass covered with a thin film of oil and exposed in the capacious 
vacuum chamber. The experiments were now phenomenally successful. Thus for the aperture $s=5$ the mean results were $n=150000$, and for $s=4.6(\mathrm{w} \mathrm{g} \mathrm{b} \mathrm{p})$, $n=140000$. The precipitation of globules was clearly seen, and they persisted even after the exhaustion was removed. The numbers being excessive and referable to globules swept in by lateral air currents, an improvement was now added by increasing the diameter of the disc $p$ to about $5 \mathrm{~cm}$. The improved apparatus gave no results whatever, and the mere addition of the wider disc wiped out all precipitation. But this capricious behavior is characteristic, for next day drops were seen to fall as follows:

$$
\begin{array}{ccc}
s=4.5 & \text { w g b p } & n=6.5 \times 10^{4} . \\
4.6 & \text { W g b p } & 4.7 \\
6.3 & \text { w g b p } & 13.3
\end{array}
$$

after which no precipitation could be caught in the 6 subsequent exhaustions by the identical method. The same unaccountable irregularity was noted in the afternoon. Next day the first experiments showed

$$
\begin{array}{clc}
s=6.0 & \text { w c g } & n=7.3 \times 10^{4} . \\
6.4 & \text { wg p p } & \text { I2.0 } \\
\text { etc. } & \multicolumn{2}{c}{\text {. }}
\end{array}
$$

after which further precipitation did not occur.

The apparatus was then again modified to the final form shown in figure $\mathrm{r}$, by inserting a thin brass tube laterally through the stopper, $C$, and firmly soldering this tube above and below at $e$ to the body of the microscope. A rod snugly fitting this tube thus provided an eccentric focussing device, abcd, with a stuffing box at $b$, and an external handle at $a$. The latter is adjustable by aid of a set screw so that the plate $s$ may be kept in focus during rotation of the rod. To catch the droplets, the plate $s$ is rotated into the position $s^{\prime}$ quite beyond the shield, for a definite time ( $15-30$ seconds), and then returned to $s$ for examination. In this way the definite results were obtained, in a manner to be further detailed below, with the apparatus free from capricious behavior. It is of particular interest that the particles caught on the oiled surface persist as brilliant round globules for a long time (sometimes ro min. or more) in a saturated atmosphere. They very gradually vanish as a rule on the readmission of air into the condensation chamber.

To remove the globules for the next experiment, the influx of air is thus not always sufficient. It is necessary to withdraw the microscope from the condensation chamber bodily and to wave it about a few times in dry air. On returning it to the chamber the plate is then again elear and white.

At first the plate was oiled by a small flat piece of blotting-paper saturated with oil and held on a stem, care being taken to remove all excess. Clean machine oil or ordinary illuminating oil or a mixture of the two subserved the purpose about equally well. Probably the best method of oiling consists in dipping the plate rotated outward to $s^{\prime}$ in very hot melted vaseline (to drive 
away moisture), rerroving the excess while hot by filter paper and when cold submerging the plate in petroleum for transparency. With solution of vaseline in benzine, etc., I have been much less successful. Damar varnish and turpentine was much used in the final work. When drops are to be counted by the method given below, the oil film n:ust be practically solid; otherwise the capillary forces produce an immediate and often startling redistribution of the precipitated granules, though they but seldom coalesce.

3. Bchavior of the precipitated droplets.-In case of a petroleum film on the plate, the water droplets were sometimes seen to fall and float on the film, which is positive evidence against spurious droplets. They are usually black and circular in outline, but when the light is intense and axial, they are bright. Fixed globules are apt to be larger and more irregular. Particles may sometimes be seen to coalesce on collision, but this is rare.

On tipping the microscope so that the light does not penetrate the vividly colored drops axially, they seem to cast shadows in opposed directions for symmetrical inclinations on both sides; but in view of the aplanatic properties of spheres, the phenomenon is probably a case of refraction, with the shadow beginning at the edge of a caustic. Similarly, on moving the lamp horizontally to either side from the position corresponding to axial illumination, the globules become opaque, and look like round shining steel beads. The diameter of the beads has but little effect. If the lamp is moved until the field is dark, the plate looks like the starry heavens. These stars seem to be above the drops.

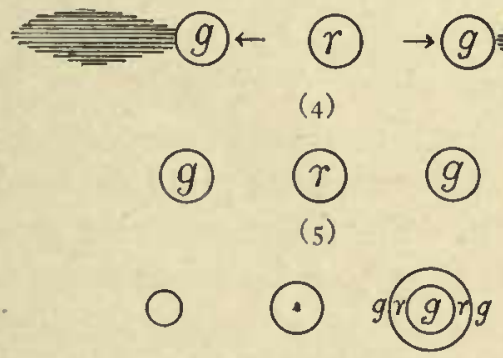

(6)

Figures 4, 5, 6. Diagrams Showing the Behavior of Fog Particles.

After remaining in the plate for some minutes the fixed droplets often become rosette-shaped (apparently), at first showing a mere black spot in the center of the color disc, which gradually enlarges to a ring-shaped appearance slowly moving radially outwards. As a rule, the color ${ }^{-1}$ is eventually the same on the inside and the outside of the enlarged ring, the ring itself appearing red with black demarcations in the surrounding green field, as shown in the figure. On influx of air the structure becomes washed. This ring-shape may be merely apparent, but the small globules when at first deposited never show the same color within and without, the former being uniformly red and the latter white.

The colors observed were afterwards found to be due to chromatic aberration of the microscope. 
As the rings are not easily produced with a very viscous oil, it is probable that the droplet has here penetrated to the glass and that the oil film is drawn over it by the capillary forces at the common edge of the three media.

4. Preliminary data.-Before adopting the eccentric focussing device, many experiments were made to ascertain the cause of the uncertainty in catching the drops on the plate when kept in place, sceing that sometimes the precipitation was abundant, while at other times under the same apparent conditions drops did not fall. Failures occurred both for high and for low nucleation. From the outset it was improbable that radiation from the outside could affect the result. It was eventually ascertained that on tipping the condensation chamber after the fog had formed, so that the subsidence would reach the plate obliquely, a precipitate would usually appear. Again, an oblique current within the chamber and passing across the plate usually produced a deposit. Hence the drops actually exist within the fog, and success in bringing them down upon the plate is probably conditioned by very close isothermal adjustment of the plate to its surroundings, added to the advantages gained from incidental and favorable air currents. Hence a little time must always elapse before the drops persist at the plate, and hence the droplets from a shallow capsule do not appear. Using a film of mica as a plate the result was the same, and it is useless to attempt to enumerate the drops by this method. Those which fall are carried in by grazing air currents, while no drops are obtained from the fiducial space under the objective.

Nevertheless, the measurement of the dia'ceters, $d$, of the drops obtained by the above method without modification is an excellent test of the results obtained elsewhere by computation. The factor of the ocular micrometer described above was $.002 \mathrm{~cm}$. per turn of the serew, or $.00004 \mathrm{~cm}$. per scale part of the drum divided into 50. The extent of plate covered by the breadth of the spider lines was about $.0003 \mathrm{~cm}$. The finest particles are of about this diameter, so that such measurements must at best be much inferior to photography with a scale attachment. The results are given in the following table, in which only those results among many are inserted for which the observations were clear and satisfactory. The coronal color with its diameter, $s$ (chord of a radius of $30 \mathrm{~cm}$. ), are as observed when the eye and the source of light (Welsbach mantle seen through a small circular hole) were at distances $85 \mathrm{~cm}$. and $250 \mathrm{~cm}$, respectively, from the center of the condensation chamber. The exhaustion was usually to a pressure difference of $I_{7} \mathrm{~cm}$., but this is of no significance when diameters are alone to be observed. The particles were collected by tipping the chamber, sometimes in large numbers, but at other times sparsely distributed without apparent cause. Nuclei were conveniently obtained from burning charcoal. Both floating and fixed globules were examined with strong microscopic illumination. It was difficult to retain a clear image without frequently removing the plate, as the adjustment for focussing the plate within the chamber had not yet been adopted. A table showing the results from coronal measurements under the same circumstances is added. 
TABLE I.-DIAMETERS OF CLOUD PARTICLES. PRELIMINARY RESUlTS, FIXED PLATE.

\begin{tabular}{|c|c|c|c|c|}
\hline$s$ & Corona. & Computed $d$. & Measured $d$. & Remarks. \\
\hline $\begin{array}{l}\mathrm{cm} \\
6.5 \\
6.5 \\
- \\
6.4 \\
9.1 \\
3.6 \\
3.8 \\
5.8 \\
4.8 \\
2.4\end{array}$ & $\begin{array}{l}\text { w b p } \\
\text { w|b|p } \\
\text { olive } \\
\text { olive } \\
g^{\prime}|\mathrm{b}| \mathrm{p} \\
\text { w r bg } \\
\text { corona } \\
\text { " } \\
\text { w c g' } \\
\text { corona }\end{array}$ & $\begin{array}{r}\mathrm{cm} . \\
.00046 \\
46 \\
32 \\
32 \\
46 \\
37 \\
82 \\
76 \\
50 \\
60 \\
120\end{array}$ & $\begin{array}{c}\mathrm{cm} \\
.00060 \\
084 \\
050 \\
040 \\
084 \\
052 \\
096 \\
116 \\
088 \\
088 \\
222\end{array}$ & $\begin{array}{c}\text { Fixed } \\
\text { "“ } \\
\text { Floating } \\
\text { Fixed } \\
\text { ". } \\
\text { Floating. } \\
\text { Fixed. } \\
\text { ". } \\
\text { ". } \\
\text { ". }\end{array}$ \\
\hline
\end{tabular}

TABLE 2.-DIAMETERS OF CLOUD PARTICLES. PLATE ADJUSTABLE.

\begin{tabular}{|c|c|c|c|c|}
\hline & $s$ & Corona. & Computed $d$. & Measured $d$ \\
\hline $\begin{array}{c}\text { Phosphorus } \\
\text { nuclei. }\end{array}$ & $\begin{array}{r}\mathrm{cm} . \\
10.0 \\
8.2 \\
7.0 \\
5.6 \\
3.3\end{array}$ & $\begin{array}{l}\text { olive } \\
\text { w o bg } \\
\text { w c g } \\
\text { w p b g } \\
\text { w rg } \\
\text { corona }\end{array}$ & $\begin{array}{r}\mathrm{cm} \\
.00030 \\
35 \\
39 \\
44 \\
52 \\
90\end{array}$ & $\begin{array}{r}\mathrm{cm} \\
.00042 \\
04 \mathrm{I} \\
060 \\
050 \\
060 \\
\text { I I } 7\end{array}$ \\
\hline
\end{tabular}

Floating globules were often observed in the act of coalescing; but this is much rarer than the passage of a floating droplet over a fixed one without interference. A distinct central bright area shading off into darkness was seen even in the floating globules when axially illuminated by intense light. The larger drops were often metallically green. The colors vanish after long standing, and they are particularly vivid immediately after falling. It was not even now possible, in spite of all precautions in tipping the vessel, to obtain an abundant crop of drops at pleasure.

The results are given graphically on the chart, figure 7 , in comparison with the computed data of my earlier experiments (upper curve) as well as with my later experiments (lower curve). The observed results lie below the former where adiabatic conditions were assumed, and above the more recent experiments where the effect of the successive expansions was computed isothermally In other words, the observed diameters are intermediate, but nearer the older results. I will pass over these preliminary data, as they are probably too high because of the difficulty in focussing among others, to resume the subject in connection with the results obtained under much more favorable conditions below. 


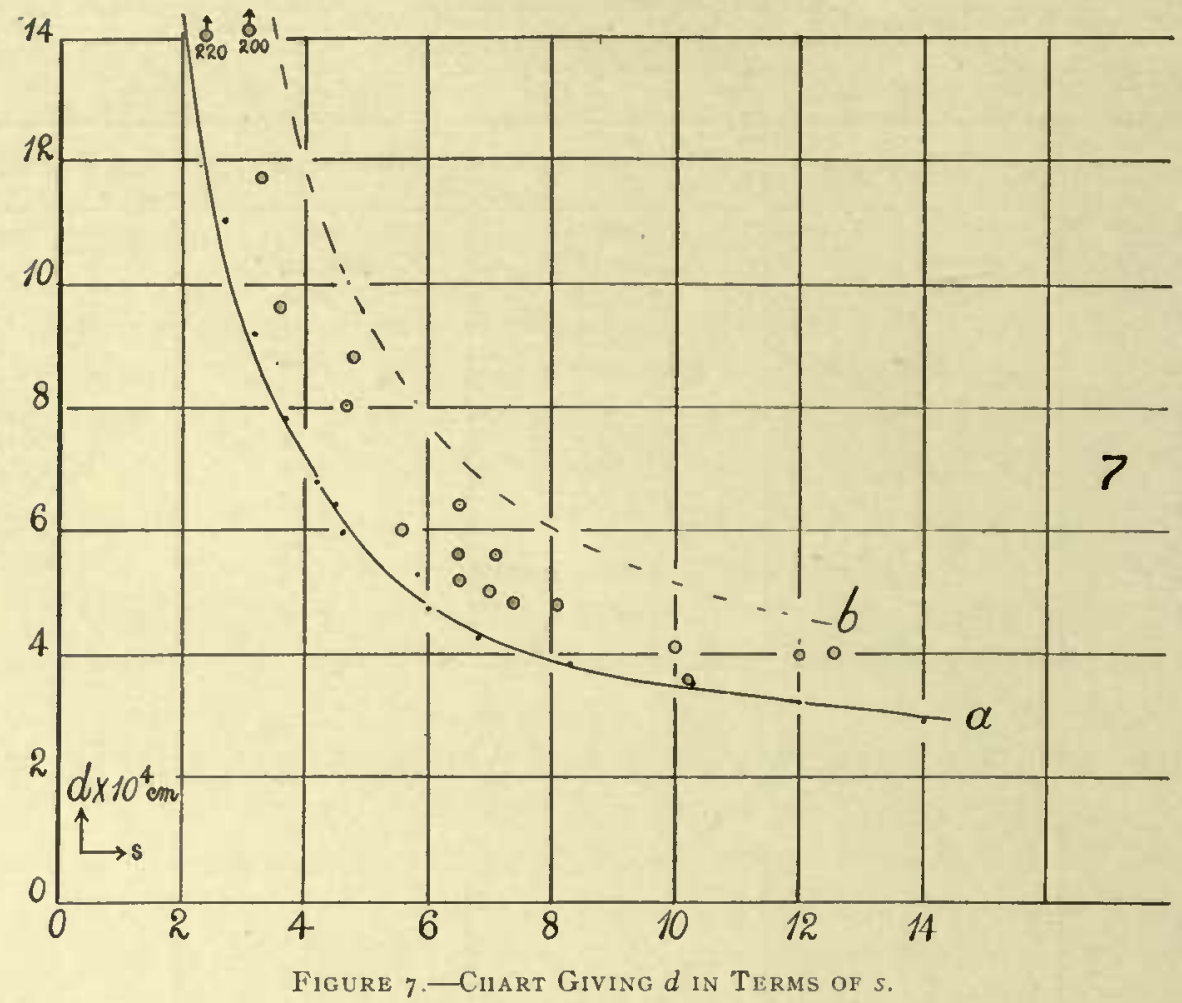

\section{Improved Method.}

5. Number of droplets. - The following results were obtained with the definite form of apparatus shown in figure I. A method of estimating the nucleation from a direct count made under the microscope is obtained as follows: Let the plate be so rotated eccentrically as to catch the descending fog particles for a definite interval of time, $t$. If $v$ be their velocity of subsidence, all particles within a height, $h$, will be caught, if

and

$$
\begin{aligned}
& h=v t \\
& v=10^{6} d^{2} / 3.24
\end{aligned}
$$

where the usual value of the viscosity of air has been inserted. Furthermore, $m$ grams are precipitated per cub. $\mathrm{cm}$. by the given exhaustion, and if $n$ be the nucleation

$$
n=6 m / \pi d^{3} \text {. }
$$

Finally, if $c$ is the area of the field seen in the microscope and $n^{\prime}$ the number of particles falling into this field

$$
n^{\prime}=\text { nhc. }
$$

From these equations $n$ is obtained by eliminating $v$ as

$$
\sqrt[3]{n}=\frac{2.11 \times n^{\prime}}{1 \mathrm{~cm}^{2 / 3} 106}
$$

The values of the constants usually adopted were $t=30 \mathrm{sec}, c=144 \times 10^{-6} \mathrm{sq}$. cm., $m^{2 / 3}=2.8 \times 10^{-4}$, whence $\sqrt[3]{n}=1.75 \times n^{\prime}$. The experiments to test this 
method often gave serviceable results, some of which are inserted in the following tables; but at times the $n$-values are out of proportion. The reason of this is threefold: In the first place $n$ is found from $n^{\mathrm{r} / 3}$ with the usual difficulty. Again, in a simple arrangement like the above, air currents cannot be quite excluded. They may arise incidentally in the apparatus or the motion of the plate cven if parallel to itself may stir the air unless some form of guard ring attachment is added. Particles are thus swept down upon the disc before and after the exposure, as was actually observed. The difficulty may be removed by adding a capsule above the plate or simply by decreasing the distance between the shield and the plate to a millimeter or less. Finally, if the oil film is semi-fluid and not quite fixed, if there is slight creeping, as was usually the case, the particles are redistributed after falling along stream lines where they cohere in strings and bunches, but without coaleseing. This was also observed, and in fact the capillary forces involved are apt to be strong enough to counteract viscosity.

I have not thus far spent much time in correcting these defects, chiefly because the new results for the diameters of fog particles are more immediately interesting. The data are given in table 3 .

TABLE 3.-OBSERVED DIAMETERS AND NUMBERS (per cub. cm.) OF CLOUD PARTICLES. $m=4.7 \times 10^{-6} \mathrm{~g}$ per cub.cm.; If $c=144 \times 10^{-6} \mathrm{sq} . \mathrm{cm}$., and $t=30 \mathrm{sec}$; $v^{3} \bar{n}$ $=\mathrm{r} .75 n^{\prime}$. Gencrally $i^{3 / n}=2.1 \mathrm{I} n^{\prime} / t c m^{2 / 3} \mathrm{IO}^{6}$. Micrometer factor, .00004 $\mathrm{cm}$.

\begin{tabular}{|c|c|c|c|c|c|c|c|c|c|}
\hline & $s$ & Corona. & $c \times 10^{6}$ & $t$ & $n^{\prime}$ & $\begin{array}{c}\text { Observed } \\
n\end{array}$ & $\underset{n}{\text { Computed }}$ & $\begin{array}{l}\text { Observed } \\
d\end{array}$ & ${ }_{d}^{C o m p u t e d}$ \\
\hline & $\frac{\mathrm{cm}}{6.0}$ & $\begin{array}{l}\text { olive } \\
\text { w r g }\end{array}$ & $\begin{array}{l}\text { cm. }^{2} \\
140 \\
140\end{array}$ & $\begin{array}{l}\text { sec. } \\
30 \\
30\end{array}$ & $\begin{array}{l}37 \\
27\end{array}$ & $\begin{array}{l}271000 \\
105000\end{array}$ & $\begin{array}{r}250000 \\
90000\end{array}$ & $\frac{\mathrm{cm} .}{-}$ & $\frac{\mathrm{cm} .}{-}$ \\
\hline $\begin{array}{l}\text { Phospliorus } \\
\text { nuclei. }\end{array}$ & $\begin{array}{r}10.2 \\
7.4 \\
7.1 \\
4.7\end{array}$ & $\begin{array}{l}\text { w o bg' } \\
\text { w p b g } \\
w^{\prime} \dot{b} \text { p } \\
\text { corona }\end{array}$ & $\begin{array}{l}70 \\
70 \\
\end{array}$ & $\begin{array}{l}30 \\
\text { I } 5 \\
-\end{array}$ & $\begin{array}{r}17 \\
6 \\
- \\
\end{array}$ & $\begin{array}{c}210000 \\
- \\
-\end{array}$ & $\begin{array}{c}210000 \\
- \\
-\end{array}$ & $\begin{array}{r}.00036 \\
48 \\
56 \\
80\end{array}$ & $\begin{array}{r}.00036 \\
4 I \\
.42 \\
6 I\end{array}$ \\
\hline
\end{tabular}

Particles as small as $.0003 \mathrm{~cm}$. present throughout.

\begin{tabular}{|c|c|c|c|c|c|c|c|c|}
\hline $\begin{array}{l}\text { Phosphorus } \\
\text { nuclci. }\end{array}$ & $\begin{array}{l}6.5 \\
6.5 \\
6.1\end{array}$ & $\begin{array}{l}\text { g b p } \\
g^{\prime} \text { b p } \\
\text { w r } / g\end{array}$ & ${ }^{\text {I } 40}{ }^{-}$ & $\frac{10}{-}$ & $\frac{43000}{-}$ & $\begin{array}{c}100000 \\
-\end{array}$ & $\begin{array}{r}.00064 \\
5^{6} \\
72\end{array}$ & $\begin{array}{r}.00046 \\
46 \\
48\end{array}$ \\
\hline
\end{tabular}

Particles as small as $.0003 \mathrm{~cm}$. always present.

\begin{tabular}{|c|c|c|c|c|c|c|c|c|c|}
\hline Air nuclci. & $\begin{array}{l}4.5 \\
6.5 \\
8.1 \\
8.2\end{array}$ & $\begin{array}{l}\text { cor } \\
g^{\prime} \text { b p } \\
\text { w c bg' } \\
\text { w c bg' }\end{array}$ & $\begin{array}{l}140 \\
70 \\
70\end{array}$ & $\begin{array}{l}\text { I } 5 \\
\text { I } 5 \\
\text { I } 5\end{array}$ & $\begin{array}{l}9 \\
\text { I } 4 \\
16\end{array}$ & $\begin{array}{l}30000 \\
\text { I } 20000 \\
180000\end{array}$ & $\begin{array}{c}40000 \\
\text { I } 50000 \\
\text { I } 50000\end{array}$ & $\begin{array}{r}.00064 \\
52 \\
4^{8} \\
-\end{array}$ & $\begin{array}{r}.00064 \\
46 \\
39 \\
39\end{array}$ \\
\hline
\end{tabular}

Particles graded as usual. 
6. Diamelers of fog particles. - If the diameters measured are plotted in a chart together with the results computed from successive exhaustions in the older and in the more recent experiments, the present values again lie between the two eurves, but now much nearer the lower (recent) curve than before. I shall not pause to interpret the differences which remain, but only to remark that the capillary forces at the area of contact of the droplet even with the liquid oil film may transform it to an oblate spheroid, and that diffraction at the circular edges of the drops is not excluded. If the nucleation, $\boldsymbol{n}_{\mathrm{o}}$, obtained from successive isothermal exhaustions and subsidence measurement, be accepted as correct (lower curve), the ratios of the nucleation found from the different methods tested will then be

\begin{tabular}{|c|c|c|}
\hline From subsidence, $a=.0029$; & $d / d_{0}=1.0$ & $n / n_{0}=\mathrm{I} . \mathrm{O}$ \\
\hline From lycopodium $(d=.003 \mathrm{~cm}),. a=.0034$; & $"=\mathbf{I} .2$ & $"=.6 \mathrm{I}$ \\
\hline From diffraction (blue), $a=.0034$; & $=\mathrm{x} .2$ & $"=.6 \mathrm{I}$ \\
\hline From micrometer measurement, $a=.003$ & $=\mathrm{I} \cdot 3$ & $"=.48$ \\
\hline Old results (adiabatic conditions assumed), & $=1.6$ & $"=.24$ \\
\hline
\end{tabular}

Since $n$ is obtained from the cube of $d$, large differences of this kind are as yet inevitable, particularly as the particles measured in these different cases are not the same.

7. Sizes of particles graded.-The point of particular interest which comes out on using the eccentric plate to catch the subsidence during 15 or 30 seconds, and at once examining the deposit, is the result that particles of all sizes are present. By far the greater number, however, have the maximum diameter. These particles are caught from the fog without interference, and it is not probable that coalescence or evaporation have been appreciably operative, so long as the corona remains the same throughout the micrometer measurement. The probable explanation is this: while the pressure decrement is growing from zero to the maximum $\delta p$, condensation is taking place on the greater number of particles throughout the whole of this interval. In other words, although the nuelei are graded in size, the greater number exceed a certain dimension and require altrost no pressure decrement to induce condensation. These are the particles (diameter exceeding a certain inferior limit) which give character to the persistent corona. A minority of the graded particles are below the dimension in question, and upon these condensation does not take place until the higher values of the pressure difference are reached; sorre may even require the full decrement, $\delta p$. Thus it is that in the deposit of fog particles, one finds those of diameter .oor cm. intermixed with others of smaller diameter, even as far as $.0002 \mathrm{~cm}$. or less, all shining like beads. When fresh phosphorus nuclei are first introduced into the condensation chamber the result is a gray fog, but a relatively small white reddish corona is nevertheless discernible. Accordingly, the crop of droplets seen under the microseope contains not only surprisingly s'nall but also relatively large droplets, with all intermediate diameters. Hence the indefinite fog and the small corona. The large olive ( $\mathrm{g} b \mathrm{p}$ ) corona and 
other of the early coronas are very apt to fade into a coarse white reddish corona. This is the evaporation of the smaller particles into the larger, which accounts, moreover, for the loss of nuclei during the first precipitation, to be caught in subsequent exhaustions. The successive coronas in a series gradually become sharper and the larger particles more uniform, but extremely fine particles are still present even when one approaches the normal coronas. The fine particles, however, belong to coronas so large and diffuse that their coronal effect scarcely modifies the strong coronas of the large particles even before the former vanish by evaporation.

When I first observed these different sizes of drops caught on a single plate, it seemed not irp probable that a difference of the condensational effect of the negative and the positive ions might here be actually in evidence; but as all intermediate sizes are present at the outset, and particularly as large and small droplets still appear together long after all electrification has certainly vanished, this conclusion is not warranted. What continually favors uniformity is subsidence of fog. As the phosphorus nuclei are graded, it is probable that the very fine droplets are due to the initial or primitive nuclei from which the larger nuclei have grown by coalescence; or the fine droplets may be due to air nuclei associated with the phosphorus nuclei. All this will appear in the more minute photographic study of the subject detailed in the next section, and it will be further interesting to decide whether the nuclei generated by the $\mathrm{X}$-rays are not also graded below a certain usually much smaller maximum diameter. That this maximum diareter will increase with the lapse of time allowed for coherence may be inferred.

The coarse and washed type of coronas obtained with nuclei produced by the X-rays is evidence of graded size, while the fog particles, so far as I have yet caught them, are of varied dimensions. In these cases the $\mathrm{X}$-rays reached the inside of the condensation chamber through its waxed wood walls lined with wet cloth. To obtain a fairly strong and large corona an exposure to the rays lasting 5 to ro minutes was needed, as the radiation was not very intense. In this interval the original extremely small nuclei are probably undergoing continuous growth, for instance, by cohering, so that on exhaustion particles of all sizes are revealed. In addition to the ragged coronas there is copious rain. Under these circumstances it seems reasonable that the time loss of nuclei must at the outset be proportional to the square but finally to the first power of the number, assuming that eventually the large nuclei do most of the catching.

\section{MICRO-PHOTOGRAPHY OF FOG PARTICLES.}

8. Preliminary.-In the preceding section ${ }^{1} \mathrm{I}$ described a series of experiments in which the diameters of fog particles were microscopically measured, directly. In the present section these particles are micro-photographed, and the negatives subsequently measured. The results, though interesting as a whole,

$$
\text { ' See also American fourn. (4), xvir, p. I60, } 1904 .
$$


are not as immediately available for quantitative discussion as was hoped. The number of nuclei per cubic centimeter and the diameters of the fog particles are respectively below and above the computed nucleation (Chap. VI), and the globules photographed are rarely of the same size for a given corona.

Very curious results, apparently capillary in character, were obtained, showing permanent pitting effects of the subsidence of fog on a film of viscous oil and persistent motion of globules in liquid oil.

9. Apparatus and method.-The apparatus needed is a modification of that described in $\$ 2$ of this chapter for the micrometry of fog particles, with the addition of a camera above the microscope. The ustual form of camera attached to a substantial eccentric axis so as easily to be rotated into place or removed therefrom for inspection is satisfactory. The revoluble condensation chamber must be clamped in place. The camera may be focussed by the stage focussing screw of the microscope, so that a lens is apparently not necessary in the camera; but it is essential that the magnified fog particles be seen very clearly with the eye at the microscope, before photographing them, and this procedure is therefore not quick enough. It was thus found necessary to add a good lens to the camera adjusted for parallel rays and to adapt the eye for the same infinite focus by concave glasses. In this case, when the particles had been caught and put in place under the objective by the eccentric stage device clescribed above ( $\$ 2)$, the camera could be at once swung into position for photography. The endeavor to adapt a small kodak for the work was not very successful.

Magnification may be secured either at the objective, or at the ocular. Some space between the objective and the plate is desirable for safe manipulation, and therefore a half-inch objective will serve the present purposes better than a quarter-inch lens. The illumination must be axial to avoid astigmatism, but the use of condensers has not as yet been tried, and would probably promote evaporation of the fog particles. In general, reasonably small magnification, much light, and rapid photography are best conducive to success. In this way the time of exposure was gradually reduced from 20 seconds to 2 seconds or less. The positive ocular seen in the plates was used merely in the absence of a suitable negative ocular, inasmuch as the former was provided with a filar ocular micrometer. But a negative ocular containing a plate ruled in square millimeters would be far preferable.

The plate in these experiments was covered with an even coating of Damar varnish, neither too moist nor too dry. A clean microscope cover-plate of glass is dipped in the varnish, and the excess removed by placing it on edge. The film, which must be smooth, clear, and even, will be ready for use in a few hours. If too dry it should be soaked in turpentine, otherwise the particles adhere broadly to the plate and rapidly evaporate. If the plate is too moist, the particles float and cannot be photographed. No precise rules can be given. Naturally, the conditions surrounding the plate should be as nearly as possible isothermal, which implies a capacious air chamber for condensation.

Io. Incidental phenomena. Pitting.-A curious phenomenon sometimes 
accompanies the deposition of the fog particles on the film of viscous varnish, inasmuch as they leave a permanent impression. In other words, the plate becomes more or less permanently pitted after the fog particles are gone, appearing washed or dull to the naked eye, and not regaining the clear state until after the lapse of 12 hours or more. The effect occurs only in the case of fog deposition and is never present on the plate in the absence of a precipitate of fog. Hence it cannot be an air bubble effect.

The cause is probably to be associated with surface tension since the weight of the particle is negligible. If the particles were to break through and reach the bottom of the film of varnish, there is no obvious reason why the pitting should varish like a viscous phenomenon in the course of time.

It is conceivable (Fig. 8) that the surface tension of the varnish is locally lessened by slight admixture, or at lcast the proximity of water, and that an alveolar structure of the surface is the result. In certain slides (No. 22) the presence of these "craters," as they may be called, seems to be clear in the photograph. At other times droplets shrinking in their cavity by evaporation were actually observed. But the phenomenon is rare and observation therefore uncertain.

I I. Dew.-When the plate is dry, the beginnings of the formation of dew on its surface are apparent after long exposure. The dew particles are very fine even as compared with fog particles, the former as observed lying within $.000 \mathrm{I}$ centim. Their number is enormous, aggregating to fully 2 million or more per square centim. of the surface (slide No. 27). They do not further seem to interfere with the deposition of fog particles than by promoting adhesion, but this is naturally objectionable.

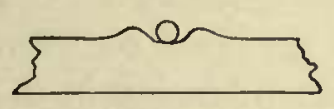

Figure 8.-Diagram of "Crater."

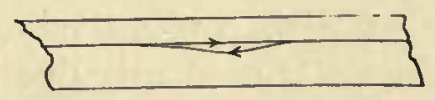

Figure 9.-Diagram Showing Circulation

12. Evaporation.-The droplets, originally sharp in outline, become vague and washed on evaporation, doubtless because their curvature decreases, while the area of adhesion remains the same, to the detriment of the nearly spherical curvature at the beginning. Floating globules are usually much more uniform in size and remain more uniform, because the differences due to adhesion are absent (see $\$ \$ 9, \mathrm{I}_{3}$ ). It has been stated that when there is evaporation the photograph fails and a blank plate results. It may be assumed that a successful photograph implies as little evaporation of fog particle before the taking of the picture as during this interval.

13. Floating and moving globules.-A final very interesting phenomenon is met with in the case of fog particles floating in a liquid film of oil. It frequently happens under these circumstances that there is a sharp line of demarkation in the field of the microscope, probably an edge of contact of the semi-fluid matter on the plate. In all such cases there is apt to be continuous 
motion of the submerged or partially submerged fog particles, to and from the edge on one side. They approach and leave in great armies, at first, gradually dwindling in number as they pass out of the field of view, eventually to be lost by evaporation. Sometires both advancing and retreating fog particles are in sharp focus at once. At other times the advancing set is obviously above or below the other, to the extent of one or more tenths of a millimeter. Cases also occur in which there are two edges or a sort of geographical strait in the field of view, in which case particles are frequently seen moving from edge to edge until they vanish in number, probably from evaporation. Vortical or involved orbits of fog particles also occur.

In explanation of these phenomena it is necessary to bear in mind that the edge of retrogression mentioned is always nearly fixed, under the microscope. Hence it is not probable that that motion of the mixed oil (varnish-turpentine) due to concentration on evaporation can be the cause; for in this case the liquid would visibly gather itself up into a drop showing a shifting edge in the field. The possible motion of a liquid on its surface skin or similar capillary phenomenon is equally hard to reconcile with the stationary edge. The most probable explanation, it seems to me, is given by the annexed diagram, figure 9 . If the film on the plate of glass is microscopically uneven and slightly inclined, the motion of a relatively liquid layer over a fixed layer may enclose a shallow region of liquid, in which eddying is kept up, as shown in the diagram, remembering, of course, that all motion is observed under the microscope. The particles indicate the motion of the skin circulation.

I4. Graded particles.--Suggestion may finally be made as to the cause of the observed gradation of particles, where such gradation appears simultaneously with clear-cut coronas. In case of the $\mathrm{X}$-rays the coronas are vague and washed, accompanied with copious rain. What is seen is a coarse red-rimmed fog. Here gradation is obviously due to the corresponding gradation in the sizes of the nuclei, as explained above. Something similar shows itself in the initial phosphorus or sulphur fogs.

The grading in question cannot be due to coalescence, not only because such coalescence is but very rarely observed, but because the volume increase is as the third power of the diameter. Sizes as I to 3 being very common, this would mean an equally frequent coalescence of 27 droplets, which could not escape detection. Moreover, the coronas in air retain a nearly fixed diameter until they are lost by subsidence.

Some difference of size must be due to a difference of surface adhesion; but if the oil stratum is of the same character throughout, this is not liable to be large without being detected in the picture.

The final cause for gradation, apart from original differences of nuclei, is evaporation. The occurrence of marked evaporation is at times beyond question, and it is then impossible, or nearly so, to obtain a photograph. In general, however, evaporation is obscure, and one may argue, as already suggested, that in the time (about 60 seconds) needed for adjustment and photography, 
the evaporation should be no less than during the anterior i 5 or 30 seconds of subsidence during which the fog particles were caught. This would seem to be especially true where the particles persist for several minutes after photography, as is usually the case. Nevertheless, it is not impossible that the first precipitate prepares the plate (by evaporating partially) for the subsequent precipitation. The subject will be resumed in connection with definite results below.

\section{Results.}

15. Photographic plates. - Unfortunately, it is impossible to reproduce the photographic negatives obtained, except in certain cases; for not only are the fog particles frequently too small, but slides suitable for the measurement of number are indistinct in relation to diameter. To obtain a plate in which the particles actually stand out is naturally a matter of chance, as this will depend on many conditions (light, evaporation, focus, etc.) beyond the observer's control in expeditious work. Curiously enough, the plates obtained for fog particles condensed on the persistent nuclei produced by the X-rays in dust-free air are the best of my series, and are therefore reproduced.

To measure the sizes of the particles on the plates a filar micrometer of low power was used. To count them, the slide was divided off into fields of convenient size, and the number then enumerated under a lens, taking all the fields in clear focus in succession.

I6. Tabulated results.-The following tables show the general results for about 50 photo-micrographs. The kind of nuclei used are given in the first column, the coronas and their angular diameters $(s / 30$, nearly) in the second and third. The mean diameter, $d$, of fog particle measured from the photograph and the limits of $d$ observed, follow. The next two columns show the number of nuclei, $n$, obtained from the photograph by the equation of this chapter, $s 5$, and the observed limits of $n$ for the different fields counted. The last columns are explanatory and usually show the film of oil or varnish used to catch the precipitate and the character of the plate.

17. Remarks on the tables.-The use of rough oil films (tallow, paraffine, wax, etc.) is naturally unsatisfactory, but the particles are easily recognized (No. 3). Plates Nos. 4, 6, 22, though very perfect as photographs (large camera magnification) showed a permanent impression which lasted 6- 12 hours, as already stated. In No. 8 only a few particles were caught. Nos. 9 and ro were taken with a $\frac{1}{4}$-inch objective, and though good in themselves were hard to obtain, and showed the advisability of the weaker objective ( $\frac{1}{2}$-inch), favorable to shorter times of exposure. No. II, and particularly No. 22, gave evidence of the occurrence of "craters" (Fig. 8) in the varnish filn left after the evaporation of the fog globule. As a whole, the photographs on the X-ray nuclei are the most successful, and in No. 23 in particular the particles stand out on the photograph. In No. 3 I particles were deposited on the plate during the vortical motion occurring on influx of air while in No. $3^{2}$ the motion of the particles (film too liquid) shows streaks on the plate. 

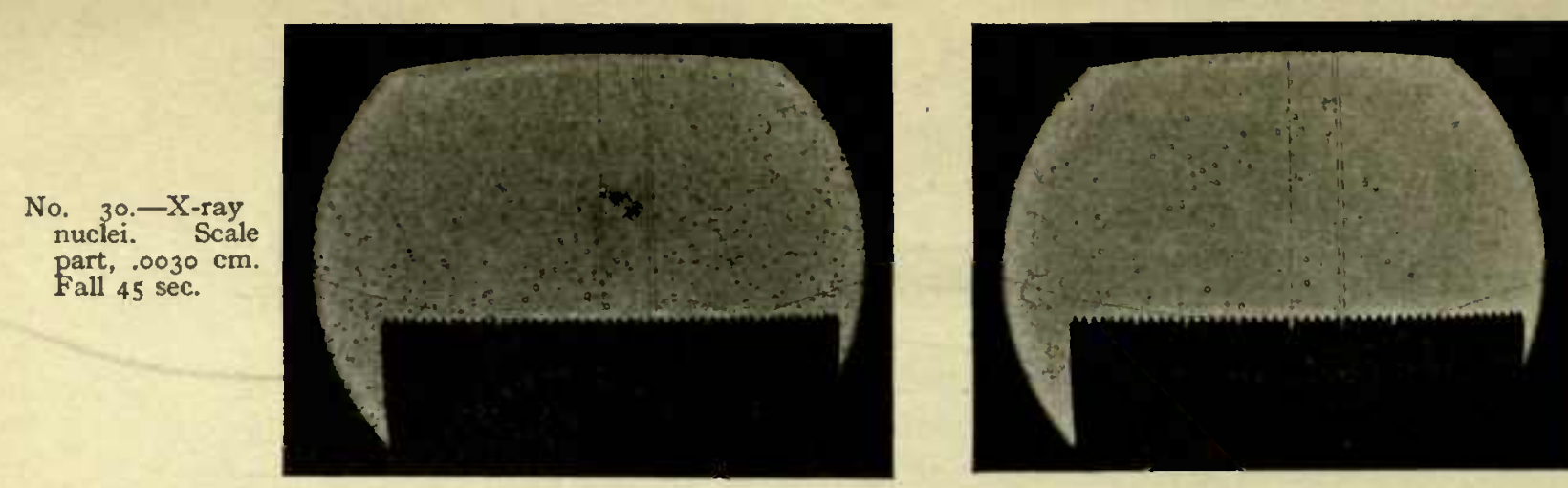

No. $27 .-X-$ ra: nuclei. Scal part, .0030 cm
Fall i 5 sec. Dev in negative.

No. Io.-Air nuclei. Scale part $.0012 \mathrm{~cm}$. Fall $30 \mathrm{sec}$
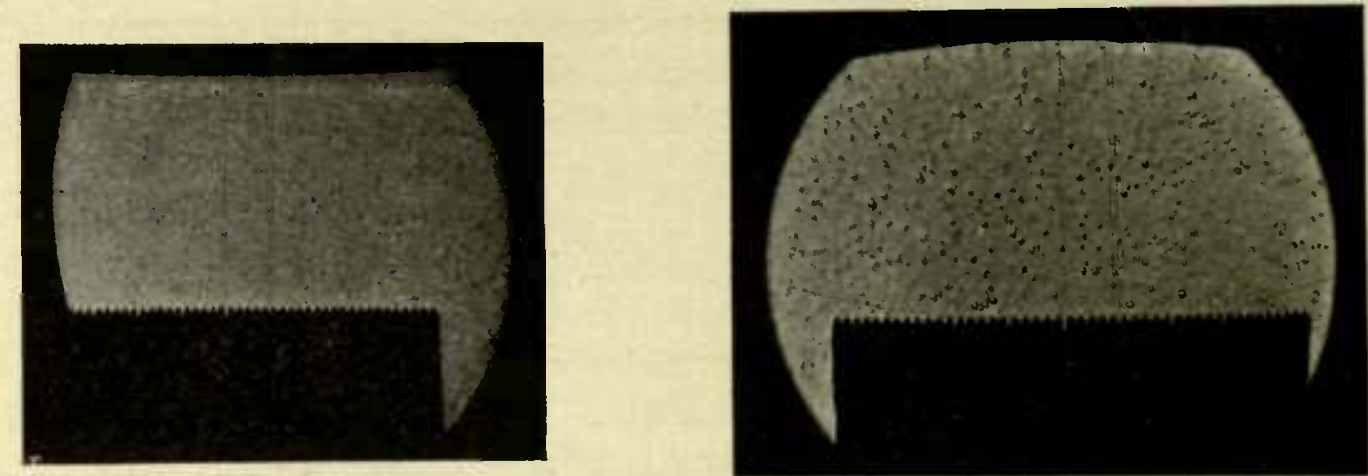

No. 23. - X-ra nuclei. Scale part, .0030 cm Fall 30 sec.
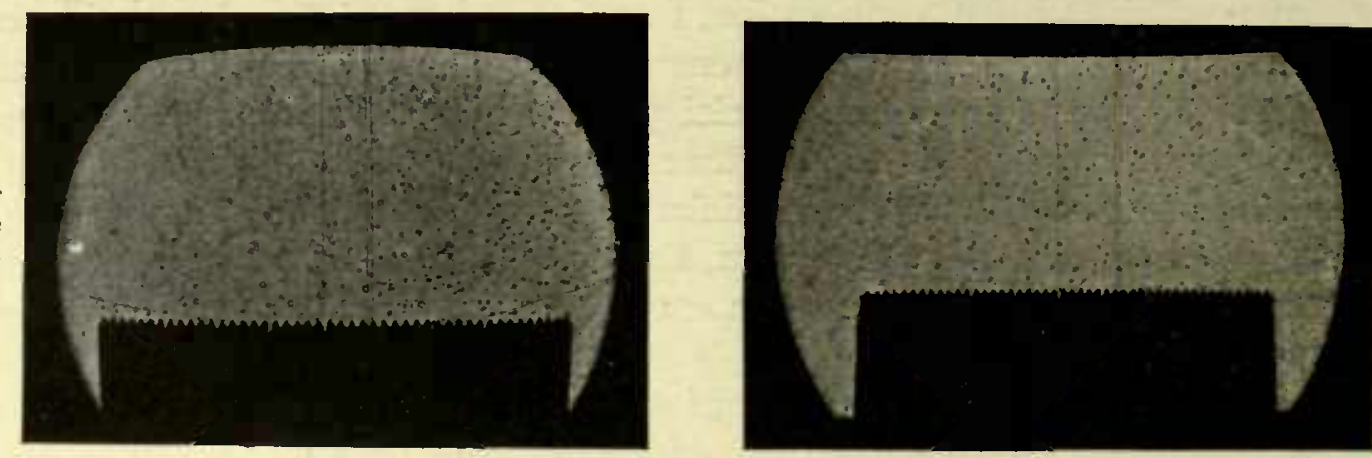

No. 9.-P nucle Scale part, .00 $\mathrm{cm}$. Fall 45 sec.

No. 22.-Scale

part, .0030 cm.

30 sec. X-ray nuclei.

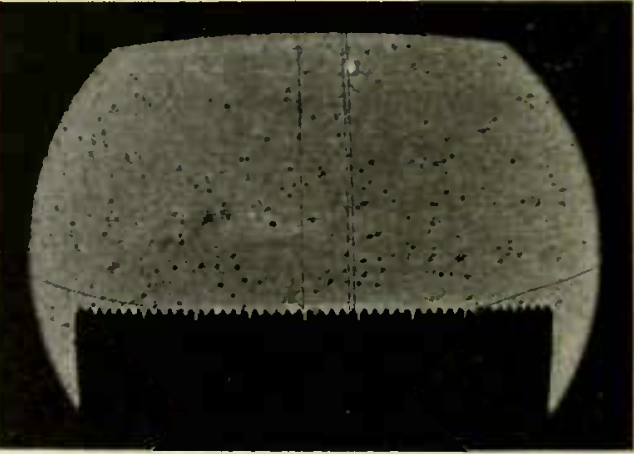

No. 4.-P nuclei. Scale part, .0044 $\mathrm{cm}$. Fall $20 \mathrm{sec}$.

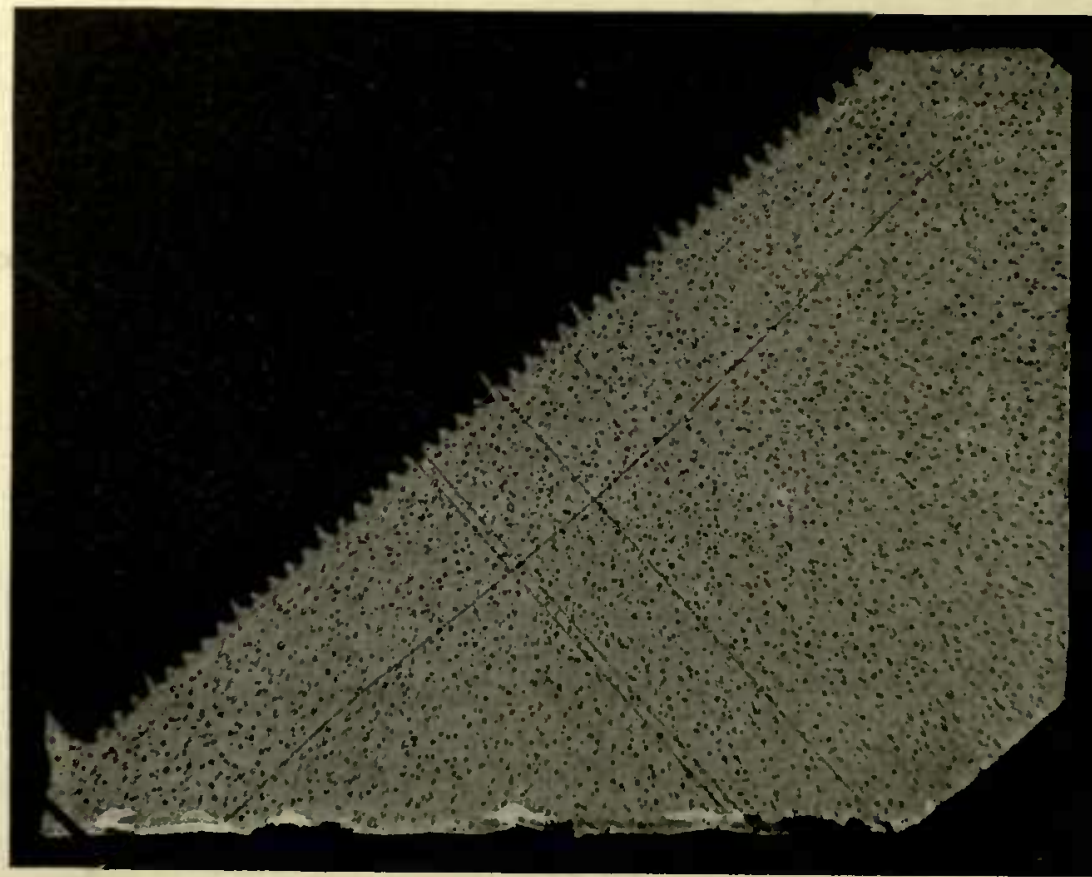

Figure io.-Micro-photographs of Fog Particles, Precipitated on Persistent Nuclei usually Produced by tile X-Rays in DUSt-free Air. 
TABLE 4-FIRST SERIES OF NEGATIVES MEASURED FOR $d$ AND $n . m=4.7 \times 10^{-6}$. $\sqrt[3]{n}=2$. I I $n^{\prime} / \mathrm{tcm}^{2 / 3} \mathrm{IO}^{6} . \quad t=20^{5} t 0.30^{5} . \quad \delta p=17 \mathrm{~cm}$.

\begin{tabular}{|c|c|c|c|c|c|c|c|c|c|c|c|c|}
\hline Nuclei. & $t$ & No. & Corona. & $s$ & $\begin{array}{l}0 \\
x \\
x \\
0\end{array}$ & 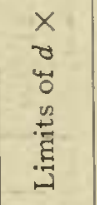 & 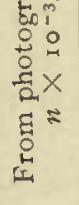 & $\begin{array}{c}\text { Limits of } \\
n \times 10^{-3}\end{array}$ & 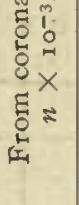 & 芽 & Remarks. & Film \\
\hline $\mathrm{P}$ & Sec. & 2 & Lycopod. & $\mathrm{cm}$. & $3 \mathrm{~cm}$. & $\mathrm{cm}$ & & & & & & \\
\hline$P$ & & 3 & w c g' & 8.9 & 55 & $4-7$ & - & - & & & Rough. & Tállow. \\
\hline$P$ & $20 ?$ & 4 & w c $g^{\prime}$ & 8.6 & 76 & $5^{-10}$ & 250 & -7000 & 160 &. .6 & Persistent. & Dämar. \\
\hline $\mathrm{P}$ & 20 & 5 & "olive" & $>\mathrm{I}_{2}$ & 57 & $4-9$ & $5 \mathrm{I}$ & $36-220$ & 250 & 5 . & Fine. & "“ \\
\hline & $30 ?$ & 6 & w c g $g^{\prime}$ & 8.4 & 69 & $3-10$ & 33 & -270 & I 5 I & 5. & Persistent. & “ \\
\hline $\begin{array}{l}\text { Anr } \\
\text { (stale) }\end{array}$ & 30 & 7 & corona & 5.6 & 109 & $10-13$ & I 8 & $-I_{500}$ & 56 & 3 & $\begin{array}{l}\text { Good but in- } \\
\text { jured. }\end{array}$ & .. \\
\hline $\mathrm{P}$ & - & 8 & fine fog & - & $50 !$ & 一 & - & $-\cdots$ & - & $\div$ & Evaporated: & .. \\
\hline $\mathrm{P}$ & $\begin{array}{l}45 \\
30 ?\end{array}$ & $\begin{array}{r}9 \\
10\end{array}$ & $\begin{array}{l}\mathrm{w} \mathrm{p} \\
\text { corona }\end{array}$ & $\begin{array}{l}7 \cdot 3 \\
5.6\end{array}$ & $\begin{array}{l}90 \\
84\end{array}$ & $\begin{array}{l}5-14 \\
7-10\end{array}$ & $\begin{array}{r}75 \\
? 250\end{array}$ & $\begin{array}{r}-1400 \\
I 75-2600\end{array}$ & $\begin{array}{r}{ }^{1} 30 \\
56\end{array}$ & $\begin{array}{r}\text { I. } \\
.2\end{array}$ & $\begin{array}{l}\text { Clear. } \\
\text { Clear. }\end{array}$ & \\
\hline . & & I I & w c g' & 7.6 & - & - & - & - & - & $\div$ & $\begin{array}{l}\text { Failed. } \\
\text { Craters. }\end{array}$ & $f^{2}$ \\
\hline P. & 30 & I 2 & w r $r^{\prime}$ & $>\mathrm{I}_{2}$ & 54 & $4-7$ & $?_{4}$ & $=$ & - & - & $\begin{array}{l}\text { Vaguc and } \\
\text { faint. }\end{array}$ & \\
\hline$\underset{\text { (stale) }}{\text { Air }}$ & “ & I 3 & corona & 4.9 & $77^{\circ}$ & $5^{-10}$ & 8 & $1-125$ & 45 & 6. & & \\
\hline " $" 1$ & “ & I 4 & " & 4.6 & 96 & $7-\mathrm{II}$ & 7 & -170 & 42 & 6. & Clear. & \\
\hline " " & “" & I 5 & & 4.6 & 97 & $9^{-10}$ & I3 & I $2-33^{\circ}$ & 42 & 3. & $\begin{array}{l}\text { Clear but not } \\
\text { all focussed. }\end{array}$ & ? \\
\hline $\mathrm{P}$ & "6 & I 6 & $y \circ g^{\prime}$ & 9.2 & 62 & $4^{-I O}$ & ? 16 & $7-230$ & I 80 & I I. & Clear but dark. & \\
\hline $\mathrm{P}$ & $"$ & 17 & w c g' & 8.3 & 82 & $5^{-12}$ & $6 I$ & $3-1300$ & I 50 & 2.5 & Good. & \\
\hline $\mathrm{P}$ & " & I 8 & $g^{\prime}$ b p & 6.5 & 62 & $3^{-10}$ & 6 & -340 & 105 & - & Evaporated. & \\
\hline $\mathrm{P}$. & " & I9 & $w \subset g^{\prime}$ & 8.3 & 55 & $3^{-\mathrm{I}} \mathbf{I}$ & 34 & $2-970$ & I 50. & $4 \cdot 4$ & $\begin{array}{l}\text { Clear but un- } \\
\text { certain. }\end{array}$ & \\
\hline
\end{tabular}

The results with fog particles condensed on nuclei of atmospheric air (table 6) and photographed by a small kodak were not very successful, due to secondary causes. In the small vessel used, the tendency to evaporation was accentuated, and the fog particles had, in many instances evaporated before the photograph could be taken. Hence $d$ was not measured.

In table 7 the chief purpose was a comparison of the precipitates obtained when using an ordinary stopcock to effect the exhaustion and on using the instantancous valve described above,

\section{Inferences.}

I8. Precipitation per cubic centimeter. The precipitation, $m$, computed from the plates Nos. 4, 5, and, 6, for instance, would be $57 \times 10^{-6}, 4.8 \times 10^{-6}$, $5.7 \times 10^{-6}$ grams per cubic centimeter, respectively. In the first case the deposit is excessive, probably due to eddy currents, in the second nearly correct, in the third too large. It is preferable, however, to compare the values of $d$ and $n$ obtained from the photographs directly with the data from coronas. 
TABLE 5.-NUCLEI DUE TO X-RAYS OR TO FILTERED P EMANATION; DATA FOR $d$ AND $n . \quad m=4.7 \times 10^{-6} ; \sqrt[9]{n}=2.11 n^{1} / t m^{2 / 3} 10^{6} ; t=15^{5}-45^{5} ;$ $\delta p=17 \mathrm{~cm} . ;$ FILM, SOFT DAMAR VARNISH.

\begin{tabular}{|c|c|c|c|c|c|c|c|c|c|c|}
\hline Nuclei. & Fall. & No. & Corona. & $s$ & $\begin{array}{l}0 \\
\stackrel{y}{1} \\
x \\
0 \\
0\end{array}$ & 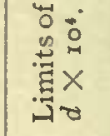 & $\begin{array}{l}i \\
\vdots \\
0 \\
x \\
\approx\end{array}$ & 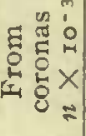 & 胥 & Remarks. \\
\hline $\begin{array}{c}\text { X-ray } \\
\text { " } \\
" 1 \\
\text { ", } \\
\text { " } \\
\text { " } \\
\text { " }\end{array}$ & $\begin{array}{l}\text { s. } \\
30 \\
30 \\
30 \\
30 \\
30 \\
30 \\
30 \\
15 \\
15 \\
30 \\
45\end{array}$ & $\begin{array}{l}20 \\
21 \\
22 \\
23 \\
24 \\
25 \\
26 \\
27 \\
28 \\
29 \\
30\end{array}$ & 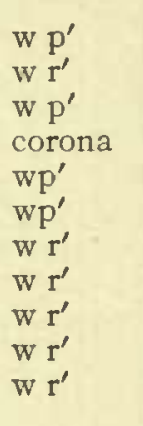 & $\begin{array}{l}6.9 \\
5 \cdot 7 \\
5 \cdot 7 \\
2.8 \\
5 \cdot 3 \\
5 \cdot 3 \\
3.6 \\
3.1 \\
4.4 \\
2.8 \\
4.6\end{array}$ & $\begin{array}{l}\text { I0I } \\
100 ? \\
\text { I33 } \\
129 \\
125 \\
126 \\
130 \\
105 \\
210 \\
139\end{array}$ & $\begin{array}{c}6-11 \\
7-16 \\
70-20 \\
- \\
5^{-21} \\
5-10 \\
7-22 \\
8-15 \\
16-24 \\
9^{-20}\end{array}$ & \begin{tabular}{r|}
\multicolumn{1}{c|}{-} \\
4.3 \\
$\mathrm{I} .3$ \\
24.4 \\
$24 . \mathrm{I}$ \\
4.1 \\
19.7 \\
$? .17$ \\
1.9
\end{tabular} & $\begin{array}{r}60 \\
8 \\
- \\
52 \\
18 \\
11 \\
36 \\
8 \\
42\end{array}$ & $\begin{array}{r}14 \\
6 \\
- \\
2.2 \\
5.8 \\
2.7 \\
1.8 \\
? 47 \\
22\end{array}$ & $\begin{array}{l}\text { Fogged plate. } \\
\text { Do. } \\
\text { Pitted. Shows crater. } \\
\text { Good. } \\
\text { Lost. } \\
\text { Good. } \\
\text { Too late. } \\
\text { Good. Shows dew. } \\
\text { Evaporated? } \\
\text { Good. }\end{array}$ \\
\hline $\begin{array}{c}\mathrm{P} \\
\mathrm{P} \\
\mathrm{P} \text { (stale) } \\
\mathrm{P} \\
\mathrm{P} \\
\mathrm{P} \\
\mathrm{P} \\
\mathrm{P} \text { (stale) } \\
\mathrm{P}\end{array}$ & $\begin{array}{l}-\overline{30} \\
30 \\
30 \\
30\end{array}$ & $\begin{array}{l}31 \\
32 \\
33 \\
34 \\
35\end{array}$ & $\begin{array}{l}\text { corona } \\
\text { wp } \\
\text { w } r^{\prime} \\
w^{\prime} c^{\prime} g\end{array}$ & $\begin{array}{c}>12 \\
10.4 \\
5.5 \\
4.5 \\
>12\end{array}$ & $\begin{array}{l}65 \\
65 \\
86 \\
65\end{array}$ & $\begin{array}{l}5-10 \\
-10 \\
5-7 \\
7-12 \\
4-11 \\
6-20 \\
8-11 \\
2-6 \\
8-10\end{array}$ & $\begin{array}{r}- \\
- \\
27.0 \\
4.9 \\
? .5 .8 \\
5.8 \\
\text { ? } 3.8 \\
? 3.1 \\
? 3.1 \\
? .10\end{array}$ & $\begin{array}{r}- \\
55 \\
40 \\
250\end{array}$ & $\begin{array}{l}\text { I.2 } \\
3.6 \\
-\end{array}$ & $\begin{array}{l}\text { On influx. } \\
\text { Streaks. } \\
\text { Good. } \\
\text { Good (mixed small and } \\
\quad \text { large). } \\
\text { Good. } \\
\text { Mixed small and large. } \\
\text { Out of focus. } \\
\text { Out of focus. }\end{array}$ \\
\hline
\end{tabular}

TABLE 6.-AIR NUCLEI; DATA FOR $n . \quad m=4.7 \times 10^{-6} \mathrm{~g} / \mathrm{cm}^{3} ; \sqrt[3]{n}=2.1 \mathrm{In}^{1} / \mathrm{lcm} \mathrm{cm}^{2 / 3} \mathrm{Io}^{6}$;

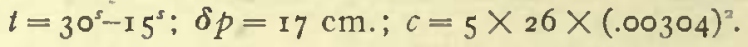

\begin{tabular}{|c|c|c|c|c|c|c|c|c|}
\hline No. & Corona. & $s$ & $n^{\prime}$ & $t$ & $\begin{array}{c}\text { From } \\
\text { photograph } \\
n \times 10^{-3}\end{array}$ & $\begin{array}{c}\text { From } \\
\text { coronas } \\
n \times 10^{-3}\end{array}$ & Ratio. & Remarks. \\
\hline 40 & $y^{\prime} \circ g^{\prime}$ & 6.8 & 105 & 30 & 10.8 & 117 & 10 & \\
\hline $4 \mathrm{I}$ & $w^{\prime} \circ g^{\prime}$ & 6.8 & 87 & 30 & 6.7 & 117 & 17 & \\
\hline 42 & wog & 6.8 & Faile & & 一 & I 17 & - & Accident. \\
\hline 43 & $\mathrm{w}$ br cor & 4.8 & 102 & 30 & $7 \cdot 4$ & 45 & 6.1 & Good. \\
\hline 44 & $\mathrm{w}$ br cor & 4.8 & 53 & I 5 & 9.0 & 45 & 5.0 & \\
\hline 45 & $\mathrm{w}^{\prime}|\mathrm{b}| \mathrm{r}^{\prime}$ & 4.6 & 54 & I 5 & I 2.3 & 42 & 3.4 & Very good. \\
\hline $4^{6}$ & $\mathrm{wrg}$ & 5.7 & 67. & I 5 & 6.1 & $5^{8}$ & $9 \cdot 5$ & Vague. \\
\hline 47 & wog & 6.8 & Faile & & - & - & - & \\
\hline 48 & wrg & 5.8 & Faile & & 一 & - & - & \\
\hline 49 & $g$ b p & 6.4 & 75 & I 5 & 31.3 & roo & $3 \cdot 3$ & Good. \\
\hline $5^{\circ}$ & $\mathrm{g} \mathrm{b} \mathrm{p}$ & 6.4 & 105 & I 5 & 85.8 & $100^{\circ}$ & 1.2 & Good. \\
\hline
\end{tabular}

I9. Diameters and numbers. - The $d$-values given by the photographs are almost without exception larger than the corresponding diameters computed 
TABLE 7.-MISCELLANEOUS EXPERIMENTS. ROOM AIR FROM FLOOR. LARGE VESSEL. $\sqrt[3]{n}^{n}=6.23^{n \prime}$. EXHAUST THROUGH STOPCOCK.

\begin{tabular}{|c|c|c|c|c|c|c|c|}
\hline No. & Corona. & $s$ & $n^{\prime}$ & $t$ & $\begin{array}{c}\text { By photograph } \\
n \times \mathrm{IO}^{-3}\end{array}$ & $\begin{array}{c}\text { From coronas } \\
n \times 10^{-3}\end{array}$ & $\frac{n_{\text {cor }}}{n_{\text {obs }}}$ \\
\hline $\begin{array}{l}53 \\
54 \\
55 \\
56\end{array}$ & $\begin{array}{l}\text { wp cor } \\
\text { w o g' } \\
\text { w ro g } \\
\text { w o g' }\end{array}$ & $\begin{array}{l}7.3 \\
9.8 \\
8.8 \\
9.8\end{array}$ & $\begin{array}{r}144 \\
\text { I } 68 \\
\text { I } 47 \\
73\end{array}$ & $\begin{array}{l}30^{5} \\
30 \\
30 \\
30\end{array}$ & $\begin{array}{l}26 \\
42 \\
28 \\
? 3 \cdot 4\end{array}$ & $\begin{array}{l}127 \\
200 \\
170 \\
200\end{array}$ & $\begin{array}{r}4.9 \\
4.7 \\
6.1 \\
? 59 \\
\text { Mean, } 5.2\end{array}$ \\
\hline
\end{tabular}

EXHAUST THROUGH INSTANTANEOUS VALVE.

\begin{tabular}{|c|c|c|c|c|c|c|c|}
\hline 57 & $w \circ g^{\prime}$ & 9.8 & I 38 & $30^{5}$ & 24 & 200 & 8.4 \\
\hline $5^{8}$ & wo & 4.6 & 92 & 30 & 7.2 & 42 & 5.8 \\
\hline 59 & w c g $g^{\prime}$ & 8.3 & 130 & 30 & ?I9 & I 35 & $7 \mathrm{I}$. \\
\hline 60 & w r $g^{\prime}$ & 8.9 & - & 30 & - & - & - \\
\hline $6 \mathrm{I}$ & w c g $g^{\prime}$ & 7.8 & I 70 & 30 & 45 & 140 & 3.1 \\
\hline 62 & $\mathrm{wcg} \mathrm{g}^{\prime}$ & 7.9 & 126 & 30 & 18 & 145 & $\begin{array}{r}8.1 \\
\text { Mean, } 6.3\end{array}$ \\
\hline
\end{tabular}

from coronas. No doubt this is in part due to adhesion; but the chief reason is the occurrence of so many large particles with the small, so that the average value of $d$ given by the photographs is within certain limits an arbitrary quantity, depending on the distribution of particles selected for measurement. If the values of $d$ be plotted graphically in terms of $s$, they do not make a smooth curve, and these values are from 1.5 to 2 times larger than the coronal values of $d$. For this reason the measurement of $d$ in the photographs was abandoned. The data are nuch inferior to the above micrometer measurements of floating globules.

The $n$-values in the photographs, being obtained independently and not conditioned by so perfect a reproduction of form, etc., as are imperative in measuring diameter, may be considered more trustworthy. Nevertheless, they also fail to suggest a smooth curve, though they are, as a whole, very much smaller than the coronal $n$-values. If observations obviously in error be omitted, the following are the mean values of the ratio $n$ (coronal) to $n$ (photographic) obtained from the successive tables. Only in two or three instances are these ratios less than one.

$$
\begin{array}{cccc}
\text { Table } 4 . & \text { Ratio } & =4.1, \\
\text { “ } & 5 . & “ & =3.8, \\
\text { " } & 6 . & “ & =4.4, \\
\text { " } & 7 . & " & =5.9,
\end{array}
$$

or on the average the coronal $n$-values are about $4 \frac{1}{2}$ times as large as the $n$ values obtained from the photographs. 
20. Explanation of discrepancies. - The reason for this result would at first sight be obviously given by the evaporation of the fog particles on the plate, before and during the photographic exposure. But as the $d$-values were from $I .5$ to 2 times too large, it is nccessary to guard against accepting this explanation too hastily: for inasmuch as the $d$-values and the $n$-values are independently given by the photograph, their relation must be

$$
n d^{3}=6 m / \pi \text {. }
$$

Now it is rather curious that while the $n$-values are 4.5 times too small, the independent $d^{3}$-values should be 3 to 6 times too large. In other words, on using the average $d$ - and the average $n$-values given by the photographs, an approximately correct value for $m$, the precipitation per eub. centim., follows, even though $d$ and $n$ are found quite independently. Add to this the fact that if evaporation occurs the photograph usually fails entirely. It is quite improbable that on the average a definite number, about 78 per cent., of the fog particles should incidentally evaporate.

Before proceeding further it will contribute to clearness if some of the better data of table 4 be summarized. The $d$-values show the marked occurrence of larger particles on the photograph, whereas the smallest particles more nearly correspond in size.

TABLE 8.--SUMMARY OF CER'TAIN DATA FROM TABLE 4.

\begin{tabular}{|c|c|c|c|c|c|c|c|c|}
\hline No. & Nuclei. & $\begin{array}{c}\text { Time of } \\
\text { subsidence. }\end{array}$ & $s$ & Corona. & $\begin{array}{l}\text { Diameter } \\
\text { from } \\
\text { photograph } \\
d \times \text { I }\end{array}$ & $\begin{array}{l}\text { Diameter } \\
\text { from } \\
\text { coronas } \\
d \times 10^{4} .\end{array}$ & $\begin{array}{c}\text { Number } \\
\text { from } \\
\text { photograph } \\
n \times 10-3 .\end{array}$ & $\begin{array}{c}\text { Number from } \\
\text { coronas } \\
n \times 10^{-3}\end{array}$ \\
\hline 4 & $\mathrm{P}$ & $\begin{array}{l}\text { sec. } \\
20\end{array}$ & $\begin{array}{l}\mathrm{cm} . \\
8.6\end{array}$ & w c g' & $\begin{array}{l}\mathrm{cm} . \\
5^{-10}\end{array}$ & $\begin{array}{l}\mathrm{cm} . \\
3.8\end{array}$ & $? 250$ & 160 \\
\hline 5 & $\mathrm{P}$. & 20 & $>I_{2}$ & olive & $4-9$ & 3.2 & $5 \mathrm{I}$ & 2.50 \\
\hline 6 & Air & 30 & 8.4 & w c g' & $3-10$ & 3.8 & 33 & 160 \\
\hline 9 & $\mathrm{P}$ & 45 & $7 \cdot 3$ & $w p$ & $5^{-14} 4$ & 4.1 & 75 & 130 \\
\hline 10 & & 30 & 5.6 & cor & $7-10$ & 5.2 & $? 250$ & 60 \\
\hline 23 & X-ray & 30 & 2.8 & cor & $5^{-20}$ & 10.6 & 1.3 & 8 \\
\hline 25 & ", & 30 & $5 \cdot 3$ & $\mathrm{w} \mathrm{r}^{\prime}$ & $5^{-2 I}$ & $5 \cdot 5$ & 24 & 50 \\
\hline 27 & $"$ & I 5 & 3.1 & “ & $7-22$ & $7 \cdot 5$ & 4 & I I \\
\hline 30 & “ & 30 & 4.6 & $"$ & $9^{-20}$ & 6.2 & 2 & 42 \\
\hline
\end{tabular}

If the micrometer data $\left(\$ \S_{4}-6\right)$ be used for the $d$-values, the ratios of coronal and measured diameters are

\begin{tabular}{|c|c|c|c|c|}
\hline$s=$ & & 6 & 8 & Io \\
\hline Ratio $=$ & $\begin{array}{l}1.3 \text { to } \\
\text { I. } 6\end{array}$ & $\begin{array}{l}1.2 \\
\text { I. } 6\end{array}$ & ${ }^{\mathrm{I} .2}$ & I.2 \\
\hline
\end{tabular}

or the $d^{3}$-values are $\mathrm{x} .7$ to $4 . \mathrm{I}$ times too large. Now in these instances the fog particles were often measured while floating, so that adhesion in these instances must have been a negligible factor. Nevertheless the $d$-values are not all incompatible with the $n$-values. 
21. Summary.-The curious state of the case mentioned is not reassuring. Briefly, the photographic $n$-values and the photographic values for diameter, $d$, do not make an incompatible system, though both differ materially from the coronal values, the former $(d)$ being larger, the $(n)$ smaller. If one ascribes the large visible diameters to adhesion, and the small numbers counted to evaporation, the compatibility of the two sets of independent data $(d$ and $n$ ) is not explained.

I have therefore concluded that the results in question suggest that the smaller particles evaporate rapidly into the larger. Hence while the mass of water precipitated per cubic centim. remains constant, the diameter of the fog particles soon increases while their number decreases (by evanescence of the smaller) in such a way that $n \times d^{3}$ remains very nearly constant throughout. In place of evaporation, capillary coalescence of the initial very minute droplets is an even greater probability; and such coalescence $I$ have often seen under the nicroscope on observing dew droplets ${ }^{1}$ very fine and close together. It is rather interesting that the evidence in favor of this complicated behavior is so strong.

That such evaporation occurs appreciably in the coronas is not probable, because in a good apparatus they retain their character during the whole of the subsidence. The plate of glass being slightly warmer contributes to the effect observed.

Hence neither the micrometric nor the photometric method can be relied upon for undistorted results. Whenever graded particles are present the evidence must be sought in the washed and blurred coronas. If the coronas are clear and multi-annular, the gradation observed under the microscope must be a secondary effect not present in the coronas themselves.

\section{RESULTS FROM SUBSIDENCE.}

22. Object and method.-The difficulties mentioned in the last sections induced me to look for corroborative data in the evidence obtainable from special experiments with subsiding fog particles. These are easily made in the apparatus for measuring atmospheric nucleation as described in Chapter VIII, Fig. I. The observer, provided with a stop-watch, simply determines the time during which the straight horizontal line of fog descends a given distance, say 5 centimeters, from the top. In other cases there were two marks between which subsidence was noted.

23. Results.- In the experiments given in the following tables, the watch was started simultaneously with the exhaustion and stopped when the given fall of fog line had been reached. The equations are then successively

$$
{ }_{10}^{4} d=18 \sqrt{v}, \quad d s=a, \quad n=9 /\left(d^{3} \times 10^{6}\right)=1550 / v^{3 / 2},
$$

where $n$ is the number of particles per cubic centim., $d$ the diameter of each, $v$ the rate of subsidence.

- If a microscope plate is dipped into thin, rapidly drying methyl alcohol varnish, a milky deposit of dew is often seen on the film when solidifying. This film behaves under the microseope as stated in the text. 
TABLE 9.-VALUES OF $a$ AND $n$. AIR NUCLEI. $n=6 m / \pi d^{3}=9 / 10^{6} d^{3} ; 10^{4} d=I 8 V \bar{v}$; $d s=a$.

\begin{tabular}{|c|c|c|c|c|c|c|}
\hline Corona. & $s$ & $10^{3} \times v$ & $104 \times d$ & $a$ & $10^{-3} \times n$ & $\begin{array}{c}\text { Constant } \\
10^{3} \times v\end{array}$ \\
\hline & $\mathrm{cm}$. & $\mathrm{cm} . / \mathrm{sec}$. & $\mathrm{cm}$. & & & $\mathrm{cm} . / \mathrm{sec}$ \\
\hline w olg & 5.8 & 77 & 5.0 & .0029 & 72 & 70 \\
\hline wrg & $5 \cdot 4$ & 84 & $5 \cdot 4$ & 34 & 56 & 80 \\
\hline gy o & 6.5 & 72 & $5 \cdot 3$ & 34 & 62 & 60 \\
\hline w ro $g$ & 6.2 & 89 & $5 \cdot 5$ & 34 & 53 & 65 \\
\hline wog & 6.2 & 89 & $5 \cdot 5$ & 34 & 53 & 65 \\
\hline wrg & $5 \cdot 9$ & 85 & $5 \cdot 4$ & 32 & $5^{6}$ & 70 \\
\hline $\mathrm{g}^{\prime}|\mathrm{b}| \mathrm{p}$ & 4.5 & I 80 & 7.6 & 34 & 20 & I 23 \\
\hline Do. & $4 \cdot 5$ & II 6 & 6.1 & 28 & 39 & I 23 \\
\hline Do. & $4 \cdot 5$ & I 30 & 6.5 & 29 & 33 & I 25 \\
\hline$w|b| p$ & 4.6 & I 22 & 6.3 & 29 & 36 & I I 5 \\
\hline w rg & 5.9 & 107 & 5.9 & 35 & 44 & 70 \\
\hline wrg & 5.7 & 102 & $5 \cdot 7$ & 33 & 48 & 75 \\
\hline $\mathrm{g} \mathrm{b} \mathrm{p}$ & 4.6 & I 28 & 6.4 & 30 & 34 & I I 5 \\
\hline$y^{\prime} b g$ & 6.2 & 77 & $5 \cdot 3$ & 33 & 59 & 65 \\
\hline wrg & 6.2 & 79 & $5 \cdot 4$ & 33 & 59 & 65 \\
\hline$g|b| p$ & 4.6 & 156 & 7.1 & 33 & 25 & I I 5 \\
\hline$w g \mid r$ & $4 \cdot 5$ & 192 & $7 \cdot 9$ & 35 & I 8 & 123 \\
\hline wrg & 4.5 & 122 & 6.3 & 28 & 36 & I 23 \\
\hline wrg & 5.6 & 94 & $5 \cdot 5$ & $3 I$ & 53 & 75 \\
\hline w r g & $5 \cdot 5$ & 104 & 5.8 & 32 & 46 & 80 \\
\hline$y^{\prime} \circ g$ & 5.8 & 107 & 5.9 & 34 & 44 & 70 \\
\hline wrg & $5 \cdot 7$ & I 25 & 6.4 & 36 & 35 & 75 \\
\hline w c g & $5 \cdot 4$ & 86 & $5 \cdot 5$ & 30 & 55 & 80 \\
\hline$y \circ b^{\prime}$ & 6.2 & 76 & $5 \cdot 3$ & 33 & 60 & 65 \\
\hline $\mathrm{g}|\mathrm{b}| \mathrm{p}$ & 4.8 & 140 & 6.7 & 32 & 29 & 105 \\
\hline $\mathrm{g} \mathrm{b} \mathrm{p}$ & 6.0 & 72 & $5 \cdot 3$ & 32 & 60 & 65 \\
\hline
\end{tabular}

Mean $a$ in decades .00322

$$
\begin{aligned}
& \left.\begin{array}{l}
304 \\
327 \\
320
\end{array}\right\} a=.00320 \\
& \begin{array}{l}
320 \\
329
\end{array}
\end{aligned}
$$

TABLE Io.-FURTHER RESULTS FOR SUBSIDENCE. $n=6 m / \pi d^{3}=9 / 10^{6} d^{3} ; \quad 10^{4} d=$

\begin{tabular}{|c|c|c|c|c|c|c|c|}
\hline Corona. & $s_{\mathrm{xg} .5}$ & $10^{3} \times v$ & $104 \times d$ & $s_{30}$ & $a$ & $n$ & $\begin{array}{c}\text { Constant } \\
\mathrm{x}^{3} \times v\end{array}$ \\
\hline y br bg & 4.2 & 79 & 5.I & 6.5 & .0033 & 68000 & 58 \\
\hline wog & 4. I & 74 & 4.9 & 6.3 & $3 \mathrm{I}$ & 76000 & 62 \\
\hline w br cor & 3.1 & I 35 & 6.6 & 4.8 & $3^{2}$ & 31000 & 105 \\
\hline $\mathrm{w}|\mathrm{b}| \mathrm{p}$ & 2.9 & I I 4 & 6.1 & $4 \cdot 5$ & 27 & 40000 & I 22 \\
\hline w y cor & 3.0 & I 25 & 6.4 & 4.6 & 29 & 34000 & I I 7 \\
\hline $\mathrm{w}|\mathrm{b}| \mathrm{r}$ & 3.2 & 106 & 5.9 & 4.9 & 29 & 44000 & 100 \\
\hline w y cor & 2.3 & 167 & $7 \cdot 4$ & $3 \cdot 5$ & 26 & 22000 & 215 \\
\hline w c g & 2.7 & I 43 & 6.8 & 4.1 & 28 & 28000 & I 50 \\
\hline w br cor & 2.2 & 200 & 8. I & $3 \cdot 4$ & 28 & 17000 & 230 \\
\hline wo g & 2.8 & I 25 & 6.4 & $4 \cdot 3$ & 27 & 35000 & I 35 \\
\hline w rg & 2.7 & I 40 & 6.7 & 4.2 & 28 & 30000 & I 45 \\
\hline
\end{tabular}
I $8 V \bar{v} ; d s=a$. 
A CONTINUOUS RECORD OF ATMOSPHERIC NUCLEATION.

\begin{tabular}{|c|c|c|c|c|c|c|c|}
\hline Corona. & $s_{19.5}$ & $10^{3} \times v$ & $104 \times d$ & $s_{30}$ & $a$ & $n$ & $\begin{array}{c}\text { Constant } \\
10^{3} \times v\end{array}$ \\
\hline cor & 2.2 & I 57 & 7.1 & $3 \cdot 4$ & 24 & 25000 & 235 \\
\hline wp cor & $3 \cdot 3$ & 94 & $5 \cdot 5$ & $5 \cdot I$ & 28 & 54000 & 90 \\
\hline $\mathrm{w}|\mathrm{b}| \mathrm{p}$ & 3.0 & 102 & $5 \cdot 7$ & 4.6 & 26 & 49000 & II 7 \\
\hline cor & 2.3 & 230 & 8.6 & 3.6 & $3 I$ & 14000 & 210 \\
\hline cor & 2.9 & ? 109 & 5.9 & $4 \cdot 5$ & 27 & 44000 & 123 \\
\hline wbp & 2.8 & I I I & 6.0 & $4 \cdot 3$ & 26 & 42000 & I35 \\
\hline wog & 2.8 & I 35 & 6.6 & $4 \cdot 3$ & 28 & 24000 & 135 \\
\hline $\mathrm{w}|\mathrm{b}| \mathrm{r}$ & 3.0 & $9^{8}$ & 5.6 & 4.6 & 26 & $5^{1000}$ & I I 7 \\
\hline $\mathrm{g} b \mathrm{p}$ & 3.0 & 102 & $5 \cdot 7$ & 4.6 & 26 & 49000 & I I 7 \\
\hline $\mathrm{wrg}$ & 3.8 & $8 \mathrm{I}$ & 5.1 & $5 \cdot 9$ & 30 & 68000 & 68 \\
\hline$g \mathrm{~b} p$ & 3.0 & 105 & 5.8 & 4.6 & 27 & 45000 & I I 7 \\
\hline w r g & 2.9 & I I 5 & 6.1 & $4 \cdot 5$ & 27 & 39000 & 123 \\
\hline wp cor & $3 \cdot 4$ & 9 I & $5 \cdot 4$ & 5.2 & 28 & 57000 & 90 \\
\hline wog & 3.0 & 122 & 6.3 & 4.6 & 29 & 36000 & I I 7 \\
\hline w rg & 2.8 & 140 & 6.7 & $4 \cdot 3$ & 29 & 30000 & 135 \\
\hline wog & 2.8 & 109 & $5 \cdot 9$ & $4 \cdot 4$ & 26 & 44000 & I 30 \\
\hline $\mathrm{gbp}$ & 2.9 & 122 & 6.3 & $4 \cdot 5$ & 28 & 36000 & 123 \\
\hline$g|b| p$ & 3.1 & 105 & 5.8 & 4.8 & 28 & 46000 & 105 \\
\hline wp cor & $3 \cdot 1$ & 100 & $5 \cdot 7$ & 4.8 & 28 & 48000 & 100 \\
\hline $\mathrm{gb} p$ & $4 \cdot I$ & 68 & 4.7 & 6.3 & 30 & 86000 & 62 \\
\hline $\mathrm{g} \mathrm{b} \mathrm{p}$ & 2.9 & 100 & 5.7 & $4 \cdot 5$ & 26 & 48000 & I 23 \\
\hline $\mathrm{w}$ br cor & 3.0 & 100 & 5.7 & 4.6 & 26 & 48000 & I I 7 \\
\hline $\mathrm{w}$ br $\mathrm{r}$ & 2.9 & 105 & 5.8 & $4 \cdot 5$ & 26 & 46000 & I 23 \\
\hline wo cor & 2.9 & 120 & 6.2 & 4.5 & 28 & 38000 & I 23 \\
\hline wog & 2.8 & 140 & 6.7 & 4.3 & 29 & 30000 & I 35 \\
\hline w rg & 2.8 & 143 & 6.8 & $4 \cdot 3$ & 29 & 28700 & I 35 \\
\hline wp cor & 3.0 & I I I & 6.0 & 4.6 & 28 & 41000 & I I 7 \\
\hline w rg & 2.9 & I 43 & 6.8 & $4 \cdot 5$ & $3 I$ & 28700 & I 23 \\
\hline$g \mathrm{br}$ & 3.0 & 109 & 5.9 & 4.7 & 28 & 44000 & I IO \\
\hline $\mathrm{w}$ br cor & 3.0 & I 28 & 6.4 & $4 \cdot 7$ & 30 & 34000 & I 10 \\
\hline $\mathrm{g} \mathrm{b} \mathrm{p}$ & 4.0 & 60 & 4.4 & 6.2 & 27 & 106000 & 64 \\
\hline $\mathrm{g} \mathrm{p} \mathrm{b}$ & 4.2 & 64 & $4 \cdot 5$ & 6.5 & 29 & 99000 & $5^{8}$ \\
\hline $\mathrm{g}$ b p & 4.0 & 69 & $4 \cdot 7$ & 6.2 & 29 & 87000 & 64 \\
\hline w rg & 4.0 & 85 & $5 \cdot 3$ & 6.2 & 33 & 60000 & 64 \\
\hline w y g & 4.2 & 79 & 5.1 & 6.5 & 33 & 68000 & $5^{8}$ \\
\hline w y cor & 2.9 & $128^{\circ}$ & 6.4 & $4 \cdot 5$ & 29 & 34000 & I 23 \\
\hline wo cor & 2.9 & I $5^{2}$ & 7.0 & 4.5 & $3 I$ & 26000 & I 23 \\
\hline w ro g & 4.0 & 76 & 5.0 & 6.2 & $3 I$ & 72000 & 64 \\
\hline w p cor & 3.2 & 98 & 5.6 & 4.9 & 27 & 5 I000 & 100 \\
\hline$w$ br cor & 3.0 & I 20 & 6.2 & 4.6 & 29 & 38000 & I I 7 \\
\hline cor & 2.9 & I 20 & 6.2 & $4 \cdot 5$ & 28 & 38000 & 120 \\
\hline w p cor & $3 \cdot 3$ & $9 I$ & 5.4 & 5.2 & 28 & 57000 & 88 \\
\hline $\mathrm{w} b \mathrm{p}$ & 3.0 & 96 & 5.6 & 4.6 & 26 & 51000 & I I 7 \\
\hline w rg & 2.9 & I 32 & 6.5 & 4.5 & 29 & 32000 & I 23 \\
\hline w r g & 2.7 & 140 & 6.7 & 4.2 & 28 & 30000 & I 35 \\
\hline w p cor & $3 \cdot 3$ & 98 & 5.6 & $5 . I$ & 29 & 51000 & 90 \\
\hline w r g & 2.9 & I 32 & 6.5 & $4 \cdot 5$ & 29 & 32000 & I 23 \\
\hline $\operatorname{cor}$ & 3.0 & I 6 & 6.I & 4.6 & 28 & 40000 & I I 7 \\
\hline wp cor & 3.6 & $9 I$ & $5 \cdot 4$ & $5 \cdot 5$ & 30 & 57000 & 80 \\
\hline w r g & 4.0 & 78 & 5.0 & 6.2 & $3 I$ & 72000 & 65 \\
\hline $\mathrm{g} \mathrm{b} \mathrm{p}$ & 3.0 & 105 & 5.8 & 4.6 & 27 & 46000 & I I 7 \\
\hline wg cor & I. 8 & 2 I 5 & 8.3 & 2.8 & 24 & I 5800 & 330 \\
\hline wr cor & 2.0 & 200 & 8.0 & 3.1 & 25 & I 7300 & 280 \\
\hline wr cor & 2.0 & 200 & 8.0 & 3.1 & 25 & I 7300 & 280 \\
\hline cor & 1.9 & 210 & 8.2 & 2.9 & 24 & I 6400 & 320 \\
\hline cor & 2.4 & I 56 & $7 \cdot I$ & 3.7 & 26 & 25000 & I95 \\
\hline cor & 2.1 & 205 & 8.2 & 3.2 & 26 & 16700 & 265 \\
\hline
\end{tabular}


24. Remarks on the tables.-Constructed graphically, the results of table 9 are irregularly below the coronal results. The results of table ro are somewhat smoother and in the main agree with the coronal data. Thus for the green coronas (see Chapter VI, $\$ 29$, table I7)

coronal datum, g b p, $n=96,000$,

from table $10, \mathrm{~g}$ b p, $n=106,000,99,000,87,000,86,000$; mean, $n=95,000$.

or the mean values are about the same. In case of low nucleations and small coronas the subsidence results are much too high as compared with the coronal results, implying too small a value of the $v$ of subsidence.

The only explanation which suggests itself for this unexpected behavior is the occurrence of acceleration in the motion of the fog particles. Accordingly the following experiments with very small coronas (large particles) were tried as a test.

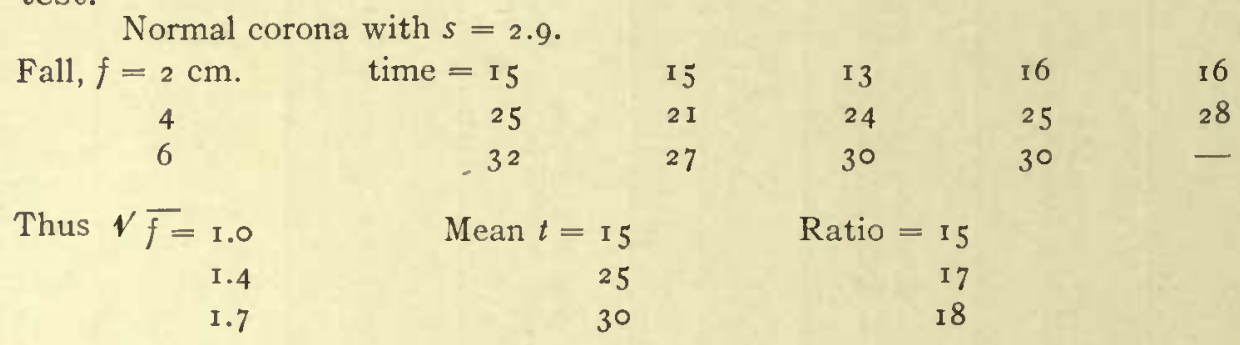

indicating that the uniformly varied motion is only very gradually retarded by the air resistance encountered. The following table contains similar data for larger coronas, in none of which the evidence of acceleration is absent.

TABLE it.-MEAN RATES FOR SUCCESSIVE DISTANCES OF $2 \mathrm{~cm}$. EACH.

\begin{tabular}{|c|c|c|c|c|}
\hline \multirow[b]{2}{*}{ Corona. } & \multirow[b]{2}{*}{$s_{30}$} & \multicolumn{3}{|c|}{ Time for successive falls of $2 \mathrm{~cm}$. cach. } \\
\hline & & $t \mathrm{sec}$ & $t \mathrm{sec}$ & $t \mathrm{sec}$ \\
\hline $\begin{array}{l}\text { w rg } \\
\text { w|b|p } \\
\text { w rog } \\
\text { wrg } \\
\text { wp cor } \\
\text { w br cor } \\
\text { w b p } \\
\text { w rg } \\
\text { wrg } \\
\text { w b p } \\
\text { cor }\end{array}$ & $\begin{array}{l}5 \cdot 7 \\
4.6 \\
4.6 \\
4.4 \\
3.4 \\
4.6 \\
4.7 \\
4.7 \\
4.5 \\
4.6 \\
1.9\end{array}$ & $\begin{array}{l}18 \\
20 \\
20 \\
17 \\
13 \\
19 \\
19 \\
15 \\
23 \\
13\end{array}$ & $\begin{array}{r}23 \\
18 \\
15 \\
12 \\
8 \\
14 \\
14 \\
14 \\
14 \\
15 \\
8\end{array}$ & $\begin{array}{r}22 \\
16 \\
12 \\
10 \\
5 \\
- \\
- \\
12 \\
11 \\
17 \\
6\end{array}$ \\
\hline
\end{tabular}

These results rob the subsidence method of much of its trustworthiness except when the particles are very small, when other difficulties step in: for large coronas are not persistent in character. 
The case may be stated by computation as follows: The velocity at which force is annulled by the resistance is

$$
v=\left(10^{4} d / 18\right)^{2}=.31 d^{2} \times 10^{6}
$$

from which the following table has been computed.

TABLE I 2 .

$s=\mathbf{I} .5 \mathrm{~cm}$.
2
3
4
5
6
7

$d=.00180 \mathrm{~cm}$.
143
098
072
055
047
042

$v=1.00 \mathrm{~cm} . / \mathrm{sec}$.

.63

.29

. 6 I

.093

.068

.053

If the results of this table are laid off graphically in a chart, the column marked "constant $10^{3} \times v$ " in tables 9 and Io above may be obtained from it. It will then be seen that in most of the instances of small coronas in table 10 , the observed velocity is less than the limiting velocity. Hence these data are to be rejected, and it follows that the subsidence method is scarcely applicable until the middle $\mathrm{g}$-b-p corona $(s=6.2-6.5)$ has been reached. The mean value of $a$ computed from the admissible data of table Io is in successive decades, .00284, $.00290, .00293, .00290$, giving a mean value of $a=.00289$. This agrees closely with the datum (.0029) accepted in the above coronal tables, but is much below the value in table 9. Hence the following experiments were made with large coronas in the cubical apparatus.

25. Further results. - The data of the following table 13 were obtained by observing the time of fall for three successive distances, of $2 \mathrm{~cm}$. each (usually), with three stop-watches. The results taken were those cases only where the three intervals observed are nearly the same. The fog was usually precipitated in the long vessel for observing atmospheric nucleation on air nuclei. If the fog line became billowy before the last observation was reached, this was discarded. The mean results are all given at a temperature of about $20^{\circ}$ centigrade.

The new results like the above fail to suggest a smooth curve; though it would be difficult to obtain them under more generally trustworthy conditions than in this large vessel, showing a fog line half a meter long. The slightest variation of temperature, etc., gives rise to an undulatory fog line or produces a washed upper surface to the fog-bank. After a lapse of time of about i min. the demarcation is rarely available. In the very large coronas above $g-b-p$, the color and the character of the coronas is fleeting. In fact, a total change is liable to occur within a fall of one centimeter. Such results have no meaning.

If these data be compared graphically with the results of tables I, 8, and I 3 , Chapter VI, they will be found to agree with them as well as these results 
agree with each other. In fact, the subsidence data fall very nearly on the curve corresponding to the fourth part of table 13.

TABLE I3.-DIAMETER AND NUMBER OF FOG PARTICLES FROM SUBSIDENCE MEASUREMENTS. $d \times 10^{4}=18 \sqrt{v} ; n=9 / d^{3} \times 10^{6} ; \delta p=17 \mathrm{~cm}$.

\begin{tabular}{|c|c|c|c|c|c|}
\hline Corona. & $s$ & Mean $v \times 10^{3}$. & Mean $d \times 10^{4}$ & Mean $a \times 10^{4}$ & Mean $n \times 10^{-8}$ \\
\hline & $\mathrm{cm}$. & $\mathrm{cm} / \mathrm{sec}$. & $\mathrm{cm}$. & & per $\mathrm{cm}^{3}$. \\
\hline w r g & 5.8 & 86 & $5 \cdot 3$ & $3 \mathrm{I}$ & 62 \\
\hline w r $g$ & $5 \cdot 9$ & 82 & 5.2 & 30 & 66 \\
\hline wog & 6.2 & 74 & 4.9 & 30 & 76 \\
\hline w o bg & 6.6 & 68 & 4.7 & $3 \mathrm{I}$ & 86 \\
\hline w o bg & 6.8 & 69 & 4.7 & 32 & 85 \\
\hline$w g b p$ & I 2. & 34 & $3 \cdot 3$ & - & 250 \\
\hline $\mathrm{g} \mathrm{bp}$ & 6.3 & 62 & 4.5 & 28 & 99 \\
\hline g.b p & 6.3 & 62 & $4 \cdot 5$ & 28 & 100 \\
\hline yg br bg & 6.6 & 66 & 4.6 & 3 I & $9 \mathrm{I}$ \\
\hline w r g & 5.9 & 80 & 5.1 & 30 & 68 \\
\hline w r g & $5 \cdot 7$ & $8 \mathrm{I}$ & $5 \cdot I$ & 29 & 66 \\
\hline y $\circ \mathrm{bg}$ & 6.2 & 72 & 4.8 & 30 & 80 \\
\hline Do. & 6.2 & $7 \mathrm{I}$ & 4.8 & 30 & 82 \\
\hline w c g & 5.8 & 83 & 5.2 & 30 & 64 \\
\hline w p cor & 5.2 & 97 & 5.6 & 29 & $5^{\mathrm{I}}$ \\
\hline w o bg & 9.2 & 37 & $3 \cdot 5$ & 32 & 220 \\
\hline yobg & I I.6 & $3 \mathrm{I}$ & 3.2 & 37 & 280 \\
\hline w o bg & $9 \cdot 4$ & 34 & $3 \cdot 3$ & $3 \mathrm{I}$ & 240 \\
\hline wobg & 9.4 & 34 & $3 \cdot 3$ & $3 \mathrm{I}$ & 240 \\
\hline wobg & $9 \cdot 9$ & 36 & $3 \cdot 4$ & 34 & 225 \\
\hline w c $\mathrm{cg}^{\prime}$ & 8.0 & 43 & 3.7 & 30 & I 70 \\
\hline w yollbg & 6.2 & 74 & 4.9 & 30 & 76 \\
\hline
\end{tabular}

The nucleation for the $\mathrm{g} \mathrm{b} \mathrm{p}$ corona $(n=100,000)$ agrees closely with the mean results above $(n=96,000)$, table $I 7$.

Beyond the $\mathrm{g} \mathrm{b}$ p corona $(n=100,000)$, however, the new data for $n$ are with few exceptions much larger than the coronal values, at least with the interval $s=6$ to ro $\mathrm{cm}$. From the difficulty encountered in observing this very slow subsidence and the variable coronas, the discrepancies are much more liable to rest with the subsidence data than with the other (coronal) group.

\section{SUMMARY OF RESULTS FOR NUCLEATION.}

26. Preparation of a table for deducing the nucleation from the observed coronal aperture.-In conclusion, it will be useful to collect all the data obtained for the nucleation on a single sheet. Accordingly a chart was drawn up (too large for convenient reproduction here) containing the results of Chapter. VI, table I, series I and 2, table 8, series I and 2, and table I 3 , series I, 2, and 4, as well as the best subsidence data of the last section. The latter are to be distinguished by a special symbol. The curves are found to lie closely together until the lower $\mathrm{g}$ b p corona $(s=4 \cdot 3-4 \cdot 5)$ is reached, where the first periodicity occurs. They then pass with wider divergence through the middle $\mathrm{g} b \mathrm{p}$ corona 
$(s=6-6.5)$, finally with still wider divergence to the upper $\mathrm{g}$ b p corona $(s=\mathrm{I} 2)$. The nucleations corresponding to these double inflections are about as $1,2,3$ $(n=4,000$ to $5,000,10,000,300,000)$. The mean curve as far as the middle $g$ b $p$ corona is to be separately constructed with the ordinates (nucleations $n$, while the abscissas are apertures, $s$ ) enlarged ro times. The lower periodicity must be ignored from the crowded condition of coronas here; the upper periodicity preferably presented as a discontinuity of the curve.

The graph so obtained was used for all the reductions of the nucleation of Chapter IX and elsewhere. From it table I4 was constructed. The nucleation $n$ may finally be reduced to air at normal pressure and temperature.

TABLE I 4.-FOR THE REDUCTION OF NUCLEATIONS ( $n$ nuclei per cub. $\mathrm{cm}$. of the partially exhausted fog chamber). FROM APERTURES $s$ AND $S$. PRESSURE DIFFERENCE, I $7 \mathrm{~cm}$. of $\mathrm{Hg}$.

s, chord in $\mathrm{cm}$., when the goniometer arms are $19.5 \mathrm{~cm}$. long.

\begin{tabular}{|c|c|c|c|c|c|c|c|}
\hline s. & S. & $n \times 10^{-3}$ & Corona. & $s$. & $S$. & $n \times 10^{-3}$ & Corona. \\
\hline $\begin{array}{l}\text { em. } \\
1.0\end{array}$ & $\begin{array}{l}\mathrm{cm} . \\
\mathrm{I} .5\end{array}$ & I. 5 & \multirow{18}{*}{ normal } & $\begin{array}{l}\text { em. } \\
2.8\end{array}$ & $\begin{array}{c}\mathrm{cm} \text {. } \\
4.3\end{array}$ & 28 & \multirow{4}{*}{ w r } \\
\hline I.I & I. 7 & I. 7 & & 2.9 & 4.5 & $3 \mathrm{I}$ & \\
\hline 1.2 & 1. 8 & 2.0 & & 3.0 & 4.6 & 35 & \\
\hline 1.3 & 2.0 & 2.5 & & 3.I & 4.8 & 38 & \\
\hline I. 4 & 2.2 & 3.0 & & 3.2 & 4.9 & 42 & \multirow{3}{*}{$g^{\prime} B p$} \\
\hline I. 5 & 2.3 & 4.0 & & $3 \cdot 3$ & $5 . \mathrm{I}$ & 45 & \\
\hline 1.6 & 2.5 & 5.0 & & 3.4 & 5.2 & 49 & \\
\hline I. 7 & 2.6 & 6.0 & & $3 \cdot 5$ & $5 \cdot 4$ & 53 & \multirow[t]{2}{*}{ w P cor } \\
\hline 1.8 & 2.8 & 7.0 & & 3.6 & $5 \cdot 5$ & 57 & \\
\hline I.9 & 2.9 & $8: 5$ & & 3.7 & 5.7 & $6 I$ & \multirow{3}{*}{$\mathrm{w} \mathrm{c}$} \\
\hline 2.0 & $3 . \mathrm{r}$ & 10.0 & & 3.8 & 5.8 & 66 & \\
\hline 2.1 & 3.2 & I I 5 & & 3.9 & 6.0 & 70 & \\
\hline 2.2 & $3 \cdot 4$ & 13.5 & & 4.0 & 6.2 & 75 & \multirow{2}{*}{$\mathrm{w} \mathrm{r}$} \\
\hline 2.3 & 3.5 & 15.5 & & 4.1 & 6.3 & 80 & \\
\hline 2.4 & $3 \cdot 7$ & 17.5 & & 4.2 & 6.5 & 85 & \multirow{2}{*}{ ygb o g } \\
\hline 2.5 & 3.8 & 20 & & 4.4 & 6.8 & 95 & \\
\hline 2.6 & 4.0 & 22 & & 4.2 & 6.5 & 100 & \\
\hline 2.7 & 4.2 & 25 & & 4.5 & 6.9 & 120 & w P cor \\
\hline
\end{tabular}

Not E. - The photographs of $\$ \$ 15-2$ I werc made from fog particles eondensed (as stated) on persistent nuclei. No attempt was made to photograph the fog particles condensed on ions or fleeting nuclei, becausc the high exhaustions needed would have endangered the large eondensation chamber. I shall therefore return to the subject elsewhere with a modification of apparatus and with this specific point in view. 


\section{CHAPTER VIII.}

\section{THE CORONAL METHOD OF ESTIMATING ATMOSPHERIC NUCLEATION.}

I. Introductory.-To produce coronas the nuclei must be very closely of the same size, for in a large trough a rigorous uniformity of diameter of fog particle and possibly of distribution is implied, if the corona is to be sharp and brilliant. Particles of even slightly different sizes would give a blurred effect or a mere fog. Therefore, as I understand it, the effect of ordinary dust to some degree vanishes from the corona, and the nucleation observed is probably something more definite. It is for this reason that in spite of very discouraging drawbacks my interest in the subject has not waned, though I am well aware that the effect of chemical products of combustion in winter, such as sulphuric acid, or ionized matter in general, has not been eliminated. One may note, for instance, that the distribution of atmospheric electrical potential is a maximum in winter and falls off in its yearly period in a way similar to the observed nucleation; that there is frequent occurrence of day minima in both cases; that maximum nucleation occurs, as shown in Chapter IX, during the winter months, when one must certainly anticipate the maximum of dust contents during the summer.

The subject of atmospheric nucleation, as a whole, has received enhanced interest in view of its bearing on Arrhenius's theory of the geophysical importance of cosmical, and in particular of solar dust. Some limitation has been put on the light-pressure theory of Schwarzschild, but this has rather stimulated Arrhenius to give a sharper expression of his views, and the theory now appears as the central feature of an admirable discussion of cosmical physics. ${ }^{x}$

I hoped therefore by aid of the present method to eventually add a contribution of my own.

2. Apparatus.--This consists of a rectangular box, $A A, 50 \mathrm{~cm}$. long, I 5 $\mathrm{cm}$. in thickness, and $10 \mathrm{~cm}$., or preferably less, in height, made of some material impervious to water. Wood covered while warm with a thick coating of wax and burgundy pitch answers the purpose very well, and is much lighter than rigid metallic vessels. The front and rear faces of the box are of thick plate glass. This must be kept clean on the inside, and suitable scrapers with a vertical straight edge of soft rubber movable to and fro along the glass by aid of a long horziontal rod should be provided within the box. The rods pass

\section{'Lehrbuch der Kosmischen Physik, Vols. 1. and 11. Leipzig, Hirzel, I903.}


out through perforated corks in the tubes $e$, one on each side, with additional protection to secure an air-tight joint when not in use.

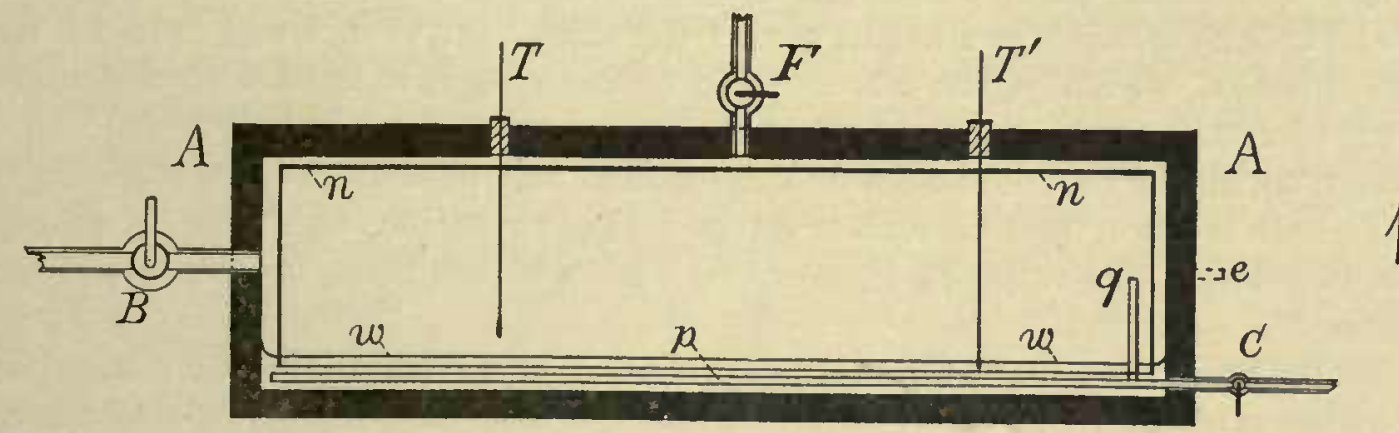

Figure I.-Condensation Chamber. Sectional Elevation.

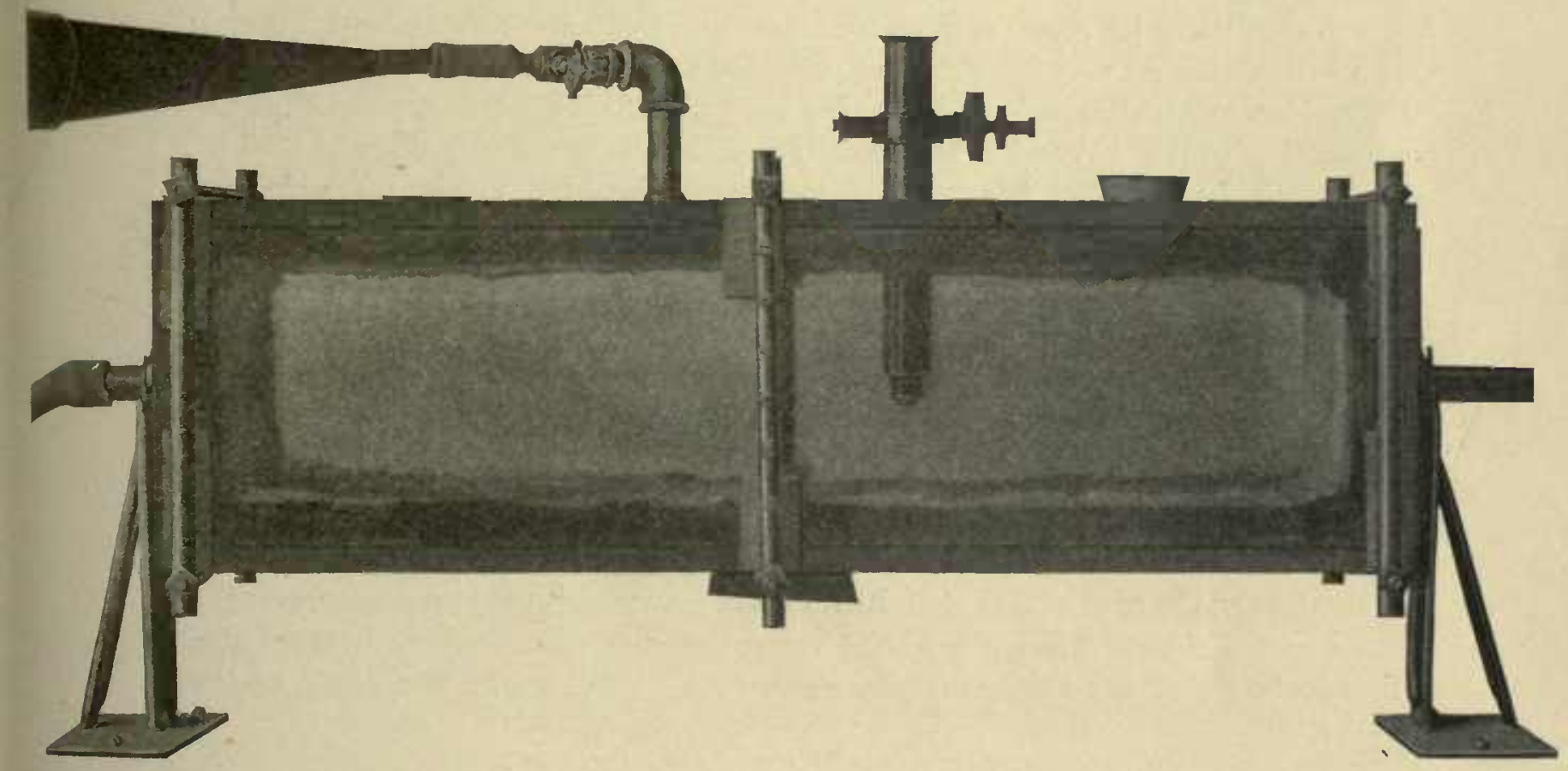

Figure ia.-The Same in Full

The air within the box communicates with the outside by three or more stopcocks, of which $B$ is very wide (more than $\frac{1}{2}$ inch in bore) in order that sudden exhaustion may be made through it. The stopcock $C$ communicates with the atmosphere at a place free from local nucleation, through a length of $\frac{1}{4}$-inch lead pipe; $C$ furthermore communicates with the interior of the box through a flat coil of the same lead pipe, $p$, lying in the bottom of the trough below the water-level, wre. A coil of lead pipe in a water bath may also be inserted 
on the outside of the box, the object being to heat the air to room temperature, especially in winter. ${ }^{1}$ Two thermometers, $T, T^{\prime}$, with their bulbs respectively in the air and the water within the trough, register the temperature. The end of the influx pipe rises to a height $q$, near the axis of the trough and opposite to the outlet, $C$. Finally, the whole inside of the trough is lined with a double layer of cotton cloth, $n n$, supported on a framework of stout copper wire. The trough should be mounted with its longitudinal axis on trunnions in order that the whole interior may be moistened in a single rotation, as shown in figure I $a$, in detail. The stopcock $F$, provided with a cotton filter, is often useful in testing.

The horizontal diameter of the coronas is observed, the point source of light being about 2 meters off on one side and a suitable goniometer about one meter on the other side of the trough. The distances used were $85 \mathrm{~cm}$. and $250 \mathrm{~cm}$. With the eye at about I meter from the fog chamber the apertures of coronas are relatively independent of this distance and at the same time large enough for satisfactory measurement.

An ordinary jet pump suffices for aspiration (with the cocks $C$ and $B$ open); and with an added vacuum chamber provided with a vacuum or mercury gauge, for sudden exhaustion ( $C$ and $B$ having been closed), care being taken that the connecting tubing beyond $B$ is wide. These details are shown above.

3. Diffusion from two opposed surfaces. - The high values of nucleation observed during the winter months will not be received without misgiving, since the air during the very cold weather is nearly dry, and after being heated to $20^{\circ}$ very far from saturation. Deficient saturation, however, would decrease the size of the fog particles and, cat. par., increase the size of the coronas, in this way showing the same result as excessive nucleation. Hence it is necessary to estimate the time which is needed to saturate dry air in the apparatus described in $\$ 2$.

Given a rectangular trough at the bottom of which is a surface of water and at the top of which a surface of saturated eloth, the diffusion problem is equivalent to the case of an indefinite air plate into which the vapor enters on the two exposed sides. Thus if $p$ be the vapor pressure relative to saturation at a distance $x$ above the surface of the water or $a-x$ below the wet cloth, at the time $t$,

$$
p=I-\frac{4}{\pi}\left(\sin \frac{\pi x}{a} \varepsilon^{-(\pi / a)^{2} k t}+\frac{1}{3} \sin \frac{3 \pi x}{a} \varepsilon^{-(3 \pi / a)^{2} k t}+\ldots\right)
$$

where $k$ is the coefficient of diffusion.

If diffusion takes place into a partially saturated atmosphere at an initial pressure $p_{0}$, the factor $4 / \pi$ in the last equation is to be replaced by $(4 / \pi)$ $\left(I-p_{0}\right)$.

${ }^{1}$ In case of a moderately long (20-30 feet), thin ( $t$ inch) influx pipe, experience showed that both the internal coil, $p$, and the external coil are superfluous. They were therefore discarded. 
The question is most conveniently stated for the middle plane, $x=a / 2$, inasmuch as the saturation here is least. Since at $20^{\circ}, k=.23 \mathrm{~cm}^{2} / \mathrm{sec}$, about, the results, if $p_{0}=0$ initially, are shown in table $I$. If the initial saturation is $p_{0}=\mathrm{I} / 3$ or $2 / 3$, the data, as the table shows, imply successively greater saturation throughout than in the preceding case. As the relative saturation left after any exhaustion would not probably be less than $2 / 3$ even in the absence of convection, the inferior limit of saturation shown by these data is excessive.

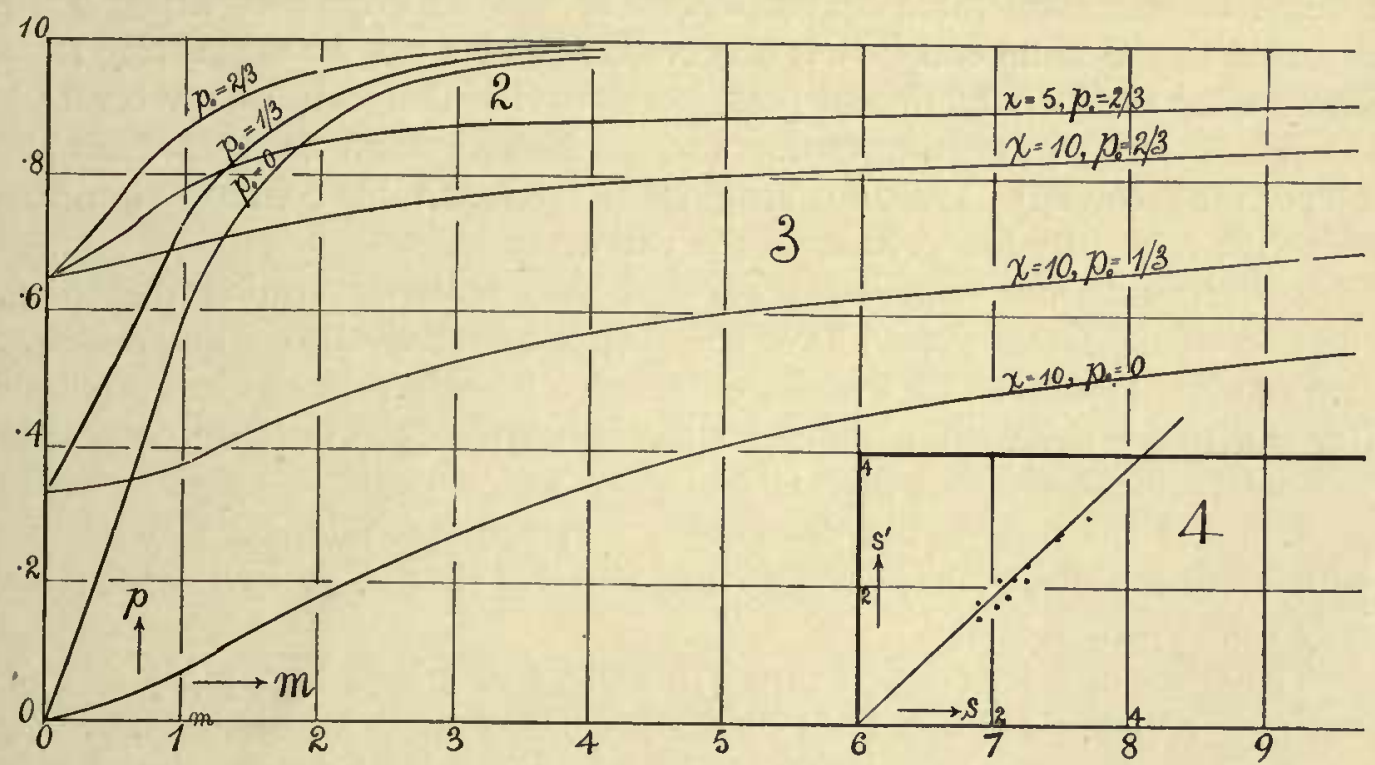

Figures 2, 3, 4.-Graphs Showing the Progress of Diffusion in tile Lapse of Time.

The upper graphs, figure 2 , contain the relative saturation pressures as ordinates at the times given by the abscissas in minutes, according as the initial pressure is $p_{0}=0, p_{0}=I / 3$, or $p_{0}=2 / 3$.

TABLE I.-DIFFUSION OF WATER VAPOR BETWEEN OPPOSED SURFACES; $a=\mathrm{II} \mathrm{cm} ., x=5.5 \mathrm{~cm} ., k=.23$.

\begin{tabular}{|c|c|c|c|c|c|}
\hline \multicolumn{2}{|c|}{$p_{0}=0$} & \multicolumn{2}{|c|}{$p_{0}=1 / 3$} & \multicolumn{2}{|c|}{$p_{0}=2 / 3$} \\
\hline$t$ & $p$ & $t$ & $p$ & $t$ & $p$ \\
\hline $30 \mathrm{sec}$ & .277 & $30 \mathrm{sec}$. & $.5^{16}$ & $30 \mathrm{sec}$. & .762 \\
\hline $\begin{array}{r}60 \\
T 20\end{array}$ & $\begin{array}{l}.587 \\
.866\end{array}$ & $\begin{array}{r}60 \\
\text { I } 20\end{array}$ & $\begin{array}{l}.722 \\
.010\end{array}$ & $\begin{array}{r}60 \\
\text { I } 20\end{array}$ & $\begin{array}{r}.864 \\
.056\end{array}$ \\
\hline 180 & .957 & 180 & $.97 \mathrm{I}$ & I 80 & .986 \\
\hline
\end{tabular}

Thus in 3 or 4 minutes the air plate in the trough may be considered saturated under the most unfavorable conditions. In the aspiration of fresh air through the trough the maximum rate was about 4.5 lit./min., the usual rate 
I. $5 \mathrm{lit} / \mathrm{min}$. The capacity of the trough, including parts above the cloth lining, was about II. 3 liters. Hence 3 to 5 minutes suffice to renew the air, and each particle remains in the trough from 1.5 to 4 minutes. The time needed for adjustments after the influx pipe was shut off and prior to sudden exhaustion was about 1.25 minutes. Therefore the total time for saturation was 2.7 to 5 minutes, which should fully suffice even in case of diffusion alone.

The conditions, however, are much more favorable as the influx and efflux currents evoke considerable convection. The lightness of water vapor is itself favorable to the same end. It is observed, for instance, on exhausting immediately after the introduction of phosphorus nuclei, that filamentary condensation is in evidence, denoting currents upward axially and downward near the walls of the receiver. ${ }^{1}$ These fog strands are the inevitable convection currents due to the relatively low density of the vapor.

4. Miscellaneous tests. - If there had been under-saturation the coronas found on condensation should have been larger in non-saturated and smaller in more saturated parts, which was not observed. Sudden exhaustion immediately after shutting off the influx showed a somewhat enlarged uniform corona, but even enlargement was not invariable.

Whether the fast or the slow influx specified was adopted proved to be without marked effect for reasonable differences of time, $i . e$, such as would not imply time losses of nuclei.

The effect of a long influx pipe (ro meters of $\frac{1}{4}$-inch lead pipe) and of a short pipe I meter long could not be sharply differentiated, owing in a measure to the cotemporaneous variation of atmospheric nucleation. So the presence compared with absence of the coil in the water bath (24 turns each about 3.5 $\mathrm{cm}$. in diameter) showed a negligible difference.

Experiments were made with regard to the usefulness of this coil for keeping the influx air at room temperature both by filling the bath with abnormally hot water $\left(40^{\circ} \mathrm{C}\right.$.) and with broken ice. The effect on the apertures of the coronas was in both cases of little importance. Hence, except on very cold days, the water bath and coil may be withdrawn altogether. The atmospheric air after traversing the ro meters of influx pipe is already sufficiently heated to be introduced into the condensation chamber directly.

Tests were also made with a U-tube loosely filled with wet sponges and with a drying tube one meter long containing phosphorus pentoxide. In neither case was a definite effect on the coronas ascertained.

Freedom from leakage was finally tested by filtering the air. The coronas on sudden exhaustion showed a gradual decrease to complete evanescence.

5. Diffusion from a single surface. - The case is naturally less favorable if the upper wet surface (double cotton cloth) is omitted. The computation may be made from an expansion of Kramp's integral, so that

$$
\begin{aligned}
p= & \mathrm{I}-\frac{2}{\pi}\left(\frac{x}{(4 k t)^{1 / 2}}-\frac{x^{3}}{3 \times \mathrm{I}(4 k t)^{3 / 2}}+\frac{x^{\mathrm{s}}}{5 \times 21(4 k t)^{3 / 2}} \ldots\right) \\
& { }^{1} \text { Smithsonian Contributions, No. }{ }^{\mathrm{I}} 373 \text {, I } 902 .
\end{aligned}
$$


where $p$ is the vapor pressure relative to saturation at a distance $x$ above the surface of the liquid at the time $t$ for the diffusion coefficient $k_{.}=23$. The data computed suffice to locate the curves which are sufficiently given in figure 3 for $x=5 \mathrm{~cm}$. the middle plane, and $x=10 \mathrm{~cm}$. the top plane of the trough. When the initial saturation is $1 / 3$ or $2 / 3$, the coefficient $2 / \pi$ is to be modified as stated above. Figure 3 shows the corresponding results.

If it were not for convection, therefore, such an apparatus would be unsuitable, for even after waiting 5 minutes, the air at the top, $x=10 \mathrm{~cm}$., for an initial saturation of $2 / 3$ is but .8 saturated from diffusion alone.

One might therefore expect to obtain distorted coronas campanulate in outline, small below and large above, whenever condensation is produced within a few minutes after closing the inlet. Yet such is never the case if less than a minute is allowed after influx ceases. Granting that 2 to 4 minutes are needed on the average for a particle to pass through the trough, as stated above, if exhaustion is made immediately after closing, the top layer is but $2 / 3$ saturated. Under these conditions there is in fact an unusually large green centered faint corona with a horizontal band of crimson color running through it. Half a minute later, however, the figure is quite regular again, showing that the convection of light vapor must be very active. After 2 minutes subsequent to the closing of the influx pipe, the air may be regarded saturated except in the coldest weather.

6. Absorption and decay of muclei.-The losses in the influx pipe are difficult to determine because of the variation of atmospheric nucleation. The observer is left in doubt whether a given difference is due to absorption in the pipe or to causes without. The experiments incidentally made throughout the long experience of Chapter IX showed no serious discrepancy.

The possibility of loss of nuclei on contact of dry air with the saturated gas in the condensation chamber is an independent question. It is also to be borne in mind that nuclei may possibly be produced by the sudden contact within the chamber. No evidence is forthcoming.

If the nuclei after being introduced into the receiver are solutions, some estimate of their persistence may be formed from my experiments on solutional nuclei, by treating the loss as if it occurred at the boundary of the vessel only. If the nucleation falls off from $n_{\mathrm{o}}$ to $n$ in the time $t$, and $k$ is the absorption coefficient,

$$
n / n_{0}=\varepsilon^{-2(\mathrm{r} / r+\mathrm{r} / \mathrm{h}) k t},
$$

where $r$ and $l$ denote the radius and length of the cylinder in which absorption takes place. In case of comminuted pure water, $k=5$ to $10 \mathrm{~cm} . / \mathrm{min}$., and the nuclèi should quite vanish in a few minutes.

$$
\begin{array}{cr}
t=\mathrm{I} \min . & n / n_{o}= \\
2 & . \mathrm{T} 54 \\
3 & .023 \\
& .003
\end{array}
$$


If the nuclei are derived from very dilute solutions, like river water, an average value $k=$.I may be taken, whence if

$\begin{array}{rr}t=\mathrm{I} \min . & n / n_{0}=.96 \\ 3 & .89 \\ 5 & .83 \\ \text { I0 } & .69 \\ 50 & .15 \\ 100 & .02\end{array}$

The reduction within 3 minutes will not exceed so per cent., which would usually lie within a given type of corona. The datum $k=. \mathrm{I}$, moreover, corresponds closely to the values found for phosphorus and other nuclei. Hence, if the type of corona changes after I or 2 minutes' waiting, it may be considered certain evidence that the air is not saturated and the diffusion error predominates. Owing to the difficulty of avoiding either insufficient saturation or excessive time losses, some of my observations in Chapter IX contain data for two different aspirating currents, the faster corresponding to about 3 minutes' sojourn of the nuclei in the receiver, the other to a time longer than 5 minutes. In this way the effects of under-saturation which are most to be feared are guarded against, while the faster current gives data falling short of the absolute nucleation by not more than ro per cent. In the course of time this also was abandoned as superfluous.

7. Effect of pressure difference. - It is next to be considered whether the pressure difference, $\delta p$, used in the exhaustions is pronounced enough to catch all the nuclei. This is of particular interest as a safeguard against low numbers in the nucleations obtained.

The usual value, $\delta p=17 \mathrm{~cm}$., corresponds to the following pressure ratios and adiabatic temperature reductions in air, $\left.\left(\left(76-p^{\prime}\right) / 76-p^{\prime}-\delta p\right)\right)-41273=9^{\prime}$ if $p^{\prime}$ is the vapor pressure of water and $q^{\prime}$ the reduced absolute temperature.

$\begin{array}{rrr}10^{\circ} & \text { Pressure ratio, } \mathrm{I} .292 & 9^{\prime}=254.7 \\ 20^{\circ} & \text { I.297 } & 263.4 \\ 30^{\circ} & \text { I.34 } & 268.8\end{array}$

For comparison data were gathered with a larger pressure difference, $\delta p=$ $22 \mathrm{~cm}$., for which the values are

$\begin{array}{lll}\text { I0 } & \text { I. } 4 \text { I } 4 & 245.5 \\ 20 & \text { I.4 I6 } & 254 . \mathrm{I} \\ 30 & \text { I.432 } & 26 \mathrm{I} .5\end{array}$

Clearly, the coronas for the larger temperatures and temperature differences must be smaller, ccet. par., in view of the greater quantity of moisture precipitated. The data for $m$, the quantity of moisture precipitated per cubic centimeter of saturated air, have been computed by the method of C. T. R. Wilson and J. J. Thomson and are given in the following table 2. Here $t_{\mathrm{r}}$ is the initial temperature, $t_{2}$ the temperature before and $t$ after condensation. 
A CONTINUOUS RECORD OF ATMOSPHERIC NUCLEATION.

TABLE 2.-PRECIPITATIONS AT DIFFERENT TEMPERATURES AND PRESSURE DIFFERENCES. $\quad y=\left(p-p^{\prime}\right) /\left(p-p^{\prime}-\delta p\right) ; p=76$

\begin{tabular}{|c|c|c|c|c|c|c|c|c|c|c|c|c|}
\hline \multicolumn{5}{|c|}{$\delta p=8.5 \mathrm{~cm}$} & \multicolumn{4}{|c|}{$\delta p=17 \mathrm{~cm}$} & \multicolumn{4}{|c|}{$\delta p=22 \mathrm{~cm}$} \\
\hline$t_{z}$. & $t_{2}$ & $t$ & $m \times 10^{6}$ & $y$ & $t_{2}$ & $t$ & $m \times 10^{6}$ & $y$ & $t_{2}$ & $t$ & $m \times 10^{6}$ & $y$ \\
\hline${ }^{\circ} \mathrm{C}$ & ${ }^{\circ} \mathrm{C}$ & ${ }^{\circ} \mathrm{C}$ & grams & & ${ }^{\circ} \mathrm{C}$ & ${ }^{\circ} \mathrm{C}$ & grams & & ${ }^{\circ} \mathrm{C}$ & ${ }^{\circ} \mathrm{C}$ & grams & \\
\hline $10^{\circ}$ & $-3 \cdot 4$ & $+3 \cdot 4$ & 2.1 & I. I 2 & $-\mathrm{I} 8.3$ & -4.5 & 3.7 & I. 27 & -27.5 & - IO.I & 4.2 & I. 39 \\
\hline $20^{\circ}$ & +5.8 & I 4.7 & 2.6 & I. I I & -9.6 & +8.8 & 4.6 & ×. 26 & - I 8.9 & -4.6 & $5 \cdot 5$ & I. 38 \\
\hline $30^{\circ}$ & I 5.8 & 25.7 & 2.8 & I.10 & -4.2 & I 9.6 & $5 \cdot 7$ & I. 24 & - I I. 5 & 17.0 & 6.7 & I. 35 \\
\hline
\end{tabular}

Since $m=n \pi d^{3} / 6$, if there are $n$ fog particles per cubic centim. each of the diameter $d$, and since $s d=D$ where $s$ is the aperture of the coronas with an arbitrary goniometer and $D$ the corresponding constant,

$$
s \sqrt[3]{m}=.524 D \sqrt[3]{n}
$$

which is constant for a given nucleation. Thus the relation between $s^{\prime}$ and $s$ at $\delta p=22 \mathrm{~cm}$. and $\mathrm{I} 7 \mathrm{~cm}$., respectively, may be written

$$
s^{\prime} / s=\left(m / m^{\prime}\right)^{1 / 3}=.95^{2} .
$$

In figure 4 the line $s^{\prime} / s$ has been constructed and the observations grouped with reference to it, showing that the curve reproduces the experiments fairly well. The exceptional cases are all too low; or, in other words, at the higher pressure difference, $\delta p=22$, which requires a longer period of waiting after influx ceases, relatively fewer nuclei are entrapped. From this one concludes not only that from the medium, if saturated, all the nuclei are precipitated at $\delta p=17$, but that at the higher pressure difference the time needed for adjustment is excessive and that the time loss of nuclei in the receiver frequently becomes appreciable.

On the other hand, if $\delta p$ exceeds $22 \mathrm{~cm}$. for the given apparatus, the conditions of spontaneous condensation of dust-free moist air are initiated and continue thereafter with increasing intensity for higher pressure differences.

8. Precipitation per cubic centimeter.-To determine $m$, I have heretofore proceeded as follows: In a mixture of $x$ grams of vapor, $y$ grams of air, and $\mathrm{I}-(x+y)$ grams of water, the absorption of heat due to a rise of temperature $d 9$ at constant volume was taken as $C(1-(x+y))+h x+r d x / d 9+c y$, per degree, where $C, c$, and $h$ are the specific heats of water, air at constant volume, and saturated vapor, respectively, and $r$ the latent heat. Since $h-C=d r / d g$ $-r / s, h$ may be eliminated. Again the absorption of heat due to a volume increase $d v$, at constant temperature is if $\gamma$ is the heat ratio $r(d x / d v) d v+y^{2} c$ $(y-\mathrm{I}) d v / v$. If the expansion is adiabatic the total heat absorption is nil and the equation thus obtained may be reduced eventually to

$$
\frac{d}{d \theta}(r x / 9+(C(\mathrm{I}-y)+c y) \lg 9) d \rho+y c(\gamma-\mathrm{I}) d \lg \vartheta
$$


As this is not a perfect differential I assumed the relation of $v$ and 9 to be approximately that of air, $v^{\gamma-r} \Omega=$ const., supposing that I could subsequently correct for the precipitated water by successive approximation. In this way one obtains at the beginning and the end of the exhaustion for any two temperatures 9 and $q^{\prime}$ (using accents throughout for the latter case), after integrating, $x^{\prime} / x=\left(9^{\prime} / r^{\prime}\right)\left(r / 9-\lg \left(9^{\prime} / 9\right)\right)$, where $\left(x-x^{\prime}\right) / x$ is the mass ratio of precipitated liquid to the original vapor. In my work thus far ${ }^{1}$ the results computed in this way and for $\delta p=17 \mathrm{~cm}$. were at $10^{\circ}, 20^{\circ}, 30^{\circ}, \mathrm{m} \times 10^{6}=$ .59 , I.I 3, I.85 grams, respectively, where the corrections for precipitated moisture have been applied and $\delta p$ is an isothermal value. If $\delta p$, the observed pressure reduction, were treated adiabatically the corresponding values of $m \times 10^{6}$ would be $.42, .76,1.28$.

The results for $m$ so obtained will have to be rejected as they are much too small (probably because the pressure coefficients were overlooked) when compared with the more direct approximation of Wilson and Thomson. The value of $m$ is here found as an intersection by making the $m$ values compatible with the vapor density curve for water. These data have already been computed for the pressure difference $\delta p=17$ above, table 2, and will be used in Chapter IX.

9. Relation of nucleation to aperture of corona.-A summary of the method of deducing the nucleation (number of nuclei per eubic centimeter) from the observed coronal aperture for an observed pressure difference $(\delta p=\mathrm{I} 7 \mathrm{~cm}$.), in the given apparatus, has already been filly explained in Chapter VII, $\$ 26$, and needs but little further reference here.

Measurements of the aperture $s$ would naturally be made as far as the inside of the red ring or the circumference of the eventually white disc; but in such a case they bring out very strong periodicity in the first place, and are soon subject to large errors due to the increasingly vague and washed outline of the dise. Hence measurement is more appropriately made to the dark blue ring which limits the green coronas or to the dark interior of the green ring in the crimson coronas. These lines are not only sharper but they reduce the periodicity. It is understood that with air nuclei and $\delta p=I 7$, the green corona is seldom exceeded. Otherwise it would be necessary to increase the uniform pressure difference, against which there is no objection other than the increased practical inconvenience, provided the pressure difference for which saturated air condenses spontaneously (above $\delta p=22 \mathrm{~cm}$. in the above apparatus) is not exceeded.

ro. Absence of electrification in cases of sudden condensation and of sudden eraporation.-It has long been known as the result of most painstaking observations, that neither in cases of ordinary evaporation nor of condensation is there an accompaniment of electrification. On the other hand, when a mass of water is suddenly shattered, as in jets, the electrification is marked, while it is

${ }^{1}$ Smithsonian Contributions to Knowledge, No. ${ }^{1} 373$. 
not obvious that the underlying eause is friction. The electrification soon vanishes, however, whereas the nueleaticn persists. The question thus arises, therefore, whether in ordinary slow evaporation the absence of an electrical effect may not be due to the possibility of charges vanishing too quickly to be noticeable. It seened worth while, therefore, to examine the case for the sudden condensation and the rapid evaporation of fog particles.

To test this question a graduated electroseope was introduced into the condensation chamber. To insulate it in the saturated atmosphere, the stem was enclosed in a hard rubber tube, as in figure 5 , open below and only in contact with the stem at the top by a sealing-wax joint. The tube was thoroughly dried before insertion into the condensation chamber, and the instrument showed satisfactory insulation for a half-hour or more, after which it was removed for desiceation prior to new experiments.

Condensation was produced by exhaustion as usual, and the nuclei used were obtained from air as well as from phosphorus. The method of procedure consisted in observing the normal leakage of the eharged electroscope by observations made every half-minute. Nuclei were then introduced and the experiment repeaterl with the alternate sudden production and sudden dissipation of fogs between each observation.

The two curves of leakage were not distinguish-

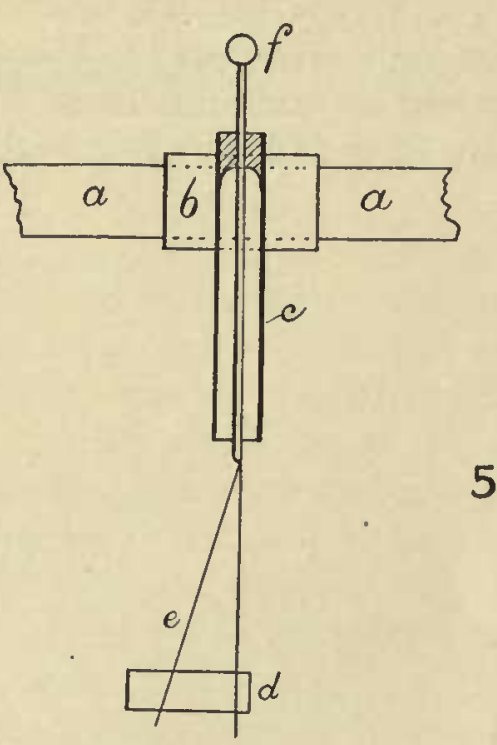

Figure 5-Apparatus to Detect Ionization Produced by SUdden Condensation.

able, even when the fogs were of the densely opaque character due to phosphorus, and the error of the method was about 5 per cent., with somewhat larger uncertainty for the ease of fog evaporation.

Pressure differences, however, were kept below the limit (much above $\delta p=22 \mathrm{~cm}$. in the given apparatus) at which saturated air spontaneously condenses without nuclei. This phase of the question, which in fact is rather the more interesting one, is thus left at issue. If, for instance, dust-free air is always slightly ionized and this contains unstable systems, the inerease of ionization by sudden exhaustion in virtue of the unstable molecules referred to is by no means excluded. The question has been touched above.

II. Conclusion.- In the above paragraphs I have endeavored to present the complications to which the method of coronal registry of atmospheric nucleation is incident, complications which were not anticipated and for which I was altogether unprepared. In the course of my work I made several unfortunate blunders in endeavoring to reduce the data to absolute values, but apart from these the greater number of discrepancies (as, for instance, the periodic distribution of nucleation in terms of aperture) could not have been forescen at the outset. 
With regard to atmospheric nucleation, it seems to me, in addition to the remarks made in $\$ \mathrm{I}$, that the variety and importance of the phenomena which are now attributed to the invasion of solar and cosmical dust into the atmosphere, such as certain variations of atmospheric pressure, of atmospheric electricity, of terrestrial magnetism, of auroral display, etc., induce one to wonder why continuous and systenuatic records of atmospheric nucleation, other than the series obtained at Ben Nevis during the period when Aitken's observations gave to the subject widespread interest, have not long since been included among the records of observatories. Surely in discharging its remarkable and varied cosmical functions, this dust from afar, if at all persistent, must some day be detected undisguised. 


\section{CHAPTER IX.}

THE NUClEATION OF THE ATMOSPHERE OF THE CITY OF PROVIDENCE.

\section{INTRODUCTION.}

I. Preliminary.-In May, igo2, Mr. Harvey Davis, at my request, put up an apparatus in the laboratory of Brown University for counting the number of nuclei in the atmosphere, by measuring the coronas producible with such air under appropriate conditions. The apparatus gave promise at once; but $\mathrm{Mr}$. Davis was unexpectedly called away before the observations became fruitful, and the project was temporarily abandoned. Believing that an instantaneous method of at least estimating the degree of atmospheric nucleation is a desideratum, and must throw light eventually on the origin and character of the nuclei in the atmosphere, I have undertaken the furtherance of the work myself, and the results obtained since October, I902, after the indications of the apparatus had become warrantable, are given in the present chapter. I may add that Mr. Davis and Mr. R. Pierce, Jr., had been at work for some time on the measurement of the daily variation of the solar constant (a project then set on fost by the U. S. Weather Bureau), and that I had hoped from the coordination of the two classes of data to reach conclusions of interest.

The chapter, therefore, contains nearly two years of continuous record of the nucleation of Providence, R. I. The observations were made in the park of Brown University, which is surrounded, however, on all sides by the city. The density of population lies to the west and sonthwest. A station entirely away from the habitations of man would naturally have been preferable, but this desideratum was at the outset out of the question.

2. Apparatus. - The apparatus and method by which the present results were obtained are fully given in the chapters above, particularly in Chapter VIII, and need not therefore be further considered here. In figure I, however, I have shown a simplified train of apparatus used in some of my early work (October 2, 1902, to March I 5, I903). Here $d$ is the influx tube, $B$ the water-bath at room temperature, $t$ the thermometer, $A$ the condensation chamber (the water $w$ is made to wet the sides by rotating $A$ before expansion), $R$ the vacuum chamber, $G$ the gauge, $S$ the pipe to the suction pump. Stopcocks $b$ and $c$ control the exhaustion, together with $a$.

3. Classification of results. - The results themselves may be embraced in two groups. The first group of observations, from October 2, 1902, to March 
I 5, I 903 , were originally made with reference to an arbitrary scale of nucleation. There are many reasons why it would be difficult to reduce them accurately to the uniform scale of the present chapter. Among these, frequent changes of apparatus, measurement of aperture to the red ring, and the absence of a variety of details of record discouraged such an attempt. The reduction, therefore, is approximate; but the values of nucleation are roughly absolute and quite

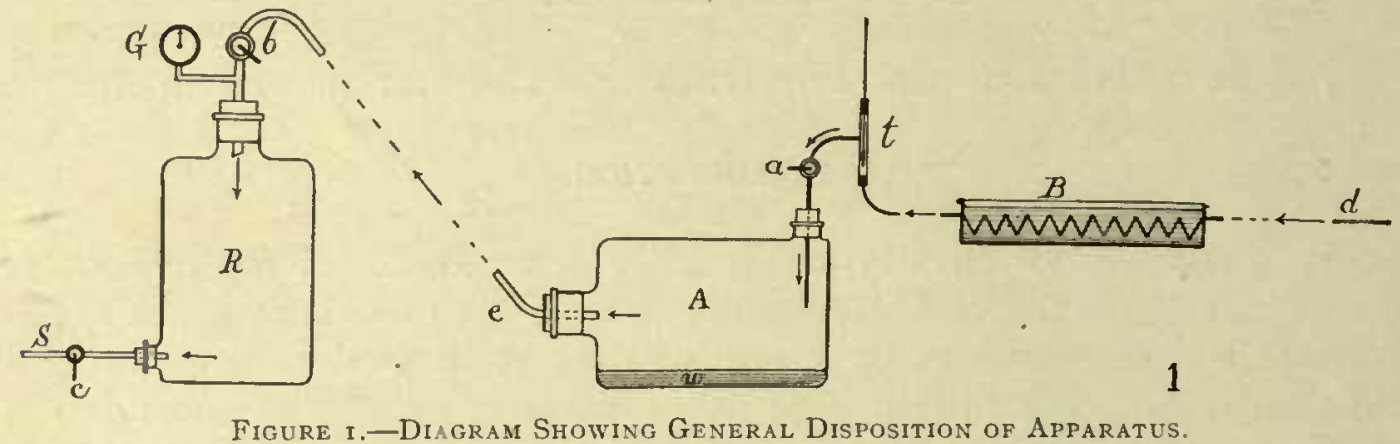

satisfactory as relations, and they thus meet most of the demands made upon them. In view of their preliminary character they will be given in charts only. In these cases the condensation chamber was a large aspirator flask (fig. I), placed horizontally so that the measurement of aperture was made parallel to the axis of the cylinder.

The second group of observations (by far the larger number) from March I 5, I903, to Oetober 3I, I904, were obtained with apparatus perfected as explained above. These will therefore be given both graphically and in tables. The latter will contain a variety of relevant data.

\section{FIRST GROUP OF OBSERVATIONS.}

4. Early observations. - The charts Nos. I-I2, comprehending the first group of results, follow. The nucleations, $n$, are laid off vertically, and in charts $\mathrm{I}-8$ they are given in full ( $n$ nuclei per cubic centimeter). In the remaining charts (Nos. 9, et seq.) the number of nuclei are given in thousands $\left(n \times 10^{-3}\right)$ for brevity. The temperature and winds (for $\left.8 \mathrm{~A} . \mathrm{M}.\right)$, taken from the weather maps of Block Island and Nantucket, will often be entered, but as the work progressed superfluous data were gradually dropped. In some of the charts the changes of temperature, $\delta t$, and of barometric pressurc, $\delta p$, are also given, with the occurrence of clear weather, $\odot$, eloudy, $\odot$ and partly cloudy weather, sunshine $\mathrm{S}$, rain $\mathrm{R}$, snow $\mathrm{Sn}$, cold wave e.w. or -9 , etc. References to Fahrenheit and to centigrade are indicated.

The nucleation (chart I) begins low on the $2 \mathrm{~d}$ of October, Igo2, with the rain, but thereafter increases nearly fourfold with the bright weather of the succeeding days. Note the prevailing winds from the north on the 5 th and 6 th, clouds and rain usher in a second minimum. On the fair days succeeding the 
nucleation is not as high as before until the Ioth, when a sudden enormous increase occurs, cotemporaneously with fall of temperature. The high maximum is succeeded by an equally low minimum, brought on by the rainy weather of October I I and I2. The nucleation then rises during the succeeding fair days, to fall to a fourth minimum during the rain of October 14 . No doubt
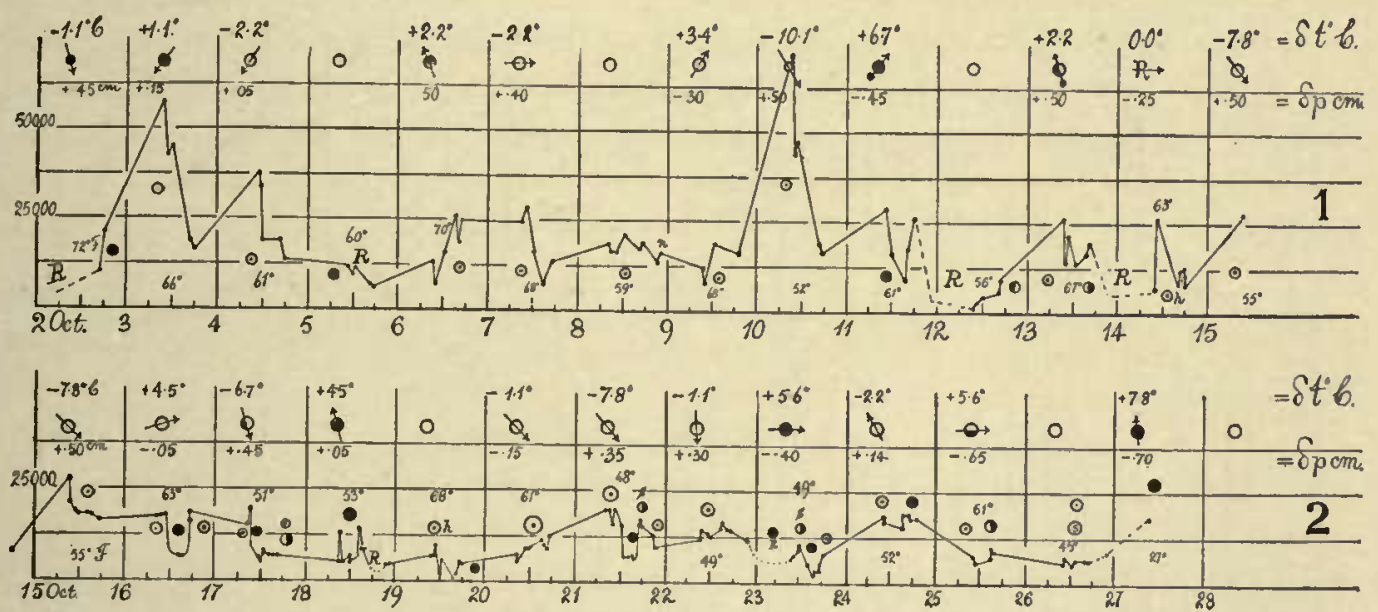

Charts i-48.-Daily Observations of the Atmospheric Nucleation of Providence. Number of Nuclei $(n)$ per Cubic Centimeter, Laid off Vertically. In Charts $1-8, n$ is given in Full In Charts $9-48, n$ is given in Tirousands per Cubic Centimeter. Charts i-i2, are Reduced from an Older Arbitrary Scale. Local Winds, Rain (R), Temperature, etc., are Given in the Charts, Arrows Showing tue Direction in which the Winds Blow. Clear ๑, Partly Cloudy a, and Cloudy Weather, etc., are Indicated with the Usual Signs.

some variation is due to the variation of the temperature of the apparatus, for which no correction ${ }^{1}$ was made, but the rain and fair weather effects as a whole are unmistakable. The maxima on the 4 th and Ioth correspond to anticyclonal conditions.

The apparatus was now modificd in a way which need not here be instanced. What is noteworthy in the next data (chart 2) is the occurrence of sharp minima on October I6, I7, 2I, 23, cotemporaneous with the passage of dense cloud masses over the sky. In three of these cases the curve rises as soon as the sky clears; on October I 7 it does not do so, but the curve runs into the overcast condition of October 18 . The pronounced minimum on October 19 during clear weather shows that sunshine can not be a reason for an abundance of nuclei. Similarly there is high nucleation on October 24 and 27 , simultaneously with an overcast sky. A number of night observations on October I 8 give no evidence of unexpected behavior.

On October 27 the apparatus was again modified, by substituting a long cylinder for the aspirator flask thus far used as a condensation chamber. The data (chart. 3) begin with high nucleation for an overcast sky and fall off to the rain storm on October 28 . The high nucleations are very fluctuating,

${ }^{1}$ Correction for temperature was applicd at a later date, but thereafter again abandoned as untrustworthy. 
which would be even more apparent if night observations had been made. The cause is obviously convection, and the diagram necessarily presents marked similarity to a wind curve.

The feature of the data from November I-Io (charts 3 and 4 ) is the ten-

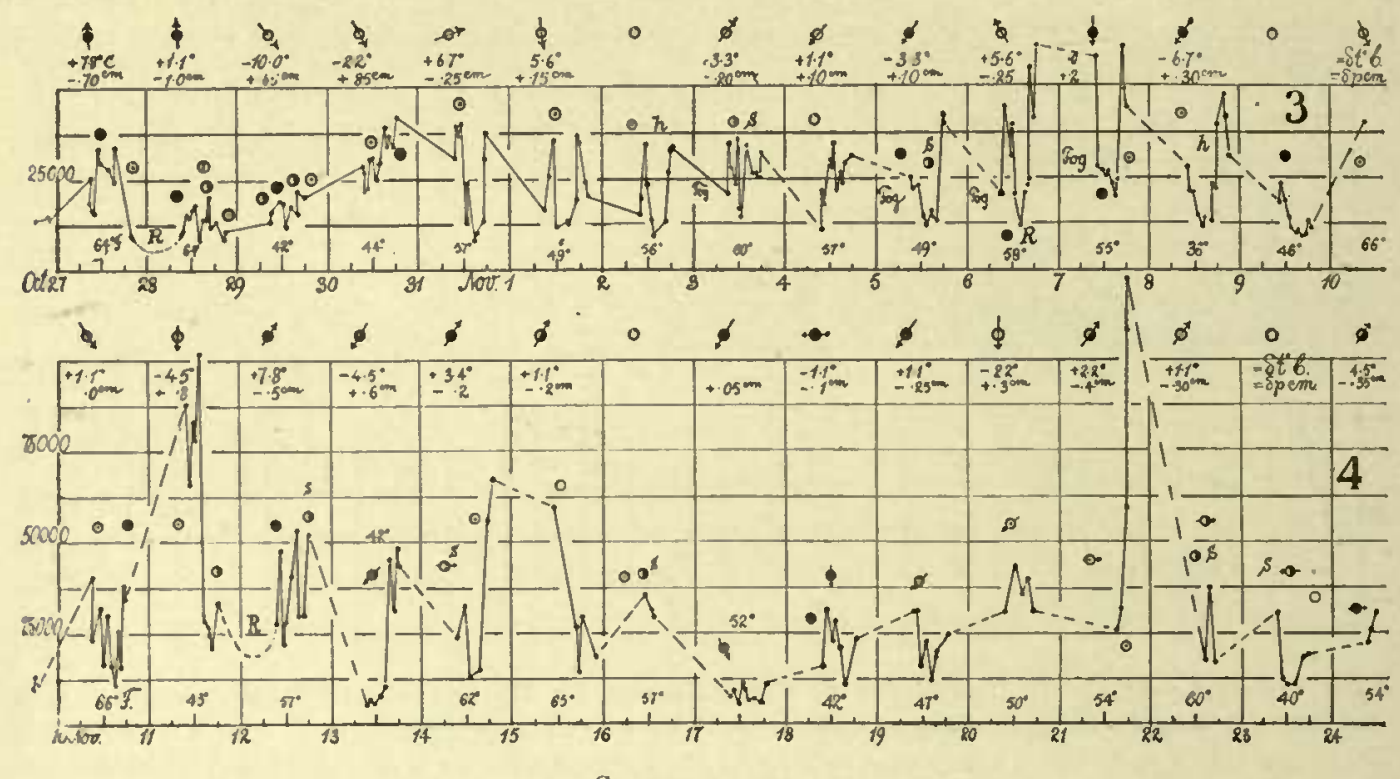

dency to notched minima at mid-day or in the early afternoon. The maximum on the IIth coincides with a slight drop in temperature, but beyond this the temperature is so nearly constant until November 24 that reasons for the variations of the figure are not forthcoming. The remarkable rise on the 2rst may

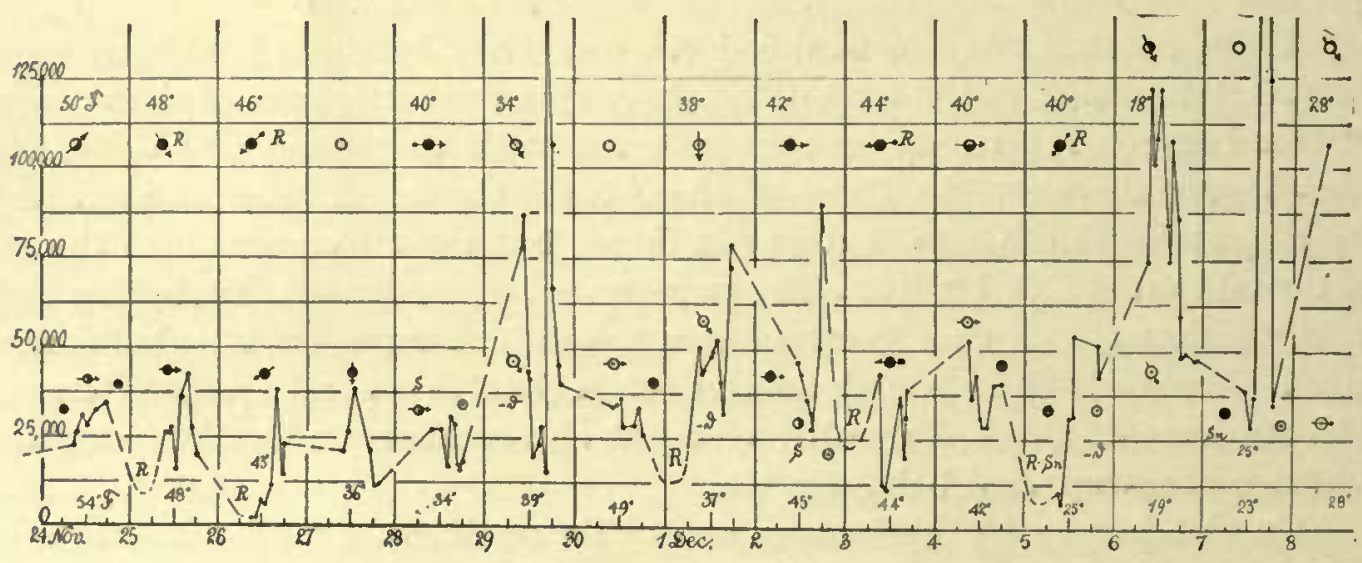

CHART 5 .

be noticed. On November 14 and 23 there are mid-day minima, but others are not apparent. During the remainder of November (chart 5), rain minima occur on November 25, 26, and at the end of November 29. The maximum on 
November 29 is clearly associated with the drop in temperature, and there is here one mid-day minimum but no others.

Throughout December cold waves associated with northwesterly winds are productive of maxima. After the rain of December I the nucleation rapidly rises, accompanying a second drop in temperature. The maximum is moderately sustained until the $3 \mathrm{~d}$, where there is fall into the rain minimum, partial recuperation thereafter, and then another drop into the rain of the 5 th.

This rain soon changes into snow and from here the nucleation (chart 6) of the cold wave begins, lasting from the 6th to the thaw on December i I. One

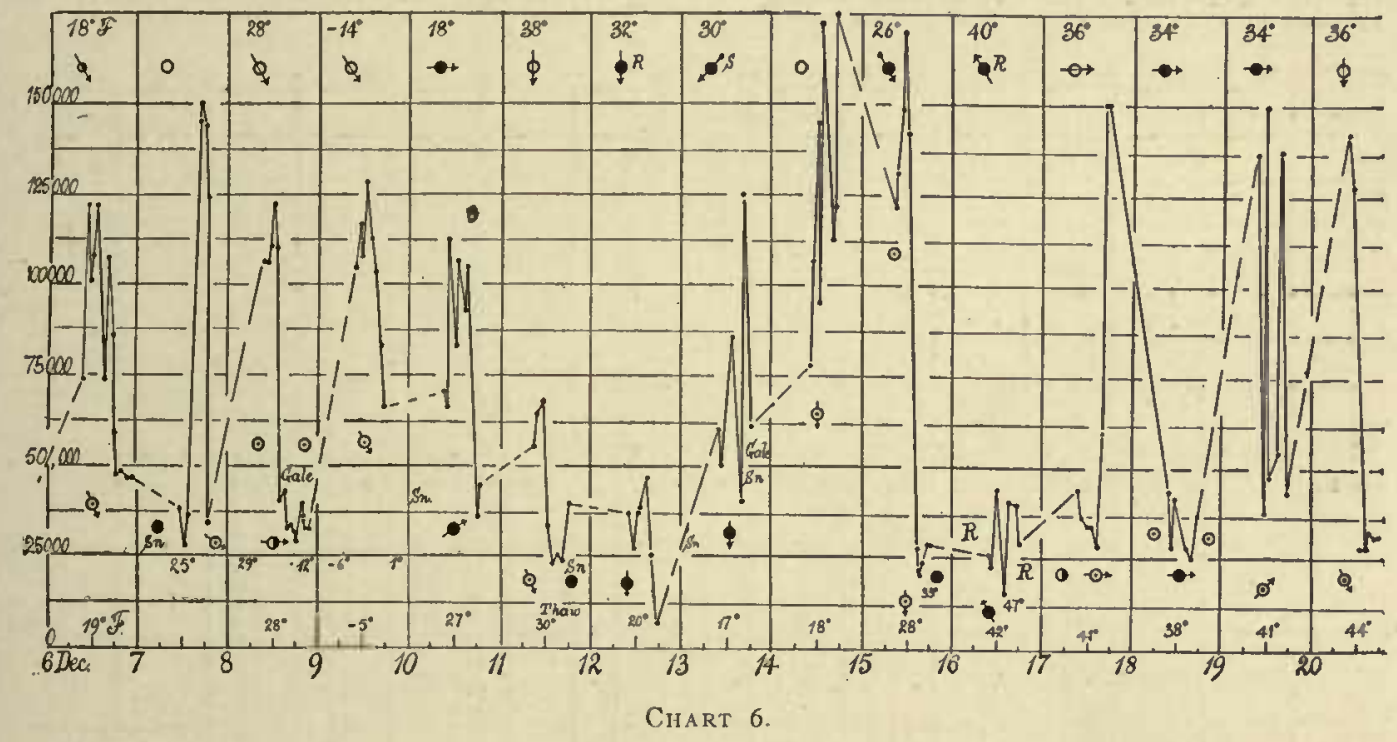

may note the reduction due to very cold snow on December 7 , and the curious minimum accompanying the gale on the evening of December 8 . The maxima on the 9 th and roth accompany northerly winds and a drop in temperature. The very remarkable maximum from December I 3 to December 16 follows the fall of temperature after the thaw on December 15 , introducing a rain minimum. This interesting region is no doubt referable to the snow-storm on the evening of December I 3 , the winds remaining northerly. The ground was frozen on December $\mathrm{I}_{3}$ and $\mathrm{I}_{4}$. The region is not due to temperature. The maxima on December $\mathrm{I} 7$ and 20 are less sustained, but they come with temperatures above freezing and westerly winds. The absence of night observations is a dilemma in these cases. The low nucleation indicated on December 20 continued for several days (December 20 to 23 ) beyond the limits of chart 6 , but is shown on chart 7 .

One may regard it as a general rule established by these charts, that the nucleation increases when temperature suddenly decreases, but that temperature is not the sole or ultimate factor involved. To see whether this view would work out in detail, I compared the observatory thermograph data for Providence during the three months (kindly placed at my disposal by my 
colleague, Prof. Winslow Upton) with the data for nucleation given in the above charts. A limited degree of similarity was in this way made out for cold weather, both in the general march of temperature throughout longer intervals and in its detailed variations. Thus the mid-day minima are obviously counterparts of corresponding temperature maxima. There were, however, many outstanding discrepancies, and it seemed probable that much of this would be referable to local differences, as the observatory is differently situated and at a distance from the laboratory. Beginning with December 28 , I have, therefore, been making daily temperature observations simultaneously with the coronal observations, using a long mercury thermometer placed be-

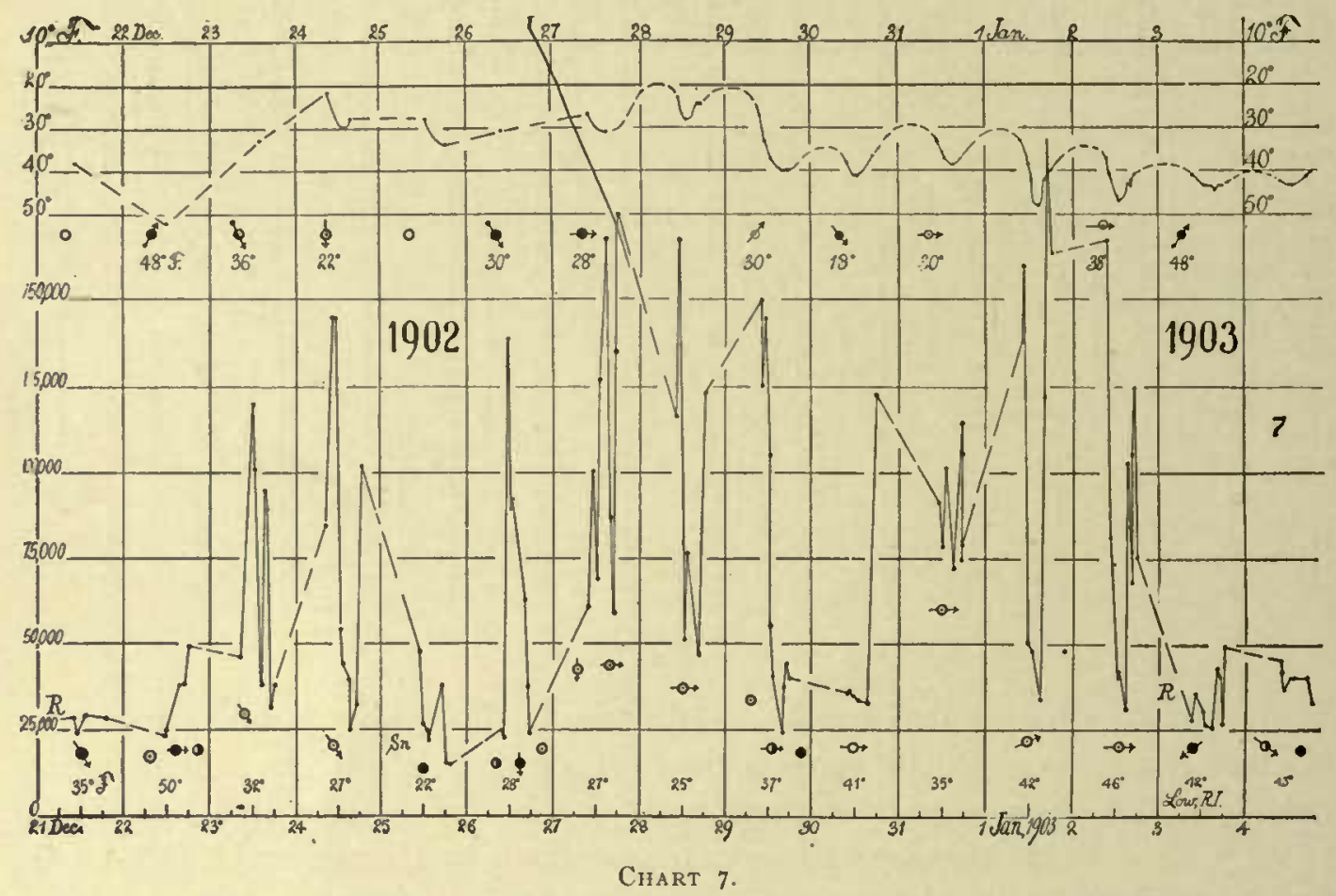

side the influx pipe. The temperatures are given on the tops of charts 7 and 8, and positive temperatures in degrees Fahrenheit are laid off downwards to insure an easier recognition of coincidences, remembering that nucleation maxima and temperature minima correspond.

In the fragmentary temperature observations before December 28 (chart 7) some relation is seen; thus the maximum between December 23 and 25 has a temperature equivalent, the broad minimum on December 22 and the midday minimum on December 24 correspond, etc. Turning to the cases under detailed observation, the mid-day minimum on December 28 , the fall on December 29 , and, to a smaller extent, the data on December 30 and $3^{I}$ (in the latter case other factors are active) agree. The marked minimum on both cases of January $\mathrm{I}$ and, to some extent, January 2 are especially striking.

In chart 8 the general maxima marked "c.w." may be noted, and the 
details are similar. One may point out the marked fall on January II and the day minimum on January I4. On this curve there is, however, one good

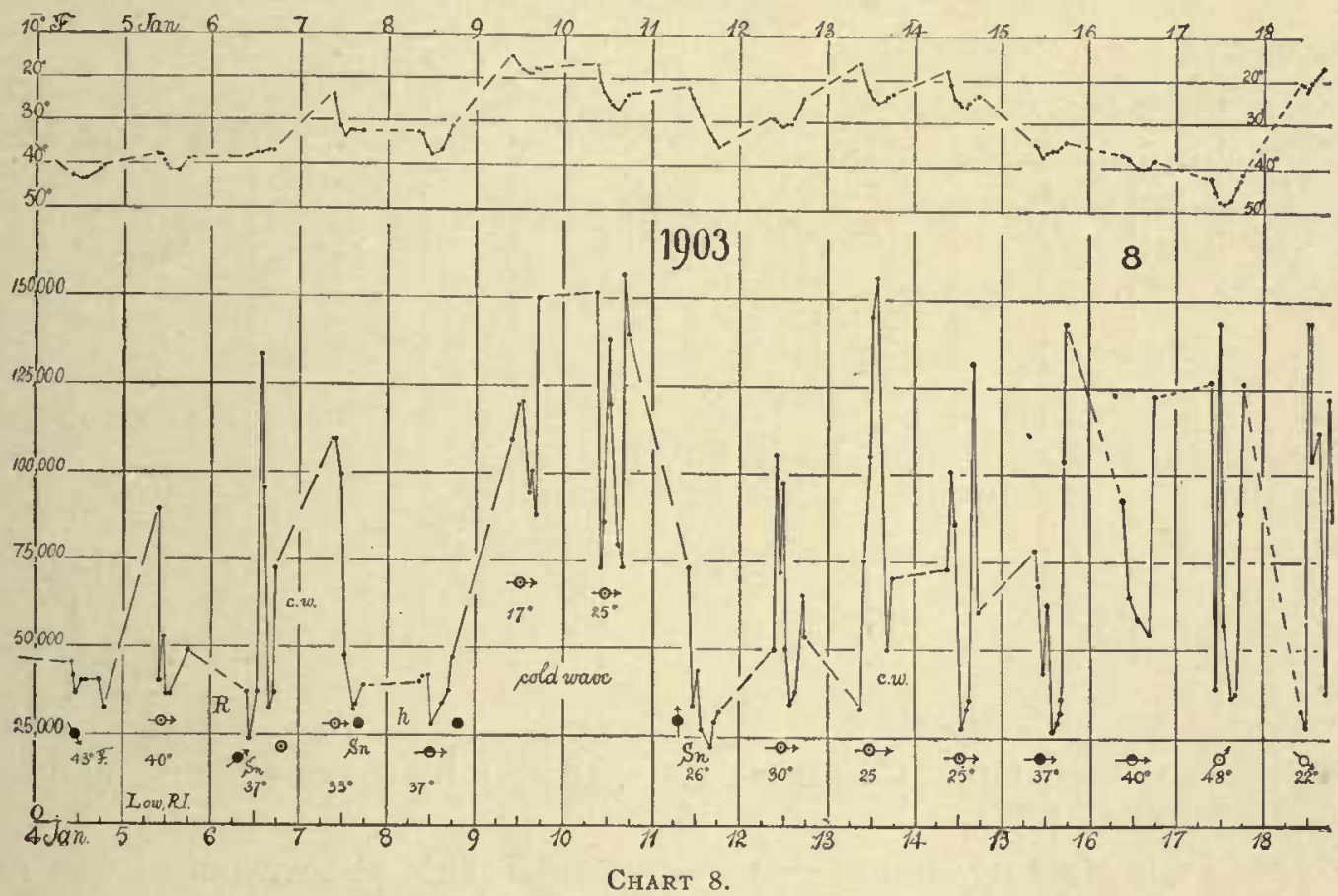

example of the opposition so frequently to be observed in the summer time, for on January I $_{3}$ a pronounced minimum of the temperature curve occurs simultaneously with a maximum of nucleation. Again, the marked fall of

1903

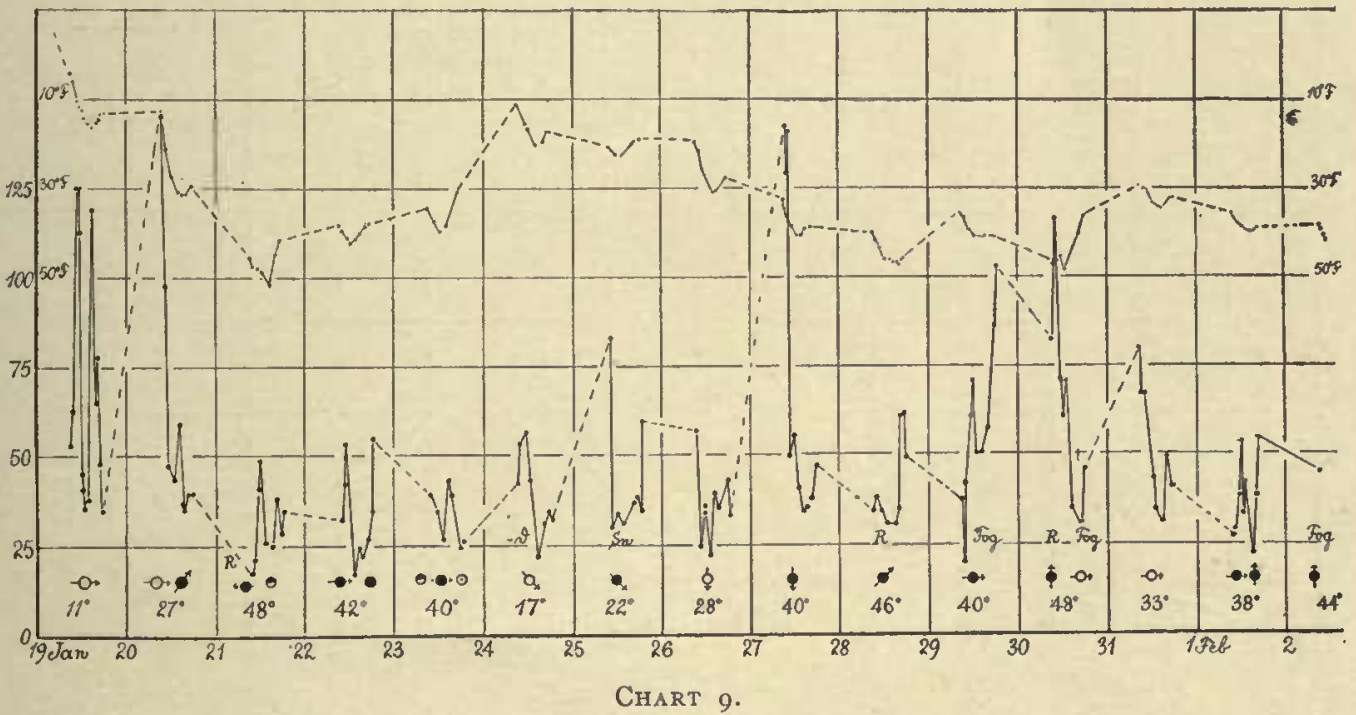

temperature on January $\mathrm{I} 7-\mathrm{I} 9$, both as a whole and in its details, is unaccompanied by a corresponding nuclear effect, and this is true throughout the 
remainder of January. Leaving the high rain minimum on January 2I (chart 9), the cold wave from January $23^{-26}$ produces but a small effect, while the

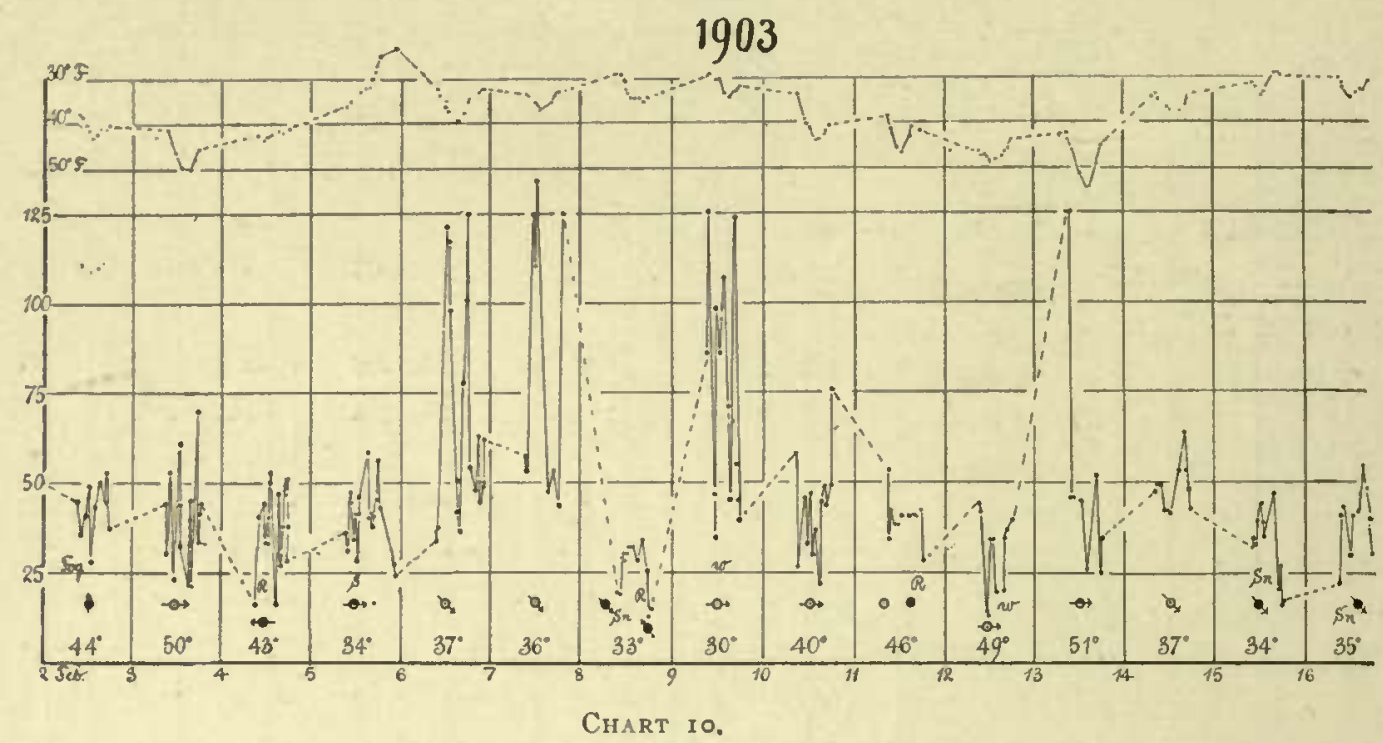

high nucleation on January 27 is not foreshadowed by the corresponding march of temperature.

5. Plate glass apparatus. - Apparatus with plate glass windows was first
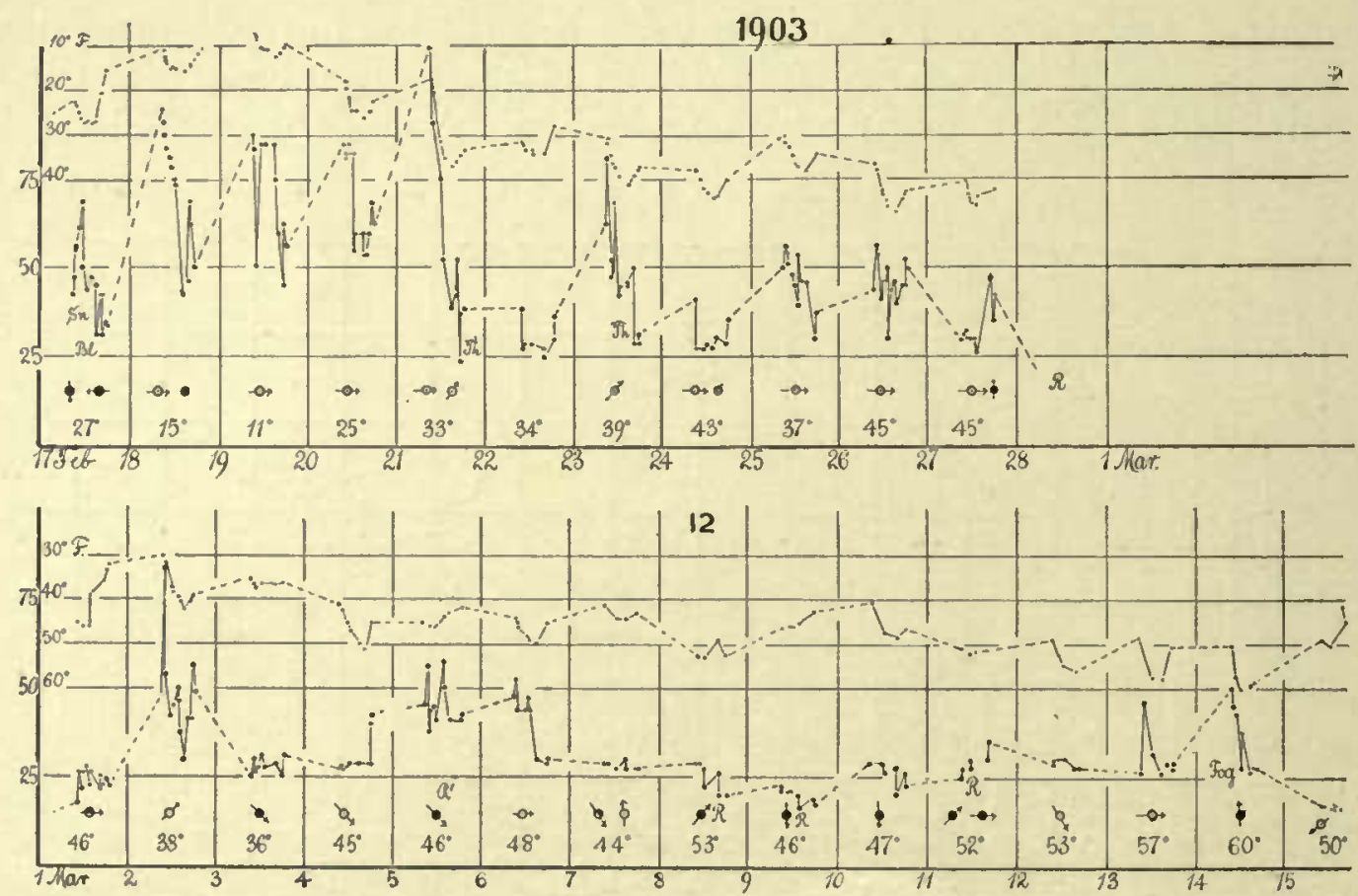

Charts it AND I 2 .

installed on January 29 and thereafter used permanently. The beginning of February (charts 9 and ro), with its frequent dark fogs and relatively warm 
weather, shows lowered winter nucleation. The data are numerous because many subsidiary experiments were made, but little of new import suggested.

The method of measuring coronas to the outside of the first red ring was adopted on February i6.

The cold wave beginning on February I 8 (chart II) shows moderately high nucleation until it breaks with the thaw on February $2 x$. Thereafter gradual but fluctuating fall of the temperature and nucleation curves appears from February 22 to the intense rain on February 28. During much of the time the ground was covered with snow. Mid-day minima are, of common occurrence.

March I-I 5 (chart I2), with its relatively high temperatures, has nearly wiped out the characteristic winter nucleation. The daily nucleation is not very variable and the rain minima are curiously high. The temperature effect is quite vague, there being as much opposition as correspondence. This sudden drop of the winter nucleation in March, I903, is particularly noteworthy and it will not be repeated in I904. The Sunday nucleations in March are not exceptionally low until the $5^{\text {th, }}$, when an abnormally small value for a clear day appears. As there is a general cessation of work in factories on this day, the corresponding nucleations are to be carefully scrutinized throughout the period of warm weather following. During cold weather there is no reduced nucleation on Sunday.

\section{SECOND GROUP OF OBSERVATIONS.}

6. Arrangement of tables and graphs.-On the I 2 th of March the same apparatus was installed in a new position, and on March I $_{5}$ the results referred to above, $\S 3$, as the "second gruop," begin. The scale is uniform throughout, though somewhat enlarged, and the plan is the same as above. Local winds and temperatures only are entered.

The data are also given in tables, as it is believed that the method is now sufficiently definite to make these available for other purposes than the immediate ones of the present chapter. The tables contain the date and hour, the state of the weather, the temperature of the atmosphere where the nucleation is observed in degrees Fahrenheit, and of the air within the apparatus in degrees centigrade; furthermore, the aperture, $s$, of the coronas when the goniometer radius is $19.5 \mathrm{~cm}$. and the distance of the goniometer and source of light $85 \mathrm{~cm}$. and $250 \mathrm{~cm}$, respectively, from the condensation chamber. Thus the angular diameter is $\phi=2 \sin ^{-1}(s / 39)$. The remaining columns contain the coronas with the colors recorded from within outward, abbreviated as stated, and the absolute nucleation, $n$, or number of nuclei per cubic centimeter of air. Certain obvious remarks will be added.

The correction for the temperature of the condensation chamber (purposely kept as nearly constant as possible) was not applied. This would have enormously increased the labor of reduction, without materially changing the 
distribution of the nucleation observed. It is to be remembered, moreover, that the true law of reduction is periodic, and unless the periodicity is fully worked out for the small coronas, the temperature correction has little meaning. It is because of this complexity that the tables are very specific as to the color type of the coronas, their size, the temperature of the fog particles, etc. In any special case, therefore, the rigorous reduction (if of interest) may be attempted, but at present there is no call for it.

As to the accuracy of the results so far as the readings are concerned, an error of 2000 to 3000 nuclei per cubic centimeter is unnecessary in the extreme case of large coronas; whereas the whole range of variation is about from 2000 to above 100,000 . For the small coronas the reading is correspondingly sharp, and the same is true if a larger goniometer is used.

TABLE I.-Continuous record of atmospheric nucleation from March I 5 , I 903 , to November $I, I 904$. The time $(t)$ is given in hours and tenths of an hour, the temperature of the fog chamber in degrees centigrade, the local atmospheric temperature (approximate) in degrees Fahrenheit. The aperture of the coronas is $s$, where the angular aperture is $\phi=2 \sin s / 39$, as the arms of the goniometer are $19.5 \mathrm{~cm}$. long. The last column but one shows the number of nuclei, $n$, per cubic centimeter of air, using the table in Chapter VII, $\S 26$, for the reduction from $s$ to $n$. Temperature corrections were not applied. The pressure difference was uniformly $\delta p=I 7 \mathrm{~cm}$. of mercury. The distances of the eye and the source of light were $85 \mathrm{~cm}$. and $250 \mathrm{~cm}$. (I:3), on opposite sides of the condensation chamber. The weather abbreviations are $f$ fair, $f^{\prime}$ partly fair, c' partly cloudy, c cloudy, $R^{\prime}$ slight rain, $R$ rain, Sn snow, S sun, etc. The coronal color abbreviations are $c$ (crimson) deep red, r orange red, br brown, o orange, y yellow, g green, b blue, $v$ violet, $\mathrm{p}$ purple, etc. A vertical line denotes an indeterminate color, as $\mathrm{w} r \mid \mathrm{g}$; an accent an approach to the color, as $r^{\prime}$ reddish; a capital, a deep dark color, as B deep blue, etc. Mixed colors are written together, as bg, blue-green, or, orange-red. Subsidence measurements when entered (November, I903) are usually abbreviated $a / b$, meaning that $a$ seconds are required for a fall of the fog line of $b$ centimeters. Sometimes the abbreviation is $b / a$, as will be stated (April, I g04).

\begin{tabular}{|c|c|c|c|c|c|c|c|c|c|c|}
\hline હ & 苞 & 芴 & 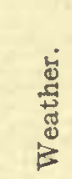 & 节 & 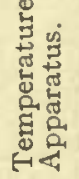 & 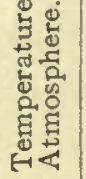 & 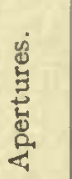 & 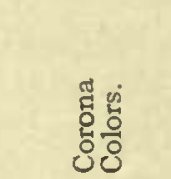 & 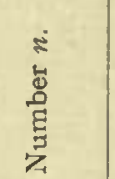 & Remarks. \\
\hline 1903 & Mar. 15 & \begin{tabular}{r|}
10.3 \\
10.6 \\
I 2.8 \\
1.0 \\
1.2 \\
1.4 \\
5.2
\end{tabular} & $\begin{array}{l}f \\
f \\
f \\
f \\
f \\
f \\
f\end{array}$ & N.E. & $\begin{array}{l}20.5 \\
20 \\
2 \mathrm{I} \\
2 \mathrm{I} \\
2 \mathrm{I} \\
2 \mathrm{I} \\
20\end{array}$ & $\begin{array}{c}\frac{49.2}{50.4} \\
\overline{5} \\
\overline{4} \\
44.8\end{array}$ & \begin{tabular}{l|} 
I. \\
1.8 \\
1.8 \\
1.9 \\
2.0 \\
2.0 \\
1.9
\end{tabular} & $\begin{array}{c}\text { w br B } \\
\text { Do. } \\
\text { Do. } \\
\text { Do. } \\
\text { Do. } \\
\text { w br B g } \\
\text { w br B g r r }\end{array}$ & $\begin{array}{r}7000 \\
8000 \\
8000 \\
8500 \\
10000 \\
10000 \\
8500\end{array}$ & \\
\hline
\end{tabular}


TABLE $\mathrm{I}-$ Continued.

\begin{tabular}{|c|c|c|c|c|c|c|c|c|c|c|}
\hline 焉 & ڤึ & $\Xi$ & $\begin{array}{l}\text { 苛 } \\
\vdots \\
\vdots\end{array}$ & 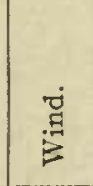 & 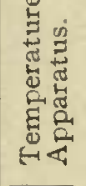 & 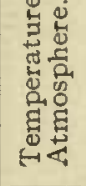 & 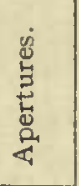 & 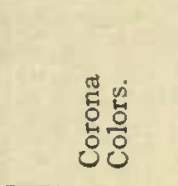 & 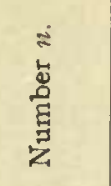 & Remarks. \\
\hline 1903 & $\begin{array}{l}\text { Mar. I } 5 \\
\text { Mar. I6 }\end{array}$ & 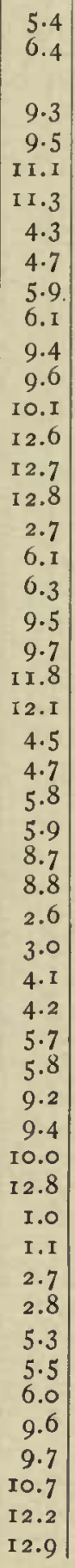 & 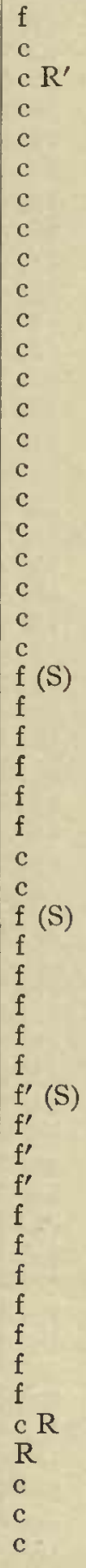 & $\begin{array}{l}\text { S.W. } \\
\text { N.W. }\end{array}$ & $\begin{array}{l}20 \\
20 \\
18 \\
18 \\
18 \\
19 \\
19 \\
19 \\
19 \\
20 \\
20 \\
20 \\
20 \\
19 \\
20 \\
21 \\
21 \\
21 \\
23 \\
23 \\
20 \\
20 \\
21 \\
21 \\
22 \\
22 \\
22 \\
19 \\
19 \\
21 \\
21 \\
22 \\
22 \\
22 \\
22 \\
20 \\
20 \\
20 \\
21 \\
21 \\
21 \\
22 \\
22 \\
23 \\
23 \\
23 \\
20 \\
20 \\
20 \\
21 \\
21\end{array}$ & 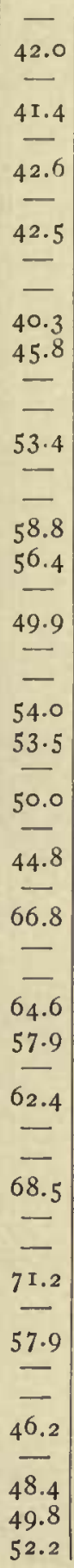 & $\begin{array}{l}1.9 \\
1.8 \\
1.8 \\
2.4 \\
2.6 \\
2.4 \\
2.5 \\
2.8 \\
2.9 \\
2.5 \\
2.5 \\
3.6 \\
3.5 \\
3.1 \\
2.9 \\
4.0 \\
3.0 \\
3.1 \\
3.1 \\
3.1 \\
2.1 \\
2.3 \\
2.3 \\
2.3 \\
2.3 \\
2.3 \\
2.2 \\
2.3 \\
3.0 \\
3.0 \\
2.9 \\
3.4 \\
3.0 \\
3.0 \\
3.5 \\
3.5 \\
3.0 \\
3.8 \\
3.5 \\
2.8 \\
3.5 \\
3.5 \\
2.7 \\
2.7 \\
2.8 \\
2.8 \\
2.8 \\
2.4 \\
2.2 \\
3.0 \\
2.4 \\
2.3\end{array}$ & $\begin{array}{c}\text { Do. } \\
\text { Do. } \\
\text { Do. } \\
\text { w br B g } \\
\text { Do. } \\
\text { Do. } \\
\text { Do. } \\
\text { w br B p } \\
\text { w br B r } \\
\text { w br B g } \\
\text { Do. } \\
\text { w c g } \\
\text { w cg } \\
\text { w br B|p } \\
\text { w br B p } \\
\text { w rg } \\
\text { w br B p } \\
\text { w br B|p } \\
\text { Do. } \\
\text { Do. } \\
\text { w br B g r } \\
\text { w br B } \\
\text { Do. } \\
\text { Do. } \\
\text { Do. } \\
\text { Do. } \\
\text { Do. } \\
\text { Do. } \\
\text { g br B p } \\
\text { Do. } \\
\text { y br|b } \\
\text { w br } \\
\text { w br } \\
\text { w br|b } \\
\text { w } c \mathrm{~g} \\
\text { Do. } \\
\text { w br B p } \\
\text { w rg } \\
\text { w cg } \\
\text { y' br b p } \\
\text { w rg } \\
\text { Do. } \\
\text { g br B p } \\
\text { w br|b } \\
\text { w br B p } \\
\text { Do. } \\
\text { w c|g } \\
\text { cor } \\
\text { Do. } \\
\text { w br B p } \\
\text { w br b' } \\
\text { w br B } \mid r\end{array}$ & $\begin{array}{r}8500 \\
8000 \\
8000 \\
18000 \\
22000 \\
18000 \\
21000 \\
28000 \\
31000 \\
20000 \\
20000 \\
57000 \\
53000 \\
40000 \\
31000 \\
78000 \\
37000 \\
38000 \\
38000 \\
38000 \\
12500 \\
16500 \\
15500 \\
16500 \\
15500 \\
16500 \\
13500 \\
15500 \\
35000 \\
35000 \\
31000 \\
49000 \\
35000 \\
35000 \\
55000 \\
53000 \\
35000 \\
66000 \\
53000 \\
30000 \\
53000 \\
53000 \\
27000 \\
27000 \\
28000 \\
28000 \\
28000 \\
19000 \\
14500 \\
35000 \\
17500 \\
15500 \\
\end{array}$ & . \\
\hline
\end{tabular}


TABLE I-Continued.

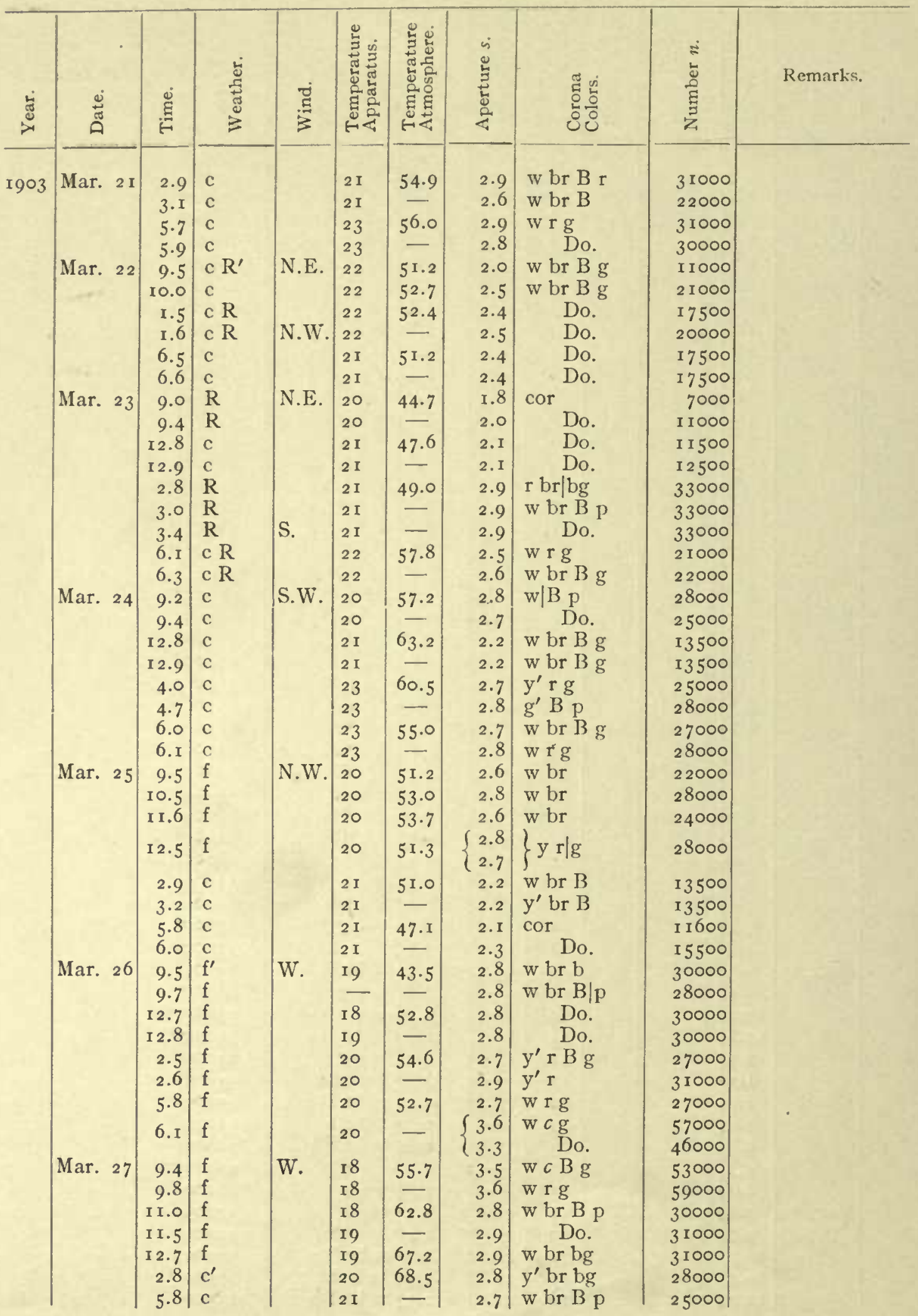


TABLE I-Continued.

\begin{tabular}{|c|c|c|c|c|c|c|c|c|c|c|}
\hline 薦 & 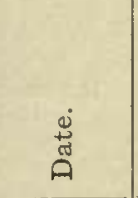 & $\underset{\sharp}{\stackrel{\Xi}{\sharp}}$ & 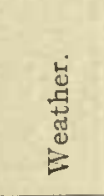 & $\stackrel{\Xi}{Z}$ & 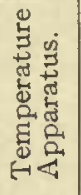 & 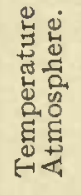 & 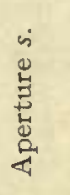 & 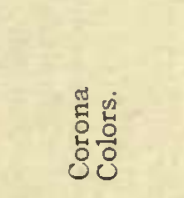 & 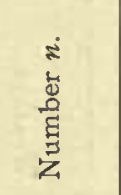 & Remarks. \\
\hline I903 & $\begin{array}{ll}\text { Mar. } & 27 \\
\text { Mar. } & 28\end{array}$ & $\begin{array}{r}5.9 \\
\text { IO.2 } \\
\text { IO.4 } \\
\text { I } 2.8 \\
\text { I } .0 \\
2.6 \\
3.3 \\
5.7 \\
5.9 \\
\text { IO.I } \\
\text { IO.7 } \\
\text { I.2 } \\
\text { I.5 } \\
4.3 \\
4.4 \\
5.0 \\
6.6 \\
6.7 \\
9.9 \\
\text { IO.9 } \\
\text { I I.I } \\
\text { I I.5 } \\
\text { I } 2.7 \\
\text { I } 2.9 \\
\text { I.I } \\
2.5 \\
2.7 \\
\text { I.7 } \\
\text { I } 2.8 \\
\text { I } 2.8 \\
9.4 \\
9.5 \\
\text { I } 2.9 \\
\text { I } 2.6 \\
3.0 \\
3.7 \\
3.8 \\
7.0 \\
7.7 \\
7.8 \\
5.0 \\
5.6 \\
9.4 \\
9.5 \\
\end{array}$ & $\begin{array}{l}\mathrm{c} \\
\mathrm{f} \\
\mathrm{f} \\
\mathrm{f} \\
\mathrm{f} \\
\mathrm{f} \\
\mathrm{f} \\
\mathrm{f} \\
\mathrm{f} \\
\mathrm{f} \\
\mathrm{f} \\
\mathrm{f} \\
\mathrm{f} \\
\mathrm{f} \\
\mathrm{f} \\
\mathrm{f} \\
\mathrm{f} \\
\mathrm{f} \\
\mathrm{c} \\
\mathrm{c} \\
\mathrm{c} \\
\mathrm{c} \\
\mathrm{c} \\
\mathrm{c} \\
\mathrm{c} \\
\mathrm{c} \\
\mathrm{c} \\
\mathrm{c} \\
\mathrm{c} \\
\mathrm{c} \mathrm{R} \\
\mathrm{c} \\
\mathrm{c} \\
\mathrm{c} \\
\mathrm{c} \\
\mathrm{c} \\
\mathrm{f}^{\prime} \\
\mathrm{f} \\
\mathrm{f} \\
\mathrm{f} \\
\mathrm{f} \\
\mathrm{c} \\
\mathrm{c} \\
\mathrm{f} \\
\mathrm{f} \\
\mathrm{f} \\
\mathrm{f} \\
\mathrm{f} \\
\mathrm{f} \\
\mathrm{f} \\
\\
\mathrm{f}\end{array}$ & $\begin{array}{l}\text { N.W. } \\
\text { N.W. }\end{array}$ & $\begin{array}{l}21 \\
21 \\
21 \\
22 \\
22 \\
23 \\
23 \\
23 \\
23 \\
20 \\
19 \\
20 \\
20 \\
20 \\
20 \\
20 \\
20 \\
20 \\
17 \\
17 \\
17 \\
17 \\
18 \\
18 \\
18 \\
19 \\
19 \\
20 \\
20 \\
21 \\
21 \\
21 \\
21 \\
21 \\
21 \\
19 \\
20 \\
21 \\
21 \\
22 \\
23 \\
19 \\
19 \\
20 \\
20 \\
22 \\
\end{array}$ & 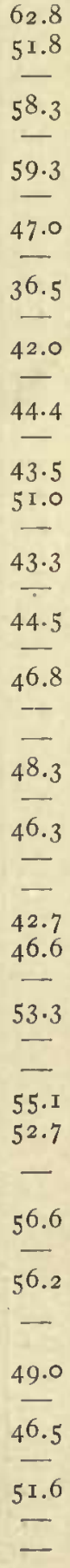 & $\begin{array}{l}3.2 \\
2.0 \\
2.0 \\
2.3 \\
2.8 \\
2.6 \\
2.6 \\
2.2 \\
2.2 \\
3.1 \\
3.3 \\
2.7 \\
2.8 \\
2.6 \\
2.7 \\
2.9 \\
2.8 \\
2.8 \\
2.7 \\
3.8 \\
3.7 \\
3.7 \\
2.8 \\
3.5 \\
3.5 \\
3.2 \\
3.5 \\
2.4 \\
2.3 \\
2.8 \\
2.3 \\
2.7 \\
2.9 \\
2.7 \\
2.8 \\
2.9 \\
2.7 \\
2.7 \\
2.7 \\
2.7 \\
2.7 \\
2.3 \\
2.8 \\
2.0\end{array}$ & 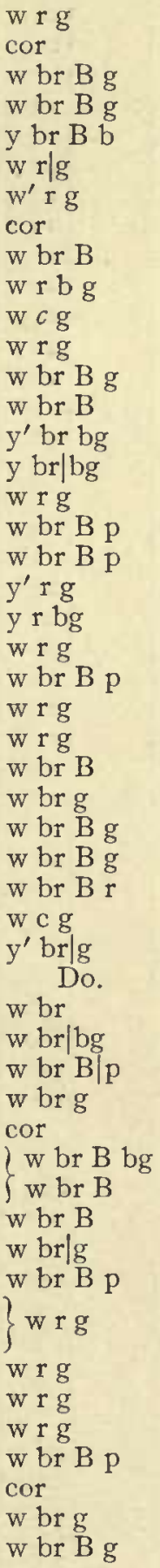 & 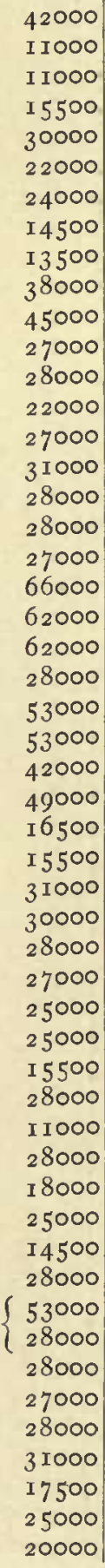 & \\
\hline
\end{tabular}


TABLE I-Continued.

\begin{tabular}{|c|c|c|c|c|c|c|c|c|c|c|}
\hline 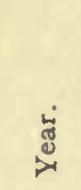 & 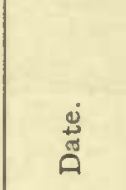 & $\underset{\Xi}{\stackrel{\Xi}{E}}$ & 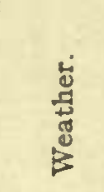 & $\stackrel{\square}{\square}$ & 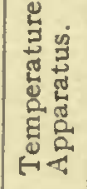 & 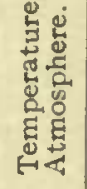 & 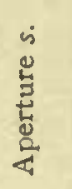 & $\begin{array}{l}\text { है } \\
\text { 영 } \\
8\end{array}$ & 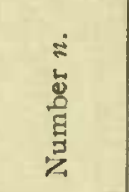 & Remarks. \\
\hline 1903 & $\begin{array}{l}\text { Apr. } 2 \\
\text { Apr. } 3 \\
\text { Apr. } 4\end{array}$ & 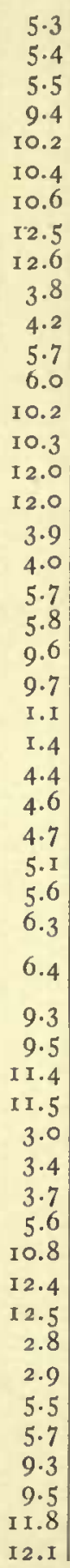 & 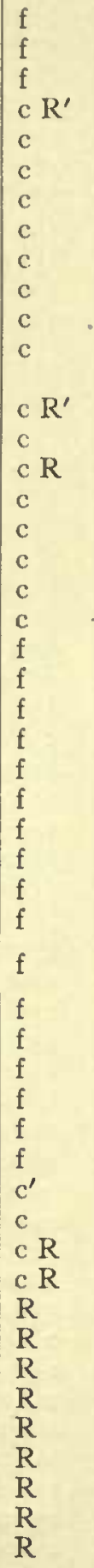 & $\begin{array}{l}\text { S.W. } \\
\text { N.W. }\end{array}$ & 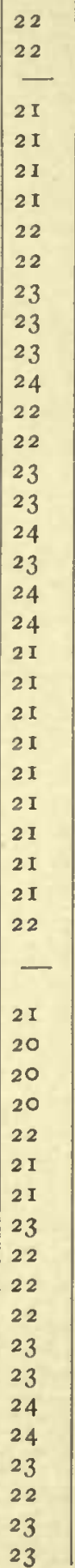 & 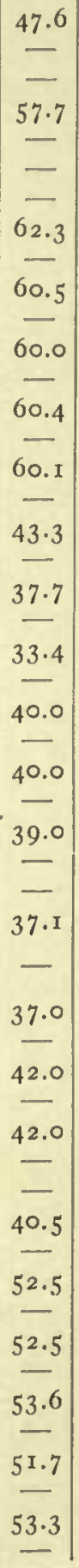 & $\begin{array}{l}2.7 \\
2.7 \\
2.8 \\
2.5 \\
2.7 \\
2.7 \\
2.8 \\
2.6 \\
2.8 \\
2.7 \\
2.7 \\
2.8 \\
2.7 \\
2.8 \\
2.8 \\
2.9 \\
2.8 \\
2.8 \\
2.8 \\
2.5 \\
2.5 \\
3.0 \\
3.2 \\
3.2 \\
3.1 \\
2.8 \\
3.8 \\
4.1 \\
2.7 \\
3.2 \\
3.7 \\
3.1 \\
3.4 \\
2.8 \\
2.9 \\
2.9 \\
2.4 \\
2.7 \\
2.6 \\
3.0 \\
2.9 \\
2.7 \\
2.7 \\
2.6 \\
2.7 \\
2.6 \\
2.6 \\
2.4 \\
2.3 \\
2.2 \\
2.2\end{array}$ & 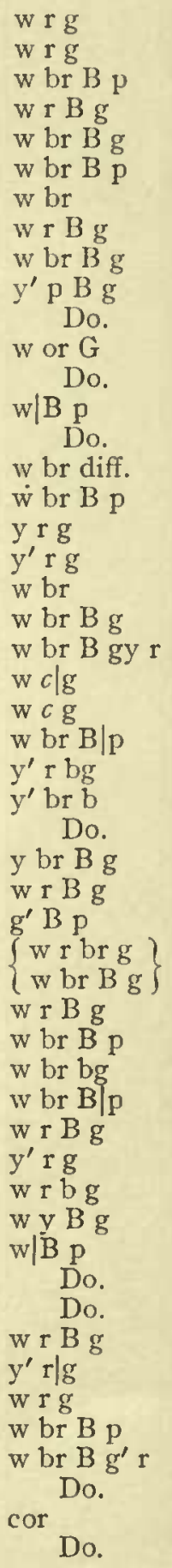 & $\begin{array}{l}27000 \\
27000 \\
28000 \\
21000 \\
26000 \\
26000 \\
28000 \\
22000 \\
28000 \\
24000 \\
24000 \\
30000 \\
27000 \\
28000 \\
28000 \\
31000 \\
28000 \\
28000 \\
28000 \\
20000 \\
20000 \\
37000 \\
44000 \\
44000 \\
38000 \\
30000 \\
68000 \\
83000 \\
27000 \\
42000 \\
27000 \\
40000 \\
139000 \\
13500 \\
14500\end{array}$ & \\
\hline
\end{tabular}


TABLE $\mathrm{x}$-Continued.

\begin{tabular}{|c|c|c|c|c|c|c|c|c|c|c|}
\hline હँ & 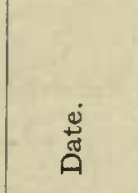 & $\underset{H}{\stackrel{\Xi}{E}}$ & 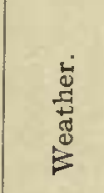 & 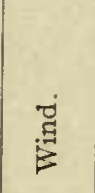 & 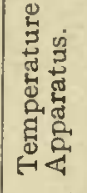 & 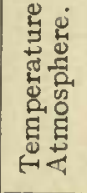 & 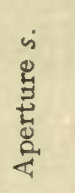 & 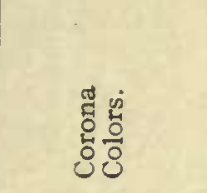 & 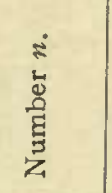 & Remarks. \\
\hline 1903 & $\begin{array}{l}\text { Apr. } 8 \\
\text { Apr. } 9\end{array}$ & $\begin{array}{r}4.2 \\
4.3 \\
4.4 \\
9.1 \\
9.4 \\
9.6 \\
12.2 \\
\text { I } 2.3 \\
3.9 \\
4.1 \\
5.4 \\
5.6 \\
9.4 \\
9.6 \\
9.9 \\
\text { I } 2.4 \\
12.5 \\
\text { I } 2.7 \\
3.1 \\
3.4 \\
5.8 \\
5.9 \\
9.4 \\
9.6 \\
\text { I } 2.1 \\
\text { I } 2.2 \\
\text { I } 2.5 \\
2.8 \\
3.0 \\
\text { I } 2.1 \\
3.5 \\
3.6 \\
5.8 \\
5.9 \\
9.2\end{array}$ & 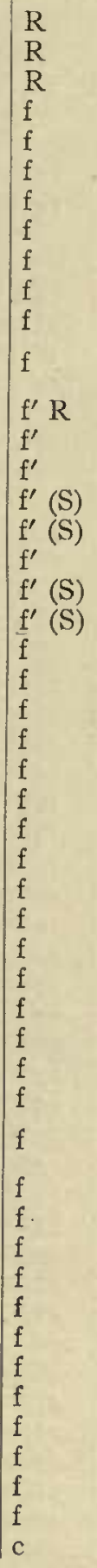 & $\begin{array}{l}\text { W. } \\
\text { N. }\end{array}$ & 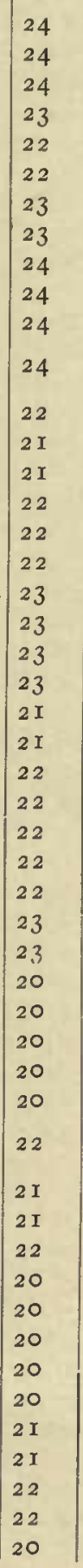 & 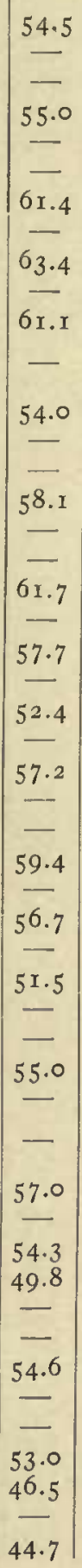 & 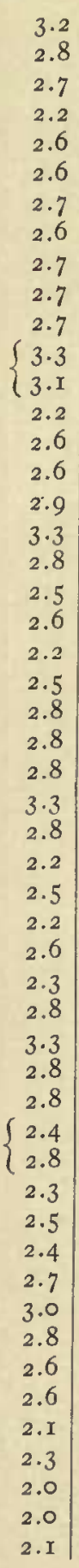 & 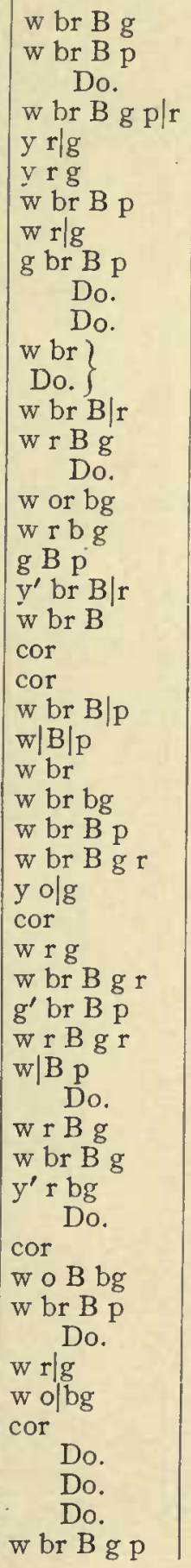 & 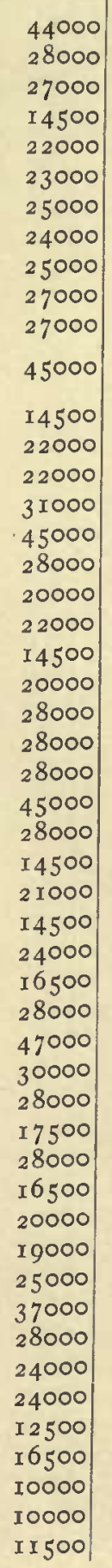 & \\
\hline
\end{tabular}


TABLE I-Continued.

\begin{tabular}{|c|c|c|c|c|c|c|c|c|c|c|}
\hline 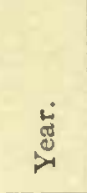 & 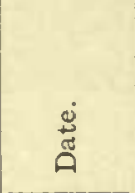 & $\stackrel{\Xi}{E}$ & 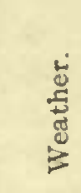 & 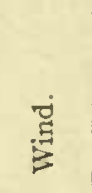 & 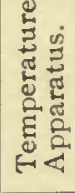 & 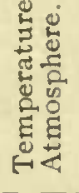 & $\begin{array}{l}n \\
0 \\
\frac{0}{3} \\
\frac{5}{0} \\
\frac{0}{4}\end{array}$ & 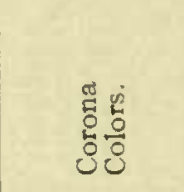 & $\begin{array}{l}E \\
\stackrel{\Xi}{0} \\
\frac{E}{E} \\
z\end{array}$ & Remarks. \\
\hline I9०3 & $\begin{array}{ll}\text { Apr. } 16 \\
\text { Apr. } 17\end{array}$ & 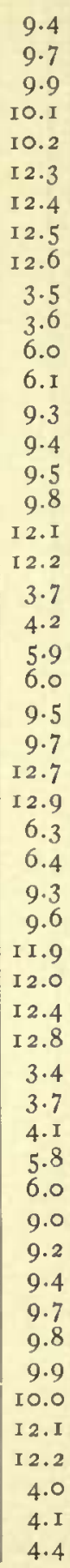 & 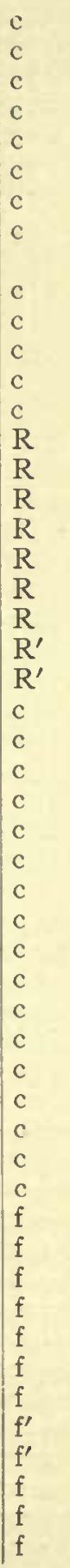 & N.E. & $\begin{array}{l}\text { I9 } \\
\text { I9 } \\
\text { I } 9 \\
\text { I9 } \\
\text { I9 } \\
20 \\
20 \\
20 \\
2 \\
2 \mathrm{I} \\
2 \mathrm{I} \\
2 \mathrm{I} \\
2 \mathrm{I} \\
2 \mathrm{I} \\
\mathrm{I} 9 \\
\mathrm{I} 9 \\
\mathrm{I} 9 \\
20 \\
20 \\
2 \mathrm{I} \\
2 \mathrm{I} \\
2 \mathrm{I} \\
2 \mathrm{I} \\
\mathrm{I} 9 \\
\mathrm{I} 8 \\
20 \\
\mathrm{I}\end{array}$ & 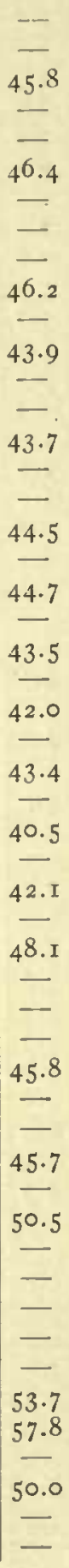 & $\begin{array}{l}2.5 \\
2.2 \\
2.3 \\
2.1 \\
2 . I \\
2.1 \\
2.2 \\
2.9 \\
1.8 \\
2.3 \\
2.2 \\
2.0 \\
2.0 \\
1.7 \\
2.1 \\
1.8 \\
1.7 \\
2 . I \\
2.0 \\
1.8 \\
1.9 \\
1.8 \\
2 . I \\
2 . I \\
2 . I \\
2.2 \\
2.3 \\
2.2 \\
2.2 \\
2.5 \\
2.4 \\
3.1 \\
2.9 \\
2.7 \\
2.8 \\
2.3 \\
2.6 \\
2.6 \\
2.7 \\
2.6 \\
2.9 \\
3.2 \\
2.9 \\
3.4 \\
3.0 \\
3.8 \\
3.3 \\
2.5 \\
2.7 \\
2.9 \\
3.3 \\
2.8\end{array}$ & $\begin{array}{l}\text { w r B g } \\
\text { cor } \\
\text { cor } \\
\text { cor } \\
\text { cor } \\
\text { cor } \\
\text { w br B g p } \\
\text { w br B } \\
\text { cor } \\
\text { cor } \\
\text { cor } \\
\text { cor } \\
\text { cor } \\
\text { cor } \\
\text { cor } \\
\text { cor } \\
\text { cor } \\
\text { cor } \\
\text { cor } \\
\text { cor } \\
\text { cor } \\
\text { cor } \\
\text { cor } \\
\text { cor } \\
\text { w br B|p } \\
\text { cor } \\
\text { w br B } \mid \text { p } \\
\text { cor } \\
\text { cor } \\
\text { cor } \\
\text { w br B } \mid p \\
\text { w br } \\
\text { g'|B p } \\
\text { w r B } \\
\text { g'|B p } \\
\text { cor } \\
\text { y r|g } \\
\text { y r|g } \\
\text { y' r|g } \\
\text { w br B bg } \\
\text { w br B g r } \\
\text { g'br B|p } \\
\text { w c g } \\
\text { w' br B|p } \\
\text { w c|g } \\
\text { w c|g } \\
\text { cor } \\
\text { w br B } \mid p \\
\text { w br B|p } \\
\text { w r g } \\
\text { cor }\end{array}$ & 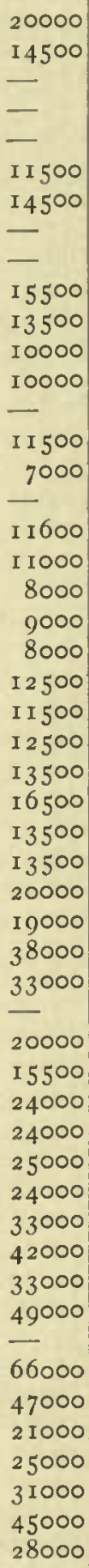 & $\begin{array}{l}\{\delta p=22 \\
\delta p=9 \\
\delta p=22 \\
\delta p=22 \\
\delta p=22\end{array}$ \\
\hline
\end{tabular}


TABLE I-Continued.

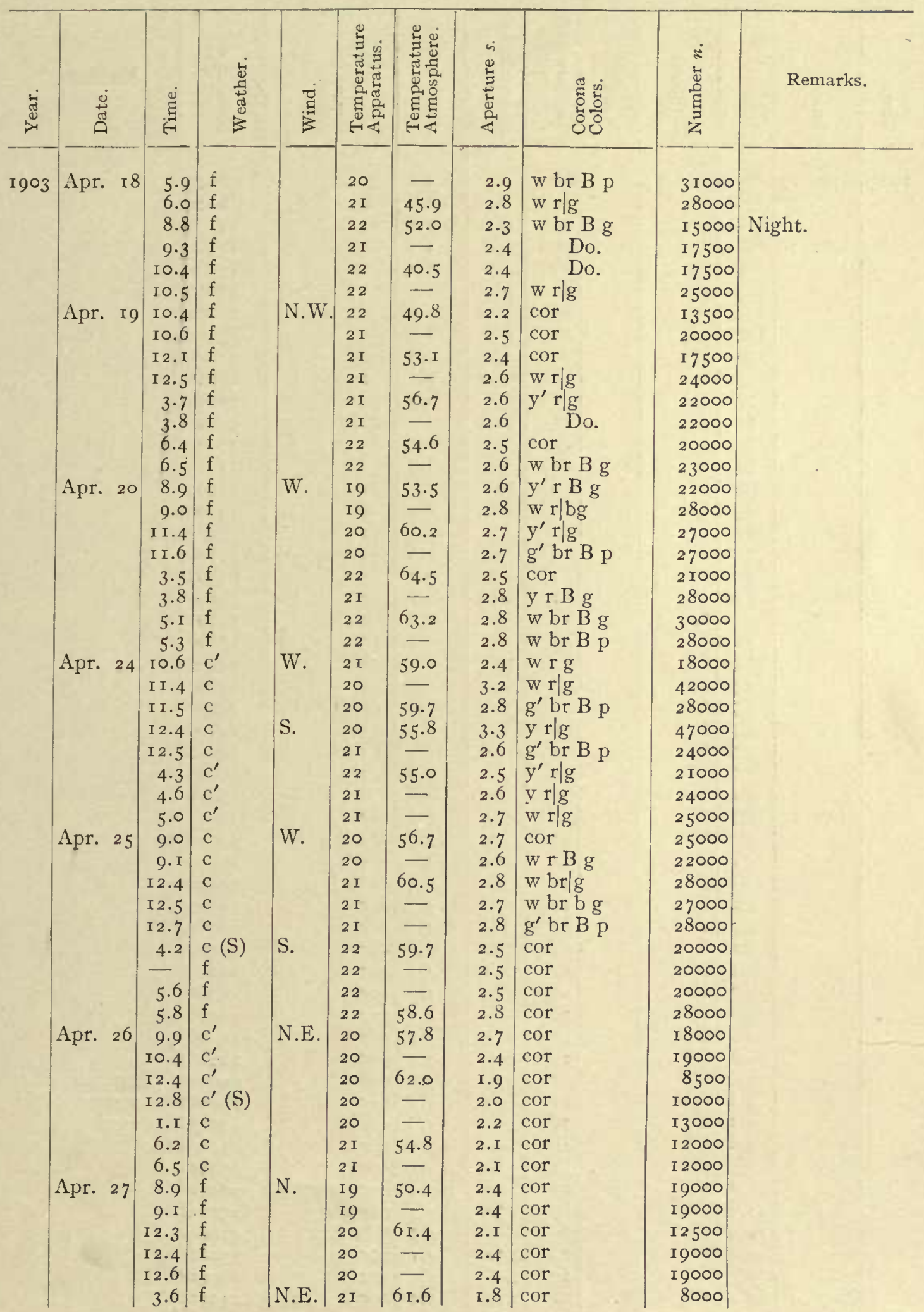


TABLE I-Continued.

\begin{tabular}{|c|c|c|c|c|c|c|c|c|c|c|}
\hline 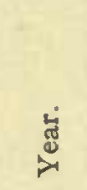 & 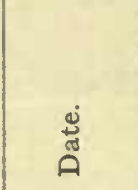 & 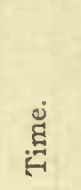 & 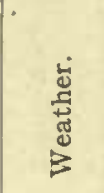 & $\stackrel{3}{3}$ & 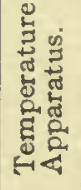 & 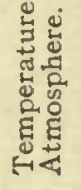 & $\begin{array}{l}0 \\
0 \\
5 \\
\frac{5}{3} \\
\frac{5}{4} \\
\frac{0}{4}\end{array}$ & 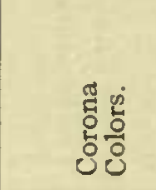 & 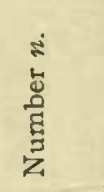 & Remarks. \\
\hline 1903 & $\begin{array}{l}\text { Apr. } 30 \\
\text { May } \quad \\
\text { May } 2 \\
\text { May } 3\end{array}$ & 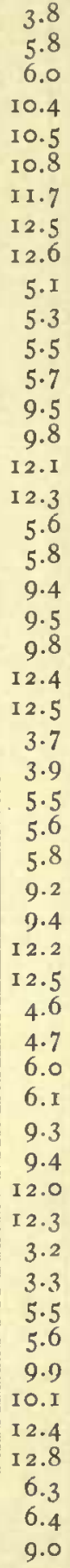 & 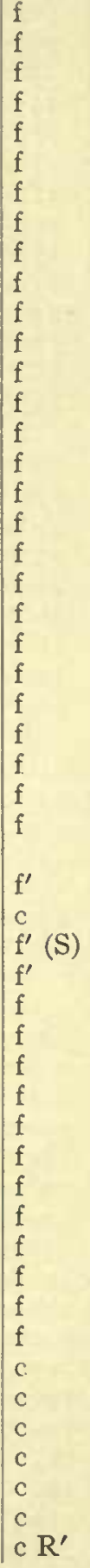 & $\begin{array}{l}\text { W. } \\
\text { N.W. }\end{array}$ & $\begin{array}{l}21 \\
22 \\
22 \\
20 \\
20 \\
20 \\
20 \\
20 \\
20 \\
20 \\
22 \\
20 \\
22 \\
22 \\
22 \\
20 \\
20 \\
21 \\
21 \\
21 \\
23 \\
23 \\
23 \\
23\end{array}$ & 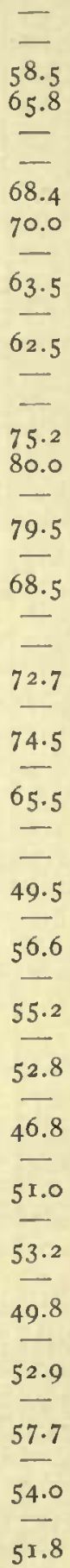 & $\begin{array}{l}1.8 \\
1.8 \\
2.1 \\
2.1 \\
2.4 \\
2.7 \\
2.8 \\
2.3 \\
2.6 \\
2.5 \\
2.8 \\
2.6 \\
2.9 \\
2.7 \\
2.7 \\
2.2 \\
2.1 \\
2.6 \\
2.6 \\
2.2 \\
2.6 \\
2.4 \\
2.2 \\
2.2 \\
2.6 \\
2.6 \\
2.4 \\
2.8 \\
2.2 \\
2.5 \\
2.5 \\
2.5 \\
2.2 \\
2.5 \\
2.1 \\
2.7 \\
2.3 \\
1.9 \\
2.3 \\
2.1 \\
2.3 \\
2.5 \\
2.3 \\
2.8 \\
2.7 \\
2\end{array}$ & $\begin{array}{l}\text { cor } \\
\text { cor } \\
\text { cor } \\
\text { cor } \\
\text { cor } \\
\text { w r b g } \\
g^{\prime} \text { br B p } \\
\text { cor } \\
\text { cor } \\
\text { cor } \\
\text { w br B g } \\
\text { w r } \mid g \\
\text { w o b } \\
\text { w br B g } \\
\text { w c B g } \\
\text { cor } \\
\text { cor } \\
\text { w } c \text { B g } \\
\text { cor Do. } \\
\text { w } c \text { B g } \\
\text { w } c \mid g \\
\text { cor } \\
\text { cor } \\
\text { cor|g } \\
\text { w r g } \\
\text { w r g } \\
\text { w r g g } \\
\text { cor Do. } \\
\text { w } c \text { B g } \\
\text { w r b g } \\
\text { w br B g } \\
\text { cor } \\
\text { w c|g } \\
\text { cor } \\
\text { cor } \\
\text { cor } \\
\text { cor } \\
\text { cor } \\
\text { cor } \\
\text { cor } \\
\text { cor } \\
\text { cor } \\
\text { cor } \\
\text { cor } \\
\text { cor } \\
\text { cor w r|g } \\
\text { g br B p } \\
\text { cor } \\
\text { cor } \\
\text { cor }\end{array}$ & 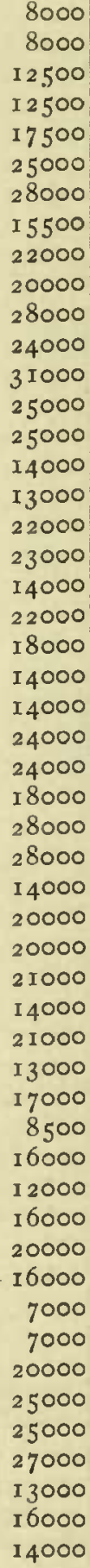 & $\left\{\begin{array}{l}\text { Clear air after } \\
\text { western bliz- } \\
\text { zard. }\end{array}\right.$ \\
\hline
\end{tabular}


TABLE I-Continued.

\begin{tabular}{|c|c|c|c|c|c|c|c|c|c|c|c|}
\hline 矛 & 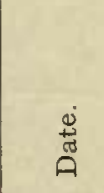 & & 㤩 & 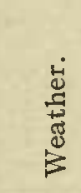 & 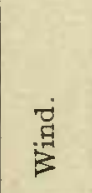 & 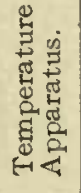 & 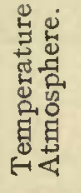 & 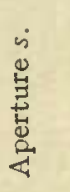 & 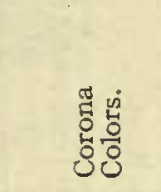 & 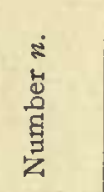 & Remarks, \\
\hline 1903 & May & I 2 & 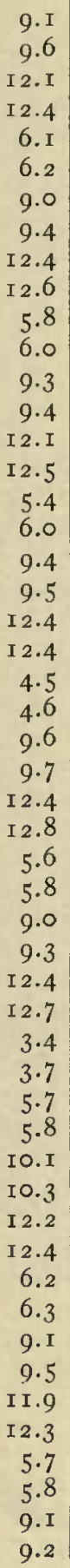 & $\begin{array}{l}c \\
c \\
c \\
c \\
c \text { R } \\
c \\
c \\
c \\
c \\
c \\
c \\
c \\
c \\
c \\
c \\
c \\
f^{\prime} \\
f^{\prime} \\
\text { f } \\
\text { f } \\
\text { f } \\
\text { f } \\
c \\
c \\
\text { f } \\
\text { f } \\
\text { f } \\
\text { f } \\
\text { f } \\
\text { f } \\
\text { f } \\
\text { f } \\
\text { f } \\
\text { f } \\
\text { f } \\
\text { f } \\
\text { f } \\
\text { f } \\
\text { f } \\
\text { f } \\
\text { f } \\
\text { f } \\
\text { f } \\
\text { f } \\
\text { f } \\
\text { f } \\
\text { f } \\
\text { f } \\
\text { f }\end{array}$ & $\begin{array}{l}\text { N.W. } \\
\text { S.E. } \\
\text { N.E. }\end{array}$ & 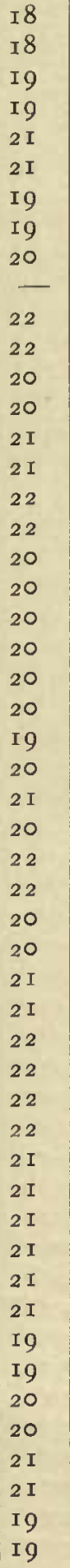 & 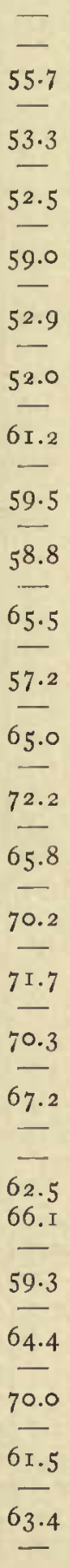 & $\begin{array}{l}2.8 \\
2.6 \\
2.6 \\
2.7 \\
2.5 \\
2.7 \\
2.0 \\
2.5 \\
2.4 \\
2.7 \\
2.2 \\
2.7 \\
2.2 \\
2.1 \\
2.1 \\
2.6 \\
2.0 \\
2.2 \\
2.7 \\
2.8 \\
2.7 \\
2.8 \\
2.8 \\
2.8 \\
2.4 \\
2.2 \\
2.4 \\
2.2 \\
2.2 \\
2.5 \\
2.2 \\
2.2 \\
1.7 \\
1.7 \\
1.8 \\
1.7 \\
1.6 \\
1.8 \\
2.6 \\
2.8 \\
2.7 \\
2.4 \\
2.0 \\
2.1 \\
2.9 \\
3.3 \\
2.9 \\
2.9 \\
2.8 \\
\end{array}$ & $\begin{array}{l}\text { cor } \\
\text { cor } \\
\text { w } c \text { B g } \\
\text { w r b g } \\
\text { cor } \\
\text { cor } \\
\text { cor } \\
\text { cor } \\
\text { w } c \mid g \\
\text { w br B g } \\
\text { cor } \\
\text { - } \\
\text { cor } \\
\text { cor } \\
\text { cor } \\
\text { cor } \\
\text { cor } \\
\text { cor } \\
\text { w br B } \\
\text { w br|g } \\
\text { w br B } \\
\text { w br B g } \\
\text { w br|g } \\
\text { Do. Do. } \\
\text { w br B|r } \\
\text { cor } \\
\text { cor } \\
\text { cor } \\
\text { cor } \\
\text { cor } \\
\text { cor } \\
\text { cor } \\
\text { cor } \\
\text { cor } \\
\text { cor } \\
\text { cor } \\
\text { cor } \\
\text { cor } \\
\text { cor } \\
\text { w br|g } \\
\text { w br } \\
\text { cor } \\
\text { cor } \\
\text { cor } \\
\text { g' br B|p } \\
\text { w } c \text { B } \\
\text { w br B p } \\
\text { w br B p } \\
\text { w r B g } \\
\text { w olg } \\
\text { g' br B p } \\
\text { w r } \mid g \\
\text { g }\end{array}$ & $\begin{array}{r}28000 \\
24000 \\
22000 \\
25000 \\
20000 \\
27000 \\
10000 \\
20000 \\
19000 \\
23000 \\
14000 \\
25000 \\
14000 \\
12000 \\
12000 \\
23000 \\
10000 \\
14000 \\
27000 \\
30000 \\
25000 \\
28000 \\
28000 \\
28000 \\
18000 \\
14000 \\
19000 \\
14000 \\
14000 \\
21000 \\
14000 \\
14000 \\
6000 \\
6000 \\
7000 \\
6500 \\
5000 \\
8000 \\
22000 \\
28000 \\
25000 \\
18000 \\
11000 \\
13000 \\
31000 \\
45000 \\
33000 \\
33000 \\
25000 \\
28000 \\
30000 \\
45000 \\
\end{array}$ & - \\
\hline
\end{tabular}


TABLE I-Continued.

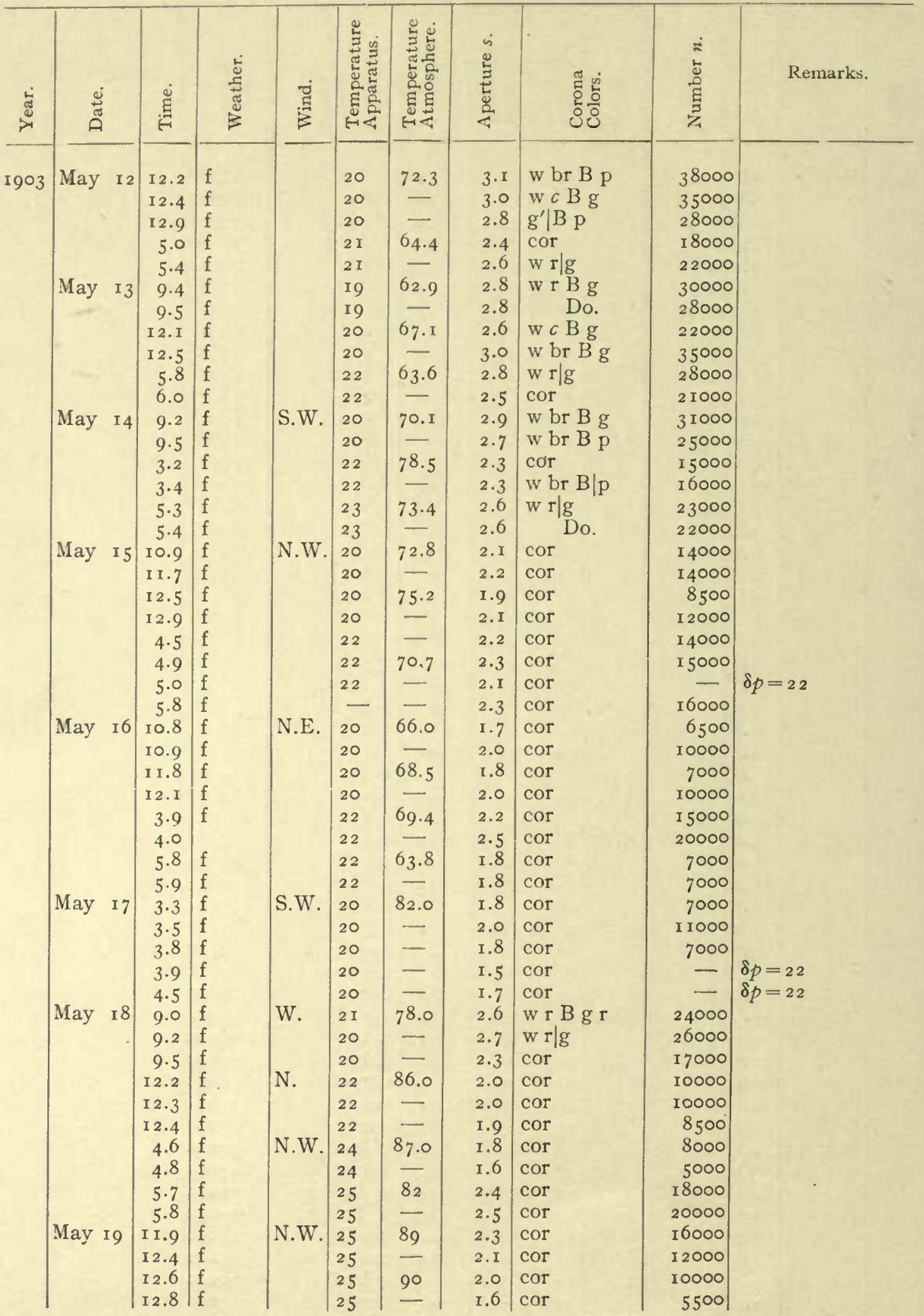


TABLE I-Continued.

\begin{tabular}{|c|c|c|c|c|c|c|c|c|c|c|}
\hline 崩 & 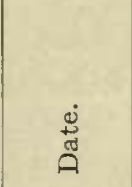 & 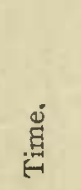 & 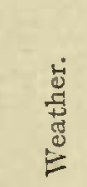 & 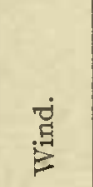 & 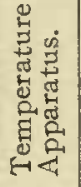 & 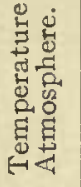 & 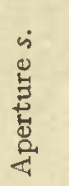 & $\begin{array}{l}\text { 范 } \\
0 \\
80 \\
80 \\
80\end{array}$ & 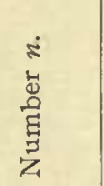 & Remarks. \\
\hline 1903 & $\begin{array}{l}\text { May } 21 \\
\text { May } 22 \\
\end{array}$ & 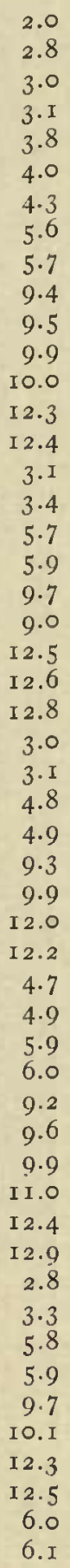 & 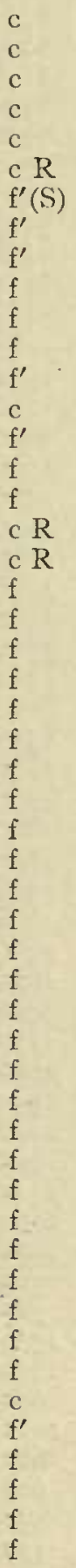 & $\begin{array}{l}\text { N.W. } \\
\text { S. }\end{array}$ & $\begin{array}{l}25 \\
25 \\
25 \\
25 \\
25 \\
25 \\
25 \\
25 \\
25 \\
23 \\
23 \\
23 \\
23 \\
24 \\
24 \\
26 \\
26 \\
27 \\
27 \\
24 \\
24 \\
25 \\
25 \\
25 \\
26 \\
26 \\
26 \\
26 \\
24 \\
24 \\
25 \\
25 \\
28 \\
27 \\
28 \\
28 \\
22 \\
21 \\
21 \\
21 \\
22 \\
22 \\
23 \\
23 \\
23 \\
23 \\
22 \\
22 \\
22 \\
32 \\
21 \\
21\end{array}$ & 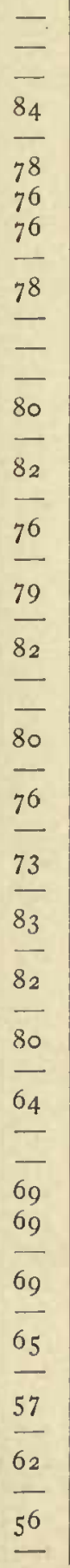 & $\begin{array}{r}2.2 \\
2.0 \\
1.8 \\
1.8 \\
2.4 \\
1.8 \\
2.0 \\
2.3 \\
2.1 \\
2.7 \\
2.5 \\
2.8 \\
2.2 \\
2.2 \\
2.3 \\
2.5 \\
2.9 \\
2.6 \\
2.1 \\
2.0 \\
2.4 \\
2.2 \\
2.5 \\
2.4 \\
2.4 \\
2.6 \\
2.6 \\
2.3 \\
2.2 \\
2.5 \\
2.0 \\
2.1 \\
2.1 \\
2.1 \\
105 t \\
2.9 \\
2.0 \\
2.0 \\
2.9 \\
2.6 \\
2.4 \\
2.4 \\
2.8 \\
2.2 \\
2.2 \\
2.7 \\
2.0\end{array}$ & $\begin{array}{l}\text { cor } \\
\text { cor } \\
\text { cor } \\
\text { cor } \\
\text { cor } \\
\text { cor } \\
\text { cor } \\
\text { w br B } \mid \mathrm{p} \\
\text { cor } \\
\text { cor } \\
\text { w br } \\
\text { w r } \\
\text { w r } \mid g \\
\text { cor } \\
\text { cor } \\
\text { cor } \\
\text { cor } \\
\text { w r } \mid g \\
\text { w r } \mid g \\
\text { cor } \\
\text { cor } \\
\text { cor } \\
\text { g br B } \mid \text { p } \\
\text { w br } \\
\text { w br } \\
\text { cor } \\
\text { w r } \mid g \\
\text { w r g } \\
\text { cor } \\
\text { cor } \\
\text { cor } \\
\text { cor } \\
\text { cor } \\
\text { cor } \\
\text { cor } \\
\text { g' B p } \\
\text { g' br B p } \\
y^{\prime} \text { br b } \\
\text { w r } \mid g \\
\text { w br } \\
\text { w r } \mid g \\
\text { cor } \\
\text { cor } \\
\text { cor } \\
\text { cor } \\
\text { cor } \\
\text { w r g } \\
\text { cor } \\
\text { cor }\end{array}$ & 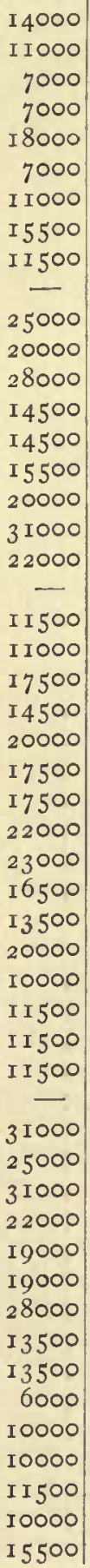 & $\begin{array}{l}\text { Storm coming. } \\
\text { Thunder. } \\
\text { Hail. }\end{array}$ \\
\hline
\end{tabular}


TABLE I-Continued.

\begin{tabular}{|c|c|c|c|c|c|c|c|c|c|c|}
\hline 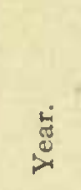 & 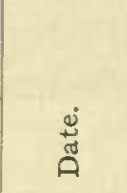 & E્. & 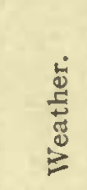 & $\stackrel{己}{\Xi}$ & 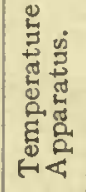 & 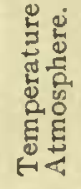 & 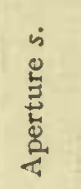 & 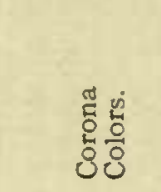 & $\frac{2}{\text { 岕 }}$ & Remarks. \\
\hline I 903 & $\begin{array}{l}\text { May } 28 \\
\text { May } 29\end{array}$ & 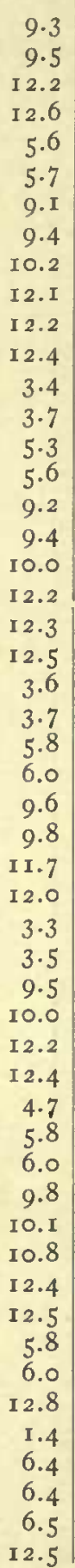 & 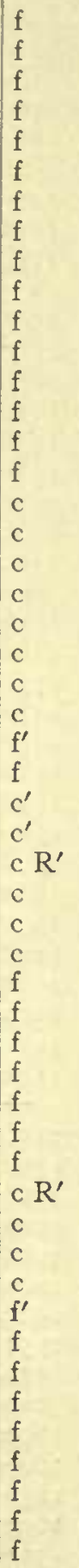 & $\begin{array}{l}\text { N.W. } \\
\text { N.E. }\end{array}$ & $\begin{array}{l}20 \\
19 \\
21 \\
20 \\
22 \\
22 \\
19 \\
19 \\
19 \\
20 \\
20 \\
20 \\
20 \\
20 \\
21 \\
20 \\
20 \\
25 \\
20 \\
21 \\
21 \\
21 \\
21 \\
21 \\
22 \\
22 \\
21 \\
21 \\
22 \\
22 \\
23 \\
23 \\
21 \\
20 \\
22 \\
22 \\
24 \\
23 \\
23 \\
20 \\
21 \\
21 \\
21 \\
20 \\
23 \\
23 \\
20 \\
20 \\
20 \\
20 \\
20 \\
19\end{array}$ & 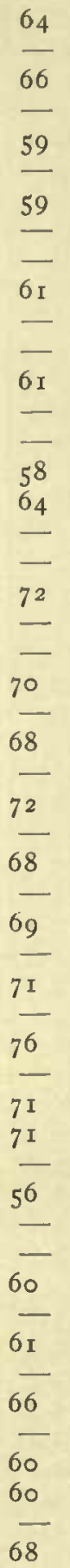 & $\begin{array}{l}2.7 \\
2.7 \\
2.1 \\
2.1 \\
2.4 \\
2.4 \\
2.1 \\
2.6 \\
2.6 \\
2.2 \\
2.3 \\
2.2 \\
2.2 \\
2.3 \\
2.5 \\
2.5 \\
2.7 \\
2.9 \\
2.6 \\
2.6 \\
3.0 \\
2.7 \\
2.3 \\
2.3 \\
2.3 \\
2.6 \\
1.7 \\
2.6 \\
2.8 \\
2.0 \\
2.0 \\
2.7 \\
2.7 \\
2.7 \\
2.8 \\
2.4 \\
2.6 \\
2.3 \\
2.5 \\
2.7 \\
2.0 \\
2.0 \\
2.0 \\
2.8 \\
2\end{array}$ & $\begin{array}{l}\text { w r } \mid g \\
\text { w o b } \\
\text { cor } \\
\text { cor } \\
\text { w r B p } \\
\quad \text { Do. } \\
\text { cor } \\
\text { w r } \mid g \\
\quad \text { Do. } \\
\text { cor } \\
\text { w br B p } \\
\text { cor } \\
\text { cor } \\
\text { cor } \\
\text { cor } \\
\text { cor } \\
\text { w r } \mid g \\
\text { w } r \text { B } \\
\text { cor } \\
\text { cor } \\
\text { w br B p } \\
\text { w r } \mid g \\
\text { w br B p } \\
\text { w br B } \mid \text { p } \\
\text { Do. } \\
\text { g' br B p } \\
\text { Do. } \\
y^{\prime} r \mid g \\
g^{\prime} \text { br B p } \\
\text { w r } \mid g \\
\text { w r g } \\
\text { w r g } \\
y^{\prime} \text { or bg } \\
\text { cor } \\
\text { w r } \mid g \\
\text { w br B } \mid p \\
\text { w br B } \\
\text { w r } \mid g \\
\text { cor } \\
\text { cor } \\
\text { cor } \\
\text { cor } \\
\text { cor } \\
\text { cor } \\
\text { cor } \\
\text { cor } \\
\text { cor } \\
\text { cor } \\
\text { cor } \\
\text { cor } \\
\text { cor }\end{array}$ & 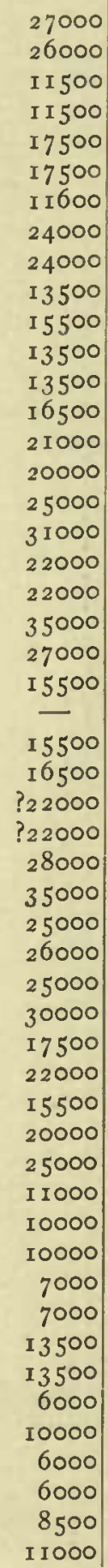 & Rain at night. \\
\hline
\end{tabular}


TABLE I-Continued.

\begin{tabular}{|c|c|c|c|c|c|c|c|c|c|c|}
\hline$\overbrace{-1}^{d}$ & 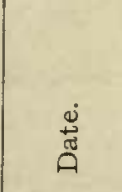 & 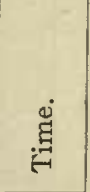 & 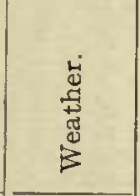 & $\bar{E}$ & 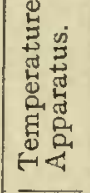 & 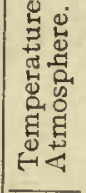 & 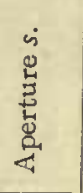 & 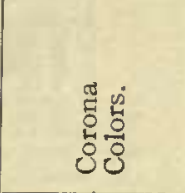 & 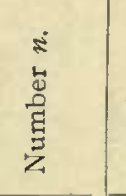 & Remarks. \\
\hline $9 \circ 3$ & June 2 & $\begin{array}{r}\mathrm{I} 2.7 \\
4.6 \\
5.4 \\
5.6 \\
9.4 \\
9.7 \\
\mathrm{I} 2.2 \\
\mathrm{I} 2.4 \\
5.7 \\
6.0 \\
7.6 \\
7.8 \\
\mathrm{I} 0.2 \\
9.3 \\
9.4 \\
\mathrm{I} 2.2 \\
\mathrm{I} 2.6 \\
3.1 \\
3.3 \\
5.7 \\
5.8 \\
9.0 \\
9.5 \\
9.8 \\
\mathrm{I} 2.0 \\
\mathrm{I} 2.2 \\
2.4 \\
2.5 \\
2.6 \\
2.6 \\
5.9 \\
6.0 \\
9.5 \\
9.7 \\
9.7 \\
\mathrm{I} 2 . \mathrm{I} \\
\mathrm{I} 2.3 \\
3.7 \\
3.8 \\
5.5 \\
5.7\end{array}$ & \begin{tabular}{|l|l|} 
& f \\
f \\
f \\
f \\
f \\
f \\
f \\
f \\
f \\
f \\
f \\
f \\
f \\
f \\
f \\
f \\
f \\
f \\
f \\
f \\
f \\
f \\
fog \\
" \\
red sun \\
fog, yel. \\
red sun \\
fog \\
red sun \\
fog \\
red sun \\
fog \\
fog \\
haze, \\
sun yel. \\
Do. \\
Do. \\
Do. \\
Do. \\
Do. \\
Do. \\
Do. \\
haze \\
sun \\
ruby \\
haze \\
haze \\
c \\
c \\
c \\
c \\
c
\end{tabular} & . & $\begin{array}{l}\text { I9 } \\
2 \mathrm{I} \\
2 \mathrm{I} \\
2 \mathrm{I} \\
\mathrm{I} 9 \\
\mathrm{I} 9 \\
20 \\
20 \\
22 \\
22 \\
22 \\
22 \\
21 \\
20 \\
20 \\
24 \\
24 \\
25 \\
25 \\
25 \\
25 \\
23 . \mathrm{I} \\
23 . \mathrm{I} \\
23 . \mathrm{I} \\
24 . \mathrm{I} \\
2 \mathrm{I} . \mathrm{I} \\
22 . \mathrm{I} \\
22 . \mathrm{I} \\
\\
22 . \mathrm{I} \\
23 . \mathrm{I} \\
23 . \mathrm{I} \\
19 . \mathrm{I} \\
18 . \mathrm{I} \\
18 . \mathrm{I} \\
20 . \mathrm{I} \\
20 . \mathrm{I} \\
22 . \mathrm{I} \\
22 . \mathrm{I} \\
2 \mathrm{II} \\
22 . \mathrm{I} \\
\\
\mathrm{I} 9 . \mathrm{I} \\
19 . \mathrm{I} \\
22 . \mathrm{I} \\
22 . \mathrm{I} \\
22 . \mathrm{I} \\
22 . \mathrm{I} \\
22 . \mathrm{I}\end{array}$ & 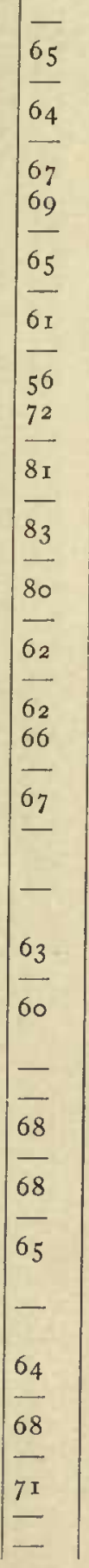 & $\begin{array}{r}2.3 \\
2.2 \\
2.6 \\
2.6 \\
105 t \\
1.7 \\
2.5 \\
2.8 \\
2.7 \\
2.7 \\
2.3 \\
2.3 \\
2.4 \\
2.0 \\
2.1 \\
1.9 \\
2.0 \\
2.0 \\
2.0 \\
2.5 \\
2.9 \\
3.0 \\
2.9 \\
2.8 \\
2.5 \\
2.4 \\
2.4 \\
2.5 \\
2.4 \\
2.3 \\
2.3 \\
3.0 \\
2.6 \\
2.5 \\
2.0 \\
1.8 \\
2.2 \\
2.2 \\
2.4 \\
2.5 \\
2.8 \\
2.8 \\
2.2 \\
2.5 \\
2.4 \\
2.6 \\
2.4\end{array}$ & 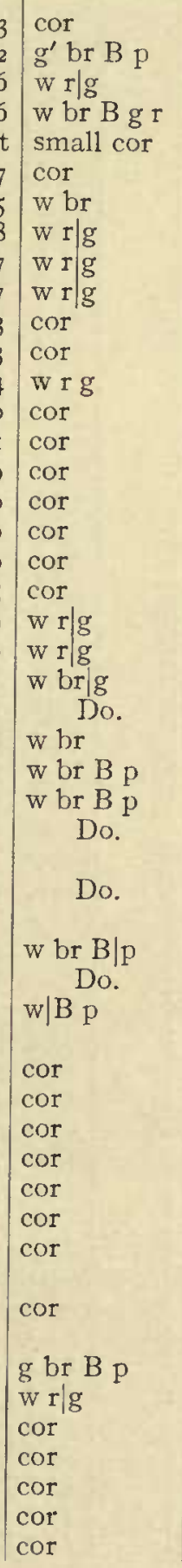 & 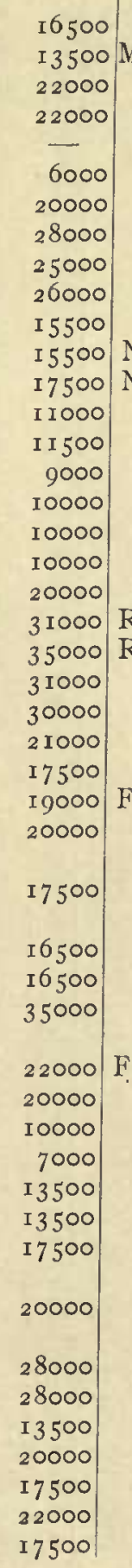 & \begin{tabular}{|l|l} 
& Maine forest fires. \\
Night. \\
mounting. \\
Red moon. \\
Red sun. \\
Forest fires on \\
Cape Cod. \\
Forest fires on \\
Cape Cod.
\end{tabular} \\
\hline
\end{tabular}


TABLE I-Continued.

\begin{tabular}{|c|c|c|c|c|c|c|c|c|c|c|}
\hline 常 & 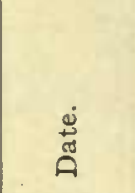 & $\underset{E}{\stackrel{\Xi}{E}}$ & 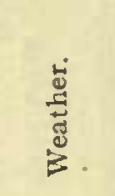 & 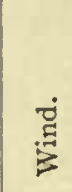 & 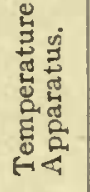 & 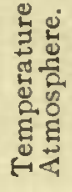 & 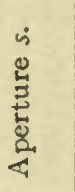 & 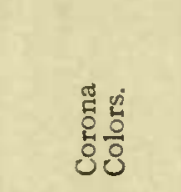 & 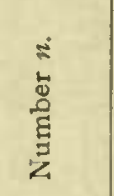 & Remarks. \\
\hline 1903 & $\begin{array}{l}\text { June I I } \\
\text { June I } 2\end{array}$ & $\begin{array}{r}5.8 \\
5.9 \\
\text { I0.3 } \\
\text { I0.8 } \\
\text { I } 2.0 \\
\text { I } 2.1 \\
4.6 \\
4.8 \\
6.1 \\
6.3 \\
\text { I0.4 } \\
\text { I I } 11 \\
\text { I I } 2.5 \\
\text { I } 2.8 \\
\text { I } 2.5 \\
2.5 \\
2.7 \\
3.1 \\
6.8 \\
6.9 \\
9.0 \\
9.2 \\
\text { I } 2.4 \\
\text { I } 2.5 \\
3.1 \\
3.3 \\
5.5 \\
5.6 \\
9.3 \\
9.5 \\
\text { I } 2.0 \\
\text { I } 2.2 \\
3.4 \\
3.6 \\
5.8 \\
6.0 \\
9.5 \\
9.6 \\
\text { I } 2.2 \\
\text { I } 2.3 \\
4.0 \\
4.1 \\
6.1 \\
6.2 \\
9.2 \\
9.4 \\
\text { I } 2.7 \\
\text { I } 2.8 \\
4.2 \\
4.8 \\
6.0 \\
6.1\end{array}$ & $\begin{array}{l}c \\
c \\
R^{\prime} \\
R^{\prime} \\
c R^{\prime} \\
c \\
f^{\prime} \\
f \\
f \\
f \\
c \\
c \\
c \\
c \\
c \\
c \\
c \\
c \\
c \\
R \\
R \\
c \\
c \text { wet } \\
c \\
c \\
c \\
c \\
c \\
c \\
c \\
c \\
c \\
c \\
c \\
c \\
c \\
c \\
c \\
c \\
c \\
c \\
c \\
c \\
R \\
\text { C } \\
c \\
c \\
c \\
c \\
c \\
c \\
c \\
R \\
R \\
R \\
R \\
R \\
c \\
c \\
c \\
c \\
c \\
c\end{array}$ & E. & 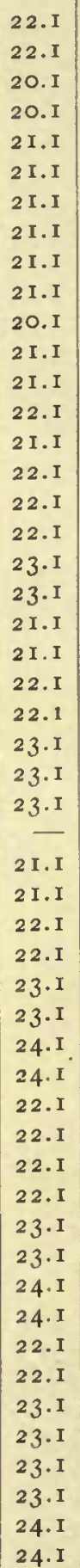 & $\begin{array}{l}\frac{69}{65} \\
\frac{66}{68} \\
\frac{66}{66} \\
\frac{72}{73} \\
\frac{75}{70} \\
\frac{67}{67} \\
\frac{68}{74} \\
\frac{72}{67} \\
\frac{70}{75} \\
\frac{75}{71} \\
\frac{75}{67} \\
\frac{70}{60} \\
\frac{70}{60} \\
\frac{65}{70}\end{array}$ & $\begin{array}{l}2.3 \\
2.3 \\
2.7 \\
2.0 \\
2.2 \\
2.0 \\
2.0 \\
2.1 \\
2.1 \\
1.9 \\
2.1 \\
2.5 \\
2.3 \\
2.0 \\
2.4 \\
2.1 \\
1.9 \\
2.2 \\
2.1 \\
2.2 \\
2.3 \\
2.1 \\
2.7 \\
2.7 \\
2.1 \\
2.3 \\
2.3 \\
2.2 \\
1.4 \\
1.5 \\
1.7 \\
1.8 \\
2.0 \\
2.3 \\
1.8 \\
2.0 \\
1.8 \\
1.7 \\
1.9 \\
1.9 \\
1.6 \\
1.6 \\
1.7 \\
1.7 \\
1.3 \\
1.3 \\
1.4 \\
1.6 \\
1.8 \\
1.7 \\
1.7 \\
1.7\end{array}$ & 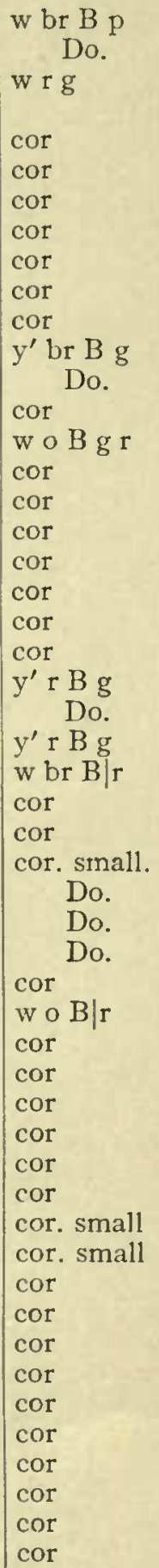 & 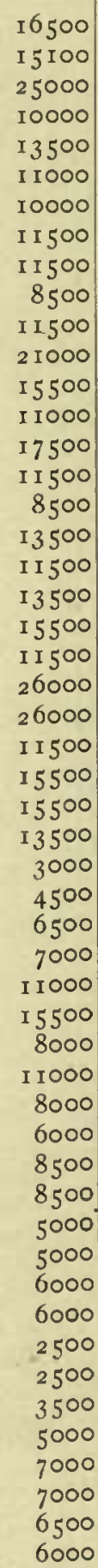 & $\begin{array}{l}\text { New axis and } \\
\text { support. }\end{array}$ \\
\hline
\end{tabular}


A CONTINUOUS RECORD OF ATMOSPHERIC NUCLEATION.

TABLE I-Continued.

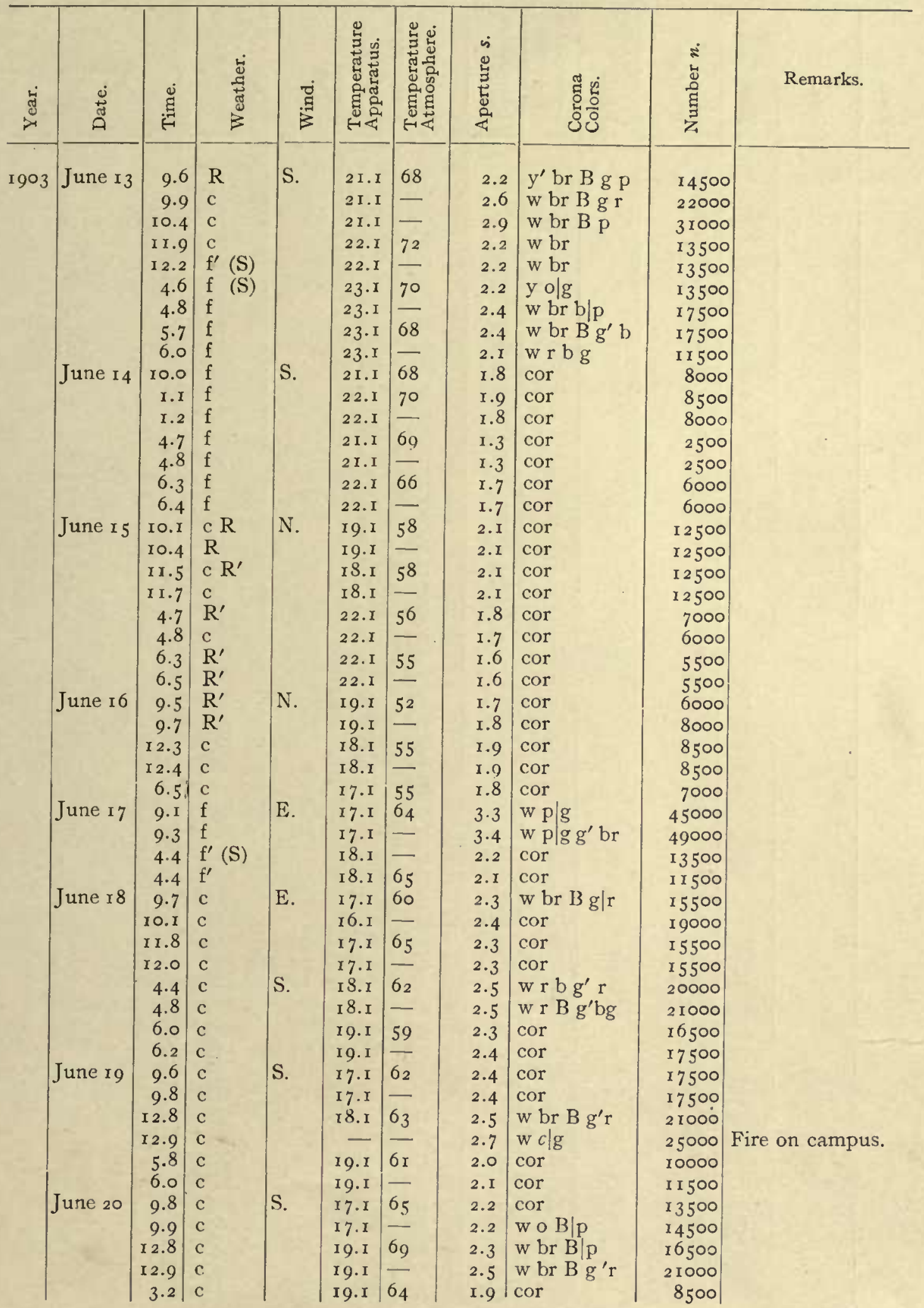


TABLE $\mathrm{x}$-Continued.

\begin{tabular}{|c|c|c|c|c|c|c|c|c|c|c|}
\hline है & ڤ̆ & 总 & 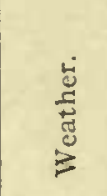 & 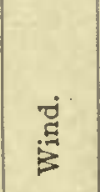 & 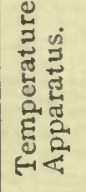 & 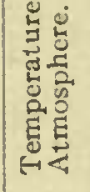 & 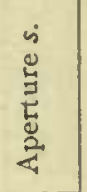 & 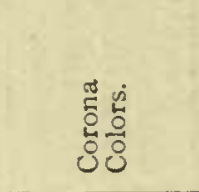 & 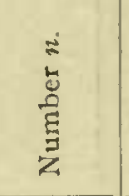 & Remarks. \\
\hline $9 \circ 3$ & $\left|\begin{array}{l}\text { June } 20 \\
\text { June } 2 \mathrm{I}\end{array}\right|$ & $\begin{array}{r}5.8 \\
6.0 \\
10.5 \\
\text { I0.6 } \\
\text { I } 2.2 \\
\text { I } 2.3 \\
4.3 \\
4.8 \\
6.4 \\
6.6 \\
9.5 \\
9.7 \\
12.6 \\
2.8 \\
5.6 \\
5.9 \\
9.6 \\
9.8 \\
12.5 \\
1.0 \\
5.8 \\
6.0 \\
9.8 \\
9.9 \\
1.3 \\
5.8 \\
6.0 \\
9.6 \\
10.7 \\
\text { I } 2.8 \\
\text { I } 2.9 \\
3.7 \\
3.8 \\
5.9 \\
6.0 \\
9.5 \\
9.8 \\
12.2 \\
\text { I } 2.4 \\
4.3 \\
6.0 \\
9.7 \\
9.8 \\
\text { I } 2.0 \\
4.4 \\
6.4 \\
10.3 \\
\text { I0.8 } \\
1.3 \\
1.4 \\
5.0 \\
6.8\end{array}$ & 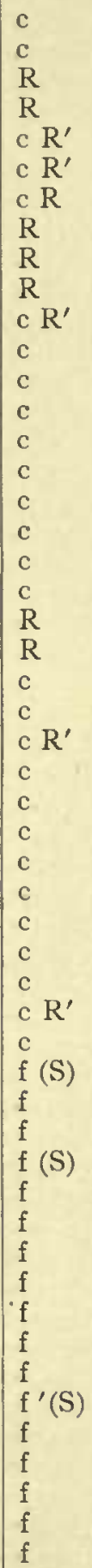 & N. E. & $\begin{array}{l}19.1 \\
19.1 \\
18.1 \\
18.1 \\
18.1 \\
18.1 \\
18.1 \\
18.1 \\
19.1 \\
19.1 \\
17.1 \\
17.1 \\
18.1 \\
19.1 \\
19.1 \\
19.1 \\
17.1 \\
17.1 \\
18.1 \\
18.1 \\
19.1 \\
19.1 \\
17.1 \\
17.1 \\
18.1 \\
19.1 \\
19.1 \\
16.1 \\
16.1 \\
17.1 \\
17.1 \\
18.1 \\
18.1 \\
18.1 \\
18.1 \\
16.1 \\
17.1 \\
18.1 \\
18.1 \\
20.1 \\
20.1 \\
18.1 \\
18.1 \\
19.1 \\
20.1 \\
21.1 \\
19.1 \\
19.1 \\
20.1 \\
20.1 \\
20.1 \\
20.1\end{array}$ & $\begin{array}{l}\frac{63}{62} \\
\frac{62}{62} \\
60 \\
\frac{58}{58} \\
\frac{57}{79} \\
59 \\
\frac{59}{59} \\
\frac{59}{78} \\
\frac{58}{54} \\
55 \\
57 \\
\frac{54}{57} \\
\frac{57}{59} \\
60 \\
59 \\
70 \\
74 \\
76 \\
73 \\
\frac{75}{81} \\
80 \\
75 \\
\frac{72}{75} \\
75 \\
69 \\
65\end{array}$ & $\begin{array}{l}2.5 \\
2.4 \\
1.8 \\
1.7 \\
1.5 \\
1.5 \\
1.8 \\
1.8 \\
1.8 \\
1.8 \\
1.7 \\
1.7 \\
1.8 \\
1.9 \\
2.8 \\
3.6 \\
1.6 \\
4.1 \\
1.8 \\
1.8 \\
2.0 \\
1.9 \\
1.6 \\
1.8 \\
1.6 \\
1.8 \\
1.9 \\
2.1 \\
2.0 \\
2.3 \\
2.5 \\
2.1 \\
2.1 \\
2.3 \\
2.3 \\
2.4 \\
2.3 \\
2.8 \\
2.8 \\
2.5 \\
2.3 \\
1.8 \\
2.4 \\
2.5 \\
2.7 \\
2.3 \\
3.0 \\
2.8 \\
2.3 \\
2.3 \\
2.2 \\
1.9\end{array}$ & $\begin{array}{l}\text { cor } \\
\text { w br B } \mid \mathrm{p} \\
\text { cor } \\
\text { cor } \\
\text { cor } \\
\text { cor } \\
\text { cor } \\
\text { cor } \\
\text { cor } \\
\text { cor } \\
\text { cor } \\
\text { cor } \\
\text { cor } \\
\text { cor } \\
\text { w|B p } \\
\text { w r g } \\
\text { cor } \\
\text { g B p } \\
\text { cor } \\
\text { cor } \\
\text { cor } \\
\text { cor } \\
\text { cor } \\
\text { cor } \\
\text { cor . } \\
\text { cor } \\
\text { cor } \\
\text { cor } \\
\text { cor } \\
\text { w o B g'r } \\
\text { w br B g r } \\
\text { cor } \\
\text { w o b g } \\
\text { w br B g } \\
\text { Do. } \\
\text { y' br B g' r } \\
\text { w br B g r } \\
\text { w orlg } \\
\text { w or b g r } \\
\text { w br B p } \\
\text { w br B g r } \\
\text { cor } \\
\text { w br B g'r } \\
\text { Do. } \\
\text { w br b g' r } \\
\text { w br|g } \\
\text { y br|g } \\
\text { Do. } \\
\text { w br B g'p } \\
\text { w br B g'p } \\
\text { w brig } \\
\text { cor }\end{array}$ & $\begin{array}{r}20000 \\
19000 \\
7000 \\
6000 \\
4000 \\
4000 \\
7000 \\
7000 \\
7000 \\
7000 \\
6000 \\
6500 \\
7000 \\
9000 \\
30000 \\
57000 \\
5500 \\
100000 \\
7000 \\
7000 \\
10000 \\
8500 \\
5500 \\
7000 \\
5500 \\
7000 \\
8500 \\
12500 \\
11000 \\
15500 \\
20000 \\
11500 \\
11500 \\
16500 \\
15500 \\
17500 \\
15500 \\
28000 \\
28000 \\
21000 \\
15500 \\
77000 \\
17500 \\
21000 \\
25000 \\
15500 \\
35000 \\
30000 \\
16500 \\
16500 \\
14500 \\
8500\end{array}$ & $\begin{array}{l}\text { Fire on campus. } \\
\text { Fire on campus. }\end{array}$ \\
\hline
\end{tabular}


TABLE I-Continued.

\begin{tabular}{|c|c|c|c|c|c|c|c|c|c|c|}
\hline 岕 & 离 & 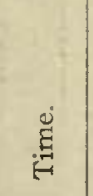 & 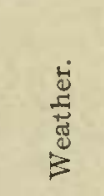 & 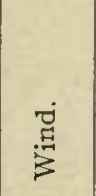 & 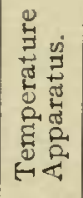 & 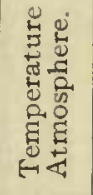 & 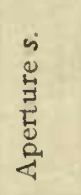 & 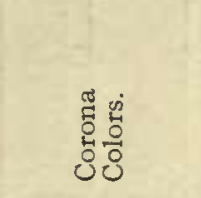 & 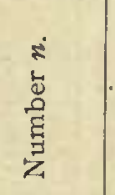 & Remarks. \\
\hline 1903 & $\begin{array}{l}\text { July } 6 \\
\text { July } 7\end{array}$ & $\begin{array}{r}9.6 \\
\text { I I.9 } \\
3.4 \\
5.8 \\
\\
9.6 \\
9.8 \\
\\
\text { IO.0 } \\
\text { I } 2.2 \\
2.5 \\
5.3 \\
5.5 \\
9.3 \\
\text { IO.5 } \\
\text { I } 2.7 \\
2.3 \\
4.7 \\
5.5 \\
9.5 \\
9.7 \\
\text { I I.3 } \\
\text { I.8 } \\
4.3 \\
5.5 \\
9.7 \\
\text { I0.7 } \\
\text { I.0 } \\
2.8 \\
4.8 \\
4.8 \\
5.5 \\
9.8\end{array}$ & 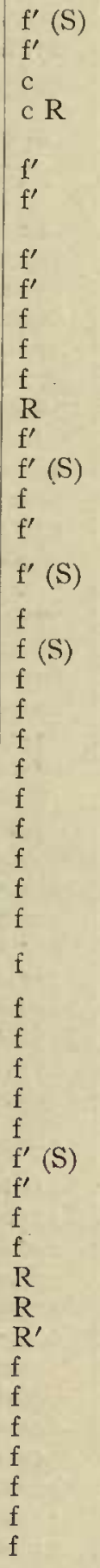 & S. W. & 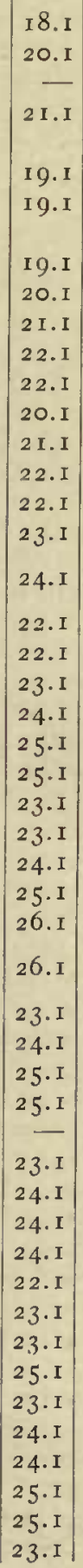 & $\begin{array}{l}72 \\
75 \\
71 \\
72 \\
77 \\
85 \\
87 \\
87 \\
87 \\
90 \\
92 \\
90 \\
89 \\
82 \\
82 \\
84 \\
85 \\
84 \\
82 \\
74 \\
78 \\
79 \\
81 \\
71 \\
74 \\
78 \\
77 \\
73 \\
66 \\
66 \\
68 \\
71 \\
79 \\
83 \\
85 \\
85 \\
84 \\
83\end{array}$ & 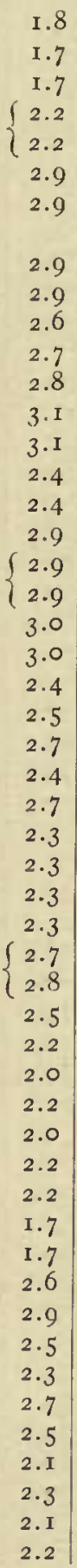 & 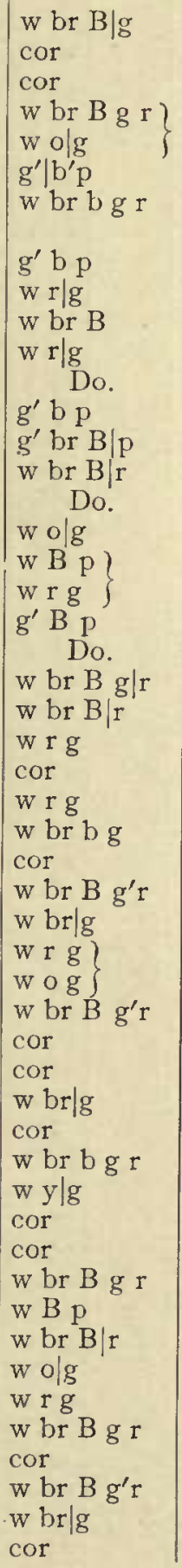 & 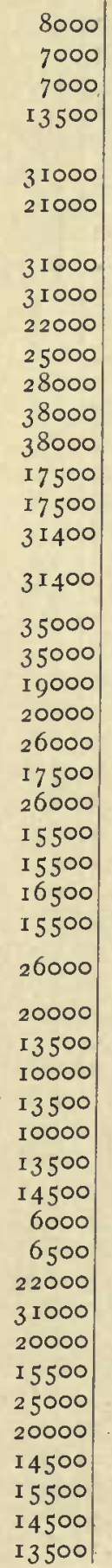 & $\begin{array}{l}\text { Runs to } \mathrm{w} \mathrm{g} \text { for } \\
\text { large } d \text {. } \\
\text { Fire on campus. }\end{array}$ \\
\hline
\end{tabular}


TABLE I-Continued.

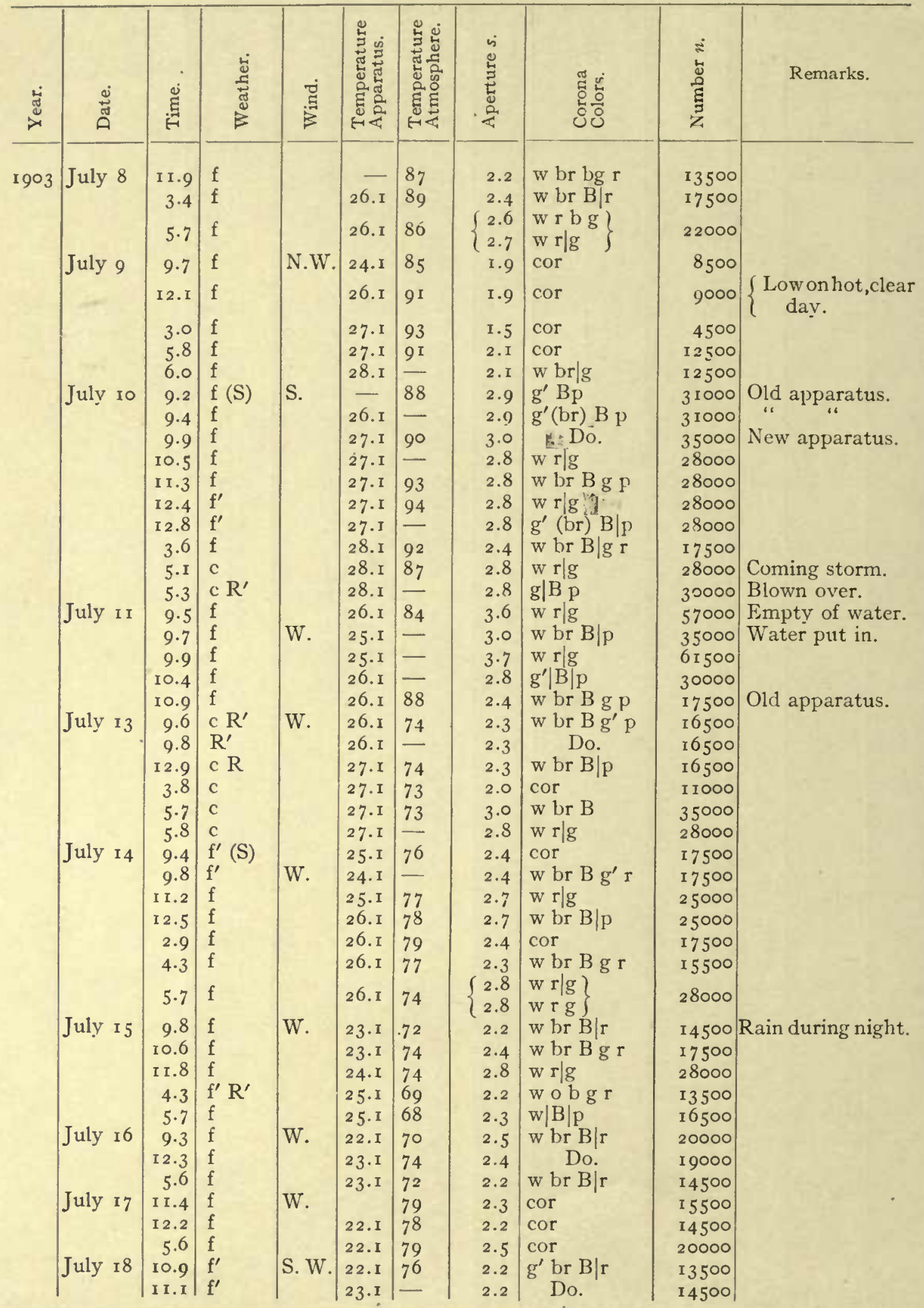


TABLE I-Continued.

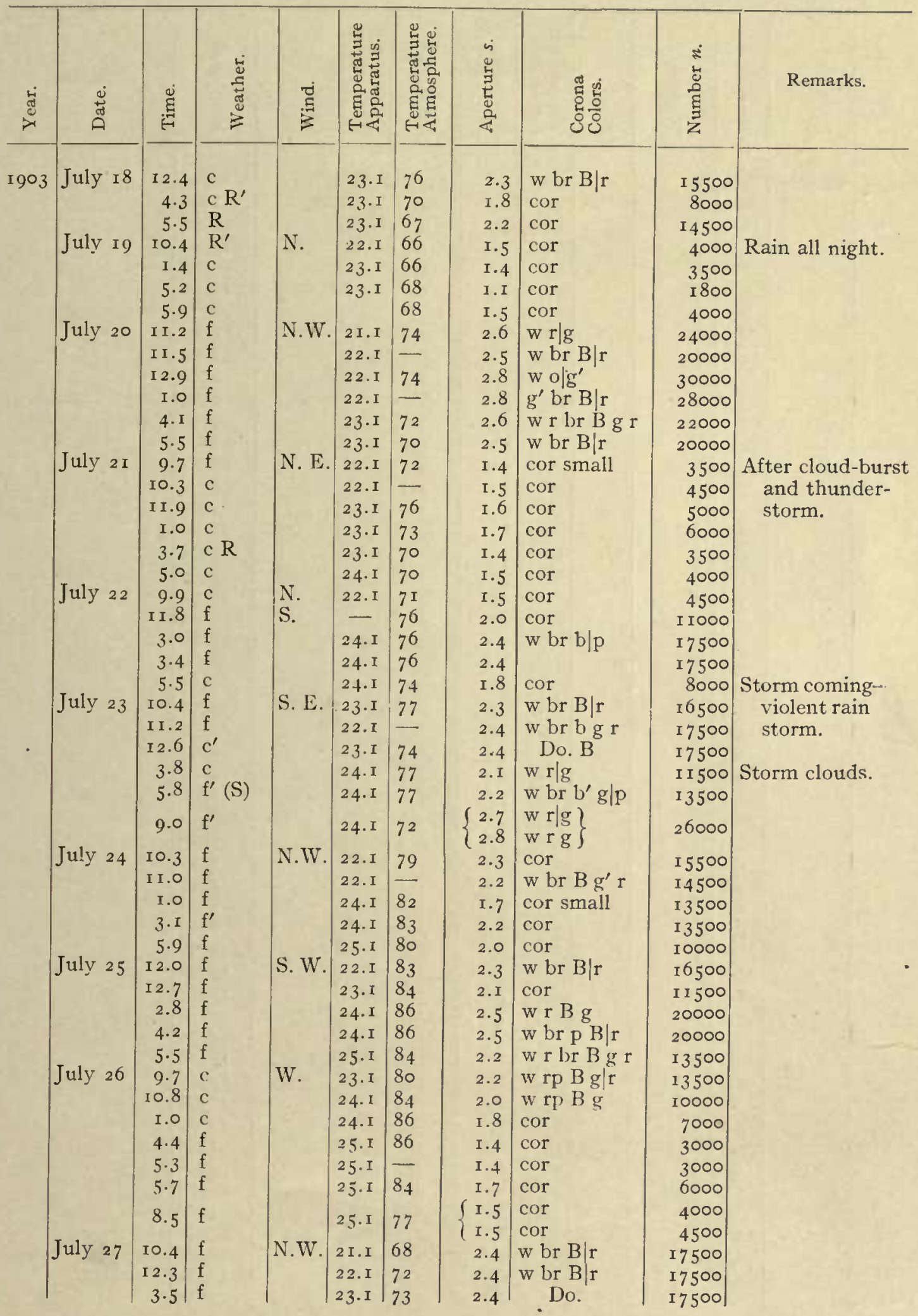


TABLE I-Continued.

\begin{tabular}{|c|c|c|c|c|c|c|c|c|c|c|}
\hline 苟 & อัँ & हूँ & 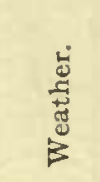 & 5 & 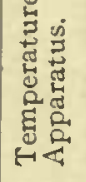 & 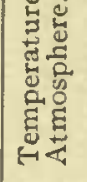 & 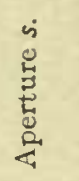 & 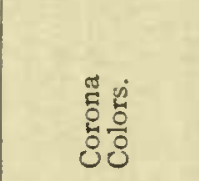 & 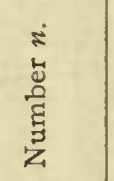 & Remarks. \\
\hline 1903 & $\begin{array}{l}\text { July } 29 \\
\text { July } 30\end{array}$ & \begin{tabular}{r|}
5.9 \\
9.8 \\
11.0 \\
$\mathrm{I} 2.5$ \\
3.0 \\
5.3 \\
6.0 \\
9.5 \\
$\mathrm{I} 2.3$ \\
$3 . \mathrm{I}$ \\
5.3 \\
9.7 \\
10.3 \\
$\mathrm{I} 2.9$ \\
3.0 \\
4.9 \\
5.5 \\
9.5 \\
11.9 \\
3.0 \\
4.4 \\
5.6 \\
9.4 \\
9.7 \\
$\mathrm{I} 2.4$ \\
3.1 \\
5.5 \\
9.3 \\
9.7 \\
$\mathrm{I} .4$ \\
$\mathrm{I} .4$ \\
4.8 \\
6.0 \\
9.5 \\
9.8 \\
10.8 \\
$\mathrm{I} 2.4$ \\
2.7 \\
4.8 \\
5.8 \\
9.6 \\
$\mathrm{I} 2.3$ \\
3.6 \\
5.8 \\
$\mathrm{I} 2.3$ \\
1.0 \\
4.7 \\
6.0 \\
$\mathrm{I} 2.3$ \\
1.2 \\
4.4 \\
5.1
\end{tabular} & $\begin{array}{l}f \\
f f \\
f f \\
f \\
f^{\prime}(S) \\
f \\
f \\
c \\
c \\
c \\
c \\
\text { f } \\
f \\
f \\
f \\
f \\
f \\
f \\
f \\
c^{\prime} \\
c \\
c \\
f \\
f \\
f \\
f \\
f \\
f \\
f \\
f \\
f \\
f \\
f \\
f \\
f \\
c^{\prime} \\
c^{\prime} \\
c^{\prime} \\
c^{\prime} \\
c^{\prime} \\
c \\
c \\
c \\
R \\
R \\
R \\
R \\
c R^{\prime} \\
c \\
c \\
c \\
c \\
c\end{array}$ & S. W. & 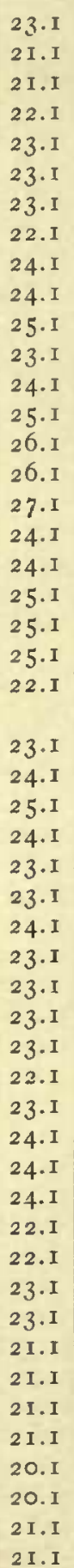 & $\begin{array}{l}70 \\
70 \\
72 \\
74 \\
76 \\
74 \\
75 \\
77 \\
78 \\
75 \\
84 \\
89 \\
89 \\
87 \\
85 \\
75 \\
78 \\
76 \\
72 \\
71 \\
70 \\
74 \\
76 \\
75 \\
66 \\
73 \\
76 \\
78 \\
78 \\
75 \\
70 \\
73 \\
76 \\
76 \\
72 \\
70 \\
66 \\
68 \\
65 \\
62 \\
60 \\
60 \\
60 \\
69 \\
68 \\
67 \\
65\end{array}$ & $\begin{array}{l}2.3 \\
2.9 \\
2.6 \\
2.3 \\
2.3 \\
2.6 \\
2.6 \\
2.5 \\
2.2 \\
2.3 \\
2.3 \\
2.8 \\
2.8 \\
2.4 \\
2.3 \\
2.2 \\
2.1 \\
2.1 \\
2.3 \\
2.3 \\
2.2 \\
2.1 \\
1.7 \\
2.3 \\
2.3 \\
2.1 \\
2.1 \\
2.4 \\
1.7 \\
1.9 \\
1.7 \\
1.5 \\
1.3 \\
1.8 \\
1.9 \\
3.2 \\
2.3 \\
2.2 \\
2.0 \\
1.6 \\
2.2 \\
2.2 \\
2.1 \\
2.0 \\
1.2 \\
1.5 \\
2.0 \\
1.5 \\
1.4 \\
1.1 \\
2.1 \\
2.0\end{array}$ & 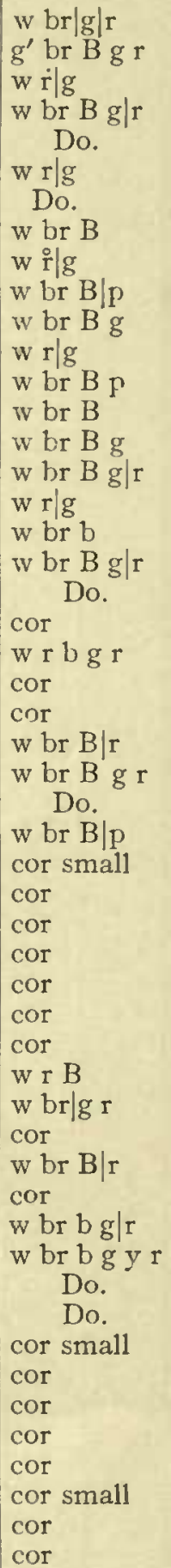 & 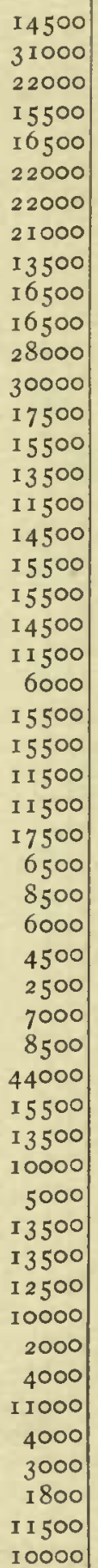 & $\begin{array}{l}\text { Storm coming. } \\
\text { After night's rain. }\end{array}$ \\
\hline
\end{tabular}


TABLE I-Continued.

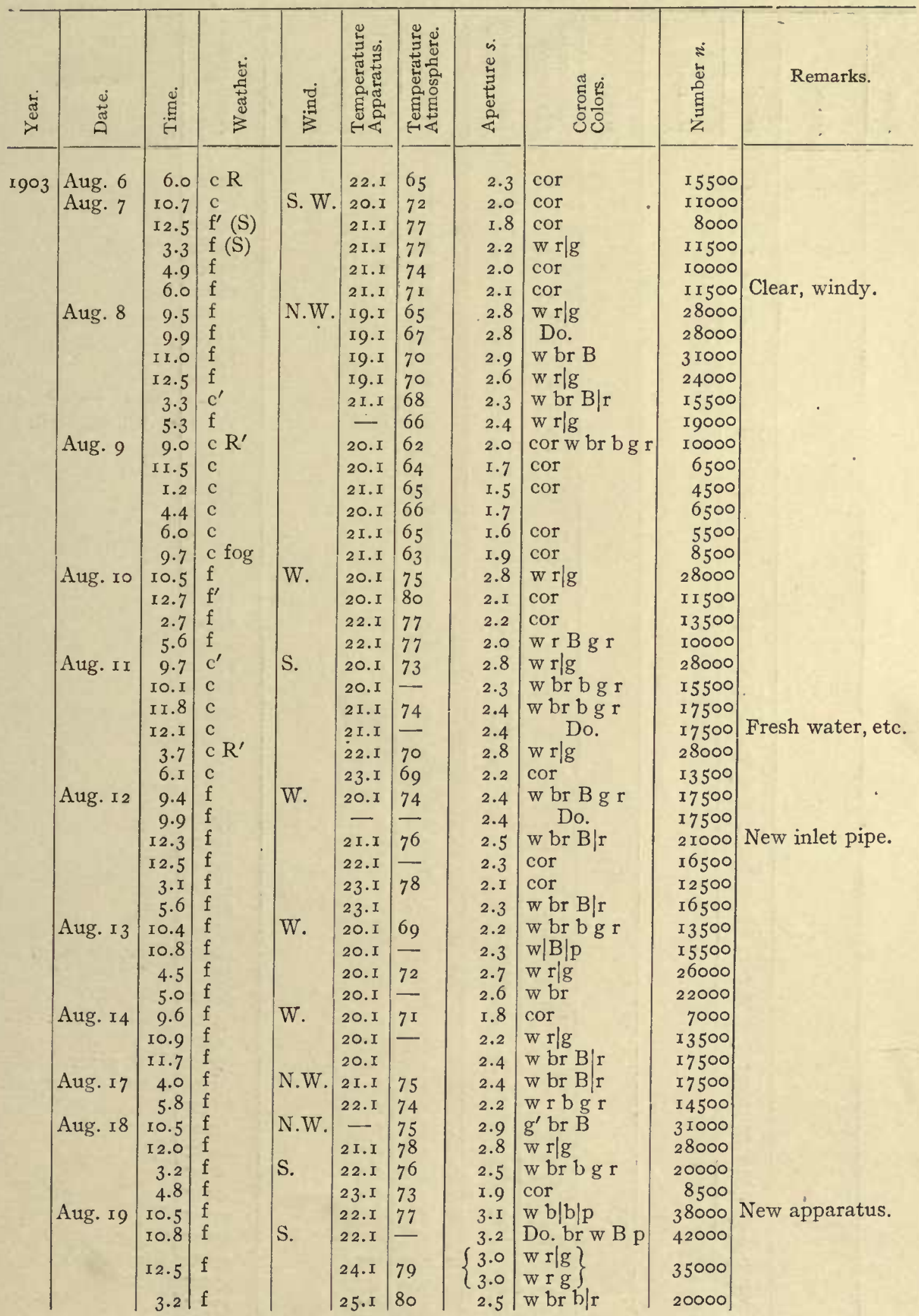


TABLE I-Contimued.

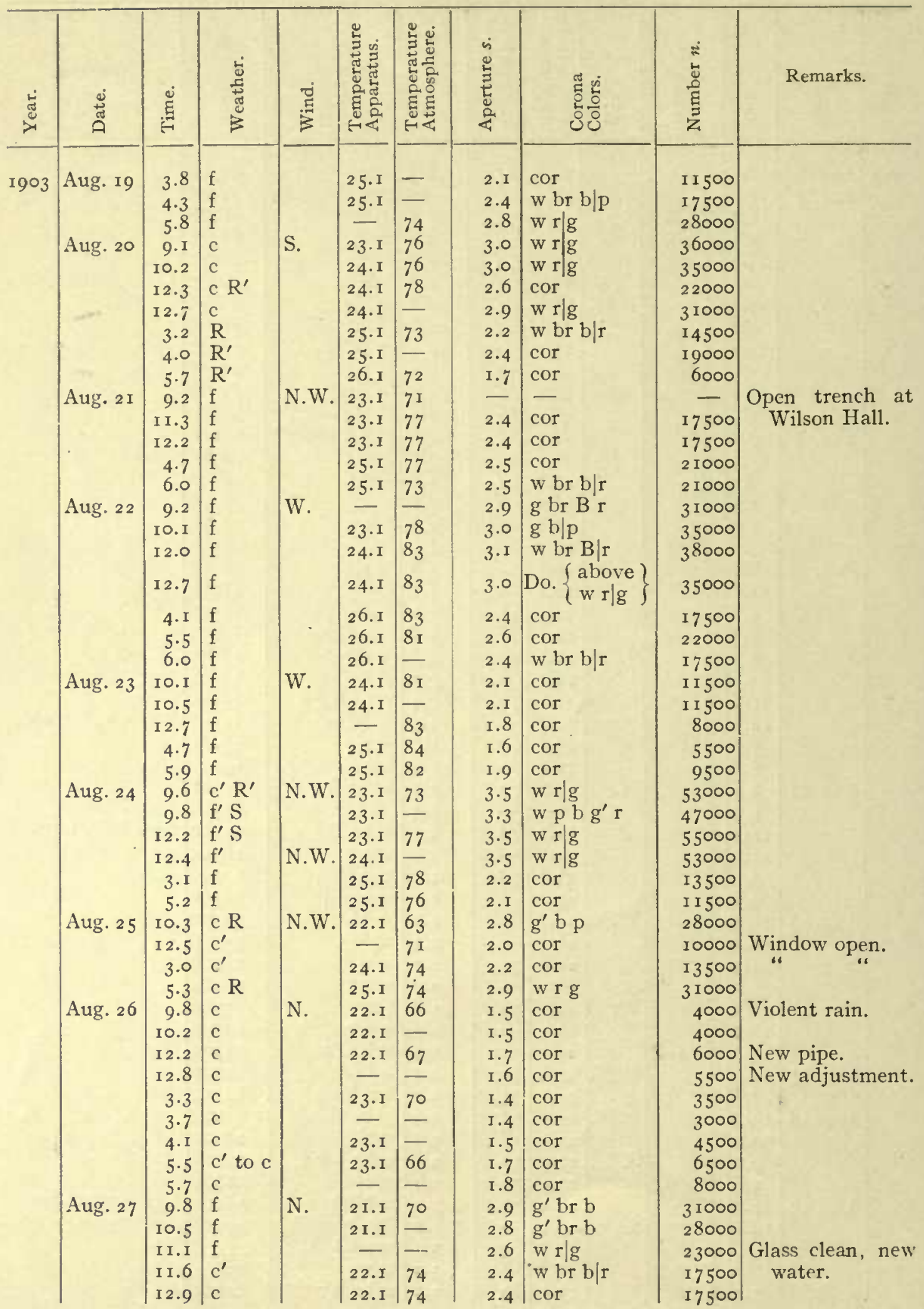


TABLE I-Contrnued.

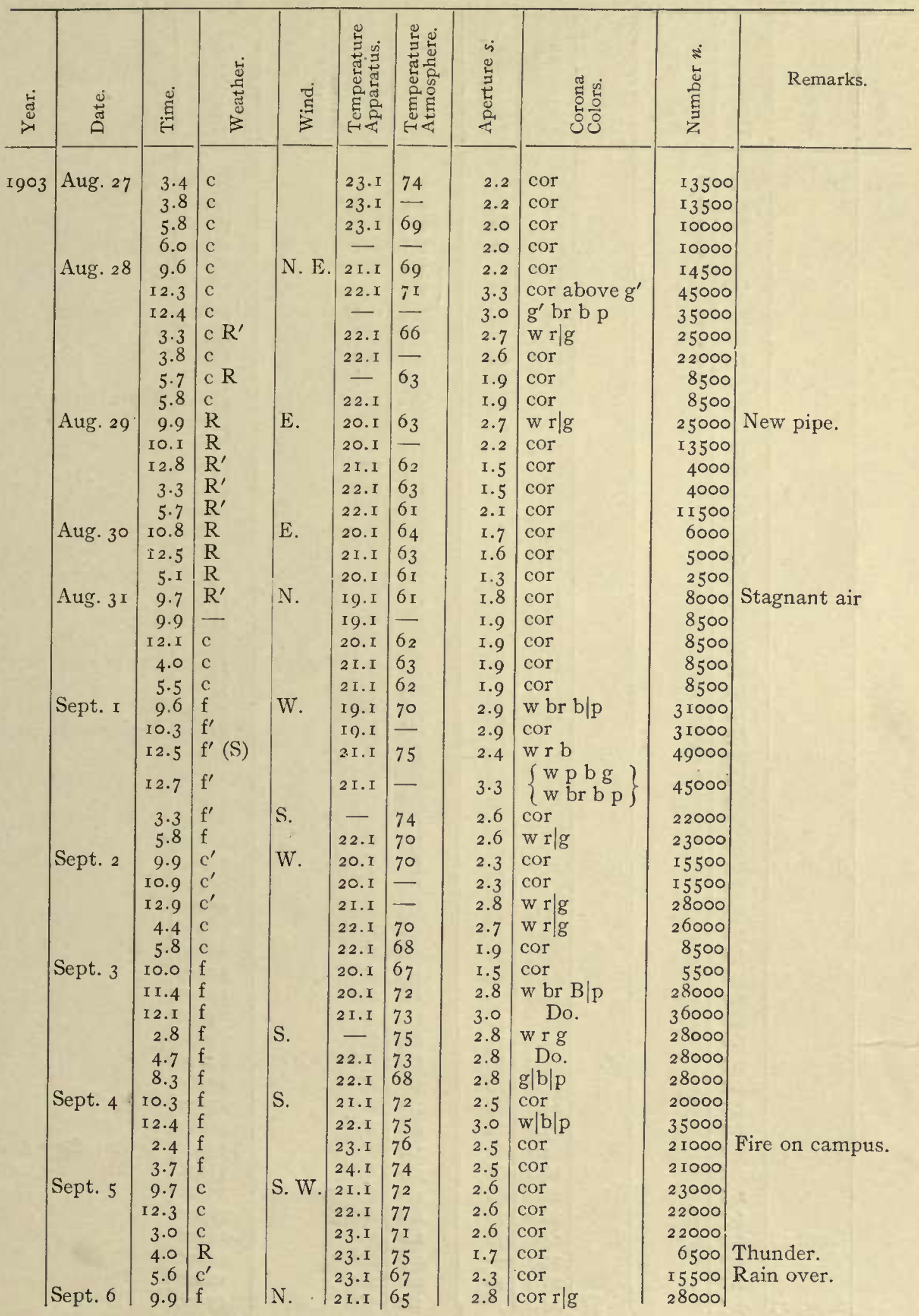


TABLE I-Continued.

\begin{tabular}{|c|c|c|c|c|c|c|c|c|c|c|}
\hline 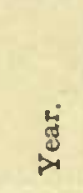 & ڤึ & 苟 & 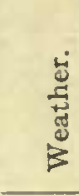 & 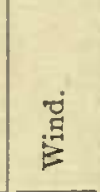 & 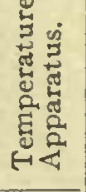 & 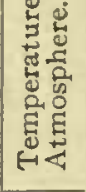 & 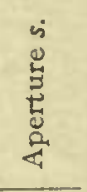 & 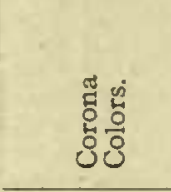 & 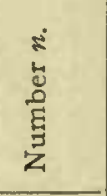 & Remarks. \\
\hline \multirow{50}{*}{ I9०3 } & Sept. 6 & Iо.8 & f & & $2 \mathrm{I} . \mathrm{I}$ & 67 & 2.8 & w r $/ g$ & 28000 & 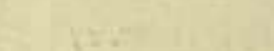 \\
\hline & & 12.8 & $\mathrm{f}$ & & $22 . \mathrm{I}$ & 69 & 2.4 & cor & I 9000 & \\
\hline & & 5.5 & $\mathrm{f}$ & & $2 \mathrm{I} . \mathrm{I}$ & 68 & 2.1 & cor & II 500 & \\
\hline & Sept. 7 & 10.7 & $\mathrm{f}$ & N.W. & 20.1 & 66 & 2.3 & cor & 16500 & \\
\hline & & I.O & $c^{\prime}$ & & 2 I.I & 68 & $\left\{\begin{array}{l}3.2 \\
2.8\end{array}\right.$ & $\left.\begin{array}{l}\mathrm{w} \text { br } \\
\text { Do. }\end{array}\right\}$ & 42000 & \\
\hline & & $3 \cdot I$ & c & N. E. & 22.1 & 69 & $\begin{array}{r}2.0 \\
2.8\end{array}$ & cor & 28000 & \\
\hline & & 4.2 & c & & $2 I . I$ & - & 2.4 & cor & I 9000 & \\
\hline & & $5 \cdot 5$ & c & & 22.1 & 66 & 2.4 & cor & I9000 & \\
\hline & Sept. 8 & 10.4 & f & N. & I9.I & 63 & 2.9 & w br b & 31000 & \\
\hline & & $\begin{array}{r}12.4 \\
2.8\end{array}$ & $\begin{array}{l}f \\
f\end{array}$ & & $\begin{array}{l}19.1 \\
20.1\end{array}$ & $\begin{array}{l}65 \\
66\end{array}$ & 3.0 & $\mathrm{w}$ br b|r & $\begin{array}{l}35000 \\
26000\end{array}$ & $\begin{aligned} \text { Repeated } s & =3.0 . \\
& s=2.7 .\end{aligned}$ \\
\hline & & 3.0 & $\mathrm{f}$ & & 20.1 & - & 2.7 & wrg & 26000 & \\
\hline & & 5.8 & $\mathrm{f}$ & & $2 \mathrm{I} . \mathrm{I}$ & 6I & 2.7 & cor & 25000 & \\
\hline & Sept. 9 & 10.2 & $\mathrm{f}$ & S. & I 8.1 & 64 & $3 \cdot 3$ & w p & 47000 & \\
\hline & & 10.4 & f & & I8.I & - & $3 \cdot 4$ & w p & 49000 & \\
\hline & & II.9 & $\mathrm{f}$ & & 19.1 & 67 & 3.I & $\mathrm{w}$ br b $/ \mathrm{r}$ & 38000 & \\
\hline & & 1.0 & f & & I9.I & - & 3.1 & $\mathrm{~g}^{\prime} \mathrm{br} \mathrm{b} \mid \mathrm{p}$ & 38000 & \\
\hline & & 2.8 & $\mathrm{f}$ & & 20.1 & 67 & 2.9 & w r $/ g$ & 33000 & Repeated $s=2.7$ \\
\hline & & $5 \cdot 4$ & $\mathrm{f}$ & & 2 I.I & 64 & 2.8 & w r g & 28000 & \\
\hline & & 9.6 & c & & 20.1 & $6 I$ & 3.0 & w r $\mid g$ & 35000 & Night. \\
\hline & Sept. Iо & 9.9 & c & & Ig.I & 72 & & & & Glass broke. \\
\hline & & 10.7 & c & & 19.1 & 73 & 3.0 & $w$ br b $/ p$ & 35000 & Old apparatus. \\
\hline & & II. 2 & c & & 21.1 & 74 & 2.9 & Do. & 31000 & \\
\hline & & 12.4 & c & & $2 \mathrm{I} . \mathrm{I}$ & 74 & 2.6 & cor & 22000 & \\
\hline & & 7.5 & $\mathrm{f}^{\prime}$ & & $2 I . I$ & 70 & 2.6 & cor & 22000 & Repeated $s=2.3$. \\
\hline & Sept. I I & $9 \cdot 5$ & f & W. & $2 I . I$ & 78 & 2.9 & W r $\mid g$ & 31000 & \\
\hline & & II.7 & $\mathrm{f}$ & & 22.1 & 82 & 2.7 & cor & 25000 & \\
\hline & & 12.4 & f & & 23.1 & 82 & 2.9 & & 31000 & \\
\hline & & 2.9 & f & & $24 . I$ & 83 & 3.0 & $\mathrm{w}(\mathrm{br}) \mathrm{b} \mid \mathrm{r}$ & 35000 & \\
\hline & & 5.6 & f & & $24 . I$ & 77 & 2.8 & w r $/ g$ & 28000 & \\
\hline & Sept. I 2 & 10.5 & $\mathrm{f}$ & W. & 21.1 & 73 & 4.0 & $g^{\prime} \circ \mathrm{b}$ & 75000 & \\
\hline & & II. 3 & f & & $2 \mathrm{I} . \mathrm{I}$ & 75 & 4.0 & Do. & 75000 & \\
\hline & & I2.5 & f & & 22.1 & 76 & $3 \cdot 3$ & $\mathrm{w}$ br & 45000 & \\
\hline & & 3.2 & f & S. & 23.1 & 77 & 2.8 & w r $\mid g$ & 28000 & \\
\hline & & 5.0 & f & & $24 . I$ & 72 & 2.8 & w r $/ \mathrm{g}$, & 28000 & \\
\hline & Sept. I3 & II.O & f & S. W. & $2 \mathrm{I} . \mathrm{I}$ & 79 & 2.8 & $\mathrm{w} b r \mathrm{~b} / \mathrm{r}$ & 30000 & \\
\hline & & I.7 & f & & 23.1 & 83 & 2.0 & cor & 10000 & \\
\hline & & 6.8 & f & & $23 . I$ & 76 & I.6 & cor & 5000 & \\
\hline & & 7.0 & f & & 23.1 & 74 & 2.0 & cor & 10000 & \\
\hline & Sept. I 4 & 9.7 & f & W. & $22 . I$ & 83 & $3 \cdot 3$ & w br ... & 45000 & \\
\hline & & I 2.4 & f & & $25 . I$ & 88 & 3.0 & $\mathrm{w}$ br b g r & 35000 & \\
\hline & & 2.5 & f & & $25 . I$ & 88 & 2.7 & & 25000 & \\
\hline & & $5 \cdot 7$ & f & & 27.1 & 84 & 2.8 & w r $/ g$ & 30000 & \\
\hline & Sept. I 5 & 9.7 & f & W. & $24 . I$ & $8 \mathrm{I}$ & 3.0 & w r b g & 35000 & Repeated $s=3.0$ \\
\hline & & I 2.5 & f & & 26.1 & 86 & 3.6 & $\mathrm{w} r \mathrm{r}$ & 57000 & $s=3.2$ \\
\hline & & 2.5 & f & & 26.1 & 86 & 3.0 & $\mathrm{~g}^{\prime}|\mathrm{b}| \mathrm{r}$ & 35000 & \\
\hline & & $5 \cdot 7$ & f & & $26 . I$ & 77 & 2.8 & $\mathrm{w} r \mid \mathrm{g}$ & 28000 & \\
\hline & Sept. I6 & 2.3 & c & S. & $24 . I$ & 78 & 2.0 & cor & 10000 & \\
\hline & & 3.7 & c & & 25.1 & & 2.0 & cor & 10000 & \\
\hline & & 6.1 & c & & 26.1 & 76 & 2.0 & cor & 10000 & \\
\hline & $\mid$ Sept. $17 \mid$ & 9.5 & c & S. W. & $24 . I$ & 76 & 2.2 & cor & I 3500 & \\
\hline
\end{tabular}


A CONTINUOUS RECORD OF ATMOSPHERIC NUCLEATION.

TABLE $\mathrm{x}$-Continued.

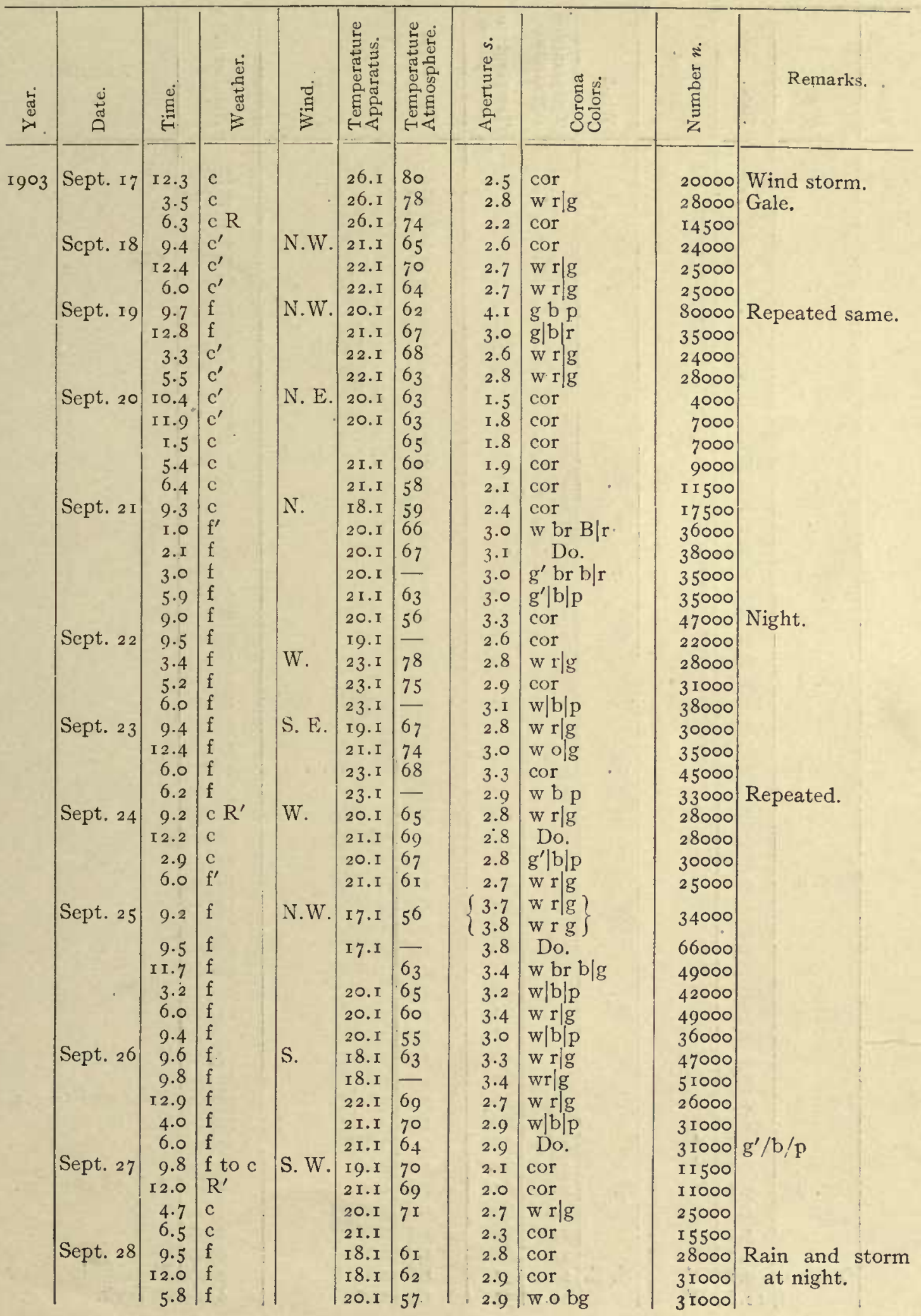


TABLE I-Contimued.

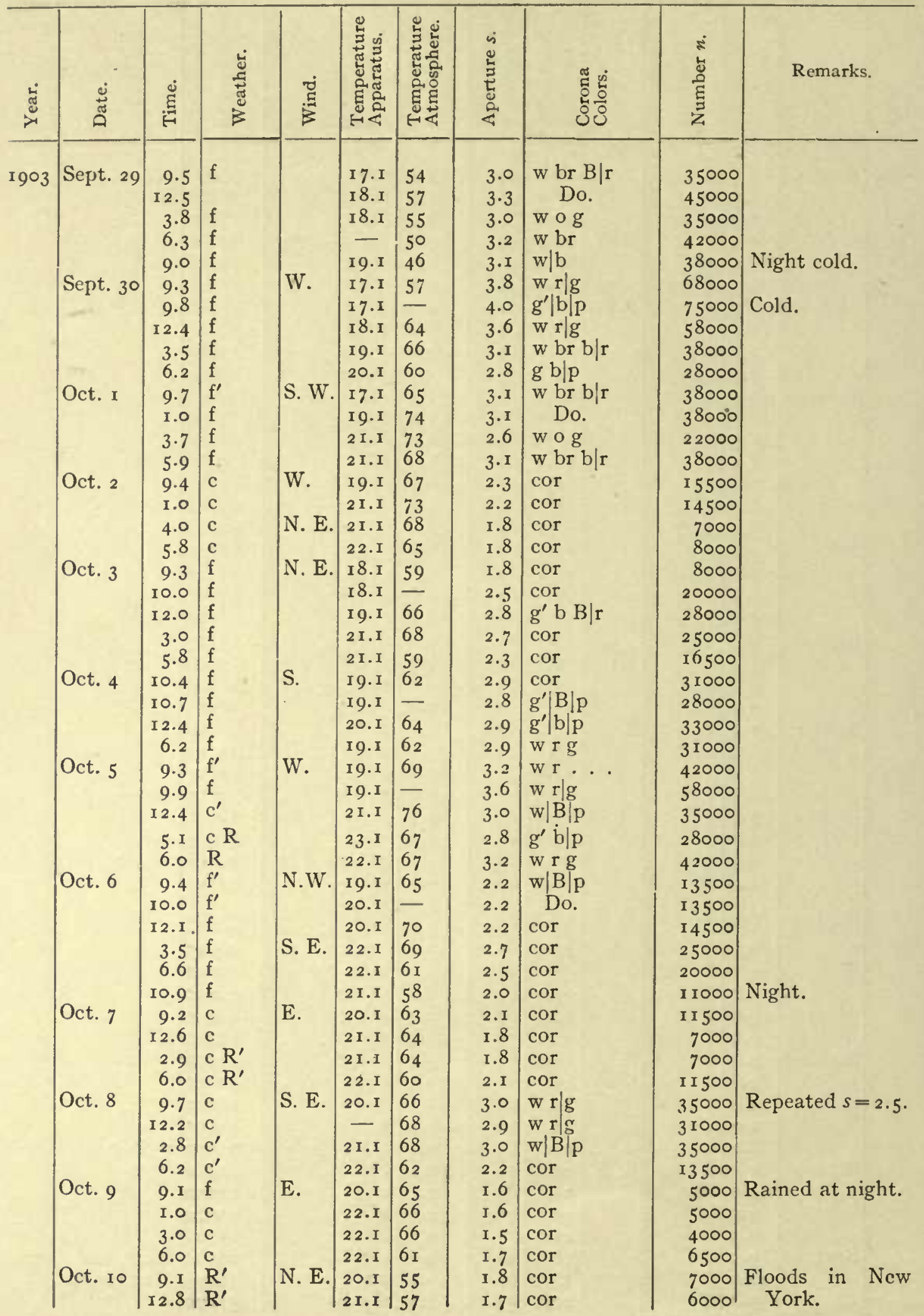


TABLE I-Continued.

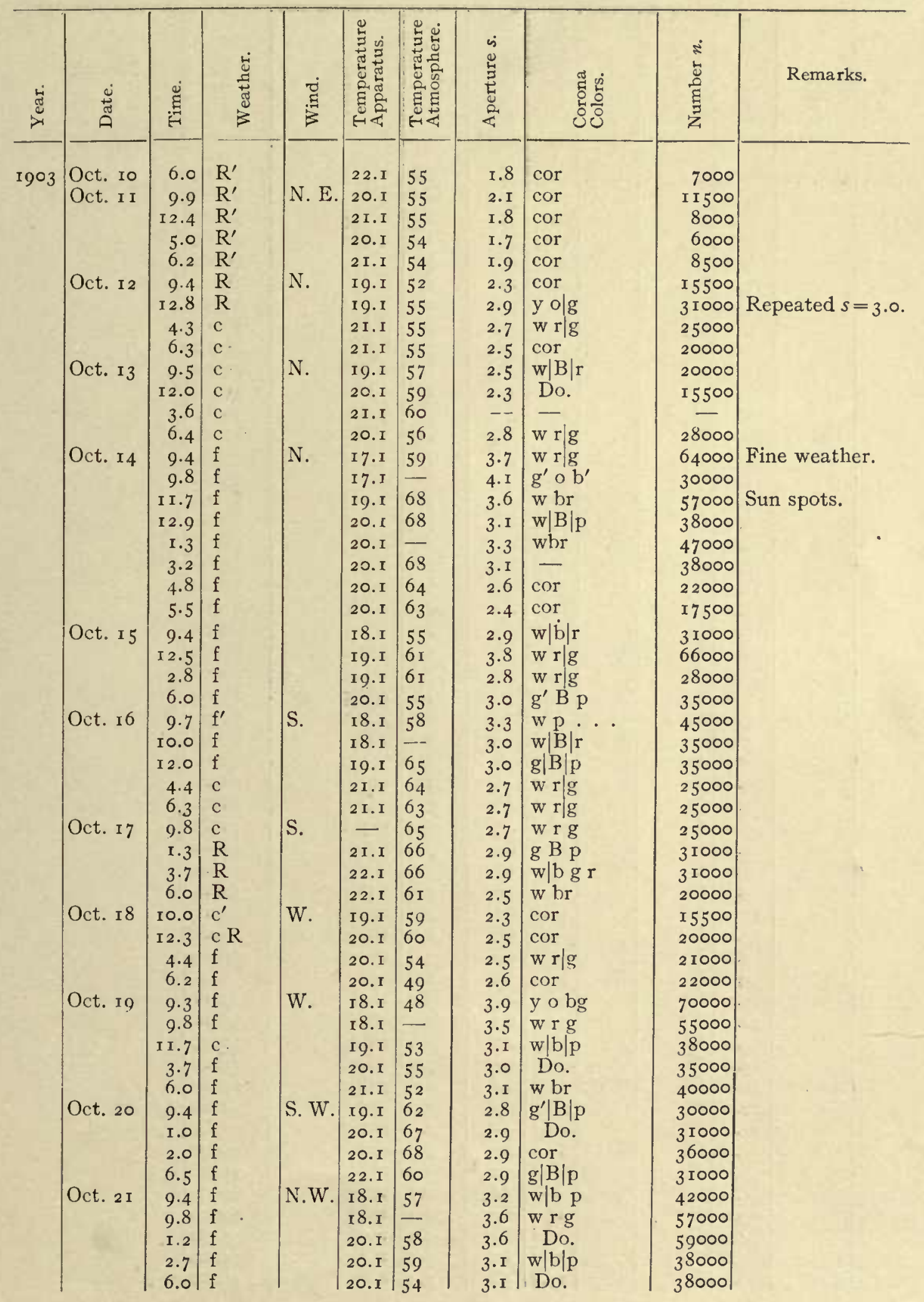


TABLE $\mathrm{r}$-Continued.

\begin{tabular}{|c|c|c|c|c|c|c|c|c|c|c|}
\hline 岂 & 苞 & 胥 & 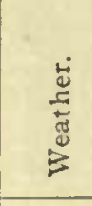 & $\underset{B}{\vec{E}}$ & 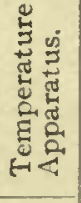 & 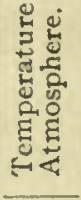 & 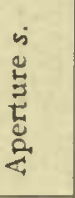 & 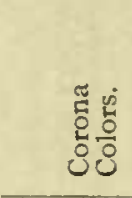 & 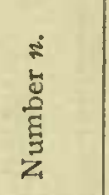 & Remarks. \\
\hline \multirow[t]{52}{*}{1903} & Oct. 22 & $9 \cdot 3$ & $\mathrm{f}$ & N.W. & I $8 . \mathrm{I}$ & 50 & 2.8 & $w r \mid g$ & 30000 & \\
\hline & & I. 4 & f & & 20.1 & 57 & 2.7 & cor & 27000 & \\
\hline & & 4.0 & f & & 20.1 & 56 & 2.8 & w r $\mid g$ & 28000 & \\
\hline & & $5 \cdot 7$ & $\mathrm{f}$ & & $2 \mathrm{I} . \mathrm{I}$ & 55 & 3.2 & w r $/ g$ & 44000 & \\
\hline & Oct. 23 & $9 \cdot 3$ & $c^{\prime}$ & S. W. & rg.r & 65 & 3.0 & cor & 36000 & \\
\hline & & I 2.0 & c & & 20.1 & 69 & 3.0 & w r $\mid g$ & 35000 & \\
\hline & - & $4 \cdot 2$ & c $\mathrm{R}^{\prime}$ & & 2 I.I & 57 & 2.4 & cor & 17500 & \\
\hline & & 5.8 & c & & $2 \mathrm{I} . \mathrm{I}$ & $5^{6}$ & 2.4 & cor & 17500 & \\
\hline & Oct. 24 & $9 \cdot 4$ & c & N.W. & I 9.1 & 48 & 3.0 & $\mathrm{~g}|\mathrm{~b}| \mathrm{p}$ & 35000 & \\
\hline & & 12.0 & $c^{\prime}$ & & 20.1 & $5^{\mathrm{I}}$ & $3 \cdot 4$ & w p cor & 49000 & \\
\hline & & $3 \cdot 5$ & $c^{\prime}$ & & 20.1 & $5 \mathrm{I}$ & 2.9 & $g|\mathrm{~b}| \mathrm{p}$ & 31000 & \\
\hline & & 6.0 & $c^{\prime}$ & & 20.1 & $4^{8}$ & 2.9 & $g B p$ & 31000 & \\
\hline & Oct. 25 & 10.4 & $\mathrm{f}$ & N. & I 8. I & 45 & $4 \cdot 3$ & gy o bg & 90000 & \\
\hline & & 10.8 & f & & I 8. I & 45 & 4.0 & wog & 75000 & \\
\hline & & I 2.3 & c & & I8. I & 48 & 3.0 & $\mathrm{~g}|\mathrm{~b}| \mathrm{p}$ & 35000 & \\
\hline & . & 5.0 & c & & I 7.I & 45 & 2.8 & w r $/ g$ & 30000 & \\
\hline & & 6.5 & & & I 7.1 & 45 & 2.8 & Do. & 30000 & \\
\hline & Oct. 26 & $9 \cdot 4$ & & W. & I 6.1 & 46 & 3.1 & w B g & 38000 & \\
\hline & & 10.0 & $c(S)$ & & I 6.1 & - & 2.7 & cor ${ }^{\circ}$ & 25000 & Clouds. \\
\hline & & $\mathrm{I} \times .7$ & & & I 6.1 & 44 & 2.4 & rop cor & 70000 & \\
\hline & & 3.4 & & & I9.I & 43 & 2.9 & $\mathrm{w}|\mathrm{b}| \mathrm{p}$ & 31000 & \\
\hline & & 6.0 & $f$ & & 20.1 & 38 & 2.8 & $\mathrm{w}|\mathrm{b}|_{\mathrm{p}}^{\mathrm{p}}$ & 28000 & \\
\hline & Oct. 27 & 9.6 & $\mathrm{f}$ & W. & I9.I & 39 & $3 \cdot 3$ & rop. & 47000 & \\
\hline & & $\times 2.8$ & f & & $21 . I$ & 42 & 3.4 & w r $/ g$ & 49000 & \\
\hline & & 5.8 & f & & $22 . I$ & $3^{8}$ & 3.2 & $\mathrm{w}|\mathrm{b}| \mathrm{g}$ & 42000 & \\
\hline & Oct. 28 & 9.4 & $\mathrm{f}$ & & - & 39 & 4.0 & y o bg & 75000 & Snow. \\
\hline & & 9.9 & f & N.W. & I9.I & - & $4 \cdot \mathrm{I}$ & Do. & 80000 & \\
\hline & & I. 4 & f & & 20.1 & 46 & $3 \cdot 7$ & w rg & 62000 & \\
\hline & & 3.2 & f & & $2 \mathrm{I} . \mathrm{I}$ & 46 & $3 . \mathrm{I}$ & $\mathrm{w} \mathrm{B} \mid \mathrm{p}$ & 40000 & \\
\hline & & 6.0 & $\mathrm{f}$ & & 22.1 & $4 x$ & 3.1 & Do. & 38000 & \\
\hline & Oct. 29 & $9 \cdot 3$ & $\mathrm{f}$ & & I9.I & 45 & $3 \cdot 4$ & $\mathrm{w} p \mathrm{~b} \mid \mathrm{r}$ & 49000 & \\
\hline & & 2.8 & $\mathrm{f}$ & & 23.1 & 62 & $3 \cdot 5$ & Do. & 53000 & \\
\hline & & 6.0 & $\mathrm{f}$ & & 23.1 & 56 & 3.5 & $\mathrm{w} r \mid \mathrm{g}$ & 53000 & \\
\hline & Oct. 30 & $9 \cdot 5$ & f & W. & $2 x . I$ & 59 & $3 . x$ & $\mathrm{w}|\mathrm{b}| \mathrm{p}$ & 38000 & \\
\hline & & $\times 2.7$ & $\mathrm{f}$ & & $22 . \mathrm{I}$ & 68 & 2.5 & cor & 20000 & \\
\hline & & 3.1 & $\mathrm{f}$ & & 23.1 & 68 & 2.7 & $\mathrm{w} \mid \mathrm{b} / \mathrm{p}$ & 25000 & \\
\hline & & 5.8 & f & & 23.1 & 62 & 3.6 & w rg & 57000 & \\
\hline & Oct. $3 \mathrm{I}$ & 9.2 & $\mathrm{f}$ & W. & $21 . \mathrm{I}$ & 63 & 2.6 & cor & & \\
\hline & & 9.9 & $\mathrm{f}$ & & 20. I & - & 3.2 & w r & 42000 & \\
\hline & & 12.2 & $\mathrm{f}$ & & $2 \mathrm{r} . \mathrm{I}$ & 69 & 2.9 & $\mathrm{~g}^{\prime}\left|\mathrm{b}^{\prime}\right| \mathrm{p}$ & 33000 & \\
\hline & & 4.2 & f & & 22. I & 68 & 3.0 & $\mathrm{w} / \mathrm{b} \mid \mathrm{p}$ & 35000 & \\
\hline & & 5.8 & $\mathrm{f}$ & & - & 64 & 3.0 & Do. & 35000 & \\
\hline & Nov. I & 10.8 & $\mathrm{f}$ & N.W. & I9.I & 62 & 3.0 & $w|b| r$ & 37000 & \\
\hline & & 12.0 & $\mathrm{f}$ & & 19.1 & 63 & 3.0 & cor & 35000 & Apparatus \\
\hline & & 12.4 & $\mathrm{f}$ & & 20. I & - & 2.9 & w r $\mid g$ & 33000 & cleaned. \\
\hline & & 4.5 & f & & 20.I & $6 I$ & 2.5 & cor & 20000 & \\
\hline & & 6.3 & $\mathrm{f}$ & & - & 57 & 3.0 & $\mathrm{~g}^{\prime} \mathrm{b} \mid \mathrm{p}$ & 37000 & \\
\hline & Nov. 2 & 9.4 & f & W. & - & $5^{8}$ & $3 \cdot 7$ & w rg & 61000 & \\
\hline & & I.O & $\mathrm{f}$ & & 20.1 & 65 & 3.2 & $\mathrm{w} b \mid \mathrm{p}$ & 42000 & \\
\hline & & $4 \cdot 1$ & f & & $2 \mathrm{I} . \mathrm{I}$ & 64 & 3.0 & w ofg & 35000 & \\
\hline & & 5.8 & $\mathrm{f}$ & & $2 \mathrm{I} . \mathrm{I}$ & 60 & $3 \cdot 5$ & w r $g$ & 53000 & \\
\hline & Nov. 3 & 9.2 & $\mathrm{f}$ & W. & 17.1 & $5^{8}$ & 3.4 & w r g & 49000 & \\
\hline
\end{tabular}


TABLE I.-Continued.

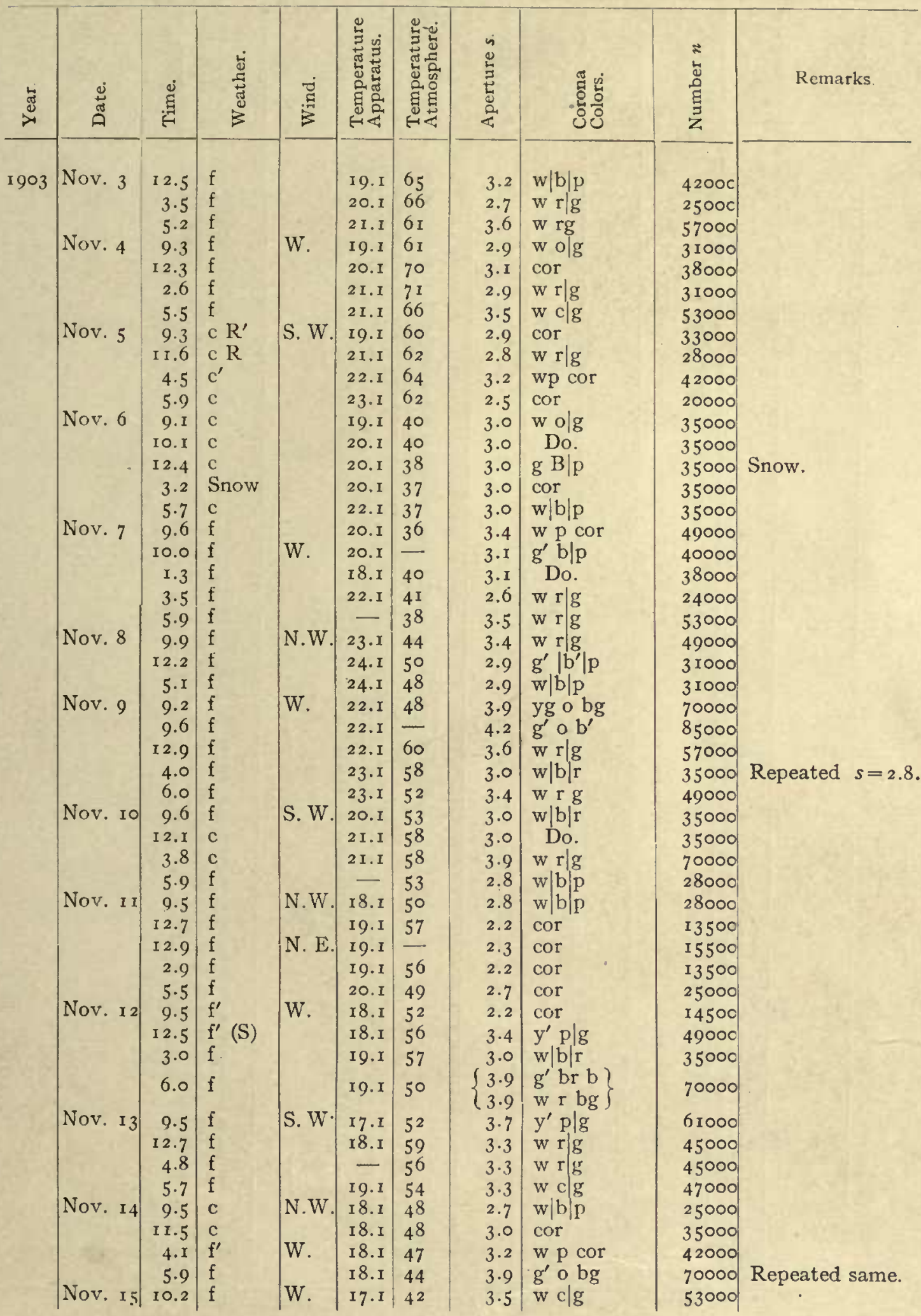


TABLE I-Contimued.

\begin{tabular}{|c|c|c|c|c|c|c|c|c|c|c|}
\hline 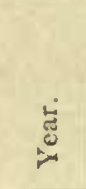 & 苞 & 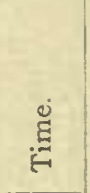 & 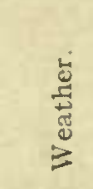 & 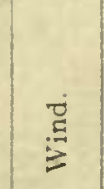 & 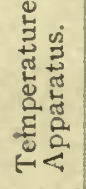 & 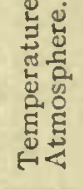 & 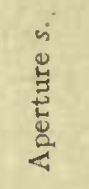 & 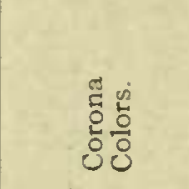 & 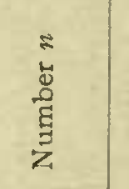 & Remarks. \\
\hline 1903 & $\begin{array}{l}\text { Nov. } 18 \\
\text { Nov. } 19 \\
\text { Nov. } 20\end{array}$ & 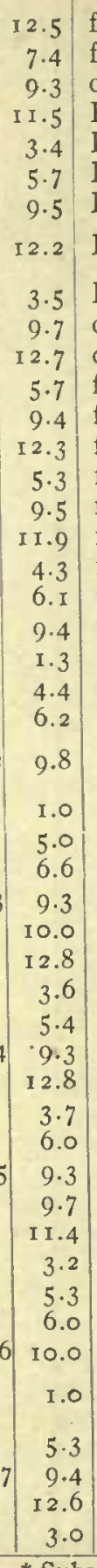 & $\begin{array}{l}f \\
f \\
c \\
R \\
R \\
R \\
R \\
R \\
R \\
c \\
c \\
f^{\prime} \\
f \\
f \\
f \\
f \\
f \\
f \\
f \\
f \\
f \\
f \\
f \\
c \\
c \\
c \\
c \\
c^{\prime} \\
c \\
c \\
c \\
c ~ R \\
f \\
f^{\prime} \\
c \\
c \\
f \\
f \\
f \\
f \\
f \\
f \\
f \\
f \\
f \\
f \\
c \\
c \\
\end{array}$ & $\begin{array}{l}\text { N.W. } \\
\text { N.E. } \\
\text { N.W. } \\
\text { N.W. } \\
\text { W. } \\
\text { N.W. } \\
\text { N. } \\
\text { N. }\end{array}$ & 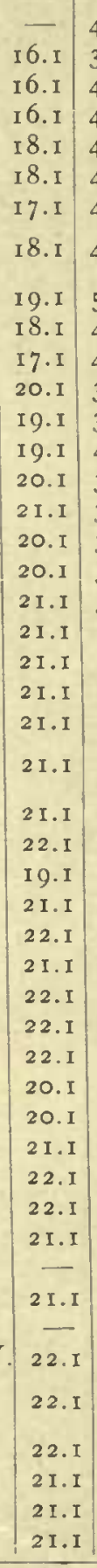 & 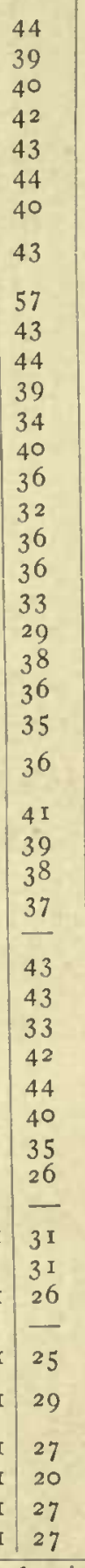 & $\begin{array}{r}2.8 \\
3.4 \\
3.2 \\
2.8 \\
2.7 \\
2.7 \\
2.8 \\
3.5 \\
3.5 \\
3.4 \\
2.8 \\
2.8 \\
2.8 \\
3.3 \\
3.3 \\
3.3 \\
3.1 \\
3.4 \\
3.3 \\
3.8 \\
3.2 \\
3.2 \\
3.2 \\
2.8 \\
3.1 \\
3.0 \\
2.7 \\
3.2 \\
3.2 \\
3.8 \\
4.0 \\
2.7 \\
2.7 \\
3.1 \\
3.3 \\
2.8 \\
2.7 \\
2.5 \\
3.0 \\
3.7 \\
3.9 \\
2.9 \\
2.9 \\
2.9 \\
3.6 \\
4.0 \\
4.0 \\
3.5 \\
3.8 \\
3.9 \\
3.5 \\
\end{array}$ & $\left.\begin{array}{l}\text { g|b|p } \\
\text { w r|g } \\
\text { w p } \\
\text { w o|g } \\
\text { w br b|r } \\
g^{\prime} \text { b p } \\
g^{\prime}|\mathrm{b}| \mathrm{p} \\
\text { wp } \\
\text { w c g }\end{array}\right\}$ & $\begin{array}{l}28000 \\
49000 \\
42000 \\
28000 \\
25000 \\
25000 \\
28000 \\
53000 \\
49000 \\
28000 \\
28000 \\
28000 \\
47000 \\
45000 \\
45000 \\
40000 \\
49000 \\
45000 \\
66000 \\
42000 \\
42000 \\
42000 \\
28000 \\
38000 \\
25000 \\
42000 \\
42000 \\
66000 \\
75000 \\
26000 \\
25000 \\
40000 \\
45000 \\
30000 \\
25000 \\
21000 \\
35000 \\
61000 \\
70000 \\
31000 \\
31000 \\
31000 \\
57000 \\
78000 \\
53000 \\
66000 \\
70000 \\
55000 \\
\end{array}$ & 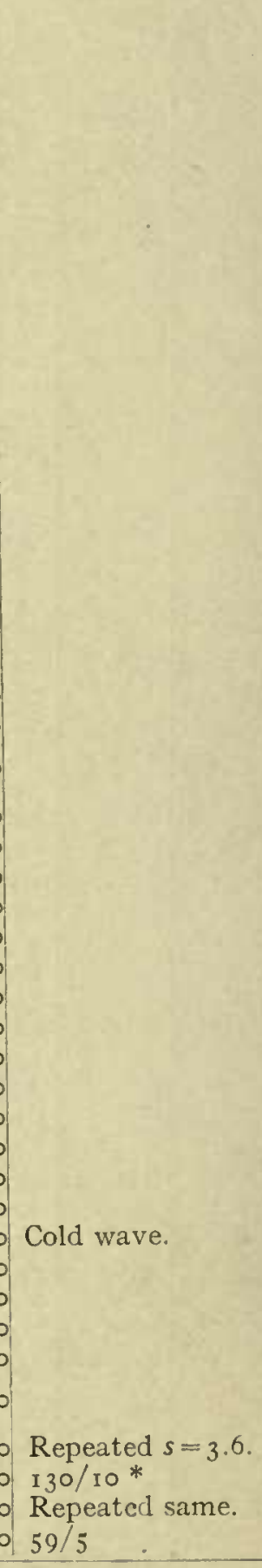 \\
\hline
\end{tabular}

* Subsidence data, meaning that in $130 \mathrm{sec}$. the fog line has fallen $10 \mathrm{~cm}$. 
TABLE I-Continued.

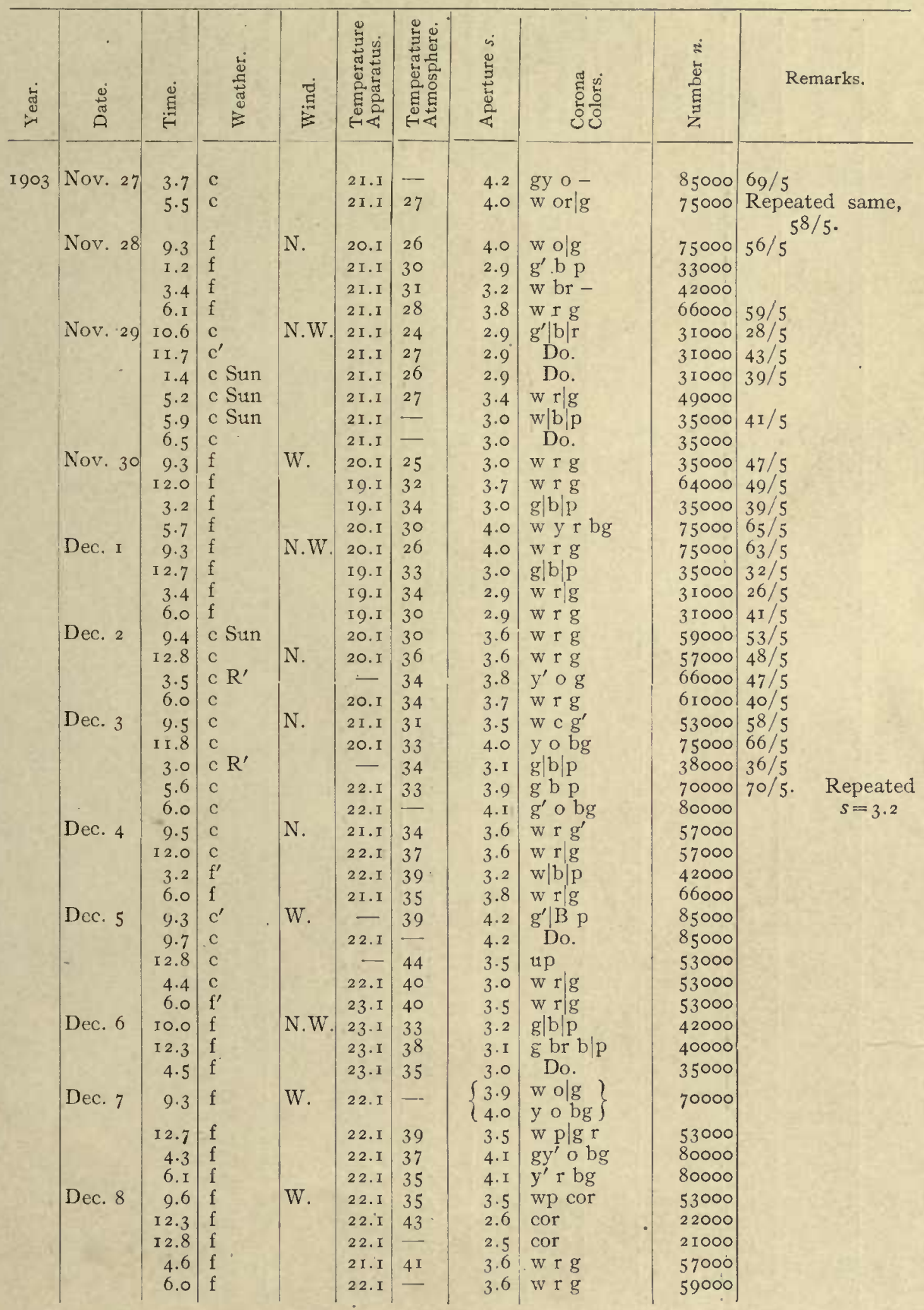


TABLE I-Continued.

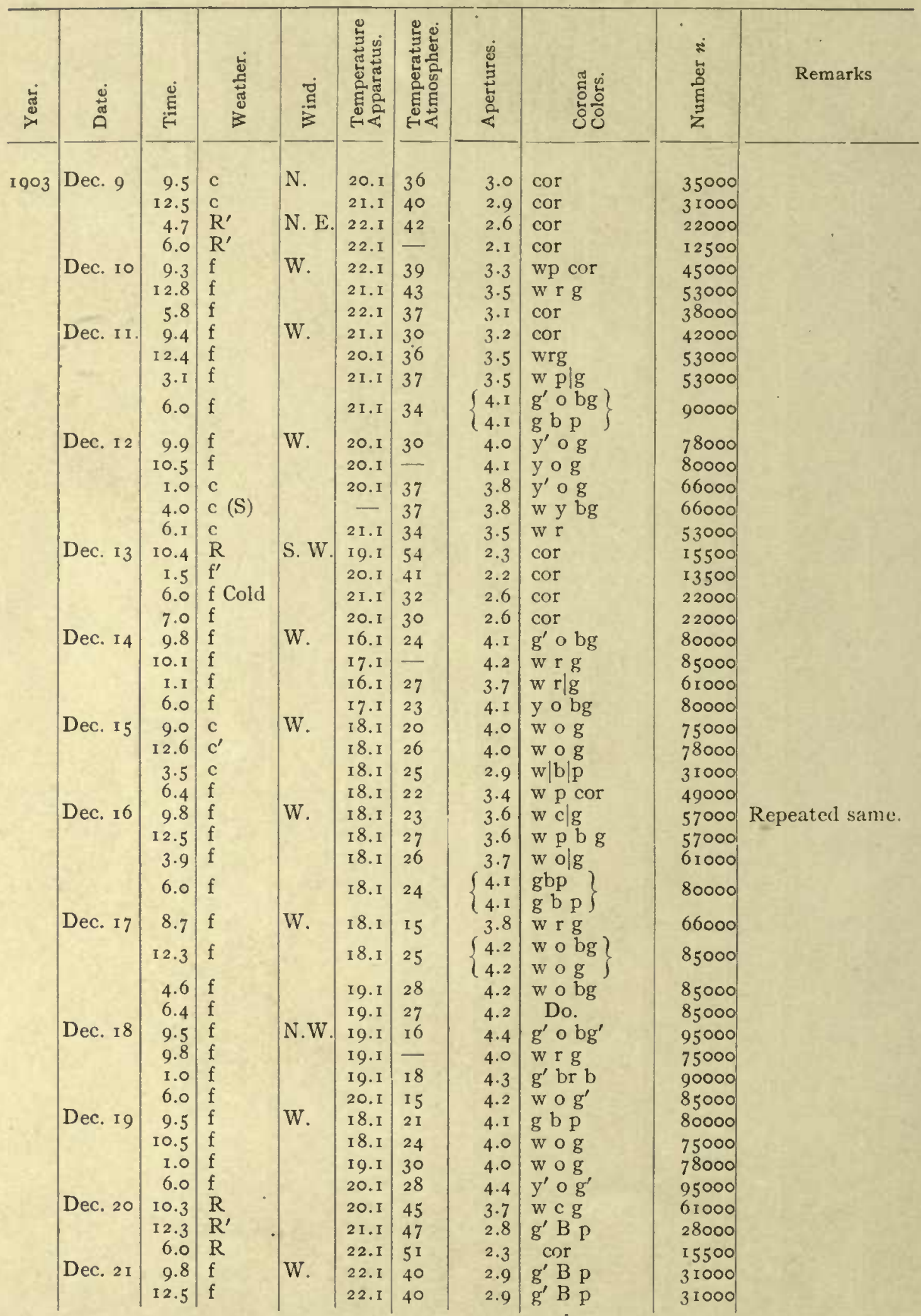


TABLE I-Continued.

\begin{tabular}{|c|c|c|c|c|c|c|c|c|c|c|}
\hline 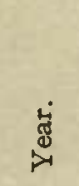 & 苞 & 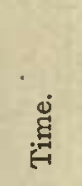 & 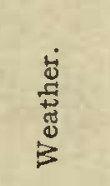 & 晃 & 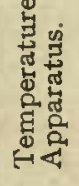 & 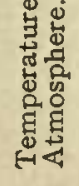 & 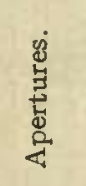 & 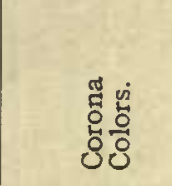 & $\begin{array}{l}\text { z } \\
\text { 岁 } \\
\text { g } \\
\text { z }\end{array}$ & Remarks. \\
\hline \multirow[t]{38}{*}{1903} & Dec. $2 \mathrm{I}$ & 6.3 & $f$ & & $23 . \pi$ & 37 & $3 \cdot 5$ & w r g & 53000 & \\
\hline & Dec. 22 & $9 \cdot 5$ & $c \mathrm{R}^{\prime}$ & & $20 . \mathrm{I}$ & 39 & 3.6 & $w r g$ & 57000 & \\
\hline & & I 2.8 & c & W. & 21.1 & 40 & 3.1 & $\mathrm{w}|\mathrm{b}| \mathrm{r}$ & 38000 & \\
\hline & & & $\mathrm{f}$ & & $21 . \mathrm{I}$ & 28 & 3.1 & Do. & 38000 & \\
\hline & Dec. 23 & 10.1 & $\mathrm{f}$ & S. W. & $2 \mathrm{I} . \mathrm{I}$ & 28 & 4.0 & wog & 75000 & \\
\hline & & 10.5 & $\mathrm{f}$ & & $2 \mathrm{I}, \mathrm{I}$ & & 4.I & $\mathrm{g} b \mathrm{p}$ & 80000 & \\
\hline & & I T. 5 & $\mathrm{f}$ & & - & $3 \mathrm{I}$ & 4.2 & $\mathrm{~g}^{\prime} \circ \mathrm{b}$ & 85000 & \\
\hline & & $\begin{array}{l}4.0 \\
6.0\end{array}$ & $\mathrm{f}$ & & 2 T.I & 35 & 4.0 & $y^{\prime} \circ g$ & 75000 & \\
\hline & Dec. 24 & IO.I & $\mathrm{f}$ & S. W. & $\begin{array}{l}22.1 \\
22.1\end{array}$ & $\begin{array}{l}35 \\
38\end{array}$ & $\begin{array}{l}4.2 \\
4.2\end{array}$ & $\begin{array}{l}\text { wo } \circ g^{\prime} \\
g^{\prime} \circ \mathrm{bg}\end{array}$ & $\begin{array}{l}88000 \\
85000\end{array}$ & \\
\hline & & 10.8 & c & & 22.1 & 43 & 4.2 & $\mathrm{~g}^{\prime}$ o bg & 85000 & \\
\hline & & 12.7 & c & & 22.1 & 47 & 3.4 & w p cor & 49000 & \\
\hline & Dec. 25 & 10.9 & c & W. & 23.1 & 47 & 2.9 & $\mathrm{~g}^{\prime} \mathrm{b} \mathrm{p}$ & 31000 & \\
\hline & & I. 3 & c & & 23.1 & 43 & 2.8 & $g^{\prime} b p$ & 30000 & \\
\hline & Dec. 26 & 10.2 & & N.W. & $23 . I$ & 35 & 2.4 & & I7500 & Repeated same. \\
\hline & & $\begin{array}{l}\text { I.3 } \\
\end{array}$ & Snow & & 22.1 & 25 & 3.8 & $w b p$ & 66000 & \\
\hline & & 4.0 & c & & $\begin{array}{l}22 . I \\
23 . I\end{array}$ & 20 & $\begin{array}{l}3.9 \\
4.0\end{array}$ & $\begin{array}{l}g \text { b p } \\
g \text { b p p }\end{array}$ & $\begin{array}{l}70000 \\
75000\end{array}$ & \\
\hline & & $5 \cdot 3$ & c & & 23.1 & 18 & $3 \cdot 4$ & w r b g & 49000 & \\
\hline & & 6.0 & c & & $22 . I$ & 77 & $\left\{\begin{array}{l}3.0 \\
2.9\end{array}\right.$ & $\left.\begin{array}{l}\mathrm{g}^{\prime}|\mathrm{B}| \mathrm{p} \\
\text { cor }\end{array}\right\}$ & 35000 & \\
\hline & Dec. 27 & 9.4 & c & S. W. & $2 \mathrm{I} . \mathrm{I}$ & I3 & $4 \cdot \mathrm{I}$ & y० bg & 80000 & \\
\hline & & 10.8 & $\mathrm{c}$ & & 2 I.I & I 5 & 3.9 & $y^{\prime} \circ b g$ & 73000 & \\
\hline & & I 2.9 & Sun & & 20. I & I 7 & $3 \cdot 4$ & up cor & 49000 & Repeated same. \\
\hline & & 5.2 & & & $2 \mathrm{I} \cdot \mathrm{I}$ & 22 & 3.6 & w r g & 57000 & \\
\hline & & 7.0 & c & & $2 \mathrm{I} . \mathrm{I}$ & 23 & 3.6 & w r g & 57000 & \\
\hline & Dec. 28 & 9.6 & f & N.W. & 20.1 & I 7 & $3 \cdot 5$ & up cor & 55000 & \\
\hline & & I.० & $\mathrm{f}$ & & 20.1 & I9 & $\left\{\begin{array}{l}4.2 \\
4.2\end{array}\right.$ & $\left.\begin{array}{l}\begin{array}{l}y \circ \\
g^{\prime} \circ \mathrm{bg}\end{array} \\
\text { bg }\end{array}\right\}$ & 88000 & \\
\hline & & $3 \cdot 4$ & $f$ & & $20 . \pi$ & 17 & $\begin{array}{r}(4.2 \\
4 . I\end{array}$ & y r g & 80000 & \\
\hline & & $6 . \mathrm{I}$ & $\mathrm{f}$ & & $2 \mathrm{~T} . \mathrm{I}$ & I 4 & $4 . \mathrm{I}$ & y r g & 80000 & \\
\hline & Dec. 29 & 10.9 & & S. E. & I9. I & I3 & 4.0 & $y^{\prime}$ r g & 75000 & \\
\hline & & $I 2.8$ & Snow & & I8. I & I6 & 3.1 & $\mathrm{~g} \mathrm{~b} \mathrm{p}$ & 38000 & \\
\hline & & $3 \cdot 3$ & c Sun' & & I8. I & 20 & $3 \cdot 4$ & w r g & 49000 & \\
\hline & & 6.2 & Sun & & I9.I & 20 & $3 \cdot 5$ & wrg & 53000 & \\
\hline & Dec. 30 & 9.8 & f & N.W. & I9. I & I9 & 4. I & $g^{\prime} \circ b g$ & 80000 & \\
\hline & & IO.I & f & & ז9.I & 一 & 4.0 & $\mathrm{~g}$ b p & 75000 & \\
\hline & & I 2.8 & $\mathrm{f}$ & W. & 20.1 & 29 & $3 \cdot 7$ & w r g & 62000 & \\
\hline & & $\begin{array}{l}3.7 \\
6.0\end{array}$ & $f$ & W. & $\begin{array}{l}20 . I \\
20 . I\end{array}$ & $\begin{array}{l}28 \\
25\end{array}$ & 3.0 & $g|\mathrm{~b}| \mathrm{p}$ & 35000 & \\
\hline & Dec. 3 I & 9.9 & f & W. & $\begin{array}{l}20.1 \\
\text { I } 9.1\end{array}$ & $\begin{array}{l}25 \\
22\end{array}$ & 3.8 & w r g & $\begin{array}{l}.3000 \\
66000\end{array}$ & \\
\hline & & I. 2 & $\mathrm{f}$ & & 2 I.I & 24 & $3 \cdot 7$ & wrg & 64000 & \\
\hline & & 6.5 & $\mathrm{f}$ & & 20.1 & 22 & 4.2 & wo bg & 85000 & Repeated same. \\
\hline \multirow{7}{*}{1904} & Jan. $\mathbf{I}$ & I0.4 & f & W. & $20 . \mathrm{I}$ & 29 & $3 \cdot 2$ & $\mathrm{wpbgr}$ & 42000 & \\
\hline & & I.3 & $f$ & & $2 \mathrm{I} . \mathrm{I}$ & 33 & 3.6 & w rg & 59000 & \\
\hline & Tan. 2 & $\begin{array}{l}5 \cdot 9 \\
0.8\end{array}$ & f & & $2 \mathrm{I} . \mathrm{I}$ & $2 \mathrm{I}$ & $\begin{array}{l}3.2 \\
4.1\end{array}$ & $\mathrm{w}$ br b g r & 44000 & \\
\hline & & $\begin{array}{r}9.8 \\
10.2\end{array}$ & $\begin{array}{l}\mathrm{c} \\
\mathrm{c}\end{array}$ & N. & $\begin{array}{l}20 . \mathrm{I} \\
20 . \mathrm{I}\end{array}$ & $\begin{array}{l}9 \\
8\end{array}$ & $\begin{array}{l}4 \cdot I \\
4 \cdot I\end{array}$ & $\begin{array}{l}g^{\prime} \circ \text { bg } \\
\text { Do. }\end{array}$ & $\begin{array}{l}80000 \\
80000\end{array}$ & Snow. \\
\hline & & $\mathrm{x} .5$ & c & & $2 I . I$ & 9 & 4.1 & 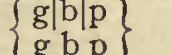 & 90000 & “ \\
\hline & & 3.6 & c Sun & & 20.1 & II & 3.9 & wrg & 70000 & Repeated same. \\
\hline & & 6.0 & & & 20. I & I 2 & 4.0 & w r g & 75000 & \\
\hline
\end{tabular}


TABLE I-Continued.

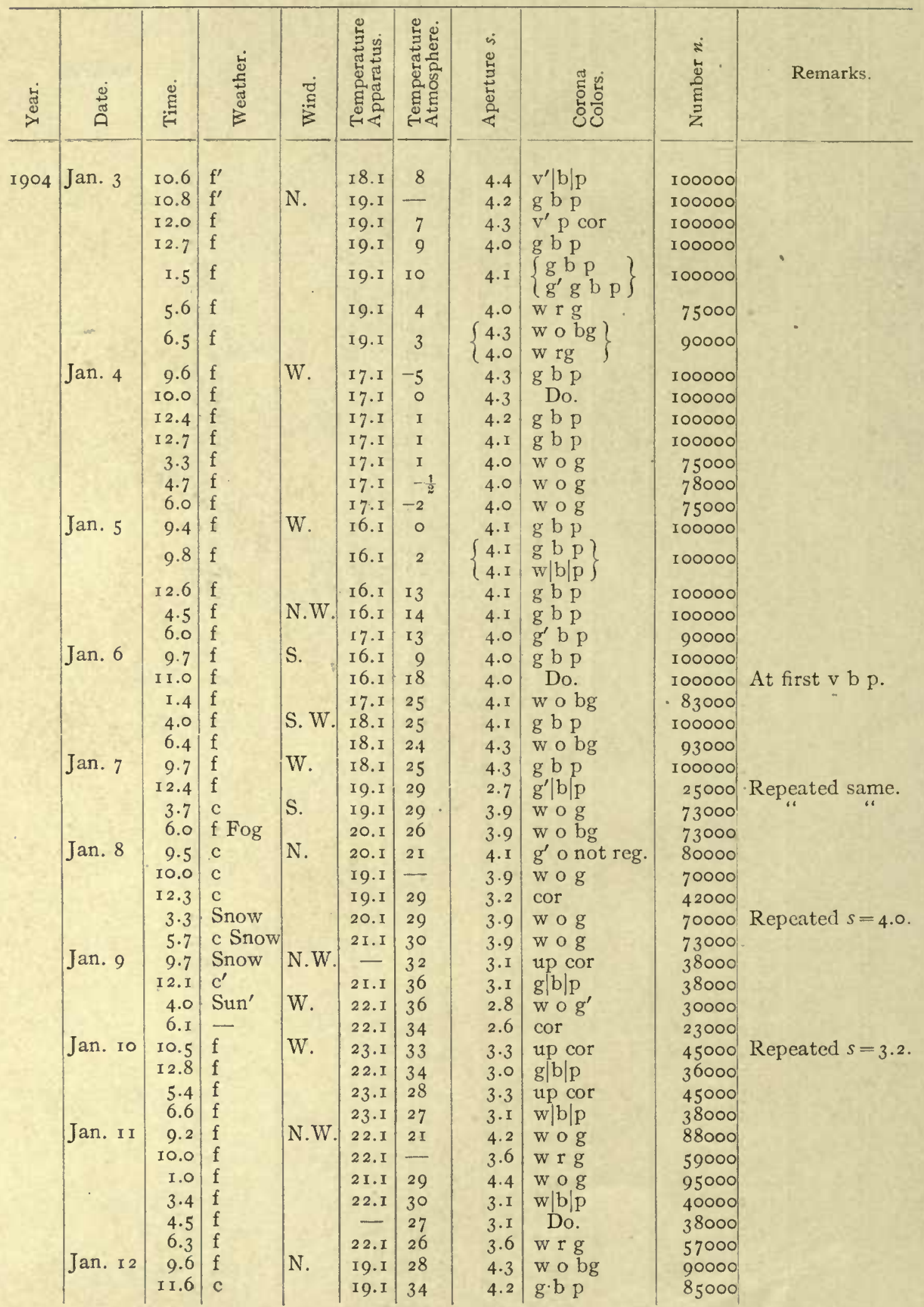


TABLE I-Continued.

\begin{tabular}{|c|c|c|c|c|c|c|c|c|c|c|}
\hline 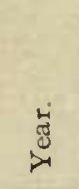 & $\begin{array}{l}\dot{0} \\
\text { 苟 } \\
\text { A }\end{array}$ & 导 & 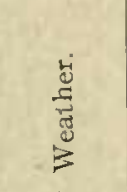 & 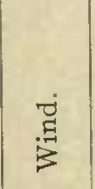 & 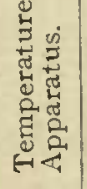 & 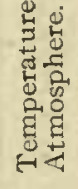 & 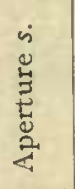 & 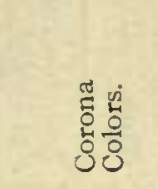 & 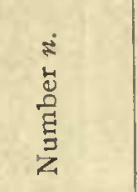 & Remarks. \\
\hline \multirow[t]{51}{*}{ I 904} & Jan. 12 & I. 3 & $\mathrm{f}$ & & $2 I . I$ & 33 & 3.4 & w r g & 49000 & \\
\hline & Jan. $\mathrm{I}_{3}$ & $9 \cdot 4$ & c & N.E. & 22.1 & 35 & 2.8 & w br $\mid g$ & 29500 & \\
\hline & & 1.5 & c R & E. & $22 . \mathrm{I}$ & 37 & 2.8 & W o cor & 28000 & \\
\hline & & $3 \cdot 7$ & c R & & 23.1 & $3^{8}$ & 2.6 & W. $r g$ & 22000 & \\
\hline & & 6.4 & c R & & 22.1 & 40 & 2.6 & w rg & 23000 & \\
\hline & Jan. I 4 & $9 \cdot 5$ & $\mathrm{f}^{\prime} \mathrm{S}^{\prime}$ & S. W. & 2 I. I & 34 & $3 \cdot 4$ & w c $g$ & 49000 & \\
\hline & & I 2.5 & f & & I9.I & 37 & 4.2 & w o bg & $85000 ?$ & \\
\hline & & $\mathrm{r} .5$ & & & I9.r & 37 & $3 \cdot 7$ & $\mathrm{wrg}$ & 62000 & \\
\hline & & $3 \cdot 7$ & $\mathrm{f}^{\prime} \mathrm{S}^{\prime}$ & & 20.1 & $3^{6}$ & 3.9 & $w \circ g$ & 73000 & \\
\hline & & 5.6 & $\mathrm{f}$ & & $2 I . I$ & 35 & $3 \cdot 7$ & w rg & 62000 & \\
\hline & Jan. I 5 & 9.4 & f & W. & $2 \mathrm{I} . \mathrm{I}$ & 27 & $4 . I$ & $\mathrm{~g}^{\prime}$ br bg & 80000 & \\
\hline & & I. 3 & $f$ & & $2 \mathrm{I} . \mathrm{I}$ & 30 & 3.8 & w c g & 68000 & \\
\hline & & $3 \cdot 3$ & $\mathrm{f}$ & & $2 \mathrm{I} . \mathrm{I}$ & 28 & 3.4 & w r g & 49000 & \\
\hline & & 6. I & $\mathrm{f}$ & & $2 \mathrm{I} \cdot \mathrm{I}$ & 24 & 3.0 & $\left.\begin{array}{l}\mathrm{w}|\mathrm{b}| \mathrm{r} \\
\mathrm{cor}\end{array}\right\}$ & 36000 & \\
\hline & Jan. 16 & 9.8 & $\mathrm{f}^{\prime}$ & S. W. & 2I.I & 23 & $\begin{array}{r}2.9 \\
3.4\end{array}$ & up cor & 49000 & \\
\hline & & I I . 3 & & & $2 \mathrm{r} . \mathrm{I}$ & 25 & 3.9 & wo g & 70000 & \\
\hline & & $4 \cdot 4$ & c Snow & S. & $2 \mathrm{I} . \mathrm{I}$ & - & $3 . \mathrm{I}$ & cor & 38000 & \\
\hline & & 6.2 & $\mathrm{R}$ & & $2 \mathrm{I} . \mathrm{I}$ & 38 & 2.8 & $\mathrm{w}|\mathrm{b}| \mathrm{p}$ & 28000 & \\
\hline & Jan. I 7 & I0.6 & $\mathrm{f}$ & W. & 22.1 & 28 & 2.9 & $\mathrm{~g}^{\prime}|\mathrm{b}| \mathrm{p}$ & 31000 & \\
\hline & & I. 5 & f & & 22.1 & 29 & $3 \cdot 3$ & w br cor & 47000 & \\
\hline & & $5 \cdot 7$ & $\mathrm{f}$ & & $22 . I$ & 22 & 3.0 & $\mathrm{w} \mid \mathrm{b} / \mathrm{p}$ & 35000 & \\
\hline & & 6.5 & $\mathrm{f}$ & & 22.1 & 20 & 3.0 & $\mathrm{w}|\mathrm{b}| \mathrm{p}$ & 35000 & \\
\hline & Jan. 18 & 9.3 & f & N.W. & 20. I & ro & $4 \cdot I$ & $\mathrm{~g} \mathrm{~b} \mathrm{p}$ & 100000 & \\
\hline & & 9.7 & $\mathrm{f}$ & & 20.1 & - & 4. $\mathrm{r}$ & $\mathrm{g} \mathrm{b} \mathrm{p}$ & 100000 & \\
\hline & & r 2.5 & $\mathrm{f}$ & & I 9.1 & I 4 & $4 \cdot I$ & $\mathrm{~g} \mathrm{~b} \mathrm{p}$ & 100000 & \\
\hline & & 4.0 & $\mathrm{f}$ & & 20.1 & 14 & 4. I & $\mathrm{g} \mathrm{b} \mathrm{p}$ & 100000 & \\
\hline & & $5 \cdot 9$ & f & & 20.1 & 12 & 4.2 & w o bg & 85000 & \\
\hline & Jan. I9 & 9.6 & $\mathrm{f}$ & N.W. & I 8.1 & o & $4 \cdot 4$ & $\mathrm{~g} \mathrm{~b} \mathrm{p}$ & 100000 & \\
\hline & & 10.0 & $\mathrm{f}$ & & I $8 . \mathrm{I}$ & - & $4 \cdot 3$ & Do. up & 100000 & \\
\hline & & I I.9 & $\mathrm{f}$ & $N$. & I 8. I & 8 & 4. I & $\mathrm{gbp}$ & 100000 & \\
\hline & & 3.8 & $\mathrm{f}$ & & I 8. I & I3 & 4.0 & wog & 75000 & \\
\hline & & 6.0 & $\mathrm{f}$ & & I9.I & I 2 & 4.I & $\mathrm{g} \mathrm{b} \mathrm{p}$ & 100000 & \\
\hline & Jan. 20 & 9.5 & Snow & N. & I 8.1 & I 6 & 4.6 & wp cor & 100000 & To wbp \\
\hline & & 9.8 & Snow & & I 8.I & I 8 & 4.2 & Do. & 100000 & \\
\hline & & I 1.8 & Snow & S. W. & 17.1 & 24 & 4.2 & $g$ b p & 100000 & \\
\hline & & 2.0 & c & & I 8.1 & 26 & $4 \cdot r$ & $\mathrm{~g} b \mathrm{p}$ & 100000 & \\
\hline & & $4 \cdot I$ & c & & I8.I & 29 & 4.2 & $y^{\prime} \circ b g$ & 90000 & \\
\hline & & 6.0 & c & & I9.I & 28 & 4.0 & w r g & 75000 & \\
\hline & Jan. 2 I & $9 \cdot 4$ & c & N. E. & ro.r & 32 & 4.0 & w r g & 75000 & \\
\hline & & I 1.5 & Snow & & I9.I & 33 & 2.2 & cor & 13000 & \\
\hline & & I 1.9 & Snow & & I 9.I & - & 2.8 & cor & 28000 & \\
\hline & & 3.8 & Snow & & I9.I & 32 & 2.8 & $\mathrm{~g}^{\prime} \mathrm{b}$ p & 29500 & \\
\hline & & 6.0 & Snow & & 20.1 & 32 & 3.6 & w c g & 57000 & Repeated $s=3.7$ \\
\hline & Jan. 22 & 9.8 & $\mathrm{R}$ & N.E. & $2 \mathrm{I} . \mathrm{I}$ & 38 & 2.5 & cor & 20000 & \\
\hline & & $\mathrm{r} 2.9$ & $\mathrm{R}$ & & $2 \mathrm{r} . \mathrm{I}$ & 36 & 2.9 & w o g & 31000 & \\
\hline & & $3 \cdot 4$ & $\mathrm{R}$ & & 22.1 & 30 & 2.9 & $\mathrm{~g} \mathrm{~b} \mathrm{p}$ & 31000 & \\
\hline & & 6.0 & $\mathrm{R}^{\prime}$ & & $2 \mathrm{I} . \mathrm{I}$ & 30 & 3.1 & $\mathrm{w}$ br b|r & 38000 & \\
\hline & Jan. 23 & 9.8 & Fog & S. & $22 . I$ & 44 & 4.0 & wrg & 75000 & \\
\hline & & IO.I & Fog & & $一$ & - & $3 \cdot 5$ & w c g & 53000 & \\
\hline & & I.I & Fog & S. W. & 22. I & 50 & 3.4 & w br cor & 49000 & \\
\hline & & 4.8 & Fog & W. & 23.1 & 47 & 3.2 & Do. & 42000 & \\
\hline
\end{tabular}


TABLE I-Continued.

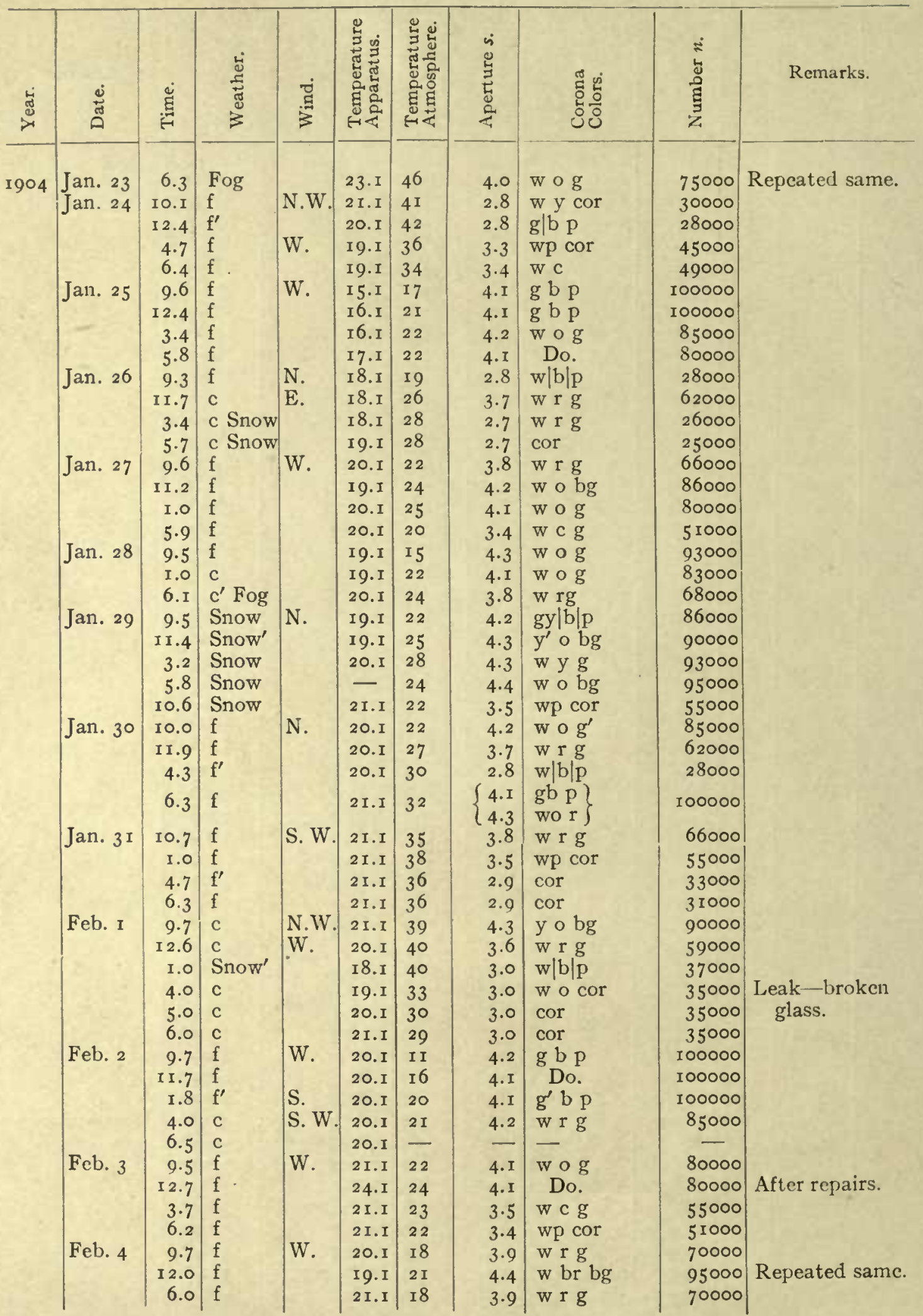


TABLE I-Continued.

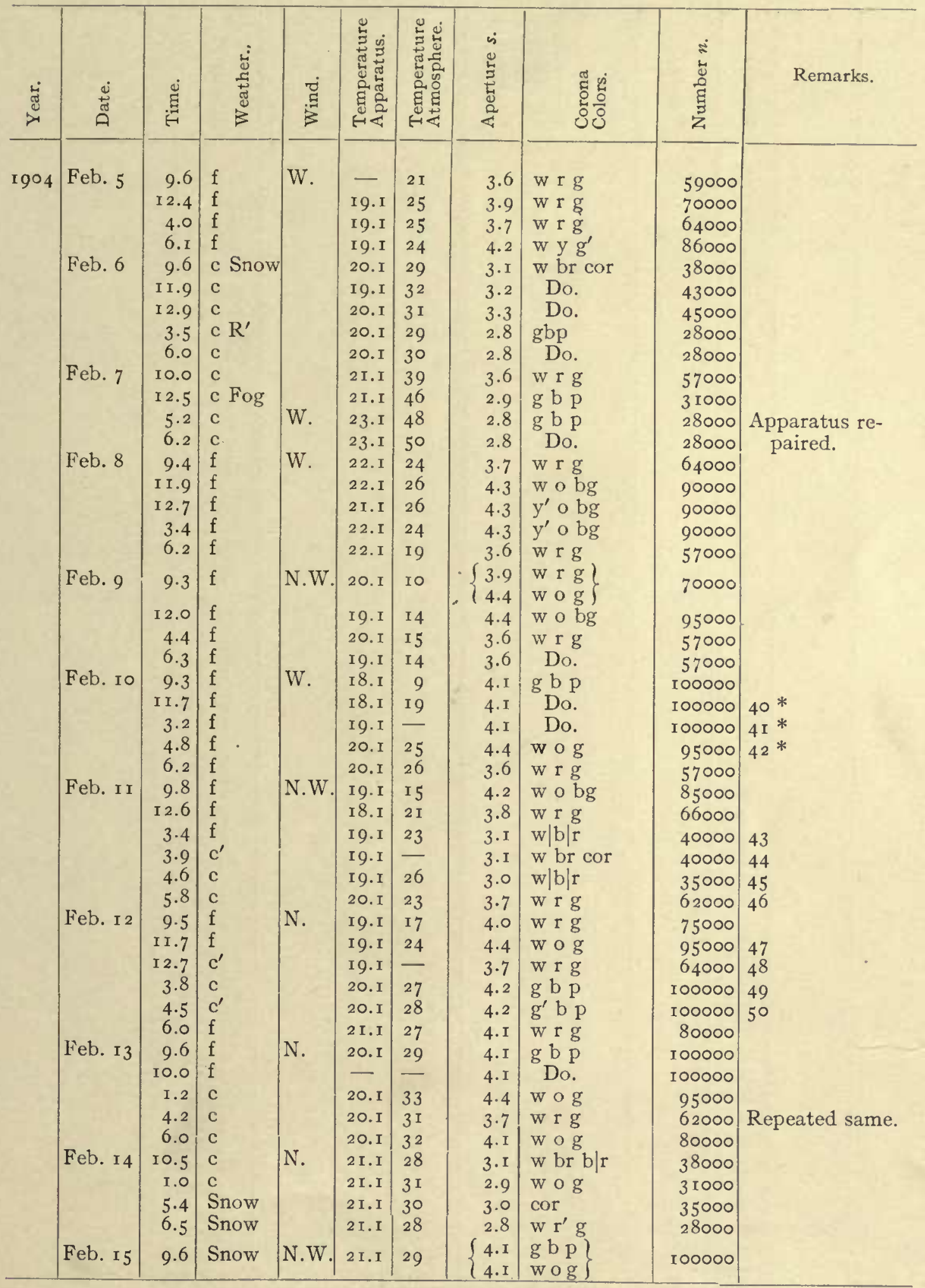

* These numbers refer to photographic slides of the fog particles. 
TABLE I-Continued.

\begin{tabular}{|c|c|c|c|c|c|c|c|c|c|c|}
\hline ֻّ & ڤ્ّ & 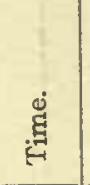 & 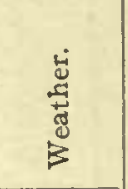 & 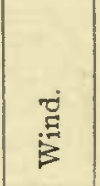 & 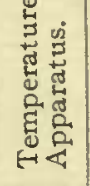 & 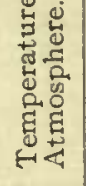 & 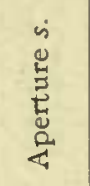 & 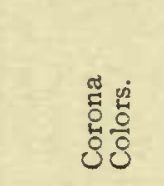 & 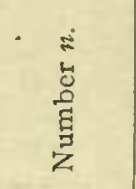 & Remarks. \\
\hline I 904 & Feb. I7 & 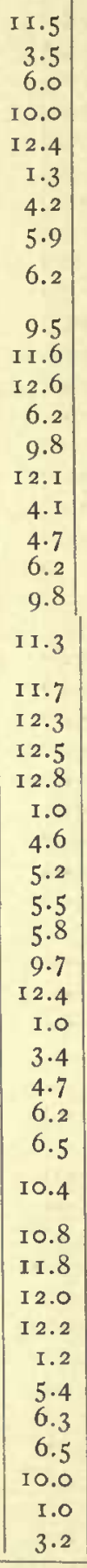 & $\begin{array}{l}\text { c } \\
f \\
f \\
f \\
f \\
f \\
f \\
f \\
f \\
f \\
f \\
f \\
f \\
f \\
f \\
c \\
c \\
c \\
\text { cSnow' } \\
\text { c } \\
\text { c } \\
\text { c } \\
c \\
\text { Snow } \\
\text { Do. } \\
\text { Do. } \\
\text { Do. } \\
\text { Do. } \\
\text { Do. } \\
\text { f } \\
\text { f } \\
\text { f } \\
\text { f } \\
f \\
f \\
f \\
f \\
\text { f }\end{array}$ & N.W. & 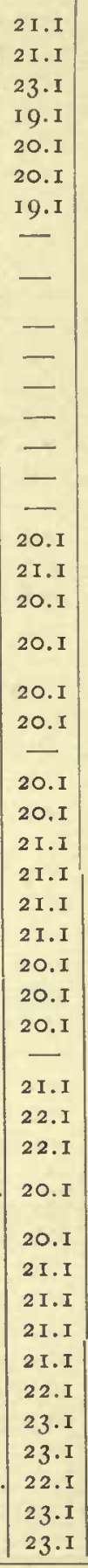 & $\begin{array}{l}3 \text { I } \\
30 \\
22 \\
6 \\
9 \\
9 \\
9 \\
8 \\
\\
12 \\
18 \\
19 \\
18 \\
18 \\
25 \\
30 \\
29 \\
28 \\
26 \\
27 \\
28 \\
27 \\
- \\
26 \\
- \\
23 \\
31 \\
33 \\
36 \\
35 \\
34 \\
29 \\
31 \\
33 \\
36 \\
35 \\
35 \\
49 \\
49 \\
43 \\
\end{array}$ & 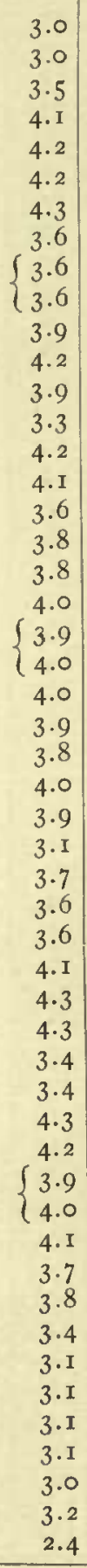 & $\begin{array}{l}\left.\begin{array}{l}g^{\prime} \text { b p } \\
g^{\prime} \text { b p } \\
\text { w r g } \\
\text { g b p } \\
\text { g b p } \\
\text { Do. } \\
\text { w yo bg } \\
\text { w c g } \\
\text { wc g } \\
\text { wc g } \\
\text { w r g } \\
\text { w o bg } \\
\text { w r g } \\
\text { w br cor } \\
\text { gy br b' } \\
\text { w o g } \\
\text { w c g } \\
\text { w r g } \\
\text { w rg } \\
\text { w o g } \\
\text { w o g } \\
\text { w o g } \\
\text { w o g } \\
\text { w r g } \\
\text { w c g } \\
\text { w y g } \\
\text { w o g } \\
\text { w br b|r } \\
\text { w r g } \\
\text { w r cor } \\
\text { w p cor } \\
\text { gbp } \\
\text { w o bg } \\
\text { w y g } \\
\text { w br cor } \\
\text { w br cor } \\
\text { w o bg } \\
\text { w o g } \\
\text { w r g } \\
\text { w o g }\end{array}\right\} \\
\text { w o g } \\
\text { w rg } \\
\text { w rg } \\
\text { w br cor } \\
\text { w|b|p } \\
\text { w br b|r } \\
\text { w b } \\
\text { w b p } \\
\text { g'|b } \\
\text { wp co } \\
\text { wo cor } \\
\text { cor }\end{array}$ & $\begin{array}{r}35000 \\
35000 \\
53000 \\
100000 \\
100000 \\
100000 \\
93000 \\
57000 \\
57000 \\
70000 \\
85000 \\
73000 \\
45000 \\
88000 \\
83000 \\
57000 \\
66000 \\
66000 \\
75000 \\
73000 \\
78000 \\
70000 \\
66000 \\
78000 \\
70000 \\
38000 \\
62000 \\
58000 \\
57000 \\
100000 \\
93000 \\
90000 \\
49000 \\
49000 \\
90000 \\
85000 \\
73000 \\
80000 \\
62000 \\
66000 \\
51000 \\
38000 \\
40000 \\
38000 \\
38000 \\
35000 \\
42000 \\
17000\end{array}$ & 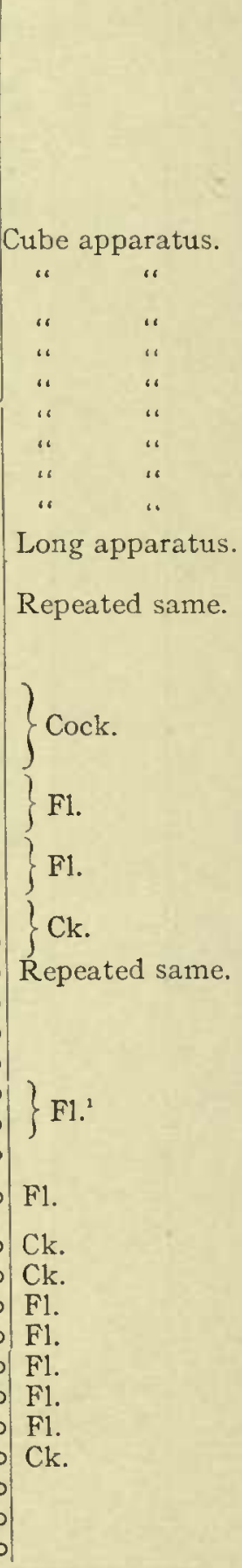 \\
\hline
\end{tabular}

1 Fl. refers to the instantaneous valve (Chap. VI, \& 33); Ck., to a half-inch plug cock. 
TABLE I-Continued.

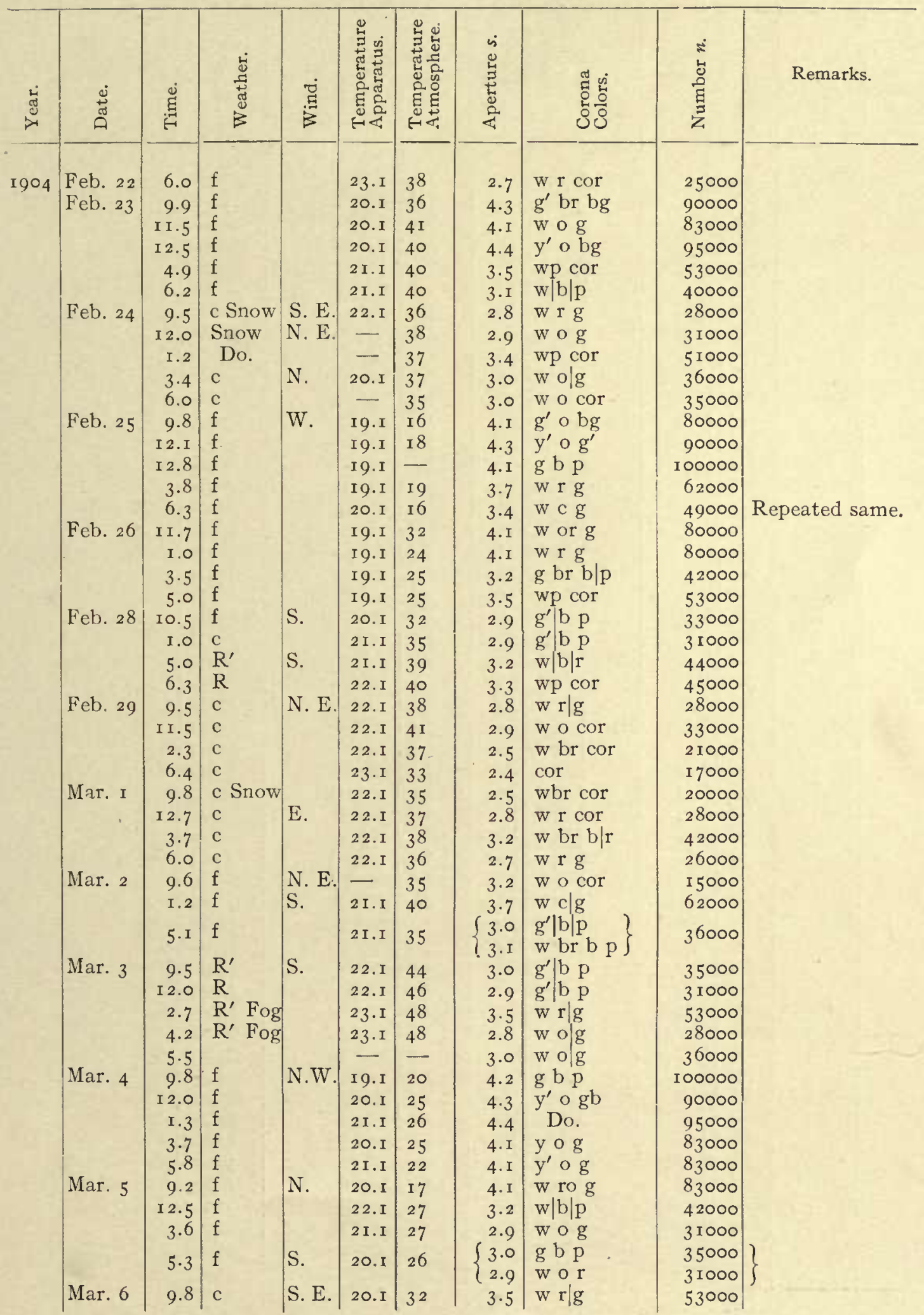


TABLE 1 -Continued.

\begin{tabular}{|c|c|c|c|c|c|c|c|c|c|c|}
\hline$-\underbrace{\circ}$ & ڤే & $\underset{\xi}{\stackrel{g}{g}}$ & 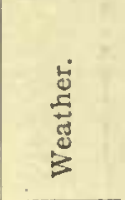 & $\vec{E}$ & 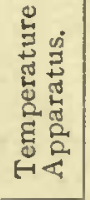 & 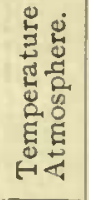 & 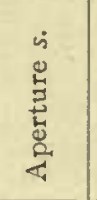 & 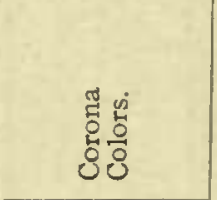 & 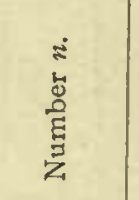 & Remarks. \\
\hline $190_{4} 1$ & $\begin{array}{l}\text { Mar. 8 } \\
\text { Mar. 9 } \\
\text { Mar. Io } \\
\text { Mar. II }\end{array}$ & 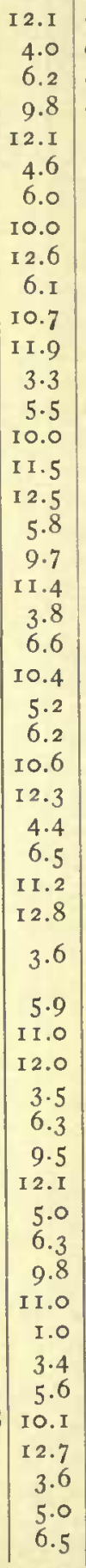 & $\begin{array}{l}c \\
c^{\prime} \\
c^{\prime} \\
c \\
R^{\prime} \\
R \\
R ! \\
f^{\prime} \\
f^{\prime} \\
f \\
f \\
f \\
f \\
f \\
f \\
f \\
f \\
f \\
c \\
c \\
\text { Snow } \\
c \\
f \\
f^{\prime} \\
f \\
f \\
f \\
f \\
f \\
f \\
f \\
c \\
c^{\prime} \\
\text { Snow } \\
\text { Do. } \\
\text { Do. } \\
c \\
f \\
f \\
f \\
f \\
f \\
f \\
f \\
f \\
f \\
\text { Snow } \\
\text { Snow' } \\
R^{\prime} \\
R \\
R^{\prime} \\
\text { f }\end{array}$ & $\begin{array}{l}\text { N.W. } \\
\text { N. }\end{array}$ & $\begin{array}{l}2 I . I \\
2 I . I \\
2 I . I \\
22 . I \\
2 I . I \\
22 . I \\
22 . I \\
2 I . I \\
22 . I \\
22 . I \\
I 9 . I \\
I 9 . I \\
20 . I \\
I 8 . I \\
I 8 . I \\
I 8 . I \\
I 9 . I \\
20 . I \\
20 . I \\
20 . I \\
20 . I \\
20 . I \\
2 I . I \\
21 . I \\
2 I . I \\
20 . I \\
2 I . I \\
2 I . I \\
20 . I \\
2 I . I \\
2 I . I \\
22 . I \\
2 I . I \\
2 I . I \\
2 I . I \\
22 . I \\
2 I . I \\
2 I . I \\
22 . I \\
2 I . I \\
2 I . I \\
2 I . I \\
2 I . I \\
2 I . I \\
22 . I \\
22 . I \\
22 . I \\
22 . I \\
23 . I \\
\end{array}$ & $\begin{array}{l}34 \\
35 \\
23 \\
44 \\
46 \\
44 \\
46 \\
51 \\
55 \\
43 \\
40 \\
35 \\
33 \\
27 \\
30 \\
32 \\
31 \\
34 \\
34 \\
31 \\
30 \\
31 \\
38 \\
36 \\
33 \\
35 \\
37 \\
34 \\
37 \\
40 \\
37 \\
35 \\
35 \\
37 \\
38 \\
36 \\
37 \\
38 \\
35 \\
33 \\
30 \\
33 \\
37 \\
40 \\
35 \\
35 \\
39 \\
41 \\
39 \\
37\end{array}$ & $\begin{array}{l}3.0 \\
2.6 \\
3.2 \\
3.2 \\
3.7 \\
2.7 \\
2.5 \\
3 . \mathrm{I} \\
3.0 \\
2.5 \\
4.0 \\
3.9 \\
3.3 \\
3.2 \\
4 . \mathrm{I} \\
3.9 \\
3.8 \\
3.4 \\
2.8 \\
2.5 \\
2.6 \\
2.9 \\
4 . \mathrm{I} \\
3.2 \\
3.2 \\
3.5 \\
3.5 \\
3.0 \\
3.0 \\
3.9 \\
3.8 \\
\begin{array}{l}4.1 \\
3.8\end{array} \\
4 . \mathrm{I} \\
2.9 \\
2.9 \\
2.9 \\
2.9 \\
3.6 \\
3.8 \\
3.0 \\
3.0 \\
4 . \mathrm{I} \\
4 . \mathrm{I} \\
3.7 \\
3.0 \\
3.4 \\
2.9 \\
2.8 \\
3.4 \\
3 . \mathrm{I} \\
2.8\end{array}$ & 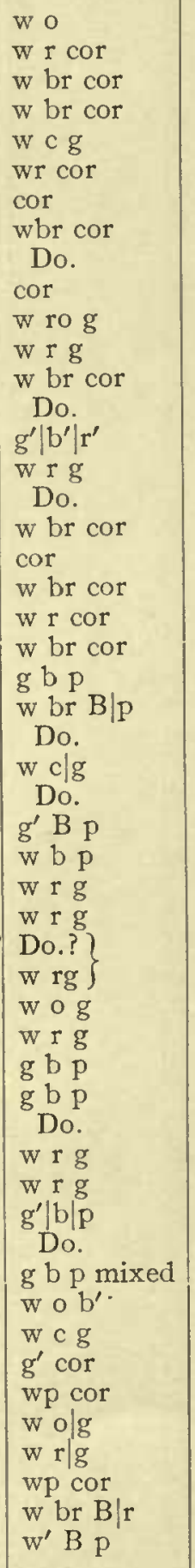 & $\begin{array}{r}35000 \\
22000 \\
44000 \\
44000 \\
64000 \\
26000 \\
20000 \\
38000 \\
35000 \\
21000 \\
75000 \\
70000 \\
47000 \\
42000 \\
100000 \\
70000 \\
68000 \\
49000 \\
30000 \\
20000 \\
23000 \\
33000 \\
100000 \\
42000 \\
42000 \\
53000 \\
53000 \\
36000 \\
35000 \\
73000 \\
68000 \\
70000 \\
80000 \\
31000 \\
31000 \\
33000 \\
31000 \\
59000 \\
68000 \\
35000 \\
35000 \\
100000 \\
83000 \\
64000 \\
35000 \\
49000 \\
31000 \\
30000 \\
49000 \\
40000 \\
30000\end{array}$ & Cold wave. \\
\hline
\end{tabular}


TABLE I-Continued.

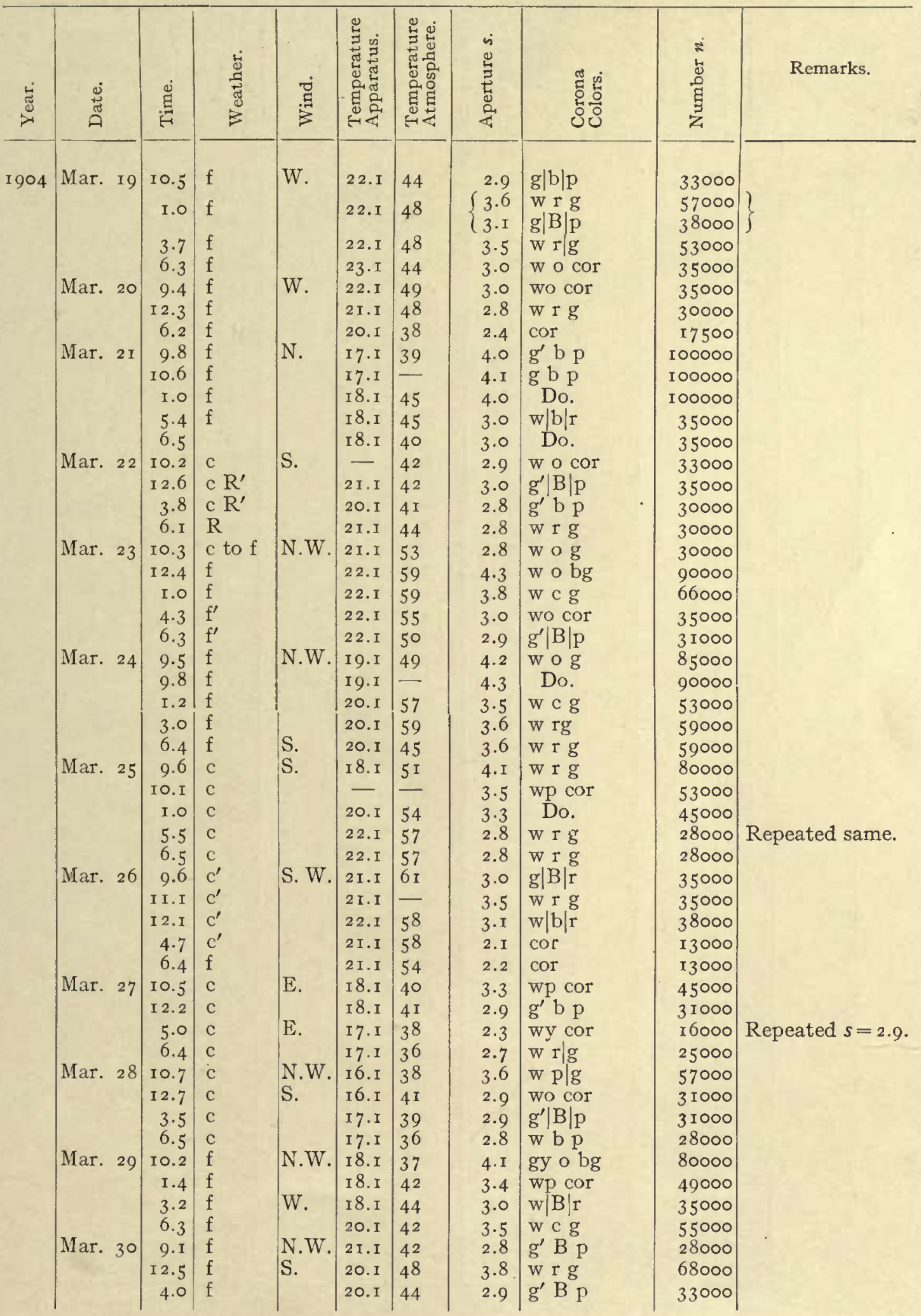


TABLE I-Continued.

\begin{tabular}{|c|c|c|c|c|c|c|c|c|c|c|}
\hline 怨 & ڤึ & $\underset{\forall}{\stackrel{\Xi}{\sharp}}$ & 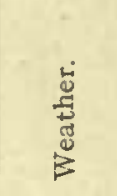 & 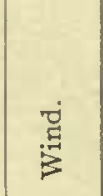 & 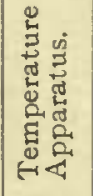 & 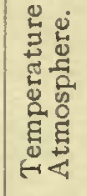 & 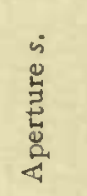 & 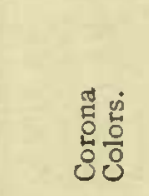 & $\begin{array}{l}\dot{z} \\
\dot{U} \\
\dot{E} \\
\dot{E} \\
z\end{array}$ & Remarks \\
\hline I 904 & $\begin{array}{l}\text { Apr. } 7 \\
\text { Apr. 9 } \\
\text { Apr. I0 } \\
\text { Apr. I I } \\
\text { Apr. I 2 }\end{array}$ & $\begin{array}{r}6.0 \\
9.5 \\
\text { I } 1.4 \\
3.7 \\
6.5 \\
9.5 \\
\text { I2.7 } \\
6.8 \\
9.3 \\
\text { II.5 } \\
3.4 \\
6.3 \\
\text { I0.3 } \\
\text { I.3 } \\
4.7 \\
6.3 \\
6.5 \\
9.5 \\
\text { I } 2.7 \\
3.5 \\
5.4 \\
9.0 \\
\text { I } 2.5 \\
6.0 \\
9.7 \\
\text { I1 } 4 \\
3.5 \\
3.9 \\
6.6 \\
9.5 \\
1.0 \\
4.4 \\
5.5 \\
8.0 \\
9.2 \\
10.7 \\
1.5 \\
5.4 \\
6.7 \\
9.2 \\
\text { I } 2.4 \\
4.3 \\
5.6 \\
0.4 \\
9.3 \\
\text { I1.7 } \\
5.0 \\
6.1 \\
9.3 \\
\text { I } 2.5\end{array}$ & 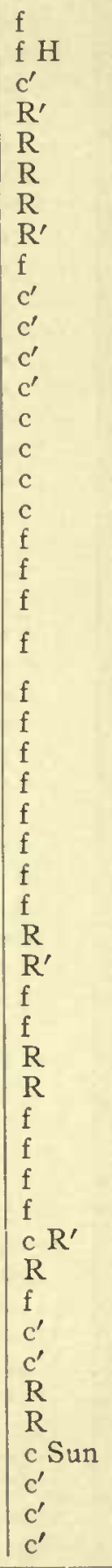 & $\begin{array}{l}\text { W. } \\
\text { S. } \\
\text { E. } \\
\text { S. }\end{array}$ & 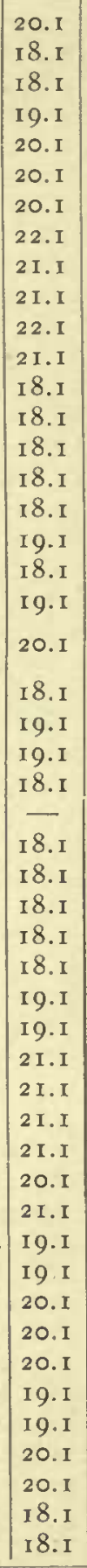 & 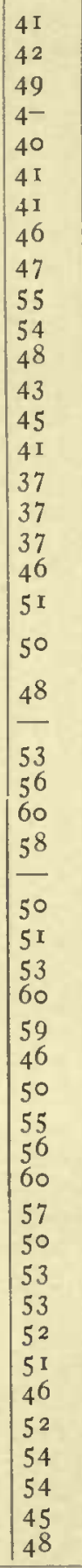 & $\begin{array}{r}3.2 \\
2.9 \\
3.5 \\
2.9 \\
2.4 \\
2.4 \\
2.6 \\
3.0 \\
2.9 \\
3.0 \\
2.9 \\
2.8 \\
3.7 \\
3.7 \\
2.9 \\
4.1 \\
3.7 \\
4.2 \\
3.8 \\
3.2 \\
3.2 \\
3.0 \\
4.1 \\
3.6 \\
3.8 \\
4.2 \\
4.1 \\
3.5 \\
3.1 \\
2.9 \\
3.0 \\
3.2 \\
2.3 \\
2.7 \\
2.8 \\
2.8 \\
2.2 \\
2.8 \\
2.7 \\
2.2 \\
3.3 \\
3.0 \\
2.3 \\
2.9 \\
2.8 \\
2.8 \\
3.0 \\
3.0 \\
3.8 \\
3.0 \\
2.9\end{array}$ & $\begin{array}{l}\text { wp cor } \\
\text { wo g } \\
\text { wp cor } \\
\text { w o g } \\
\text { w br cor } \\
\text { wbr cor } \\
\text { Do. } \\
\text { w B p } \\
g^{\prime} \text { b p } \\
\text { w o cor } \\
\text { w o g } \\
\text { w|b|p } \\
\text { w r g } \\
\text { Do. } \\
\text { g b p } \\
\text { w o g } \\
\text { w c g } \\
\text { y' o bg } \\
\text { w rg } \\
\text { w p cor } \\
\text { w|b|r } \\
\text { w|b|p } \\
\text { g b p } \\
\text { w r g } \\
\text { w c g } \\
\text { y br bg } \\
\text { w o g } \\
\text { wp cor } \\
\text { wbrcor } \\
\text { w|b|p } \\
\text { w y cor } \\
\text { w|B|r } \\
\text { wy cor } \\
\text { wc g } \\
\text { w r g } \\
\text { Do. } \\
\text { wbr cor } \\
\text { w o g } \\
\text { w rg } \\
\text { cor } \\
\text { wp cor } \\
\text { w b|p } \\
\text { cor } \\
\text { cor } \\
\text { w b p } \\
\text { w o g } \\
\text { w|b|r } \\
g^{\prime} \text { b p } \\
\text { w r g } \\
\text { g' b p } \\
\text { w r g }\end{array}$ & $\begin{array}{l}42000 \\
31000 \\
55000 \\
31000 \\
17000 \\
19000 \\
22000 \\
35000 \\
31000 \\
35000 \\
33000 \\
29000 \\
64000 \\
61000 \\
33000 \\
80000 \\
61000 \\
85000 \\
66000 \\
44000 \\
42000 \\
80000 \\
57000 \\
66000 \\
83000 \\
80000 \\
53000 \\
38000 \\
33000 \\
35000 \\
42000 \\
15000 \\
25000 \\
28000 \\
28000 \\
14000 \\
28000 \\
25000 \\
13500 \\
45000 \\
35000 \\
16000 \\
31000 \\
28000 \\
28000 \\
35000 \\
37000 \\
66000 \\
35000 \\
31000\end{array}$ & $\begin{array}{l}6.5 / 82{ }^{1} \\
5 \cdot 5 / 74 \\
5 / 37 \\
5 / 44 \\
5 / 40 \\
5 / 47 \\
5 / 30 \\
5 / 35 \\
\text { Night. } \\
5 / 25 \\
5 / 40 \\
5 / 35 \\
5 / 32 \\
5 / 53 \\
5 / 49 \\
5 / 22 \\
5 / 46 \\
5 / 45 \\
5 / 37 \\
5 / 51 \\
5 / 49 \\
5 / 62 \\
4 / 38 \\
5 / 44\end{array}$ \\
\hline
\end{tabular}

\footnotetext{
1. Subsidence data, showing a fall of fog level of $6.5 \mathrm{~cm}$. in $82 \mathrm{sec}, 5.5 \mathrm{~cm}$. in $74 \mathrm{sec}, 5 \mathrm{~cm}$. in $37 \mathrm{sec}$., etc.
} 
TABLE I-Continued.

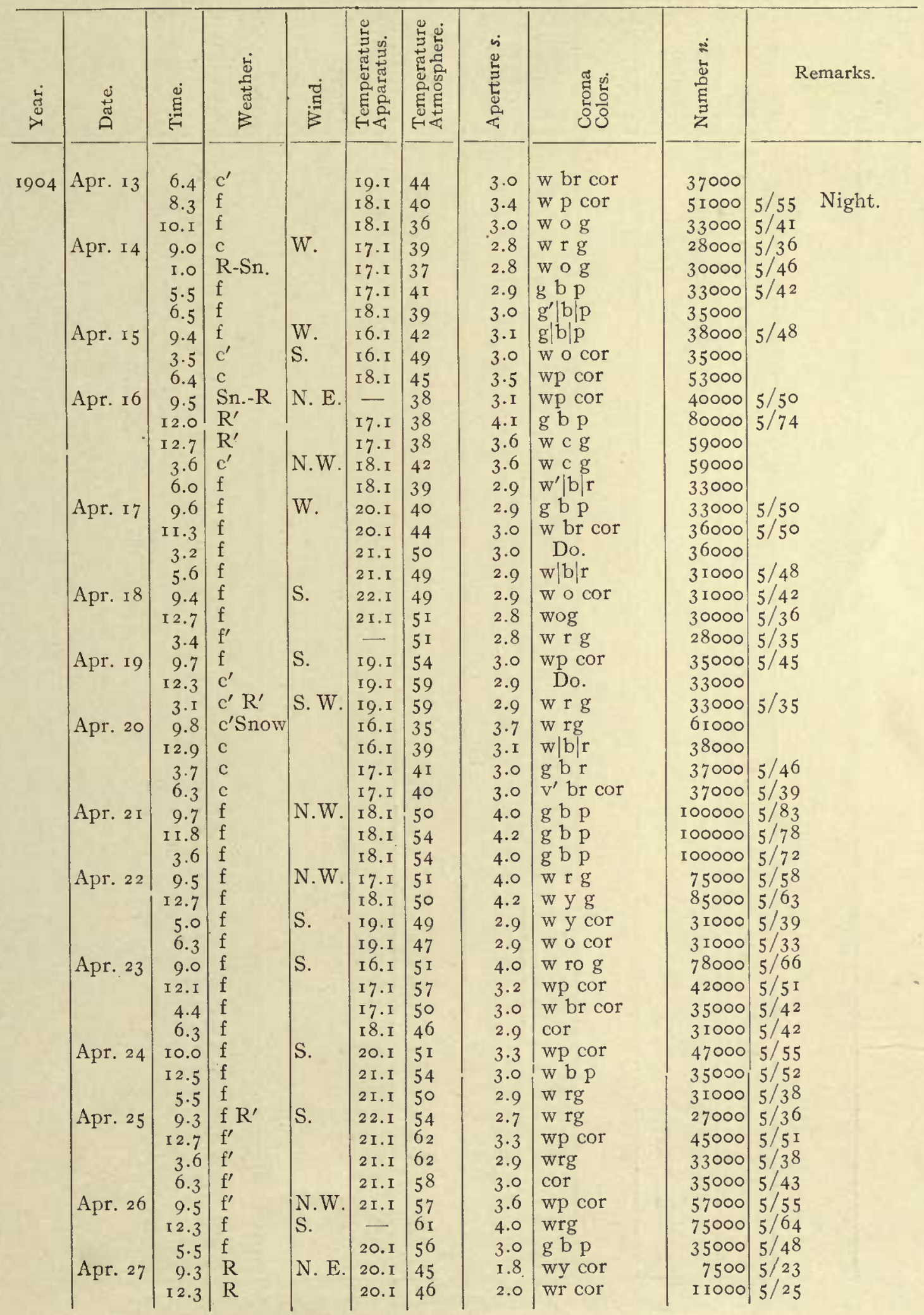


TABLE I-Continued.

\begin{tabular}{|c|c|c|c|c|c|c|c|c|c|c|}
\hline$\stackrel{\nu}{J}$ & ڤ̆ & 苞 & 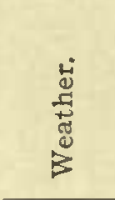 & 晃 & 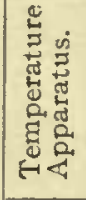 & 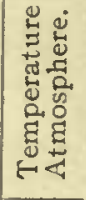 & 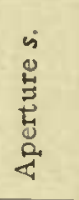 & 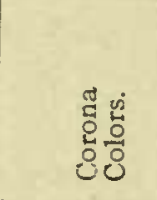 & 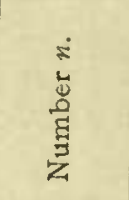 & Remarks. \\
\hline 1904 & $\begin{array}{l}\text { Apr. } 30 \\
\text { May I } \\
\text { May } 2 \\
\text { May } 3 \\
\text { May } 4 \\
\text { May } 5 \\
\text { May } 6\end{array}$ & $\begin{array}{r}3.4 \\
6.0 \\
9.4 \\
\text { II.5 } \\
4.0 \\
4.7 \\
4.9 \\
5.5 \\
5.7 \\
5.9 \\
8.8 \\
9.3 \\
9.7 \\
\text { II.5 } \\
3.5 \\
6.1 \\
\text { I0.0 } \\
\text { II.0 } \\
2.3 \\
6.0 \\
9.6 \\
\text { I } 2.0 \\
3.1 \\
6.4 \\
9.0 \\
\text { I } 2.3 \\
3.3 \\
5.4 \\
9.2 \\
\text { II.2 } \\
5.4 \\
9.3 \\
\text { II.7 } \\
3.1 \\
6.3 \\
9.2 \\
\text { I } 2.3 \\
3.4 \\
9.4 \\
\text { I } 2.2 \\
3.3 \\
6.3 \\
8.8 \\
9.7 \\
9.3 \\
\text { II.9 } \\
4.0 \\
5.2 \\
\text { I } 2.0 \\
4.0 \\
6.0 \\
\end{array}$ & $\begin{array}{l}R \\
R \\
R \\
R \\
R \\
R \\
R \\
R \\
R \\
R \\
R^{\prime} \\
R^{\prime} \\
c \\
c \\
R^{\prime} \\
c \\
c^{\prime} \\
c^{\prime}(S) \\
R \\
c^{\prime}(S) \\
f \text { to } c \\
c \\
c^{\prime} \\
c^{\prime} \\
f \\
f \\
f \\
f \\
f \\
f \\
f \\
f \\
f \\
f \\
f \\
f \\
f \\
f \\
f \\
f \\
f \\
f \\
f \\
f \\
f \\
f \\
f \\
f \\
f \\
f \\
f \\
\end{array}$ & $\begin{array}{l}\text { N.W. } \\
\text { N. E. } \\
\text { E. } \\
\text { N.W. } \\
\text { S. } \\
\text { S. }\end{array}$ & 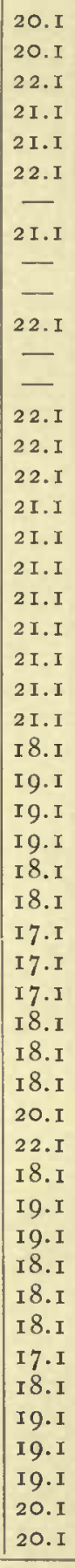 & $\begin{array}{l}45 \\
42 \\
46 \\
48 \\
52 \\
- \\
53 \\
- \\
51 \\
- \\
55 \\
57 \\
57 \\
63 \\
63 \\
64 \\
62 \\
59 \\
62 \\
64 \\
55 \\
59 \\
63 \\
62 \\
60 \\
57 \\
63 \\
54 \\
61 \\
65 \\
74 \\
67 \\
72 \\
79 \\
82 \\
67 \\
70 \\
68 \\
61 \\
53 \\
52 \\
59 \\
67 \\
74 \\
71 \\
74 \\
74 \\
67 \\
\end{array}$ & $\begin{array}{l}2.0 \\
1.9 \\
2.4 \\
2.1 \\
2.1 \\
1.9 \\
1.9 \\
1.9 \\
1.9 \\
3.7 \\
3.0 \\
3.0 \\
2.8 \\
2.2 \\
3.0 \\
3.0 \\
3.0 \\
2.9 \\
3.0 \\
1.9 \\
2.7 \\
2.5 \\
2.3 \\
3.4 \\
3.0 \\
2.8 \\
3.1 \\
4.1 \\
3.8 \\
2.8 \\
4.0 \\
3.8 \\
3.1 \\
3.1 \\
4.0 \\
2.9 \\
2.4 \\
2.8 \\
2.9 \\
3.0 \\
3.0 \\
3.0 \\
2.7 \\
2.6 \\
3.1 \\
2.8 \\
2.6 \\
3.0 \\
2.9 \\
2.1 \\
\end{array}$ & $\begin{array}{l}\text { w r cor } \\
\text { cor } \\
\text { cor } \\
\text { cor } \\
\text { cor } \\
\text { cor } \\
\text { cor } \\
\text { cor } \\
\text { wrcor } \\
\text { w r g } \\
\text { w|b|p } \\
\text { w ro g } \\
\text { w r g } \\
\text { wp cor } \\
\text { wbr cor } \\
\text { w|b|p } \\
\text { w r g } \\
\text { w r g } \\
\text { w b p } \\
\text { cor } \\
\text { w r g } \\
\text { cor } \\
\text { cor } \\
\text { wp cor } \\
\text { cor } \\
\text { w rg } \\
\text { w|B|p } \\
\text { g b p } \\
\text { w r g } \\
\text { w r g } \\
\text { y o bg } \\
\text { w c g } \\
\text { w br cor } \\
\text { g|b|p } \\
\text { w y bg } \\
\text { w o cor } \\
\text { cor } \\
\text { w br|g } \\
\text { w br cor } \\
\text { w o } \mid g \\
\text { g|b|p } \\
\text { w b p } \\
\text { w rg cor } \\
\text { wbr cor } \\
\text { w|b|r } \\
\text { w o cor } \\
\text { w br cor } \\
\text { g' br cor } \\
\text { w y|g } \\
\text { cor } \\
\end{array}$ & $\begin{array}{r}10000 \\
8500 \\
17000 \\
12000 \\
12000 \\
8500 \\
8500 \\
8500 \\
8500 \\
81000 \\
35000 \\
35000 \\
30000 \\
13000 \\
35000 \\
37000 \\
37000 \\
33000 \\
35000 \\
9000 \\
25000 \\
21000 \\
16000 \\
49000 \\
36000 \\
28000 \\
38000 \\
100000 \\
68000 \\
28000 \\
78000 \\
66000 \\
40000 \\
38000 \\
78000 \\
31000 \\
17000 \\
29000 \\
31000 \\
35000 \\
35000 \\
35000 \\
25000 \\
23000 \\
38000 \\
29000 \\
22000 \\
35000 \\
31000 \\
12000 \\
\end{array}$ & 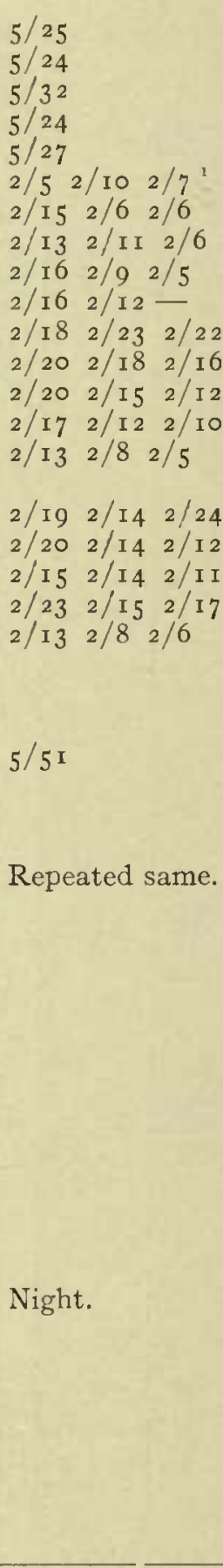 \\
\hline
\end{tabular}

. Subsidence over consecutive distances of $2 \mathrm{~cm}$. each. 
TABLE $\mathrm{x}$-Continued.

\begin{tabular}{|c|c|c|c|c|c|c|c|c|c|c|}
\hline 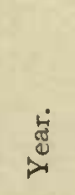 & 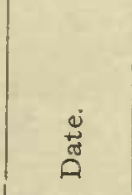 & $\underset{⿱ 乛}{\stackrel{\mathscr{E}}{\Xi}}$ & 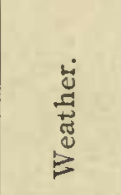 & 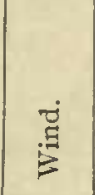 & 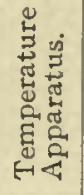 & 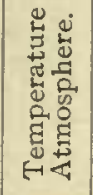 & 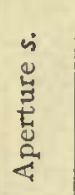 & 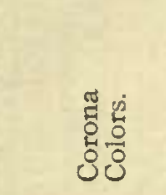 & 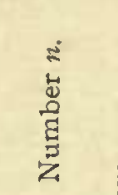 & Remarks. \\
\hline \multirow[t]{52}{*}{1904} & May 9 & $9 \cdot 5$ & $\mathrm{R}$ & E. & I9. I & 63 & I. 8 & cor & 7000 & \multirow{8}{*}{$2 / 3^{6} 2 / 24$} \\
\hline & & 12.3 & $\mathrm{R}$ & & I9.I & 65 & 2.0 & cor & 10000 & \\
\hline & & 3.6 & $\mathrm{R}^{\prime}$ & & $20 . \mathrm{I}$ & 66 & $2 . I$ & $\mathrm{w} r \mathrm{cor}$ & I 2000 & \\
\hline & May Io & $9 \cdot 4$ & f & W. & 19.1 & 70 & $4 \cdot 3$ & $\mathrm{y}^{\prime}$ br bg' & 90000 & \\
\hline & & 9.8 & $\mathrm{f}$ & & I9.I & - & 4.2 & yobg & 88000 & \\
\hline & & 12.5 & $\mathrm{f}$ & & 20.1 & 74 & 3.0 & cor & 35000 & \\
\hline & & $3 \cdot 5$ & $\mathrm{f}$ & S. & 20.1 & 72 & 2.9 & $\mathrm{w}|\mathrm{b}| \mathrm{r}$ & 31000 & \\
\hline & & 6.2 & c & & 20.1 & 71 & 2.9 & Do. & 33000 & \\
\hline & \multirow[t]{4}{*}{ May II } & $9 \cdot 3$ & c & N.W. & 20.1 & 64 & 2.2 & $\mathrm{wrg}$ & I 3000 & \multirow[t]{8}{*}{ Rain at night. } \\
\hline & & 12.6 & c & & 20.1 & 68 & 3.4 & wp cor & 49000 & \\
\hline & & $4 \cdot 5$ & $f^{\prime} f$ & & 20.1 & 70 & 3.0 & $\mathrm{w}|\mathrm{b}| \mathrm{r}$ & 36000 & \\
\hline & & 6.3 & c & & 20.1 & 69 & 2.9 & $\mathrm{~g}^{\prime} \mathrm{b} p$ & 31000 & \\
\hline & \multirow[t]{3}{*}{ May I 2} & 9.4 & $f^{\prime}$ & N.W. & I9.I & 63 & 2.4 & cor & 19000 & \\
\hline & & 3.2 & f & N. E. & I9.I & 67 & 2.6 & cor & 22000 & \\
\hline & & 5.6 & $\mathrm{f}$ & S. & I9.I & 64 & 3.0 & $\mathrm{w} / \mathrm{b} \mid \mathrm{r}$ & 35000 & \\
\hline & May I 3 & 9.2 & $\mathrm{f}$ & N.W. & I9.I & 69 & $3 \cdot 5$ & wrg & 53000 & \\
\hline & (1) & $4 \cdot 7$ & $f$ & & I 9.1 & 72 & 2.0 & cor & I I 000 & \multirow[t]{8}{*}{ Repeated $s=2$. I. } \\
\hline & & 6.4 & & & 19.1 & 66 & I. 9 & cor & 8000 & \\
\hline & \multirow[t]{3}{*}{ May I4 } & 9.0 & $c^{\prime}$ Fog & & 19.1 & $6_{3}$ & 2.I & cor & I 2000 & \\
\hline & & 12.5 & & & I9.I & 66 & I.9 & cor & 8000 & \\
\hline & & 6.2 & $\mathrm{c}^{\prime}$ & & I9.I & 60 & I. 8 & cor & 7000 & \\
\hline & \multirow[t]{3}{*}{ May I 5} & $9 \cdot 5$ & $\mathrm{c}$ & N. E. & I 8 I & $5^{8}$ & I. 8 & cor & 7000 & \\
\hline & & I2. 5 & $\mathrm{c}$ & & I 8.1 & 62 & 2.0 & cor & 10000 & \\
\hline & & 6.2 & & & I $9 . \mathrm{I}$ & 55 & 2.0 & cor & To000 & \\
\hline & \multirow[t]{4}{*}{ May I 6} & $9 \cdot 5$ & $c^{\prime}(\mathrm{S})$ & & I 8.1 & 60 & 3.2 & wp cor & 43000 & \multirow{28}{*}{ Rain at night. } \\
\hline & & I2.I & & & I $8 . I$ & 62 & 3.0 & wog & 35000 & \\
\hline & & $3 \cdot 3$ & $c^{\prime} R^{\prime}$ & - & I 8. I & 59 & 2.9 & wo $g$ & 33000 & \\
\hline & & 6.0 & $\mathrm{f}$ & & I 7.1 & 60 & 3.0 & $\mathrm{w}$ br $\mathrm{b} \mid \mathrm{r}$ & 35000 & \\
\hline & \multirow[t]{4}{*}{ May I 7} & $9 \cdot 3$ & c & S. W. & 17.1 & $5^{8}$ & $3 \cdot 3$ & $\mathrm{w}|\mathrm{b}| \mathrm{r}$ & 45000 & \\
\hline & & 12.5 & c & & $\mathrm{x} 7 . \mathrm{I}$ & $6_{3}$ & 3.2 & Do. & 42000 & \\
\hline & & 2.3 & $c^{\prime}$ & & 17.1 & 65 & 3.0 & $w \circ g$ & 35000 & \\
\hline & & 6.1 & c & & 17.1 & 62 & 3.0 & $\mathrm{w}|\mathrm{b}| \mathrm{r}$ & 35000 & \\
\hline & \multirow[t]{3}{*}{ May I 8} & 9.2 & c $\mathrm{R}^{\prime}$ & N. E. & I 7.1 & 63 & I.9 & cor. & 8000 & \\
\hline & & x 2.5 & $\mathrm{R}^{\prime}$ & & 20.1 & 63 & 1.9 & cor & $8_{500}$ & \\
\hline & & $5 \cdot 5$ & $c$ & & I8.I & $5^{8}$ & I.9 & cot & 8500 & \\
\hline & \multirow{4}{*}{ May rg } & $9 \cdot 5$ & $c$ & $N$. & I 7.1 & 55 & .2 .9 & w r g & 31000 & \\
\hline & & I 1.9 & c & & I 8 . I & 59 & I.9 & cor & 8000 & \\
\hline & & 3.0 & $\mathrm{R}$ & & I8. I & 56 & 2.3 & cor & I 5000 & \\
\hline & & 5.6 & $\mathrm{R}$ & & Ig.I & 55 & 2.8 & w r g & 28000 & \\
\hline & \multirow{4}{*}{ May 2o } & 9.I & & S. W. & I $8 . x$ & 63 & 2.8 & w r g & 28000 & \\
\hline & & I 2.5 & $c^{\prime} \cdot(\mathrm{S})$ & & I 8.1 & 69 & 3.2 & wp cor & 42000 & \\
\hline & & 3.0 & $\mathrm{R}^{\prime}$ & W. & I 8. I & $6 I$ & 2.8 & $\mathrm{w} \mathrm{rg}$ & 30000 & \\
\hline & & 6.1 & $f$ & S. & - & 62 & 2.8 & w r g & 28000 & \\
\hline & \multirow[t]{4}{*}{ May 2 I } & $9 \cdot 5$ & $f$ & N.W. & I 8.1 & 67 & 3.8 & $\mathrm{y}^{\prime} \circ \mathrm{g}^{\prime}$ & 66000 & \\
\hline & & I 2.7 & $\mathrm{f}$ & & I9.I & 73 & 3.0 & W I g ? & 35000 & \\
\hline & & 4.8 & f & S. & 20.1 & 69 & 2.9 & w $\circ \mathrm{g}$ & 31000 & \\
\hline & & 6.0 & $\mathrm{f}$ & & - & 68 & 3.2 & $\mathrm{w} p$ cor & 42000 & \\
\hline & \multirow[t]{3}{*}{ May 22} & 9.8 & $\mathrm{f}$ & S. & I9.I & 68 & 2.4 & cor & I7 500 & \\
\hline & & I0. 8 & $\mathrm{f}$ & & 19.1 & 70 & 2.4 & $\operatorname{cor} b$ & 18500 & \\
\hline & & I 2.3 & $\mathrm{f}$ & & I 9.1 & $7 \mathrm{I}$ & 2.2 & cor & 13500 & \\
\hline & \multirow{2}{*}{ May 23} & 9.7 & $\mathrm{f}$ & S. & I9.1 & 67 & 3.0 & $\mathrm{~g} b \mid \mathrm{p}$ & 36500 & \\
\hline & & I 2.3 & f & & I9.I & 68 & 3.0 & Do. & 35000 & \\
\hline
\end{tabular}


TABLE I-Continued.

\begin{tabular}{|c|c|c|c|c|c|c|c|c|c|c|}
\hline 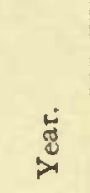 & 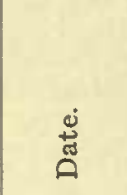 & $\underset{E}{\stackrel{E}{E}}$ & 离 & $\underset{⿱ 乛}{\stackrel{D}{Z}}$ & 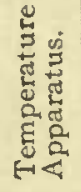 & 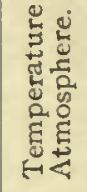 & 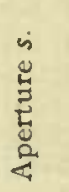 & 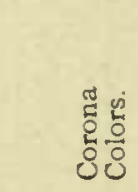 & 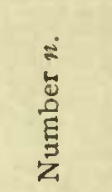 & Remarks. \\
\hline \multirow[t]{46}{*}{1904} & May 23 & 6.3 & $\mathrm{f}$ & & I9. I & 66 & 2.9 & w r g & 31000 & \multirow{11}{*}{ Rain at night. } \\
\hline & May 24 & $9 \cdot 3$ & $c^{\prime}$ & S. W. & I9.I & 69 & 2.9 & $\mathrm{w}|\mathrm{b}| \mathrm{p}$ & 31000 & \\
\hline & & I 2.5 & $\mathrm{f}$ & & 20. I & 75 & 2.9 & wog & 31000 & \\
\hline & & 3.2 & f & \multirow{3}{*}{ N.W. } & 20.1 & 76 & 2.7 & w r g & 25000 & \\
\hline & \multirow{2}{*}{ May 25} & $9 \cdot 3$ & f & & 20. I & 75 & $3 \cdot 5$ & w c g & 53000 & \\
\hline & & I 2.7 & $\mathrm{f}$ & & 20. I & 78 & 2.8 & w r g & 30000 & \\
\hline & \multirow{5}{*}{ May 26} & $3 \cdot 4$ & $\mathrm{f}$ & \multirow{5}{*}{ S. } & 20. I & 73 & 2.3 & cor & I 5000 & \\
\hline & & 6.3 & f & & $2 \mathrm{I} . \mathrm{I}$ & 70 & 2.3 & cor & I 6000 & \\
\hline & & $9 \cdot 3$ & f & & $2 \mathrm{I} . \mathrm{I}$ & 78 & 2.9 & cor b & 31000 & \\
\hline & & 2.4 & $\mathrm{f}^{\prime}$ & & $22 . I$ & 78 & 2.8 & w rg & 28000 & \\
\hline & & 6.4 & f & & 22.I & 74 & 2.3 & wycor & I 5000 & \\
\hline & \multirow{4}{*}{ May 27} & 9.8 & $\mathbf{f}^{\prime}$ & \multirow[t]{4}{*}{ S. W. } & $2 \mathrm{I} . \mathrm{I}$ & 76 & 2.9 & wog & 31000 & \multirow[t]{5}{*}{ Rain at night. } \\
\hline & & I 2.0 & f & & 22.1 & 82 & $3 \cdot 5$ & wp cor & 53000 & \\
\hline & & 3.2 & $\mathrm{R}$ & & $23 . I$ & 71 & 3.0 & $\mathrm{~g}|\mathrm{~b}| \mathrm{p}$ & 36000 & \\
\hline & & $5 \cdot 5$ & f & & 22.1 & $7 \mathrm{I}$ & 2.4 & cor & 17000 & \\
\hline & \multirow{4}{*}{ May 28} & 9.5 & $\mathrm{f}$ & \multirow[t]{4}{*}{ W. } & 20.1 & 68 & 4. I & w o bg & 80000 & \\
\hline & & 9.7 & f & & 20.1 & 一 & $3 \cdot 3$ & $\mathrm{~g}|\mathrm{~b}| \mathrm{p}$ & 45000 & \multirow[t]{9}{*}{ Repeated. } \\
\hline & & 1.0 & $\mathrm{f}$ & & 20.1 & 73 & 3.1 & w $y^{\prime} g$ & 38000 & \\
\hline & & $5 \cdot 7$ & f & & 22.1 & 74 & 3.0 & $w|b| r$ & 35000 & \\
\hline & \multirow[t]{6}{*}{ May 29} & 9.6 & f & \multirow[t]{4}{*}{ S. } & 21.1 & $7 \mathrm{I}$ & $3 \cdot 5$ & wp cor & 53000 & \\
\hline & & IO.I & $\mathrm{f}$ & & $21 . I$ & 72 & $3 \cdot 4$ & Do. & 50000 & \\
\hline & & 10.8 & $\mathrm{f}$ & & $2 \mathrm{I} . \mathrm{I}$ & 74 & $3 \cdot 4$ & $w r g$ & 49000 & \\
\hline & & 12.0 & $\mathrm{f}$ & & 22.1 & 74 & 3.2 & wp cor & 42000 & \\
\hline & & 4.5 & $\mathrm{f}$ & \multirow[t]{6}{*}{ S. W. } & 22.1 & 75 & I. 8 & $\operatorname{cor}$ & 7000 & \\
\hline & & 6.4 & $\mathrm{f}$ & & $23 . I$ & 70 & 2.8 & $\mathrm{w} r \mathrm{~g}$ & 28000 & \\
\hline & \multirow[t]{4}{*}{ May 30} & $9 \cdot 5$ & $c^{\prime}$ & & $2 \mathrm{I} . \mathrm{I}$ & 72 & 2.4 & cor & I 7000 & \multirow[t]{4}{*}{ Repeated same. } \\
\hline & & 1.0 & c & & 22.1 & 74 & 2.4 & cor & I 9000 & \\
\hline & & 2.5 & c & & 22.1 & 73 & 1.9 & cor & 8500 & \\
\hline & & 6.4 & c & & 22.1 & 69 & 2.4 & cor & I 7000 & \\
\hline & \multirow[t]{5}{*}{ May 3 I } & 9.5 & & N. E. & 20.1 & 65 & 3.0 & w r g & 35000 & Rain at night. \\
\hline & & I 2.3 & $\mathrm{c}^{\prime}(\mathrm{S})$ & & 20.1 & 70 & 2.7 & w rg & 26000 & $\begin{array}{l}\text { New apparatus } \\
\text { same. }\end{array}$ \\
\hline & & $3 \cdot 5$ & $\mathrm{f}^{\prime}(\mathrm{S})$ & & 20.1 & 71 & 2.7 & w r g & 26000 & New brass apja- \\
\hline & & 6.3 & & & - & 65 & 2.1 & w r g & I 2000 & $\begin{array}{l}\text { ratus. } \\
\text { peated } s=2.8 \text {. }\end{array}$ \\
\hline & & 8.4 & $\mathrm{f}^{\prime}$ & & $2 \mathrm{I} . \mathrm{I}$ & 60 & 2.I & Do. & I 2000 & Night. \\
\hline & June $\mathrm{I}$ & 9.0 & $\mathrm{c}$ & N. E. & 20. I & 62 & 2.8 & $\mathrm{w}|\mathrm{b}| \mathrm{p}$ & 28000 & \\
\hline & & I I. 5 & $\mathrm{c}$ & & I9.I & 65 & 2.4 & cor & I 9000 & \\
\hline & & 4.5 & $\mathrm{R}$ & & I9.I & 57 & I. 9 & cor & 8500 & \\
\hline & & 5.8 & $\mathrm{R}$ & & I9.I & 56 & r. 8 & cor & 7000 & \\
\hline & June 2 & I0.4 & $\mathrm{c}$ & N. & I 7.1 & 57 & I.9 & cor & 8500 & \\
\hline & & I 2.9 & $\mathrm{c}$ & & 17.1 & 60 & 2.1 & $\mathrm{w} \mathrm{rg}$ & I 2500 & \\
\hline & & 4.7 & $\mathrm{c}$ & & 18.1 & $5^{8}$ & I. 8 & cor & 7000 & \\
\hline & & $\begin{array}{l}4 \cdot 7 \\
6.3\end{array}$ & c & & I 8.1 & $5^{6}$ & 1. 8 & cor & 7000 & \\
\hline & June 3 & 9.8 & c & N. E. & 18.1 & 59 & 2.2 & cor & I 3000 & \\
\hline & & 10.8 & & & 18.1 &. & 2.2 & cor & I 3000 & \\
\hline & & I $\mathrm{x} .5$ & $\mathrm{f}^{\prime}(\mathrm{S})$ & & 18.1 & 67 & 2.6 & w r g & 24000 & \\
\hline & & $\mathrm{I} 2.3$ & $f^{\prime}$ & & - & - & 2.8 & $\mathrm{w}|\mathrm{b}| \mathrm{p}$ & 28000 & \\
\hline$\theta$ & & $3 \cdot 3$ & $\mathrm{f}^{\prime}(\mathrm{S})$ & & I g.I & 74 & 2.8 & w T g & 28000 & \\
\hline & & 6.0 & $\mathrm{f}$ & & I 9.1 & 70 & 2.8 & wrg & 28000 & \\
\hline & June 4 & $9 \cdot 5$ & $f$ & N. E. & I9.I & 73 & 2.9 & $w|b| r$ & 31000 & \\
\hline & & I 2.5 & $\mathbf{f}^{\prime}$ & & I 9.1 & 78 & 2.3 & cor & I 5000 & \\
\hline
\end{tabular}


TABLE I-Continued.

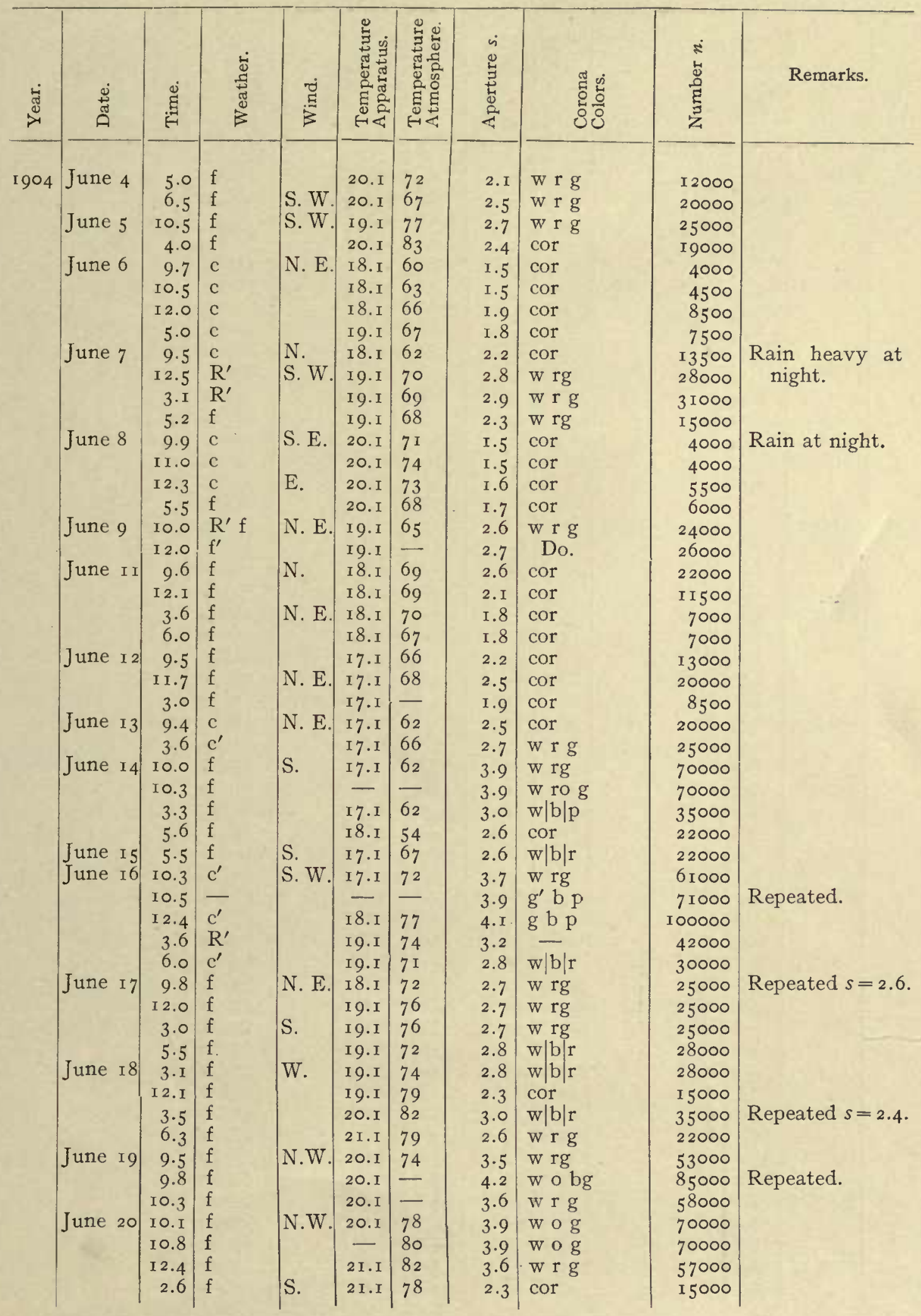


TABLE I-Continued.

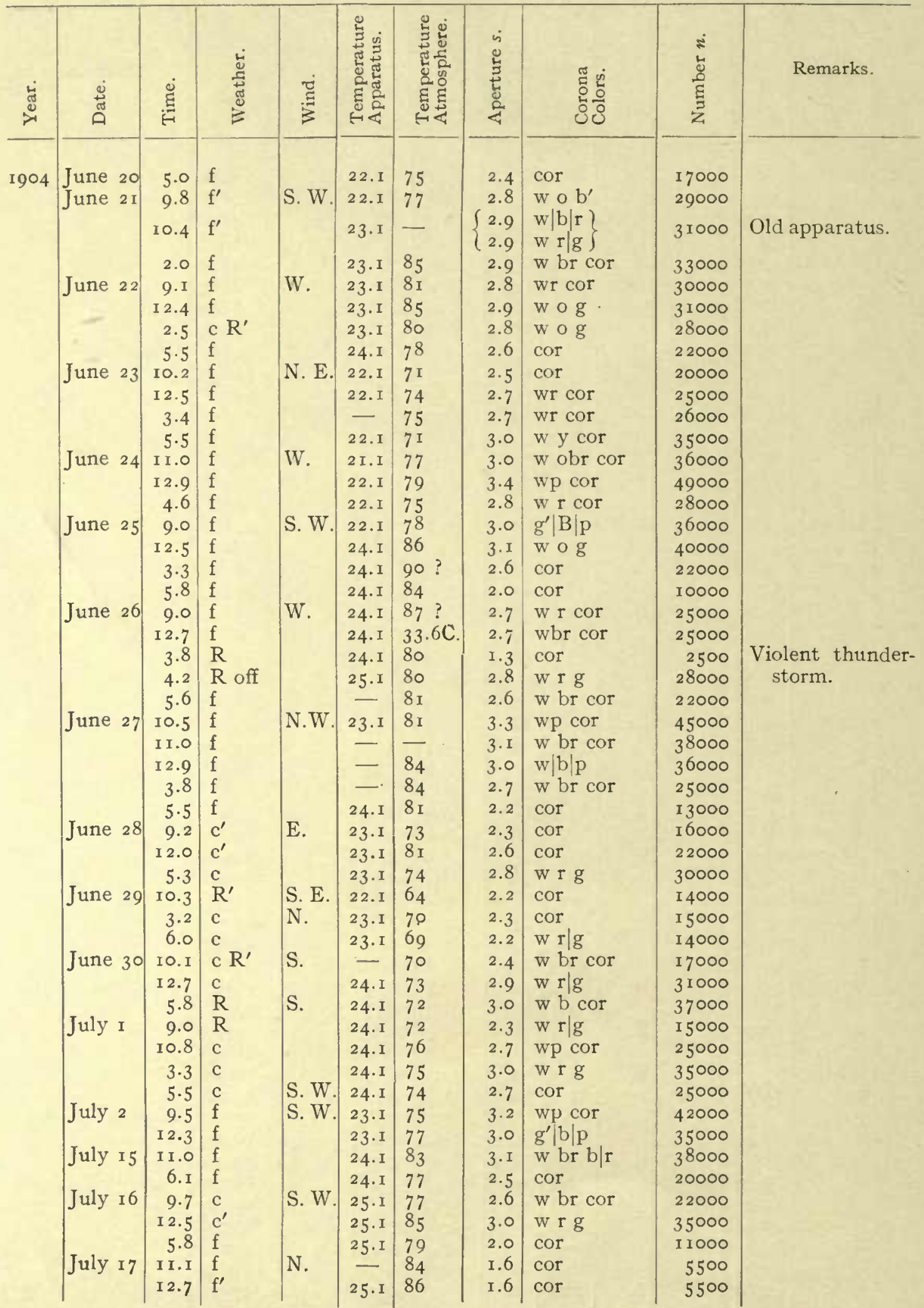


A CONTINUOUS RECORD OF ATMOSPHERIC NUCLEATION.

TABLE I-Continued.

\begin{tabular}{|c|c|c|c|c|c|c|c|c|c|c|}
\hline 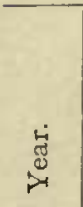 & 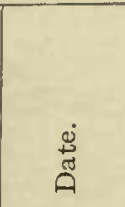 & 㟧 & 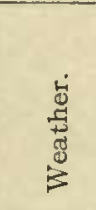 & 苞 & 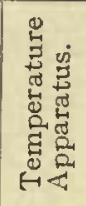 & 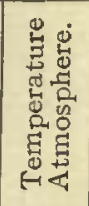 & 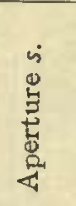 & 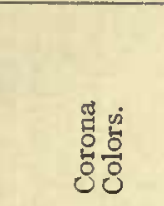 & 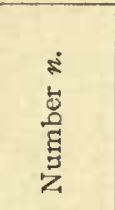 & Remarks. \\
\hline \multirow[t]{50}{*}{ I 904} & July I & 2.5 & $f^{\prime}$ & & - & - & & & & \\
\hline & & 5.0 & $\mathrm{f}$ & & $26 . \mathrm{I}$ & 85 & 1.8 & cor & $\begin{array}{l}4500 \\
7000\end{array}$ & \\
\hline & July i 8 & $9 \cdot 5$ & f & N.W. & $24 . \mathrm{I}$ & 84 & 3.6 & w r g & 59000 & \\
\hline & & 10.8 & & & $25 . I$ & & 3.4 & wp cor & 51000 & \\
\hline & Julv Io & I. 6 & $\mathrm{f}$ & S. & $25 . \mathrm{I}$ & $89 ?$ & 2.8 & wrg & 28000 & \\
\hline & July I9 & II. 3 & $\mathrm{f}$ & S. & $25 \cdot \mathrm{I}$ & $8 \mathrm{I}$ & 2.3 & wo cor & I6002 & \\
\hline & & $\begin{array}{r}\text { I } 2.9 \\
2.4\end{array}$ & $\begin{array}{l}f \\
f\end{array}$ & & 26.1 & 86 & 2.3 & $\begin{array}{l}\text { Do. } \\
\text { Do. }\end{array}$ & $\begin{array}{l}\text { I6000 } \\
\text { I } 60000\end{array}$ & \\
\hline & & 5.7 & $\mathrm{f}$ & & $26 . I$ & $\begin{array}{l}87 \\
78\end{array}$ & $\begin{array}{l}2.3 \\
2.3\end{array}$ & Do. & 16000 & \\
\hline & July 20 & 10.2 & f & N.W. & $26 . I$ & 87 & 3.0 & w rolg & 35000 & \\
\hline & & 2.0 & f & W. & $26 . I$ & 88 & 2.7 & w br cor & 25000 & \\
\hline & & $5 \cdot 7$ & f & & $25 . I$ & 84 & 2.2 & cor & 14000 & \\
\hline & July 2 I & 9.6 & f & W. & $25 \cdot I$ & $8 \mathbf{r}$ & 3.0 & w r $\mathbf{r} g$ & 35000 & \\
\hline & & T 2.0 & f & & $25 \cdot \mathrm{I}$ & 84 & 3.0 & wog & 35000 & \\
\hline & & 3.2 & f & & $25 \cdot I$ & 84 & 2.3 & w r $\mid g$ & 15000 & \\
\hline & & 5.6 & $\mathrm{f}$ & & $25 \cdot \mathrm{I}$ & 82 & 2.2 & cor & I 3000 & \\
\hline & July 22 & 10.0 & $c^{\prime}$ & E. & $24 . I$ & 77 & 2.3 & w rbr cor & ז 5000 & \\
\hline & & I 2.5 & c & & $24 \cdot I$ & $8 \mathrm{I}$ & x. 6 & cor & 5000 & \\
\hline & & 4.6 & $\mathrm{R}^{\prime}$ & & $24 \cdot I$ & 71 & т.6 & $\operatorname{cor}$ & 5500 & \\
\hline & & 6.0 & c & & $24 . I$ & 69 & I. 8 & cor & 7500 & \\
\hline & July 23 & 9.7 & $\mathrm{R}^{\prime}$ & N. E. & $24 \cdot I$ & 66 & I. 4 & cor & 3000 & Rain at night. \\
\hline & & 12.3 & c & & $24 . I$ & 64 & I. 4 & cor & 3000 & \\
\hline & & $3 \cdot 5$ & c & N. & $23 . \mathrm{I}$ & 66 & $x .6$ & cor & 5000 & \\
\hline & & 5.9 & c & & 23.1 & 63 & I. 6 & cor & 5500 & \\
\hline & July 24 & 12.2 & c & N. & - & 68 & $\mathrm{I} .4$ & cor & 3000 & Repeated $s=\mathrm{I} .3$. \\
\hline & & 6.0 & $\mathrm{R}$ & & $23 . I$ & $6_{5}$ & T. 2 & $\operatorname{cor}$ & 2000 & “ $\quad s=$ I.I. \\
\hline & July 25 & $9 \cdot 5$ & c & S. & $23 \cdot I$ & 72 & 2.3 & cor & I 5000 & \\
\hline & & 12.5 & $f^{\prime}$ & & $23 . \mathrm{I}$ & 79 & 2.4 & cor & I 9000 & \\
\hline & & 6.0 & \pm & & - & 78 & 2.6 & cor & 22000 & \\
\hline & July 26 & 10.7 & $c^{\prime} R^{\prime}$ & S. & $23 \cdot \mathrm{I}$ & 79 & $3 \cdot \mathrm{I}$ & $\mathrm{w}|\mathrm{b}| \mathrm{p}$ & 40000 & \\
\hline & & $\mathrm{T} 2.4$ & & & $24 . \mathrm{I}$ & 80 & 3.I & Do. & 38000 & \\
\hline & & 4.0 & & & $24 . \mathrm{I}$ & 80 & $3 . \mathrm{I}$ & Do. & 38000 & \\
\hline & & 6.0 & & & $24 . I$ & 77 & 2.9 & w r g & 31000 & \\
\hline & July 27 & $9 \cdot 5$ & $c^{\prime} R^{\prime}$ & W. & $24 . I$ & 78 & 3.1 & $\mathrm{~g}^{\prime}|\mathrm{b}| \mathrm{p}$ & 38000 & Rain at night. \\
\hline & & I 2.I & & & $24 . \mathrm{I}$ & 80 & 3.0 & w yog & 35000 & \\
\hline & & 3.4 & $c^{\prime}$ & & $24 . I$ & 82 & 3.0 & Do. & 35000 & \\
\hline & & 6.0 & & & $24 \cdot \mathrm{I}$ & 77 & 2.9 & wog & 33000 & \\
\hline & July 28 & 2.I & $c^{\prime}$ & S. W & - & 83 & 2.8 & wrg & 28000 & \\
\hline & & 6.5 & c & & $25 . I$ & 76 & 2.4 & w rcor & I 7 500 & \\
\hline & July 29 & T0.4 & c & W. & $25 \cdot \mathrm{I}$ & 78 & 2.7 & wp cor & 25000 & \\
\hline & & I 2.7 & $\mathrm{f}$ & & $24 \cdot \mathrm{I}$ & 83 & 3.0 & $\mathrm{w}|\mathrm{b}| \mathrm{p}$ & 35000 & \\
\hline & & $3 \cdot 3$ & $\mathrm{f}$ & & $24 \cdot I$ & 82 & 3.0 & w r g & 35000 & \\
\hline & & 5.6 & $\mathrm{f}$ & & $25 \cdot I$ & 78 & 2.4 & & I 7500 & \\
\hline & July 30 & $9 \cdot 7$ & f & N. & $23 . I$ & $7 x$ & 3.8 & w r g & 66000 & \\
\hline & & $\begin{array}{l}10.0 \\
\text { I } 2.6\end{array}$ & $\begin{array}{l}t \\
f\end{array}$ & & $\overline{23.6}$ & $\overline{77}$ & $\begin{array}{l}3.6 \\
3.1\end{array}$ & $\begin{array}{l}\text { w r g } \\
\text { w o g }\end{array}$ & $\begin{array}{l}59000 \\
40000\end{array}$ & \\
\hline & & 4.2 & $\mathrm{f}$ & & $24 . I$ & 74 & 2.7 & wp cor & 25000 & \\
\hline & & $5 \cdot 7$ & $\mathrm{f}$ & & $24 \cdot I$ & $7 \mathrm{I}$ & 2.6 & w br cor & 22000 & Repeated same. \\
\hline & July $3 \mathrm{I}$ & $9 \cdot 4$ & $f^{\prime}$ & S. W. & $24 . I$ & 78 & 2.2 & $\operatorname{cor}$ & I 4500 & \\
\hline & & I 2.1 & f & & $24 . I$ & 84 & 2.0 & cor & 10000 & \\
\hline & & 4.0 & f & & $24 . I$ & 84 & I. 5 & cor & 4000 & \\
\hline & & 6.0 & $f$ & & 25.1 & 80 & I. 5 & cor & 4000 & \\
\hline
\end{tabular}


TABLE I-Continued.

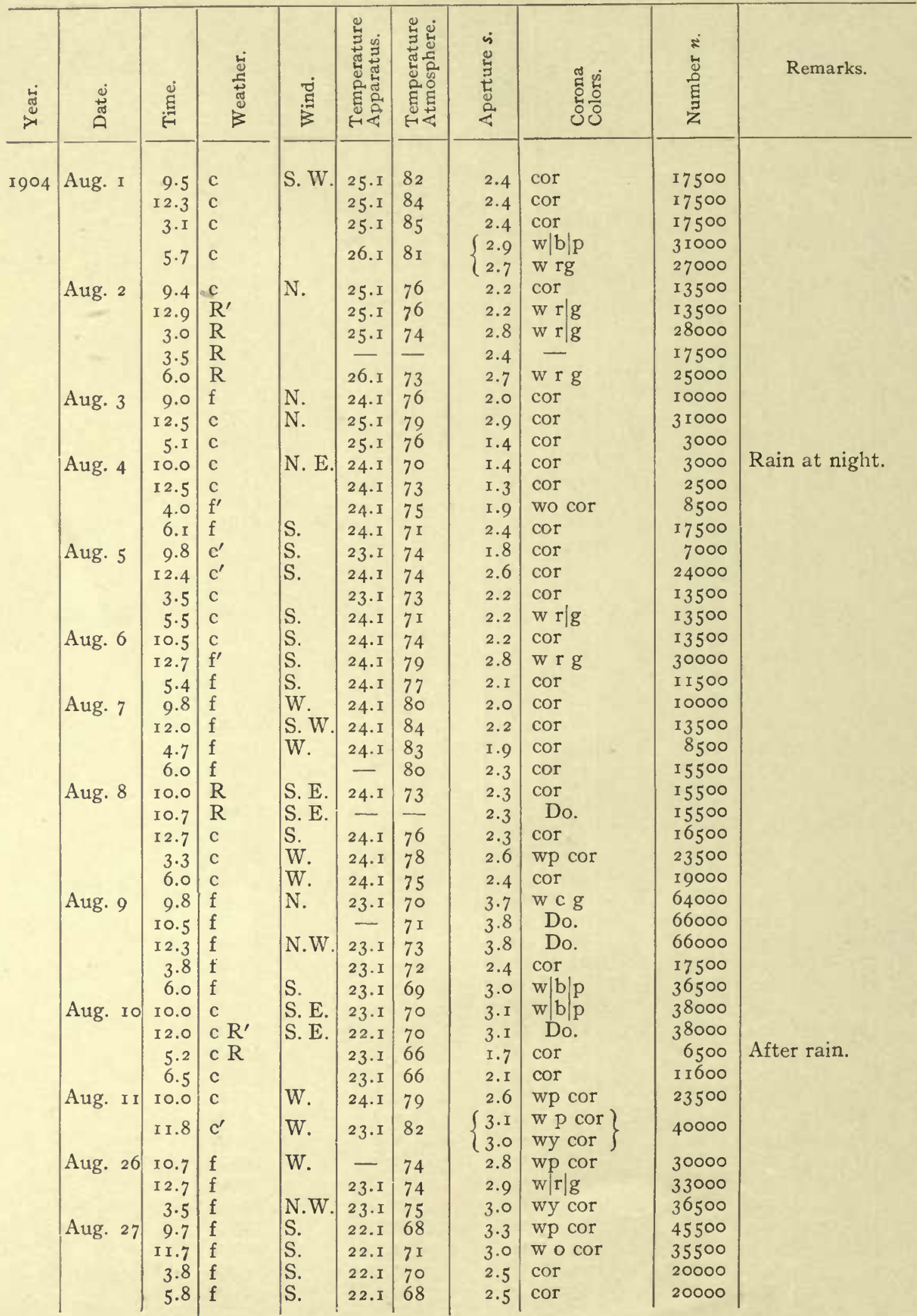


TABLE I-Continued.

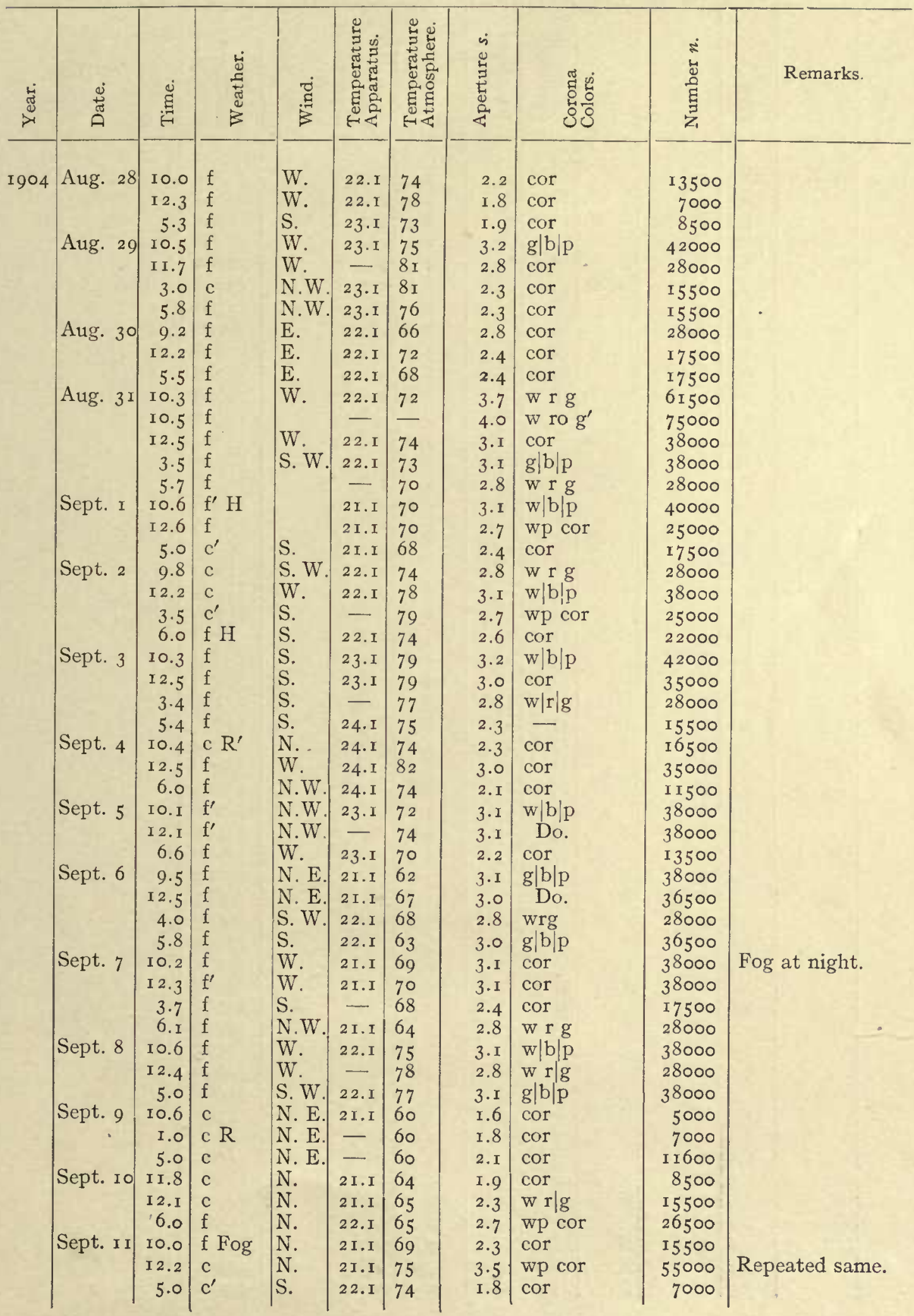


TABLE I-Continued.

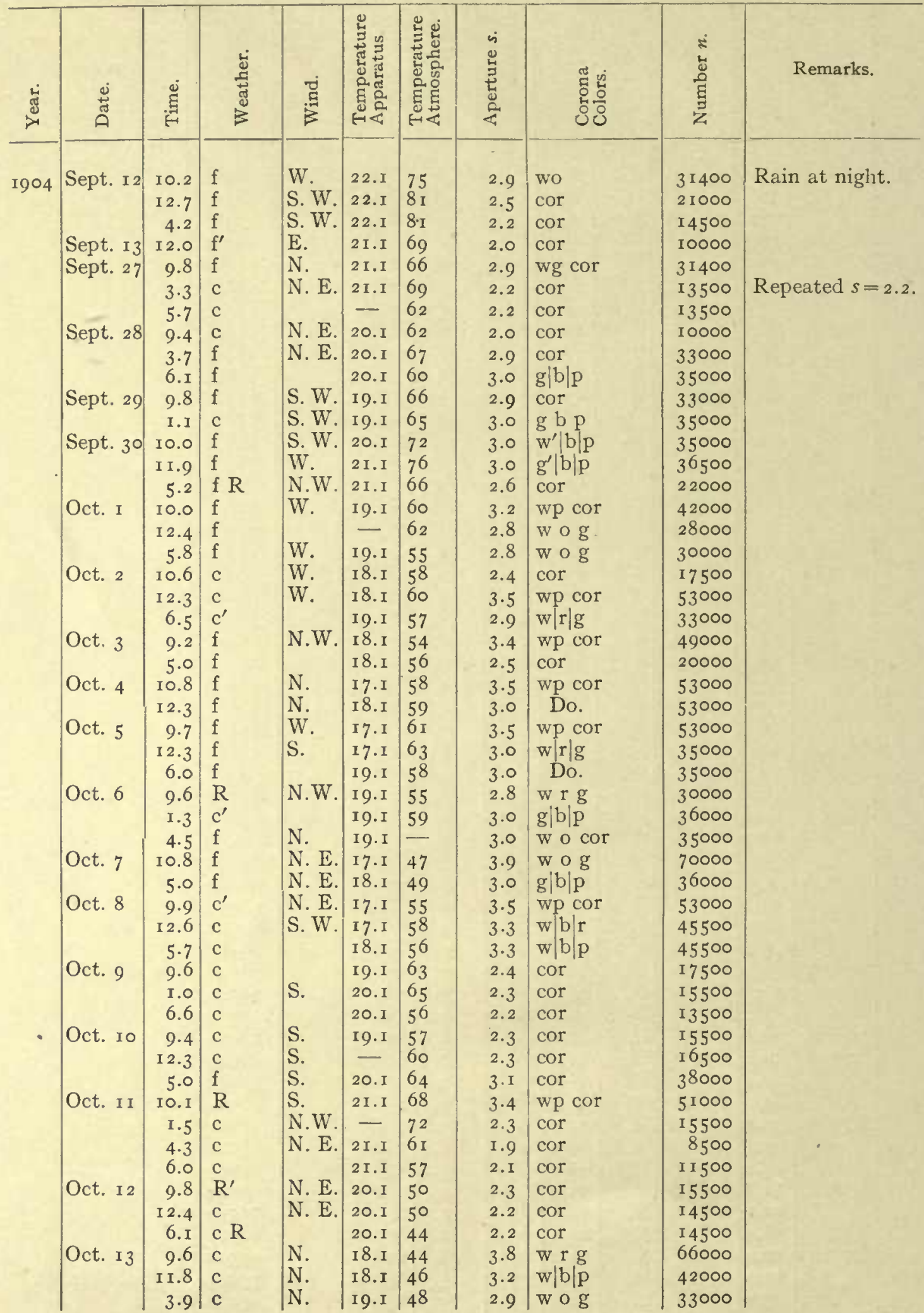


TABLE I-Continued.

\begin{tabular}{|c|c|c|c|c|c|c|c|c|c|c|}
\hline 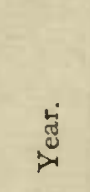 & 苞 & 芫 & 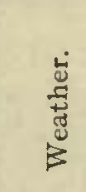 & $\frac{\vec{g}}{3}$ & 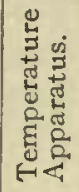 & 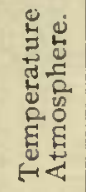 & 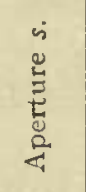 & 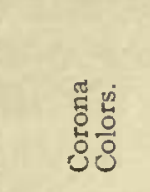 & 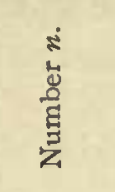 & Remarks. \\
\hline \multirow[t]{52}{*}{1904} & Oct. 14 & 9.5 & $\mathrm{f}$ & N. & - & 50 & 3.6 & & 57000 & \\
\hline & & I 2.4 & f & & 2 I.I & 58 & $4 . I$ & w o bg & 81000 & \\
\hline & & 4.0 & f & & $20 . \mathrm{I}$ & 57 & 2.6 & & 22000 & \\
\hline & & 6.0 & f & & $20 . \mathrm{I}$ & $5^{2}$ & 3.1 & w r g & 40000 & \\
\hline & Oct. I 5 & $9 \cdot 5$ & f & N. & I9. I & 49 & $3 \cdot 7$ & wp cor & 61500 & \\
\hline & & 12.3 & f & N. & I9.I & 54 & $3 . \mathrm{I}$ & $\mathrm{g}^{\prime}|\mathrm{b}| \mathrm{p}$ & 40000 & \\
\hline & & & $\mathrm{f}$ & & $20 . \mathrm{I}$ & 50 & 3.0 & $\mathrm{~g} \mathrm{~b} \mathrm{p}$ & 35000 & \\
\hline & Oct. I 6 & 10.0 & $\mathrm{f}$ & N.W. & I8.I & 55 & 4.I & wo 0 gg & 83000 & \\
\hline & & I 2.5 & f & N. & I 9.1 & 62 & $3 \cdot 9$ & $\mathrm{wrg}$ & 71000 & \\
\hline & & $5 \cdot 5$ & f & & I 9.1 & $5^{6}$ & 3.4 & wp cor & 51000 & \\
\hline & Oct. 17 & $9 \cdot 5$ & $\mathrm{f}$ & S. W. & 18.1 & $5^{6}$ & 3.9 & w r g & 71000 & \\
\hline & & $\mathrm{I} 2.2$ & f & S. & I8.I & 65 & 3.7 & w c g & 64000 & \\
\hline & & 5.0 & f & N.W. & I9. I & $6 I$ & 3.2 & wp cor & 42000 & \\
\hline & Oct. I 8 & 9.7 & f & S. & 二 & 60 & 3.8 & w clg & 66000 & \\
\hline & & 12.0 & f & W. & I 8.1 & 74 & 2.9 & - & 31000 & \\
\hline & Oct. I9 & $9 \cdot 5$ & c & N. & I9. I & 57 & 2.0 & cor & 10000 & \\
\hline & & 6.2 & c & & I 9.1 & 57 & 2.0 & cor & I 1000 & \\
\hline & Oct. 20 & 9.6 & c & N. E. & I 9.1 & 60 & 2.5 & cor & 20000 & \\
\hline & & 1.0 & c & S. E. & 19.1 & $6_{3}$ & 3.0 & cor & 35000 & \\
\hline & & 4.I & c & S. & 20.1 & 63 & 2.8 & cor & 28000 & \\
\hline & Oct. 2 I & $9 \cdot 3$ & c & S. & 20.1 & 67 & 2.2 & cor & I 4500 & \\
\hline & & 4.0 & $\mathrm{R}$ & S. & - & 64 & 2.9 & w r $\mid \mathrm{g}$ & 31000 & Gale. \\
\hline & & 6.3 & $\mathrm{f}$ & & $2 \mathrm{I} . \mathrm{I}$ & 60 & $3 \cdot I$ & $\mathrm{w} b r \mathrm{~b} / \mathrm{p}$ & 38000 & \\
\hline & Oct. 22 & 9.8 & $\mathrm{f}$ & S. & 22.1 & 59 & 2.5 & cor & 20000 & \\
\hline & & 12.5 & $\mathrm{f}$ & S. & $2 \mathrm{I} . \mathrm{I}$ & 62 & 2.3 & cor & 16500 & \\
\hline & & 6.0 & $\mathrm{f}$ & & - & $5^{8}$ & 3.0 & wrg & 35000 & \\
\hline & Oct. 23 & 9.8 & f & W. & 20.1 & 53 & $3 \cdot 5$ & wp cor & 53000 & \\
\hline & & I.I & f & S. W. & 20.1 & $5^{8}$ & $3 \cdot 5$ & wpcor & 53000 & \\
\hline & & 5.9 & f & & 20.1 & 53 & 2.9 & $\mathrm{w} \mathrm{rg}$ & 31400 & \\
\hline & Oct. 24 & $9 \cdot 3$ & f & W. & I9.I & 47 & $3 \cdot 3$ & w & 47000 & \\
\hline & & 4.4 & f & N.W. & 18.1 & $5 \mathrm{I}$ & $3 . I$ & cor & 38000 & \\
\hline & Oct. 25 & 10.0 & f & W. & 18.1 & $5^{6}$ & $3 . \mathrm{I}$ & $\mathrm{g}^{\prime}|\mathrm{b}| \mathrm{p}$ & 38000 & \\
\hline & & I.O & f & S. W. & I 8.1 & 6 I & $\{3 \cdot 5$ & $\mathrm{wrg}\}$ & 55000 & \\
\hline & & & $f$ & & IQ.I & 56 & $\begin{array}{r}3.1 \\
3.4\end{array}$ & $\begin{array}{l}\text { gbp } \int \\
\text { wp cor }\end{array}$ & 40000 & \\
\hline & Oct. 26 & $\begin{array}{l}3.5 \\
9.4\end{array}$ & c & S. W. & $\begin{array}{l}19.1 \\
\mathrm{I} 8.1\end{array}$ & $\begin{array}{l}50 \\
57\end{array}$ & $\begin{array}{l}3.4 \\
3.4\end{array}$ & $\begin{array}{l}\text { wp cor } \\
\text { wpcor }\end{array}$ & $\begin{array}{l}49000 \\
40000\end{array}$ & \\
\hline & & 12.4 & $\mathrm{R}$ & S. W. & 18.1 & 58 & 3.1 & $\mathrm{w} b \mathrm{p}$ & 38000 & \\
\hline & & 6.0 & c & & I9.I & 54 & 2.7 & cor & 25000 & \\
\hline & Oct. 27 & 9.0 & $\mathrm{f}$ & N.W. & 17.1 & 44 & 3.I & $\mathrm{w}|\mathrm{b}| \mathrm{p}$ & 40000 & \\
\hline & & 1.7 & c & N.W. & 17.1 & 48 & 3.2 & wp cor & 44000 & \\
\hline & & & $c^{\prime}$ & $\mathrm{N}$. & & 53 & 3.1 & $\mathrm{~g}|\mathrm{~b}| \mathrm{p}$ & 38000 & \\
\hline & Oct. 28 & 9.6 & f & N. E. & 16.1 & 39 & 3.1 & $\mathrm{~g}|\mathrm{~b}| \mathrm{p}$ & 38000 & \\
\hline & & 12.4 & f & N.W. & 16.1 & 45 & 3.6 & w c g & 57000 & \\
\hline & & 5.8 & f & & 17.1 & 43 & 3.7 & w c g & 61500 & \\
\hline & Oct. 29 & 10.2 & f & S. & 17.1 & $5^{2}$ & 3.0 & $\mathrm{w}|\mathrm{b}| \mathrm{p}$ & 36000 & \\
\hline & & I.0 & $\mathrm{f}$ & W. & 18.1 & 57 & 3.7 & w C g & 61500 & \\
\hline & & 6.4 & 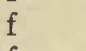 & & 18.1 & 50 & 3.6 & w c g & 57000 & \\
\hline & Oct. 30 & 9.9 & f & N. & 17.1 & 44 & 3.9 & $\mathrm{wrg}$ & 70500 & \\
\hline & & 12.0 & f & N. & 17.1 & 46 & 3.4 & wp cor & 49000 & \\
\hline & & & $\mathrm{f}$ & & 18.1 & $4^{\mathrm{I}}$ & 3.0 & - & 35000 & \\
\hline & Oct. 3 I & $9 \cdot 3$ & f & N. & I 4.1 & 35 & 4.3 & $g \mathrm{~b} \mathrm{p}$ & 90500 & \\
\hline & & 9.9 & f & N W & $\overline{6}$ & $\overline{43}$ & $4 \cdot 3$ & $\mathrm{y}^{\prime} \circ \mathrm{bg}$ & 90500 & \\
\hline & & & & & I6.I & 43 & & & 90500 & \\
\hline
\end{tabular}


7. Successive monthly data.-The temperature effect during the remainder of March (charts I3, I4) is rather in opposition to the above inferences for winter. Thus the maximum on March I9-2I corresponds to a hot wave while the other cases are vague. In other words, there is quite apt to be a rise of nucleation with rise of temperature. The reason for this is frequently to be ascribed to the rain minimum which in winter corresponds to warmer and in spring and summer to colder weather. The Sunday nucleation during clear weather is low on March I 5 and high on March 29. The maxima occur for

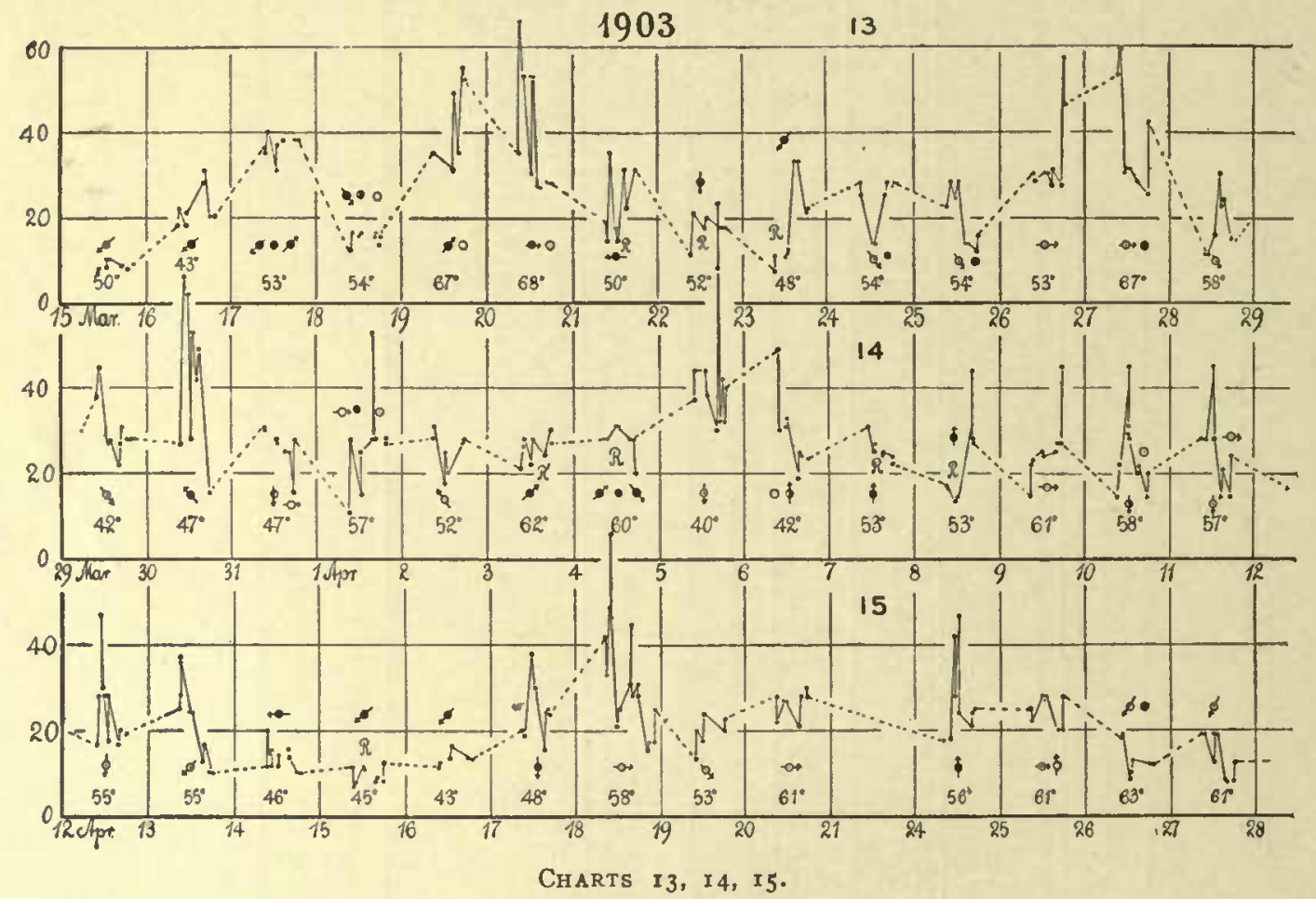

cloudy (March I 7), partially cloudy (March I9-2 I), and for clear (March 26-29) weather. On March 29-3I the wind and weather change is a complete reversal, and yet the maximum is sustained. Cloud effects appear on March 19, 25, 27, but on the I 8 th clear weather is ineffective.

8. Throughout the whole of April (charts 14, I5, I6) the nucleation is moderate. The maximum on April $5^{-7}$ clearly corresponds to cold weather. The falling off to the minima on April $14-16$ is due to the rain-storm, and the subsequent maximum (April I7-I9) has no temperature equivalent. The minima on April 26-27 are cloud effects, the air having been purified elsewhere. Remarkably high rain nucleations occur on April 3-5. Night observations on April i 8 show no exceptional values. The cusps on April I, 5, 8, 9, I0, I I, I2, I 3 I 8 are peculiar results.

9. May (charts $16,17,18$ ) opens with clear weather but with relatively low nucleation, due probably to the western blizzard. On May 7 and 8 change 
of wind and weather is without effect on the nucleation. On the 20 th there is a cloud effect. No specific result marks the hail-storm of May 19. On May I 7 and 3 I there are low Sunday nucleations for clear weather, but the other Sunday nucleations are high.

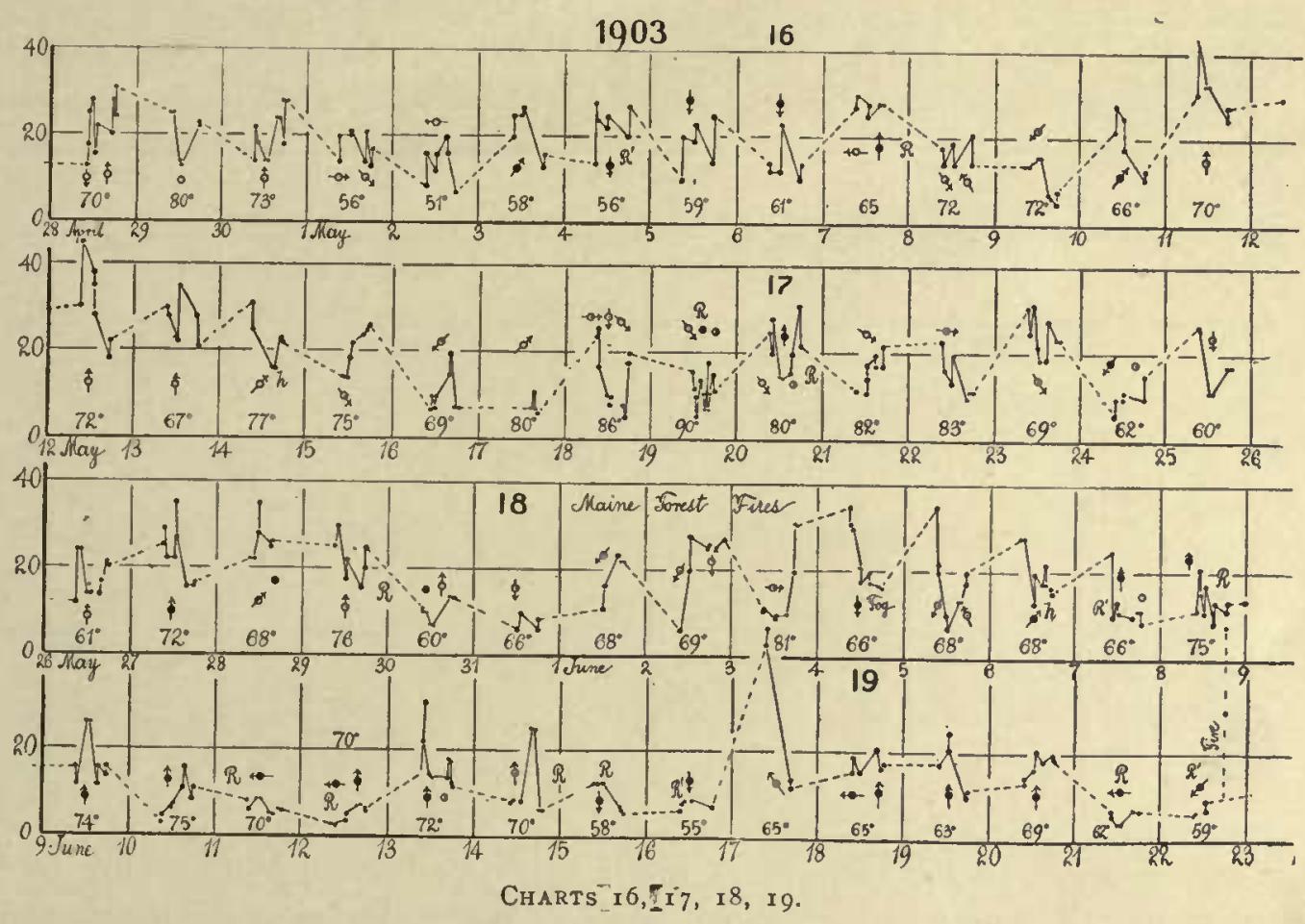

10. In the beginning of June (charts I8, 19, 20) hazy weather and relatively high nucleation are due, no doubt, to the New England forest fires, in Maine and elsewhere, which occurred during a period of drought. Intensely yellow fogs, red moons, and greenish suns were observed. The nucleation, however, is by no means remarkably high, not more than twice the normal summer values and scarcely one-fifth of the large winter values-in spite of the excessive fogs. The copious rains which followed the period of droughts gradually reduced the atmospheric nucleation to very small values (June $7-16$ ). The sharp maxima on June $17,22,23$, are probably due to local fires.

II. In the end of June and beginning of July (charts $20,21,22$ ) the nucleation is remarkably high and sustained, changes of wind and weather (June 30, July I), and even of high temperature (July 3), notwithstanding. Marked rises of temperature and of nucleation concur. Sunday minima with clear weather occur on July 5 only, July 26 being partly cloudy. The effect of gunpowder smoke on July 3 and 4 seems to be quite absent. Rain on July 7, 22, 30 (the latter is even followed by a maximum) scarcely reduces the nucleation, but low values follow the rain-storms on July 18 and $2 \mathrm{I}$. The thunder-storms on July Io and 15 without rain seem actually to be compatible with a rise of nucleation. Incidental night observations on July 23,26 , and August I and 2 show no 
determinable peculiarity. Throughout the month the temperature effect is vague or in opposition to the winter effect.

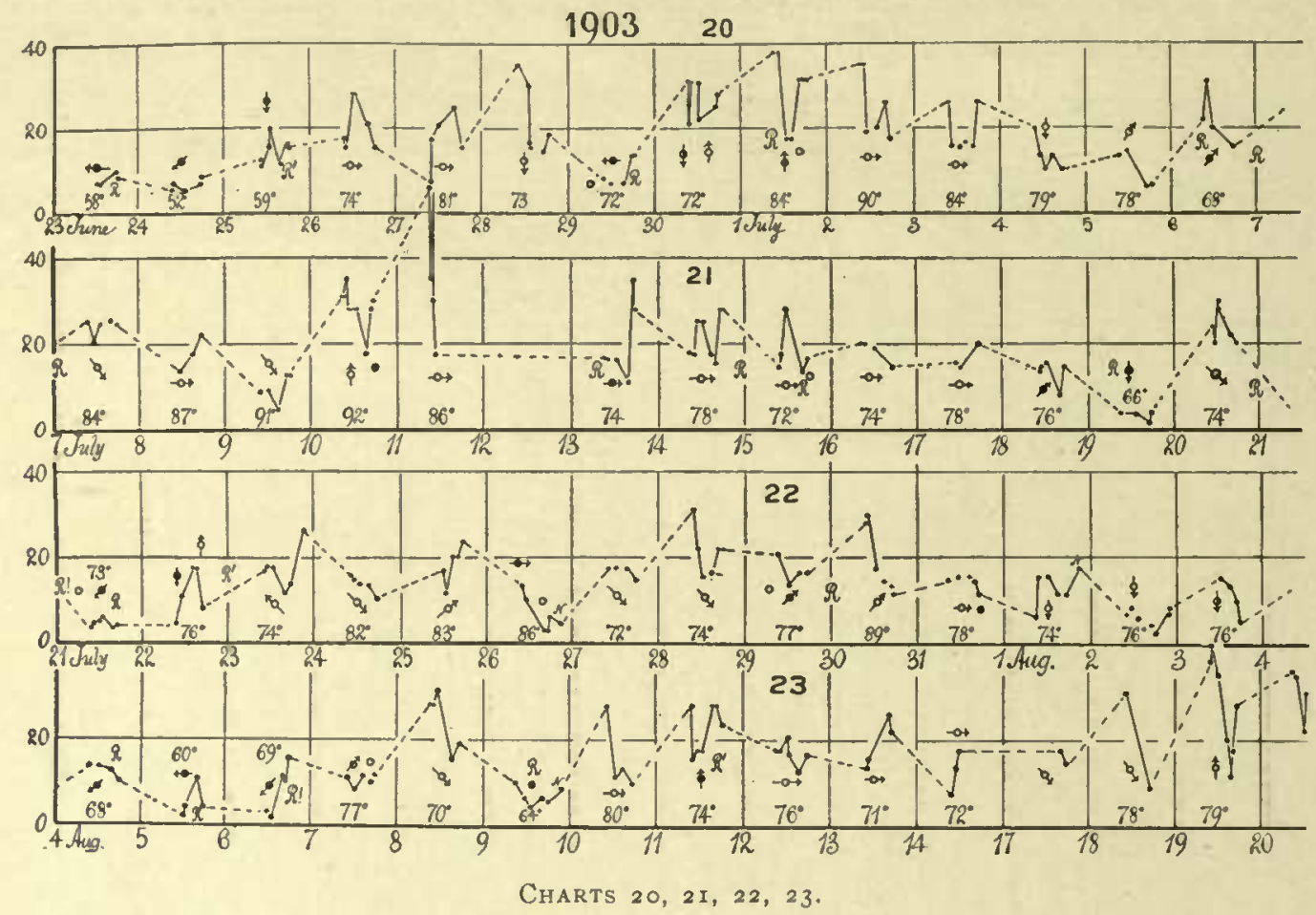

1 2. August (charts 22, 23, 24) at first shows low nucleation due to frequent rains. The rest of the month is somewhat uncertain in consequence of building operations on the college green. Thus the maxima on the 24 th and $25^{\text {th }}$ are due to fires on the campus. Low Sunday nucleations occur on August 2 and 23 , the latter being phenomenal. Rapid increase after rain is frequently noticeable, as on August 10, 21, 27, and similar effects may be found in the earlier months.

I3. September (charts $24,25,26$ ), in spite of high temperature, clearly ushers in the high winter nucleations, just as March had removed them. The suddenness in both cases is surprising: one naturally asks whether there is here a return to the locality of the nuclei-producing civil activity which had left it in March. But the data of 1904 do not bear this out. The high maxima on September I, 9, I 2, I 5, 19, 22, 25, 30 are all striking. The Sunday minima on the clear days of September 6 and I 3 are not low. The gale on September I 7 and the thunder-storms on September 5 and 27 are followed by high nucleation, particularly in the latter case, but the assertion that nuclei are produced in this way would be unwarranted.

I4. October (charts $27,28,29$ ) sustains the high nucleation of September. Sunday minima (October 4 , et seq.) quite vanish, but the winter temperature effect is not yet resumed. The rain effect on October 5, I 2, I 7 is obscure, and in the latter case even the rain-storm is ineffective. Rise of nucleation on October 19 
is broken by a cloud effect. The occurrence of marked sun-spot disturbances, as announced during the month, may be noted. After October 23 the winter
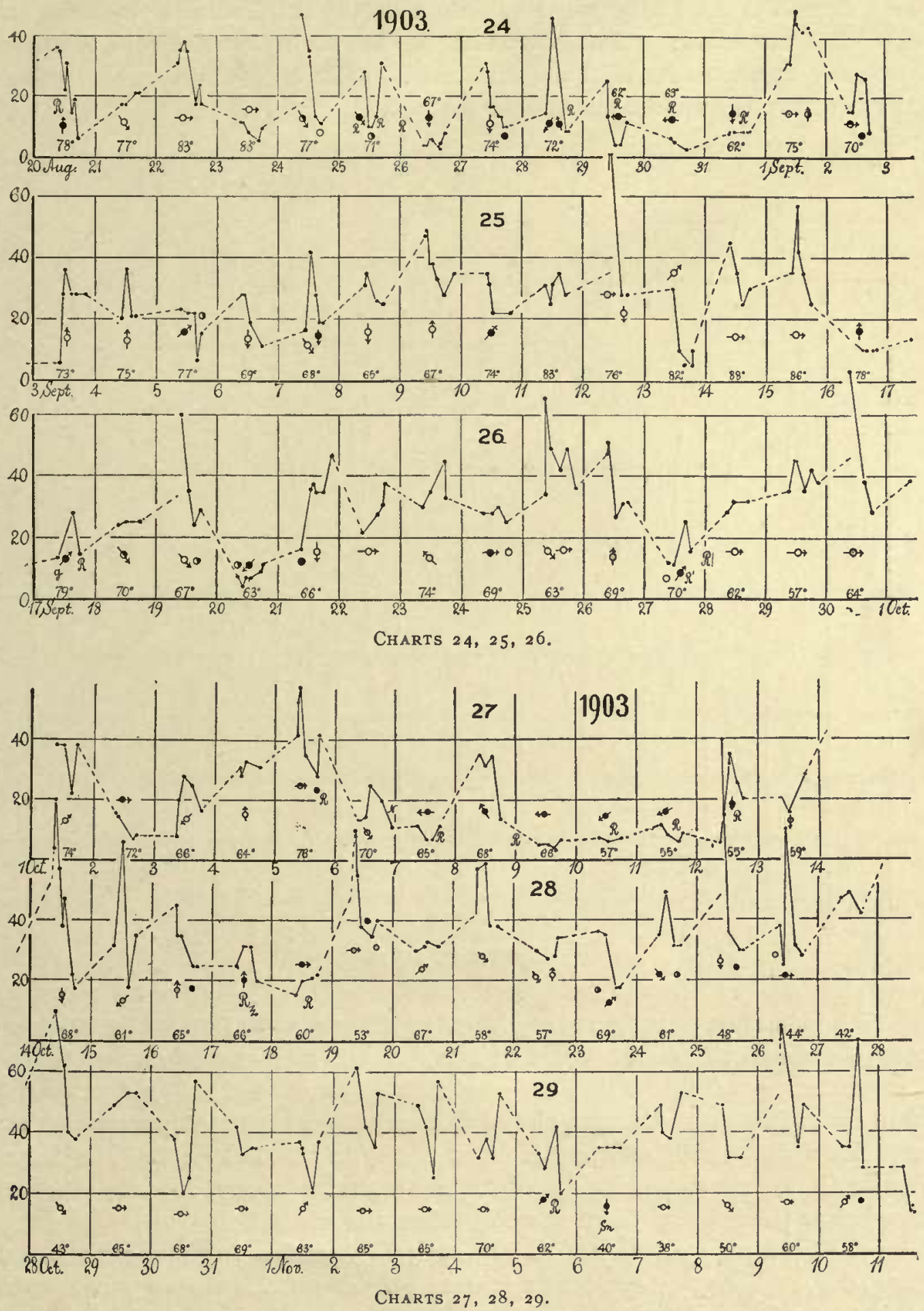

temperature effect is again reinstated, both for the general march through the weeks and for many daily variations. 
15. In November (charts $29,30,31$ ) the cold wave on the 7 th has no counterpart in the nucleation, which is throughout nearly uniformly high. A curious result is the unexplained minimum on the $\mathrm{I}$ ith for clear weather, much below the rain effect. After November 22 the sweep toward cold weather on November 27 shows itself in the nucleation. The cold snow on November 29 has but slight, if any, denucleating tendency.

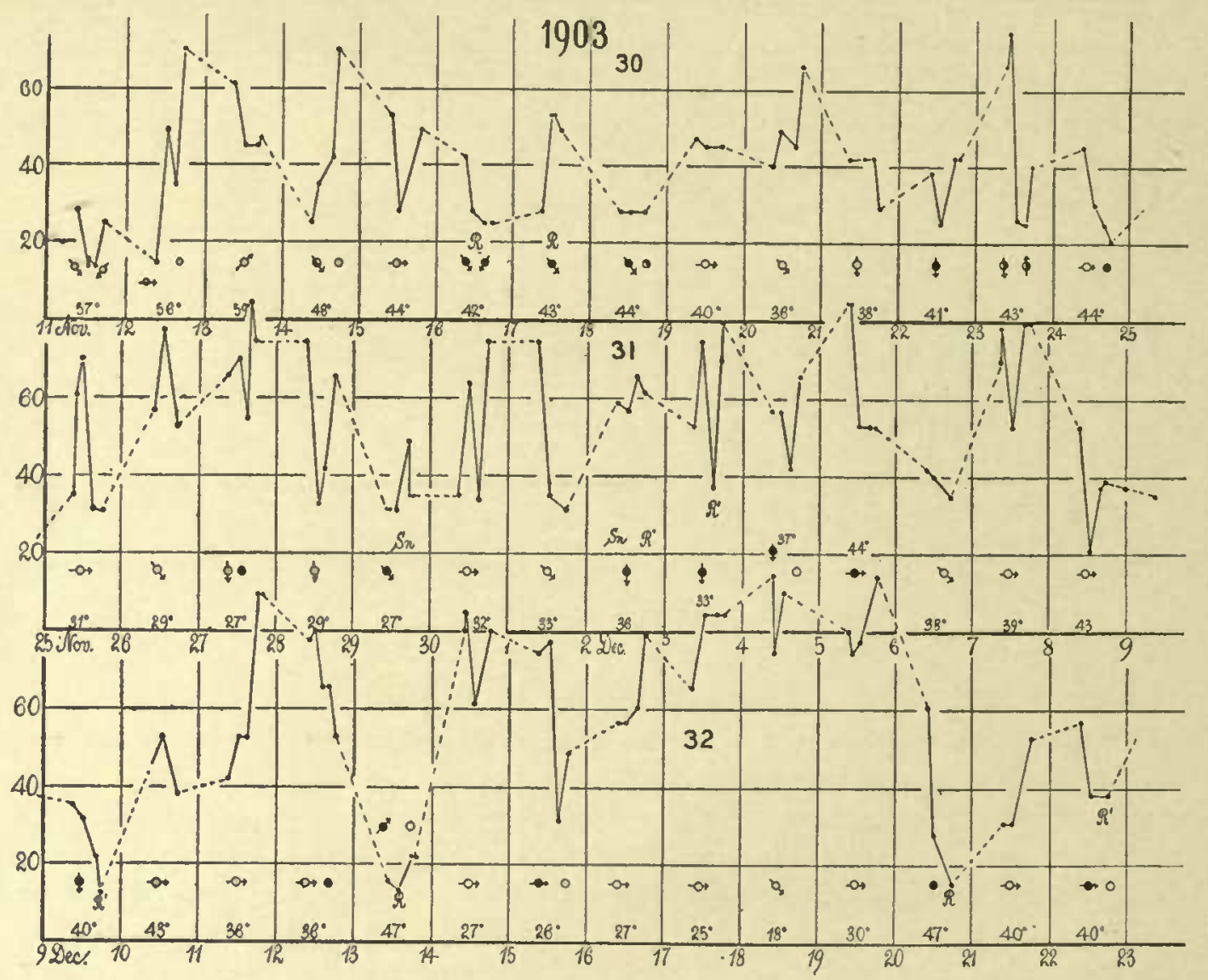

Charts $30,31,32$.

16. So in December (charts $31,32,33$ ) the precipitation on the $2 \mathrm{~d}$ and $3 \mathrm{~d}$ scarcely reduces the nucleation, and this remains high except during the rains of December 9 and I3. The clear weather minimum on the 8th is noteworthy. From December 10 to the rain-storm of December 21 , the temperature effect is very sharply marked, as appears particularly in charted data. The same is true for the remainder of the month. On December $26,27,28$, precipitation of snow below the freezing-point actually raises the nucleation.

I7. Successive monthly data for I904.-With the beginning of the new year, I904, and very cold weather (charts $33,34,35$ ), there is striking parallelism between the temperature curve and the excessively high nucleation, as far as the minimum introduced by the thaw and wet snow on the 9 th. The con- 
trast between the negligible dry snow effect on January 2 and the wet snow minimum on January 9 is to be noted. The maximum reaches its height (the diffractions actually surpassing the large green-blue-purple corona) during the cold blizzard and snow drift of January 3. As the coronal method breaks down for nucleations exceeding the case for the $\mathrm{g}-\mathrm{b}-\mathrm{p}$ corona, the higher nucleations are merely suggested in value on the charts. There is a fog effect on January 7. Moreover, the maximum is well sustained while the winds shift from northerly to southerly on January 6.

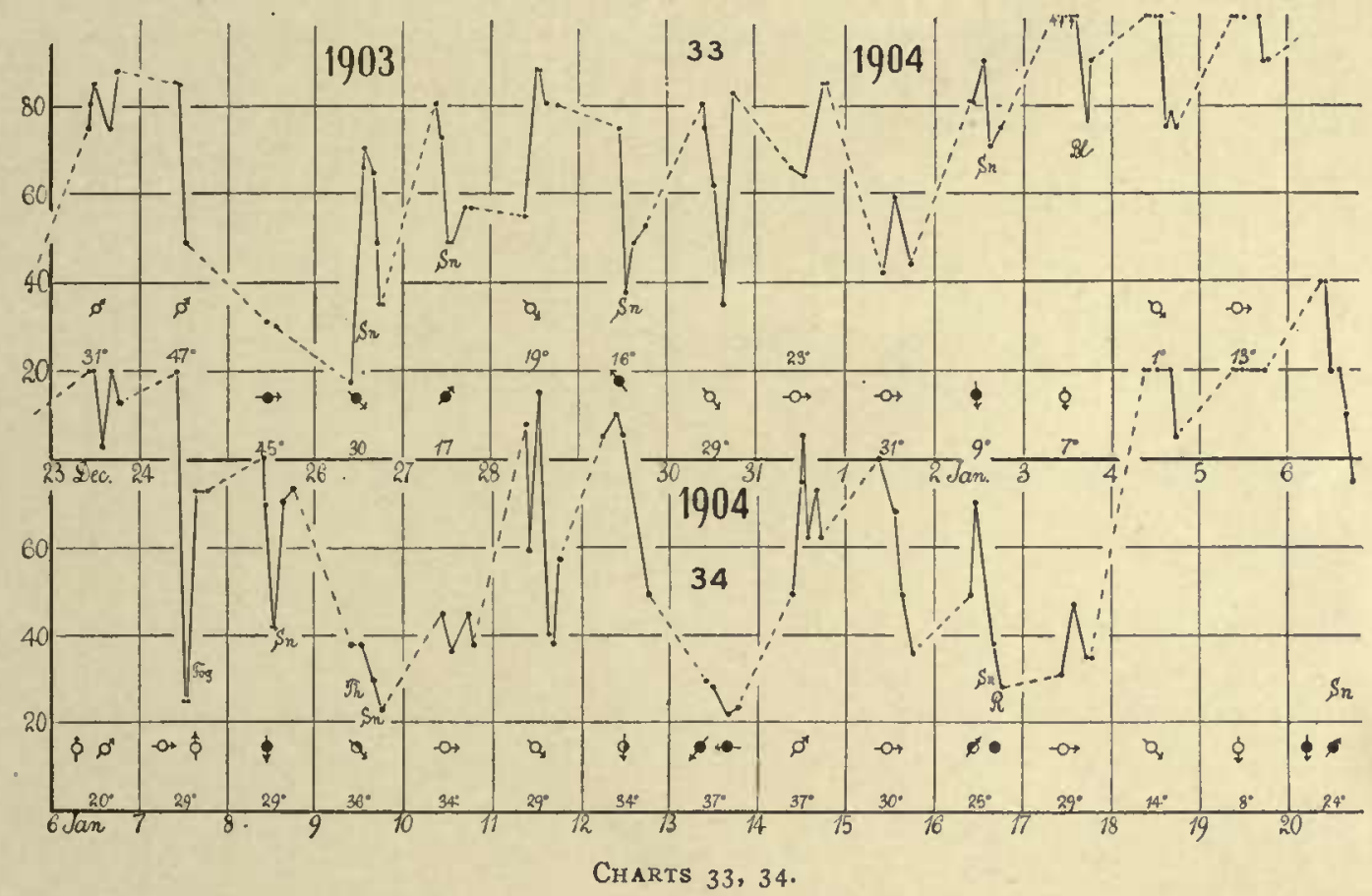

After the rains on January 13 and 16 a second march of nucleation into extremely high maxima (January I 8-2 I) coincides with the sweep of very cold weather, ultimately to be broken by the wet snow on January $2 \mathrm{~s}$. During the remainder of the month there are some suggestive data on the nucleation accompanying a strong fog, though but few of the active nuclei are probably entrapped in the latter. Cold snow effects on January 26 and 29 may be noticed.

I 8 . February (charts $35,36,37$ ) begins with a sweep of cold weather and high nucleation, terminating in the thaw and fog of the 7 th. The next cycle extends to the snow on the $\mathrm{I} 4 \mathrm{th}$, a low minimum, remarkable as being below freezing. The temperature effect from February $\mathrm{I}_{2-\mathrm{I}} 4$ is obscure. High nucleation again prevails until the rain and thaw on February 22. The large number of observations entered are due to a number of subsidiary experiments made during this interval. There is cold snow on February ${ }_{15}$ and 19 . Even the dense snow-storm on February 24 only partially removes the nucleation, as this falls off gradually into the minimum following the rain of February 29. 

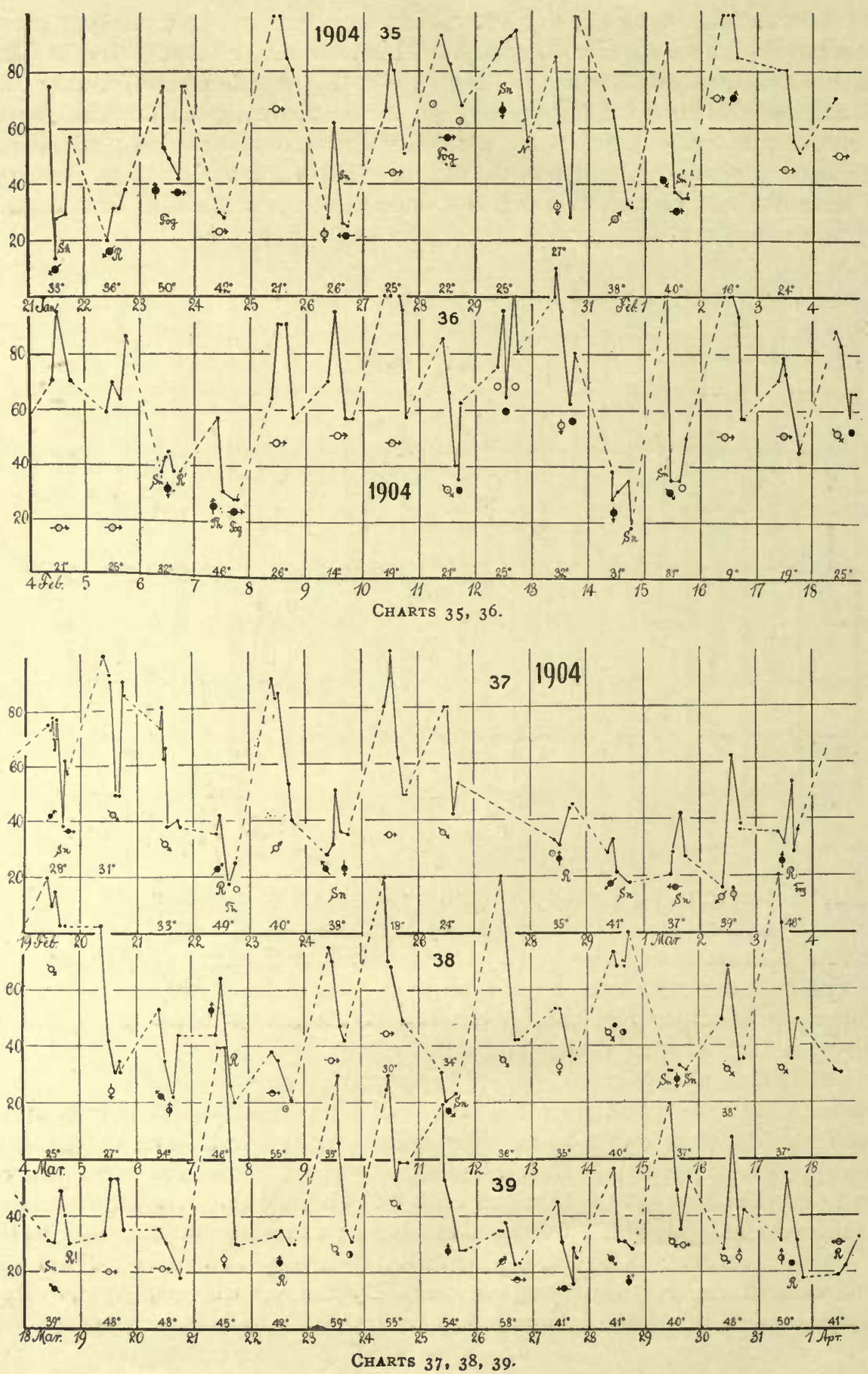
I 9. The high nucleation of March, I904 (charts $37,38,39$ ), is in distinct contrast to the relatively low nucleation of March, I903. This is true in like measure for April, May, and even June. In I904, the winter nucleations are in all cases very gradually replaced by the low summer nucleations. The sweep of temperature from March $\mathrm{I}^{-\mathrm{I}} 5$ is in very full accord with the changes of nucleation. Remarkably high rain minima occur on March 3, 7, 18, 22, and the same is true of the snow minima on March II, I 5, I 8, and of the Sunday minima on March 6, 13, 20, 27. On the I 4th there is a cloud effect. Toward the end of the month temperature correspondence ceases and the nucleation remains high, in spite of weather much above freezing.

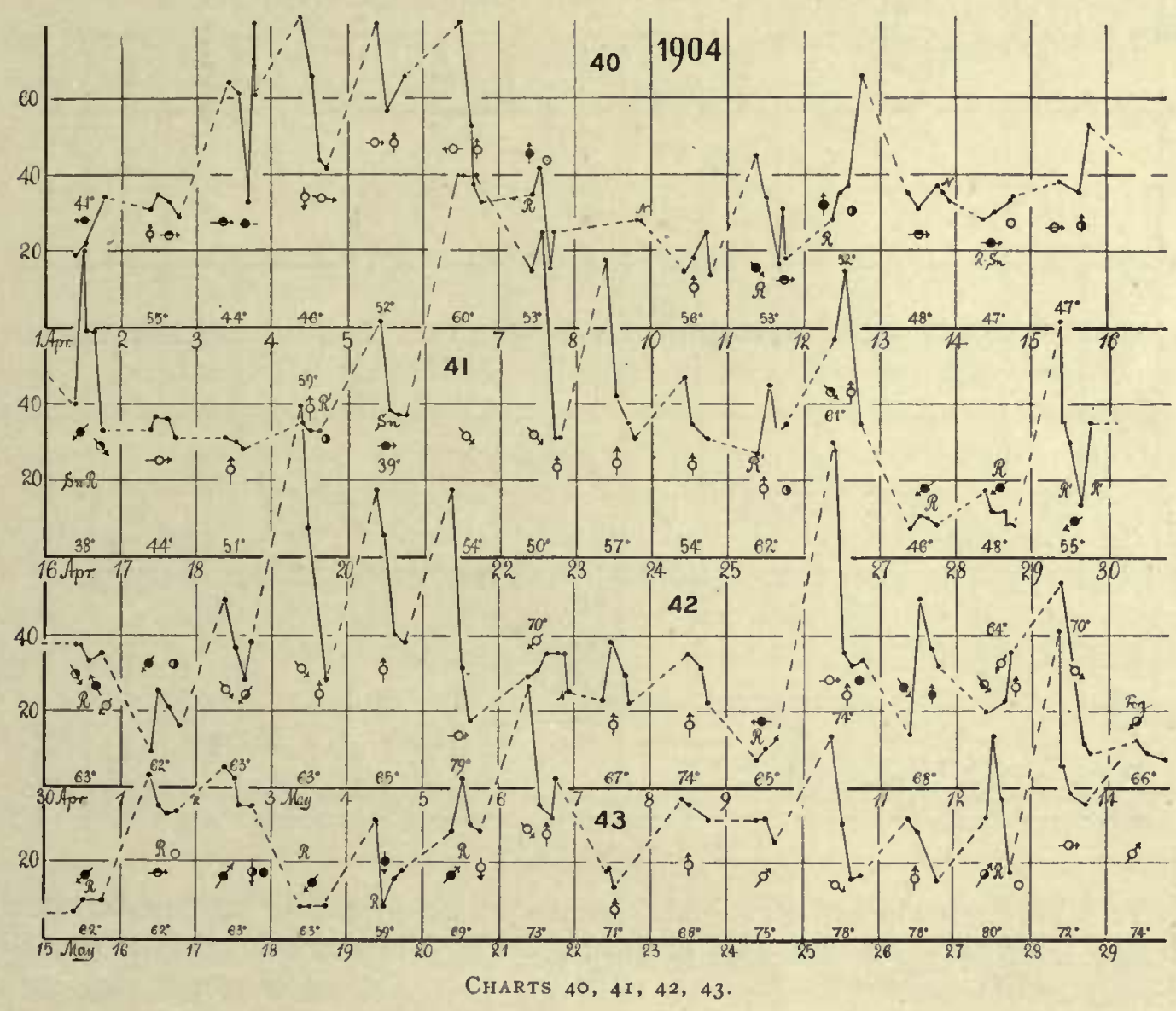

20. The first period of nucleation, beginning with the rain of April I (charts 39, 40, 4I) and terminating in the rain of April 7, partakes of the relatively high character for the season already instanced. It is possibly referable to the corresponding sweep of temperature which approaches freezing on April 4, supposing that the nucleation due to fall of temperature outlasts the latter. The period from April Io-I 8 is less pronounced, but high nucleation corresponds to the moist snows on April 14 and I6. The night observations betray nothing unusual. The final cycle of the month from April is to the very low minimum 
on April 27 and 28 is again high in nucleation and here there is a reversal of the winter temperature effect, both in the high maximum of April 2I (which comes too late) and the minimum of April 27 (which is also too late). In relation to temperature, both regions have been shifted to the right. The rise of nucleation during the rain of the 29 th may be pointed out. There are many results during the month which seem to imply a change of nucleation with the direction of the wind.

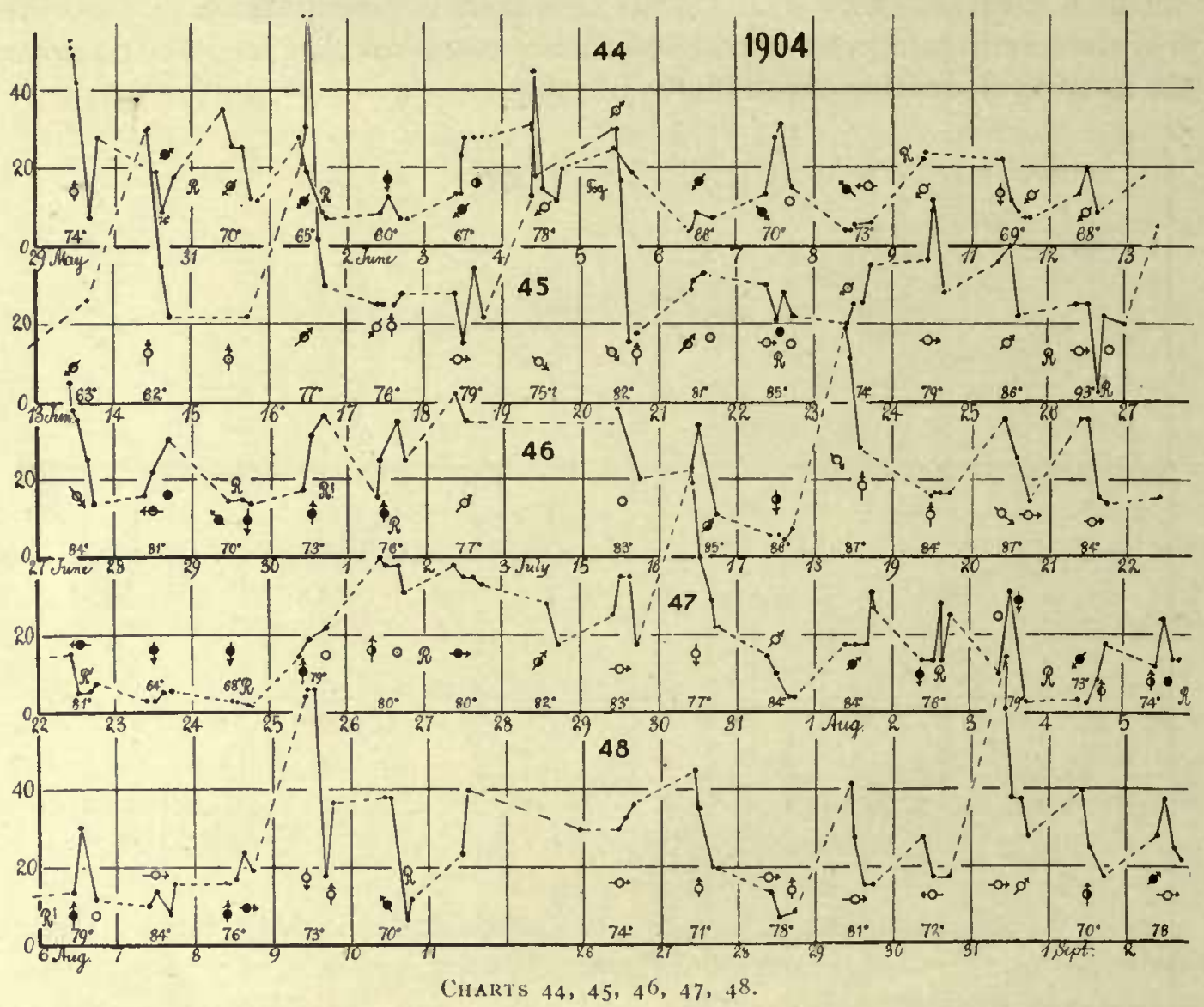

21. May (charts $42,43,44$ ) also begins with a period of high nucleations, reaching from May 2 to the rain of May 9. The temperature effect is usually reversed or obseure. The contrast with the low nucleation of the preceding year is marked. Rain minima as a whole are low. Strong nucleations (as was the case in April) mark the close of the month.

22. June (charts $44,45,46$ ) at last introduces the characteristic summer nucleation, during the first half of the month. The temperature effect is reversed. Sunday nucleations are low on June 5, I 2 , but not on June 19, which shows phenomenally high values. Abrupt high maxima occur on June 14 and I6. On the 17 th there is change of nucleation with the direction of the wind. One may note the sharp reduction of nucleation due to the thunder-storm of June 26. After June 20 the values are as a whole seasonable. 
23. Observations during the first half of July (charts 46,47 ) were suspended. High rain values occur on July $16, \pm 8,27,30$. Thus the rain on July 27 has but partially wiped out the maximum. On the other hand, the rain minima on July 23, 24, like those of August 4, are excessively low, being among the lowest nucleations observed (1000-2000 per cub. cm.). Sunday nucleations are not remarkable.

24. The nucleations of August (charts 47,48) are low at the outset, preceding the abrupt maximum of August 9. The temperature effect is vaguc. During the middle of the month observations were suspended. The maximum on August 3 I suggests the approach of the winter nucleations.

25. September nucleations (chart 48) present no new features and but few are charted. The observations were suspended from September $13^{-27}$, during my absence in St. Louis.

GENERAL INFERENCES.

26. Efficiency of apparatus. - The experiments as a whole are in the first place to be regarded as a severe test, amounting almost to a strain of the method employed. Since both maxima and minima are registered with equal facility at exceedingly low as well as at high atmospheric temperatures, the temperature error of the method is not menacing. Rain minima, snow minima, and other exceedingly low nucleations have all been recognized. The high maxima require a long apparatus, since the diameters of the corona occupy nearly half a meter at the condensation chamber; but the maximum of atmospheric nucleation does not, except in very rare cases, exhaust the limit of measurable coronas. Nevertheless a higher pressure difference would make it possible to confine the measurements to normal coronas exclusively - a desideratum, inasmuch as Chapters VII. and VIII. have shown that after the $\mathrm{g}-\mathrm{b}-\mathrm{p}$ corona is reached, the data lying in the region of the cusp are estimates. Higher pressure differences are somewhat less convenient, and in regions remote from cities it is improbable that the normal coronas will ever be exceeded. Care must be taken to have the influx pipe far enough from the walls to guard against exhalations from the building in summer. If this pipe is sufficiently long and thin (influx current rapid), the air arrives in the condensation chamber almost at room temperature and without appreciable loss of nuclei during the passage.

27. Variations of mucleation.-Mere inspection of the charts shows the extreme variability of atmospheric nucleation, though quiescent periods are also met with. A part of this must be a local effect, even if the changes correspond to the weather. Work in cities where there is so much chance for the pollution of the atmosphere is to some extent unsatisfactory, but the method could hardly have been developed in the country. It is probable that even the small variations of the chart (if they exceed 2000 nuclei per cub. cm.) are real. If the nuclei were colored, the atmosphere would look like mottled soap with 
the clear regions usually but by no means always accompanying rain or lying under clouds.

28. Wind effect.-Changes of wind velocity were not observed. The nucleation consistently follows certain changes in the directions of the winds, as will appear from a detailed examination of the charts as a whole. The following table, which is a summary of this kind, shows the distribution of maxima above $n=60000$ with the wind direction.

TABLE II.

Wind Directions for Maxima Exceeding $n=60000$.

\begin{tabular}{c|c|c|c|c|c|c|c|c}
\hline Time. & East. & S-East. & South. & S-West. & West. & N-West. & North. & N-East. \\
\hline Oct., 1902-Jan., I904... & $\circ$ & I & 0 & I4 & 54 & 25 & I 4 & 2 \\
Jan., 1904-Sept., 1904... & I & I & I I* & I0 & 27 & 26 & I I & 3 \\
\hline
\end{tabular}

By far the greater number of these maxima occur for nearly westerly winds and but few for easterly winds, if stress is laid on the winter nucleations. In 1904, where high maxima occurred in the spring and even in the summer, the distribution is more southerly, but in the main like the preceding. It by no means follows, however, that nucleation is inherently associated with these particular winds, inasmuch as the winds observed are merely those prevailing in Providence. Moreover, the density of population, etc., is far greater towards the southwest than towards the northwest of the University, whereas the northwest winds prevail during the period of maxima.

Considered as a whole, therefore, it is improbable that any real variation of the nucleation with the direction of the winds is in question. What has been observed is the obviously more frequent occurrence of maxima during the prevailing winds.

29. Rain effect.- The observation next in importance is the occurrence of pronounced minima during rain, of which the charts contain examples in great abundance. There is rarely an exception to this rule. It implies a faster removal of nuclei by precipitation in a saturated atmosphere (the result of fall of temperature probably) than the supply of nuclei to the same region by either diffusion, or subsidence, or convection, or other more occult causes. Whether this deficiency is eventually made up from the lower air strata in contact with the surface of the earth or from the higher air strata is at the outset left open.

Rain minima never fall quite down to the zero of nucleation, in cities rarely below $n=1000$, and they are themselves quite variable. Thus the summer minimum is as a rule much lower than the winter minimum.

Minima quite as low as the rain minima are sometimes observed in clear weather, but they are very rare. 
One may note that the tendency of rain to change the normal air potential from positive to negative values would thus be accompanied by relative absence of nuclei. In other words minimum nucleation exists here cotemporaneously with maximum negative ionization.

30. Snow effect.-Wet snow usually acts similarly to rain, but less powerfully. The effect is particularly noticeable during a thaw. There are cases, however, in which the nucleation is high with a fresh fall of wet snow. Dry snow may even increase the nucleation.

The present experiments, moreover, show that ordinary terrestrial dust, properly so-called, has no bearing on the nucleation; for this is frequently a maximum immediately after a rain, when the earth is blanketed with water, or after the earth has been covered with snow or with sleet to the exclusion of all dissemination of dust as such.

3I. Cloud effect.-Another interesting feature are the cloud minima as seen on October I 5, I 7, 21, 23, 1902; March 19, 25, 27, April 1, May 20, August 27, September 27, October I 8, 19, 26, December I 5, I903, etc. These observations are incidental, and a larger number would have been found if systematically sought for. Usually a higher nucleation is again established after the cloud train has passed the sky, the phenomenon beginning and ending in periods of clear weather. At other times, however, the minimum remains, as on October 17 , when many observations were made with this end in view. The explanation of this result is at hand: the air has moved bodily with the cloud, the whole constituting a region of deficient nucleation. The nuclei may have been precipitated by rain elsewhere and the cloud may even have vanished from the region.

32. Solar nucleating effect absent. - Since the nuclei cannot re-enter a region by diffusion as quickly as is usually observed, one may be tempted to infer that solar radiation is the cause by which the nucleation of a deficient region is re-established. There is no evidence of this in the, chart and much against it. Thus remarkably low minima are frequently maintained throughout the day in full sunlight. The minimum may be part of a cloud region with which the day closed, but sunlight is powerless to replenish it. Similar references may be made to the notched midday minima which so often occur. By contrast high nucleation develops in spite of an overcast sky. Finally night observations show neither increase nor decrease of nucleation, but are usually normal in character. Hence there is no evidence, so far as these observations go, that ultra-violet light or other solar radiation has any potency in producing the nucleations here immediately in question, and cloud effects have therefore been explained as purely convective. Indeed, compatibly with the final summary $(37)$ of mean monthly nucleations, the sun must be regarded rather in the light of a denucleating agency.

33. Temperature effect.-An important general result is the frequent occurrence of maxima of nucleation, cotemporaneously with the sudden fall of atmospheric temperature in cold weather. So far as the drop of temperature 
is concerned, one would have the conditions for the formation and growth of water nuclei, remembering that persistent nuclei may be made in an atmosphere not too far from saturation, from solutions, or in the presence of a solute. Without actual accession to their numbers, however, there could be no increment of nuclei in the condensation chamber at about $20^{\circ}$. centigrade. In other words, an actual rise and fall of the number of nuclei per cubic centimeter must occur in the atmosphere at the place of observation.

This temperature or cold-weather effect is very striking in winter where both the daily and the periodic effects frequently coincide. In warm weather the temperature effect is vague or may be even quite reversed. In relation to this one may note that rains in winter usually correspond to rising temperatures, but in summer to falling temperatures. This would accentuate the winter effect, which is otherwise quite independent of rain, but would obscure or even reverse the summer effect, if it exists.

If a polar air current or a current from the upper atmosphere is associated with the cold waves in question, a part of the region where there is continual production of nuclei may be bodily transported to the place of observation.

34. Local effect. - The prevailing westerly winds sweeping over a part of the city of Providence carry the products of combustion and other local impurities along with them. A large part of the nucleation at the place of observation must therefore be of artificial origin. The local effect should be greater as the wind velocity is smaller and as the temperature is lower. In this way the winter temperature effect is in a manner explained, seeing that fuel will be consumed more rapidly during the cold waves. Observations of wind velocity are inadequate.

The difficulty with this view is that it does not consistently explain all of the observed facts. Thus cold waves occur in winter without maximum nucleation, as, for instance, on November I I, December 8, I 903. The Sunday nucleations during clear weather in summer are not uniformly low. Maxima during relatively warm weather are often quite as high as during very cold weather, the temperature effect is often reversed even in winter, etc. But the most direct reason for caution will be given in the summary of monthly nucleations where it appears that the highest nucleations do not occur in midwinter so far as cold weather is concerned. Speculation as to the origin of the nuclei is thus premature. Whether it is the residue of the ionized products of combustion, or whether ultra-violet light or other radiation at the boundary of the atmosphere is the efficient and preponderating source will only be made clear in a series of correlative observations obtained in a wilderness remote from cities or on isolated mountains. Work of this character is now actively under way.

\section{SUMMARY AND CONCLUSIONS.}

35. Mean daily nucleations.-Meanwhile it is expedient to deduce from the above data, both the daily and the monthly average nucleations or number 
of nuclei per cubic centimeter. Incidental disturbances will thus be to some extent removed at least from the latter, so that the sweep of nucleation throughout the year may be morc clearly apparent. The rain effect will be more uniformly distributed during the summer months, though it cannot of course be eliminated.

The data for the mean daily nucleations are given in Table III, and require but little explanation. The symbols for the state of the weather have been abbreviated, $\mathrm{f}$ denoting fair, $\mathrm{f}^{\prime}$ slightly cloudy, $\mathrm{c}^{\prime}$ partly cloudy, $\mathrm{c}$ cloudy, $\mathrm{R}^{\prime}$ slightly rainy, $\mathrm{R}$ rainy, etc. Sn refers to snow, $\mathrm{Sn}^{\prime}$ to a light snow.

TABLE III.

Mean Daily Nucleations from Oct. 2, igo2, to November i, igo4.

\begin{tabular}{|c|c|c|c|c|c|c|c|c|c|}
\hline 岂 & 㫕 & 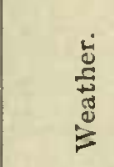 & 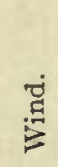 & 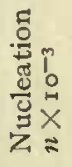 & 可 & 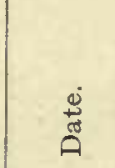 & 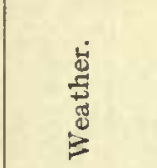 & $\vec{Z}$ & 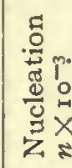 \\
\hline 1902 & \begin{tabular}{|r} 
Oct. 2 \\
3 \\
4 \\
5 \\
6 \\
7 \\
8 \\
9 \\
10 \\
11 \\
12 \\
13 \\
14 \\
15 \\
16 \\
17 \\
18 \\
19 \\
20 \\
21 \\
22 \\
23 \\
24 \\
25 \\
26 \\
27 \\
28 \\
-29 \\
30 \\
31 \\
Nov. I \\
2 \\
3 \\
4 \\
5 \\
6
\end{tabular} & $\begin{array}{l}c \\
f \\
f \\
c \text { c R } \\
R \text { c } c^{\prime} \\
f \\
f \\
f \\
f \\
c \\
R \text { c } \\
f^{\prime} \\
R^{\prime} f \\
f \\
f c^{\prime} \\
f c^{\prime} \\
c \text { R' } \\
f c^{\prime} \\
f \\
f^{\prime} \\
f \\
R^{\prime} c f \\
f c \\
f^{\prime} \\
f^{\prime} c \\
c \\
R^{\prime} \\
f^{\prime} \\
f \\
f \\
f \\
f \\
f \\
f \\
f \\
c f \\
c R\end{array}$ & & $\begin{array}{r}18 \\
45 \\
31 \\
12 \\
19 \\
22 \\
21 \\
16 \\
52 \\
24 \\
6 \\
22 \\
12 \\
24 \\
18 \\
13 \\
9 \\
6 \\
12 \\
15 \\
16 \\
7 \\
21 \\
9 \\
10 \\
31 \\
18 \\
21 \\
37 \\
30 \\
22 \\
24 \\
30 \\
30 \\
27 \\
45\end{array}$ & 1902 & $\begin{array}{r}\text { Nov. } 7 \\
8 \\
9 \\
\text { I0 } \\
\text { I } 1 \\
12 \\
13 \\
14 \\
15 \\
16 \\
17 \\
18 \\
19 \\
20 \\
21 \\
22 \\
23 \\
24 \\
25 \\
26 \\
27 \\
28 \\
29 \\
30 \\
\text { Dec. } 1 \\
2 \\
3 \\
4 \\
5 \\
6 \\
7 \\
8 \\
9 \\
10 \\
11 \\
\text { I } 2\end{array}$ & $\begin{array}{l}c \\
f \text { N } \\
c c \\
f \text { c } \\
f \\
c f^{\prime} \\
c c \\
f \\
f \\
f^{\prime} \\
c \\
c \\
c \\
f \\
f \\
f^{\prime} \\
f^{\prime} \\
c^{\prime} \\
R ~ c \\
R \\
c \\
f^{\prime} f \\
f \\
f c \\
f \text { after } R \\
c f \\
R c \\
f ~ c \\
c \\
f \\
\text { Sn } \\
f \\
f \\
\text { Sn c } \\
f^{\prime} c \\
\text { Sn } c\end{array}$ & & $\begin{array}{r}52 \\
37 \\
18 \\
30 \\
75 \\
45 \\
30 \\
45 \\
37 \\
7 \\
9 \\
24 \\
24 \\
42 \\
90 \\
30 \\
22 \\
34 \\
34 \\
13 \\
30 \\
27 \\
75 \\
36 \\
60 \\
45 \\
30 \\
45 \\
60 \\
100 \\
90 \\
75 \\
100 \\
90 \\
52 \\
37\end{array}$ \\
\hline
\end{tabular}


TABLE III-Continued.

\begin{tabular}{|c|c|c|c|c|c|c|c|c|c|}
\hline 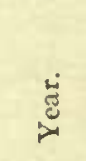 & 气̊ & 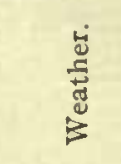 & 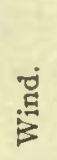 & 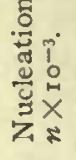 & 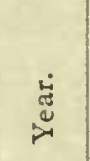 & ڤّ & 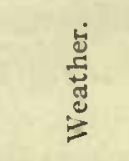 & 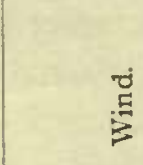 & 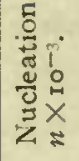 \\
\hline $19 \circ 3$ & $\begin{array}{r}\text { Dec. } 13 \\
14 \\
15 \\
16 \\
17 \\
18 \\
19 \\
20 \\
21 \\
22 \\
23 \\
24 \\
25 \\
26 \\
27 \\
28 \\
29 \\
30 \\
31 \\
\text { Jan. } \\
1 \\
2 \\
3 \\
4 \\
5 \\
6 \\
7 \\
8 \\
9 \\
10 \\
11 \\
\text { Feb. } \\
12 \\
13 \\
14 \\
15 \\
16 \\
17 \\
18 \\
19 \\
20 \\
21 \\
22 \\
23 \\
24 \\
25 \\
26 \\
27 \\
28 \\
29 \\
30 \\
\end{array}$ & 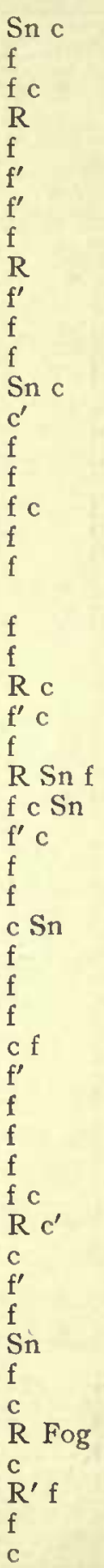 & & $\begin{array}{r}75 \\
100 \\
100 \\
36 \\
75 \\
37 \\
90 \\
45 \\
33 \\
45 \\
90 \\
90 \\
36 \\
75 \\
100 \\
100 \\
100 \\
42 \\
100 \\
\\
100 \\
100 \\
40 \\
48 \\
55 \\
75 \\
75 \\
45 \\
100 \\
100 \\
45 \\
75 \\
100 \\
90 \\
90 \\
90 \\
100 \\
100 \\
90 \\
75 \\
40 \\
45 \\
42 \\
45 \\
52 \\
45 \\
90 \\
45 \\
75 \\
75 \\
60 \\
45\end{array}$ & I 903 & $\begin{array}{r}\text { Feb. } 2 \\
3 \\
4 \\
5 \\
6 \\
7 \\
7 \\
8 \\
9 \\
10 \\
11 \\
12 \\
13 \\
14 \\
15 \\
16 \\
17 \\
18 \\
19 \\
20 \\
21 \\
22 \\
23 \\
24 \\
25 \\
26 \\
27 \\
28 \\
\text { Mar. } 1 \\
2 \\
3 \\
3 \\
4 \\
5 \\
6 \\
7 \\
8 \\
9 \\
10 \\
11 \\
12 \\
13 \\
14 \\
15 \\
16 \\
17 \\
18 \\
19 \\
20 \\
21 \\
22 \\
23 \\
24 \\
25\end{array}$ & 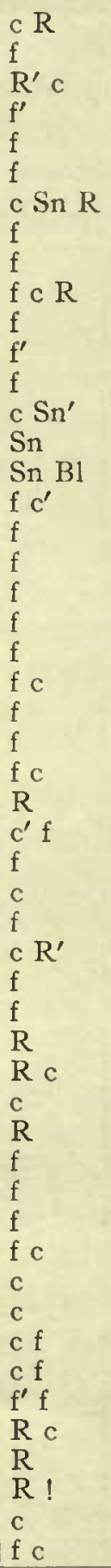 & $\begin{array}{l}\text { W. } \\
\text { E. } \\
\text { N. } \\
\text { N.E. S. } \\
\text { S.W. } \\
\text { W. }\end{array}$ & $\begin{array}{l}52 \\
45 \\
45 \\
52 \\
60 \\
90 \\
34 \\
75 \\
45 \\
45 \\
30 \\
45 \\
52 \\
45 \\
45 \\
60 \\
83 \\
92 \\
93 \\
70 \\
30 \\
60 \\
31 \\
60 \\
62 \\
39 \\
19 \\
61 \\
27 \\
31 \\
44 \\
48 \\
26 \\
20 \\
13 \\
23 \\
28 \\
32 \\
32 \\
36 \\
84 \\
22 \\
44 \\
15 \\
41 \\
36 \\
25 \\
17 \\
19 \\
23 \\
19 \\
\end{array}$ \\
\hline
\end{tabular}

* End of the approximate data. 
A CONTINUOUS RECORD OF ATMOSPHERIC NUCLEATION.

TABLE III-Continued.

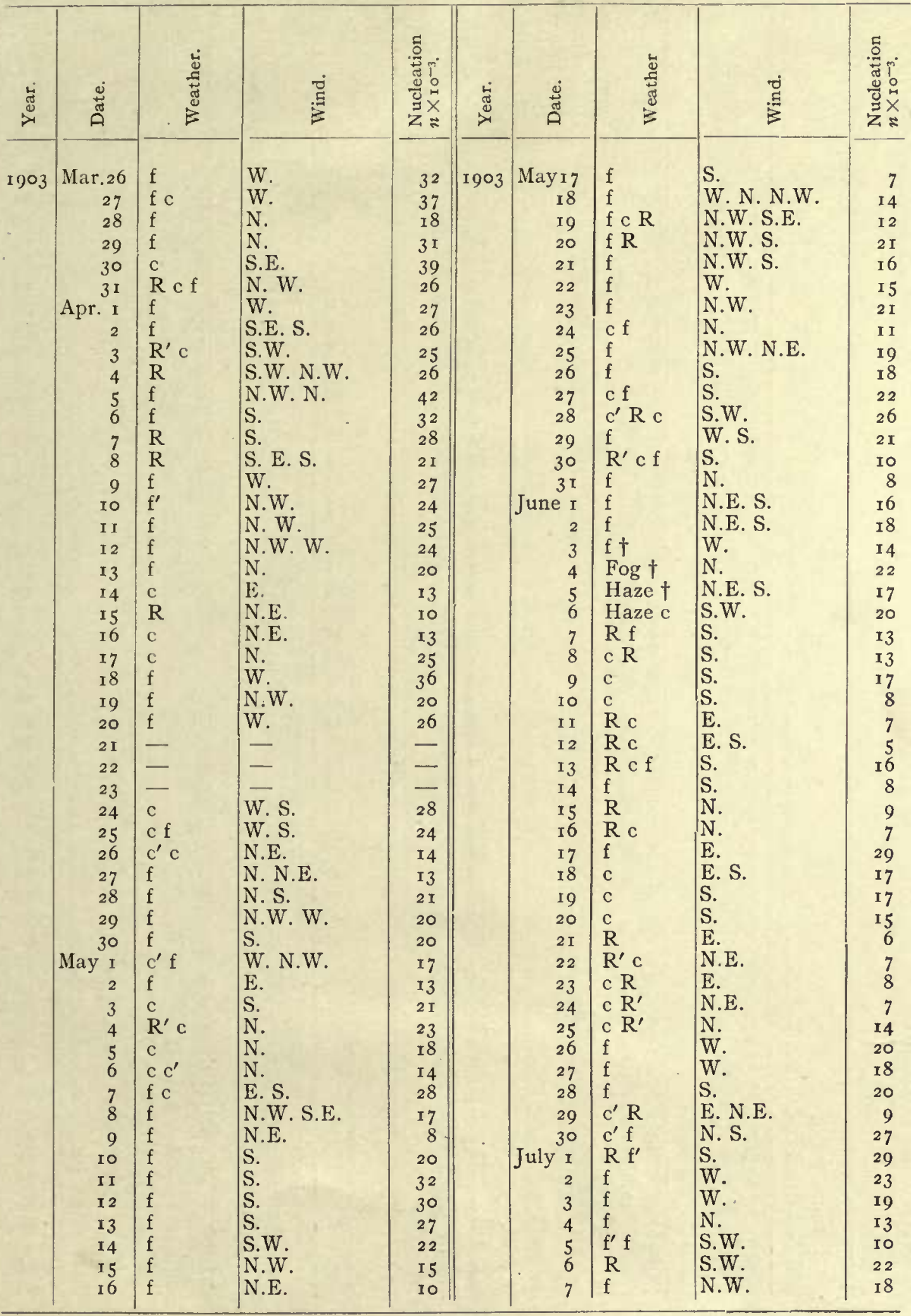

$\dagger$ Forest fires. 
TABLE III-Continued.

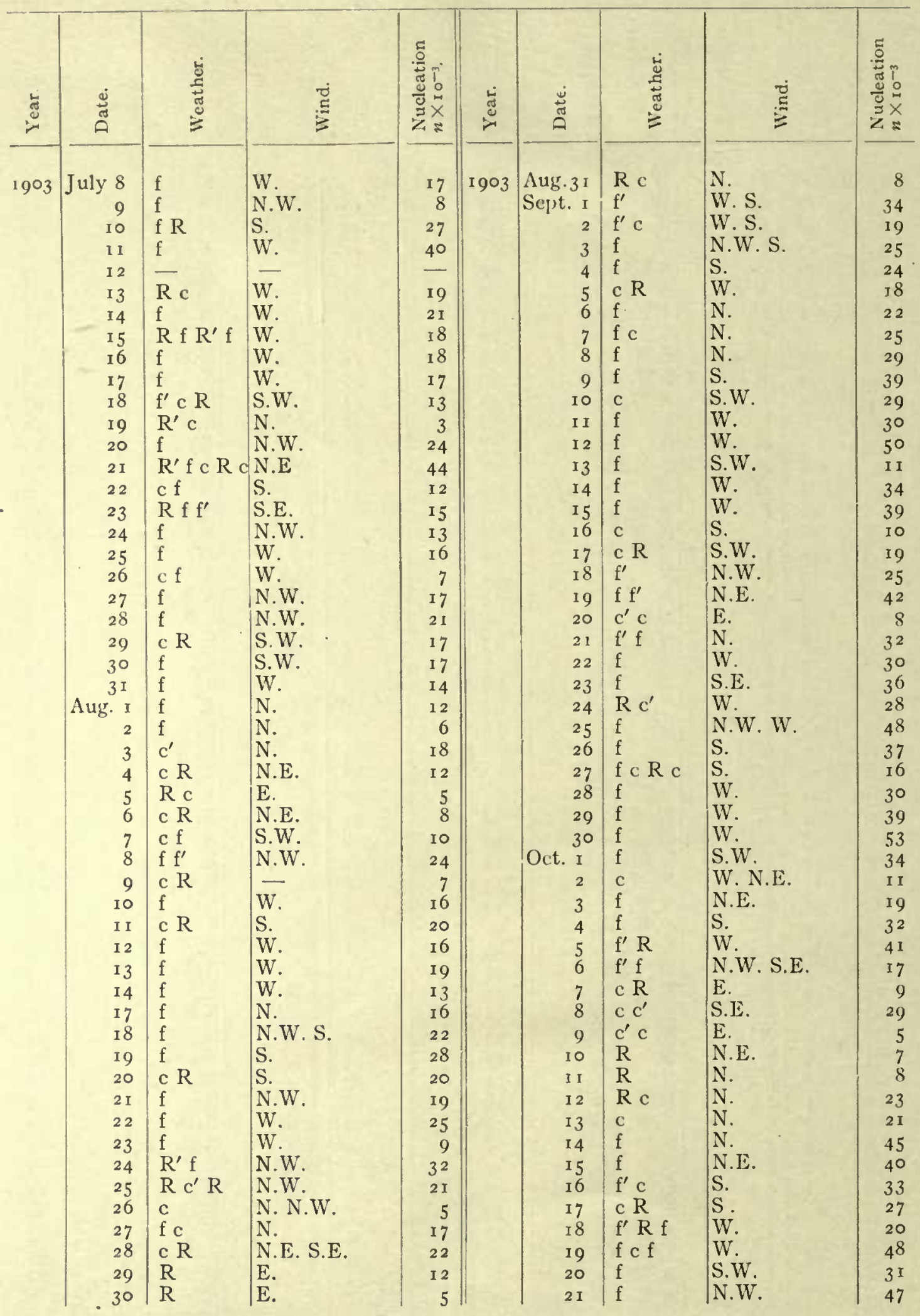


A CONTINUOUS RECORD OF ATMOSPHERIC NUCLEATION.

TABLE III-Continued.

\begin{tabular}{|c|c|c|c|c|c|c|c|c|c|}
\hline 峁 & 苞 & 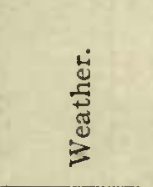 & $\stackrel{\Xi}{\Xi}$ & 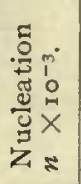 & 岕 & 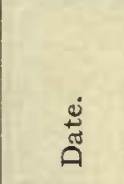 & 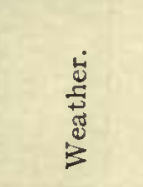 & $\stackrel{\Xi}{E}$ & 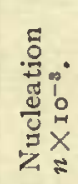 \\
\hline \multirow[t]{52}{*}{ I 903} & Oct.22 & & N.W. S. & $3^{2}$ & 1903 & Dec.13 & $\mathrm{R} \mathrm{f}$ & S.W. & 18 \\
\hline & 23 & $f^{\prime} c R$ & S.W. N.W. & 26 & & I 4 & & W. & 77 \\
\hline & 24 & c c $c^{\prime}$ & N.W. & 37 & & 15 & $c c^{\prime} f$ & W. & $5^{8}$ \\
\hline & 25 & $\mathrm{f} \mathrm{c}$ & N. & $5^{8}$ & & 16 & & W. & 64 \\
\hline & 26 & $\mathrm{f}^{\prime}$ & W. & 39 & & 17 & $\mathrm{f}$ & W. & 80 \\
\hline & 27 & $\mathrm{f}$ & W. & 46 & & 18 & $\mathrm{f}$ & N.W. & 86 \\
\hline & 28 & $f$ & N.W. & 59 & & 19 & $\mathrm{f}$ & W. & 82 \\
\hline & 29 & f & W. & $5 I$ & & 20 & $\mathrm{R}$ & $\bar{w}$ & 35 \\
\hline & 30 & $\mathrm{f}$ & W. & 35 & & 21 & & W. & 39 \\
\hline & 31 & $\mathrm{f}$ & W. & 36 & & 22 & $\mathrm{R}$ c f & W. & 44 \\
\hline & Nov. 1 & $\mathrm{f}$ & W. S.W. & 32 & & 23 & & S.W. & $8 I$ \\
\hline & 2 & f & IV. & 48 & & 24 & f c & S.W. & 73 \\
\hline & 3 & $\mathrm{f}$ & W. & 43 & & 25 & & W. & 31 \\
\hline & 4 & & W. & $3^{8}$ & & 26 & $c \operatorname{Sn} c$ & N.W. & $5^{2}$ \\
\hline & 5 & $R_{c}$ & S.W. & $3 I$ & & 27 & $\mathrm{c} S \mathrm{Sn}$ & S.W. & 63 \\
\hline & 6 & c Sn & N. & 35 & & 28 & & N.W. & 76 \\
\hline & 7 & & W. & $4 I$ & & 29 & c Sn & S.E. & 54 \\
\hline & 8 & $\mathrm{f}$ & W. & 37 & & 30 & f & N.W. W. & 69 \\
\hline & 9 & $\mathrm{f}$ & W. & 59 & & 31 & f & W. & 72 \\
\hline & 10 & $\mathrm{f} \mathrm{c} \mathrm{f}$ & S.W. & 42 & & & & & \\
\hline & I I & & N.W. N.E. & 19 & 1904 & Jan. $I$ & f & W. & $4^{8}$ \\
\hline & 12 & $f^{\prime} f$ & W. & 42 & & 2 & c. $\mathrm{Sn}$ & N. & 79 \\
\hline & I 3 & f & S.W. & 49 & & 3 & f & N. & 100 \\
\hline & 14 & $c f$ & N.W. W. & 43 & & 4 & f & W. & 100 \\
\hline & 15 & f & W. & 43 & & 5 & $\mathrm{f}$ & W. N.W. & 100 \\
\hline & 16 & c R & N.W. N.E. & 30 & & 6 & f & S. S.W. & 97 \\
\hline & 17 & $\mathrm{R}$ & N.W. & 43 & & 7 & f Fog & W. S. & 68 \\
\hline & 18 & $c f$ & N.IV. & 28 & & 8 & c $\mathrm{Sn}$ & N. & 67 \\
\hline & 19 & $\mathrm{f}$ & N.W. & 46 & & 9 & $S n f^{\prime} S n$ & N.W. & 32 \\
\hline & 20 & f & N.W. N. & 50 & & 10 & & W. & $4 \mathrm{I}$ \\
\hline & 21 & f & N. & $3^{8}$ & & 11 & f & N.W. & 63 \\
\hline & 22 & c & N. & 37 & & 12 & $\mathrm{f} \mathrm{c} \mathrm{f}$ & N. & 75 \\
\hline & 23 & c $\mathrm{R}$ & N. S. & 46 & & 13 & c R & N.E. E. & 26 \\
\hline & 24 & $f c^{\prime} c$ & W. & 30 & & 14 & $\mathrm{f}^{\prime}$ & S.W. & 66 \\
\hline & 25 & & W. & 43 & & 15 & & W. & $5^{8}$ \\
\hline & 26 & $\mathrm{f}$ & N. & 63 & & 16 & $f^{\prime} \mathrm{Sn} R$ & S.W. S. & $4^{6}$ \\
\hline & 27 & f $\mathrm{c}$ & N. & 70 & & 17 & & W. & 37 \\
\hline & 28 & & N. W. & 54 & & 18 & $f$ & N.W. & 100 \\
\hline & 29 & c Sn & N. & $3^{6}$ & & 19 & $f$ & N.W. & 100 \\
\hline & 30 & & W. & $5^{2}$ & & 20 & $\mathrm{Sn} \mathrm{c}$ & N. S.W. & IOO \\
\hline & Dec. I & & N.W. & 43 & & $2 I$ & $\mathrm{c} \mathrm{Sn}$ & N.E. & $4 I$ \\
\hline & 2 & c Sn $R^{\prime} c$ & N. & $6 I$ & & 22 & $\mathrm{R}$ & N.E. & 30 \\
\hline & 3 & c R c & N. & 63 & & 23 & Fog & S. S.W.W. & 59 \\
\hline & 4 & $\mathrm{c} \mathrm{f}$ & N. & 55 & & 24 & f & W. & 38 \\
\hline & 5 & $f^{\prime} c$ & W. & 66 & & 25 & $\mathrm{f}$ & W. & $9 \mathrm{I}$ \\
\hline & 6 & f & W. & 39 & & 26 & f c & N. E. & 35 \\
\hline & 7 & $\mathrm{f}$ & W. & 71 & & 27 & & W. & 71 \\
\hline & 8 & & W. & 42 & & 28 & $\mathrm{f} c \mathrm{c}^{\prime}$ & W. & $8 \mathrm{I}$ \\
\hline & 9) & c $R^{\prime}$ & N. N.E. & 25 & & 29 & $\mathrm{Sn}$ & N. & 91 \\
\hline & 10 & f & W. & 45 & & 30 & & N. & 69 \\
\hline & I I & $\mathrm{f}$ & W. & 59 & & $3 I$ & $f c^{\prime}$ & S. & 46 \\
\hline & 12 & $\mathrm{f} \mathrm{c}$ & W. & 69 & & Feb. I & $\mathrm{c} S n^{\prime} \mathrm{c}$ & N.W. W. & $4^{8}$ \\
\hline
\end{tabular}


TABLE III-Continued.

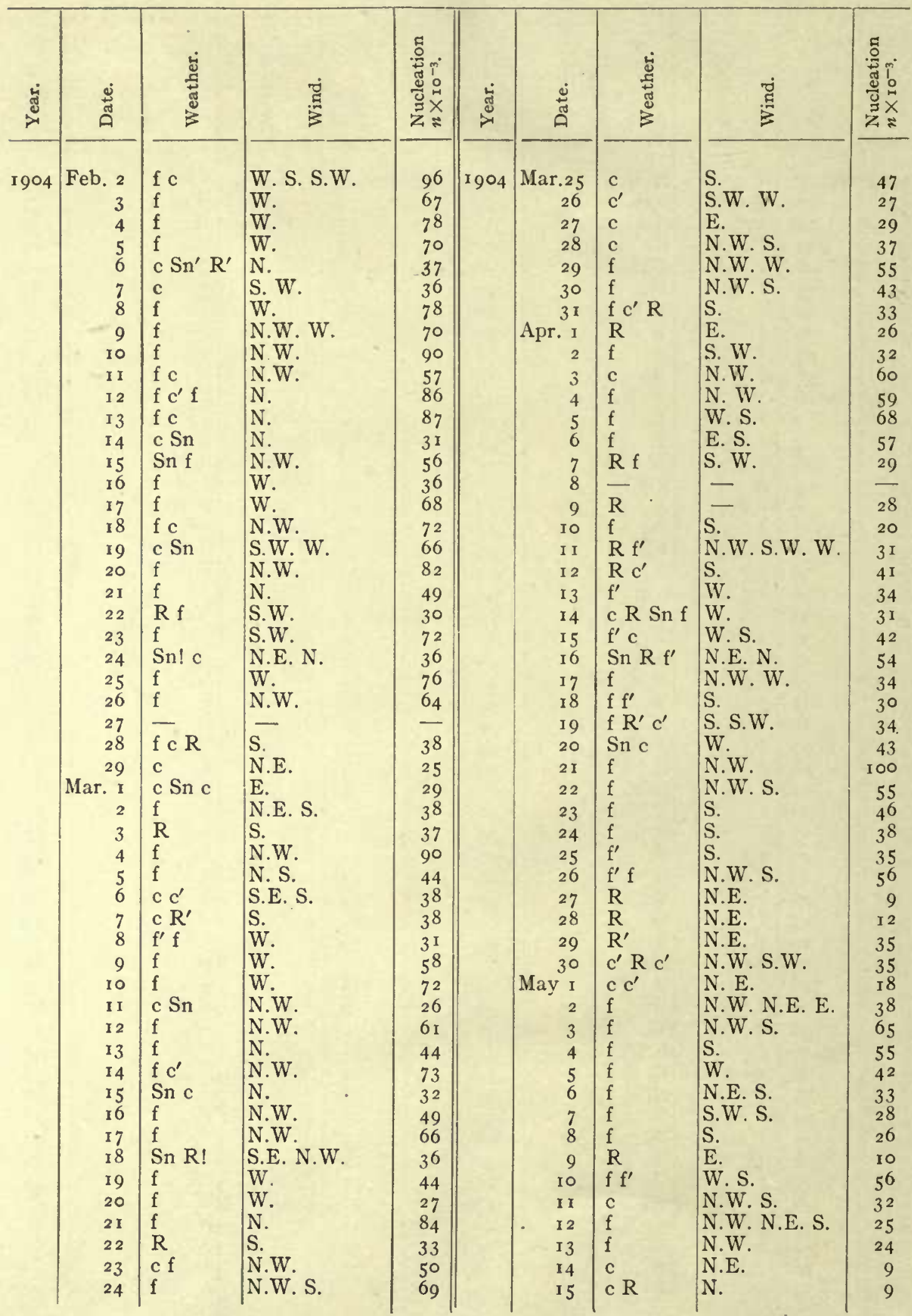


TABLE III-Continued.

\begin{tabular}{|c|c|c|c|c|c|c|c|c|c|}
\hline 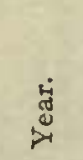 & ڤં & 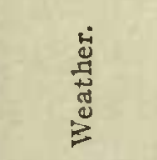 & 节 & 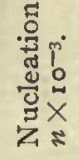 & 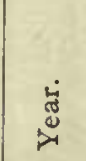 & ڤึ & 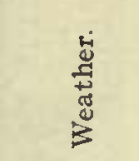 & $\stackrel{\vec{\Xi}}{\Xi}$ & 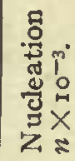 \\
\hline \multirow[t]{52}{*}{1904} & Mаух 6 & $f^{\prime} R^{\prime} f$ & W. & 36 & \multirow[t]{52}{*}{ I9०4 } & Julyig & $\mathrm{f}$ & & 16 \\
\hline & 17 & c & S.W. S. & 39 & & & $\mathrm{f}$ & N.W.W. & 25 \\
\hline & 18 & $c R^{\prime} c$ & N.E. & 8 & & 21 & f & & 25 \\
\hline & 19 & c R & & $2 \mathrm{I}$ & & 22 & $f^{\prime} R$ & & 8 \\
\hline & 20 & c R f & S.W. W. S. & 32 & & 23 & $\mathrm{R} \mathrm{c}$ & N.E. N. & 4 \\
\hline & $2 \mathrm{I}$ & & N.W. S. & 44 & & 24 & & & 2.5 \\
\hline & 22 & $\mathrm{f}$ & S. & 16 & & 25 & $\mathrm{cf}$ & & 19 \\
\hline & 23 & $\mathrm{f}$ & S. & 34 & & 26 & $f^{\prime} f$ & S. & 37 \\
\hline & 24 & f & S.W. & 29 & & 27 & $R^{\prime} f$ & W. & 37 \\
\hline & 25 & f & N.W. & 29 & & 28 & f $c$ & S. & 23 \\
\hline & 26 & & S. & 25 & & 29 & $c \mathrm{f}$ & W. & 28 \\
\hline & 27 & $\mathrm{f}^{\prime} \mathrm{R} \mathrm{f}^{\prime}$ & S.W. & 35 & & 30 & f & N. & $4^{2}$ \\
\hline & 28 & f & W. & 49 & & $3 \mathrm{I}$ & $f^{\prime} f$ & S.W. & 8 \\
\hline & 29 & $\mathrm{f}$ & S. & $3^{8}$ & & Aug. I & c & S.W. & 22 \\
\hline & 30 & c & S.W. & 16 & & 2 & $\mathrm{R}$ & & I5 \\
\hline & $3 x$ & $\mathrm{f}^{\prime}$ & N.E. & 22 & & 3 & f c & & 15 \\
\hline & June I & c R & N.E. & 16 & & 4 & & N. S. & 8 \\
\hline & 2 & c & N. & 9 & & 5 & $f^{\prime} c$ & & 14 \\
\hline & 3 & $c c^{\prime}$ & N.E. & 22 & & 6 & $(R) c f$ & & 18 \\
\hline & 4 & & N.E. S.W. & I9 & & 7 & & & I 2 \\
\hline & 5 & & S. & 22 & & 8 & $\mathrm{R} \mathrm{c}$ & S.E. S. W. & 18 \\
\hline & 6 & c (R) & N.E. & 6 & & 9 & & N.S. & 46 \\
\hline & 7 & $c \mathrm{R} f(\mathrm{R})$ & N. S.W. & 22 & & IO & $c R^{\prime} \mathrm{c}$ & S.E. E. & 23 \\
\hline & 8 & c f & S.E. E. & 5 & & I I & (R) $\mathrm{c} \mathrm{c}^{\prime}$ & W. & $? 3^{2}$ \\
\hline & 9 & $R^{\prime} f^{\prime}$ & N.E. & 25 & & 26 & & W. N.W. & 33 \\
\hline & 10 & - & - & - & & 27 & $f$ & & 30 \\
\hline & II & $\mathrm{f}$ & N. N.E. & I 2 & & 28 & $f$ & W. S. & 10 \\
\hline & J 2 & $\mathrm{f}$ & & I 4 & & 29 & $\mathrm{f}^{\prime}$ & W. N.W. & 25 \\
\hline & I 3 & $f^{\prime}$ & N.E. & 23 & & 30 & $\mathrm{f}$ & & $2 \mathrm{I}$ \\
\hline & $\mathrm{J} 4$ & f & S. & 49 & & $3 \mathrm{I}$ & f & W. S.W. & 43 \\
\hline & 15 & f & S. & 22 & & Sept. I & $f^{\prime}$ & & 27 \\
\hline & I 6 & $f^{\prime} R$ & S.W. & $6 x$ & & 2 & $\mathrm{cf}$ & S.W. W. S. & 28 \\
\hline & 17 & f & N.E. & 26 & & 3 & $\mathrm{f}$ & & 30 \\
\hline & 18 & f & W. & 25 & & 4 & $c R^{\prime} f$ & N. W. & $2 \mathrm{I}$ \\
\hline & I9 & $\mathrm{f}$ & N. & 65 & & 5 & $\mathrm{f}^{\prime} \mathrm{f}$ & N.W.W. & 30 \\
\hline & 20 & f & S. & 46 & & 6 & & N.E. S.W. S. & 35 \\
\hline & $2 \mathrm{I}$ & $\mathrm{f}^{\prime} \mathrm{f}$ & S.W. & $3 \mathrm{I}$ & & 7 & & W. S. & $3 \mathrm{I}$ \\
\hline & 22 & $f R f$ & W. & 28 & & 8 & f...R & W. S.W. & 35 \\
\hline & 23 & & N.E. & 27 & & 9 & c R & N.E. & 8 \\
\hline & 24 & & W. & 38 & & Io & $\mathrm{cf}$ & N. & 17 \\
\hline & 25 & f $(\mathrm{R})$ & S.W. & 27 & & I I & $f^{\prime} \ldots R$ & N. S. & 26 \\
\hline & 26 & $f R f$ & W: & 25 & & I 2 & & W. S.W. & 22 \\
\hline & 27 & f & N.W. & 32 & & 13 & $\mathbf{f}^{\prime}$ & E. & Io \\
\hline & 28 & $c^{\prime} c$ & & 23 & & - & - & - & 一 \\
\hline & 29 & $\mathrm{R} c$ & S.E. N. & I 5 & & 27 & f c & N.E. & $x 9$ \\
\hline & 30 & c R & & 29 & & 28 & & N.E. & 26 \\
\hline & July I & $\mathrm{R} c$ & S. S.W. & 25 & & 29 & f c ...R & & 34 \\
\hline & 2 & f & S.W. & 38 & & & f $\mathrm{Rf}$ & S.W.W. N.W. & $3 I$ \\
\hline & I 5 & f & & 29 & & Oct. I & & W. & 33 \\
\hline & 16 & $\mathrm{cf}$ & S. & 23 & & 2 & $\mathrm{c} \mathrm{c}^{\prime}$ & W. & 34 \\
\hline & 77 & $\mathrm{f}^{\prime}$ & N. & 5 & & 3 & $\mathrm{f}$ & N.W. & 35 \\
\hline & 18 & $\mathrm{f}$ & N. & 46 & & 4 & $\mathrm{f}$ & N. & 53 \\
\hline
\end{tabular}


TABLE III-Continued.

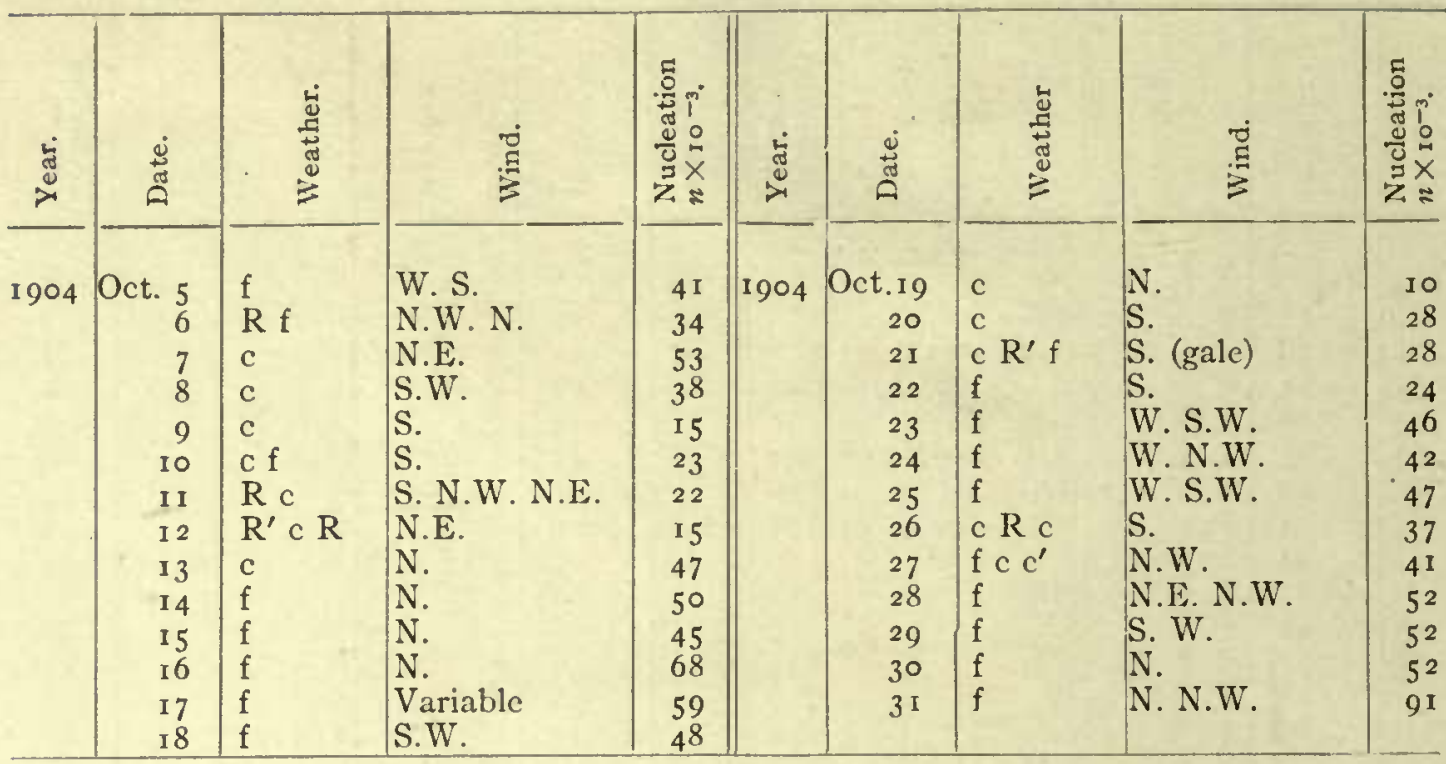

These data have been constructed in the charts 49 and 50, with the two years of observation overlying the same abscissas to bring out the contrasts. The series for October, I902-Octōber, I903, are easily distinguished from the later series, October, 1903-October, I904, by the continuous curves. The nucleations are given in thousands $\left(n^{-r / 3}\right)$. Clear, partly cloudy, and cloudy weather are indicated on the curves by the usual Weather Bureau symbols$\odot, \mathbf{O}$, - Rain is shown by $r$.

Apart from details, the striking difference of time changes of nucleation, I $902-03$ and $1903^{-04}$, are apparent. In both cases the high winter nucleations as compared with the summer nucleations are the essential feature; but in 1903 these nucleations fall off almost suddenly and permanently in March, whereas the change in 1904 beyond March is much more gradual.

36. Mean monthly nucleations.-The character of these secular changes will, however, appear much more elearly in the monthly averages given in Table IV.

TABLE IV.

Mean Monthly Nucleations from October, 1902. to October, igo4.

\begin{tabular}{|c|c|c|c|c|c|c|c|c|}
\hline Year. & Month. & $n \times 10^{-3}$ & Year. & Month. & $n \times 10^{-3}$ & Year. & Month. & $n \times 10^{-3}$. \\
\hline 1902 & $\begin{array}{l}\text { Oct. } \\
\text { Nov. } \\
\text { Dec. }\end{array}$ & $\begin{array}{l}19.9 \\
35.8 \\
69.4\end{array}$ & 1903 & $\begin{array}{l}\text { Jan. } \\
\text { Feb. } \\
\text { Mar. } \\
\text { April } \\
\text { May } \\
\text { June } \\
\text { July } \\
\text { Aug. } \\
\text { Sept. } \\
\text { Oct. } \\
\text { Nov. } \\
\text { Dec. }\end{array}$ & $\begin{array}{l}71.2 \\
55.0 \\
31.2 \\
23.3 \\
17.9 \\
14.1 \\
18.4 \\
15.4 \\
29.4 \\
30.8 \\
42.3 \\
57.8\end{array}$ & 1904 & $\begin{array}{l}\text { Jan. } \\
\text { Feb. } \\
\text { Mar. } \\
\text { April } \\
\text { May } \\
\text { June } \\
\text { July } \\
\text { Aug. } \\
\text { Sept. } \\
\text { Oct. }\end{array}$ & $\begin{array}{l}66.3 \\
60.8 \\
46.5 \\
41.5 \\
30.4 \\
26.3 \\
23.2 \\
22.6 \\
25.3 \\
40.8\end{array}$ \\
\hline
\end{tabular}




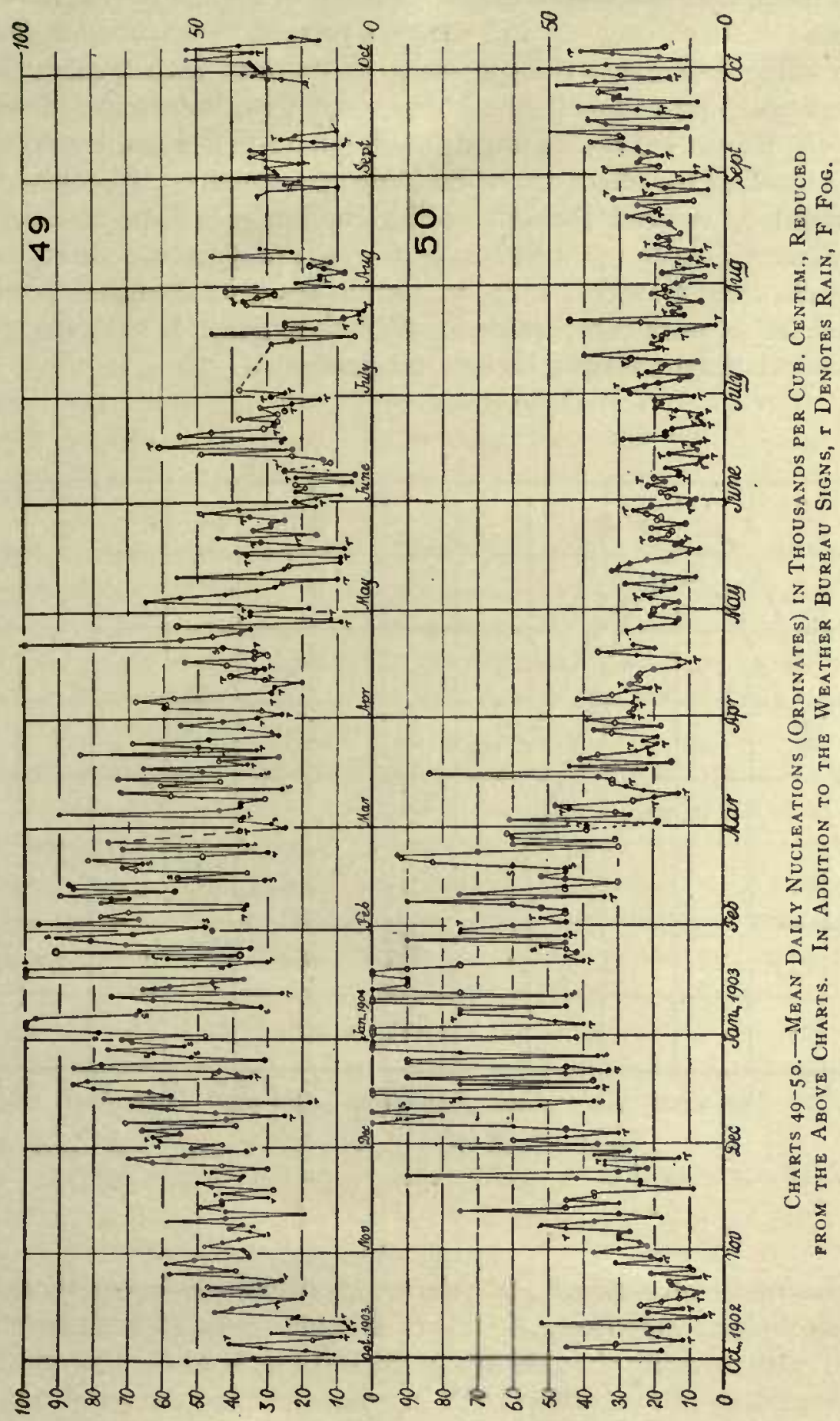


These data have been plotted in chart $5 \mathrm{I}$, the nucleations ( $n$ per eubic centimeter) being again laid off in thousands. Both the similarity and the divergences of the results of $\mathrm{I}_{902-0}$ and $1903^{-0} 4$ become apparent at a glance. It has been possible to connect the successive mean data with a curve almost at once without resorting to much smoothing, exeept in the summer observations of I903, where the dotted line is drawn to eliminate the probable and excessive rain effect, as well as the effect due to building operations on the college campus, etc. It should be noticed that the new data for Igo4 (August, September, October) fall very closely upon the curve for 1903 prolonged. As stated above, $\S 3$, the results from October, I902, to March, I 903 , are reduced from the old scale and are not at once comparable in absolute magnitude with the remaining data, but the relations are nevertheless well indicated.

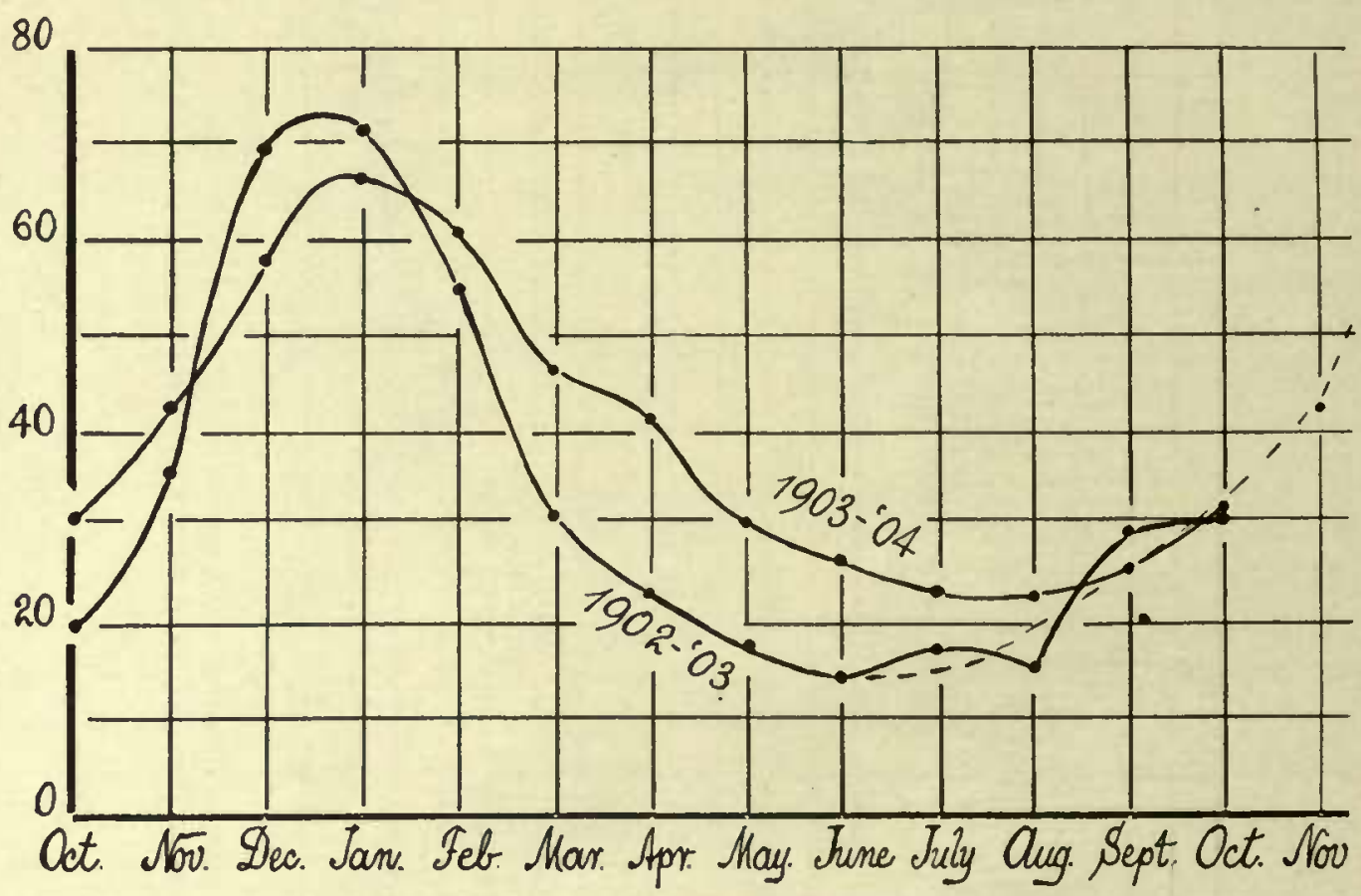

Chart 51.-Mean Monthly Nucleations (Ordinates) from October, 1902, to October, igo4, in Thousands per Cub. Centim.

37. Occurrence of maxima and minima of nucleation during the winter and the summer solstices, respectively.-The remarkable result of both curves is unmistakable: remembering that the mean nucleations hold for the middle of the month, the maxima of nucleation both in I902 and I903 occurred between the middle of December and the middle of January, nearer the latter; in other words, about at the time of the winter solstice. The minima for summer occur between the middle of June and the middle of August somewhat after the summer solstice. The winter maximum is in both cases sharply indicated and 
its coincidence with December 22 not improbable. The summer minimum is much more prolonged and uncertain, a result to be anticipated from the marked rain effect which prevails at this season.

The identification of maxima with the winter solstice and of minima with the summer solstice is alluring: for in these cases the earth is respectively nearest and farthest from the sun. At the same time the orbital velocities are respectively greatest and least, so that the path volume of the earth would have corresponding values. Both causes are qualitatively in harmony with the observed results, if the nucleation comes in great part from the sun. Quantitatively the results are less convincing.

If the data for $1903^{-04}$ (which are more nearly absolute) be taken, the ratio of greatest and least nucleation is about 3 , seeing that December 22 corresponds to about $n=66000$ and June 22 to $n=22000$. The case of $1902-03$ is even more accentuated. On the other hand, the greatest and least values of the radius vectur of the earth's orbit are 1.0168 and .9832 , in terms of the mean radius. The same effect, approximately, is attributable to the differences in velocity, making only about $6.7 \%$ by which the winter nucleation should exceed the summer nucleation, for the case of linear distribution. No easily discernible distribution of nuclei from the sun outward would account for the three- to four-fold winter nucleation as compared with the summer nucleation, seeing that the decrement of about $2 \%$ of distance corresponds to an increment of $100 \%$ of nucleation. Thus the reasonable surmise which makes the density of the solar output vary as the inverse square of radius, while the path volume per unit of time varies as the inverse radius, gives a compound law of the inverse cube of radius, which, however, is quite inadequate.

Unless some occult law of distribution is at the root of these cases, mere change of distance and velocity does not account for the facts. On the other hand, if the influence of the sun is atmospheric and productive of dissipation of nuclei (by breaking them into fragments beyond the lower limit of nuclear size, for instance), or if there is greater tendency toward supersaturation of the atmosphere when the nights are longest with the consequent precipitation of local nuclei, the effect due to greater length of days in the summer as compared with the winter will much more nearly correspond to the nucleations observed. The effect postulated is, however, wholly conjectural.

38. Conclusion.- On the other hand, the two graphs showing the distribution of mean monthly nucleation are not compatible with the postulate of a purely local or incidental origin of the nuclei. Rains in general would make the winter nucleation higher than the summer nucleation, while the products of combustion still further increase the former. But neither of these incidental effects is so distributed as to give rise to the salient maximum in Decenber, reproduced independently and sharply by both curves.

Briefly, then, while local and incidental effects enormously modify the seasonal variation of atmospheric nucleation, it is not improbable that 
its origin is in part to be traced to a more deep-seated cause. At least the results obtained are sufficient to warrant the extension of similar experiments over a wide area of territory, including regions remote from the habitations of man-researches for which I am now making extensive preparation. 



\section{5}




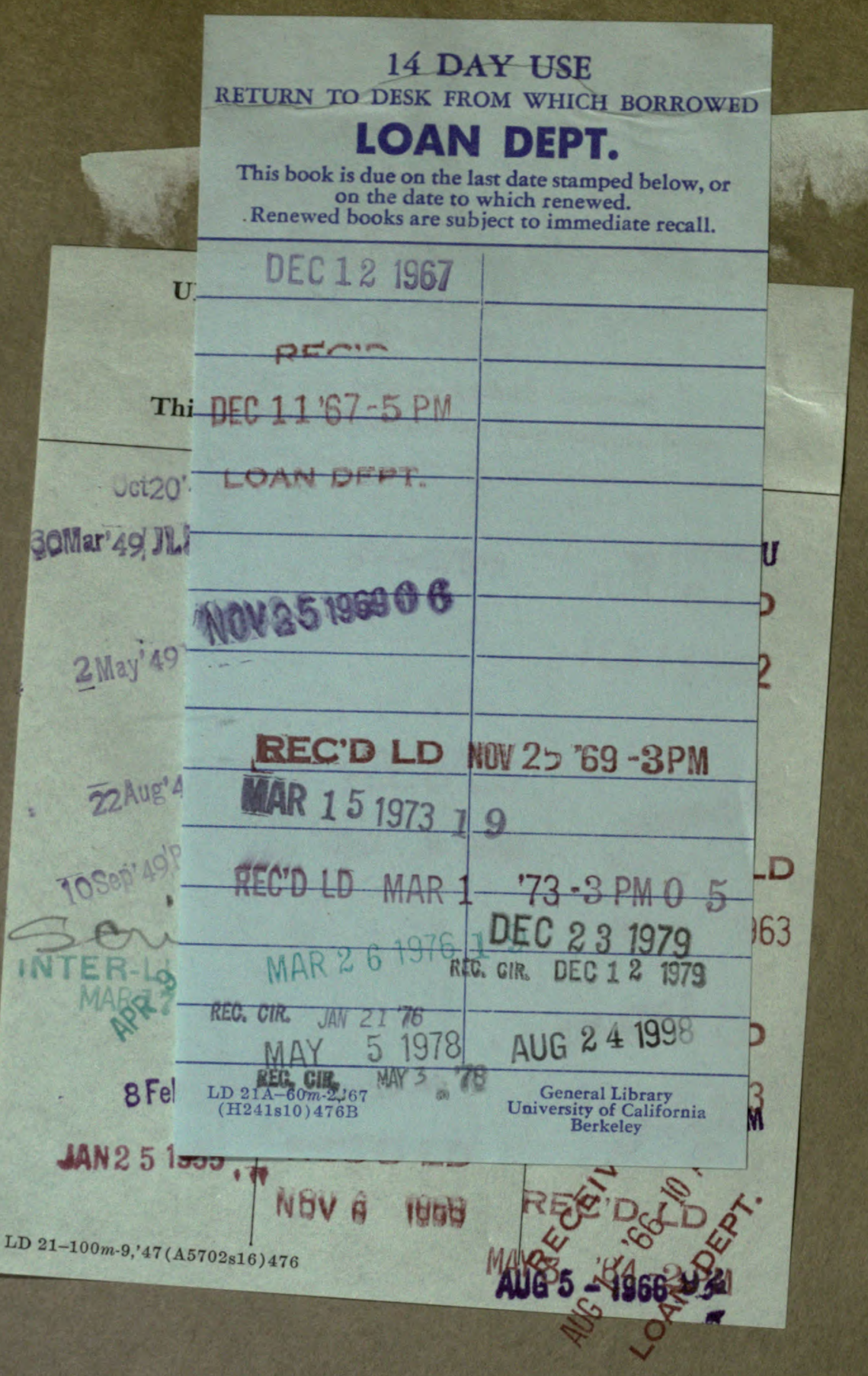


U. C. BERKELEY LIBRARIES

|| ||| || || || ||| |||||||||||||||||||||||||| 
1)

1. 